\title{
Modeling of heat transfer and reactive chemistry for particles in gas-solid flow utilizing continuum-discrete methodology (CDM)
}

Jordan M. H. Musser

West Virginia University

Follow this and additional works at: https://researchrepository.wvu.edu/etd

\section{Recommended Citation}

Musser, Jordan M. H., "Modeling of heat transfer and reactive chemistry for particles in gas-solid flow utilizing continuum-discrete methodology (CDM)" (2011). Graduate Theses, Dissertations, and Problem Reports. 4760.

https://researchrepository.wvu.edu/etd/4760

This Dissertation is protected by copyright and/or related rights. It has been brought to you by the The Research Repository @ WVU with permission from the rights-holder(s). You are free to use this Dissertation in any way that is permitted by the copyright and related rights legislation that applies to your use. For other uses you must obtain permission from the rights-holder(s) directly, unless additional rights are indicated by a Creative Commons license in the record and/ or on the work itself. This Dissertation has been accepted for inclusion in WVU Graduate Theses, Dissertations, and Problem Reports collection by an authorized administrator of The Research Repository @ WVU.

For more information, please contact researchrepository@mail.wvu.edu. 
Modeling of heat transfer and reactive chemistry for particles in gas-solid flow utilizing continuum-discrete methodology (CDM)

\author{
Jordan M. H. Musser
}

Dissertation submitted to the Eberly College of Arts and Sciences at West Virginia University in partial fulfillment of the requirements

for the degree of

Doctor of Philosophy

in

Mathematics

Approved by

\author{
Mary Ann Clarke, Ph.D., Committee Chairperson \\ Sherman Riemenschneider, Ph.D. \\ Ian Christie, Ph.D. \\ Edgar Fuller, Ph.D. \\ Janine Galvin, Ph.D.
}

Department of Mathematics

Morgantown, West Virginia

2011

Keywords: discrete element method, particle-scale modeling, gas-solids systems, particle heat transfer

Copyright: 2011 Jordan M. H. Musser 


\section{Abstract \\ Modeling of heat transfer and reactive chemistry for particles in gas-solid flow utilizing continuum-discrete methodology (CDM) by Jordan M. H. Musser}

A comprehensive multi-phase flow model requires coupled hydrodynamics, boundary conditions, heat and mass transfer, and chemical reaction kinetics. A model must also capture the multi-scale nature of these problems. Computational fluid dynamics-discrete element method (CFD-DEM) provides an accurate description of chemical reactions and heat and mass transfer at the particle scale. Currently, MFIXDEM, the existing CFD-DEM used as the foundation for this work, can only model coupled hydrodynamics. This dissertation extends the functionality of MFIX-DEM by addressing the remaining deficiencies in three separate efforts.

The first effort outlined in this dissertation focuses on the algorithmic development of discrete mass inflow and outflow boundary conditions. This approach allows for the construction of more dynamic models of gas-solid systems. It permits the amount and type of particles to fluctuate during a simulation. Examples illustrating the added functionality are provided.

The second investigation explores the three modes of heat transfer in gas-solids systems. Models for particle-particle contact conduction, particle-fluid-particle conduction, particle-gas convection, and particle-particle radiation are selected. Model selection is based on model simplicity, acceptance in existing CFD-DEM heat transfer models, extendibility to particles of different sizes, and computational expense. Modifications are made to selected models before implementing them into MFIX-DEM. The implementation of each model is verified for simple two particle test cases, or in the case of gas-particle convection, a single fixed particle in a flowing gas. Strong agreement is observed between the simulation data and the analytic or numerical solution.

Finally, a mathematical interface for managing user-defined particle-gas chemical reactions is developed. The shrinking, unreacted core model is selected as the particle reaction model for its accurate physical account of particle-gas reactions and ability to allow particles to initially contain inert material. The implementation of the reactive chemistry interface is verified for a single reacting particle. Strong agreement is observed between simulation data and the analytic solutions for the particle's mass, species mass fraction, and internal energy equations. Agreement between the simulation data and analytic solution for the shrinking, unreacted core is considered acceptable. 


\section{Acknowledgements}

I would first like to thank those responsible for funding this project. This was provided by the National Energy Technology Laboratory (NETL) Research Participation Program sponsored by the U.S. Department of Energy and administered by the Oak Ridge Institute for Science and Education (ORISE). Without this contribution, this project would not have been possible.

Collaborative support was provided by the developers of MFIX including (in alphabetical order) Sofian Benyahia, Janine Carney, Jeff Dietiker, Rahul Garg, Aytekin Gel, Chris Guenther, Tingwen Li, Philip Nicoletti, Tom O’Brien, Mehrdad Shahnam, and Madhava Syamlal. I want to especially thank Janine Carney for all of her support and insight throughout this entire project. Without her guidance and knowledge, this work would have been insurmountable.

Many thanks go to my advisor Mary Ann Clarke. Although she may be the Empress of Evil, Destroyer of Worlds to some (namely undergraduates), she has been and will continue to be, a positive influence in my life. I am greatly appreciative of the support and friendship Mary Ann and Michael have provided me over the years.

I would finally like to thank my friends and family. Thank you for not locking me up in the 'nut barn' after 10+ years of college. Joe Andria also deserves special recognition. Thank you for the countless opportunities to rant and rave about trivial details in my course work and research. Thank you for all the clean laundry, tasty dinners, and fun times we've shared over the year. You and your friendship is greatly appreciated even though I may not say it enough. 


\section{Table of Contents}

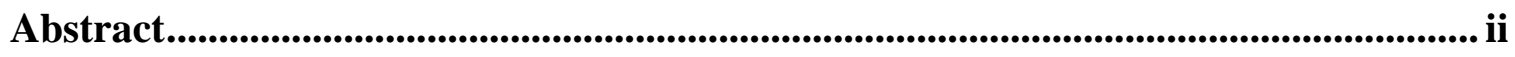

Acknowledgements .....................................................................................................ii

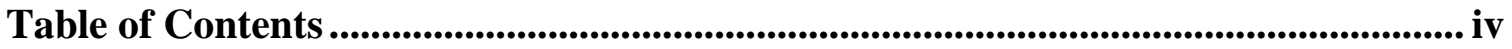

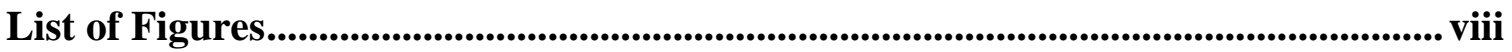

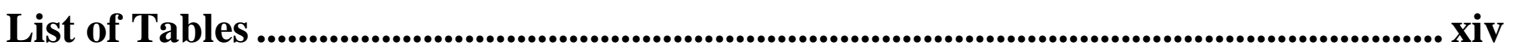

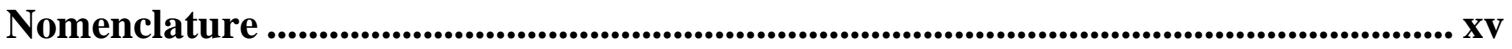

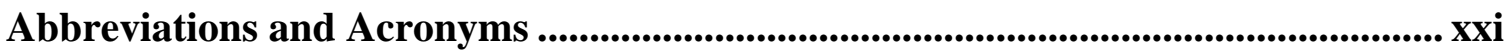

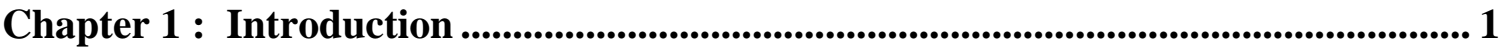

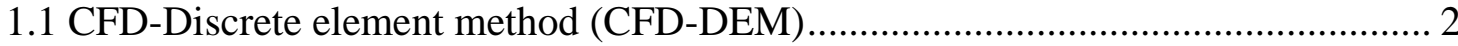

1.1.1 Gas phase mathematical model................................................................. 3

1.1.1.1 Gas phase conservation of mass (continuity equation) ........................... 4

1.1.1.2 Gas phase conservation of momentum .............................................. 5

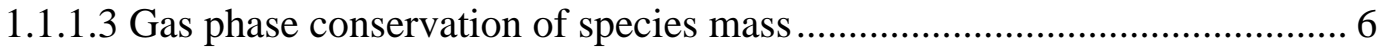

1.1.1.4 Gas phase conservation of internal energy .......................................... 7

1.1.1.5 Gas phase equations of state ........................................................ 8

1.1.2 Solids phase mathematical model ............................................................. 8

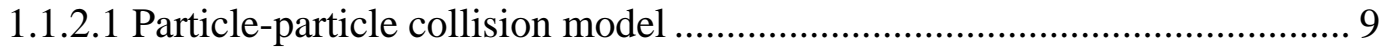

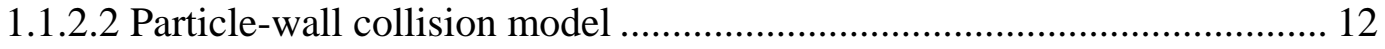

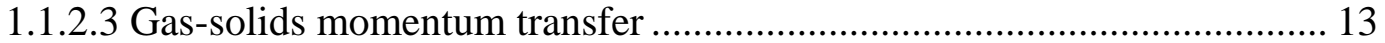

1.2 Dissertation objectives .............................................................................. 14

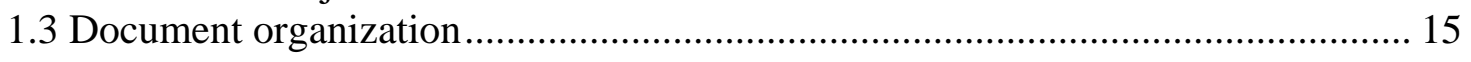

Chapter 2 : Discrete mass boundary conditions ..................................................... 16

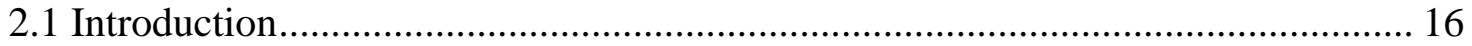

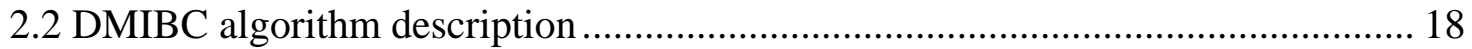

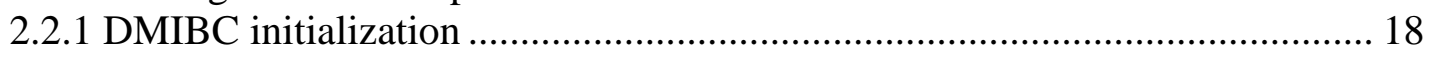

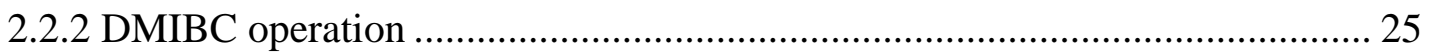

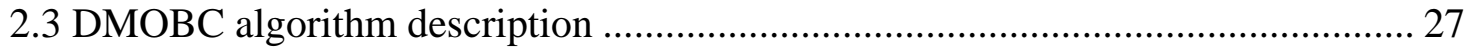

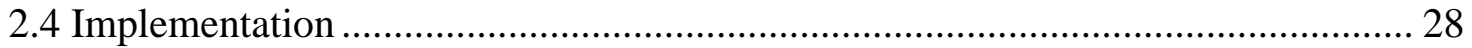

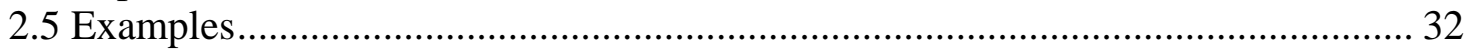

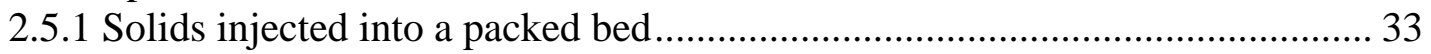

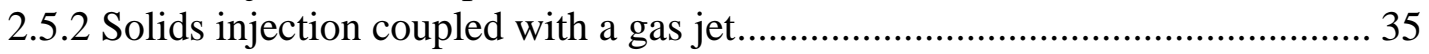

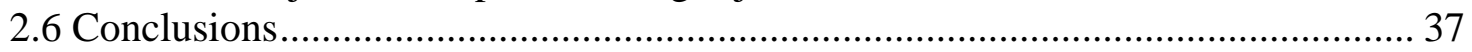

Chapter 3 : Particle heat transfer.......................................................................................... 38

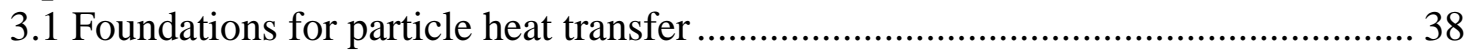

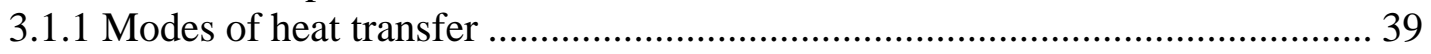

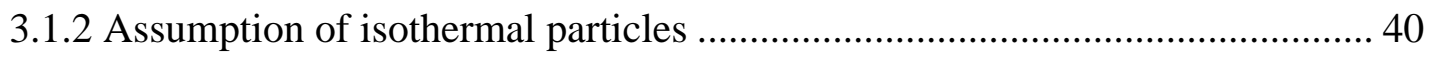

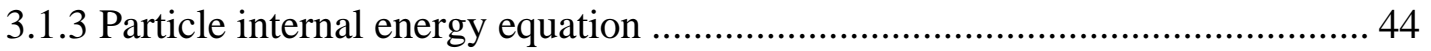

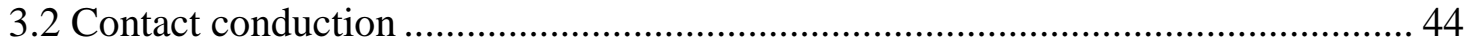

3.2.1 Survey of particle-particle contact conduction models................................ 45

3.2.1.1 Sun and Chen [49] (1988) ............................................................ 45 
3.2.1.2 Shimizu [51] (2006) ...................................................................... 49

3.2.1.3 Batchelor and O'Brien [52] (1977) ....................................................... 53

3.2.2 Modified Batchelor and O'Brien [52] two-particle contact conduction model55

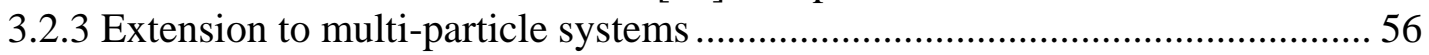

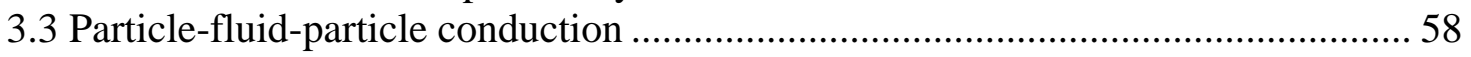

3.3.1 Survey of particle-fluid-particle conduction models ...................................... 58

3.3.1.1 Wen and Chang [63] (1967) ............................................................... 58

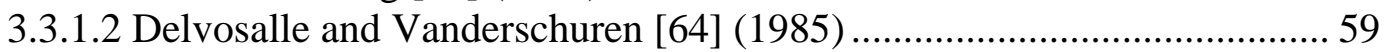

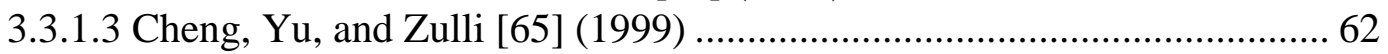

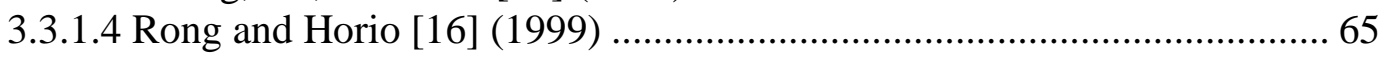

3.3.2 Modified Rong and Horio [16] particle-fluid-particle conduction model ....... 67

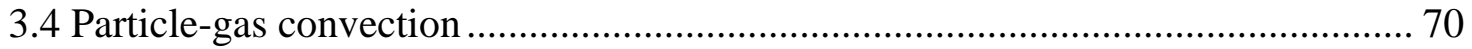

3.4.1 Background of particle-gas convection …………...................................... 70

3.4.2 Ranz and Marshall [69] (1952) ................................................................ 72

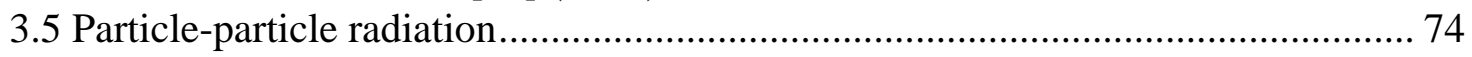

3.5.1 Background of particle-particle radiation .................................................. 75

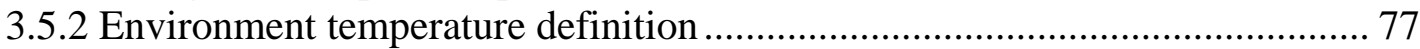

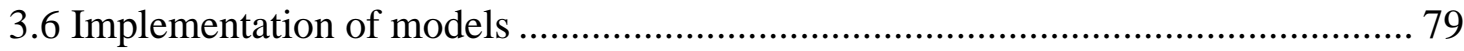

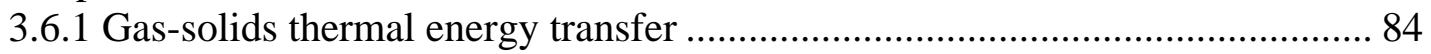

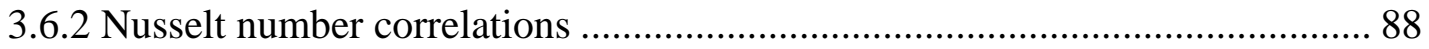

3.6.3 Temperature updating procedures................................................................. 88

3.6.4 Integral management in particle-fluid-particle conduction model.................... 89

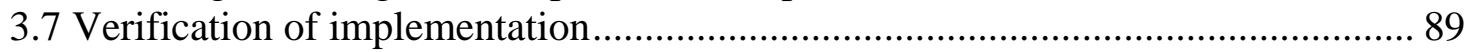

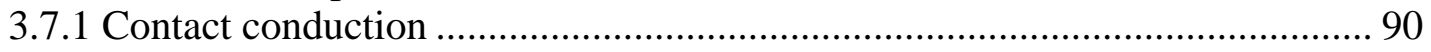

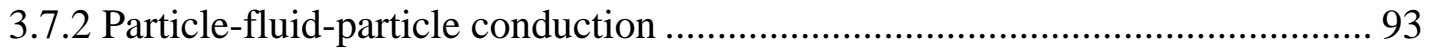

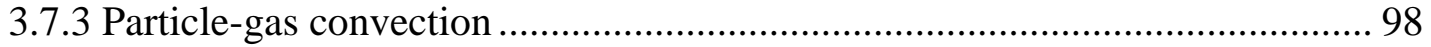

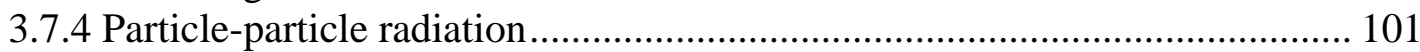

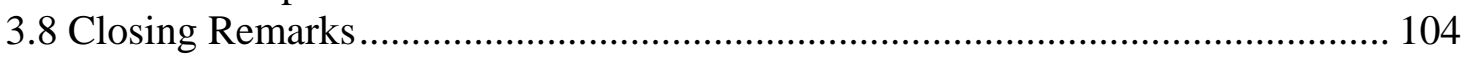

Chapter 4 : Particle-gas reactive chemistry interface ..................................................... 106

4.1 Foundations for particle-gas reactive chemistry ................................................ 106

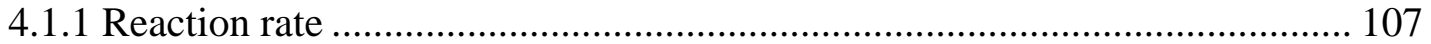

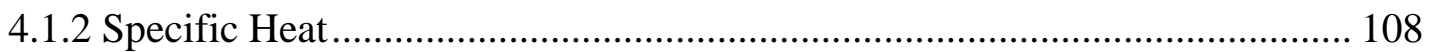

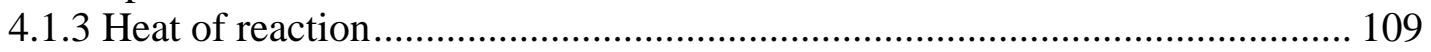

4.1.4 Mass and species equations for a particle .................................................... 111

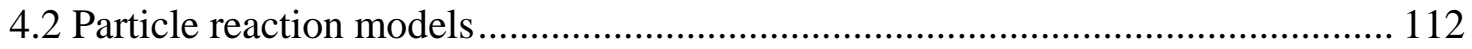

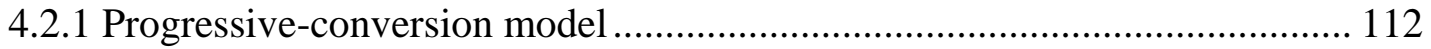

4.2.2 Shrinking particle model....................................................................... 113

4.2.3 Shrinking, unreacted-core model .............................................................. 115

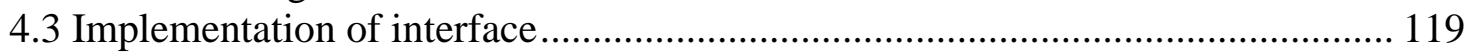

4.3.1 Gas-solids mass and species mass transfer .................................................. 124

4.3.2 Mass, species mass, and core radius updating procedures.............................. 125

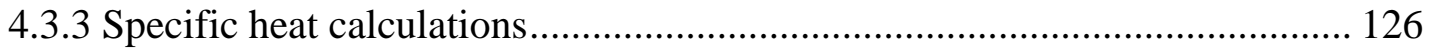

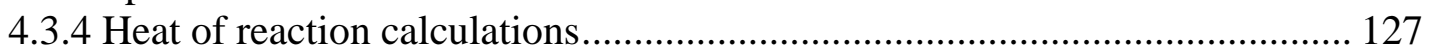

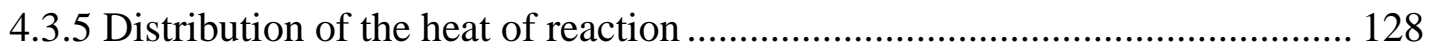

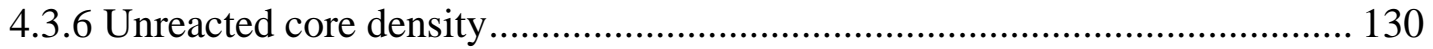

4.4 Verification of implementation..................................................................... 130 


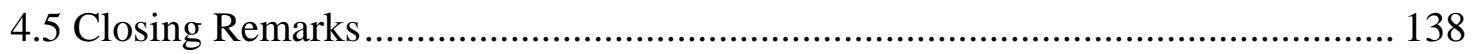

Chapter 5 : Summary and recommendations ............................................................. 139

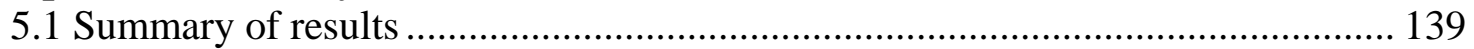

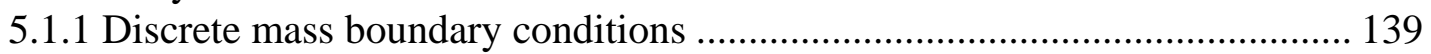

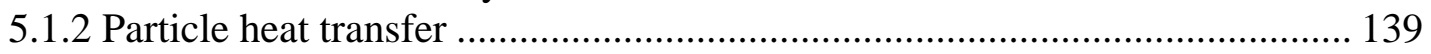

5.1.3 Particle-gas reactive chemistry interface …………....................................... 141

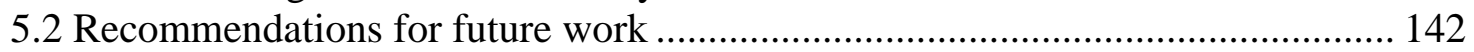

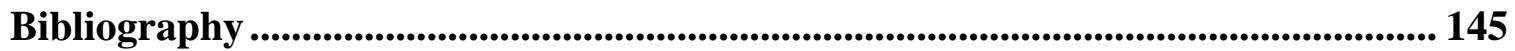

Appendix A : Geometric definitions for collision calculations ..................................... 156

Appendix B : Geometric definitions for conduction models.......................................... 159

B.1 Contact conduction definitions .................................................................. 159

B.2 Particle-fluid-particle conduction definitions ...................................................... 161

Appendix C : Discrete mass inflow boundary condition source code ......................... 163

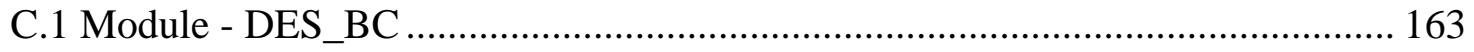

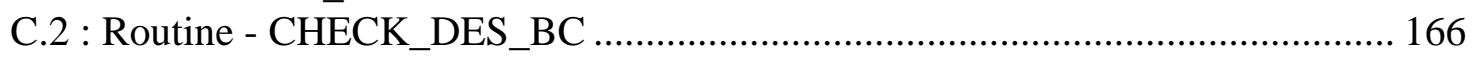

C.3 : Routine - DES_CHECK_MIO_LOCATION …………………..................... 172

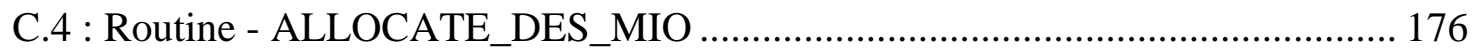

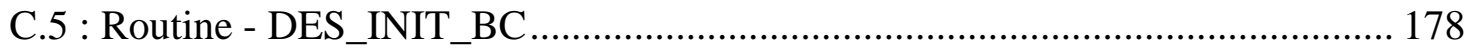

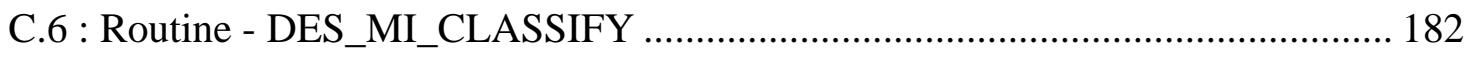

C.7 : Routine - DES_BC_VEL_ASSIGN.............................................................. 188

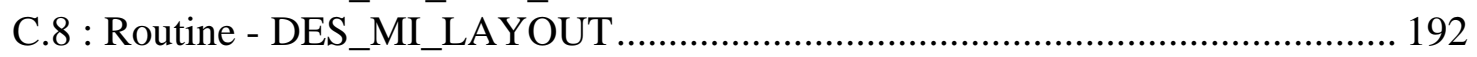

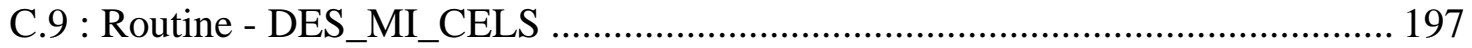

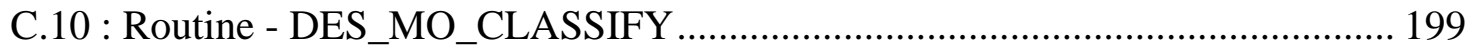

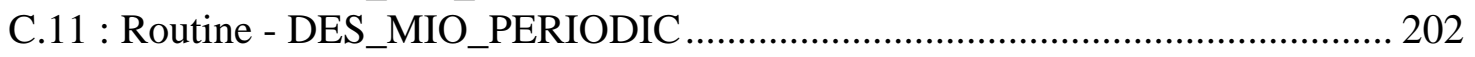

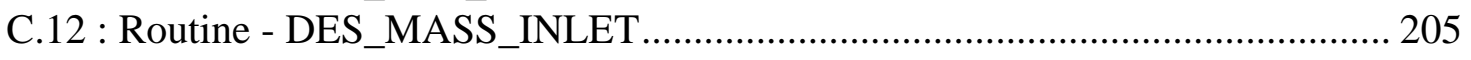

C.13 : Routine - DES_PLACE_NEW_PARTICLE ……….................................. 210

C.14 : Routine - DES_NEW_PARTICLE_TEST …………………...................... 219

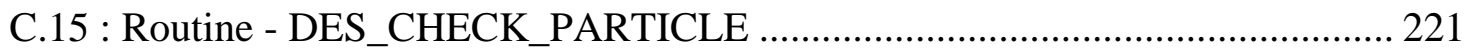

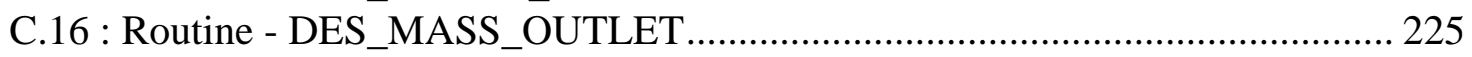

Appendix D : Particle heat transfer model source code................................................. 227

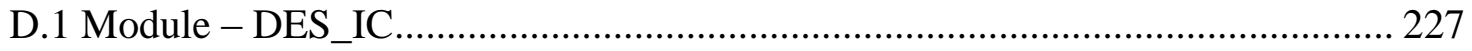

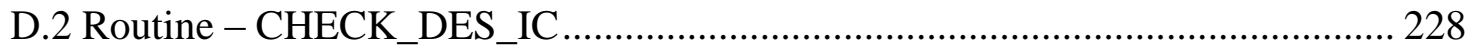

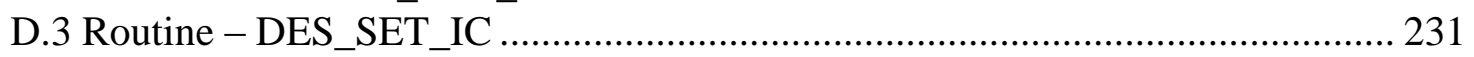

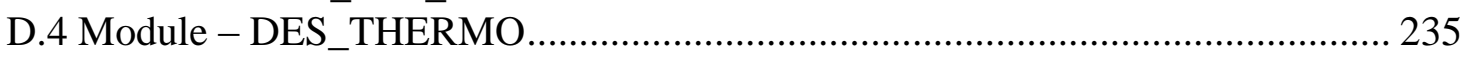

D.5 Routine - CHECK_DES_THERMO ............................................................. 237

D.6 Routine -CALC_THERMO_DES............................................................... 242

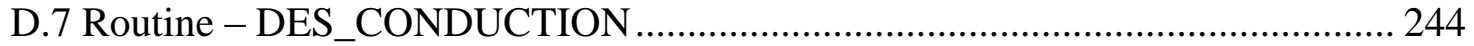

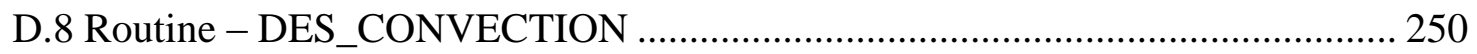

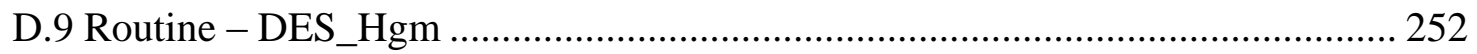

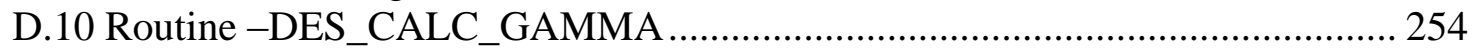

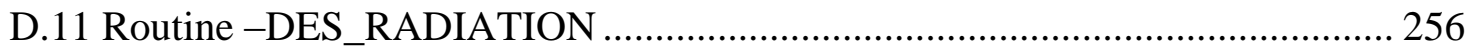

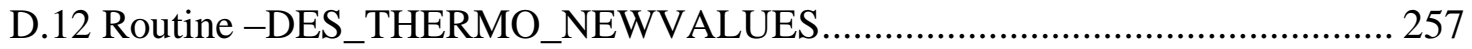

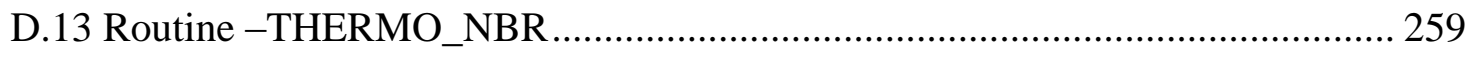

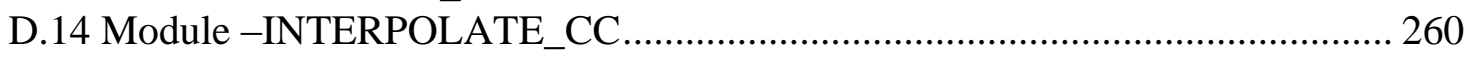


D.15 Module -SET_INTERPOLATION_STENCIL_CC .................................... 262

Appendix E : Interface for managing reactive chemistry source code .................... 266

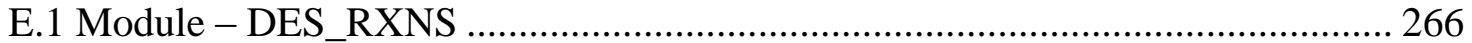

E.2 Routine-CHECK_DES_RXNS ............................................................. 268

E.3 Function - DES_CALC_H.................................................................... 271

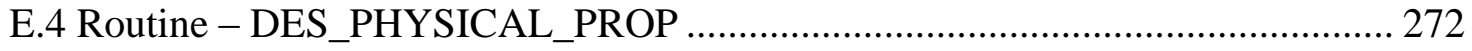

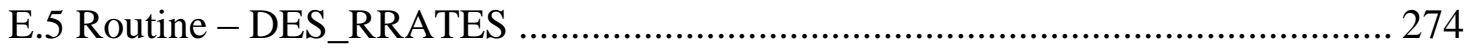

E.6 Routine - DES_REACTION_MODEL ..................................................... 281 


\section{List of Figures}

Figure 1-1: Schematic of the soft-sphere model that employs mechanical elements to simulate particle-particle and particle-wall collisions. 10

Figure 1-2: (a) During a particle-wall collision, an artificial particle the same size of the colliding particle is generated to represent the wall. The artificial particle is placed opposite the colliding particle, outside the computational domain. (b) As the position and trajectory of the colliding particle change, the artificial particles are replaced by new artificial particles to ensure that normal forces remain normal to the boundary plane..... 13

Figure 2-1: The staging area for incoming particles is located outside of the computational domain, in the ghost cells bordering the region identified with the boundary condition. 17

Figure 2-2: Example distribution array with ten elements, three mass solids phase indices $(\boldsymbol{i}, \boldsymbol{j}, \boldsymbol{k})$ with the respective distribution $(20 \%, 30 \%, 50 \%)$. The fifth array element is randomly selected, leading to the incoming particle being assigned the properties associated with solids phase- $\boldsymbol{j}$. 19

Figure 2-3: Motivation for a uniform inlet velocity. A slow moving particle is seeded behind a fast moving particle generating an artificial overlap.

Figure 2-4: Illustration of the seeding process of the grid structure. In this example, the sub-grid in the staging area contains seven cells, while two particles are placed every solids time step. Therefore, the sub-grid will overfill in four solids time steps (eight particles and only seven cells) if incoming particles do not move with sufficient speed toward the domain leaving available cells for new particles. 24

Figure 2-5: Call graph of main MFIX source code to initialization routines for the discrete particle model. Dashed lines indicate new calls and subroutines implemented for the discrete mass boundary conditions. 30

Figure 2-6: Call graph of MFIX discrete particle model routines. Dashed lines indicate new calls and subroutines implemented for the discrete mass boundary conditions........ 31

Figure 2-7: Flow chart illustrating the locations within MFIX-DEM where modifications have been made to include discrete mass boundary condition routines. 32

Figure 2-8: Time series (in seconds) of particle flow for a 2D example illustrating a DMIBC injecting into a packed bed. 34

Figure 2-9: Cumulative incoming particle distributions at times 0.5, 3.0, and 5.0 seconds during the simulation of a DMIBC into a packed particle bed. 35

Figure 2-10: Time series (in seconds) of particle flow for a 2D example illustrating a DMIBC with a gas jet. 36 
Figure 3-1: Illustration of conduction between particles. (a) Contact conduction: thermal energy is transferred from a hot particle to a cold particle through their shared contact area. (b) Particle-fluid-particle conduction: thermal energy is transferred from a hot particle to the stagnant gas separating its surface from the cold particle. The thermal energy is then transferred from the stagnant gas to the cold particle. 39

Figure 3-2: Illustration of particle-gas convection. Thermal energy is exchanged between the surface of the particle and the moving fluid. 40

Figure 3-3: Illustration of particle-environment radiative heat transfer. Electromagnetic waves transmitted by a hot particle are absorbed and reflected by the cold particle........ 40

Figure 3-4: Illustration of Biot number classification on a particle's temperature. Arrows on the surface of the particles indicate the rate of convective heat transfer, while the arrows inside the particle represent the rate of conductive heat transfer. (a) For small Biot numbers, a particle's temperature is nearly-isothermal because the redistribution of thermal energy within a particle occurs at a faster rate than convective heat transfer. (b) When the Biot number is large, a temperature gradient may exist within a particle because the redistribution of thermal energy inside a particle is slower than the rate of convective heat transfer. 43

Figure 3-5: The elastic collision model employed by Sun and Chen [49] leads to the assumption that the shared contact area is flat...... 46

Figure 3-6: Particle-particle conduction model employing a thermal pipe connecting the centers of two particles. Implemented in PFC2D/3D [56,57] and presented by Shimizu [51]. 50

Figure 3-7: Illustration of a particle assembly as defined by Shimizu [51] with interconnected thermal pipes. 51

Figure 3-8: Illustration of the thermal energy flux from a hot particle to a cold particle across the contact area. 54

Figure 3-9: Illustration depicting the heat transfer paths as defined by Delvosalle and Vanderschuren's [64]. The heat transfer paths are defined as portions of circumferences perpendicular to the surfaces of the particles. 60

Figure 3-10: Delvosalle and Vanderschuren's [64] model assumes particle-fluid-particle conduction is initiated when the laminar sub-layers surrounding two particles overlap. This configuration is used to define the parameter $\boldsymbol{\alpha}$ in Equation (3-42). 61

Figure 3-11: The model of Cheng, Yu, and Zulli [65] restricts particle-fluid-particle conduction to the region delineated by the double pyramid (3-D) generated by the Voronoi-boundary plane. 62 
Figure 3-12: Cheng, Yu, and Zulli's [65] Model A for particle-fluid-particle conduction assumes that the surface of the double tapered cone is isothermal, conduction is negligible in the outer region of the double tapered cone, and the heat transfer paths are parallel to the axis joining the particles' centers. 63

Figure 3-13: Geometric representation of quantities used by Cheng, Yu, and Zulli's [65] Model A for particle-fluid-particle conduction.

Figure 3-14: Cheng, Yu, and Zulli's [65] Model B for particle-fluid-particle conduction assumes that each particle has an isothermal core and the paths of heat transfer radiate from the particles centers.

Figure 3-15: Rong and Horio's [16] configuration for particle-fluid-particle conduction. In their model, each particle is surrounded by a gas layer and heat transfer occurs across paths parallel to the line connecting the centers of the particles. When particles engage in contact, the overlap region is assumed to have a uniform gas layer separating the particles' surfaces. 66

Figure 3-16: Illustration of geometric quantities delineating the region of particle fluid heat transfer imposed in this work. 68

Figure 3-17: A singularly arises in the modified Rong and Horio model at the interface of the contact boundary. This is corrected by prohibiting perfect contact between particles, and require a minimum separation distance of $\mathbf{4} \times \mathbf{1 0}-\mathbf{1 0 m}$. Similar assumptions are employed by Delvosalle and Vanderschuren [64] and Rong and Horio [16]. 69

Figure 3-18: A multi-particle radiation problem is reduced by assuming that the particles predominantly exposed to radiation form a spherical environment surrounding the particle of interest. 76

Figure 3-19: Illustration of the sub-domain model proposed by Zhou, Yu, and Zulli [19] for determining the environment temperature used to calculate the rate of radiative heat transfer with particle $\boldsymbol{i}$. In this example, three particles are in the sub-domain (i.e., $\boldsymbol{N p}, \boldsymbol{\Omega}=\mathbf{3}$ ) of particle $\boldsymbol{i}$. 78

Figure 3-20: Call graph of main MFIX-DEM source code for initialization routines. Dashed lines indicate new calls and subroutines implemented for the heat transfer model.

Figure 3-21: Call graph of MFIX discrete particle model routines. Dashed lines indicate new calls and subroutines implemented for the heat transfer model. 82

Figure 3-22: Flow chart illustrating the locations within MFIX-DEM where modifications have been made to include particle heat transfer. 83

Figure 3-23: Flow chart illustrating customizability of the implementation by allowing a user to select which modes of heat transfer to compute during a simulation. 84 
Figure 3-24: Example illustrating the Eulerian grid which stores scalar field variables, like temperature, at the cell-center. Particles are permitted to exist at any location within the domain defined by the Eulerian grid. 85

Figure 3-25: Example illustrating the two methods available to transmitting thermal energy between the Eulerian grid and the particles. (a) Energy is passed between a particle and the grid cell containing its center; (b) energy is distributed from a particle's position to the nearest grid cells. 86

Figure 3-26: Illustration of the fluid step forward-particle catch-up process that occurs during MFIX-DEM. First the solution to the fluid phase if obtained at time $t$, then multiple, smaller time steps are taken within the DEM to allow the particles to catch-up to the fluid phase. Here, $\boldsymbol{t}$ is the time of the fluid's most recent solution, and $\boldsymbol{t} \boldsymbol{s}$ is the time of the discrete solids most recent solution. 87

Figure 3-27: The geometric configuration of the particles used to verify the implementation of the contact conduction model implemented into MFIX-DEM. All measurements are in centimeters. 92

Figure 3-28: MFIX-DEM and analytic solutions to the internal energy equation for two particles engaged in contact conduction. Tp1 and Tp2 are the temperatures of the cold and hot particles, respectively. Relative error between the analytic and numerical solutions is indicated by open symbols and is associated with the right vertical axis...... 93

Figure 3-29: The geometric configuration of the particles used in the first simulation to verify the implementation of the particle-fluid-particle conduction model implemented into MFIX-DEM. All measurements are in centimeters. 96

Figure 3-30: MFIX-DEM and analytic solutions to the internal energy equation for two particles not touching and engaged in particle-fluid-particle conduction. Tp1 and Tp2 are the temperatures of particle 1 and particle 2, respectively (c.f. Table 3-4). Relative error between the two solutions is indicated by open symbols and is associated with the right vertical axis.

Figure 3-31: MFIX-DEM and analytic solutions to the internal energy equation for two touching particles engaged in particle-fluid-particle conduction. Tp1 and Tp2 are the temperatures of particle 1 and particle 2, respectively (c.f. Table 3-4). Relative error between the two solutions is indicated by open symbols and is associated with the right vertical axis.

Figure 3-32: The geometric configuration of a particle used to verify the implementation of the particle-gas convection model implemented into MFIX-DEM. All measurements are in centimeters. 
Figure 3-33: MFIX-DEM and analytic solutions to the internal energy equation for a single particle undergoing particle-gas convection with a constant fluid. Tp and $\mathrm{Tg}$ are the temperatures of particle and gas, respectively Relative error between the numerical and analytic solutions is indicated by open symbols and is associated with the right vertical axis. 100

Figure 3-34: The geometric configuration of the particles used in the first simulation to verify the implementation of the particle-environment radiation model implemented into MFIX-DEM. All measurements are in centimeters. 103

Figure 3-35: MFIX-DEM and fourth order Runge-Kutta (RK4) solutions to the internal energy equation for two particles not touching engaged in radiative heat exchange. Relative error between the two solutions is indicated by open symbols and is associated with the right vertical axis. 104

Figure 4-1: Illustration of the progressive-conversion model. The reaction occurs uniformly throughout a particle, leaving the particle size unchanged. In this example, the concentration of the reactant diminishes as the reaction proceeds. 112

Figure 4-2: Illustration of the shrinking particle model. The reaction occurs at the particle's surface while the composition of the particle does not change. Any solid products of the reaction are assumed to 'flake off' of the particle's surface (i.e. no solid products collect on the surface of the particle). 114

Figure 4-3: An example illustrating how the number of possible neighbor particles increases as the ratio of the neighbor's radius $(\boldsymbol{R n})$ to the particle's radius $(\boldsymbol{R} \boldsymbol{p})$ decreases.

Figure 4-4: Illustration of the shrinking core model. The reaction occurs at the surface of the particle's unreacted core with an inert solid (ash) replacing the particle's solids. Although the unreacted core shrinks as the reaction proceeds, its composition remains constant. 116

Figure 4-5: Example illustrating the connection between the radius of the unreacted core $(\boldsymbol{R} \boldsymbol{c})$ and the definition of the unreacted core's density $(\boldsymbol{R} \boldsymbol{c})$. If the unreacted core's density ( $\boldsymbol{\rho}$ core) is equated to the particle's initial density $(\boldsymbol{\rho} \boldsymbol{p})$, then the core's radius shrinks to $\boldsymbol{R} \mathbf{1}$. If the unreacted core's density is defined as the initial mass of the solid phase reactants divided by the particle's volume ( $\rho$ all reactants), the core's radius shrinks to $\boldsymbol{R} 2$. Finally, if the unreacted core's density is defined as the total mass of solid phase reactants consumed divided by particle's volume ( $\rho$ used reactants), then the core's radius shrinks to zero. 117

Figure 4-6: Call graph of main MFIX-DEM source code for initialization routines. Dashed lines indicate new calls and subroutines implemented for the reactive chemistry interface. 121 
Figure 4-7: Call graph of MFIX discrete particle model routines. Dashed lines indicate new calls and subroutines implemented for the reactive chemistry interface. 122

Figure 4-8: Flow chart illustrating the locations within MFIX-DEM where modifications have been made to include the reactive chemistry interface. 123

Figure 4-9: MFIX-DEM and analytic solutions to the particle mass equation engaged in reactive chemistry interface. $\mathrm{mp}$ is the mass of the particle. Relative error between the analytic and numerical (forward Euler method) solutions is indicated by open symbols and is associated with the right vertical axis. 135

Figure 4-10: MFIX-DEM and analytic solutions to the particle species equations engaged in reactive chemistry interface. $\mathrm{XB}, \mathrm{XD}$, and $\mathrm{XI}$ are the species mass fractions for species $\mathrm{B}, \mathrm{D}$, and I, respectively. Absolute error between the analytic and numerical (forward Euler method) solutions is indicated by open symbols and is associated with the right vertical axis. 136

Figure 4-11: MFIX-DEM and analytic solutions to the particle energy equation engaged in reactive chemistry interface. Tp is the temperature of the particle. Relative error between the analytic and numerical (forward Euler method) solutions is indicated by open symbols and is associated with the right vertical axis. 136

Figure 4-12: MFIX-DEM and analytic solutions to the radius of the shrinking, unreacted core engaged in reactive chemistry interface. Rc is the core radius. Absolute error between the analytic and numerical (forward Euler and Adams-Bashforth (AB) methods) solutions are indicated by open symbols and is associated with the right vertical axis.. 137

Figure A-1: Schematic demonstrating various terms needed in the calculation of the

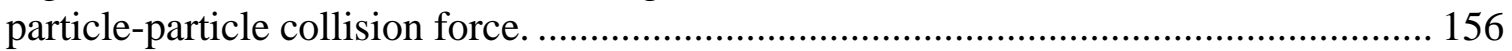

Figure B-1: Schematic demonstrating various terms needed in the calculation of the contact radius needed for computing contact conduction. In this illustration particle $\boldsymbol{i}$ is smaller than particle $\boldsymbol{j}$.

Figure B-1: Schematic demonstrating various terms needed in the calculation of the contact radius needed for computing contact conduction. In this illustration particle $\boldsymbol{i}$ is smaller than particle $\boldsymbol{j}$. 160

Figure B-2: Schematic demonstrating various terms needed in the calculation of the contact radius needed for computing contact conduction. In this illustration particle $\boldsymbol{i}$ is smaller than particle $\boldsymbol{j}$. 161

Figure B-3: Schematic demonstrating various terms needed in the calculation of the particle-fluid-particle conduction. 161 


\section{List of Tables}

Table 2-1: A list of the subroutines comprising the discrete mass boundary conditions with brief description of their use. 28

Table 2-2: Particle and feed properties of the examples outlined in Sections 2.5.1 and 2.5.2....... 33

Table 3-1: Summary of the particle internal energy equation and four forms of particle heat transfer considered in this work. 79

Table 3-2: A list of subroutines developed to set initial conditions of particles and manage the heat transfer models implemented into MFIX. 80

Table 3-3: Physical and thermodynamic properties of the particles used to verify the contact conduction model implemented into MFIX-DEM. The geometric configuration is illustrated in Figure 3-27 91

Table 3-4: Physical and thermodynamic properties of the particles used to verify the particle-fluid-particle conduction model implemented into MFIX-DEM. The geometric configuration for the first simulation is illustrated in Figure 3-29 while the geometric configuration for the second simulation is illustrated in Figure 3-27. The gas phase thermal conductivity for both simulations was set as $1.239 \times 10^{-4} \mathrm{cal} / \mathrm{sec} \cdot \mathrm{cm} \cdot \mathrm{K} \ldots \ldots \ldots \ldots . . . .95$

Table 3-5: Physical and thermodynamic properties of the gas and particle used to verify the convection model implemented into MFIX-DEM. The geometric configuration is illustrated in Figure 3-32.

Table 3-6: Physical and thermodynamic properties of the particles used to verify the radiative heat transfer model implemented into MFIX-DEM. The geometric configuration is illustrated in Figure 3-34. 102

Table 4-1: Summary of the models managed by the reactive chemistry interface considered in this work. 120

Table 4-2: A list of subroutines developed for the reactive chemistry interface implemented into MFIX. 120

Table 4-3: Physical and thermodynamic properties of the gas and particle used to verify the reactive chemistry interface implemented into MFIX-DEM. 134

Table 4-4: Physical and thermodynamic properties of the gas and particle species used to verify the reactive chemistry interface implemented into MFIX-DEM. 134 


\section{Nomenclature}

\begin{tabular}{|l|l|}
\hline$A_{B C}$ & cross-sectional inlet area \\
\hline$A_{c}$ & contact area between two colliding particles \\
\hline$A_{n}$ & coefficients of Fourier series solution \\
\hline$A_{s}$ & surface area of a particle \\
\hline $\mathrm{Bi}$ & Biot number \\
\hline $\mathrm{Bi}^{\prime}$ & Biot number associated with collision duration \\
\hline$C_{\mathrm{A}}, C_{\mathrm{B}}$ & concentration of species A and B \\
\hline$C_{p g}$ & gas phase specific heat \\
\hline$C_{p p}$ & particle specific heat \\
\hline$d$ & thickness of laminar sub-layer surrounding the indexed particle \\
\hline$D_{g n}$ & gas phase species $n$ diffusion coefficient \\
\hline$D_{m}$ & diameter of particles belonging to solids phase $m$ \\
\hline$D_{m a x}$ & maximum diameter of incoming particles over all solids phases \\
\hline$E^{*}$ & effective Young's modulus of two particles \\
\hline$E_{b}$ & rate of at which radiation is emitted from a black body \\
\hline$E r r_{v_{t e s t}}{ }^{\prime}$ & error between user-provided and computed bulk density values \\
\hline $\mathbf{F}_{c}$ & total force resulting from collisions acting on a particle \\
\hline$F$ & shape (or view) factor for radiative heat transfer \\
\hline $\mathbf{F}_{n c}$ & total force not associated with collisions acting on a particle \\
\hline$F_{p}$ & placement frequency of incoming particles \\
\hline $\mathrm{Fo}_{p}$ & Fourier number for a particle \\
\hline $\mathrm{Fo}_{p}{ }^{\prime}$ & Fourier number for a collision \\
\hline$g_{i}$ & $i^{\text {th }}$ component of the gravity vector \\
\hline $\mathbf{g}$ & gravity vector \\
\hline$H^{\circ}\left(T_{R E F}\right)$ & enthalpy of formation at reference temperature $T_{R E F}$ \\
\hline$H_{\mathrm{A}}, H_{\mathrm{B}}, H_{C}, H_{\mathrm{D}}$ & enthalpies of species A, B, C, and D \\
\hline$\dot{H}_{f r}$ & enthalpy transfer due to mass transfer \\
\hline$I$ & mass moment of inertia \\
\hline$I_{g m}$ & $\begin{array}{l}\text { interphase momentum transfer between the gas and the } m^{t h} \text { solids } \\
\text { phase }\end{array}$ \\
\hline$l_{B C}$ & length of inlet region \\
\hline$k$ & gate coefficient of a chemical reaction \\
\hline$K_{C V}$ & \\
\hline & \\
\hline
\end{tabular}




\begin{tabular}{|c|c|}
\hline$k_{n}$ & normal spring coefficient \\
\hline$k_{t}$ & tangential spring coefficient \\
\hline$L^{(i)}$ & distance from the center to the point of contact of the indexed particle \\
\hline$\ell^{(i, j)}$ & distance between the centers of the particles indexed by $i$ and $j$ \\
\hline$\ell^{(m)}$ & length of $m^{\text {th }}$ thermal pipe in the particle assembly \\
\hline$\ell_{i, r}$ & length of path of conduction through the $i^{\text {th }}$ particle $\mathfrak{L}$ \\
\hline$\ell_{g, r}$ & length of path of conduction through the gas \\
\hline$m^{*}$ & effective mass of two particles \\
\hline$m$ & mass of $i^{\text {th }}$ particle \\
\hline$M$ & total number of solids phases \\
\hline$m_{s n}$ & species $n$ mass of reactant initially in the indexed particle \\
\hline$m_{s n}^{0}$ & species $n$ mass of reactant consumed in the indexed particle \\
\hline$M_{g}$ & molecular weight of the gas phase \\
\hline$N_{B C}$ & number of cells in the staging area of the inlet \\
\hline$N_{C V}$ & number of particles in the control volume, $\mathrm{CV}$ \\
\hline$N_{g}$ & number of species comprising the gas phase \\
\hline$N_{m, t}$ & number of solids phase $m$ particles entering the system per unit time \\
\hline$N_{p}$ & number of particles in a simulation \\
\hline$N_{p s}$ & number of particles seeded into the staging area \\
\hline$N_{p, \Omega}$ & number of particles in radiation domain, $\Omega$ \\
\hline$N_{S}$ & number of species comprising the indexed particle \\
\hline$N_{t}$ & total number of particles entering the system per unit time \\
\hline $\mathrm{Nu}_{p}$ & Nusselt number of a particle \\
\hline$p_{g}$ & gas pressure \\
\hline $\operatorname{Pr}$ & Prandtl number \\
\hline$q_{A}$ & total heat flux across the surface of a type-A particle \\
\hline$q_{c}$ & thermal flux normal to the contact area of two particles \\
\hline$\dot{Q}_{p f p}$ & rate of heat transfer via particle-fluid-particle conduction \\
\hline$\dot{Q}_{p g}$ & rate of heat transfer via particle-gas convection \\
\hline$\dot{Q}_{p p}$ & rate of heat transfer via particle-particle conduction \\
\hline$\dot{Q}_{\text {rad }}$ & rate of heat transfer via particle-environment radiation \\
\hline$q_{g s}$ & rate of heat transfer between gas and solids phase \\
\hline $\mathbf{q}_{\mathfrak{R}}$ & heat flux of particle assembly $\mathfrak{L}$ \\
\hline$R^{*}$ & effective radius of two particles \\
\hline$R$ & gas constant \\
\hline
\end{tabular}




\begin{tabular}{|c|c|}
\hline$R_{C}$ & radius of the circular contact region between two spheres \\
\hline$\tilde{R}_{c}$ & geometrically derived contact radius \\
\hline$R_{f}$ & $\begin{array}{l}\text { distance delineating the upper bound of particle-fluid-particle } \\
\text { conduction }\end{array}$ \\
\hline$R_{g n}$ & gas phase species $n$ rate of production (or consumption) \\
\hline$r_{g}, r_{s}$ & distances delineating the region of particle-fluid-particle conduction \\
\hline$R_{p}$ & particle radius \\
\hline $\operatorname{Re}$ & Reynolds number \\
\hline$\left(-r_{A}\right),\left(-r_{B}\right)$ & rates of disappearance of species A and B \\
\hline$r_{\mathrm{C}}, r_{\mathrm{D}}$ & rates of formation of species $\mathrm{C}$ and $\mathrm{D}$ \\
\hline$s$ & $\begin{array}{l}\text { parameter that influences the total number of iterations used in } \\
\text { identifying the uniform inlet velocity }\end{array}$ \\
\hline 8 & distance between the surfaces of the indexed particles \\
\hline$S_{g j i}$ & gas phase stress tensor \\
\hline$t$ & time \\
\hline$t_{c}$ & particle-particle contact duration \\
\hline $\mathbf{T}$ & total torque forces acting on a particle \\
\hline$T_{A}, T_{B}$ & temperatures of type-A and type-B particles \\
\hline$T_{g}$ & temperature of the gas phase \\
\hline$T_{g, \Omega}$ & temperature of gas phase in radiation domain $\Omega$ \\
\hline$T_{R g}$ & gas phase radiation temperature \\
\hline$T_{\mathfrak{R}}$ & temperature of particle assembly $\mathfrak{L}$ \\
\hline$T_{\text {env }}$ & environment temperature surrounding a particle \\
\hline$t_{p}$ & time indicating next particle placement event \\
\hline$T_{p}$ & particle temperature \\
\hline$T_{p 0}$ & particle initial temperature \\
\hline$T_{p, r e f}$ & particle reference temperature \\
\hline$T_{R E F}$ & reference temperature \\
\hline $\mathbf{V}$ & velocity of a particle \\
\hline$v_{C V}$ & volume of a control volume (CV) \\
\hline$v_{g i}$ & $i^{\text {th }}$ component of gas phase velocity \\
\hline$v_{m}^{0}$ & initial velocity for incoming particles of solids phase $m$ \\
\hline$v_{\min }$ & lower bound on incoming particle velocity \\
\hline $\mathbf{V}_{n}$ & normal relative velocity \\
\hline$v_{\text {rand }}$ & $\begin{array}{l}\text { velocity delineating order and random placement of incoming } \\
\text { particles }\end{array}$ \\
\hline
\end{tabular}




\begin{tabular}{|l|l|}
\hline$v_{\text {test }}^{i}$ & test velocity for incoming particles \\
\hline$\dot{V}_{m}$ & volumetric flow rate of solids phase $m$ into the system \\
\hline $\mathcal{V}_{C V}$ & volume of control volume $(\mathrm{CV})$ \\
\hline$V_{p}$ & particle volume \\
\hline $\mathbf{V}_{t}$ & tangential relative velocity \\
\hline$w_{B C}$ & width of inlet region \\
\hline $\mathbf{X}$ & position of a particle \\
\hline$X_{g n}$ & gas phase's species $n$ mass fraction \\
\hline$X_{p n}$ & indexed particle's species $n$ mass fraction \\
\hline
\end{tabular}

\section{Greek Symbols:}

\begin{tabular}{|l|l|}
\hline$\alpha, \beta$ & angles delineating the region of particle-fluid-particle conduction \\
\hline$\alpha_{k}$ & polynomial coefficients for temperature dependent specific heat \\
\hline$\beta_{m}$ & local gas-solids momentum transfer coefficient \\
\hline$\gamma_{c p}$ & convective heat transfer coefficient for a particle \\
\hline$\gamma_{B A}$ & $\begin{array}{l}\text { particle-particle convective heat transfer coefficient between particle } \\
\text { types } A \text { and } B\end{array}$ \\
\hline$\gamma_{R g}$ & gas phase radiative heat transfer coefficient \\
\hline$\delta_{i j}$ & Kronecker delta \\
\hline$\delta_{n}$ & magnitude of normal overlap \\
\hline $\boldsymbol{\delta}_{t}$ & tangential displacement \\
\hline$\Delta H$ & heat of reaction \\
\hline$\Delta H_{Q}$ & change in enthalpy due to temperature change \\
\hline$\Delta H_{r g}$ & gas phase heat of reaction \\
\hline$\Delta H_{r s}$ & indexed particle's heat of reaction \\
\hline$\Delta T_{p}$ & temperature change of a particle over a solids time step of $\Delta t_{s}$ \\
\hline$\Delta t_{s}$ & solids time step size \\
\hline$\nabla T$ & $\begin{array}{l}\text { temperature gradient along indexed thermal pipe in particle assembly } \\
\mathfrak{L}\end{array}$ \\
\hline$\epsilon$ & radiation emittance \\
\hline$\varepsilon_{g}$ & gas phase volume fraction \\
\hline$\varepsilon_{s m}$ & solids phase $m$ volume fraction \\
\hline$\zeta_{p}$ & particle emissivity \\
\hline$\eta$ & non-dimensionalized radial position \\
\hline$\eta_{n}$ & normal damping coefficient \\
\hline
\end{tabular}




\begin{tabular}{|c|c|}
\hline$\eta_{t}$ & tangential damping coefficient \\
\hline$\eta_{\mathfrak{L}}$ & thermal resistance of particle assembly $\mathfrak{L}$ \\
\hline$\Theta$ & non-dimensionalized particle temperature \\
\hline$\theta_{0}, \theta_{c}$ & angles delineating the region of particle-fluid-particle conduction \\
\hline$\kappa_{g}$ & gas phase thermal conductivity \\
\hline$\kappa_{p}$ & particle thermal conductivity \\
\hline$\kappa_{p}^{*}$ & effective thermal conductivity \\
\hline $\boldsymbol{\kappa}_{\mathfrak{L}}$ & thermal conductivity of particle assembly $\mathfrak{L}$ \\
\hline $\bar{\kappa}_{\mathfrak{L}}$ & uniform thermal resistance of all thermal pipes in particle assembly $\mathfrak{L}$ \\
\hline$\lambda_{n}$ & eigenvalues of Fourier series solution \\
\hline$\mu$ & friction coefficient \\
\hline$\mu_{g}$ & gas phase viscosity \\
\hline$\mu_{g}^{\prime}$ & second coefficient of gas phase viscosity \\
\hline$v$ & Poisson's ration \\
\hline$\xi$ & unit vector directed along the indexed thermal pipe \\
\hline$\xi_{n}$ & unit normal vector relating the particles' centers \\
\hline$\xi_{t}$ & unit tangential vector relating the particles' centers \\
\hline$\xi_{t}^{\prime}$ & $\begin{array}{l}\text { unit tangential vector relating the particles' centers corrected for } \\
\text { prolonged contact }\end{array}$ \\
\hline$\rho$ & radiation reflectance \\
\hline$\rho_{b m}$ & bulk density on solids phase $m$ \\
\hline$\tilde{\rho}_{b m}\left(v_{\text {test }}^{i}\right)$ & test bulk density value \\
\hline$\rho_{c}$ & unreacted core denisty \\
\hline$\rho_{g}$ & density of gas phase \\
\hline$\rho_{p}$ & particle density \\
\hline$\rho_{s m}$ & density of solids phase $m$ \\
\hline$\sigma$ & Stefan-Boltzmann constant $\left(5.67 \times 10^{-8} \mathrm{~W} / \mathrm{m}^{2} \mathrm{~K}^{4}\right)$ \\
\hline$\varsigma$ & radiation absorbance \\
\hline$\tau_{g i j}$ & gas phase viscous stress tensor \\
\hline$\omega$ & angular velocity of a particle \\
\hline$\psi$ & resistance of thermal pipe \\
\hline$\psi_{\mathfrak{\Omega}}$ & thermal resistance for particle assembly $\mathfrak{L}$ \\
\hline$\Omega$ & domain defining environment for radiation effects \\
\hline
\end{tabular}




\section{Fraktur Symbols:}

\begin{tabular}{|l|l|}
\hline $\mathfrak{U}$ & $\begin{array}{l}\text { the set of particles engaged in particle-particle heat transfer with } \\
\text { particle } i\end{array}$ \\
\hline $\mathfrak{B}$ & $\begin{array}{l}\text { the set of particles engaged in particle-fluid-particle heat transfer with } \\
\text { particle } i\end{array}$ \\
\hline $\mathfrak{L}$ & $\begin{array}{l}\text { the set of particles contained in a specific control volume (particle } \\
\text { assembly) }\end{array}$ \\
\hline $\mathfrak{L}_{N_{C}}$ & number of particle-particle contacts in particle assembly $\mathfrak{L}$ \\
\hline $\mathfrak{L}_{N_{p}}$ & number of particles in particle assembly $\mathfrak{L}$ \\
\hline
\end{tabular}




\section{Abbreviations and Acronyms}

\begin{tabular}{|l|l|}
\hline AB & Adams-Bashforth method \\
\hline CDM & continuum discrete method \\
\hline CFD & computational fluid dynamics \\
\hline CV & control volume \\
\hline DEM & discrete element method \\
\hline DMIBC & discrete mass inflow boundary condition \\
\hline DMOBC & discrete mass outflow boundary condition \\
\hline DNS & direct numerical simulation \\
\hline DPM & discrete particle method \\
\hline DSMC & direct simulation Monte Carlo \\
\hline EE & Eulerian-Eulerian \\
\hline LBM & lattice-Boltzmann method \\
\hline MFIX & Multiphase Flow with Interphase eXchanges \\
\hline MPPIC & multiphase particle in cell \\
\hline TFM & two-fluid method \\
\hline
\end{tabular}




\section{Chapter 1: Introduction}

Gas-solids systems are found in industrial food preparation, mineral processing, pharmaceutical production, energy generation, and chemical production setups as well as academic research laboratories world-wide. Applications range from co-current flow

drying processes for food particles [1] to fluidized bed reactors for polyethylene production [2]. With such a large industrial dependence on gas-solids systems, it is imperative that they are fully understood. A greater understanding of the physical processes occurring within a gas-solids system may yield designs that are more cost effective, energy efficient, and environmentally friendly.

Traditionally, design, scaling, and optimization of gas-solids systems are guided by empirical models derived from experimental observations $[\mathbf{3 , 4}]$. The development of these models requires a large investment in both time and capital while their predictive capabilities remain limited and unreliable [5]. Consequently, multiphase computational fluid dynamics (CFD) models are gaining in popularity for testing various design considerations [6] and predicting flow characteristics [7]. This physics-based approach provides greater modeling flexibility since it is rooted in the first principles of gas-solids dynamics [8].

There are two general methods for modeling gas-solids flows. Simulations that solve continuum conservation equations for both the gas and solids phases are termed Eulerian-Eulerian (EE) or two fluid methods (TFM). Simulations that manage distinct objects for the solids phase while continuum conservation equations are solved for the gas phase are called Lagrangian-Eulerian (LE) or continuum discrete methods (CDM). 
CDMs encompass a wide range of models that resolve gas-solids flow characteristics at various length and time scales [5]. These techniques include direct numerical simulation (DNS), lattice-Boltzmann method (LBM), CFD-discrete element method (CFD-DEM), direct simulation Monte Carlo method (DSMC), and multiphase particle in cell (MPPIC) methods. Each approach leads to a trade-off between computational expense and modeling complexity. A brief overview of these methods is found in the book, Multiphase Continuum Formulation of Gas-Solids Reacting Flows by Pannala, Syamlal, and O'Brien [5]. The primary goal of this work is to further the modeling capabilities of an existing CFD-DEM. Section 1.1 provides an overview of this modeling technique so that the objectives of this work are defined more clearly.

\subsection{CFD-Discrete element method (CFD-DEM)}

CFD-DEM combines two different modeling techniques for simulating gas-solids flows. The gas phase is described by a set of conservation equations that are solved over a static grid, similar to single phase CFD. In contrast, the solids phase is described by a collection of discrete particles that obey Newton's laws of motion. Each particle is individually tracked and all particle-particle collisions are resolved. This method of solids modeling is termed the discrete element method (DEM) but often is referred to as the discrete particle method (DPM).

The original concept of the CFD-DEM is found in the pioneering work of Tsuji, Tanaka, and Ishida [9]. In their research, a simple gas phase model is coupled with a DEM to simulate plug flow in a horizontal pipe. Since then, the simulation capabilities of the CFD-DEM have advanced. Initial investigations strived to refine the gas phase model $[\mathbf{1 0 , 1 1}]$, while new developments continue to improve the coupling of the gas and solids 
phases [12]. Some research groups have incorporated Van der Waals forces to simulate cohesion between particles $[\mathbf{1 3}, \mathbf{1 4}]$ as well as the Saffman and Magnus lift forces [15]. Presently, there is a large effort to include particle scale heat transfer and reactive chemistry models $[\mathbf{2}, \mathbf{1 1}, \mathbf{1 6}, \mathbf{1 7}, \mathbf{1 8}, \mathbf{1 9}, \mathbf{2 0}, \mathbf{2 1}, \mathbf{2 2}, \mathbf{2 3}]$. Sections 1.1 .1 and 1.1 .2 present the mathematical models for the gas and solids phases employed by the current CFD-DEM. A brief discussion covering the physical interpretation of the equations' components is provided. The gas phase model is introduced first, followed by a presentation of the solids phase model.

\subsubsection{Gas phase mathematical model}

The gas phase model employed by the CFD-DEM in this work is based on the volume averaging method of Anderson and Jackson [24,25]. In this approach, continuum equations for the gas phase are derived from the point form Navier-Stokes equations by converting point variables (e.g., gas velocity) to local mean variables [8]. The transformation occurs by incorporating a weighting function when averaging point variables over a region (volume) in space. Regions are defined so that they are small in comparison to macroscopic changes in flow characteristics within the system, but large enough to contain many particles [26].

The governing equations presented below only contain the source and sink terms found in references $[\mathbf{2 4 , 2 5 , 2 7 ]}$. Constitutive relationships needed to define various terms that arise during the derivation of the continuum equations (model closure) are obtained from the gas-solids flow theory outlined by Syamlal, Rogers, and O'Brien [27]. A complete review of all possible source and sink terms as well as the many constitutive relationships presented in the literature is beyond the scope of this work. 


\subsubsection{Gas phase conservation of mass (continuity equation)}

The continuity equation (also referred to as the conservation of mass) for the gas phase is given in tensor notation in Equation (1-1). Here and throughout this document, $\varepsilon_{g}$ is gas phase volume fraction, $\rho_{g}$ is gas density, $v_{g i}$ is the $i^{\text {th }}$ component of gas velocity, and $R_{g n}$ is the rate that species $n$ of the gas phase is generated or consumed. The superscript over the summation, $N_{g}$, is the total number of species that comprise the gas phase. (For example, the composition of dry air at sea level by mass percent is approximately $\mathrm{N}_{2}: 75.52 \%, \mathrm{O}_{2}: 23.15 \%$, Ar: $1.28 \%$, and $\mathrm{CO}_{2}: 0.046 \%$ [28]. A simplified model of air is described by a combination of nitrogen and oxygen resulting in $N_{g}=2$.)

$$
\frac{\partial}{\partial t}\left(\varepsilon_{g} \rho_{g}\right)+\frac{\partial}{\partial x_{i}}\left(\varepsilon_{g} \rho_{g} v_{g i}\right)=\sum_{n=1}^{N_{g}} R_{g n}
$$

The physical interpretation of the components of Equation (1-1) are identified best in the context of a control volume (CV). For the following discussion, one can think of a CV as the unit cube. The first term on the left hand side of Equation (1-1) describes the rate at which mass accumulates within the $\mathrm{CV}$. The second component expresses the rate at which mass is convected (transported) across the boundaries of the CV. The term on the right hand side specifies the rate of mass production/consumption in the $\mathrm{CV}$ of the various gas phase species.

For simulations that do not include chemical reactions or phase changes, the term on the right hand side is zero, otherwise a constitutive relationship is needed to specify this quantity. Since the rate of production/consumption is dependent on the chemical and/or physical processes modeled, no specific constitutive relationships are proposed in this section to maintain generality. 


\subsubsection{Gas phase conservation of momentum}

The conservation of momentum for the gas phase is given in tensor notation in Equation (1-2). Here, $S_{g i j}$ is the gas phase stress tensor, $g_{i}$ is the $i^{\text {th }}$ component of the gravity vector, and $I_{g m i}$ represents the exchange of momentum between the gas and the $m^{\text {th }}$ solids phase in the $i^{\text {th }}$ direction. The superscript over the summation, $M$, is the total number of solids phases present during a simulation. The term solids phase is used in this work to identify all the particles in a system that have the same density, $\rho_{s m}$, and diameter, $D_{m}$.

$$
\frac{\partial}{\partial t}\left(\varepsilon_{g} \rho_{g} v_{g i}\right)+\frac{\partial}{\partial x_{j}}\left(\varepsilon_{g} \rho_{g} v_{g i} v_{g j}\right)=\frac{\partial}{\partial x_{j}}\left(S_{g i j}\right)+\varepsilon_{g} \rho_{g} g_{i}-\sum_{m=1}^{M} I_{g m i}
$$

The first term of Equation (1-2) represents the rate at which momentum increases within the $\mathrm{CV}$. The second component indicates the rate at which momentum is transported out of the $\mathrm{CV}$ across its boundaries. The terms on the right hand side represent various sources and sinks of momentum within a $\mathrm{CV}$. The first component accounts for viscous forces acting on the gas and is defined below. The second term accounts for gravitational effects acting on the gas. The last component indicates the amount of momentum that is transferred between the gas and $m^{\text {th }}$ solids phases. This quantity is the result of several physical phenomena including the drag, pressure, Basset, Saffman, Magnus, and virtual mass forces $[29,30]$. Further definition of this term is reserved for Section 1.1.2 where it is presented in the context of the solids phase model.

The gas phase stress tensor is defined in Equation (1-3) as a combination of the gas pressure, $p_{g}$, and the viscous stress tensor, $\tau_{g i j}$. Here, $\delta_{i j}$ is the Kronecker delta. An expression (constitutive relation) for the viscous stress tensor is obtained by assuming the 
gas is a Newtonian fluid. This assumption implies that the rate of deformation of a fluid element is proportional to the viscous stresses, defined in Equation (1-4). Here $\mu_{g}^{\prime}$ is the second coefficient of viscosity for the gas phase which is taken as $\frac{2}{3} \mu_{g}$. Additional details about viscous forces and Newtonian fluids are covered in the classic fluid dynamics text by Batchelor [31].

$$
\begin{gathered}
S_{g i j}=-p_{g} \delta_{i j}+\tau_{g i j} \\
\tau_{g i j}=\mu_{g}\left(\frac{\partial v_{g i}}{\partial x_{j}}+\frac{\partial v_{g j}}{\partial x_{i}}\right)+\mu_{g}^{\prime}\left(\frac{\partial v_{g k}}{\partial x_{k}}\right) \delta_{i j}
\end{gathered}
$$

\subsubsection{Gas phase conservation of species mass}

For simulations that include phase changes (e.g., evaporation) or reactive chemistry (e.g., combustion), one must account for the generation and/or consumption of the individual species that comprise the gas phase. This is done through solving a conservation equation for each species. For a gas comprised of $N_{g}$ species, this results in $N_{g}$ equations of the form shown in Equation (1-5). In this equation, $X_{g n}$ represents the mass fraction of the $n^{\text {th }}$ species of the gas (i.e., the ratio of the mass of species $n$ in the $\mathrm{CV}$ to the total mass of the gas in the CV), $D_{g n}$ is the diffusion coefficient of the $n^{\text {th }}$ species of the gas phase, and as before, $R_{g n}$ is the rate at which the $n^{\text {th }}$ species of the gas phase is generated or consumed.

$$
\frac{\partial}{\partial t}\left(\varepsilon_{g} \rho_{g} X_{g n}\right)+\frac{\partial}{\partial x_{j}}\left(\varepsilon_{g} \rho_{g} v_{g j} X_{g n}\right)=\frac{\partial}{\partial x_{j}}\left(D_{g n} \frac{\partial X_{g n}}{\partial x_{j}}\right)+R_{g n}
$$

The first term on the left hand side of Equation (1-5) describes the rate at which the $n^{\text {th }}$ species accumulates within the $\mathrm{CV}$, and the second component indicates the rate 
at which this species leaves the CV across its boundaries. The first term on the right hand side represents the rate at which the $n^{\text {th }}$ species enters the $\mathrm{CV}$ as a result of diffusion. The second component specifies the rate at which the species is generated or consumed through chemical and/or physical processes. As in the continuity equation, no expression for $R_{g n}$ is specified to preserve generality.

\subsubsection{Gas phase conservation of internal energy}

The conservation of internal energy for the gas phase in terms of the gas temperature is shown in Equation (1-6). Here, $C_{p g}, T_{g}$, and $\kappa_{g}$ are the specific heat, temperature, and thermal conductivity of the gas, respectively; $q_{g s}$ is the energy transferred between the gas and solids phases, $\Delta H_{r g}$ is the heat of reaction, $\gamma_{R g}$ is the gas phase radiative heat transfer coefficient, and $T_{R g}$ is the gas phase radiation temperature.

$$
\varepsilon_{g} \rho_{g} C_{p g}\left(\frac{\partial T_{g}}{\partial t}+v_{g j} \frac{\partial T_{g}}{\partial x_{j}}\right)=-\frac{\partial}{\partial x_{i}}\left(-\kappa_{g} \frac{\partial T_{g}}{\partial x_{i}}\right)+q_{g s}-\Delta H_{r g}+\gamma_{R g}\left(T_{R g}^{4}-T_{g}^{4}\right)
$$

The first term on the left hand side of Equation (1-6) represents the rate at which thermal energy accumulates within the $\mathrm{CV}$, while the second component indicates the rate at which thermal energy is transferred out of the CV across its boundaries. The first term on the right hand side is the gas phase conductive heat flux and is closed (defined) assuming Fourier's law of heat conduction. The second component indicates the heat transfer between the gas and the solids in the $\mathrm{CV}$. A detailed description of this term is reserved for Section 3.4 of Chapter 3 where it is presented in the context of particle-gas convection. The third term is the heat of reaction and is the result of an endothermic (energy absorbing) or exothermic (energy creating) chemical reaction. Further explanation of this term is postponed until Chapter 4 where it is discussed in the context 
of the mathematical interface for managing gas-solids reactions. The last component is described via a simplified model for radiative heat transfer in the gas phase.

\subsubsection{Gas phase equations of state}

In addition to the gas phase continuity and internal energy equations, specifying an equation of state establishes a relationship between the thermodynamic variables of density, pressure, temperature, and specific internal energy. The ideal gas law, shown in Equation (1-7), is used commonly in CFD for compressible fluids [32]. In this example, the density of the gas is defined by the gas constant $R$ and the pressure $p_{g}$, molecular weight $M_{g}$, and temperature $T_{g}$ of the gas, respectively. If the gas phase is assumed incompressible, then it is common to define the density of the gas as a constant value.

$$
\rho_{g}=\frac{p_{g} \mathrm{M}_{g}}{R T_{g}}
$$

This concludes the outline of the equations governing the gas phase. The interested reader is directed to $[\mathbf{2 9 , 3 3}]$ for additional theoretical treatment of gas phase modeling for multiphase flows.

\subsubsection{Solids phase mathematical model}

The solids phase in the CFD-DEM is represented by a collection of discrete particles that obey Newton's equations of motion, shown in Equation set (1-8). In these equations, for a particle indexed by the superscript $(i)$, the variables $m, \mathbf{X}, \mathbf{V}, \boldsymbol{\omega}$ and $I$ indicate the particle's mass, position, linear and angular velocities, and mass moment of inertia, respectively. $\mathbf{F}_{c}^{(i)}$ are the total forces resulting from particle-particle and particlewall collisions, $\mathbf{F}_{n c}^{(i)}$ are the total forces not associated with collisions (i.e., gas-solids 
interaction, cohesion, etc.), and $\mathbf{T}^{(i)}$ is the total torque acting on particle $i$. Bold face font is used throughout this work to identify vector quantities.

$$
\begin{gathered}
\mathbf{V}^{(i)}=\frac{d}{d t}\left(\mathbf{X}^{(i)}\right) \\
\left(m^{(i)} \mathbf{g}+\mathbf{F}_{c}^{(i)}+\mathbf{F}_{n c}^{(i)}\right)=m^{(i)} \frac{d}{d t}\left(\mathbf{V}^{(i)}\right) \\
\mathbf{T}^{(i)}=I^{(i)} \frac{d}{d t}\left(\boldsymbol{\omega}^{(i)}\right)
\end{gathered}
$$

The first equation in set (1-8) defines the velocity of the particle as the time rate of change of the particle's position. The second equation is typically referred to as Newton's Second Law of Motion and relates the forces acting on a particle to the product of its mass and acceleration. The third equation, similar to the second, relates the rotational forces (torque) to the product of the particle's mass moment of inertia and angular acceleration.

\subsubsection{Particle-particle collision model}

There are two general approaches to manage collisions within the CFD-DEM [30]. The hard-sphere model, first introduced by Allen and Tildesley [34], assumes that particle-particle collisions are binary and instantaneous. Hard sphere models usually employ an event-driven algorithm to advance the particles' positions throughout a simulation. This type of algorithm results in a time step that is inversely proportional to the volume fraction of the system [35]. This can produce prohibitively small time steps in simulations where dense regions of particles may occur. Furthermore, enduring, multiparticle collisions may arise in dense regions of a system, violating the assumption that particle collisions are binary $[\mathbf{3 5}, 36]$. These limitations lead to the adoption of the soft- 
sphere model for more complex simulations [37]. However, the interested reader is directed to references $[\mathbf{3 0}, \mathbf{3 4}, \mathbf{3 8}, \mathbf{3 9}, \mathbf{4 0}]$ for additional details about hard-sphere models.

The soft-sphere model uses mechanical spring, dashpot and slider elements to account for the elastic, damping and rotational forces generated during a collision. The total collision force is comprised of normal and tangential components which are the result of a small overlap of the colliding particles, as illustrated in Figure 1-1.

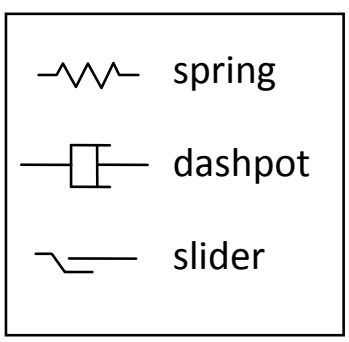

Figure 1-1: Schematic of the soft-sphere model that employs mechanical elements to simulate particle-particle and particle-wall collisions.

The normal force resulting from contact is given by Equation (1-9). The first term is the conservative (spring) force while the second term is the dissipative (damping) force. In this equation, $k_{n}$ is the normal spring stiffness, $\delta_{n}$ is the magnitude of the normal overlap, $\xi_{n}$ is the unit normal vector relating the particles' centers, $\eta_{n}$ is the normal damping coefficient, and $\mathbf{V}_{n}$ is the relative normal velocity. The algebraic and geometric definitions for these variables are reserved for Appendix A.

$$
\mathbf{F}_{n}^{(i, j)}=-k_{n} \delta_{n} \xi_{n}-\eta_{n} \mathbf{V}_{n}
$$

A Coulomb-type friction law is used for the tangential force in Equation (1-10). The first portion is the force associated with static contact between two touching particles and contains conservative and dissipative components. The second element is the force 
associated with frictional slip between the particles' surfaces. In these equations, $k_{t}$ is the tangential spring stiffness, $\boldsymbol{\delta}_{t}$ is the tangential displacement, $\eta_{t}$ is the tangential damping coefficient, $\mathbf{V}_{t}$ is the relative tangential velocity, $\mu$ is the friction coefficient, and $\xi_{t}^{\prime}$ is the tangent unit vector corrected for prolonged contact. As before, the algebraic and geometric definitions for these variables are reserved for Appendix A.

$$
\mathbf{F}_{t}^{(i, j)}=\left\{\begin{array}{lll}
-k_{t} \boldsymbol{\delta}_{t}-\eta_{t} \mathbf{V}_{t} & \text { for } & \left|\mathbf{F}_{t}\right| \leq \mu\left|\mathbf{F}_{n}\right| \\
-\mu\left|\mathbf{F}_{n}\right| \xi_{t}^{\prime} & \text { for } & \left|\mathbf{F}_{t}\right|>\mu\left|\mathbf{F}_{n}\right|
\end{array}\right.
$$

The total collision force acting on particle $(i)$ is given by Equation (1-11). Here, $\mathfrak{C}$ represents the set of particles in contact with particle $(i)$.

$$
\mathbf{F}_{c}^{(i)}=\sum_{k \in \mathbb{E}}\left(\mathbf{F}_{n}^{(i, k)}+\mathbf{F}_{t}^{(i, k)}\right)
$$

The torque acting on particle $(i)$ from contact with particle $(j)$ is given by Equation (1-12). Here, $L^{(i)}$ is the distance from the center of particle $(i)$ to the point of contact. The total torque on particle $(i)$ is given by Equation (1-13).

$$
\begin{gathered}
\mathbf{T}^{(i, j)}=L^{(i)} \xi_{n} \times \mathbf{F}_{t}^{(i, j)} \\
\mathbf{T}^{(i)}=\sum_{k \in \mathfrak{C}} \mathbf{T}^{(i, k)}
\end{gathered}
$$

The soft sphere model uses a traditional time step-driven algorithm to advance the particles' positions throughout a simulation. The step size needed for numerical stability is a function of the spring stiffness and is typically small [9]. Additional details about the soft-sphere model as well as the spring and damping coefficients are provided in references $[35,41]$. 


\subsubsection{Particle-wall collision model}

Particle-wall collisions are managed similarly to particle-particle collisions with additional complexity arising from the need to represent the wall [41]. This work uses artificial particles to act as the wall during a collision. When the surface of a particle initiates contact with a boundary plane (wall), an artificial particle of the same size is generated. The artificial particle is assigned the physical properties associated with the wall and placed across the boundary, opposite the colliding particle, outside the computational domain. This process is illustrated in Figure 1-2-(a). Once the forces generated from the particle-wall collision are calculated, the artificial particle is removed. After the position and trajectory of the colliding particle are updated, a new artificial particle is created if the colliding particle remains in contact with the wall. This procedure continues until the collision has evolved fully and the colliding particle is no longer in contact with the wall, as illustrated in Figure 1-2-(b). This method of modeling particle-wall collisions ensures that any normal forces generated during the collision are always perpendicular to the boundary plane. 


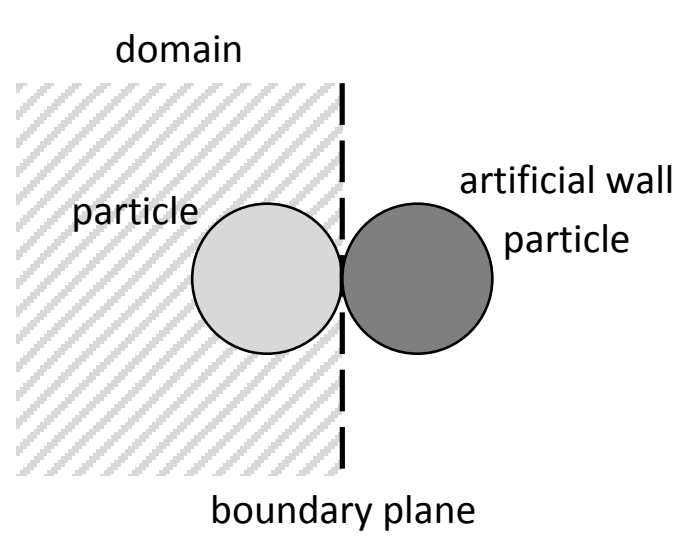

(a)

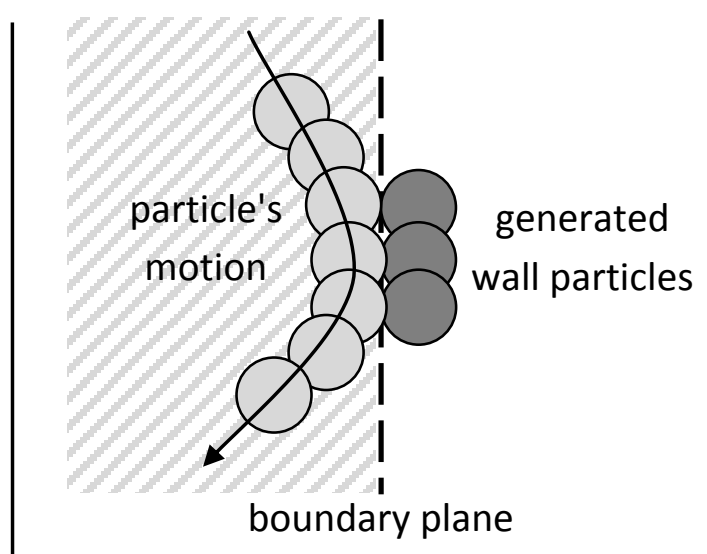

(b)

Figure 1-2: (a) During a particle-wall collision, an artificial particle the same size of the colliding particle is generated to represent the wall. The artificial particle is placed opposite the colliding particle, outside the computational domain. (b) As the position and trajectory of the colliding particle change, the artificial particles are replaced by new artificial particles to ensure that normal forces remain normal to the boundary plane.

\subsubsection{Gas-solids momentum transfer}

Interphase momentum transfer is the result of several physical phenomena including the drag, Basset, Saffman, Magnus, and virtual mass forces $[\mathbf{2 9 , 3 0 , 4 2 ]}$. This work assumes that gas-solids drag is the only significant interaction force between the gas and solids. Consequently, only the gas-solids drag term is presented below.

The gas-solids drag force acting on the particle indexed by $(i)$, belonging to solids phase $m$, and contained within the control volume (CV) is shown in Equation (1-14). Here, $\mathcal{V}^{(i)}$ is the volume of the particle $\left(\frac{4}{3} \pi\left(R_{p}^{(i)}\right)^{3}\right), p_{g}^{(i, C V)}$ is the gas phase pressure at the particle's position, $\varepsilon_{s m}^{C V}$ is the solids volume fraction of the $m^{\text {th }}$ solids phase in the $\mathrm{CV}$, and $\boldsymbol{v}_{g}^{(i, C V)}$ is the gas phase velocity at the particle's position. $\beta_{m}^{(i, C V)}$ is the local gas-solids momentum transfer coefficient for particle $(i)$ in the CV. Several expressions for $\beta_{m}^{(i, C V)}$ are available in the literature [42], therefore no specific model is presented here to preserve generality. 


$$
\mathbf{F}_{n c}^{(i)}=-\mathcal{V}_{p}^{(i)} \nabla p_{g}^{(i, C V)}+\frac{\beta_{m}^{(i, C V)} \mathcal{V}_{p}^{(i)}}{\varepsilon_{s m}^{C V}}\left(\boldsymbol{v}_{g}^{(i, C V)}-\mathbf{V}^{(i)}\right)
$$

Equation (1-15) defines the corresponding force acting on the gas phase through the interphase momentum transfer term, $\mathbf{I}_{g m}$. Here, $\mathcal{V}_{C V}$ is the volume of the $\mathrm{CV}, N_{m}$ is the number of solids phase $m$ particles contained in the $\mathrm{CV}$, and $K_{C V}^{(i)}$ is a function used to distribute the point-form drag force to the computational nodes of the gas phase Eulerian grid.

$$
\mathbf{I}_{g m}=\frac{1}{v_{C V}} \sum_{k=1}^{N_{m}} \mathbf{F}_{n c}^{(k)} K_{C V}^{(k)}
$$

This concludes the outline of the solids phase model. The interested reader is referred to $[35,37,41,42]$ for additional information concerning the DEM and its implementation.

\subsection{Dissertation objectives}

Efforts to further enhance the CDM, with a strong emphasis placed on heat transfer and specific reactive chemistry models, are published in the literature $[2,11,16,17,18,19,20,21,22,23]$. The goal of this work is to add to this body of research by introducing three new modeling capabilities to an existing CFD-DEM:

1. develop discrete mass boundary conditions;

2. construct a mathematical description of particle scale heat transfer in the context of gas-solids flows; and

3. develop a generic mathematical interface to manage user-specified gas-solids chemical reactions.

MFIX-DEM (Multiphase Flow with Interphase eXchange), an open-source multiphase flow software package $[\mathbf{2 7 , 4 3}]$, is the CFD-DEM framework wherein all work 
is conducted. Accordingly, all programmatic efforts follow the FORTRAN90/95 coding standard.

\subsection{Document organization}

Chapter 2 presents the algorithmic description of the discrete mass inflow and outflow boundary conditions. Details concerning the implementation of the boundary conditions into MFIX-DEM are discussed and two examples illustrating the behavior of the discrete mass inflow boundary condition are given. Chapter 3 begins by defining the three modes of heat transfer and the internal energy equation for a single, isothermal particle. An examination of the assumption of isothermal particles is conducted. In the subsequent sections, an analysis of contact conduction, particle-fluid-particle conduction, particle-gas convection, and particle-environment radiation are presented. Each investigation includes (1) a survey of models proposed for the specified mode of heat transfer, and (2) a discussion detailing modifications made to the selected model. Finally, the algorithmic implementation and verification of the selected heat transfer models are

given. In Chapter 4, a discussion of reactive chemistry is presented in conjunction with a survey of previous efforts to incorporate reactive chemistry into the CFD-DEM. Then the mathematical interface for managing user-defined chemical reactions and its implementation into MFIX-DEM are outlined. Finally, a brief study verifying the interface is discussed. Finally, in Chapter 5 a summary of this work's efforts is presented along with areas of interest for future investigations. 


\section{Chapter 2: Discrete mass boundary conditions}

In mathematics, boundary conditions, like those of Neumann and Dirichlet, are typically considered in the context of differential equations. However, in a discrete

element model (DEM), there is no natural analogue to specify the flux of solids into a system. This investigation aims to develop a Neumann-like boundary condition algorithm for introducing new solids into a simulation that employs the DEM. This work also describes the construction of a mass outflow boundary region. The algorithms are termed the discrete mass inflow boundary condition (DMIBC) and discrete mass outflow boundary condition (DMOBC).

\subsection{Introduction}

Recall from Chapter 1 that the contact force scheme of Cundall and Strack [44] is adopted for this work. In their model, mechanical elements are used to account for the elastic, damping and rotational forces generated during a particle-particle collision. As particles collide, the deformation of each particle's shape is captured through an overlap of their contact regions. The magnitude of the overlap is related to the scale of the forces resulting from impact. As the collision evolves, the resultant forces are used to recalculate the positions, trajectories, and rotational speeds of the particles.

Since the overlap between particles is used to determine the kinetic response to a collision, a particle entering the system cannot be placed overlapping another particle. This limitation is apparent if a DMIBC is defined along a domain region that is associated with a high particle density, such as a packed bed. To avoid improper placement of particles while retaining the ability to define a DMIBC along any region of 
the domain, a staging area is designated outside of the computational domain. This is illustrated in Figure 2-1.

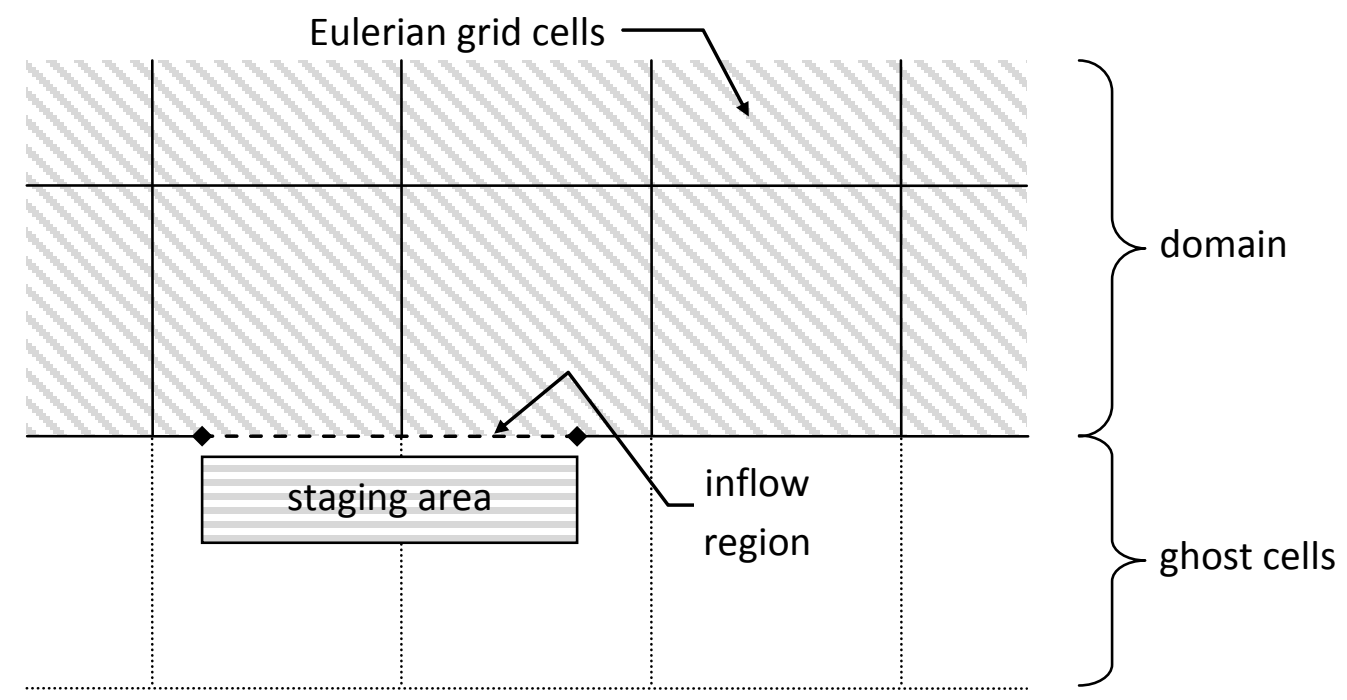

Figure 2-1: The staging area for incoming particles is located outside of the computational domain, in the ghost cells bordering the region identified with the boundary condition.

Particles are seeded into the staging area and then incrementally moved across the boundary into the domain of the system. The behavior of the particles within the staging area is controlled so that given acceptable boundary condition parameters (discussed in Section 2.2.1), a new particle can be introduced to the staging area without overlapping previously inserted particles.

As a particle passes from the staging area into the computational domain, contact forces acting on the incoming particle are dismissed temporarily but particles already within the system respond accordingly. Thus, entering particles will move particles obstructing their entry out of the way, despite any disparities in size or density. Once an incoming particle has entered the domain fully, it then responds to any forces acting on it in complete accordance to the DEM. Here it is assumed that since there is a net flux of particles into the system and the solids fraction near the inlet region is relatively low (i.e., 
not packed) for most situations $[\mathbf{4 5 , 4 6}]$, the overall effect of incoming particles neglecting contact forces is negligible.

An outflow boundary condition for the DEM results in a modification of the particle-wall collision algorithm. In its application, when a particle collides with a wall region associated with an outlet, the collision force is ignored. This allows the particle to move from the computational domain to a bordering ghost cell over a series of time steps. Once the particle has exited the computational domain fully, all instances of the particle are removed from memory.

\subsection{DMIBC algorithm description}

The DMIBC has two operating components. The first element is called during the initialization of the software, wherein user provided data is checked for consistency, and operational characteristics are determined. The second element is executed during the simulation, and controls the placement and assignment of physical properties to incoming particles. The two components of the algorithm are outlined below.

\subsubsection{DMIBC initialization}

To define the operational characteristics of a DMIBC, the physical properties and feed rates of the incoming particles are specified. The physical properties of an incoming particle are identified through its solids phase. As previously stated, the solids phase is associated with the diameter, $D_{m}$, and material density, $\rho_{s m}$, of a particle. Note, particles belonging to the solids phase indexed by $m$ are identified by a subscript $m$ and are referred to as 'solids phase $m$ ' particles. The feed rate for each solids phase is given by a volumetric flow rate and bulk density, $\dot{\mathcal{V}}_{m}$ and $\rho_{b m}$, respectively. Here $\rho_{b m}$ is the 
product of the material density and the volume fraction of the solids phase $m$ particles for the feed, $\varepsilon_{s m}$.

Next, the flow properties for each solids phase are discretized. This is done by converting the solids phase $m$ volumetric flow rate into an equivalent number of solids phase $m$ particles entering the system per unit time, $N_{m, t}$. The total number of incoming particles per unit time, $N_{t}$, is given by summing $N_{m, t}$ over all solids phases.

$$
N_{m, t}=\frac{\dot{v}_{m}}{\frac{\pi}{6} D_{m}^{3}} \quad \text { and } \quad N_{t}=\sum_{m} N_{m, t}
$$

The discrete feed rate values, $N_{m, t}$ and $N_{t}$ are used to establish the distribution of incoming particles. The distribution is stored in an array where each element contains the index of a single solids phase (see Figure 2-2). The number of elements that contain the index for solids phase $m$ is proportional to the ratio $N_{m, t} / N_{t}$. When a particle is seeded, a random element of the distribution array is selected and the properties corresponding to the associated index are assigned to the new particle. By assigning solids phase values in this manner, the local distribution of incoming particles remains random while the globally set distribution is preserved.

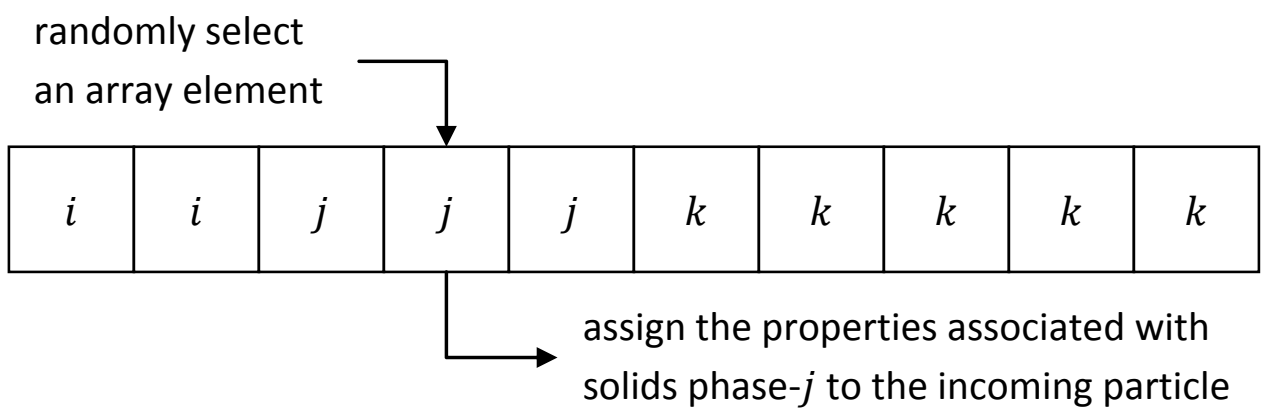

Figure 2-2: Example distribution array with ten elements, three mass solids phase indices $(i, j, k)$ with the respective distribution $(20 \%, 30 \%, 50 \%)$. The fifth array element is randomly selected, leading to the incoming particle being assigned the properties associated with solids phase-j. 
The discrete feed rate is adjusted to the solids time step, $\Delta t_{s}$, by determining the placement frequency of particles, $F_{p}$, shown in Equation (2-2). If $F_{p}$ is equal to one, one or more particles is placed every solids time step. If the value is greater than one, $F_{p}$ represents the number of solids time steps that transpire between the placement of individual particles.

$$
F_{p}=\left\lfloor\frac{1}{N_{t} \Delta t_{s}}\right\rfloor
$$

In Equation (2-2), $\lfloor\cdot]$, is the standard floor function that returns the integer portion of the provided value. The truncation results in the actual feed rate of solids differing slightly from the user provided parameters. This is an unavoidable consequence of translating a continuous description of the feed rate into a discrete representation.

Once the placement frequency is known, an initial velocity for each incoming solids phase, $v_{m}^{0}$, is determined, shown by Equation (2-3). The feed rate and solids properties are combined with the cross-section inlet area, $A_{B C}$, to calculate the corresponding initial velocities for each solids phase.

$$
v_{m}^{0}=\frac{\dot{v}_{m}}{\frac{\rho_{b m}}{\rho_{s m}} A_{B C}}
$$

The solids phase $m$ velocities are used to determine a uniform inlet velocity that is applied to all solids phases. This prevents the generation of particle overlaps within the staging area. Without this condition, an overlap might arise due to any velocity differences. This event is illustrated in Figure 2-3, where a fast moving particle is seeded behind a slower moving particle. As the particles move toward the domain, the fast moving particle overtakes the slower moving particle resulting in an overlap between the 
two particles. Once the slow moving particle fully enters the domain, the overlap is treated as the result of a collision. A sufficiently large overlap has the potential to generate an artificial spike in the total kinetic energy of the system, adversely affecting the integrity of the model. The scenario is avoided by imposing a uniform inlet velocity.
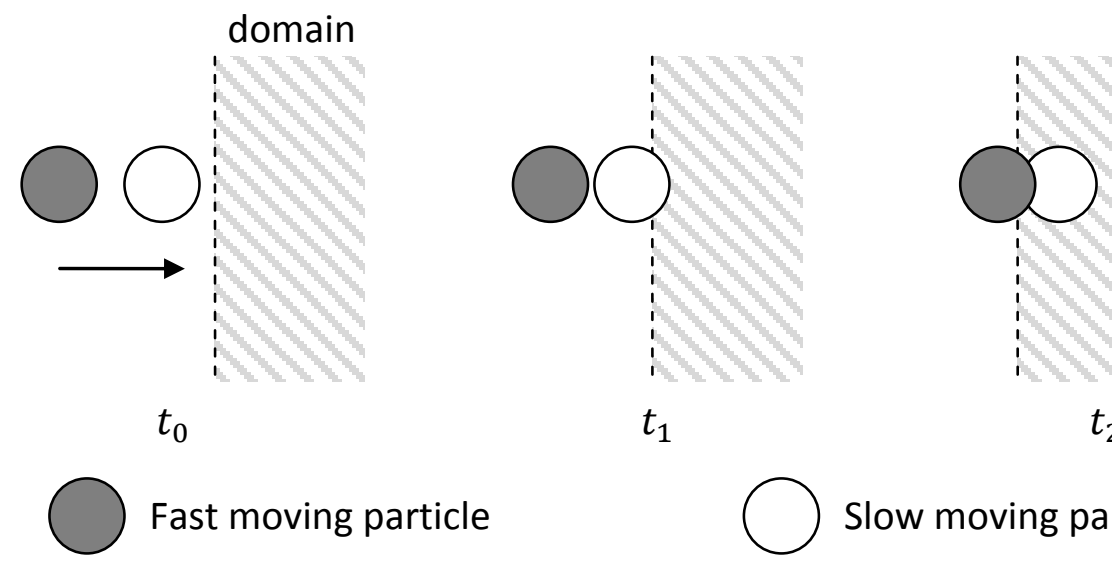

$$
t_{1}
$$$$
t_{2}
$$

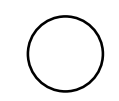

Slow moving particle

Figure 2-3: Motivation for a uniform inlet velocity. A slow moving particle is seeded behind a fast moving particle generating an artificial overlap.

The uniform inlet velocity is selected to minimize the error between userprovided and calculated bulk density values. This is accomplished through a simple iterative procedure described below.

To begin the iterative selection process for the uniform inlet velocity, the minimum and maximum phase- $m$ velocities specified by Equation (2-3), $\min _{m} v_{m}^{0}$ and $\max _{m} v_{m}^{0}$, are used to generate $(s+1)$ test velocity values, $v_{\text {test }}^{i}$. The calculation of the test velocities is shown in Equation (2-4). Here, $s$ is a parameter with integer value, like 100 , that influences the number of iterations.

$$
v_{\text {test }}^{i} \in\left\{\min _{m} v_{m}^{0}+i * \frac{\max _{m} v_{m}^{0}-\min _{m} v_{m}^{0}}{s} \mid i \in\{0,1, \ldots, s\}\right\}
$$

For a given test velocity, a corresponding test bulk density is computed for each solids phase, $\tilde{\rho}_{b m}\left(v_{\text {test }}^{i}\right)$, shown. Here, $A_{B C}$ is the area of the inlet region. 


$$
\tilde{\rho}_{b m}\left(v_{\text {test }}^{i}\right)=\frac{\rho_{\text {sm }} \dot{\mathcal{V}}_{m}}{v_{\text {test }}^{i} * A_{B C}}
$$

At this point, the total error, $E r r_{v_{t e s t}^{i}}$, between the user-defined and newly computed test bulk density values is calculated. The test velocity that produces the least amount of total bulk density error is selected. This process is repeated with a new set of test velocities established around the minimizing velocity of the previous iteration, continuing until the difference between the results of two consecutive iterations is less than a specified tolerance (e.g., $\left.10^{-4}\right)$.

$$
\operatorname{Err}_{v_{\text {test }}^{i}}=\sum_{m}\left|\rho_{b m}-\tilde{\rho}_{b m}\left(v_{\text {test }}^{i}\right)\right|
$$

Finally, this optimized uniform inlet velocity is compared to two velocity values, explained below. The first defines a lower bound on the inlet velocity, while the second identifies the method used to seed incoming particles. These comparisons assure that the user-defined inlet specifications are successfully managed, and determine which of the two seeding algorithms, also presented below, is employed.

The lower bound on the inlet velocity is the minimum operational velocity, $v_{\min }$. It coincides with an unmanageable situation where particles are seeded into the staging area faster than they leave. Below $v_{\min }$ a new particle may be seeded such that it overlaps with an existing particle still within the staging area. Again, if the overlap is sufficiently large, it may cause an artificial spike in the kinetic energy of the system.

To prevent particle overlap within the staging area, a sub-grid is constructed to keep particles fully separated. The number of cells in the sub-grid, $N_{B C}$, depends on the 
dimensions of the boundary inlet, $l_{B C}$ and $w_{B C}$, and the maximum particle diameter. The calculation used to determine the number of cells is shown in Equation (2-7).

$$
\begin{aligned}
& N_{B C}=\left\lfloor\frac{l_{B C}}{D_{\max }}\right\rfloor\left\lfloor\frac{w_{B C}}{D_{\max }}\right\rfloor \\
& \text { where } \quad D_{\max }=\max _{m} D_{m}
\end{aligned}
$$

$N_{B C}$ is equivalent to the maximum number of disks of maximum diameter, $D_{\max }$, that can be positioned in a square packing arrangement in a rectangle of the given dimensions. The cell sizes are uniform with their dimension based upon the maximum particle diameter to ensure that every cell can accommodate all specified particle diameters. This is a necessary condition because the diameter of an incoming particle is not established until the time of placement.

Next, a minimum inlet velocity is defined from the sub-grid and feed rate properties. Recall that if the placement frequency, $F_{p}$, is equal to one, then $N_{p s}$ particles are seeded every solids time step, and if $F_{p}$ is greater than one, one particle $\left(N_{p s}=1\right)$ is seeded every $F_{p}$ solids time steps. Thus, with $N_{B C}$ locations available within the sub-grid, it follows that $\left\lfloor N_{B C} / N_{p s}\right\rfloor$ placements can occur before two particles are seeded in the same cell. Therefore, the first $N_{p s}$ particles that are seeded must exit the sub-grid by the time the $\left(\left\lfloor N_{B C} / N_{p s}\right\rfloor+1\right)$ placement occurs. This assures that $\left(N_{p s}+N_{B C}-\right.$ $\left.N_{p s}\left\lfloor N_{B C} / N_{p s}\right\rfloor\right)$ cells are available to receive a new particle without that particle overlapping a previously seeded particle (see Figure 2-4). The minimum inlet velocity, presented in Equation (2-8), is the direct result of this requirement, establishing the lower bound on incoming particle velocity. 


$$
v_{\min }=\frac{D_{\max }}{\Delta t_{s} F_{p}\left\lfloor\frac{N_{B C}}{N_{p s}}\right\rfloor}
$$
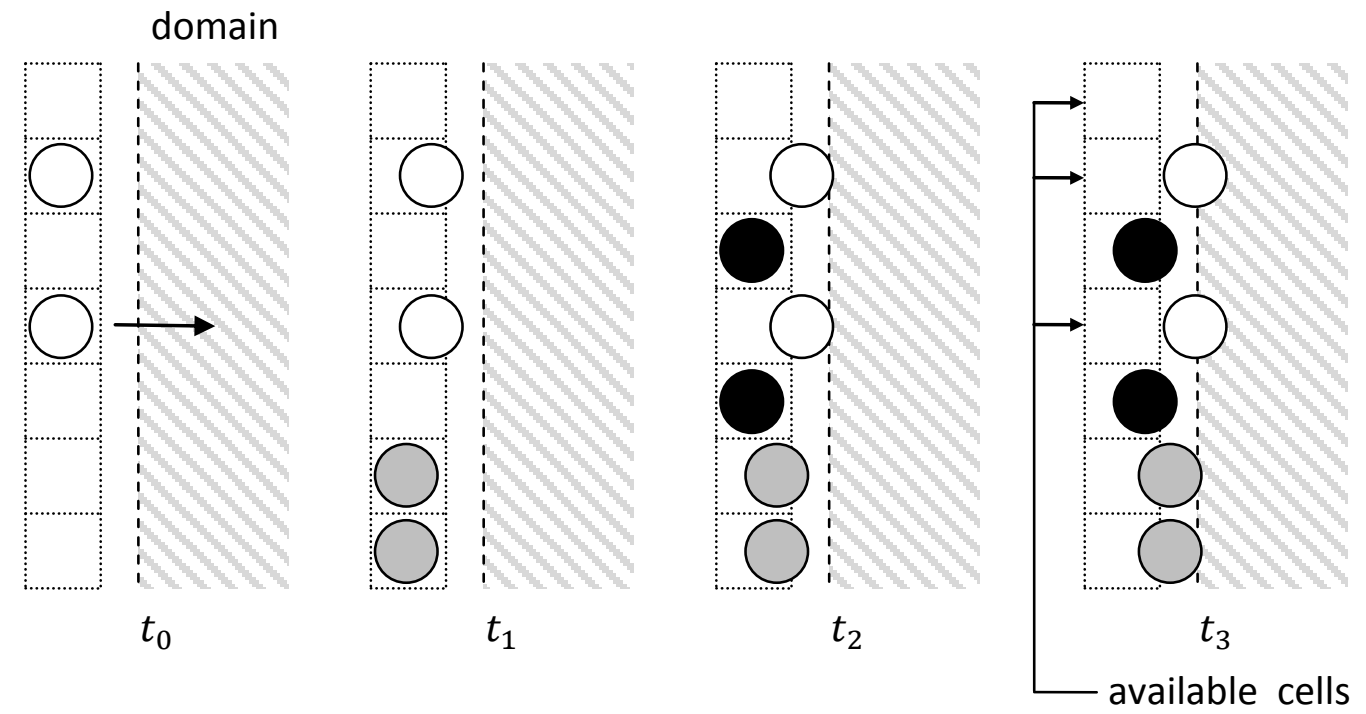

Figure 2-4: Illustration of the seeding process of the grid structure. In this example, the sub-grid in the staging area contains seven cells, while two particles are placed every solids time step. Therefore, the sub-grid will overfill in four solids time steps (eight particles and only seven cells) if incoming particles do not move with sufficient speed toward the domain leaving available cells for new particles.

Once the lower bound is defined, the manner in which particles are seeded into the staging area is determined. Particles are placed either in an ordered arrangement or randomly distributed throughout the staging area.

If the staging area is densely populated, randomly placing particles potentially becomes computationally expensive. Therefore random placement is reserved for instances when a square packing would occupy less than half of the staging area an an given instant. The lower bound for the velocity correlating to less than half occupancy is given by Equation (2-9).

$$
v_{\text {rand }}=\frac{D_{\max }}{\Delta t_{s} F_{p}\left\lfloor\left\lfloor\frac{N_{B C}}{2}\right\rfloor / N_{p s}\right\rfloor}
$$


With the velocities $v_{\min }$ and $v_{\text {rand }}$ indentified, the placement method of particles is determined. If the inlet velocity is between $v_{\min }$ and $v_{\text {rand }}$, particles are placed in the square packing arrangement defined by the grid structure imposed on the staging area. In this instance a fill order for the grid cells is generated. (This pattern is repeated cyclically to seed additional particles.)

For velocities greater than $v_{\text {rand }}$ the particles are seeded via a guess-and-check (random) method. First, a particle is tentatively assigned a random location within the staging area. This position is independent of the sub-grid imposed on the staging area. Then a check is conducted to test if the location causes particle overlap with inlet particles that were previously seeded. If an overlap occurs, the particle is assigned a new random location within the staging area. This process continues until the particle is placed without any overlaps.

After a particle is seeded into the staging area, the ghost cell that contains the new particle is determined (see Figure 2-1). The cells within the computational domain that border those ghost cells in the inlet area are identified. Finally, a local search is conducted that allows for particles already within the computational domain to identify incoming particles as possible contact partners.

\subsubsection{DMIBC operation}

During a simulation, the placement frequency is used to calculate when the next particle is seeded. During each solids time step, the current time value is compared to a placement time, $t_{p}$. If $t_{p}$ is less than or equal to the current solids time, a new particle is seeded into the inlet region, and the next seeding time updated. 


$$
t_{p}=t_{p}+F_{p} \Delta t_{s}
$$

When a new particle is seeded, a flag is set identifying it as a new particle which indicates the dismissal of any contact forces the particle is exposed to during entry. This classification is maintained only until the particle has entered the computational domain fully. As a result, entering (new) particles will push obstructing particles in the computational domain away from the inlet.

Next, physical properties are assigned to the new particle through the appointment of a solids phase index. As previously discussed, an element from the array representing the incoming particle distribution is selected randomly. That array element contains the index of a single solids phase. From this selection, the physical properties associated with that index are assigned to the incoming particle.

After the solids phase is assigned, the center location of the new particle is determined. For an inlet using an ordered placement scheme, the sub-grid cell that will contain the new particle is identified. If the dimensions of the cell are larger than the diameter of the particle, the particle is positioned randomly within the cell; the particles that have delimited the cell dimensions are placed on cell-center. For an inlet using the random placement, the sub-grid imposed on the staging area is discarded and a guessand-check process initiated. New locations within the staging area are assigned to the particle until it is placed without overlapping another particle.

Finally, the ghost cell that contains the new particle is determined. A local search is conducted allowing particles already within the computational domain to identify incoming particles as possible contact partners. 
The positions of incoming particles are monitored during each solids time step. Once a particle has fully entered the computational domain, the flag identifying the particle as new is removed. This reclassification allows the particle to react appropriately to any force that acts upon it.

\subsection{DMOBC algorithm description}

The DMOBC operates in conjunction with the existing DEM framework. Namely, during the simulation a check is performed on each particle to ascertain whether or not it is in contact with any walls of the domain. If a particle is found to be in contact with a wall that contains a discrete mass outlet, an additional check is initiated. If the contact occurs at a region identified with a DMOBC, then the resulting particle-wall collision forces are dismissed. This, in effect, makes the wall invisible to the particle, allowing it to move out of the computational domain. This is synonymous with the process that allows incoming particles to travel from the staging area into the computational domain.

The forces acting on an exiting particle are discarded once its center crosses the domain boundary. This allows particles partially carried out of the computational domain by grid-dependent forces (e.g., gas-solids drag) to continue exiting with a constant velocity once these forces are no longer calculable.

A check is conducted at the end of each solids time step to identify particles that have fully exited the computational domain. Once a particle is completely outside the domain, a logical flag indicating the existence of the particle is removed, and the array positions associated with that particle are cleared. The vacated array positions are populated by information for incoming particles as-needed. By reusing the array positions, the overall amount of system resources needed during a simulation are reduced. 


\subsection{Implementation}

The discrete mass boundary conditions were implemented into the DEM of the open-source multiphase flow software MFIX. This required the modification and creation of several subroutines. Overviews of the new subroutines are listed in Table 2-1 with their complete code included in Appendix C. Modifications to existing routines mainly consisted of logical checks associated with call statements to the new routines. A call-tree showing the invocation of the initialization routines is shown in Figure 2-5, and a calltree showing the invocation of the operation routines is shown in Figure 2-6.

Table 2-1: A list of the subroutines comprising the discrete mass boundary conditions with brief description of their use.

\begin{tabular}{|c|c|}
\hline $\begin{array}{l}\text { Subroutine } \\
\text { file name }\end{array}$ & Description \\
\hline $\begin{array}{c}\text { CHECK_DES_BC } \\
\text { check_des_bc.f }\end{array}$ & $\begin{array}{l}\text { Check the data provided for the DES mass inflow } \\
\text { boundary condition and flag errors if the data is } \\
\text { improper. Also, this module is used to convert the } \\
\text { provided information into the format necessary for the } \\
\text { dependent subroutines to function properly. }\end{array}$ \\
\hline $\begin{array}{c}\text { ALLOCATE_DES_MIO } \\
\text { des_allocate_arrays.f }\end{array}$ & $\begin{array}{l}\text { Allocate the arrays associated with the mass inflow and } \\
\text { outflow boundary conditions. }\end{array}$ \\
\hline $\begin{array}{c}\text { CHECK_DES_MIO_LOCATION } \\
\text { check_des_bc.f }\end{array}$ & $\begin{array}{l}\text { Verify that the location of the inlet or outlet is specified } \\
\text { on the wall of the system. }\end{array}$ \\
\hline $\begin{array}{c}\text { DES_BC_VEL_ASSIGN } \\
\text { des_init_bc.f }\end{array}$ & Calculate the uniform inlet velocity. \\
\hline $\begin{array}{c}\text { DES_INIT_BC } \\
\text { des_init_bc.f }\end{array}$ & $\begin{array}{l}\text { Driver program; calls the necessary routines to determine } \\
\text { the operation of the DMIBC. }\end{array}$ \\
\hline $\begin{array}{c}\text { DES_MI_CELLS } \\
\text { des_init_bc.f }\end{array}$ & $\begin{array}{l}\text { Identifies the computational fluid cells that border the } \\
\text { DMIBC. }\end{array}$ \\
\hline $\begin{array}{c}\text { DES_MI_CLASSIFY } \\
\text { des_init_bc.f }\end{array}$ & Classifies the plane used for seeding particles. \\
\hline
\end{tabular}




\begin{tabular}{|c|c|}
\hline $\begin{array}{c}\text { DES_MI_LAYOUT } \\
\text { des_init_bc.f }\end{array}$ & $\begin{array}{l}\text { Determines if the particles are randomly or orderly } \\
\text { placed in the staging area. }\end{array}$ \\
\hline $\begin{array}{l}\text { DES_MIO_PERIODIC } \\
\text { des_init_bc.f }\end{array}$ & $\begin{array}{l}\text { Verifies that an inlet or outlet has not been placed on a } \\
\text { periodic wall and that any inlet or outlet will not interfere } \\
\text { with any periodic walls. }\end{array}$ \\
\hline $\begin{array}{c}\text { DES_MO_CLASSIFY } \\
\text { des_init_bc.f }\end{array}$ & Classifies the plane that the outlet uses. \\
\hline $\begin{array}{c}\text { DES_MASS_INLET } \\
\text { des_mass_inlet.f }\end{array}$ & $\begin{array}{l}\text { Determines the physical properties of an incoming } \\
\text { particle. }\end{array}$ \\
\hline $\begin{array}{c}\text { DES_NEW_PARTICLE_TEST } \\
\text { des_mass_inlet.f }\end{array}$ & $\begin{array}{l}\text { Tests to see if a randomly seeded particle is placed } \\
\text { overlapping an existing particle. }\end{array}$ \\
\hline $\begin{array}{c}\text { DES_PLACE_NEW_PARTICLE } \\
\text { des_mass_inlet.f }\end{array}$ & Seeds a new particle in the identified location. \\
\hline $\begin{array}{c}\text { DES_BC } \\
d e s \_b c \_m o d . f\end{array}$ & $\begin{array}{l}\text { Global data storage for common boundary condition } \\
\text { variables. }\end{array}$ \\
\hline $\begin{array}{c}\text { DES_CHECK_PARTICLE } \\
\text { des_check_particle.f }\end{array}$ & $\begin{array}{l}\text { Checks to see if an entering/exiting particle has } \\
\text { entered/left the computational domain fully. }\end{array}$ \\
\hline $\begin{array}{l}\text { DES_MASS_OUTLET } \\
\text { cfwallcontact.f }\end{array}$ & $\begin{array}{l}\text { Checks to see if a particle in the system is interacting } \\
\text { with a boundary region specified with a mass outlet. }\end{array}$ \\
\hline
\end{tabular}




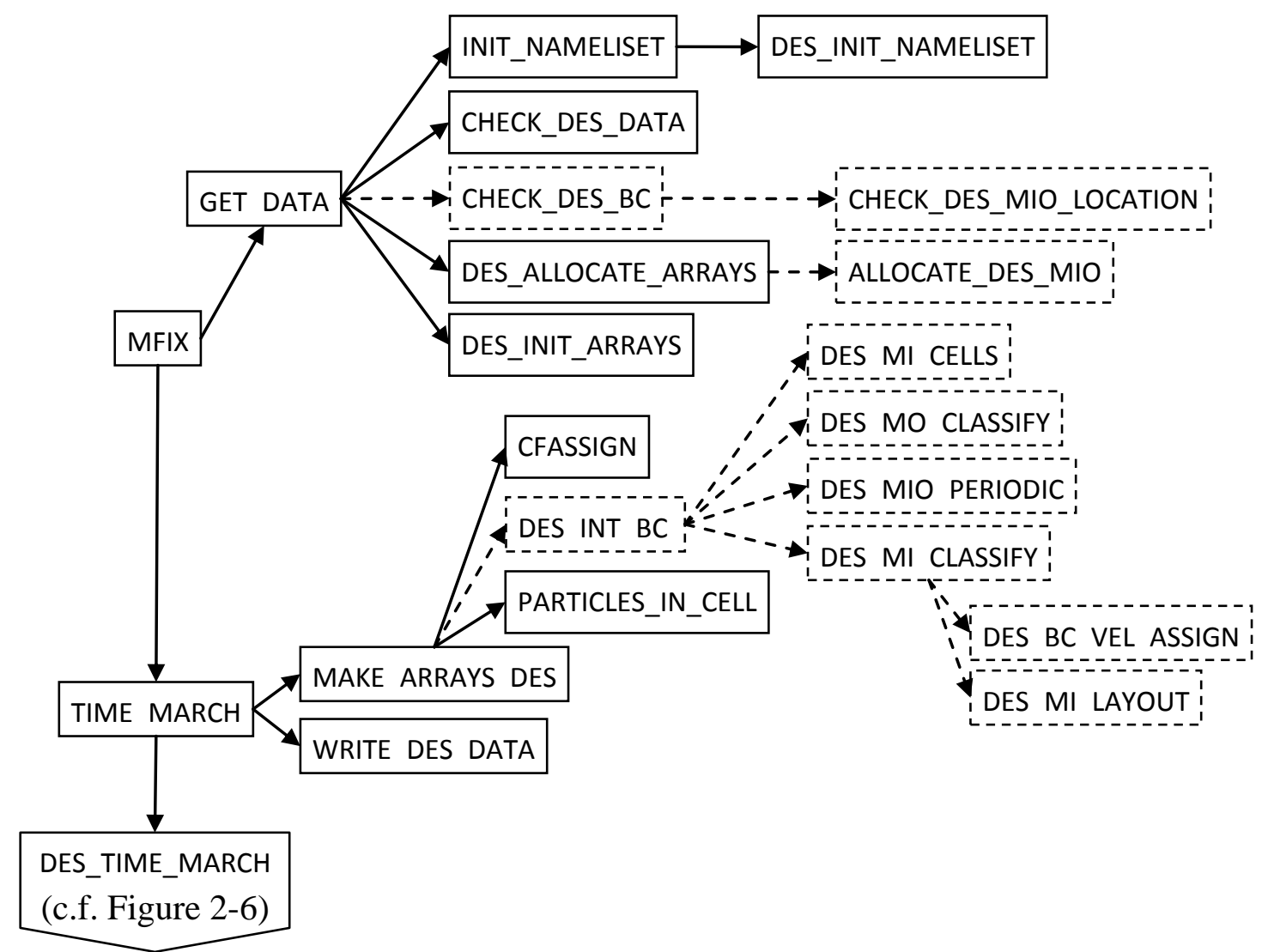

Figure 2-5: Call graph of main MFIX source code to initialization routines for the discrete particle model. Dashed lines indicate new calls and subroutines implemented for the discrete mass boundary conditions. 


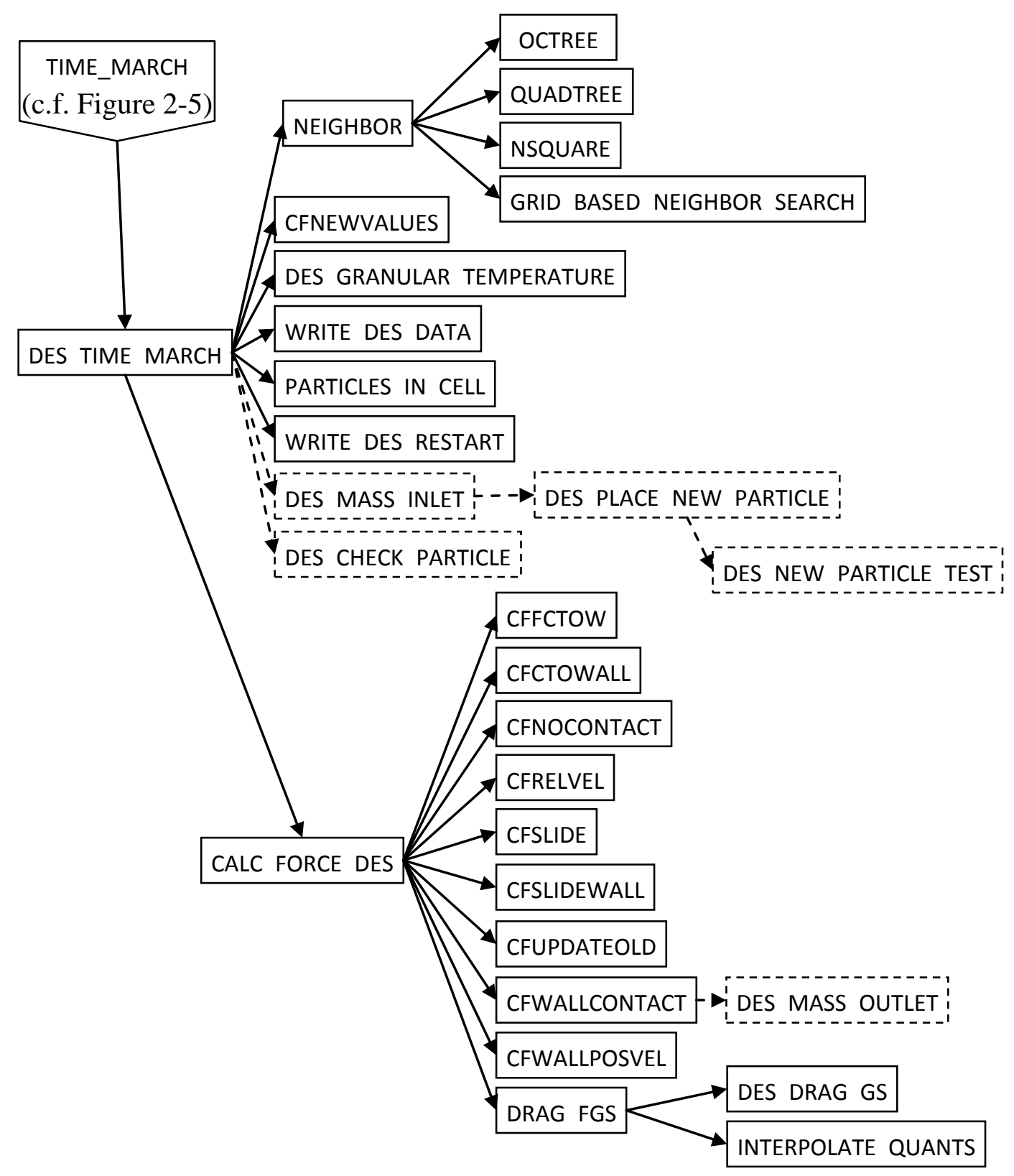

Figure 2-6: Call graph of MFIX discrete particle model routines. Dashed lines indicate new calls and subroutines implemented for the discrete mass boundary conditions.

A flow chart illustrating the overall DEM algorithm implemented in MFIX is presented in Figure 2-7. The additions to the algorithm including the effects of the discrete mass boundary conditions are indicated by dashed lines. If a simulation is conducted without a discrete mass boundary condition, these modifications are omitted, resulting in the original code structure. 


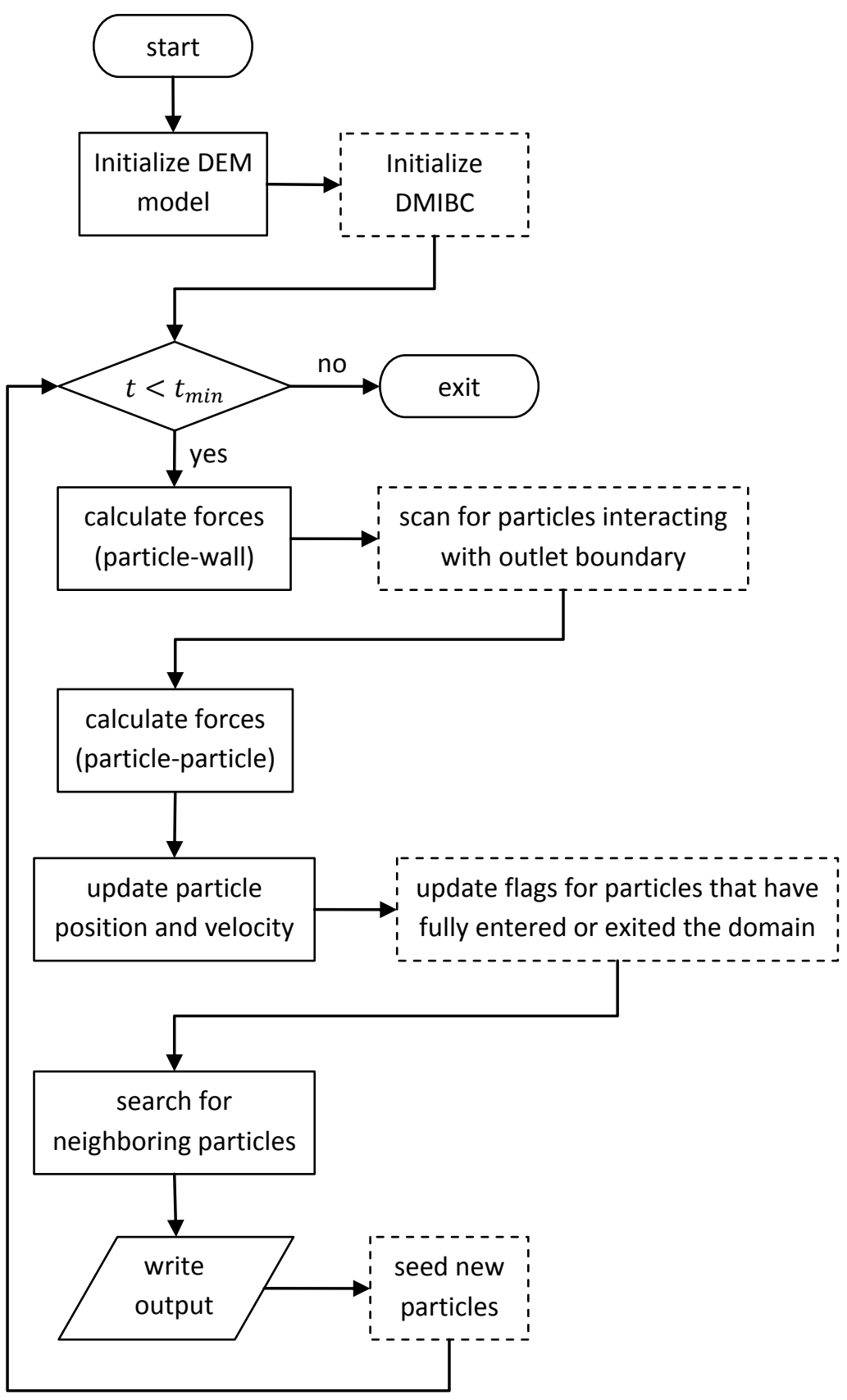

Figure 2-7: Flow chart illustrating the locations within MFIX-DEM where modifications have been made to include discrete mass boundary condition routines.

\subsection{Examples}

Provided below are two examples illustrating different applications of the DMIBC. The first example, presented in Section 2.5.1, illustrates a 2D packed bed (solids 
only, no gas phase) with a discrete mass inlet supplying new particles through the bottom of the bed. The second example, presented in Section 2.5.2, shows a 2D gas-solids system with an inlet gas jet coupled with a discrete mass inlet feeding particles into the bottom of the system. Both examples initially contain 500 particles comprised of five mass phases with a percent distribution for each phase of $15 \%, 20 \%, 30 \%, 20 \%$, and $15 \%$. Table 2-2 lists the physical and operating parameters of the DMIBC used in both simulations.

Table 2-2: Particle and feed properties of the examples outlined in Sections 2.5.1 and 2.5.2

\begin{tabular}{|c|c|c|c|c|c|}
\hline $\begin{array}{c}\text { Phase } \\
\text { Index }\end{array}$ & $\begin{array}{c}D_{m} \\
(\mathrm{~cm})\end{array}$ & $\begin{array}{c}\rho_{s m} \\
\left(\mathrm{~g} / \mathrm{cm}^{3}\right)\end{array}$ & $\begin{array}{c}\dot{\mathcal{V}}_{m} \\
\left(\mathrm{~cm}^{3} / \mathrm{sec}\right)\end{array}$ & $\begin{array}{c}\rho_{b m} \\
\left(\mathrm{~g} / \mathrm{cm}^{3}\right)\end{array}$ & $\begin{array}{c}\text { Corresponding } \\
\text { Distribution }\end{array}$ \\
\hline 1 & 0.100 & 1.75 & 0.00785 & 0.0125 & 0.15 \\
\hline 2 & 0.125 & 1.85 & 0.02045 & 0.0325 & 0.20 \\
\hline 3 & 0.150 & 1.95 & 0.05301 & 0.0950 & 0.30 \\
\hline 4 & 0.175 & 2.05 & 0.05612 & 0.1000 & 0.20 \\
\hline 5 & 0.200 & 2.15 & 0.06283 & 0.1500 & 0.15 \\
\hline
\end{tabular}

\subsubsection{Solids injected into a packed bed}

In this first example, particles are fed into the bottom of a static particle bed operating in a vacuum (i.e., particles in the bed have zero velocity and no gas phase is present). The feed rate, $N_{t}$, is approximately 100 particles per second. The incoming particle distribution (the distribution of incoming particles by solids mass phase) is the same as the initial distribution of particles in the system (i.e., the percent of incoming particles in solids phases 1, 2, 3, 4, and 5 are 15\%, 20\%, 30\%, 20\%, and 15\%, respectively). As previously stated, this is a global or target distribution for the incoming particles and is skewed on the placement frequency time scale. The computational domain is $3 \mathrm{~cm}$ wide by $20 \mathrm{~cm}$ tall. 
Snapshots of the particle configuration within the system are shown in Figure 2-8, with only the lower $8 \mathrm{~cm}$ of the domain displayed. For identification purposes, new particles are shaded darker than the particles initially in the system. This series of images demonstrates that incoming particles push particles already in the system away from the inlet region as they enter the computational domain.

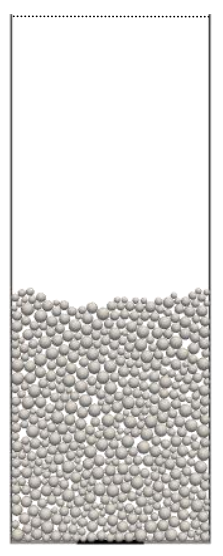

$t=0.0 \mathrm{sec}$

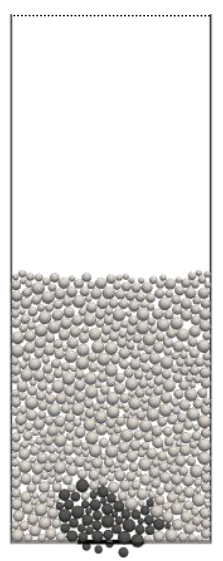

$t=0.5 \mathrm{sec}$
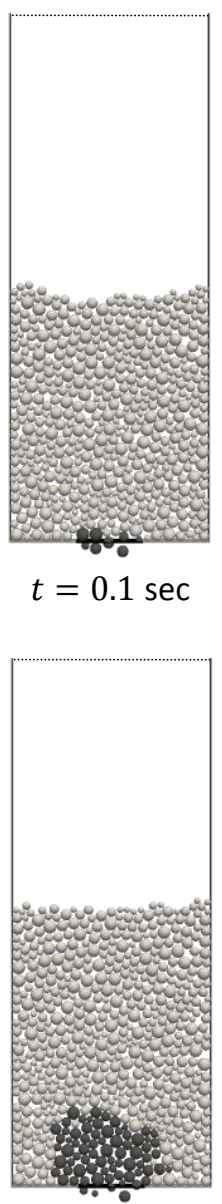

$t=0.75 \mathrm{sec}$

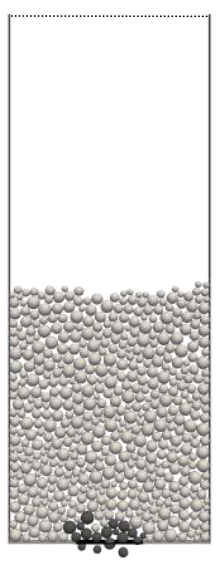

$t=0.2 \mathrm{sec}$

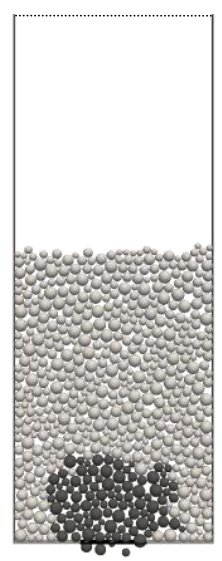

$t=1.0 \mathrm{sec}$

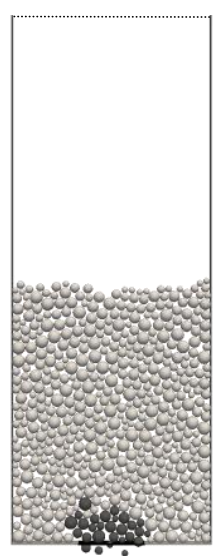

$t=0.3 \mathrm{sec}$

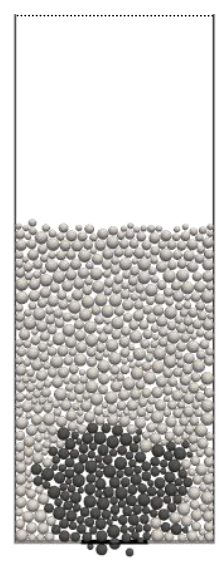

$t=1.5 \mathrm{sec}$

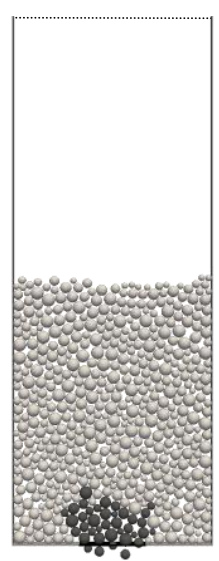

$t=0.4 \mathrm{sec}$

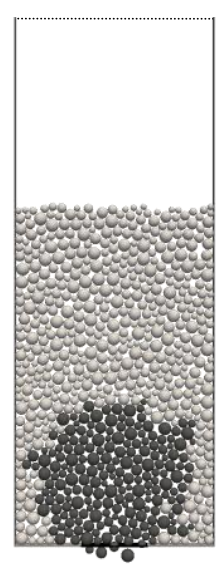

$t=2.0 \mathrm{sec}$

Figure 2-8: Time series (in seconds) of particle flow for a $2 \mathrm{D}$ example illustrating a DMIBC injecting into a packed bed.

Figure 2-9 shows the total incoming particle distributions at three times during the simulation. The user-specified distribution of incoming particles (i.e., 15\%, 20\%, 30\%, $20 \%, 15 \%)$ is shown to the right of the calculated values. This shows that the percent distribution for each solids phase is approximately maintained throughout the simulation. 
Furthermore, the distribution of the particles within the system approaches the desired distribution specified by the user as the simulation progresses through time.

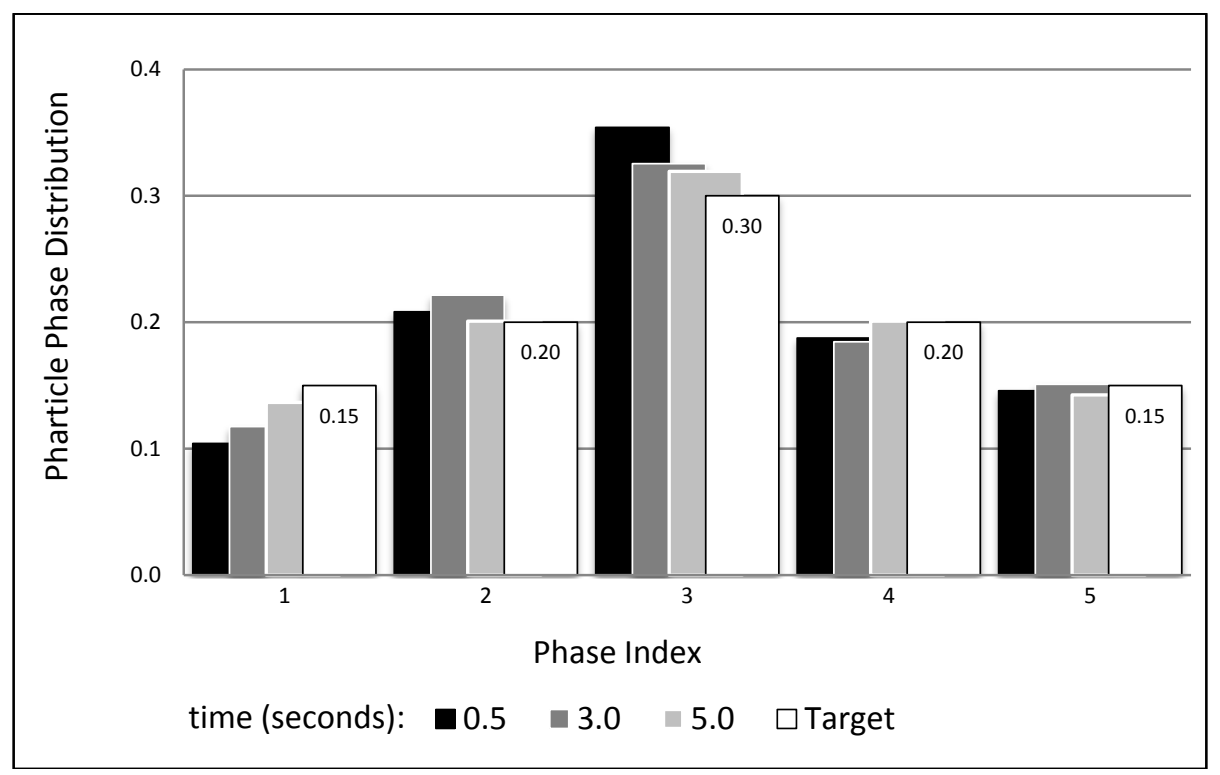

Figure 2-9: Cumulative incoming particle distributions at times 0.5, 3.0, and 5.0 seconds during the simulation of a DMIBC into a packed particle bed.

\subsubsection{Solids injection coupled with a gas jet}

This second example shows a discrete mass inlet coupled with a gas inflow boundary condition. In this simulation, the computational domain is $3 \mathrm{~cm}$ wide and $50 \mathrm{~cm}$ tall. A $1 \mathrm{~cm}$ gas jet is centered on the bottom of the domain with a velocity of $1200 \mathrm{~cm} / \mathrm{sec}$. A pressure outlet for the gas is specified along the top of the domain.

The simulation starts with an initial set of 500 particles in a packed bed arrangement. Initially, the velocities of the gas phase and particles are zero. During the first 3 seconds (not shown), a pseudo-steady-state is established between the gas flow and the initial particles, a time during which no particles are fed into the system. After this initial transient period, particles are fed into the system at a rate of 100 particles per second. As in the first example, the target incoming particle distribution for solids phases $1,2,3,4$, and 5 is $15 \%, 20 \%, 30 \%, 20 \%$, and $15 \%$, respectively (refer to Table $2-2$ ). 
Snapshots of the particle configuration within the system are shown in Figure 2-10, with only the lower $8 \mathrm{~cm}$ displayed. Again, to distinguish new particles from initial particles, the new particles are shaded darker. Here, new particles are seen entering the computational domain and mixing with particles already in the system. This demonstrates that a DMIBC can be coupled with a continuum gas boundary condition without adversely affecting qualitative flow physics, and thereby adding new physical modeling possibilities.
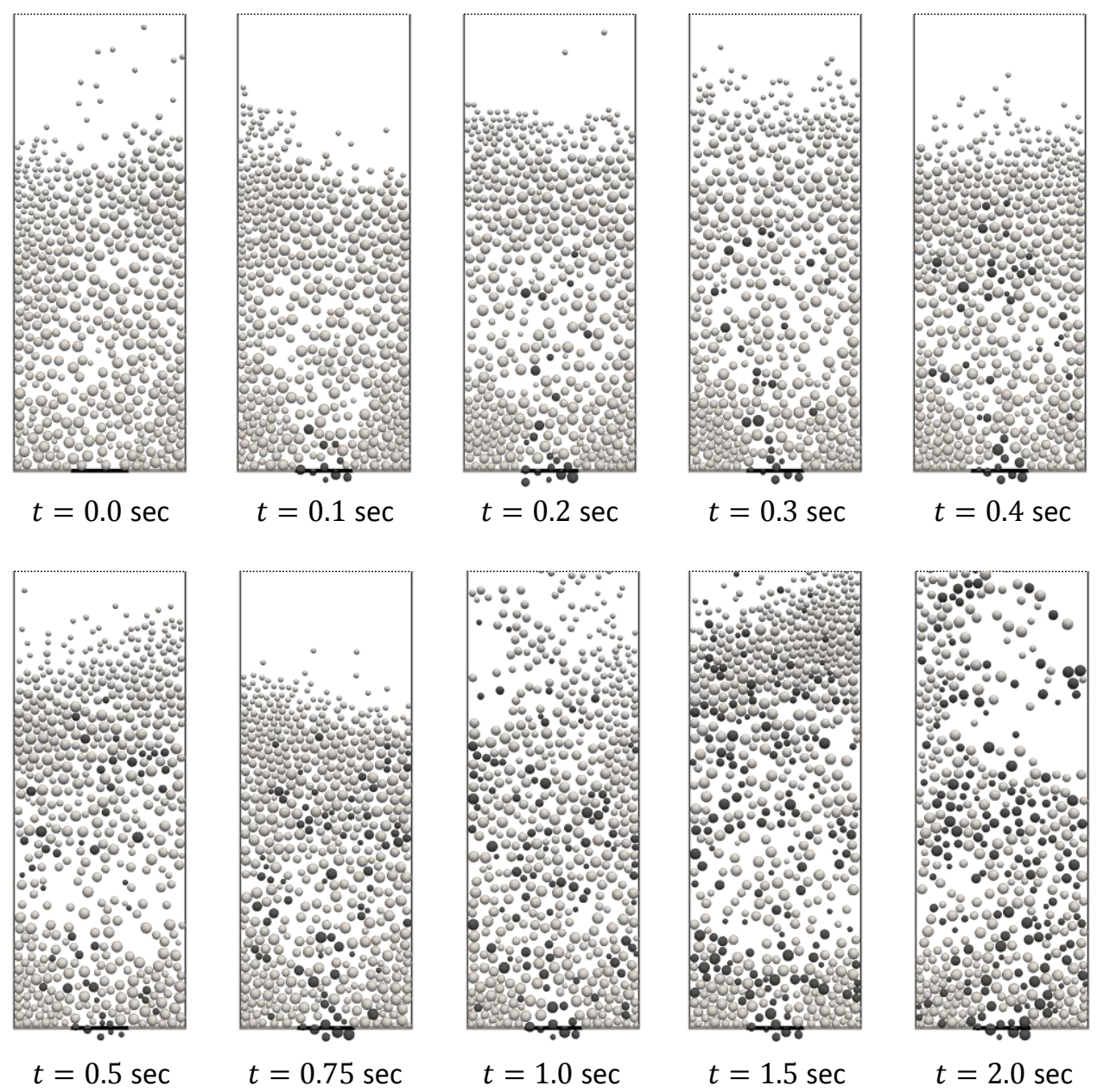

Figure 2-10: Time series (in seconds) of particle flow for a 2D example illustrating a DMIBC with a gas jet. 
The distribution of incoming particles (not shown) closely matches the results from the first example. This is expected since both inlets are defined by the same flow properties.

\subsection{Conclusions}

The current MFIX-DEM code was modified to include DMIBC and DMOBC. This allows models of more dynamic gas-solids systems by assigning user defined boundary conditions to the computational domain. The new algorithmic features have potential for use in simulations where particles enter as feedstock, like coal in a gasification model, or systems where particles are recycled, like in a circulating fluidized bed model. 


\section{Chapter 3: Particle heat transfer}

Particle-particle and particle-gas heat transfer in gas-solids systems occur in the forms of conduction, convection, and radiation. The goal of this investigation is to define a mathematical model for each type of heat transfer and successfully implement the selected models into MFIX-DEM, an existing CFD-DEM.

This chapter begins by briefly defining the three modes of heat transfer and introducing the internal energy equation for a single, isothermal particle in Section 3.1. An examination into the assumption of isothermal particles is included. In the subsequent sections, an analysis of contact conduction (Section 3.2), particle-fluid-particle conduction (Section 3.3), particle-gas convection (Section 3.4), and particle-environment radiation (Section 3.5) are presented. Each investigation includes (1) a survey of models proposed in the literature, and (2) a discussion detailing modifications made to the selected model. Finally, the algorithmic implementation and verification of the selected heat transfer models are given in Sections 3.6 and 3.7.

\subsection{Foundations for particle heat transfer}

In this section the physical mechanisms of conduction, convection, and radiation, including a characterization of their contribution to particle heat transfer in gas-solids systems are presented briefly. Additional details concerning the fundamentals of heat

transfer are found in reference [47]. The assumption of isothermal particles employed in this work is then examined. Finally, the internal energy equation of a single, isothermal particle is introduced. 


\subsubsection{Modes of heat transfer}

Conduction is the mode of heat transfer responsible for distributing thermal energy throughout matter due to a temperature gradient. Two forms of conduction are considered in this work. Contact conduction occurs when two particles at different temperatures touch. As illustrated in Figure 3-1-(a), thermal energy passes from the hot particle to the cold particle through the contact area. Particle-fluid-particle conduction arises when two particles are in close proximity to one another. First, thermal energy is transferred from the hot particle to the intermediate gas, and then passed from the gas to the cold particle. This procedure is demonstrated in Figure 3-1-(b). Additional details concerning contact conduction and particle-fluid-particle conduction are provided in sections 3.2 and 3.3 , respectively.

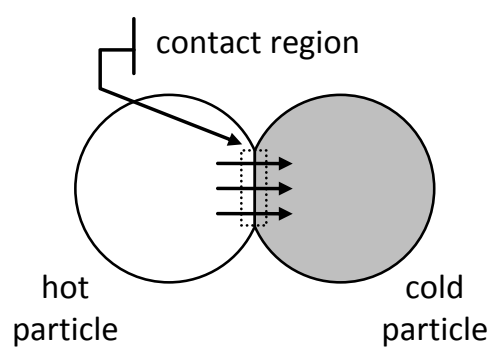

(a)

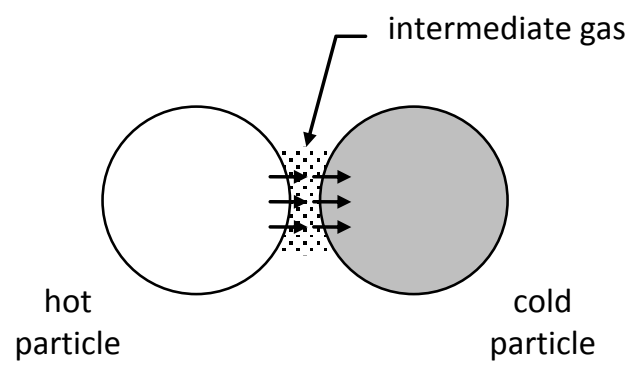

(b)

Figure 3-1: Illustration of conduction between particles. (a) Contact conduction: thermal energy is transferred from a hot particle to a cold particle through their shared contact area. (b) Particle-fluidparticle conduction: thermal energy is transferred from a hot particle to the stagnant gas separating its surface from the cold particle. The thermal energy is then transferred from the stagnant gas to the cold particle.

Convection in this work is defined as the transfer of thermal energy from a surface to a moving fluid due to the presence of a temperature gradient. As illustrated in Figure 3-2, convection may transfer thermal energy away from the surface of a hot particle by a cold gas, or transfer thermal energy to the surface of a cold particle by a hot gas. Additional details about convection are provided in Section 3.4. 


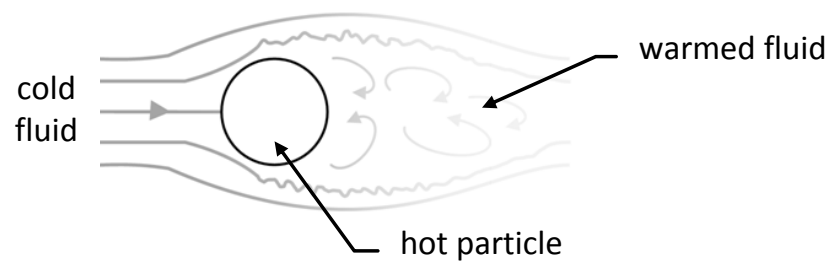

Figure 3-2: Illustration of particle-gas convection. Thermal energy is exchanged between the surface of the particle and the moving fluid.

Radiation is the mode of heat transfer that exchanges thermal energy between two objects through the absorbing, reflecting, refracting, diffracting, and transmitting of electromagnetic waves. An illustration of radiative heat transfer is shown in Figure 3-3, where thermal energy is transmitted from a hot particle to a cold particle. A portion of the energy is absorbed by the cold particle, while some of the energy is reflected away. Additional details regarding radiation are provided in Section 3.5.

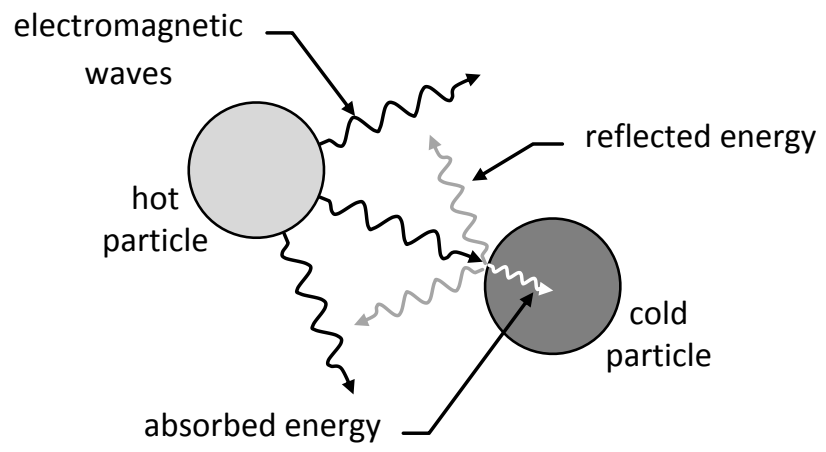

Figure 3-3: Illustration of particle-environment radiative heat transfer. Electromagnetic waves transmitted by a hot particle are absorbed and reflected by the cold particle.

\subsubsection{Assumption of isothermal particles}

In this work particles are assumed isothermal (i.e., no thermal gradient exists within a single particle). This assumption is examined by analyzing the conduction of thermal energy through a homogeneous, spherical particle without internal heat generation. 
The governing equations for a homogeneous, spherical particle with no internal heat generation are given by Equation set (3-1) [29]. The first boundary condition asserts that the temperature distribution is continuous at the center of a particle. The second boundary condition specifies that the heat loss at the surface of a particle is equivalent to the heat transfer to the surrounding gas via convection. The initial condition assigns a temperature distribution to the particle at time, $t=0$.

$$
\begin{gathered}
\rho_{p} C_{p p} \frac{\partial T_{p}}{\partial t}=\frac{1}{R_{p}^{2}} \frac{\partial}{\partial r}\left(\kappa_{p} R_{p}^{2} \frac{\partial T_{p}}{\partial r}\right)+\frac{1}{R_{p}^{2} \sin \theta} \frac{\partial}{\partial \theta}\left(\kappa_{p} \sin \theta \frac{\partial T_{p}}{\partial \theta}\right)+\frac{1}{R_{p}^{2} \sin ^{2} \theta} \frac{\partial}{\partial \phi}\left(\kappa_{p} \frac{\partial T_{p}}{\partial \phi}\right) \\
\text { with } \begin{cases}\frac{\partial T_{p}}{\partial r}=0 & \text { at } r=0 \\
\frac{\partial T_{p}}{\partial r}=\gamma_{c p}\left(T_{g}-T_{p}\right) & \text { at } r=r_{p} \\
T_{p}=T_{p 0}(r, \theta, \phi) & \text { for } t=0\end{cases}
\end{gathered}
$$

In Equation set (3-1), $T_{p}, R_{p}, \rho_{p}, C_{p p}$, and $\kappa_{p}$ are a particle's temperature, radius, density, specific heat, and thermal conductivity, respectively. $\gamma_{c p}$ is the convective heat transfer coefficient between a particle and gas and $T_{g}$ is the average temperature of the gas surrounding a particle. By assuming that thermal conductivity is constant and that the heat transfer is radially symmetric, Equation set (3-1) is transformed through a change of variables into the dimensionless form shown in Equation set (3-2). 


$$
\begin{gathered}
\frac{\partial \Theta}{\partial \mathrm{Fo}_{p}}=\frac{1}{\eta} \frac{\partial^{2}}{\partial \eta^{2}}(\eta \Theta) \quad \text { with } \begin{cases}\frac{\partial \Theta}{\partial \eta}=0 & \text { at } \eta=0 \\
\frac{\partial \Theta}{\partial \eta}+\Theta \mathrm{Bi}=0 & \text { at } \eta=1 \\
\Theta=\Theta_{0}(\eta) \quad \text { for } \mathrm{Fo}_{p}=0\end{cases} \\
\text { where } \begin{array}{c}
\eta=\frac{R}{R_{p}}, \\
\mathrm{Fo}{ }_{p}=\frac{\kappa_{p} t}{\rho_{p} C_{p p} R_{p}^{2}}
\end{array} \\
\mathrm{Bi}=\frac{\gamma_{p p}-T_{g}}{T_{p, r e f}-T_{g}} . \\
\kappa_{p} / R_{p}
\end{gathered}
$$

In Equation set (3-2), $\eta$ is the non-dimensionalized radial position in a particle of radius $R_{p}$ and $\Theta$ is the non-dimensionalized temperature based on the gas temperature and some reference temperature $T_{p, r e f} . \mathrm{Bi}$ is the Biot number and $\mathrm{Fo}_{p}$ is the Fourier number, both dimensionless parameters associated with a particle. The Fourier number, defined in Equation (3-3), relates the rate of conduction through a particle to the rate of heat storage within a particle, whereas the Biot number, defined in Equation (3-4), relates the convective heat transfer coefficient to the thermal conductivity within a particle. Thus, a small Biot number indicates that heat is redistributed throughout a particle faster than it is transferred from its surface via convection. As a result, a particle's core and surface temperatures are approximately the same (i.e., the particle is nearly-isothermal). In contrast, a large Biot number indicates that heat transfer via convection occurs faster than heat is redistributed inside a particle. Therefore, a particle may have different temperatures at its surface and core. These two cases are illustrated in Figure 3-4. 


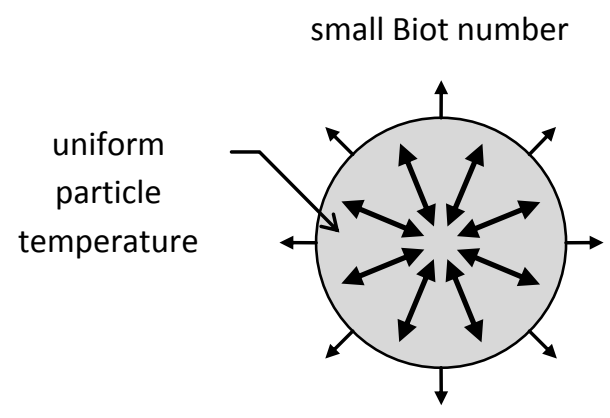

(a)

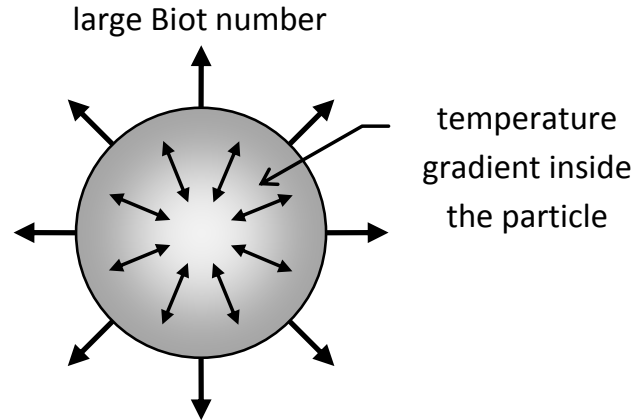

(b)

Figure 3-4: Illustration of Biot number classification on a particle's temperature. Arrows on the surface of the particles indicate the rate of convective heat transfer, while the arrows inside the particle represent the rate of conductive heat transfer. (a) For small Biot numbers, a particle's temperature is nearly-isothermal because the redistribution of thermal energy within a particle occurs at a faster rate than convective heat transfer. (b) When the Biot number is large, a temperature gradient may exist within a particle because the redistribution of thermal energy inside a particle is slower than the rate of convective heat transfer.

By inspection, note that the solution of the non-dimensionalized equation set is obtained through separation of variables. This leads to a Fourier series type solution, shown as Equation (3-5). Here $A_{n}$ are the Fourier coefficients and $\lambda_{n}$ the eigenvalues of the solution. Additional details related to obtaining this solution are presented in Mills [47] and Yovanovich [48].

$$
\Theta=\sum_{n=1}^{\infty} A_{n} e^{-\lambda_{n}^{2} \mathrm{Fo}_{p}} \frac{\sin \left(\lambda_{n} \eta\right)}{\lambda_{n} \eta}
$$

where $A_{n}=\frac{2 \mathrm{Bi}\left(\lambda_{n}^{2}+(\mathrm{Bi}-1)^{2}\right)}{\lambda_{n}^{2}+\mathrm{Bi}^{2}-\mathrm{Bi}}(-1)^{n+1} \quad$ and $\quad \lambda_{n} \cos \lambda_{n}=(1-\mathrm{Bi}) \sin \lambda_{n}$

Yovanovich [48] states that as the Biot number approaches zero, the first eigenvalue of the solution trends toward zero along $\sqrt{3 \mathrm{Bi}}$ as the higher order eigenvalues approach nonzero values. This observation leads to the conclusion that the first term of the series solution is dominant for small Biot numbers. Using this assumption, one can show that when Biot number is less than 0.1 , a condition typically satisfied for particles in a gas-solids flow [29], the difference between the center and surface temperatures of a 
particle is less than a 5\% [47]. This small temperature difference across a particle in concert with the added computational complexity required to resolve the temperature within a particle leads to the general assumption that particles are isothermal in gas-solids flows. Additional details concerning this assumption are found in references [29,47]. The following section introduces the internal energy equation for a single, isothermal particle.

\subsubsection{Particle internal energy equation}

The internal energy for a single, isothermal particle indexed by $(i)$ is shown in Equation (3-6) [30]. Here, $m, C_{p p}$, and $T_{p}$ are the particle's mass, specific heat, and temperature, respectively. $\dot{Q}_{p p}^{(i)}$ is the total rate of heat transfer resulting from contact conduction, $\dot{Q}_{p f p}^{(i)}$ is the total rate of heat transfer due to particle-fluid-particle conduction, $\dot{Q}_{p g}^{(i)}$ is the rate of heat transfer between the gas and particle via convection, and $\dot{Q}_{r a d}^{(i)}$ is the rate of radiative heat transfer between particle $(i)$ and its environment. The heat of reaction supplied to the solids phase and enthalpy transfer due to mass transfer, $\Delta H_{r s}$, are governed by the chemical reactions, if any, that the particle is undergoing.

$$
m^{(i)} C_{p p}^{(i)} \frac{d T_{p}^{(i)}}{d t}=\dot{Q}_{p p}^{(i)}+\dot{Q}_{p f p}^{(i)}+\dot{Q}_{p g}^{(i)}+\dot{Q}_{r a d}^{(i)}-\Delta H_{r s}
$$

Formal definitions (mathematical models) for $\dot{Q}_{p p}, \dot{Q}_{p f p}, \dot{Q}_{p g}$, and $\dot{Q}_{r a d}$ are provided in Sections 3.2 - 3.5. Further discussion of $\Delta H_{r s}$ is reserved for Chapter 4 where it is presented in the context of a mathematical interface for managing chemical reactions.

\subsection{Contact conduction}

Typically, heat transfer as a result of particle-particle and particle-wall contact is assumed negligible in gas-solids systems [29]. This is attributed to (1) a small contact area, and (2) the short contact duration between colliding particles [49]. However, the 
contribution from contact conduction is expected to increase for highly conductive particles, as well as particles engaged in enduring contact $[\mathbf{2 9 , 4 9 , 5 0 ]}$. Since a goal of this work is to construct a comprehensive heat transfer model for the CFD-DEM, contact conduction is included for completeness. The limited nature of contact conductance with respect to the overall heat transfer in a gas-solids system is further addressed in the model selection and implementation discussions.

\subsubsection{Survey of particle-particle contact conduction models}

This section continues by surveying several contact conduction models from the literature $[499,51,52]$ along with various considerations that are needed to implement each model in the selected CFD-DEM framework (i.e., MFIX-DEM). Batchelor and O'Brien's [52] model is selected as the basis for the default contact conduction model developed for this work. Modifications to Batchelor and O’Brien's [52] model as employed in this work are detailed in Section 3.2.2.

\subsubsection{Sun and Chen [49] (1988)}

The contact conduction model developed by Sun and Chen [49] is based on Hertzian theory which relates the size of the contact area between two particles to the particles' material properties [53]. Using Hertzian theory as the basis for several assumptions, the authors hypothesize that (1) the curvatures of the particles' surfaces are negligible over the contact area, and (2) temperature changes in the particles are confined to a small region around the contact area. Both assumptions are illustrated in Figure 3-5, and lead the authors to conclude that heat transfer between two contacting particles parallels heat transfer between two semi-infinite media. 


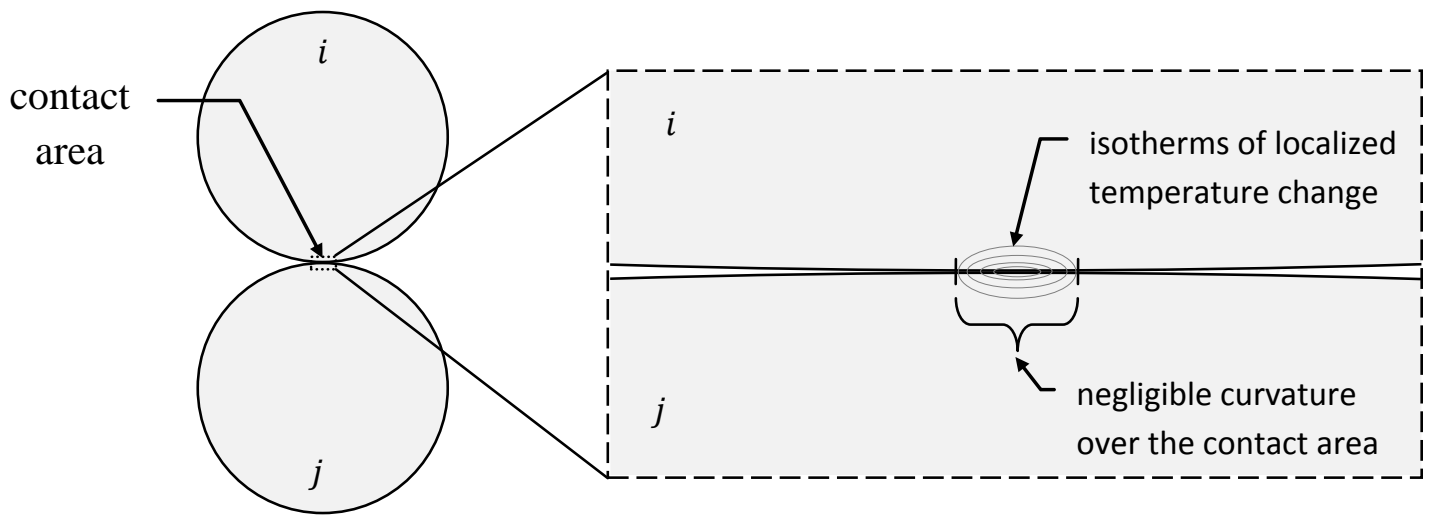

Figure 3-5: The elastic collision model employed by Sun and Chen [49] leads to the assumption that the shared contact area is flat.

Sun and Chen [49] further assume that (3) the thermal resistance across the contact area between two particles is negligible, and (4) the particles' surfaces are insulated outside their contact area. These assumptions are used by the authors to conclude that heat transfer is governed by two axially symmetric heat conduction equations with appropriate boundary and initial conditions. The authors make the further simplifications that (5) the physical properties of the particles are constant, (6) heat transfer is one-dimensional, occurring perpendicular to the contact area between two particles only, and (7) the Fourier numbers, defined by Equation (3-7) and based on the total contact time and maximum contact radius, are small compared to unity. Recall, a small Fourier number represents the case where the rate of heat conduction is small compared to the rate of thermal energy storage. The total contact time and maximum contact radius follow from Hertzian theory and are defined in Equations (3-8) and (3-9), respectively.

$$
=\mathrm{Fo}^{(i)^{\prime}}\left(\frac{\kappa_{p}^{(i)}}{\rho_{p}^{(i)} C_{p p}^{(i)}} \cdot \frac{t_{c}^{(i, j)}}{\left(R_{c}^{(i, j)}\right)^{2}}\right)
$$




$$
\begin{gathered}
R_{c}^{(i, j)}=\left(\frac{5 m^{*} R^{* 2}}{4 E^{*}}\right)^{1 / 5}\left\|\mathbf{V}_{n}^{(i, j)}\right\|_{2}^{2 / 5} \\
t_{c}^{(i, j)}=2.94\left(\frac{5 m^{*}}{4 E^{*}}\right)^{2 / 5}\left(R^{*}\left\|\mathbf{V}_{n}^{(i, j)}\right\|_{2}\right)^{-1 / 5}
\end{gathered}
$$

In these equations, $\left\|\mathbf{V}_{n}^{(i, j)}\right\|_{2}$ is the Euclidean norm (also known as the L2 norm or distance norm) of the normal impact velocity between the particles, and as before, $T_{p}$, $\rho_{p}, C_{p p}$, and $\kappa_{p}$ are the temperature, density, specific heat, and thermal conductivity of the indexed particles. $R^{*}$ and $m^{*}$ are the effective radius and mass of the particles defined by Equations (3-10) and (3-11), respectively. $E^{*}$ is the effective Young's modulus, and is defined by Equation (3-12), where $E$ and $v$ are the Young's modulus and Poisson's ratio for the indexed particles. Young's modulus, also referred to as the modulus of elasticity, and Poisson's ratio are material properties of the particle.

$$
\begin{gathered}
R^{*}=\frac{R_{p}^{(i)} R_{p}^{(j)}}{R_{p}^{(i)}+R_{p}^{(j)}} \\
m^{*}=\frac{m^{(i)} m^{(j)}}{m^{(i)}+m^{(j)}} \\
E^{*}=\frac{4}{3}\left(\frac{1-\left(v^{(i)}\right)^{2}}{E^{(j)}}+\frac{1-\left(v^{(j)}\right)^{2}}{E^{(j)}}\right)^{-1}
\end{gathered}
$$

Sun and Chen [49] use the assumptions outlined above to derive an analytical solution for the heat flux across the interface (contact area) of two touching particles. A solution for the total energy exchanged per impact for Fourier numbers approaching zero, shown as Equation (3-13), is obtained by integrating over the contact area and contact 
duration. Here, $c$ is a correction factor introduced by the authors to adjust for the assumption that heat transfer only occurs across paths perpendicular to the contact surface. Limited values for the correction factor are provided graphically by the authors, however a value is attainable by solving the conduction equations for contact spheres numerically.

$$
\dot{Q}_{p p}^{(i, j)}=c \frac{0.87 \pi\left(R_{c}^{(i, j)}\right)^{2} \sqrt{t_{c}^{(i, j)}}}{\left(\rho_{p}^{(i)} C_{p p}^{(i)} \kappa_{p}^{(i)}\right)^{-1 / 2}+\left(\rho_{p}^{(j)} C_{p p}^{(j)} \kappa_{p}^{(j)}\right)^{-1 / 2}}\left(T_{p}^{(j)}-T_{p}^{(i)}\right)
$$

Zhou, Yu, and Horio [54] show that the semi-infinite media assumption employed by Sun and Chen [49] over-predicts heat transfer when the Fourier number is large (e.g., heat transfer between the particle, and in turn, temperature changes in the particles are not confined to the small region around the contact area). By solving a finite element simulation of heat transfer during a particle-particle collision, the authors develop an analytic expression for the correction factor, shown as Equation (3-14). Zhou, Yu, and Horio [54] assert that their correction factor in conjunction with Sun and Chen's model [49] better approximates heat transfer between smooth, spherical particles with Fourier number as large as 10 . The correction is formulated for the specific case where the thermal conductivity of the colliding particles are equal, and the authors suggest conducting a finite element simulation to reformulate Equation (3-14) varying particle thermal conductivities (i.e., $\kappa^{(i)} / \kappa^{(j)} \neq 1$ ) for best results. 


$$
\begin{gathered}
c^{\prime}=\frac{0.43\left(\sqrt{c_{2}^{2}-4 c_{1}\left(c_{3}-\mathrm{Fo}^{\prime}\right)}-c_{2}\right)}{c_{1}} \\
\text { where } \quad \begin{array}{l}
c_{1}=-2.300 \beta^{2}+8.909 \beta-4.235 \\
c_{2}=8.169 \beta^{2}-33.770 \beta+24.885 \\
c_{3}=-5.758 \beta^{2}+24.464 \beta-20.511
\end{array} \quad \text { and } \quad \beta=\frac{\rho_{p}^{(i)} C_{p p}^{(i)}}{\rho_{p}^{(j)} C_{p p}^{(j)}}
\end{gathered}
$$

The original model proposed by Sun and Chen [49] is found in several CFD-DEM codes $[\mathbf{1 7 , 1 8 , 5 5}]$, as well as Zhou, Yu, and Horio's [54] correction factor [19,20]. One limitation of Sun and Chen's [49] model is the level of computational resources needed to evaluate Equations (3-7), (3-8), (3-9), (3-10), (3-11), (3-12), (3-13), and possibly (3-14) for each occurrence of particle-particle conduction. This amount of computational expense is considered excessive given that contact conduction is not anticipated to contribute substantially to the overall heat transfer in gas-solids systems $[\mathbf{2 9 , 4 9 , 5 0 ]}$. Another limitation of Sun and Chen's [49] model is its dependence on a correction factor. The authors provide two graphs for approximating the correction. Numerical experiments, like that employed by Zhou, Yu, and Horio [54], produce specific values or analytic expressions for the correction factor, but require substantial effort to obtain. Due to its computational expense and dependence on a correction factor, this model was not selected for this work.

\subsubsection{Shimizu [51] (2006)}

Another model proposed for contact conduction is employed in the software PFC2D/3D [56,57], and is presented in the work of Shimizu [51]. In this approach, illustrated in Figure 3-6, thermal energy is exchanged between two particles through a thermal pipe. The rate of heat transfer between particles $(i)$ and $(j)$ across the thermal pipe is given by Equation (3-15). Here, $\ell^{(i, j)}$ is the distance between the centers of the 
particles and delineates the length of the thermal pipe, $A_{c}^{(i, j)}$ is the contact area between the two particles and defines the cross-sectional area of the pipe, and $\psi^{(i, j)}$ is the thermal resistance of the pipe.

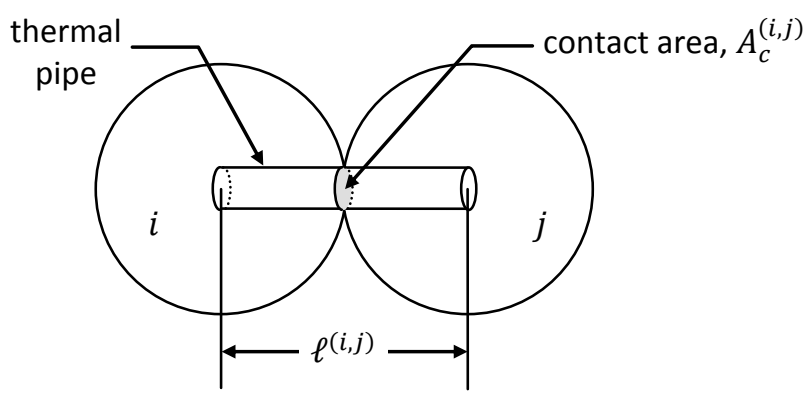

Figure 3-6: Particle-particle conduction model employing a thermal pipe connecting the centers of two particles. Implemented in PFC2D/3D [56,57] and presented by Shimizu [51].

$$
\dot{Q}_{p p}^{(i, j)}=\frac{\left(T_{p}^{(j)}-T_{p}^{(i)}\right)}{\psi^{(i, j)} \ell^{(i, j)}}
$$

The complexity of this model arises from the need to obtain a value for the resistance of the thermal pipes, $\psi^{(i, j)}$. First, the author analyzes Fourier's Law of Conduction, shown as Equation (3-16), for a particle assembly. The term particle assembly defines a collection of particles in a predefined control volume $(\mathrm{CV})$. Here, $\mathbf{\kappa}_{\mathfrak{Q}}$ and $T_{\mathfrak{L}}$ are the thermal conductivity and temperature of the particle assembly, respectively. $T_{\mathfrak{L}}$ is defined as the arithmetic average particle temperature in the assembly. The definition of $\boldsymbol{\kappa}_{\mathfrak{L}}$ is more complex as discussed below. In this work the symbol $\mathfrak{L}$ is used to denote the particle assembly containing the $(i, j)$ particle pair as illustrated in Figure 3-7.

$$
\mathbf{q}_{\mathfrak{L}}=-\mathbf{\kappa}_{\mathfrak{L}} \frac{\partial T_{\mathfrak{L}}}{\partial x_{i}}
$$




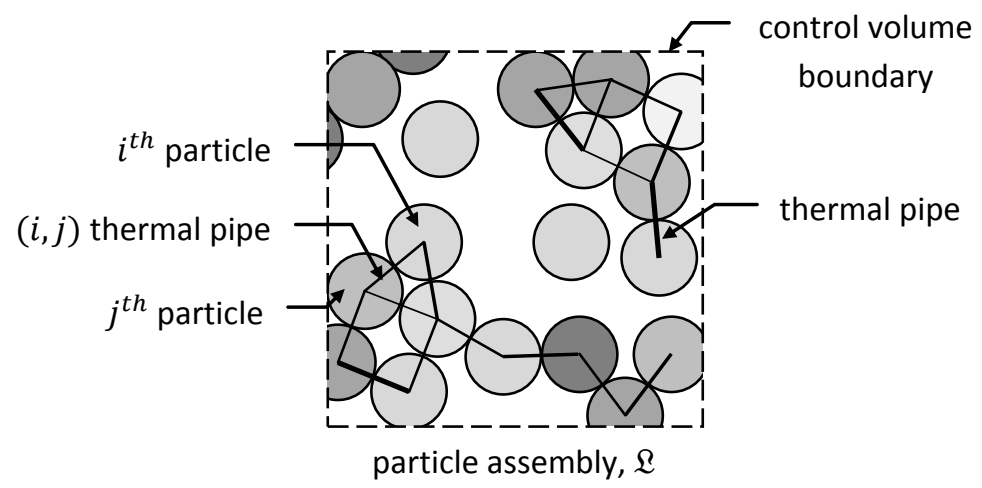

Figure 3-7: Illustration of a particle assembly as defined by Shimizu [51] with interconnected thermal pipes.

Shimizu [51] then equates the heat flux of the assembly, $\mathbf{q}_{\mathfrak{R}}$, to the volume averaged heat flux across all the thermal pipes within the assembly, shown as Equation (3-17). In this equation, $\mathcal{V}_{C V}$ is the volume of the $\mathrm{CV}$, and $N_{\mathfrak{R}, t p}$ is number of thermal pipes in particle assembly $\mathfrak{L}$. For the thermal pipe indexed by $(m), \ell, \nabla T$, and $\psi$ are its length, temperature gradient, and thermal resistance, respectively. $\xi$ is the unit vector directed along the thermal pipe indexed by $(m)$.

$$
\mathbf{q}_{\mathfrak{L}}=-\frac{1}{\mathcal{V}_{C V}} \sum_{m=1}^{N_{\mathcal{Q}, t p}} \frac{\ell^{(m)} \boldsymbol{\xi}^{(m)} \cdot \xi^{(m)^{T}}}{\psi^{(m)}} \nabla T^{(m)}
$$

Shimizu [51] goes on to replace the individual thermal pipe (microscopic) temperature gradients, $\nabla T^{(m)}$, with the assembly (macroscopic) temperature gradient, $\nabla T_{\mathfrak{R}}$. By comparing the result with Equation (3-16), Equation (3-18) is established as the definition for the thermal conductivity of the assembly.

$$
\boldsymbol{\kappa}_{\mathfrak{L}}=\frac{1}{\mathcal{V}_{C V}} \sum_{m=1}^{N_{\mathfrak{Q}, t p}} \frac{\ell^{(m)} \boldsymbol{\xi}^{(m)} \cdot \boldsymbol{\xi}^{(m)^{T}}}{\psi^{(m)}}
$$


Shimizu [51] then assumes that (1) heat transfer in the assembly is isotropic (directionally invariant) and (2) the thermal resistance is uniform for all thermal pipes in the assembly (i.e., $\psi_{\mathfrak{L}}=\psi^{(m)} \forall m \in \mathfrak{Q}$ ). Thus, the thermal conductivity of the assembly is defined as the mean value of the diagonal elements of the thermal conductivity tensor, $\boldsymbol{\kappa}_{\mathfrak{L}}$, as shown in Equation (3-19). Here, the three in the denominator arises from the spatial dimension of the given problem (in this case, 3-D).

$$
\bar{\kappa}_{\mathfrak{Q}}=\frac{1}{3 \mathcal{V}_{C V} \psi_{\mathfrak{\Omega}}} \sum_{m=1}^{N_{\mathfrak{Q}, t p}} \ell^{(m)}
$$

Equation (3-19) is then rearranged to develop an expression for the thermal resistance of the assembly, as shown in Equation (3-20). Once a value for the thermal resistance of the assembly is attained, it is used to represent the thermal resistance of each thermal pipe $\left(\psi^{(i, j)}=\psi_{\mathfrak{\Omega}}\right)$. That is, it substituted back into Equation (3-15) to evaluate the rate of heat transfer between the two contacting particles.

$$
\psi_{\mathfrak{Q}}=\frac{1}{3 \bar{\kappa}_{\mathfrak{L}} \mathcal{V}_{\mathfrak{Q}}} \sum_{m=1}^{N_{\mathfrak{Q}, t p}} \ell^{(m)}
$$

Careful examination reveals that Shimizu's [51] published equations (i.e., Equation (3-20)) are underdetermined. Specifically, a secondary expression for the thermal conductivity of the particle assembly $\left(\bar{\kappa}_{\mathfrak{L}}\right)$ is needed to obtain a meaningful model. The author does not explicitly sate how this value is obtained, or if it is taken as some average of the particles' thermal conductivity.

This model poses several limitations, most notably, the underdetermined definition for the thermal conductivity of the particle assembly $\left(\bar{\kappa}_{\mathfrak{L}}\right)$. Additionally, this 
approach appears better suited for simulations where particle movement is limited. In gas-solids flows where particles are always moving, the members comprising the particle assemblies are continually changing. In turn, the thermal resistances of the assemblies require recalculation at each solids time step. Due to the ambiguity of the model and the avoidable computational expense of calculating thermal resistances, this model is dismissed in favor of a well-defined, simpler model.

\subsubsection{Batchelor and O'Brien [52] (1977)}

Batchelor and O'Brien [52] also developed a contact conduction model based on Hertzian collision theory. Unlike the model of Sun and Chen [49] which employs a maximum contact radius, Batchelor and O'Brien [52] use the instantaneous compression force (normal contact force) to calculate the contact radius, shown in Equation (3-21). $R^{*}$ and $E^{*}$ are the effective radius and Young's modulus previously defined in Equations (3-10) and (3-12), respectively. $\mathbf{F}_{n}^{(i, j)}$ is the normal force resulting from the collision/contact between two particles indexed by $(i)$ and $(j)$.

$$
R_{c}^{(i, j)}=\left(\frac{2 R^{*}}{E^{*}}\left\|\mathbf{F}_{n}^{(i, j)}\right\|_{2}\right)^{1 / 3}
$$

By drawing a comparison between the velocity potential of an incompressible fluid through a circular hole in a plane wall, and the temperature distribution inside the particles, Batchelor and O'Brien [52] obtain an expression for the energy flux normal to the contact area of two particles. The energy flux is given by Equation (3-22), and illustrated in Figure 3-8. Integrating Equation (3-22) over the contact area gives total rate of heat transfer between the particles per unit time, shown as Equation (3-23). 


$$
q_{c}^{(i, j)}=\frac{\kappa_{p}\left(T_{p}^{(j)}-T_{p}^{(j)}\right)}{\pi \sqrt{R_{c}^{(i, j)}-r^{2}}} \text { for } r \in\left[0, R_{c}^{(i, j)}\right)
$$

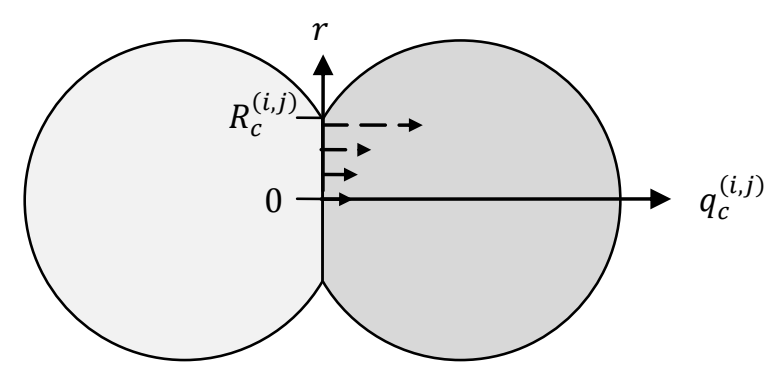

Figure 3-8: Illustration of the thermal energy flux from a hot particle to a cold particle across the contact area.

$$
\dot{Q}_{p p}^{(i, j)}=2 \kappa_{p} R_{c}^{(i, j)}\left(T_{p}^{(j)}-T_{p}^{(i)}\right)
$$

This model was selected as the foundation for developing a contact conduction model in this work. The selection was guided by (1) the algebraic simplicity of the model, and (2) the wide employment of this model for particle-particle conduction in CFD-DEM [58,59,19,22,60,61]. Specific modifications to Batchelor and O'Brien's [52] model are presented in the following section wherein limitations of this model are addressed. In particular, Batchelor and O'Brien's [52] model assumes that particles have the same thermal conductivity, $\kappa_{p}$, and employs a Hertzian collision model as a basis for calculating the contact area. Finally, assumptions needed to extend the two-particle contact conduction model to a multiple-particle contact conduction model are presented. Specifically, what assumptions need satisfied so that simultaneous instances of contact conduction for the same particle (i.e., a particle experiences contact conduction with two or more particles at the same time) are summable, resulting in an expression for the total amount of contact conduction the particle experiences. 


\subsubsection{Modified Batchelor and O'Brien [52] two-particle contact conduction model}

A constraint of Batchelor and O'Brien's [52] contact conduction model is the assumption that both particles have the same thermal conductivity, $\kappa_{p}$. This prohibits the use of the model in systems where particle types, and in turn thermal conductivities of the particles, may vary. Their model is extended in this work by defining an effective thermal conductivity between two particles, shown in Equation (3-24). As defined, the smaller thermal conductivity controls the rate of heat transfer across the contact area. This is consistent with the thermal circuit concept put forward by Mills [47], whereby heat transfer across a composite wall is limited by the material with the highest resistance to conductive heat transfer. Likewise, Zhou, Yu, and Zulli [19] impose a similar modification to Batchelor and O'Brien's [52] model in their work.

$$
\kappa_{p}^{*}=2\left(\frac{1}{\kappa_{p}^{(i)}}+\frac{1}{\kappa_{p}^{(j)}}\right)^{-1}
$$

In Batchelor and O'Brien's [52] derivation, they employ a Hertzian collision model to determine the area of contact through which heat may flow. In this effort, their model is generalized further by using the geometric configuration of the two particles to obtain the radius of their shared contact area. This approach results in a contact area representative of the specific collision model employed by the DEM (e.g., Cundall and Strack [44], Hertz [53], etc.), and promotes greater modeling consistency throughout the CFD-DEM. Equation (3-25) defines the contact radius employed in this work. The indices $k$ and $l$ are used to identify the particles by diameter size, specifically $R_{p}^{(k)}=$ $\max \left\{R_{p}^{(i)}, R_{p}^{(j)}\right\}$ and $R_{p}^{(l)}=\min \left\{R_{p}^{(i)}, R_{p}^{(j)}\right\} ;$ and, $\ell^{(i, j)}$ is the distance separating the 
particles' centers. Formal algebraic and geometric definitions of these terms are reserved for Appendix B.

$$
\tilde{R}_{c}^{(i, j)}=\sqrt{R_{p}^{(k)^{2}}-\left(\frac{R_{p}^{(k)^{2}}-R_{p}^{(l)^{2}}+\ell^{(i, j)^{2}}}{2 \ell^{(i, j)}}\right)^{2}}
$$

Substituting Equations (3-24) and (3-25) into Equation (3-23) results in the contact conduction model developed and implemented in this work, shown as Equation $(3-26)$.

$$
\dot{Q}_{p p}^{(i, j)}=\frac{4 \kappa_{p}^{(i)} \kappa_{p}^{(j)}}{\kappa_{p}^{(i)}+\kappa_{p}^{(j)}} \sqrt{R_{p}^{(k)^{2}}-\left(\frac{R_{p}^{(k)^{2}}-R_{p}^{(l)^{2}}+\ell^{(i, j)^{2}}}{2 \ell^{(i, j)}}\right)^{2}}\left(T_{p}^{(j)}-T_{p}^{(i)}\right)
$$

This model, as well as those presented in Section 3.2.1, is derived in the context of two particle systems (i.e., contact conduction only occurs between two individual particles). The following section explains the extension of this model to situations where a particle may undergo contact conduction with several neighboring particles simultaneously.

\subsubsection{Extension to multi-particle systems}

The model developed for this work and those described in Section 3.2.1 are based on two particle systems. Vargas [62] states that the individual instances of contact conduction are summable under two conditions. First, the Biot numbers associated with a collision, defined in Equation (3-27), must remain small compared to unity. Physically, a small Biot number ensures that the rate of heat transfer between two particles is slow in comparison to the rate of thermal energy redistributed inside each particle. Conversely, the resistance to heat transfer inside a particle is much smaller than the resistance 
between particles. Thus, all particles touching a particle will witness the same temperature for that particle. This is consistent with the argument used in this work to justify the assumption of isothermal particles (see Section 3.1.2).

$$
\mathrm{Bi}^{\prime}=\frac{2 \kappa_{p}^{*} \widetilde{R}_{c}^{(i, j)} / A_{p}^{(i)}}{\kappa_{p}^{*} / R_{p}^{(i)}}=\frac{2 \widetilde{R}_{c}^{(i, j)}}{\pi R_{p}^{(i)}} \ll 1 \text { for } j \in \mathfrak{U}
$$

The second assumption, according to Vargas [62], is that a particle's temperature must change gradually so that fluctuations in temperature do not propagate beyond its immediate neighbors over one solids time step. This quasi-steady temperature criterion [62] is given by Equation (3-28). Here $\Delta T_{p}^{(i)}$ represents the temperature change of particle $i$ over a solids time step of duration $\Delta t_{s}$. This is recast in Equation (3-29) to generate an upper bound on the size of the solids time step. Vargas [62] states that this bound is easily met within the framework of the CFD-DEM as it is orders of magnitude less restrictive than the bound placed on the solids time step for numerical stability of the collision model.

$$
\begin{aligned}
\frac{\Delta T_{p}^{(i)}}{T_{p}^{(j)}-T_{p}^{(i)}} & =\frac{2 \kappa_{p}^{*} \widetilde{R}_{c}^{(i, j)}}{m^{(i)} C_{p p}^{(i)}} \Delta t_{s} \ll 1 \text { for } j \in \mathfrak{A} \\
\Delta t_{s} & \ll \frac{m^{(i)} C_{p p}^{(i)}}{2 \kappa_{p}^{*} \tilde{R}_{c}^{(i, j)}} \text { for } j \in \mathfrak{A}
\end{aligned}
$$

This completes the discussion of contact conduction. Here, Equation (3-26) is established as the default contact conduction model $\left(\dot{Q}_{p p}\right)$ for the particle internal energy equation (Equation (3-6)). The subsequent section introduces various models for particlefluid-particle conduction $\left(\dot{Q}_{p f p}\right)$ presented in the literature as well as considerations for implementing them into the framework of the CFD-DEM. 


\subsection{Particle-fluid-particle conduction}

In addition to contact conduction, particles in close proximity to one another may also transfer energy by conducting heat through the stagnant gas separating their surfaces. Wen and Chang [63] suggest this form of conduction may contribute between 10-35\% of the total heat transferred among solids in some gas-solids systems.

\subsubsection{Survey of particle-fluid-particle conduction models}

This section begins by surveying several particle-fluid-particle conduction models from the literature $[\mathbf{1 6 , 6 3 , 6 4 , 6 5}]$ along with various considerations that are needed to implement each model in the selected CFD-DEM framework (i.e., MFIX-DEM). Rong and Horio's [16] model is selected as the basis for the particle-fluid-particle conduction model developed in this work. Modifications to Rong and Horio's [16] model are discussed in Section 3.3.2.

\subsubsection{Wen and Chang [63] (1967)}

Wen and Chang [63] proposed a simple analytic model for heat transfer in gassolids systems operating at low temperatures and containing two types of particles (i.e., type A and type B particles). Particles of the same type are assumed identical in physical and thermal properties as well as temperature. The authors hypothesize that heat transferred from a particle's surface primarily results from particle-gas convection and particle-fluid-particle heat transfer. Contact conduction and radiative heat transfer are assumed insignificant for their case (i.e., particulate fluidization at temperatures below $600^{\circ} \mathrm{C}$ ). They conclude that the total flux of thermal energy across the surface of a type A particle is given by Equation (3-30). The first component on the right hand side is the 
flux due to particle-gas convection, while the second component is the result of particlefluid-particle conduction.

$$
\dot{q}_{A}=\gamma_{c p}\left(T_{A}-T_{g}\right)+\gamma_{B A}\left(T_{A}-T_{B}\right)
$$

In Equation (3-30), $\gamma_{c p}$ is the convective heat transfer coefficient between the gas and a type A particle, $\gamma_{B A}$ is the particle-particle heat transfer coefficient, and $T_{A}, T_{B}$, and $T_{g}$ are the temperatures of the type A and B particles and gas, respectively. Wen and Cheng [63] determined the particle-particle heat transfer coefficient through physical experimentation.

The simplified analysis of heat transfer used to construct this model prevents it from functioning as a general particle-fluid-particle conduction model. For example, the authors hypothesize that contact conduction and radiative heat transfer are negligible, limitations not imposed in the present work. In addition, enforcing the authors' assumption that particles of the same type have the same temperature requires that each Type A particle (or Type B particle) experience the same total heat flux. Otherwise, the individual particles temperatures may diverge as the solutions to the internal energy equations for the particles (i.e., Equation (3-6)) are advanced. Finally, expressions for the particle-particle heat transfer coefficients are scarcely available and limit this model's validity to cases from which correlations are derived. As such, Wen and Chang's [63] model was not selected for this work.

\subsubsection{Delvosalle and Vanderschuren [64] (1985)}

Delvosalle and Vanderschuren [64] developed an analytic model for particlefluid-particle conduction by considering heat transfer between two isothermal particles in a stagnant medium with negligible heat capacity. They hypothesize that for large 
spherical particles of the same size, heat transfer between particles is restricted to a small region around the axis relating the particles' centers. They further assume that heat transfer paths are portions of circumferences perpendicular to the particles' surfaces, as illustrated in Figure 3-9.

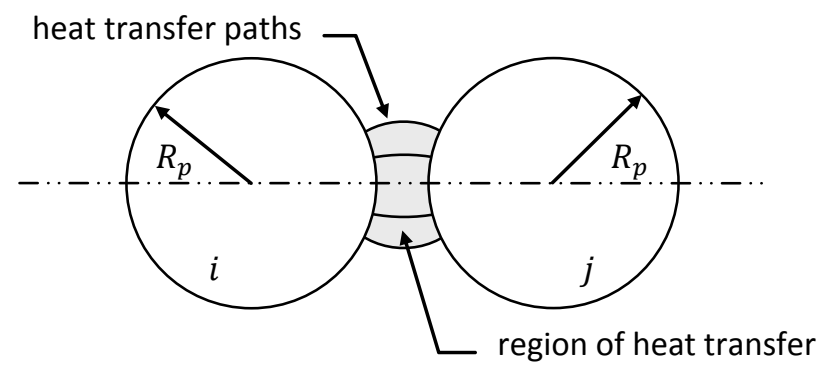

Figure 3-9: Illustration depicting the heat transfer paths as defined by Delvosalle and Vanderschuren's [64]. The heat transfer paths are defined as portions of circumferences perpendicular to the surfaces of the particles.

The resulting model for the instantaneous rate of heat transfer between the particles indexed by $(i)$ and $(j)$ is given Equation (3-31). Here, $R_{p}$ is the particle radius, $\delta$ is distance between the particles' surfaces, and $\alpha$ is the angle delineating the region of particle-fluid-particle conduction. The quantity inside the integral represents the length of the heat transfer paths through the stagnant medium.

$$
\dot{Q}_{p f p}^{(i, j)}=\pi \kappa_{g} R_{p}\left(T_{p}^{(j)}-T_{p}^{(i)}\right) \int_{0}^{\alpha} \frac{\sin ^{2} \theta}{2\left[s / R_{p}+(1-|\cos \theta|)\right] \theta} d \theta
$$

Delvosalle and Vanderschuren [64] extend this model to fluidized systems (i.e., multiple particles in the presents of a non-stagnant gas) in their definition of $\alpha$. The authors assume laminar sub-layers, spherical in shape, develop around particles due to gas flow. Particle-fluid-particle conduction initiates when the sub-layer surrounding one particle intersects the sub-layer surrounding another particle. This is illustrated in Figure 3-10. The resulting definition for $\alpha$ is given by Equation (3-32). Here, $d$ is the thickness 
of the sub-layer and is defined by the authors as the ratio of gas phase thermal conductivity to the heat transfer coefficient of the solids phase.

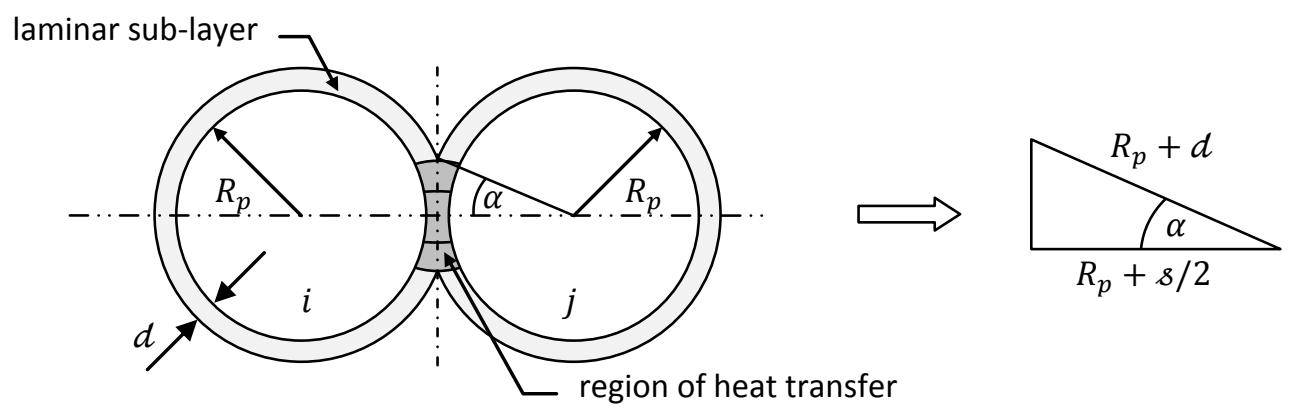

Figure 3-10: Delvosalle and Vanderschuren's [64] model assumes particle-fluid-particle conduction is initiated when the laminar sub-layers surrounding two particles overlap. This configuration is used to define the parameter $\alpha$ in Equation (3-42).

$$
\cos \alpha=\frac{R_{p}+s / 2}{R_{p}+d}
$$

In their model, Delvosalle and Vanderschuren [64] observed that the rate of heat transfer grows infinitely as the distance separating the particles' surfaces approach zero (i.e., $\left.\lim _{s \rightarrow 0} Q_{p f p}^{(i, j)}\right)$. They conclude that perfect contact between particles $(s=0)$ is prohibited by surface roughness, and therefore hypothesize the existence of a minimum separation distance, $s_{m}$. By imposing the minimum separation distance between the particles' surfaces, the rate of heat transfer between the particles is bounded.

Delvosalle and Vanderschuren's [64] model is limited to gas-solids systems where solids are characterized by equal sized particles (i.e., monodispersity). Furthermore, inspection of Equation (3-31) shows that this model does not extend to include particles of varying size (i.e., polydispersity). Specifically, the paths of heat transfer are not definable as portions of circumferences of circles perpendicular to the surfaces of the particles if the diameters of two neighboring particles are different. (It is a geometric impossibility.) Since gas-solids flows are not typically monodisperse [66], 
selecting this approach introduces unnecessary limitations into the CFD-DEM. Although Delvosalle and Vanderschuren's [64] model is implemented in various CFD-DEM codes $[\mathbf{5 9}, \mathbf{6 7}]$, it was not selected for this work in lieu of a more general approach.

\subsubsection{Cheng, Yu, and Zulli [65] (1999)}

Cheng, Yu, and Zulli [65] developed two structure-based analytic models for particlefluid-particle conduction. Both models assume that heat transfer between two neighboring particles is restricted to the region delineated by the double pyramid defined by a Voronoi tessellation boundary plane, illustrated in Figure 3-11. The authors simplify the geometry of the heat transfer region by replacing the double pyramid with a double tapered cone that has the same volume and distance between vertices (particles' centers).
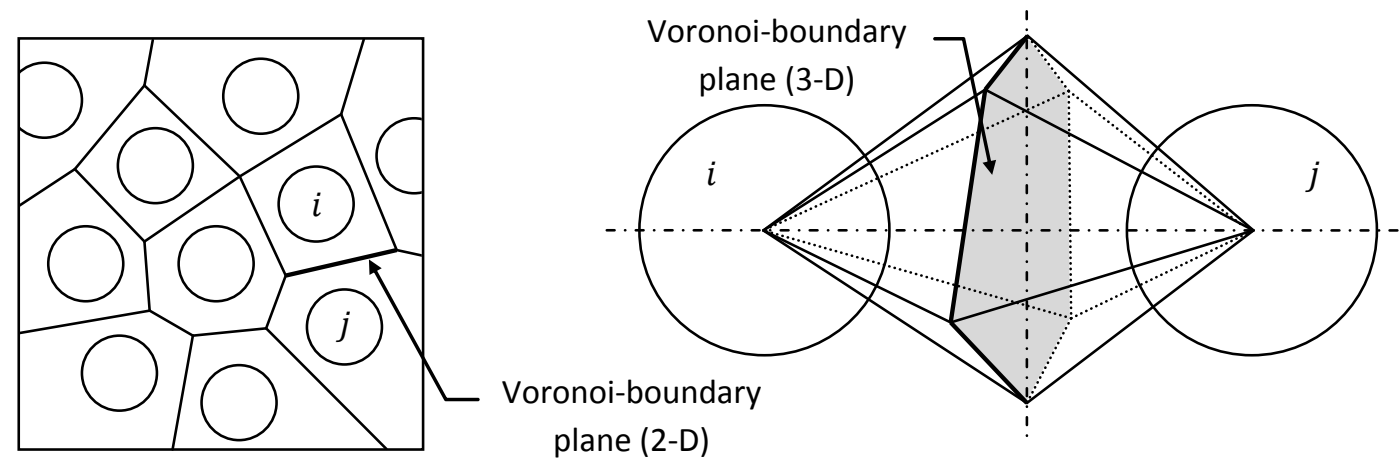

Figure 3-11: The model of Cheng, Yu, and Zulli [65] restricts particle-fluid-particle conduction to the region delineated by the double pyramid (3-D) generated by the Voronoi-boundary plane.

Cheng, Yu, and Zulli [65] employ several assumptions to construct their first model, Model A, including (1) the surface of the double tapered cone is isothermal, (2) conduction is negligible in the outer region of the double tapered cone, and (3) the heat transfer paths are parallel to the axis joining the particles' centers. These assumptions are illustrated in Figure 3-12. The resulting model for the rate of heat transfer between particles $(i)$ and $(j)$ is given as Equation (3-33). 


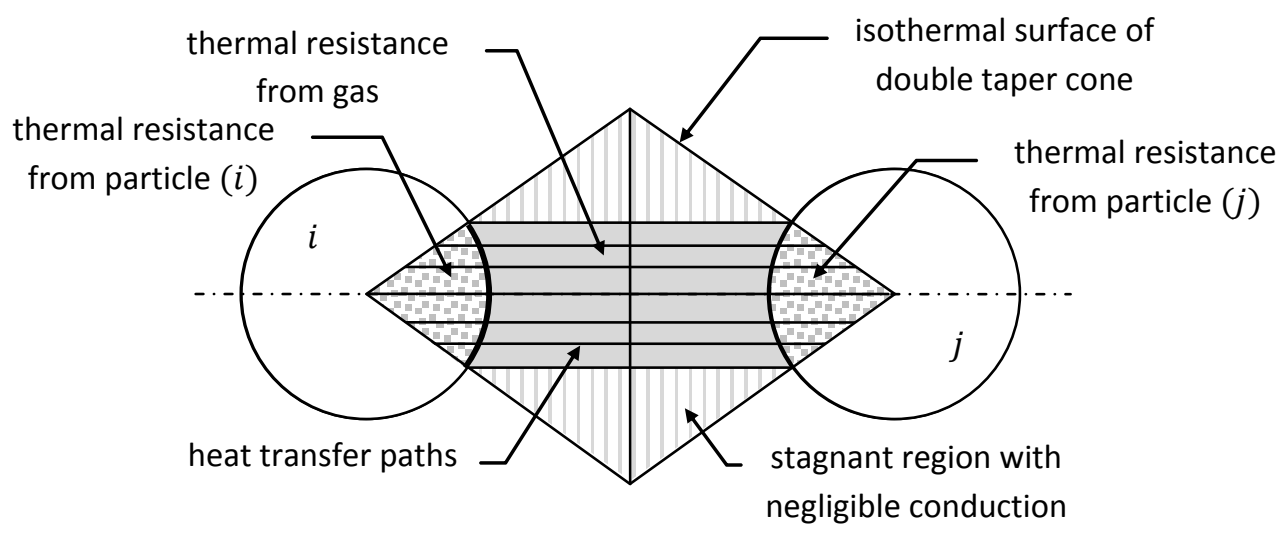

Figure 3-12: Cheng, Yu, and Zulli's [65] Model $A$ for particle-fluid-particle conduction assumes that the surface of the double tapered cone is isothermal, conduction is negligible in the outer region of the double tapered cone, and the heat transfer paths are parallel to the axis joining the particles' centers.

$$
\dot{Q}_{p f p}^{(i, j)}=\left(T_{p}^{(j)}-T_{p}^{(i)}\right) \int_{r_{s}}^{r_{g}}\left(\frac{2 \pi r}{\ell_{i r} / \kappa_{p}^{(i)}+\ell_{g r} / \kappa_{g}+\ell_{j r} / \kappa_{p}^{(j)}}\right) d r
$$

In Equation (3-33), $r_{s}$ and $r_{g}$ define the fluid region for conduction. Specifically, $r_{s}$ defines the inner boundary (zero if the particles are not touching and the radius of the contact area if touching) and $r_{g}$ defines the outer boundary. $\ell_{r i}$ and $\ell_{r j}$ are the lengths of the heat transfer paths through the $i^{\text {th }}$ and $j^{t h}$ particles, and $\ell_{r g}$ is the length of the heat transfer path through the gas, respectively. As previously indicated, $T_{p}$ and $\kappa_{p}$ are the temperatures and thermal conductivities of the indexed particles, $\kappa_{g}$ is the thermal conductivity of the gas, and $\ell^{(i, j)}$ is distance between the particles' centers. The geometric representation of these quantities is illustrated in Figure 3-13.

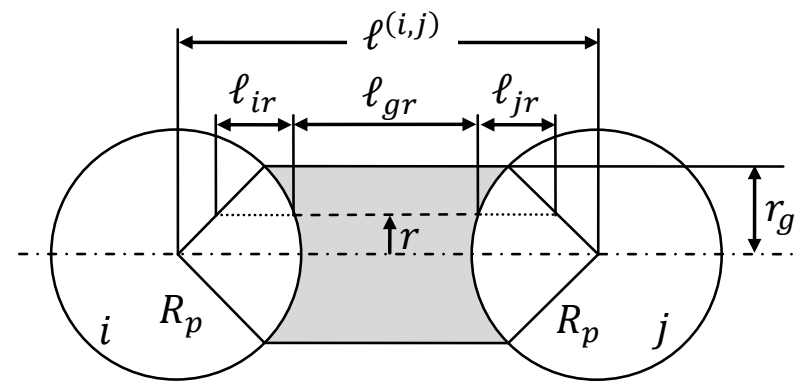

Figure 3-13: Geometric representation of quantities used by Cheng, Yu, and Zulli's [65] Model A for particle-fluid-particle conduction. 
To develop their second model for particle-fluid-particle conduction, Model B, Cheng, Yu, and Zulli [65] assume (1) each particle has an isothermal core of radius $R_{c}$, and (2) heat transfer paths radiate from the cores' surfaces. These assumptions are illustrated in Figure 3-14, and the resulting model for the rate of heat transfer between particles $(i)$ and $(j)$ is given as Equation (3-34).

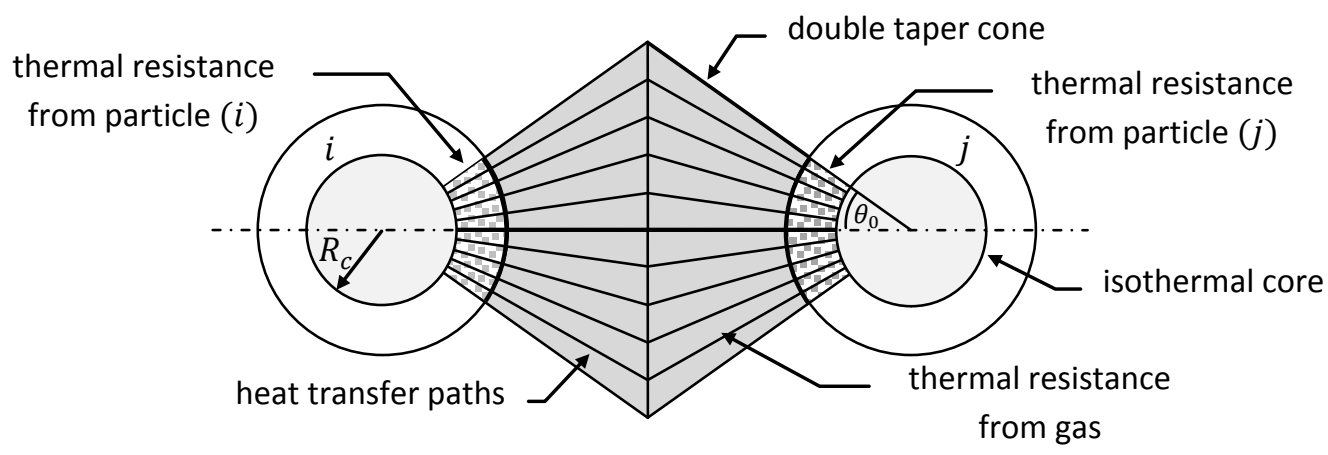

Figure 3-14: Cheng, Yu, and Zulli's [65] Model B for particle-fluid-particle conduction assumes that each particle has an isothermal core and the paths of heat transfer radiate from the particles centers.

$$
\begin{gathered}
\dot{Q}_{p f p}^{(i, j)}=\pi\left(T_{p}^{(j)}-T_{p}^{(i)}\right) \frac{1}{b} \ln \left(\frac{a-b \cos \theta_{0}}{a-b \cos \theta_{c}}\right) \\
a=\frac{1}{2}\left(\frac{1}{\kappa_{p}^{(i)}}+\frac{1}{\kappa_{p}^{(j)}}\right)\left(\frac{1}{R_{c}}-\frac{1}{R_{p}}\right)+\frac{1}{\kappa_{g} R_{p}} ; \quad b=\frac{1}{\kappa_{g}\left(R_{p}+s / 2\right)}
\end{gathered}
$$

In Equation (3-34), $\theta_{0}$ and $\theta_{c}$ define the fluid region for conduction. Specifically, $\theta_{c}$ defines the inner boundary (zero if the particles are not touching and the angle delineating the contact area if touching), $\theta_{0}$ defines the outer boundary, and $s$ is distance between the particles' surfaces.

A significant drawback to Cheng, Yu, and Zulli's [65] models is their dependence on a Voronoi tessellation to determine the region of particle-fluid-particle heat transfer. Due to the continuous movement of particles in gas-solids systems, a tessellation is required at each solids time step. Such calculations are computationally expensive. Additionally, since their models were developed for packed bed simulations, extending 
them to fluidized systems may result in unphysical behavior. Specifically, particles that are separated by large distances may share common Voronoi-boundaries, and in turn, have the potential to engage in particle-fluid-particle heat transfer. Additional constraints are needed to prevent this from occurring in the current model (i.e., a maximum distance at which particle-fluid-particle ceases to occur). Finally, introducing models which consider resistance to heat transfer through particles is contradictory to the assumption in the present effort that particles are isothermal (see Section 3.1.2). Although a simplified version of Model $A$ is found in the CFD-DEM model of Zhou, Yu, and Zulli [19], neither Model A nor Model B is selected for this work. This is due to the computational complexity of the models and disagreement with the fundamental assumption employed in this work of isothermal particles.

\subsubsection{Rong and Horio [16] (1999)}

Rong and Horio [16] introduced a particle-fluid-particle heat transfer model similar to that of Delvosalle and Vanderschuren [64]. The authors hypothesize that (1) each particle is surrounded by a gas layer with thickness $\delta=R_{p}$, and (2) particle-particle heat transfer initiates when the gas layer surrounding a particle intersects with the surface of another particle. They further assume that (3) heat transfer occurs across paths parallel to the axis joining the particles' centers, and (4) a uniform gas layer, $4.0 \times 10^{-10} \mathrm{~m}$ thick, separates the particles' surfaces as they collide. These assumptions are illustrated in Figure 3-15. The resulting model is given in Equation (3-35). 


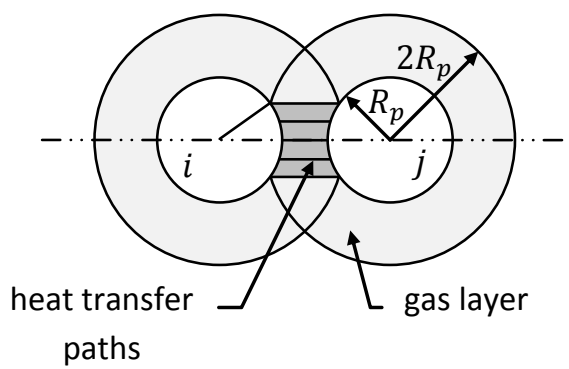

no contact

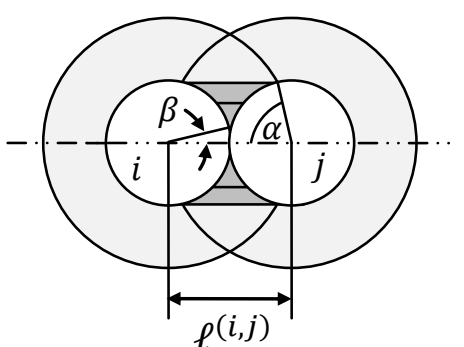

contact

Figure 3-15: Rong and Horio's [16] configuration for particle-fluid-particle conduction. In their model, each particle is surrounded by a gas layer and heat transfer occurs across paths parallel to the line connecting the centers of the particles. When particles engage in contact, the overlap region is assumed to have a uniform gas layer separating the particles' surfaces.

$$
\dot{Q}_{p f p}^{(i, j)}=\kappa_{g}\left(T_{p}^{(j)}-T_{p}^{(i)}\right) \mathcal{A}
$$

where

$$
\mathcal{A}= \begin{cases}0 & \text { for } \quad \ell^{(i, j)} \geq 3 R_{p} \\ \int_{0}^{\alpha} \frac{2 \pi R_{p} \sin \theta}{\ell^{(i, j)}-2 R_{p} \cos \theta} d\left(R_{p} \sin \theta\right) & \text { for } 3 R_{p}>\ell^{(i, j)}>2 R_{p} \\ \frac{\pi\left(R_{p} \sin \beta\right)^{2}}{4 \times 10^{-10}}+\int_{\beta}^{\alpha} \frac{2 \pi R_{p} \sin \theta}{\ell^{(i, j)}-2 R_{p} \cos \theta} d\left(R_{p} \sin \theta\right) & \text { for } \quad \ell^{(i, j)} \leq 2 R_{p}\end{cases}
$$

In Equation (3-35), $\alpha$ and $\beta$ are the angles delineating the region of particle-fluidparticle conduction, $\ell^{(i, j)}$ is the distance between the centers of the particles, $\kappa_{g}$ is the thermal conductivity of the gas, and $R_{p}$ and $T_{p}$ are the particles radius and temperature, respectively. Particles whose surfaces are separated by a distance greater than one particle radius $\left(R_{p}\right)$ do not undergo particle-fluid-particle heat transfer. When the surfaces of two particles are closer than one particle radius but not touching particle-fluid-particle heat transfer occurs in the defined region. If two particles are in direct contact, then there is a uniform rate of particle-fluid-particle heat transfer across the contact area 
$\left(\frac{\pi\left(R_{p} \sin \beta\right)^{2}}{4 \times 10^{-10}}\right)$ as particle-fluid-particle heat transfer occurs around the interface of the contact area.

This model was selected as the foundation for the particle-fluid-particle model developed in this work. Like the model proposed by Delvosalle and Vanderschuren [64], Rong and Horio's [16] approach is valid only for particles of equal diameter. However, unlike Delvosalle and Vanderschuren's [64] model, the model proposed by Rong and Horio [16] extends to particles of different sizes by modifying several geometric definitions. The extension is possible because Rong and Horio [16] assume that heat transfer paths are parallel to the axis joining the particles' centers as opposed to the arc structures used by Delvosalle and Vanderschuren [64]. The following section presents the extension of Rong and Horio’s [16] model to include particles of different sizes.

\subsubsection{Modified Rong and Horio [16] particle-fluid-particle conduction model}

As previously noted, Rong and Horio's [16] model for particle-fluid-particle conduction was developed in the context of equal size particles. Extending their model to particles of different size requires a standard point of reference to develop a consistent definition for the particle-fluid-particle region. This specification is satisfied by delineating the outer region of particle-fluid-particle heat transfer by the intersection of the gas layer surrounding the larger particle with the surface of the smaller particle. This is expressed by Equation (3-36) and illustrated in Figure 3-16. Here, the indices $(k)$ and (l) identify the particles by size, specifically $R_{p}^{(k)}=\max \left\{R_{p}^{(i)}, R_{p}^{(j)}\right\}$ and $R_{p}^{(l)}=$ $\min \left\{R_{p}^{(i)}, R_{p}^{(j)}\right\}$, and $d^{(k)}$ is the thickness of the gas layer. $R_{f}^{(i, j)}$ is similar to $\alpha$ in Equation (3-35) in that it delineates the upper bound on the region of particle-fluid- 
particle conduction. The default gas layer thickness for a particle indexed by $(i)$ is defined as $R_{p}^{(i)} / 5$ in the current effort. This value was suggested by Xavier and Davidson [68] in their surface-to-bed heat transfer studies using $0.1-8.0 \mathrm{~mm}$ particles and a vertical heater.

$$
R_{f}^{(i, j)}=\sqrt{\left(R_{p}^{(k)}+d^{(k)}\right)^{2}-\left(\frac{\left(R_{p}^{(k)}+d^{(k)}\right)^{2}-R_{p}^{(l)^{2}}+\ell^{(i, j)^{2}}}{2 \ell^{(i, j)}}\right)^{2}}
$$

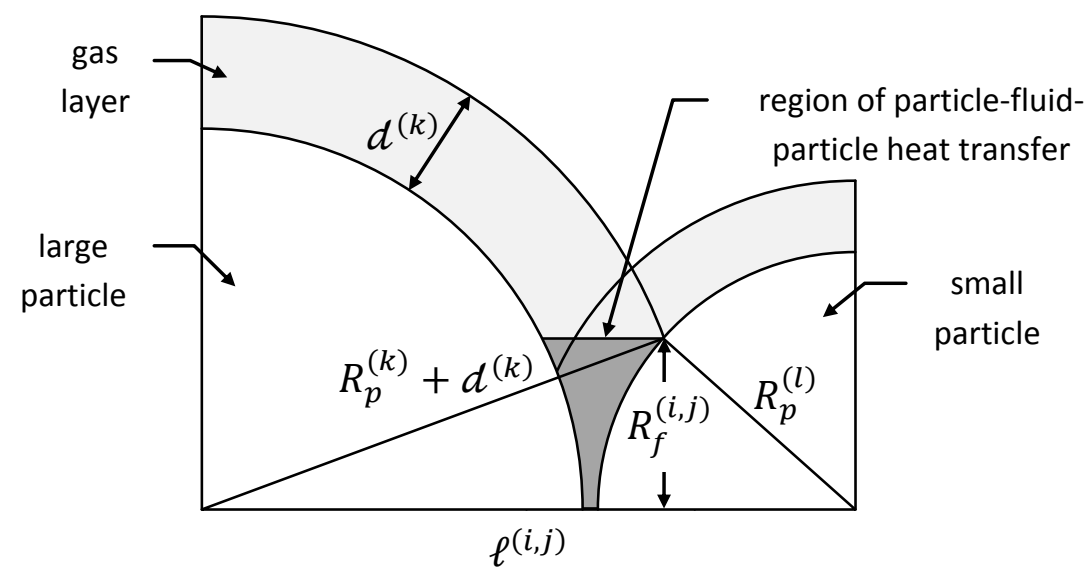

Figure 3-16: Illustration of geometric quantities delineating the region of particle fluid heat transfer imposed in this work.

A change of variable is applied to Rong and Horio's [16] model to remove its dependency on the trigonometric functions of sine and cosine. This change was established so that standard quadrature routines are capable of managing the integral term. Finally, the additional quantity added by Rong and Horio [16] to account for heat transfer in the contact region (i.e., $\pi\left(R_{p} \sin \beta\right)^{2} / 4 \times 10^{-10}$ in Equation (3-35)) is discarded in lieu of the contact conduction model defined in Section 3.2.2. The resulting model for particle-fluid-particle heat transfer is given as Equation (3-37). Here, $\tilde{R}_{c}^{(i, j)}$ is the contact radius of two touching particles defined in Equation (3-25) and $\ell^{(i, j)}$ is the distance separating the particles' centers. 


$$
\dot{Q}_{p f p}^{(i, j)}=\kappa_{g}\left(T_{p}^{(j)}-T_{p}^{(i)}\right) \int_{R_{c}}^{R_{f}^{(i, j)}} \frac{2 \pi r}{\ell^{(i, j)}-\left(\left(R_{p}^{(i)^{2}}-r^{2}\right)^{1 / 2}+\left(R_{p}^{(j)^{2}}-r^{2}\right)^{1 / 2}\right)} d r
$$

where

$$
R_{c}=\left\{\begin{array}{ccc}
0 & \text { for } \quad \ell^{(i, j)}>2 R_{p} \\
\tilde{R}_{c}^{(i, j)} & \text { for } & \ell^{(i, j)} \leq 2 R_{p}
\end{array}\right.
$$

As illustrated in Figure 3-17, a singularity in the integral term of Equation (3-37) occurs at the interface of two touching particles. This results in an infinite rate of heat transfer between the particles. Delvosalle and Vanderschuren [64] also observed this problem and avoided perfect contact between the particles by requiring a minimum separation distance. In this effort, a default minimum separation distance of $4 \times 10^{-10} \mathrm{~m}$ is specified, which corresponds to the value used for surface roughness in Rong and Horio's [16] model.

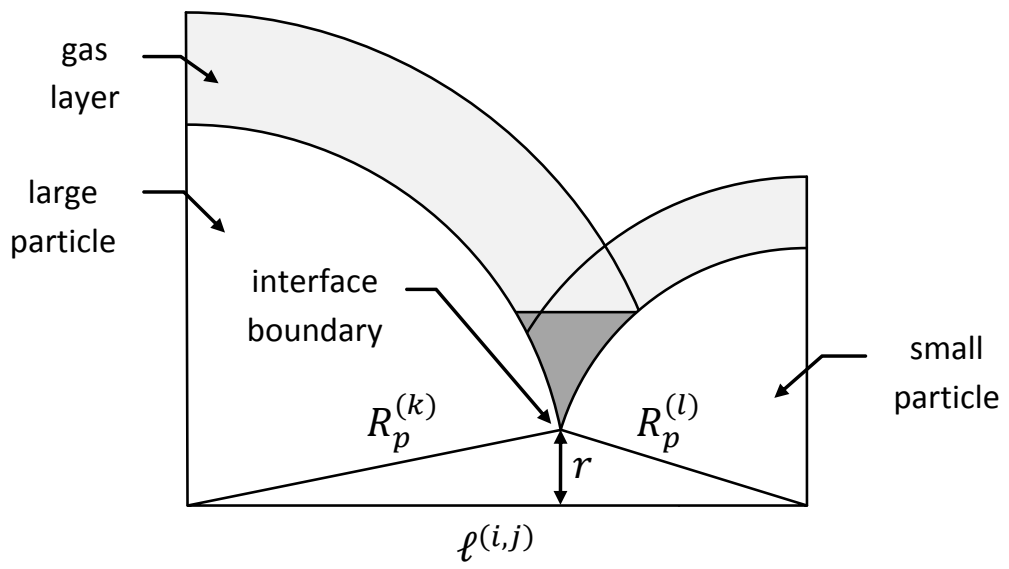

Figure 3-17: A singularly arises in the modified Rong and Horio model at the interface of the contact boundary. This is corrected by prohibiting perfect contact between particles, and require a minimum separation distance of $4 \times 10^{-10} \mathrm{~m}$. Similar assumptions are employed by Delvosalle and Vanderschuren [64] and Rong and Horio [16].

This completes the section on particle-fluid-particle heat transfer. Here, Equation (3-37) is established as the default particle-fluid-particle conduction model $\left(\dot{Q}_{p f p}\right)$ for the 
particle internal energy equation (Equation (3-6)). The following section considers particle-gas convective heat transfer $\left(\dot{Q}_{p g}\right)$ in gas-solids systems.

\subsection{Particle-gas convection}

Recall, convection is defined in this effort as heat transfer between a particle and the surrounding gas resulting from differences in temperature and velocity. This section introduces Newton's law of cooling for modeling convection, and briefly discusses the connection between the convective heat transfer coefficient and dimensionless Nusselt number. The Ranz and Marshall [69] correlation for the Nusselt number, selected as the default correlation in this work, is presented.

\subsubsection{Background of particle-gas convection}

Heat transfer between a particle and gas is assumed in this work to follow Newton's law of cooling. Newton's law of cooling, shown as Equation (3-38), hypothesizes that the rate of heat transfer between two objects is proportional their temperature difference. The constant of proportionality for convective heat transfer between a particle and gas often is given as the combination of a convective heat transfer coefficient $\left(\gamma_{c p}\right)$ and the surface area of the particle $\left(A_{s}\right)$. Therefore, the rate of convective heat transfer between a single particle and moving gas is given by Equation (3-39), where $T_{p}$ is the particle's temperature, and $T_{g}$ is the average temperature of the gas surrounding the particle.

$$
\begin{gathered}
\dot{Q}_{p g} \propto \Delta T \\
\dot{Q}_{p g}=\gamma_{c p} A_{s}\left(T_{g}-T_{p}\right)
\end{gathered}
$$


The convective heat transfer coefficient is commonly modeled by the dimensionless Nusselt number which expresses the ratio of total convective heat transfer to conductive heat transfer. The Nusselt number for a spherical particle is shown in Equation (3-40), where $R_{p}$ is the particle's radius and $\kappa_{g}$ is the thermal conductivity of the gas phase. Note that the Nusselt number's definition is similar that of the Biot number presented in Equation (3-4). However, the Nusselt number's definition uses the thermal conductivity of the fluid as opposed to the solid.

$$
\mathrm{Nu}_{p}=\frac{\gamma_{c p}}{\kappa_{g} / 2 R_{p}}
$$

A large number of experimental correlations for the Nusselt number are available in the literature $[69,70,71,72,73]$. Typically, correlations are reported in a format similar to that presented in Equation (3-41) where $a, b, c$, and $d$ are fitted empirical constants, and Re and Pr are the dimensionless Reynolds and Prandtl numbers, respectively. The Reynolds number, defined in Equation (3-42), is the ratio of inertial forces and viscous forces in the fluid. The Prandtl number, given by Equation (3-43), is the ratio of momentum and thermal diffusivity in the fluid. In these equations, $\rho_{g}, \varepsilon_{g}, \kappa_{g}, C_{p g}, \mu_{g}$, and $\boldsymbol{v}_{g}$ are the density, void fraction, thermal conductivity, specific heat, viscosity, and velocity of the gas, respectively. $R_{p}$ and $\mathbf{V}_{p}$ are the radius and velocity of the particle.

$$
\begin{gathered}
\mathrm{Nu}_{p}=a+b \operatorname{Re}^{c} \operatorname{Pr}^{d} \\
\mathrm{Re}=\frac{\rho_{g} \varepsilon_{g}\left\|\boldsymbol{v}_{g}-\mathrm{V}_{p}\right\|_{2} 2 R_{p}}{\mu_{g}} \\
\operatorname{Pr}=\frac{C_{p g} \mu_{g}}{\kappa_{g}}
\end{gathered}
$$


Nusselt number correlations of the form shown in Equation (3-41) are assumed to include both natural and forced convection. Typically, natural convection (also referred to as free convection) is associated with a change in density of the fluid. For example, consider a hot object surrounded by a cooler fluid. The density of the fluid at near the object's surface decreases as heat is transferred to it from the object. The warmer, less dense fluid then rises, allowing cooler fluid to take its place at the objects surface. In contrast, forced convection occurs when fluid is moved past an object due to some external force (e.g., a free-falling particle, fan, pump, etc.). Thus, the constant $a$ in Equation (3-41) captures the effects of natural convection as $\operatorname{Re} \rightarrow 0$, while the second term in Equation (3-41) $\left(b \operatorname{Re}^{c} \operatorname{Pr}^{d}\right)$ incorporates the contribution to heat transfer from forced convection as the Reynolds number increases.

Although numerous experimental correlations for the Nusselt number exist, this work employs the correlation of Ranz and Marshall [69], defined in the following section. The inclusion of additional correlations is addressed in Section 3.6.2.

\subsubsection{Ranz and Marshall [69] (1952)}

Ranz and Marshall's [69] correlation is given in Equation (3-44). This expression was developed by analyzing heat transfer data from evaporating water droplets, suspended in an upward-flowing gas. Recall that $\operatorname{Re}$ and $\operatorname{Pr}$ are the dimensionless Reynolds and Prandtl numbers defined in Equations (3-42) and (3-43), respectively.

$$
\mathrm{Nu}_{p}=2.0+0.6 \operatorname{Re}^{1 / 2} \operatorname{Pr}^{1 / 3}
$$

Since Ranz and Marshall's [69] investigation analyzed data from isolated particles (i.e., $\varepsilon_{g} \rightarrow 1$ in Equation (3-42)), additional considerations are needed as the solids volume fraction $\left(\varepsilon_{s}=1-\varepsilon_{g}\right)$ increases. For $\operatorname{Re}>10^{2}$, experimentally obtained heat 
transfer data from fluidized beds was shown to agree reasonably well with Equation (3-44). However, as the Reynolds number decreases ( $\operatorname{Re}<10)$, Equation (3-44) is shown to over predict the heat transfer coefficient for a bed of particles [74]. Specifically, as the Reynolds number decreases, observed values for the Nusselt number for the bed drop below two, the theoretical minimum given by Equation (3-44) as $\operatorname{Re} \rightarrow 0$. It is hypothesized that this is a result of reduced particle-gas contact as particles in a bed move closer together [74]. No bounds on void fraction were found in the literature indicating an acceptable rage in which this model is applicable. Additionally, no bounds for the Reynolds and Prandtl numbers were provided by Ranz and Marshall [69], but several recommendations are found in the literature. Fan and Zhu [29] suggest that Equation (3-44) is applicable for $\operatorname{Re}<10^{4}$ and $\operatorname{Pr} \geq 0.7$ encompassing most gas-solids flows. Li and Mason [17] claim Equation (3-44) provides acceptable results for $\operatorname{Re}<200$, while Mankad, Nixon, and Fryer [75] suggest it only applicable for Re $<100$.

Ranz and Marshall's [69] correlation is employed in numerous CFD-DEM models $[\mathbf{2}, \mathbf{1 6}, \mathbf{1 7}, \mathbf{2 1}, \mathbf{7 6}]$ as well as a variation found in $[\mathbf{1 8}]$ (i.e., $\mathrm{Nu}=2 \varepsilon_{g}+0.69 \mathrm{Re}^{1 / 2} \operatorname{Pr}^{1 / 3}$ ). Likewise, Equation (3-44) was selected as the default correlation for this work. The consideration to include additional correlations in the future is addressed in Section 3.6.2. This completes the discussion on particle-gas convective heat transfer. Here, Equation (3-39) is established as the convective heat transfer model $\left(\dot{Q}_{p g}\right)$ for the particle internal energy equation (Equation (3-6)). Ranz and Marshall's [69] Nusselt number correlation (Equation (3-44)) was selected as the default closure model for the heat transfer coefficient $\left(\gamma_{c p}\right)$ in Equation (3-39). The following section introduces particleenvironment radiative heat transfer $\left(\dot{Q}_{r a d}\right)$ in gas-solids systems. 


\subsection{Particle-particle radiation}

Radiative heat transfer occurs between the surfaces of solids [77] as well as an object's surface and the fluid surrounding it. Specifically, radiative heat transfer in gassolids systems may occur between the particles and walls, individual/groups of particles, and the particles and gas. Presently, MFIX-DEM operates with the assumption that system walls are adiabatic (i.e., perfectly insulated so that no thermal energy enters or leaves the system through the walls). As a result, radiative heat transfer between particles and walls is not considered. Information concerning particle-wall heat transfer is found in references $[\mathbf{2 9 , 7 8 , 7 9 ]}$. Additionally, this work assumes that the gas phase is perfectly transparent (i.e., the gas offers no resistance or contribution to radiative heat transfer). Therefore, particle-gas radiative heat transfer is also not considered. Information concerning particle-gas radiative heat transfer is provided in references $[\mathbf{4 7 , 8 0}]$. Finally, particle-particle radiative heat transfer is commonly omitted from CFD-DEM heat transfer models $[\mathbf{1 7}, \mathbf{2 1}, \mathbf{5 1}, \mathbf{5 9}, \mathbf{6 0}, \mathbf{6 1}, \mathbf{6 3}, \mathbf{6 7}, \mathbf{7 6}, \mathbf{8 1}]$ due to its negligible contribution to overall heat transfer at temperatures below $600^{\circ} \mathrm{C}[63]$. However, as temperatures increase, the importance of particle-environment radiation also increases $[\mathbf{1 9}, \mathbf{7 8}]$. Since a goal of this work is to construct a comprehensive heat transfer model for the CFD-DEM, particle-particle radiative heat transfer is included for completeness. The negligible effect of radiative heat transfer at moderate temperatures is addressed in the implementation discussion.

This section continues by briefly describing radiative heat transfer, whereas the interested reader is directed to references $[\mathbf{4 7 , 8 2}]$ for a thorough review of the topic. The radiative heat transfer model employed in various CFD-DEM models is presented. 
Finally, the distinguishing closer term separating these models,$T_{\text {env }}$ (i.e., environment temperature), is discussed as well as the new definition developed for this work.

\subsubsection{Background of particle-particle radiation}

Radiative heat transfer is the result of reflecting, absorbing, and emitting of thermal radiation [47]. An object that absorbs all incident radiation (i.e., no radiation is reflected) is termed a blackbody or is said to have a black surface. The rate at which radiation is emitted from a blackbody per unit surface area $\left(E_{b}\right)$ is called the blackbody emissive power and is given by the Stefan-Boltzmann law, shown as Equation (3-45). Here, $\sigma$ is the Stefan-Boltzmann constant, and $T$ is the temperature of the blackbody.

$$
E_{b}=\sigma T^{4}
$$

Real surfaces absorb and emit less radiation than black surfaces. The absorptance $(\varsigma)$ is the fraction of incident radiation absorbed by a surface. Conversely, reflectance $(\rho)$ is the fraction of incident radiation reflected by a surface. For surfaces that are not transparent, $\rho=1-\varsigma$. The emittance $(\epsilon)$ is the fraction of blackbody emissive power a surface possess (i.e., the ratio of actual to ideal emissive power). Typically, real surfaces are treated as gray surfaces, wherein the absorptance and emittance are equal $(\epsilon=\varsigma$ and $\epsilon=1-\rho)$

An energy balance between two diffuse, gray surfaces leads to Equation (3-46) (see reference [47] for details). Here, $T, A_{s}$, and $\epsilon$ are the temperatures, surface areas, and emittances of surfaces $i$ and $j$, respectively. $F_{i j}$ is the shape factor (also referred to as a view factor), which indicates the fraction of radiation emitted by surface $i$ that is absorbed by surface $j$. Various configurations for surface factors are tabulated and available in an assortment of tables and graphs $[\mathbf{4 7 , 8 3}]$. 


$$
\dot{Q}_{\text {rad }}^{(i, j)}=\frac{\sigma T_{j}^{4}-\sigma T_{i}^{4}}{\frac{1-\epsilon_{i}}{\epsilon_{i} A_{s i}}+\frac{1}{A_{s i} F_{i j}}+\frac{1-\epsilon_{j}}{\epsilon_{j} A_{s j}}}
$$

The complexity of Equation (3-46) is reduced by considering the particles surrounding a particle of interest as a complete surface. Specifically, the neighboring particles are assumed to form a spherical environment surrounding the particle, as illustrated Figure 3-18. This reduces the multiple particle radiative heat transfer problem to one of concentric spheres $\left(F_{i j}=1\right.$ [47]). Furthermore, by assuming that the environment has a nearly black surface $\left(\epsilon_{j} \approx 1\right)$, Equation $(3-46)$ reduces to Equation (3-47). Observe that this simplification also arises by assuming that the surface area of the environment is sufficiently large (i.e., $A_{s j} \gg 1$ ).
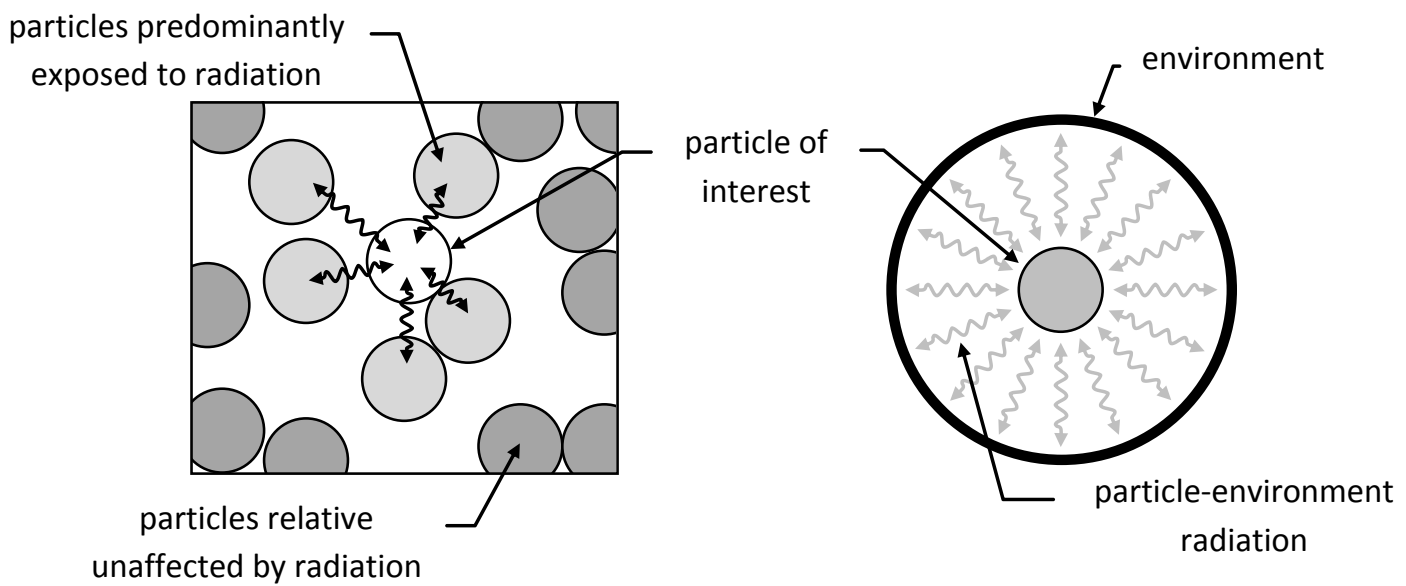

Figure 3-18: A multi-particle radiation problem is reduced by assuming that the particles predominantly exposed to radiation form a spherical environment surrounding the particle of interest.

$$
\dot{Q}_{\text {rad }}^{(i)}=\epsilon_{i} A_{s i} \sigma\left(T_{e n v}^{4}-T_{i}^{4}\right)
$$

Various CFD-DEM codes use Equation (3-47) for modeling particle-particle radiative heat transfer $[\mathbf{1 6}, \mathbf{1 8}, \mathbf{1 9}, \mathbf{2 0}]$. The main distinction separating these models is their definitions for the environment temperature, $T_{\text {env }}$. Two definitions found in the literature 
are discussed in the following section. Finally, a new definition for the environment temperature developed for this effort is presented.

\subsubsection{Environment temperature definition}

One definition for the environment temperature found in the literatures is obtained by averaging the temperature of all particles in a system $[\mathbf{1 6 , 1 8 , 2 0}]$, as shown in Equation (3-48). Here, $N_{p}$ is the total number of particles in a simulation, and $T_{p}$ is the temperature of the indexed particle. This definition invokes the assumption that every particle is exposed to the same radiative heat flux. For example, an isolated particle will experience the same radiative heat flux as a particle, at the same temperature, surrounded by several particles whose temperatures differ greatly from the average solids temperature. As a result, this definition is not employed in this work, in favor of one that computes $T_{\text {evn }}$ based upon the local conditions of a particle.

$$
T_{e n v}=\frac{1}{N_{p}} \sum_{j=1}^{N_{p}} T_{p}^{(j)}
$$

Zhou, Yu, and Zulli [19] calculate the environment temperature based on the contents of a sub-domain surrounding a particle. This is shown in Equation (3-49) and

illustrated in Figure 3-19. In this equation, $\Omega$ defines the sub-domain around the $i^{\text {th }}$ particle, $N_{p, \Omega}$ is the number of particles in the sub-domain, and $\varepsilon_{g}$ and $T_{g}$ are the void fraction and temperature of the gas, respectively. The authors define the radius of the subdomain as 1.5 times the diameter of the particle. This definition addresses the limitation observed in Equation (3-48), however, by including the temperature of the gas, Equation (3-49) suggests some consideration to particle-gas radiative heat transfer is needed. Since 
particle-gas radiative heat transfer is not considered in this work (see Section 3.5), this definition for the environment temperature was not selected.

$$
T_{e n v}^{(i)}=\varepsilon_{g} T_{g, \Omega}+\left(1-\varepsilon_{g}\right) \frac{1}{N_{p, \Omega}} \sum_{\substack{j=1 \\ j \neq i}}^{N_{p, \Omega}} T_{p}^{(j)}
$$

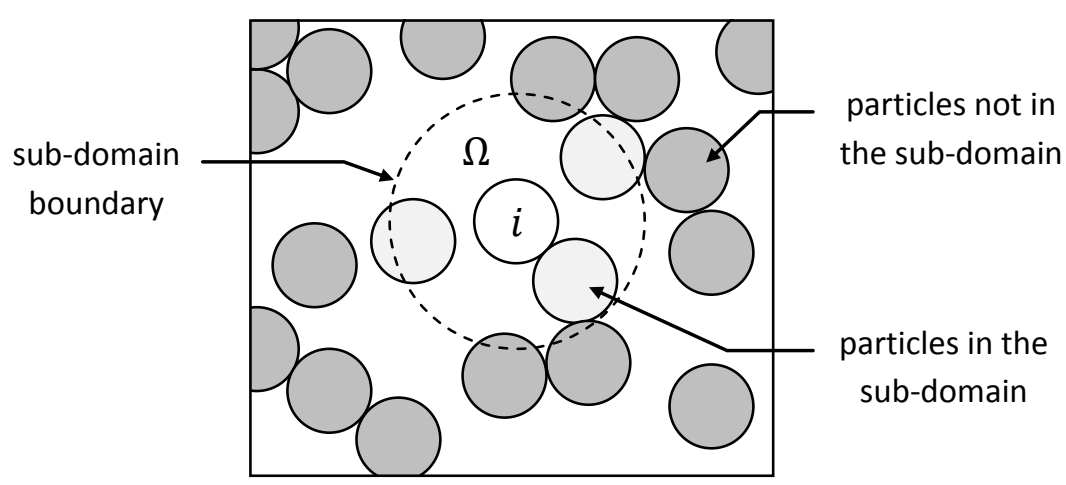

Figure 3-19: Illustration of the sub-domain model proposed by Zhou, Yu, and Zulli [19] for determining the environment temperature used to calculate the rate of radiative heat transfer with particle $i$. In this example, three particles are in the sub-domain (i.e., $N_{p, \Omega}=3$ ) of particle $i$.

In this effort, the environment temperature is defined as Equation (3-50). This definition considers the temperature of the surrounding particles and assumes negligible radiative heat transfer between the gas and particle. Note that Equation (3-50) is very similar to each of the above for environment temperature. That is, equating $N_{p, \Omega}$ to $N_{p}$ in Equation (3-50) produces Equation (3-48), while setting $\varepsilon_{g}=0$ in Equation (3-49) results in Equation (3-50).

$$
T_{e n v}^{(i)}=\frac{1}{N_{p, \Omega}} \sum_{\substack{j=1 \\ j \neq i}}^{N_{p, \Omega}} T_{p}^{(j)}
$$

This completes the discussion of particle-particle radiation $\left(\dot{Q}_{\text {rad }}\right)$. Here, Equation (3-47) is established as the radiation heat transfer model $\left(\dot{Q}_{r a d}\right)$ for the particle internal 
energy equation (Equation (3-6)). The environment temperature $\left(T_{\text {env }}\right)$ needed for closer of Equation (3-47) is defined by Equation (3-50).

This section concludes the survey on the individual mechanisms of heat transfer considered in this work and the selection of a heat transfer model for each mechanism. The following section outlines the implementation of these models into MFIX-DEM.

\subsection{Implementation of models}

The mathematical models selected to describe the four forms of particle heat transfer considered in this effort and discussed in the preceding sections, are summarized in Table 3-1. These models were implemented into MFIX-DEM, an existing CFD-DEM code.

Table 3-1: Summary of the particle internal energy equation and four forms of particle heat transfer considered in this work.

\begin{tabular}{|c|c|}
\hline $\begin{array}{l}\text { particle internal energy } \\
\text { equation (Section 3.1.3) }\end{array}$ & $m^{(i)} C_{p p}^{(i)} \frac{d T_{p}^{(i)}}{d t}=\dot{Q}_{p p}^{(i)}+\dot{Q}_{p f p}^{(i)}+\dot{Q}_{p g}^{(i)}+\dot{Q}_{r a d}^{(i)}$ \\
\hline $\begin{array}{l}\text { particle-particle contact } \\
\text { conduction } \\
\text { (Section } 3.2 .2)\end{array}$ & $\dot{Q}_{p p}^{(i, j)}=\frac{4 \kappa_{p}^{(i)} \kappa_{p}^{(j)}}{\kappa_{p}^{(i)}+\kappa_{p}^{(j)}} \sqrt{R_{p}^{(k)^{2}}-\left(\frac{R_{p}^{(k)^{2}}-R_{p}^{(l)^{2}}+\ell^{(i, j)^{2}}}{2 \ell^{(i, j)}}\right)^{2}}\left(T_{p}^{(j)}-T_{p}^{(i)}\right)$ \\
\hline $\begin{array}{l}\text { particle-fluid-particle } \\
\text { conduction } \\
\text { (Section 3.3.2) }\end{array}$ & $\begin{array}{l}\dot{Q}_{p f p}^{(i, j)}=\kappa_{g}\left(T_{p}^{(j)}-T_{p}^{(i)}\right) \int_{R_{c}}^{R_{f}^{(i, j)}} \frac{2 \pi r}{\ell^{(i, j)}-\left(\left(R_{p}^{(i)^{2}}-r^{2}\right)^{1 / 2}+\left(R_{p}^{(j)^{2}}-r^{2}\right)^{1 / 2}\right)} d r \\
\text { where } \\
\qquad R_{c}=\left\{\begin{array}{l}0 \quad \text { for } \ell^{(i, j)}>2 R_{p} \\
\text { and } \\
\qquad R_{p}^{(k)}=\max \left\{R_{p}^{(i)}, R_{p}^{(j)}\right\} \quad \text { and } \quad R_{p}^{(l)}=\min \left\{R_{p}^{(i)}, R_{p}^{(j)}\right\}\end{array}\right.\end{array}$ \\
\hline $\begin{array}{l}\text { particle-gas convection } \\
\text { (Sections 3.4.1 and 3.4.2) }\end{array}$ & $\dot{Q}_{p g}=\gamma_{c p} A_{s}\left(T_{g}-T_{p}\right) \quad$ where $\quad \mathrm{Nu}_{p}=\frac{\gamma_{c p}}{\kappa_{g} / 2 R_{p}}=2.0+0.6 \mathrm{Re}^{1 / 2} \operatorname{Pr}^{1 / 3}$ \\
\hline $\begin{array}{l}\text { particle-particle radiation } \\
\text { (Sections 3.5.1 and 3.5.2) }\end{array}$ & $\dot{Q}_{r a d}^{(i)}=\epsilon_{i} A_{s i} \sigma\left(T_{e n v}^{4}-T_{i}^{4}\right) \quad$ where $\quad T_{e n v}^{(i)}=\frac{1}{N_{p, \Omega}} \sum_{\substack{j=1 \\
j \neq i}}^{N_{p, \Omega}} T_{p}^{(j)}$ \\
\hline
\end{tabular}


This required the modification and creation of several subroutines. Descriptions of new subroutines are outlined in Table 3-2, while the source code is reserved for Appendix D. Modifications to existing routines mainly consist of logical checks associated with call statements to the new routines. A call-tree showing the invocation of the heat transfer model initialization routines is shown in Figure 3-20, and the invocation of the heat transfer model operational routines is shown by the call-tree in Figure 3-21.

Table 3-2: A list of subroutines developed to set initial conditions of particles and manage the heat transfer models implemented into MFIX.

\begin{tabular}{|c|c|}
\hline $\begin{array}{l}\text { Subroutine } \\
\text { file name }\end{array}$ & Description \\
\hline $\begin{array}{c}\text { DES_IC } \\
\text { des_ic_mod.f }\end{array}$ & Global data storage for common initial condition data. \\
\hline $\begin{array}{l}\text { CHECK_DES_IC } \\
\text { check_des_ic.f }\end{array}$ & $\begin{array}{l}\text { Checks the data provided for the DES initial conditions } \\
\text { and flag errors if data is improper. }\end{array}$ \\
\hline $\begin{array}{c}\text { DES_SET_IC } \\
\text { des_set_ic.f }\end{array}$ & $\begin{array}{l}\text { Assigns the necessary data (e.g., temperature) to each } \\
\text { particle at the start of a simulation. }\end{array}$ \\
\hline $\begin{array}{l}\text { DES_THERMO } \\
\text { des_thermo_mod.f }\end{array}$ & Global data storage for the heat transfer model. \\
\hline $\begin{array}{l}\text { CHECK_DES_THERMO } \\
\text { check_des_thermo.f }\end{array}$ & $\begin{array}{l}\text { Checks the data provided for the heat transfer model, and } \\
\text { sets various flags used by the heat transfer } \\
\text { implementation. }\end{array}$ \\
\hline $\begin{array}{c}\text { CALC_THERMO_DES } \\
\text { calc_thermo_des.f }\end{array}$ & $\begin{array}{l}\text { Calls the routines that calculate the various heat transfer } \\
\text { models. }\end{array}$ \\
\hline $\begin{array}{l}\text { DES_CONDUCTION } \\
\text { des_thermo_cond.f }\end{array}$ & $\begin{array}{l}\text { Calculates the rate of heat transfer resulting from contact } \\
\text { conduction and particle-fluid-particle heat transfer. }\end{array}$ \\
\hline $\begin{array}{l}\text { DES_CONVECTION } \\
\text { des_thermo_conv. } f\end{array}$ & $\begin{array}{l}\text { Calculates the rate of heat transfer between the particle } \\
\text { and the gas acting on the particle. }\end{array}$ \\
\hline $\begin{array}{c}\text { DES_Hgm } \\
\text { des_thermo_conv.f }\end{array}$ & $\begin{array}{l}\text { Calculates the rate of heat transfer between particles and } \\
\text { the gas acting on the gas. }\end{array}$ \\
\hline $\begin{array}{l}\text { DES_CALC_GAMMA } \\
\text { des_thermo_conv.f }\end{array}$ & $\begin{array}{l}\text { Calculates the heat transfer coefficient based on the } \\
\text { specified Nusselt number model. }\end{array}$ \\
\hline $\begin{array}{l}\text { DES_RADIATION } \\
\text { des_thermo_rad.f }\end{array}$ & $\begin{array}{l}\text { Calculates the rate of particle-environment radiative heat } \\
\text { transfer. }\end{array}$ \\
\hline $\begin{array}{l}\text { DES_THERMO_NEWVALUES } \\
\text { des_thermo_newvalues.f }\end{array}$ & $\begin{array}{l}\text { Advances the solution of the internal energy equation for } \\
\text { a particle through numerical integration. }\end{array}$ \\
\hline
\end{tabular}




\begin{tabular}{|c|l|}
\hline $\begin{array}{c}\text { THERMO_NBR } \\
\text { thermo_nbr.f }\end{array}$ & $\begin{array}{l}\text { Determines if a particle belongs in the thermodynamic } \\
\text { neighborhood of another particle. This subset of particles } \\
\text { is used to assess which particles are undergoing contact } \\
\text { conduction and radiative heat transfer. }\end{array}$ \\
\hline $\begin{array}{c}\text { INTERPOLATE_CC } \\
\text { interpolation_mod.f }\end{array}$ & $\begin{array}{l}\text { Populates the variables needed for interpolating data } \\
\text { from the Eulerian grid to a particle's position. }\end{array}$ \\
\hline $\begin{array}{c}\text { SET_INTERPOLATION_ } \\
\text { STENCIL_CC } \\
\text { interpolation_mod.f }\end{array}$ & $\begin{array}{l}\text { Calculates the indices needed to access the Eulerian grid } \\
\text { cells for interpolation. }\end{array}$ \\
\hline
\end{tabular}

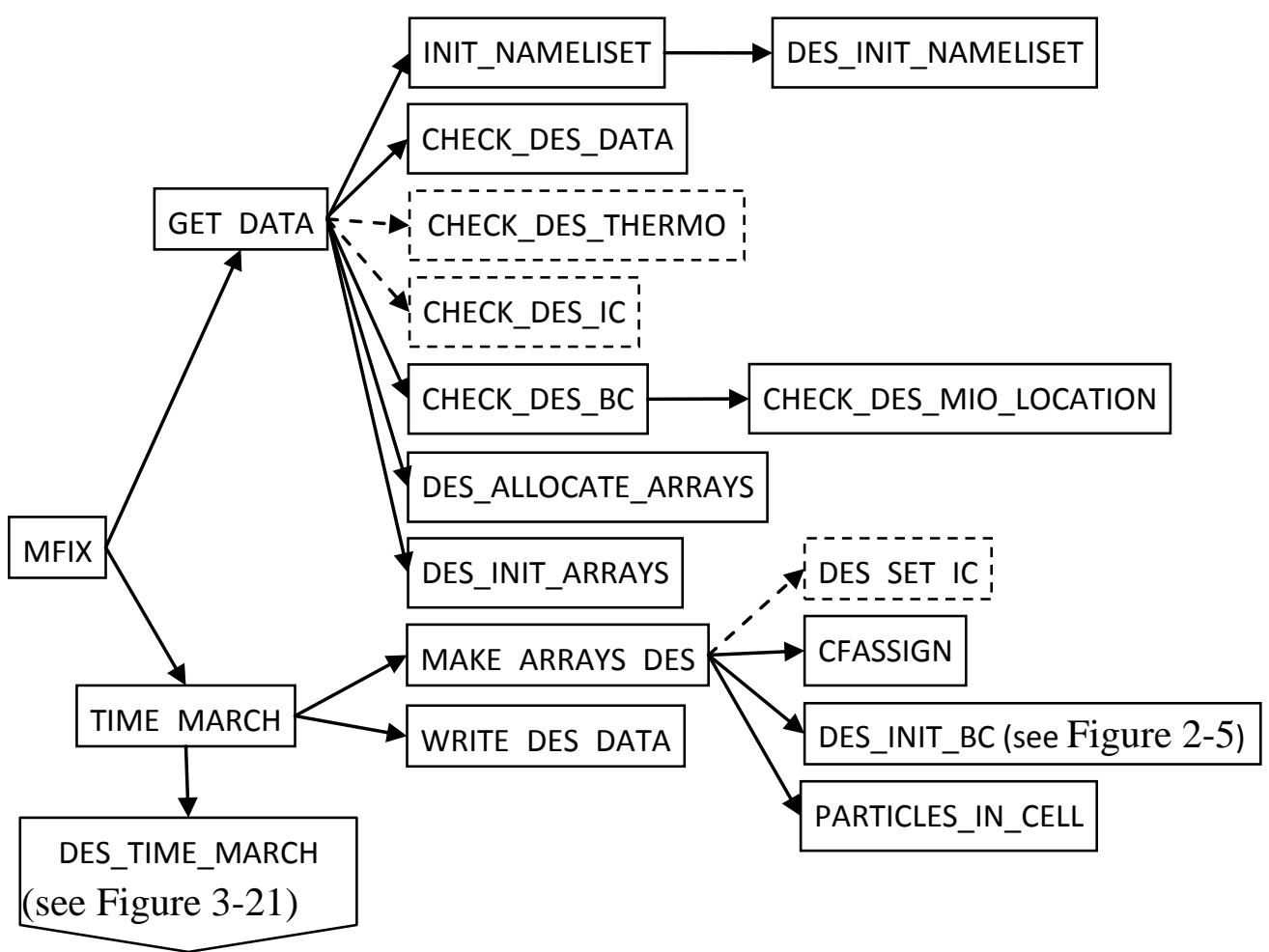

Figure 3-20: Call graph of main MFIX-DEM source code for initialization routines. Dashed lines indicate new calls and subroutines implemented for the heat transfer model. 


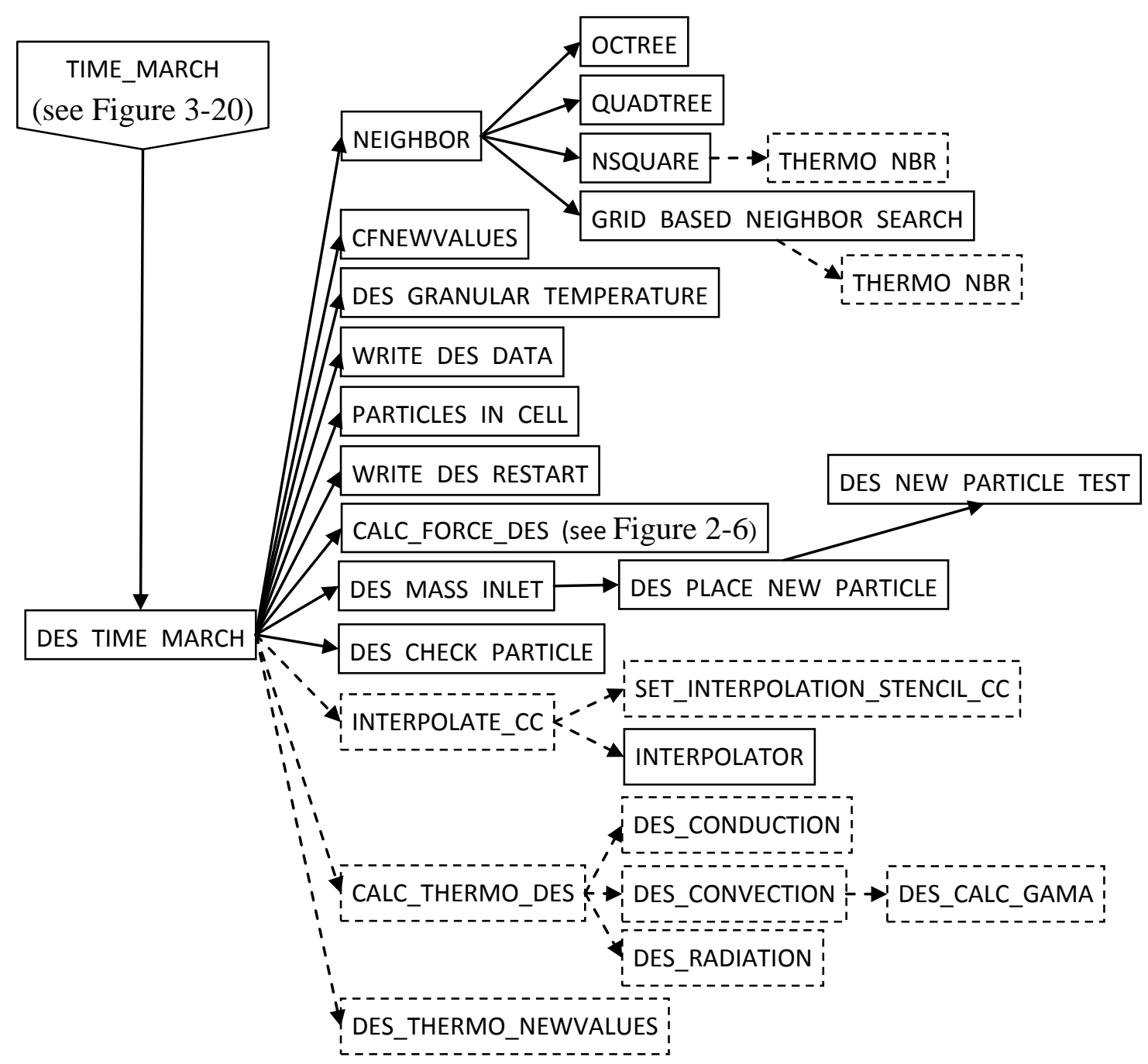

Figure 3-21: Call graph of MFIX discrete particle model routines. Dashed lines indicate new calls and subroutines implemented for the heat transfer model.

A flow chart depicting the heat transfer algorithm implemented in MFIX-DEM is presented in Figure 3-22. Processes added to the original DEM algorithm are indicated by dashed lines. The implementation is generalized by allowing a user to omit any mode of heat transfer he/she deems negligible by setting specific flags in the setup file (i.e., mfix.dat [84]). For example, a user may turn off radiative heat transfer in a simulation operating below $600^{\circ} \mathrm{C}$ to reduce computational expense. A flowchart detailing the processes for calculating the particle heat fluxes is illustrated in Figure 3-23. Discussions 
addressing interpolation and updating a particle's temperature are reserved for Sections 3.6.1 and 3.6.3, respectively.

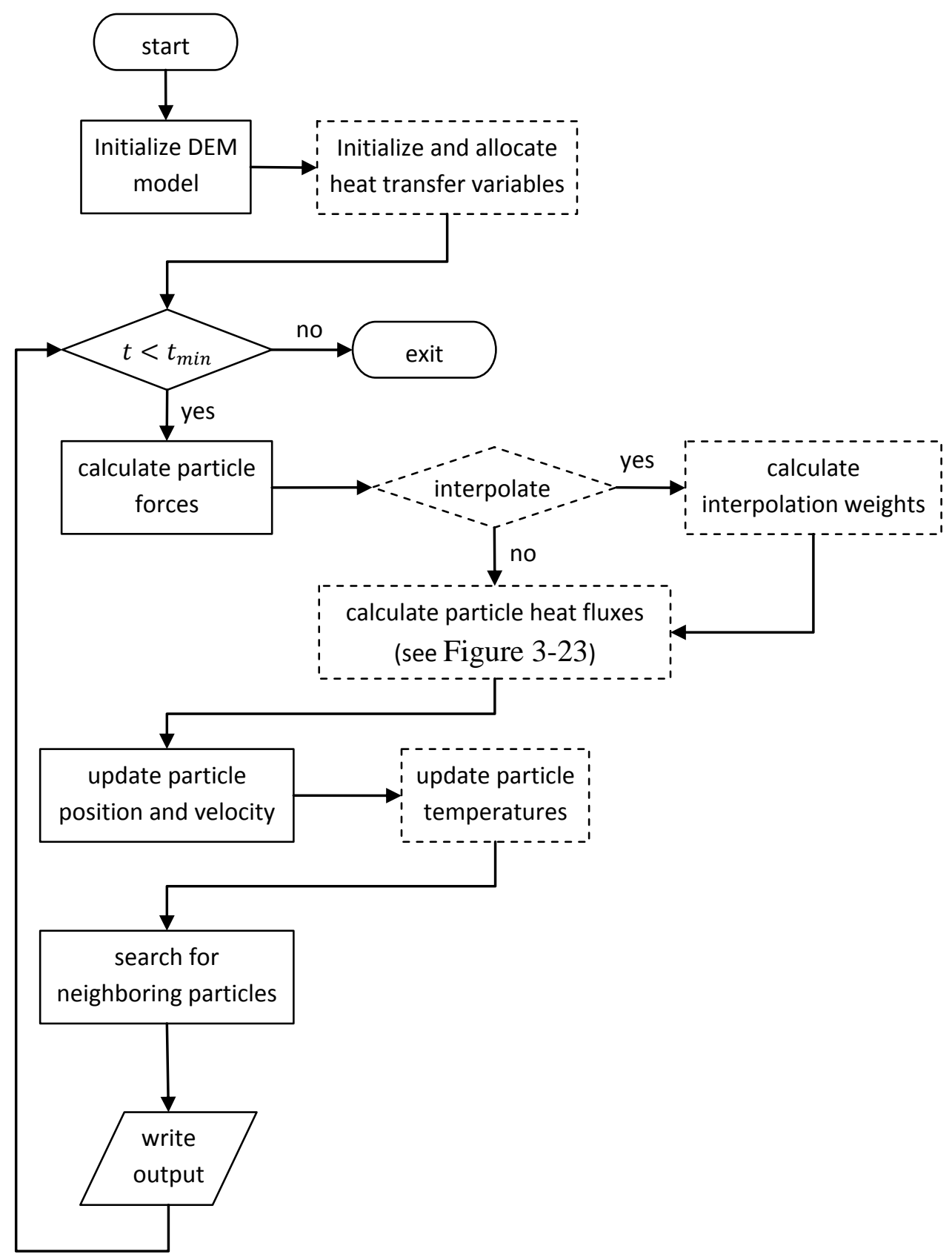

Figure 3-22: Flow chart illustrating the locations within MFIX-DEM where modifications have been made to include particle heat transfer. 


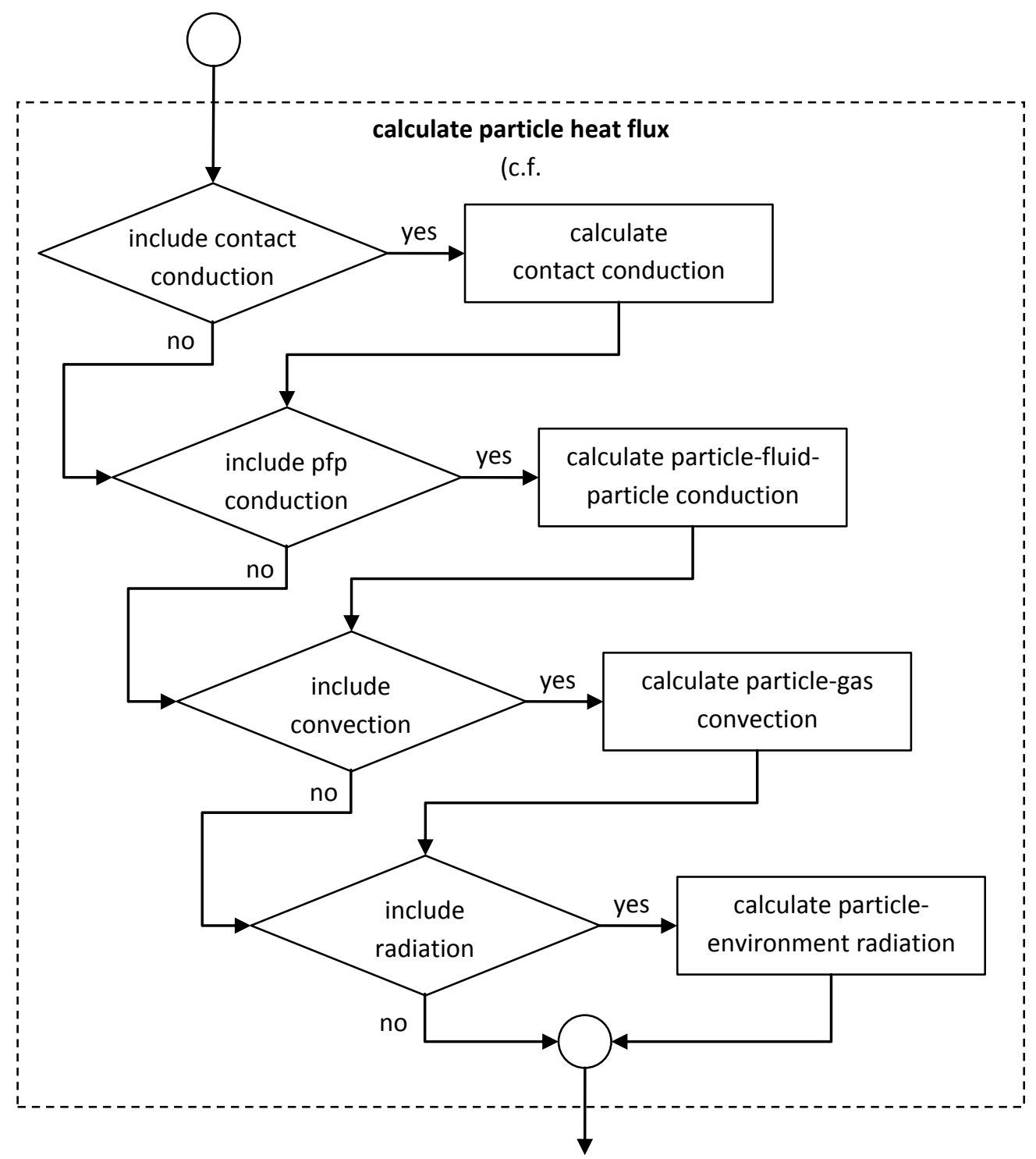

Figure 3-23: Flow chart illustrating customizability of the implementation by allowing a user to select which modes of heat transfer to compute during a simulation.

\subsubsection{Gas-solids thermal energy transfer}

Particle-gas convection, $\dot{Q}_{p g}$, introduced in Section 3.4, is an interphase heat transfer term that couples the gas and particle energy balance equations. Specifically, particle-gas convection is a source term in the internal energy equations for the gas $\left(q_{g s}\right.$ in Equation (1-6)) and particle $\left(\dot{Q}_{p g}\right.$ in Equation (3-6)). In MFIX the numerical solution of the gas phase energy equation separates $\dot{Q}_{p g}$ into the coefficient for the unknown gas 
phase temperature $\left(-\gamma_{c p} A_{s}\right)$ and a known source term $\left(\gamma_{c p} A_{s} T_{p}\right)$. (See references $[\mathbf{8 5 , 8 6}]$ for detailed solution methodology used in MFIX for continuum phases).

A consistent approach is needed to manage thermal energy transfer between the particles and gas (i.e., how information is transferred between the Eulerian grid and discrete particles). For example, gas phase temperature is a scalar variable associated with the Eulerian grid cell centers. However, particles can exist in any region of the domain defined by the Eulerian grid, as illustrated in Figure 3-24. Therefore, passing energy (information) between the Eulerian grid and particles can occur through either a direct exchange between a particle and the Eulerian cell that contains its center, or through interpolating the information to the nearest Eulerian cells surrounding the particle.

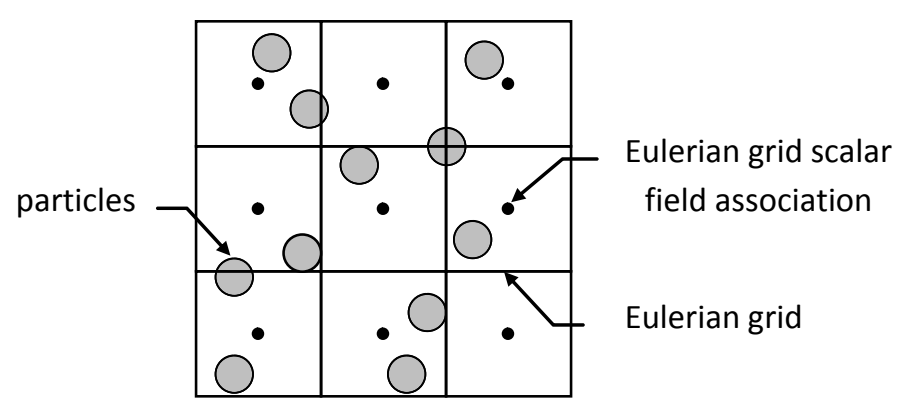

Figure 3-24: Example illustrating the Eulerian grid which stores scalar field variables, like temperature, at the cell-center. Particles are permitted to exist at any location within the domain defined by the Eulerian grid.

Equation (3-51) defines the particle-gas convective heat transfer acting on the gas phase, $q_{g s}$. Here, $\mathcal{V}_{C V}$ is the volume of the control volume (CV), $N_{C V}$ is the number of particles contained in the $\mathrm{CV}$, and $\dot{Q}_{p g}$ is the rate of particle-gas convective heat transfer given by Equation (3-39) and relations (3-40) and (3-44). $K_{C V}^{(k)}$ is a function used to map the energy flux from a particle's position to the computational nodes of the gas phase Eulerian grid. 


$$
q_{g s}=-\frac{1}{v_{C V}} \sum_{k=1}^{N_{C V}} \dot{Q}_{p g}^{(k)} K_{C V}^{(k)}
$$

Presently, there are two definitions for the mapping function, $K_{C V}^{(k)}$. By default, thermal energy transfer between the fluid and a particle is associated with the CV containing the particle's center, as illustrated in Figure 3-25-(a). Therefore, calculations that require the temperature of the gas phase (i.e., Equation (3-39)) use the temperature associated with the CV (fluid cell) containing the particle's center. Also, thermal energy taken from, or given to the fluid as the result of particle-gas convective heat transfer is done so with the fluid cell containing the particle's center. A second option distributes thermal energy flux among the control volumes surrounding the particle via linear interpolation. This technique is depicted in Figure 3-25-(b). If the interpolation method is employed, the gas phase temperature used in Equation (3-39) is interpolated to a particle's position from the surrounding control volumes for consistency. Likewise, thermal energy transfer resulting from particle-gas convective heat transfer is distributed among the control volumes surrounding the particle.

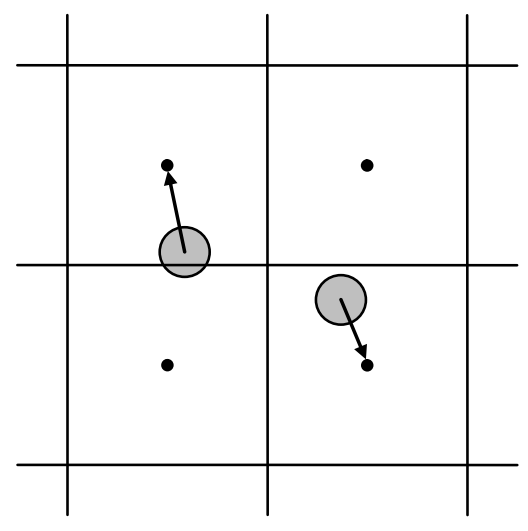

(a)

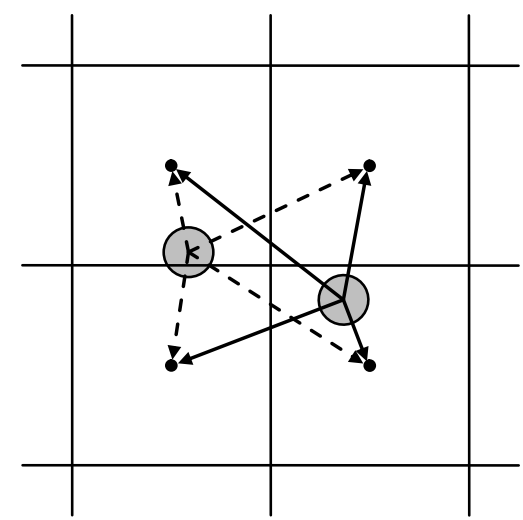

(b)

Figure 3-25: Example illustrating the two methods available to transmitting thermal energy between the Eulerian grid and the particles. (a) Energy is passed between a particle and the grid cell containing its center; (b) energy is distributed from a particle's position to the nearest grid cells. 
Finally, one must consider when information is exchanged between the gas and particles. This is a result of the solution technique used to computationally join the gas and discrete solids phases. Presently in MFIX-DEM [85], the gas phase takes a step forward in time using an extended version of the SIMPLE algorithm [86] (not presented). Once the fluid variables converge, several smaller time steps are taken within the DEM to allow the particles to catch-up to the gas phase [35]. This fluid step forward-particle catch-up process is illustrated in Figure 3-26.

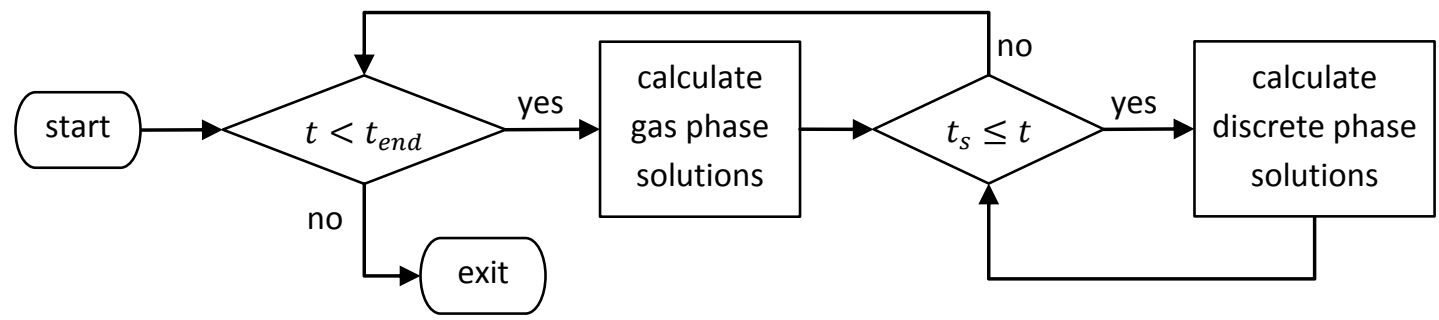

Figure 3-26: Illustration of the fluid step forward-particle catch-up process that occurs during MFIX-DEM. First the solution to the fluid phase if obtained at time $t$, then multiple, smaller time steps are taken within the DEM to allow the particles to catch-up to the fluid phase. Here, $t$ is the time of the fluid's most recent solution, and $t_{s}$ is the time of the discrete solids most recent solution.

The source terms for the internal energy equations for the particles and gas are managed differently to account for the disjoint updating procedure. When the gas phase equations are solved, all of the physical properties of the particles are constant. This is a direct result of their solutions lagging behind the solution of the gas phase. Furthermore, only the coefficient for the unknown gas phase temperature $\left(\frac{-1}{\mathcal{V}_{C V}} \sum_{k=1}^{N_{C V}} \gamma_{c p} A_{s}^{(k)} K_{C V}^{(k)}\right)$ and the known source term $\left(\frac{-1}{v_{C V}} \sum_{k=1}^{N_{C V}} \gamma_{c p} A_{s}^{(k)} T_{p}^{(k)} K_{C V}^{(k)}\right)$ are computed for each fluid cell (see Equations (3-39) and (3-51), subroutine DES_Hgm in Appendix 0, and references $[\mathbf{8 5}, \mathbf{8 6}])$. Similarly, when the particle phase equations are solved, all of the physical properties of the gas phase are constant, and only the contributions to the particle internal energy equation are calculated $\left(\dot{Q}_{p g}\right.$ in Equation (3-6)). 


\subsubsection{Nusselt number correlations}

The model for particle-gas convection, introduced in Section 3.4, requires a closure model for the heat transfer coefficient, $\gamma_{c p}$. Ranz and Marshall's [69] correlation, discussed in Section 3.4.2, was selected as the default Nusselt number correlation for this

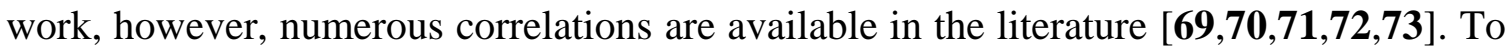
provide greater customizability of the convective heat transfer model, a general framework was created to facilitate implementation of additional correlations by users. This was achieved by providing a set location within the code (see subroutine DES_CALC_GAMMA in Appendix 0) for the user to implement their correlation. By creating a flag in the setup file (i.e., mfix.dat [84]) a user may indicate which correlation to use during a simulation. This approach allows for multiple correlations to exist within the code simultaneously, while only evaluating the desired correlation during execution.

\subsubsection{Temperature updating procedures}

There are various methodologies available for solving differential equations numerically $[\mathbf{8 7 , 8 8 , 8 9}]$. However, the time integration methods for the discrete solids phase momentum equation implemented in MFIX-DEM are limited to Euler's and Adams-Bashforth methods [35]. Therefore, the same methods were used to advance the solution of the particle internal energy equation. Euler's method, shown as Equation (3-52), is the default approach selected by this work. However, the Adams-Bashforth method (not shown) is available for higher accuracy. Details concerning these methods are provided in references $[\mathbf{8 8 , 8 9}]$.

$$
T_{p}^{(i)}\left(t+\Delta t_{s}\right)=T_{p}^{(i)}(t)+\frac{\Delta t_{s}}{m^{(i)}(t) C_{p p}^{(i)}(t)}\left(\dot{Q}_{p p}^{(i)}(t)+\dot{Q}_{p f p}^{(i)}(t)+\dot{Q}_{p g}^{(i)}(t)+\dot{Q}_{r a d}^{(i)}(t)\right)
$$


The step size for the numerical integration of the particle internal energy equation, $\Delta t_{s}$, is the same used for the numerical integration of the particle momentum equation. Although the solids time step for numerical stability of the collision model is orders of magnitude more smaller than that needed for stability of the particle internal energy (see Section 3.2.3), the same solids time step is used.

\subsubsection{Integral management in particle-fluid-particle conduction model}

The particle-fluid-particle conduction model, discussed in Section 3.3.2, contains an integral which represents the heat transfer paths joining the surfaces of the particles. A numerical approach is needed to solve the integral, shown in Equation, as no analytical solution is obvious.

$$
\int_{R_{c}}^{R_{f}^{(i, j)}} f(r) d r \text { where } f(r)=\frac{2 \pi r}{\ell^{(i, j)}-\left(\left(R_{p}^{(i)^{2}}-r^{2}\right)^{1 / 2}+\left(R_{p}^{(j)^{2}}-r^{2}\right)^{1 / 2}\right)}
$$

As noted in the discussion in Section 3.3.2, a singularity in Equation (3-53) occurs as $\ell^{(i, j)}$ approaches zero. The singularity is avoid in this effort by requiring a minimum separation distance between particles' surfaces of $4 \times 10^{-10} \mathrm{~m}$. However, the slope of $f(r)$ varies sharply across integral bounds, resulting in additional difficulty. Adaptive Simpson's method [88] (see subroutine DES_CONDUCTION in Appendix 0) was selected to numerically integrate this term due to its ability to compute the value quickly while maintaining a known bound on the amount of error generated.

\subsection{Verification of implementation}

In this section, implementation of the contact conduction, particle-fluid-particle conduction, and particle-gas convection models in MFIX-DEM are verified by comparing the numerical results to analytic solutions. Simple test cases were developed 
to isolate the heat transfer mechanisms of interest. Recall from Section 3.6, a simulation may selectively omit individual mechanisms of heat transfer. Implementation of the particle-particle radiation model is verified by comparing the results of MFIX-DEM with those of a fourth order Runge-Kutta (RK4) solution. An analytic solution for particleparticle radiation was not sought due to a nonlinear dependence on particle temperatures (see Equation set (3-61)).

\subsubsection{Contact conduction}

Implementation of the contact conduction model (see Section 3.2.2) was verified using the case of two touching particles. The particles' positions are assumed fixed in time and heat transfer is assumed to occur only via particle-particle conduction. In this case the internal energy equation for each particle (see Equation (3-6)) reduces to the expressions shown in Equation set (3-54) describing the temperatures of particles indexed by the numbers 1 and 2 . Here, $m, C_{p p}, \kappa_{p}, R_{p}$, and $T_{p}$ are the mass, specific heat, thermal conductivity, radius, and temperatures of the indexed particles, respectively.

$$
\begin{aligned}
& \frac{d T_{p}^{(1)}}{d t}=\frac{4 \kappa_{p}^{(1)} \kappa_{p}^{(2)}\left(T_{p}^{(2)}-T_{p}^{(1)}\right)}{m^{(1)} C_{p p}^{(1)}\left(\kappa_{p}^{(1)}+\kappa_{p}^{(2)}\right)} \sqrt{R_{p}^{(2)^{2}}-\left(\frac{R_{p}^{(2)^{2}}-R_{p}^{(1)^{2}}+\ell^{(2,1)^{2}}}{2 \ell^{(1,2)}}\right)^{2}} \\
& \frac{d T_{p}^{(2)}}{d t}=\frac{4 \kappa_{p}^{(2)} \kappa_{p}^{(1)}\left(T_{p}^{(1)}-T_{p}^{(2)}\right)}{m^{(2)} C_{p p}^{(2)}\left(\kappa_{p}^{(2)}+\kappa_{p}^{(1)}\right)} \sqrt{R_{p}^{(2)^{2}}-\left(\frac{R_{p}^{(2)^{2}}-R_{p}^{(1)^{2}}+\ell^{(2,1)^{2}}}{2 \ell^{(2,1)}}\right)^{2}}
\end{aligned}
$$

An analytic solution for the particles' temperature is obtained for particles with constant (but not necessarily equal) thermal and physical properties $\left(m, C_{p p}, \kappa_{p}\right.$, and $\left.R_{p}\right)$. The resulting system of ordinary differential equations is solved by diagonalization. The 
solutions for the particles' temperatures as a function of time are shown as Equation set (3-55). Here, $T_{p .0}$ is the initial temperature of the indexed particles, respectively.

$$
\begin{gathered}
T_{p}^{(1)}(t)=\frac{1}{a+b}\left[b T_{p, 0}^{(1)}+a T_{p, 0}^{(2)}+a\left(T_{p, 0}^{(1)}-T_{p, 0}^{(2)}\right) e^{-(a+b) t}\right], \\
T_{p}^{(2)}(t)=\frac{1}{a+b}\left[b T_{p, 0}^{(1)}+a T_{p, 0}^{(2)}-b\left(T_{p, 0}^{(1)}-T_{p, 0}^{(2)}\right) e^{-(a+b) t}\right], \\
\text { where } \quad a=\frac{2}{m^{(1)} C_{p p}^{(1)}}\left(\frac{\kappa_{p}^{(1)} \kappa_{p}^{(2)}}{\kappa_{p}^{(1)}+\kappa_{p}^{(2)}}\right) \sqrt{R_{p}^{(2)^{2}}-\left(\frac{R_{p}^{(2)^{2}}-R_{p}^{(1)^{2}}+\ell^{(2,1)^{2}}}{2 \ell^{(1,2)}}\right)^{2}}, \\
\text { and } \quad b=\frac{2}{m^{(2)} C_{p p}^{(2)}}\left(\frac{\kappa_{p}^{(1)} \kappa_{p}^{(2)}}{\kappa_{p}^{(1)}+\kappa_{p}^{(2)}}\right) \sqrt{R_{p}^{(2)^{2}}-\left(\frac{R_{p}^{(2)^{2}}-R_{p}^{(1)^{2}}+\ell^{(2,1)^{2}}}{2 \ell^{(2,1)}}\right)^{2}} .
\end{gathered}
$$

A simulation employing the same assumptions defined above was conducted with MFIX-DEM where only particle-particle conduction was solved. The physical and thermodynamic properties of the particles are outlined in Table 3-3, and illustrated in Figure 3-27. The values are based on those used in reference [19] who studied heat transfer in packed and bubbling beds using CFD-DEM. In this simulation, 1000 seconds of physical time was modeled to allow the particles to achieve an equilibrium temperature $\left(\left|\Delta T_{p}\right| \leq 10^{-4}\right)$. The solids time step was kept constant at $\Delta t_{s}=$ $0.856357 \times 10^{-6}$. A single AMD Opteron $265,1.8 \mathrm{GHz}$ processor was used.

Table 3-3: Physical and thermodynamic properties of the particles used to verify the contact conduction model implemented into MFIX-DEM. The geometric configuration is illustrated in Figure 3-27.

\begin{tabular}{|c|c|c|}
\hline properties & particle 1 & particle 2 \\
\hline diameter $(\mathrm{cm})$ & 0.30 & 0.36 \\
\hline density $\left(\mathrm{g} / \mathrm{cm}^{3}\right)$ & 0.42 & 8.85 \\
\hline $\begin{array}{c}\text { thermal conductivity } \\
(\mathrm{cal} / \mathrm{sec} \cdot \mathrm{cm} \cdot \mathrm{K})\end{array}$ & 0.131453 & 0.002008 \\
\hline
\end{tabular}




\begin{tabular}{|c|c|c|}
\hline specific heat $(\mathrm{cal} / \mathrm{g} \cdot \mathrm{K})$ & 0.191204 & 0.013145 \\
\hline initial temperature $(\mathrm{K})$ & 298.15 & 453.15 \\
\hline x-position $(\mathrm{cm})$ & 2.5 & 2.5 \\
\hline y-position $(\mathrm{cm})$ & 0.1499864 & 0.4799731 \\
\hline z-position $(\mathrm{cm})$ & 0.18 & 0.18 \\
\hline
\end{tabular}

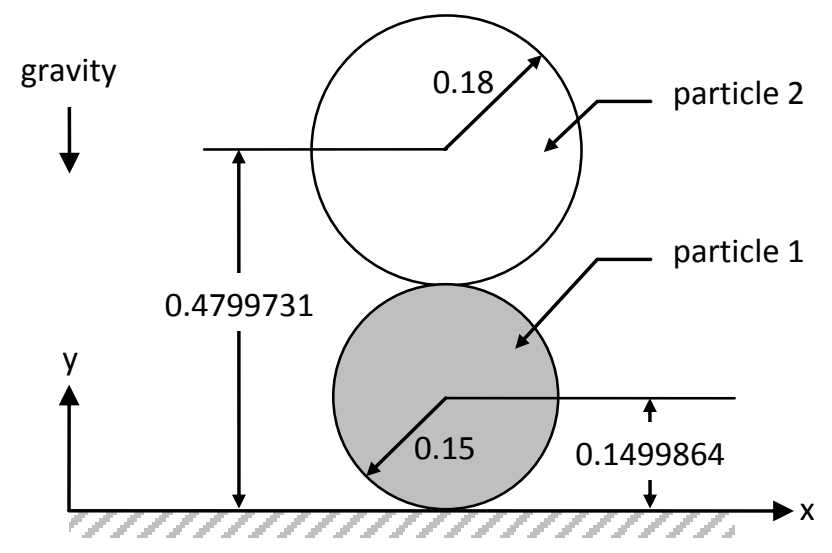

Figure 3-27: The geometric configuration of the particles used to verify the implementation of the contact conduction model implemented into MFIX-DEM. All measurements are in centimeters.

The numerical and analytical solutions for the particles' temperatures are shown in Figure 3-28. Tp1 and Tp2 indicate the temperatures of particle 1 particle 2, respectively. Solid symbols denote the particle temperatures calculated by MFIX-DEM, continuous lines represent the analytic solutions, and open symbols indicate the percent relative error between the numerical and analytical solutions as defined in Equation (3-56). Note the strong agreement between the analytic and numerical solutions. Percent relative error is bounded above by $10^{-5}$, indicating the numerical solution deviates less than $\pm 10^{-5}$ percent from the analytic solution over the entire simulation. Therefore, the implementation of the contact conduction model into the MFIX-DEM code is considered successful. 


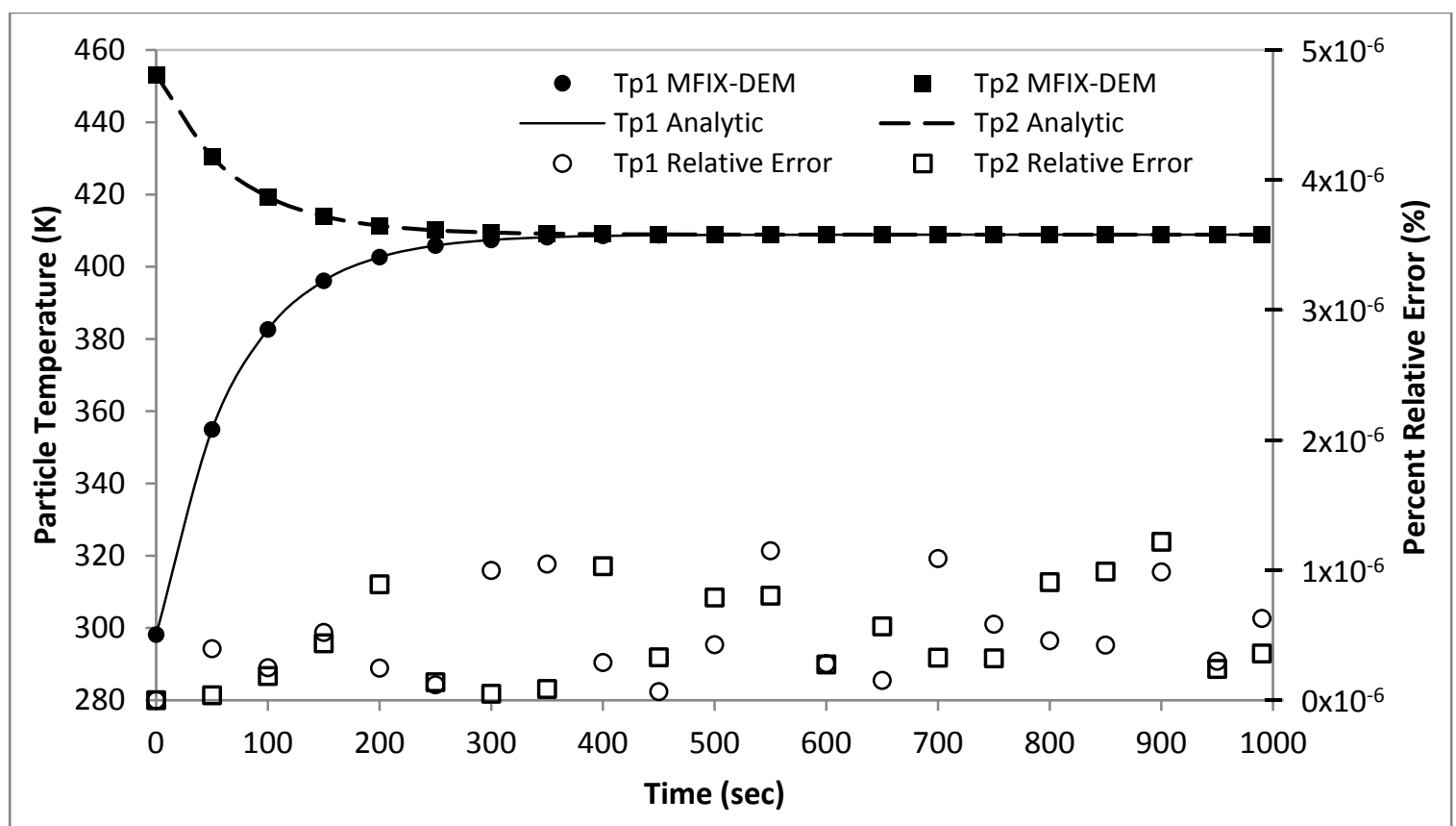

Figure 3-28: MFIX-DEM and analytic solutions to the internal energy equation for two particles engaged in contact conduction. Tp1 and $\mathrm{Tp} 2$ are the temperatures of the cold and hot particles, respectively. Relative error between the analytic and numerical solutions is indicated by open symbols and is associated with the right vertical axis.

$$
\text { Percent Relative Error }=\frac{\left|T_{p k}^{\text {analytic }}-T_{p k}^{\text {MFIX-DEM }}\right|}{\left|T_{p k}^{\text {analytic }}\right|} \times 100 \%
$$

\subsubsection{Particle-fluid-particle conduction}

Implementation of the particle-fluid-particle conduction model (see Section 3.3.2)

was verified using the same test case as in the particle-particle conduction verification study but with an additional variation. Specifically, two test cases were constructed. In the first test case, the two particles are close but not in contact, while in the second test, the particles are touching. The particles' positions are assumed fixed in time and heat transfer is assumed to occur only via particle-fluid-particle conduction. In this case the internal energy equation for each particle (see Equation (3-6)) reduces to the expressions shown in Equation set (3-57) describing the temperatures of particles indexed by the numbers 1 and 2 . Here, $\kappa_{g}$ is the thermal conductivity of the gas, and $m, C_{p p}, R_{p}$, and $T_{p}$ 
are the mass, specific heat, radius, and temperatures of the indexed particles, respectively. $R_{f}$ and $R_{c}$ delineate the region of particle-fluid-particle conduction, and are defined in Equations (3-25), (3-36), and (3-37), respectively.

$$
\begin{aligned}
& \frac{d T_{p}^{(1)}}{d t}=\frac{\kappa_{g}\left(T_{p}^{(2)}-T_{p}^{(1)}\right)}{m^{(1)} C_{p p}^{(1)}} \int_{R_{c}}^{R_{f}^{(1,2)}} \frac{2 \pi r}{\ell^{(1,2)}-\left(\left({R_{p}^{(1)}}^{2}-r^{2}\right)^{1 / 2}+\left(R_{p}^{(2)^{2}}-r^{2}\right)^{1 / 2}\right)} d r \\
& \frac{d T_{p}^{(2)}}{d t}=\frac{\kappa_{g}\left(T_{p}^{(1)}-T_{p}^{(2)}\right)}{m^{(2)} C_{p p}^{(2)}} \int_{R_{c}}^{R_{f}^{(1,2)}} \frac{2 \pi r}{\ell^{(1,2)}-\left(\left({R_{p}^{(2)}}^{2}-r^{2}\right)^{1 / 2}+\left(R_{p}^{(1)^{2}}-r^{2}\right)^{1 / 2}\right)} d r
\end{aligned}
$$

As before, analytic solutions for the particles' temperature are obtained for particles and gas with constant thermal and physical properties $\left(m, C_{p p}, \kappa_{p}, R_{p}\right.$, and $\left.\kappa_{g}\right)$. These assumptions result in a system of ordinary differential equations solved by diagonalization. The resulting solutions for the particles' temperatures are shown in Equation set (3-58). Here, $T_{p .0}$ is the initial temperature of the indexed particles.

$$
\begin{gathered}
T_{p}^{(1)}(t)=\frac{1}{a+b}\left[b T_{p, 0}^{(1)}+a T_{p, 0}^{(2)}+a\left(T_{p, 0}^{(1)}-T_{p, 0}^{(2)}\right) e^{-(a+b) t}\right], \\
T_{p}^{(2)}(t)=\frac{1}{a+b}\left[b T_{p, 0}^{(1)}+a T_{p, 0}^{(2)}-b\left(T_{p, 0}^{(1)}-T_{p, 0}^{(2)}\right) e^{-(a+b) t}\right], \\
\text { where } \quad a=\frac{\kappa_{g}}{m^{(1)} C_{p p}^{(1)}} \int_{R_{c}}^{R_{f}} \frac{2 \pi r}{\ell^{(i, j)}-\left(\left(R_{p}^{(1)^{2}}-r^{2}\right)^{1 / 2}+\left(R_{p}^{(2)^{2}}-r^{2}\right)^{1 / 2}\right)} d r, \\
\text { and } \quad b=\frac{\kappa_{g}}{m^{(2)} C_{p p}^{(2)}} \int_{R_{c}}^{R_{f}} \frac{2 \pi r}{\ell^{(i, j)}-\left(\left(R_{p}^{(1)^{2}}-r^{2}\right)^{1 / 2}+\left(R_{p}^{(2)^{2}}-r^{2}\right)^{1 / 2}\right)} d r .
\end{gathered}
$$

Simulations with the same assumptions used to construct the analytic solutions were conducted with MFIX-DEM. Accordingly, only particle-fluid-particle convection was solved. The physical and thermodynamic properties of the particles are outlined in 
Table 3-4, with the geometric configurations of the simulations illustrated in Figure 3-27 and Figure 3-25, respectively. The thermal conductivity of the gas was defined as $1.239 \times 10^{-4} \mathrm{cal} / \mathrm{sec} \cdot \mathrm{cm} \cdot \mathrm{K}$. As in the previous test case, all values follow from reference [19]. In the first simulation, 300 seconds of physical time was modeled to allow two nontouching particles to achieve an equilibrium temperature $\left(\Delta T_{p} \leq 10^{-4}\right)$.Similarly, the second simulation, with touching particles, modeled 50 seconds of physical time to allow the particles to achieve an equilibrium temperature. The integral term in Equation (3-37) and Equation set (3-58) was evaluated using adaptive Simpsons quadrature [88]. The solids time step was kept constant $\Delta t_{s}=0.856357 \times 10^{-6}$. Both simulations were carried out on a single AMD Opteron $265,1.8 \mathrm{GHz}$ processor.

Table 3-4: Physical and thermodynamic properties of the particles used to verify the particle-fluidparticle conduction model implemented into MFIX-DEM. The geometric configuration for the first simulation is illustrated in Figure 3-29 while the geometric configuration for the second simulation is illustrated in Figure 3-27. The gas phase thermal conductivity for both simulations was set as $1.239 \times 10^{-4} \mathrm{cal} / \mathrm{sec} \cdot \mathrm{cm} \cdot \mathrm{K}$.

\begin{tabular}{|c|c|c|c|c|}
\hline properties & \multicolumn{2}{|c|}{ particle 1 } & \multicolumn{2}{c|}{ particle 2 } \\
\hline diameter $(\mathrm{cm})$ & \multicolumn{2}{|c|}{0.30} & \multicolumn{2}{c|}{0.36} \\
\hline density $\left(\mathrm{g} / \mathrm{cm}^{3}\right)$ & \multicolumn{2}{|c|}{0.42} & \multicolumn{2}{c|}{0.85} \\
\hline $\begin{array}{c}\text { thermal conductivity } \\
\text { (cal/sec·cm } \cdot \mathrm{K})\end{array}$ & \multicolumn{2}{|c|}{0.131453} & \multicolumn{2}{c|}{0.002008} \\
\hline specific heat $(\mathrm{cal} / \mathrm{g} \cdot \mathrm{K})$ & \multicolumn{2}{|c|}{0.191204} & \multicolumn{2}{c|}{453.15} \\
\hline initial temperature $(\mathrm{K})$ & \multicolumn{2}{|c|}{298.15} & 2.5 & $\mathbf{2}$ \\
\hline Simulation & $\mathbf{1}$ & $\mathbf{2}$ & 2.5 \\
\hline x-position $(\mathrm{cm})$ & 2.5 & 2.5 & 1.498 & 0.4799731 \\
\hline y-position $(\mathrm{cm})$ & 1.15 & 1.1499864 & 0.18 & 0.18 \\
\hline z-position $(\mathrm{cm})$ & 0.18 & 0.18 & & \\
\hline
\end{tabular}




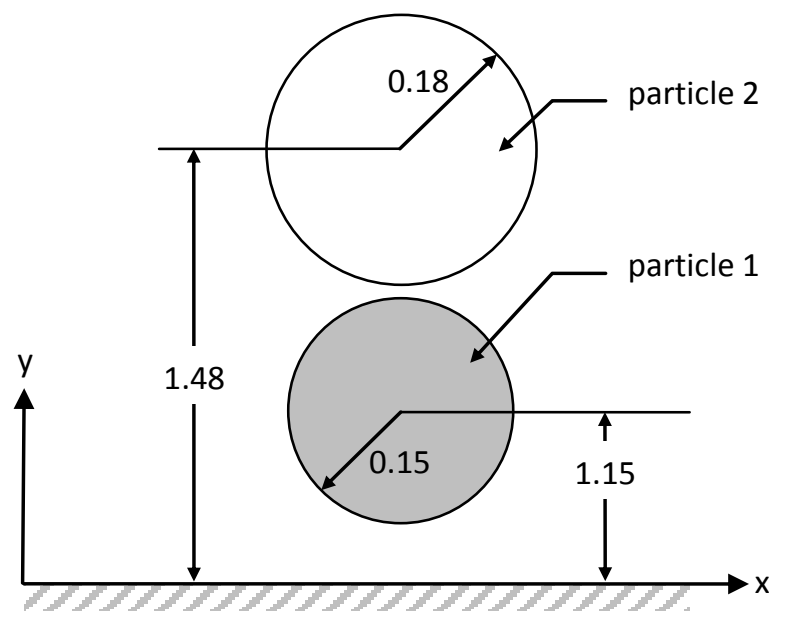

Figure 3-29: The geometric configuration of the particles used in the first simulation to verify the implementation of the particle-fluid-particle conduction model implemented into MFIX-DEM. All measurements are in centimeters.

The numerical and analytical solutions for the particles' temperatures are shown in Figure 3-30 for the non-touching case and in Figure 3-31 for the touching case, respectively. Tp1 and Tp2 indicate the temperatures of particle 1 and particle 2, respectively. Solid symbols denote the particle temperatures calculated by MFIX-DEM, continuous lines represent the analytic solutions, and open symbols indicate the percent relative error between the numerical and analytical solutions (see Equation (3-56)). Note the strong agreement between the analytic and numerical solutions. For both test cases, percent relative error is bounded above by $10^{-5}$, indicating the numerical solutions deviate less than $\pm 10^{-5}$ percent from the analytic solutions over the entire simulations. Therefore, the implementation of the particle-fluid-particle conduction model into the MFIX-DEM code is considered successful. 


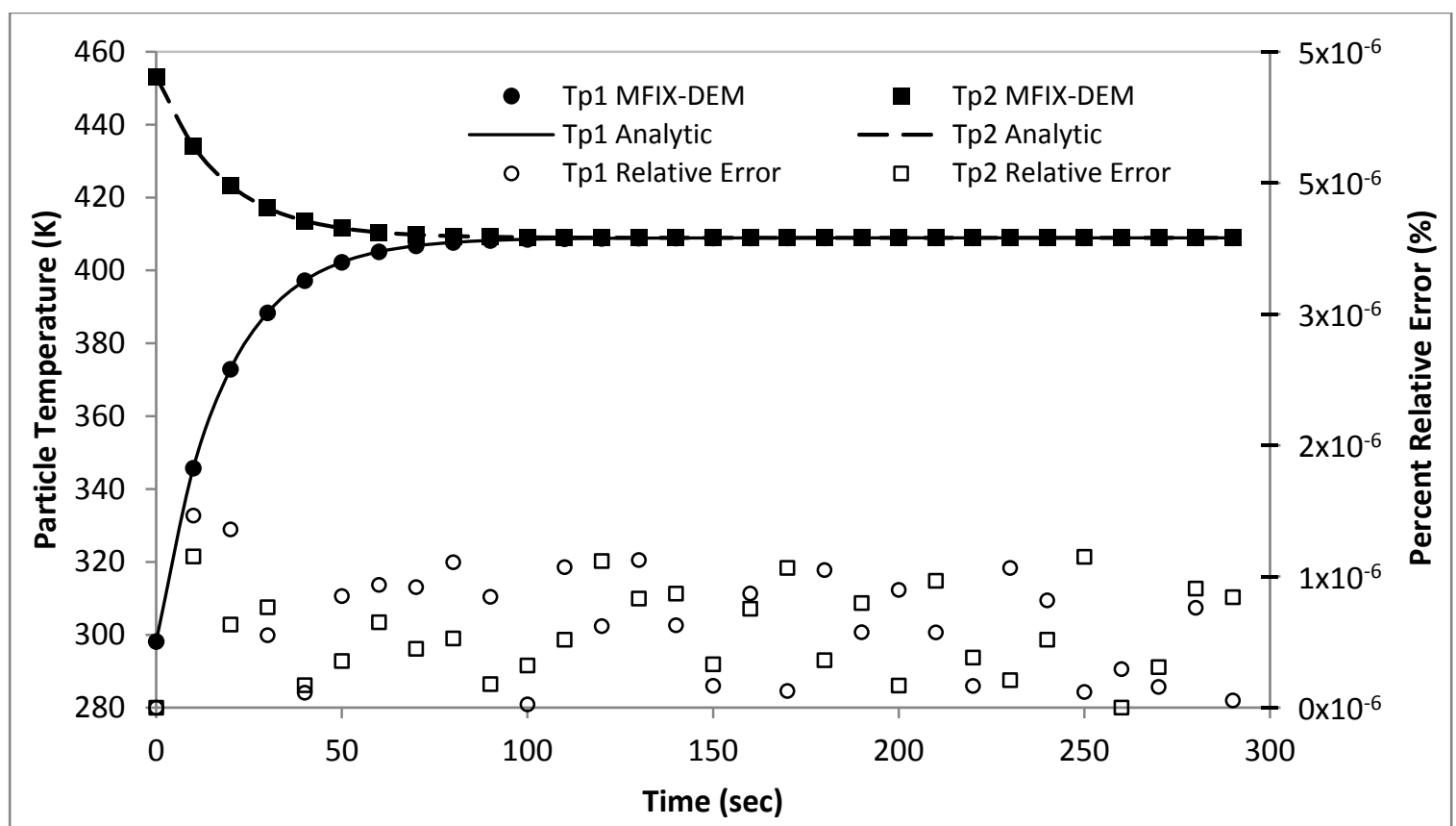

Figure 3-30: MFIX-DEM and analytic solutions to the internal energy equation for two particles not touching and engaged in particle-fluid-particle conduction. Tp1 and Tp2 are the temperatures of particle 1 and particle 2, respectively (c.f. Table 3-4). Relative error between the two solutions is indicated by open symbols and is associated with the right vertical axis.

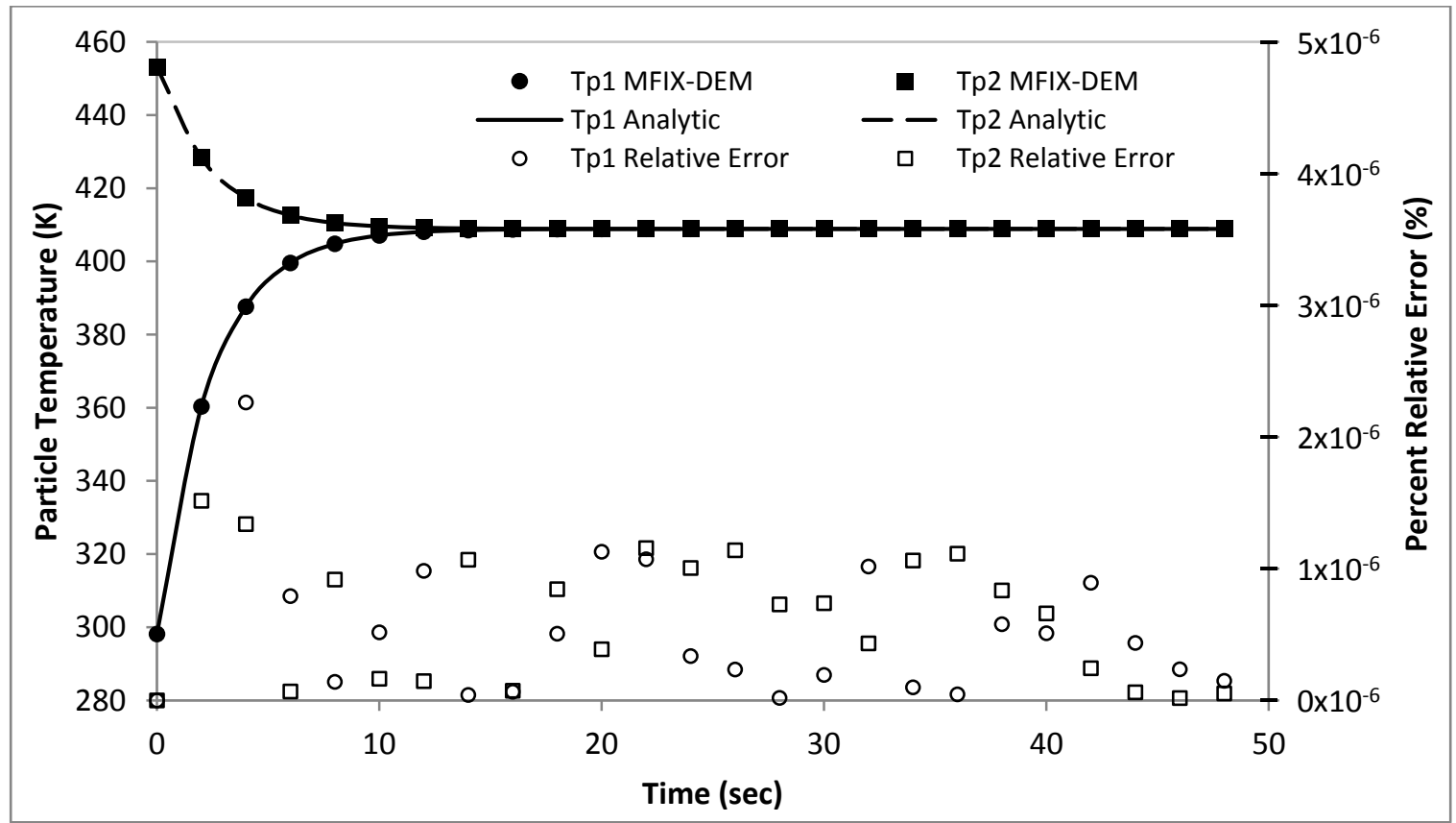

Figure 3-31: MFIX-DEM and analytic solutions to the internal energy equation for two touching particles engaged in particle-fluid-particle conduction. Tp1 and Tp2 are the temperatures of particle 1 and particle 2, respectively (c.f. Table 3-4). Relative error between the two solutions is indicated by open symbols and is associated with the right vertical axis. 


\subsubsection{Particle-gas convection}

Implementation of the particle-gas convection model (see Section 3.4) was verified using the case of a single particle fixed in a uniform gas flow. The particle's position is assumed fixed in time and heat transfer is assumed to occur only via particlegas convection. In this case the internal energy equation for the particle (see Equation (3-6)) reduces to the expression shown in Equation (3-59) describing the temperature of particle. Here, $\kappa_{g}, \rho_{g}, C_{p g}, \mu_{g}, \varepsilon_{g}, T_{g}$, and $\boldsymbol{v}_{g}$ are the thermal conductivity, density, specific heat, viscosity, void fraction, temperature, and velocity of the gas phase, and $m$, $C_{p p}, R_{p}, A_{s}, T_{p}$, and $\mathbf{V}_{p}$ are the mass, specific heat, radius, surface area, temperature, and velocity of the particle, respectively.

$$
\begin{gathered}
\frac{d T_{p}}{d t}=\frac{\gamma_{c p} A_{s}\left(T_{g}-T_{p}\right)}{m C_{p p}} \\
\text { where } \gamma_{c p}=\frac{\kappa_{g}}{2 R_{p}}\left(2.0+0.6\left(\frac{\rho_{g} \varepsilon_{g}\left\|\boldsymbol{v}_{g}-\mathbf{v}_{p}\right\|_{2} 2 R_{p}}{\mu_{g}}\right)^{1 / 2}\left(\frac{C_{p g} \mu_{g}}{\kappa_{g}}\right)^{1 / 3}\right)
\end{gathered}
$$

As before, an analytic solution for the particle's temperature is obtained for a particles and gas with constant thermal and physical properties $\left(m, C_{p p}, R_{p}, A_{s}, \mathbf{V}_{p}, \kappa_{g}\right.$, $\left.\rho_{g}, C_{p g}, \mu_{g}, \varepsilon_{g}, T_{g}, \boldsymbol{v}_{g}\right)$. These assumptions result in a ordinary differential solved by separation of variables. The resulting solution for the particle's temperature is shown in Equation (3-60), where $T_{p, 0}$ is the particle's initial temperature.

$$
\begin{gathered}
T_{p}(t)=T_{g}-\left(T_{g}-T_{p, 0}\right) e^{\frac{-\gamma_{c p} A_{s}}{m c_{p}} t} \\
\text { where } \gamma_{c p}=\frac{\kappa_{g}}{2 R_{p}}\left(2.0+0.6\left(\frac{\rho_{g} \varepsilon_{g}\left\|\boldsymbol{v}_{g}-\mathbf{V}_{p}\right\|_{2} 2 R_{p}}{\mu_{g}}\right)^{1 / 2}\left(\frac{C_{p g} \mu_{g}}{\kappa_{g}}\right)^{1 / 3}\right)
\end{gathered}
$$


A simulation employing the same assumptions defined above was conducted with MFIX-DEM where only particle-gas convection was solved. The physical and thermodynamic properties of the particle and gas are outlined in Table 3-5, and illustrated in Figure 3-32. As in the previous test cases, all values follow from reference [19]. The simulation was run for 25 seconds of physical time to allow the particle's temperature to equilibrate with the gas temperature $\left(\left|T_{p}-T_{g}\right| \leq 10^{-4}\right)$. The solids time step was kept constant $\Delta t_{s}=0.856357 \times 10^{-6}$. The simulation was performed on a single AMD Opteron 265, $1.8 \mathrm{GHz}$ processor.

Table 3-5: Physical and thermodynamic properties of the gas and particle used to verify the convection model implemented into MFIX-DEM. The geometric configuration is illustrated in Figure 3-32.

\begin{tabular}{|c|c|c|}
\hline properties & gas & particle \\
\hline diameter $(\mathrm{cm})$ & - & 0.36 \\
\hline density $\left(\mathrm{g} / \mathrm{cm}^{3}\right)$ & $1.1854 \times 10^{-3}$ & 8.85 \\
\hline $\begin{array}{c}\text { thermal conductivity } \\
(\mathrm{cal} / \mathrm{sec} \cdot \mathrm{cm} \cdot \mathrm{K})\end{array}$ & $1.2396 \times 10^{-4}$ & 0.002008 \\
\hline specific heat $(\mathrm{cal} / \mathrm{g} \cdot \mathrm{K})$ & 0.23878 & 0.013145 \\
\hline initial temperature $(\mathrm{K})$ & 298.15 & 453.15 \\
\hline velocity $(\mathrm{cm} / \mathrm{sec})$ & 600 & 0 \\
\hline
\end{tabular}

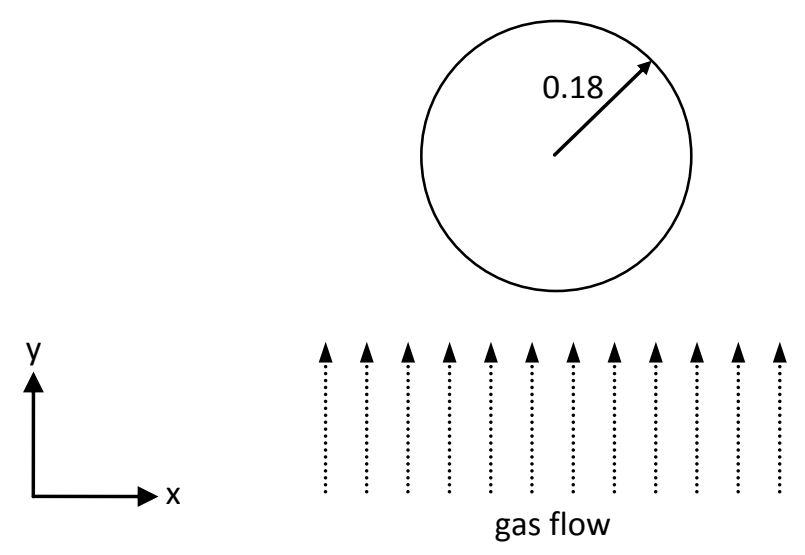

Figure 3-32: The geometric configuration of a particle used to verify the implementation of the particle-gas convection model implemented into MFIX-DEM. All measurements are in centimeters. 
The numerical and analytical solutions for the particle's temperature is shown in Figure 3-33. $\mathrm{Tp}$ and $\mathrm{Tg}$ indicate the temperatures of the particle and gas, respectively. Solid symbols denote the particle's temperature calculated by MFIX-DEM, a continuous line represents the analytic solution for the particle's temperature, and open symbols indicate the percent relative error (see Equation (3-56)). The constant gas phase temperature is indicated with a dashed line. Note the strong agreement between the analytic and numerical solutions. Percent relative error is bounded above by $10^{-5}$, indicating the numerical solution deviates less than $\pm 10^{-5}$ percent from the analytic solution over the entire simulation. Therefore, the implementation of the particle-gas convection model into the MFIX-DEM code is considered successful.

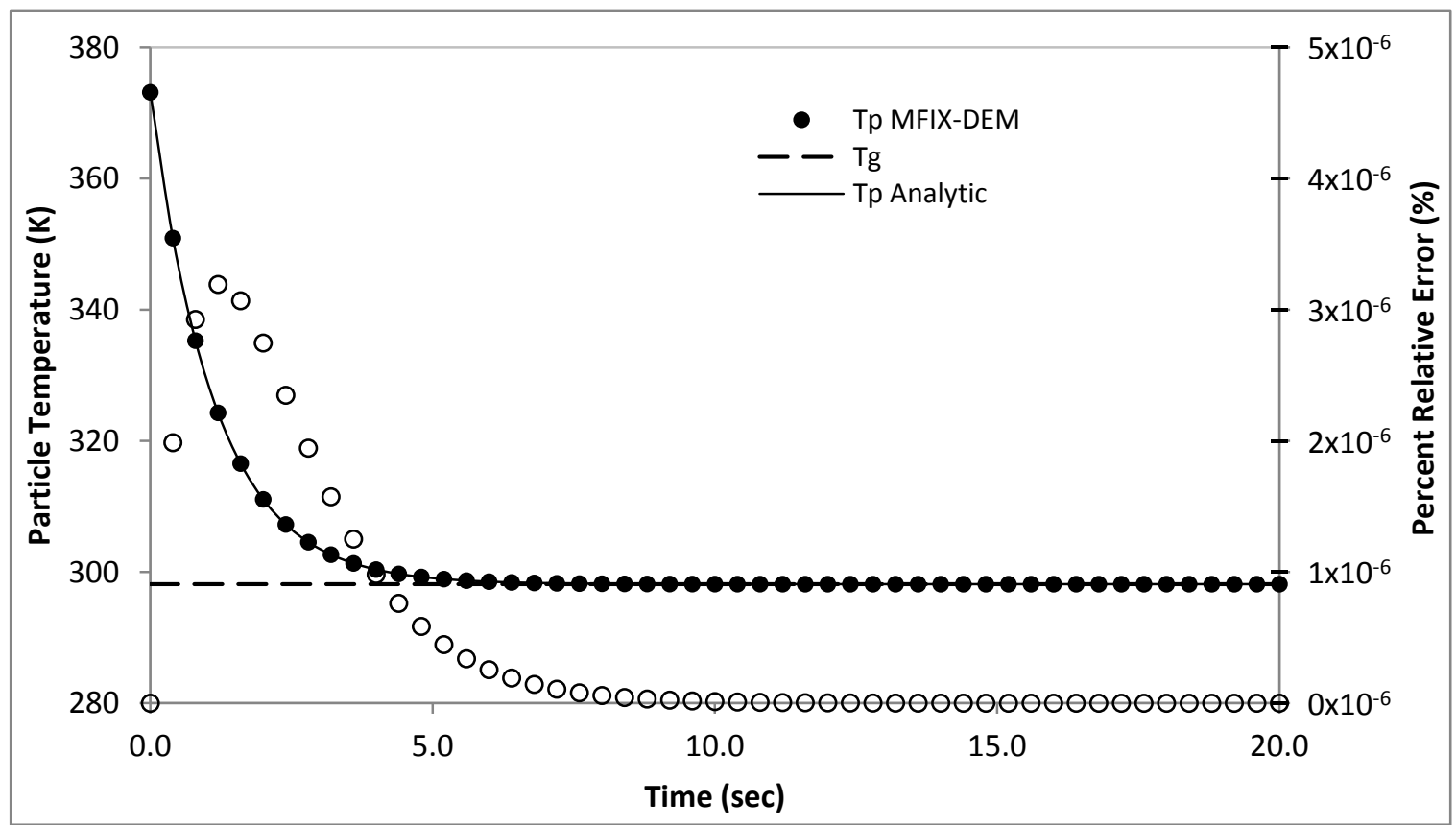

Figure 3-33: MFIX-DEM and analytic solutions to the internal energy equation for a single particle undergoing particle-gas convection with a constant fluid. $\mathrm{Tp}$ and $\mathrm{Tg}$ are the temperatures of particle and gas, respectively Relative error between the numerical and analytic solutions is indicated by open symbols and is associated with the right vertical axis. 


\subsubsection{Particle-particle radiation}

Implementation of the radiative heat transfer model (see Section 3.5) was verified using the case of two particles in close proximity to one another. The particles' positions are assumed fixed in time and heat transfer is assumed to occur only via particle-particle radiation. In this case the internal energy equation for each particle (see Equation (3-6)) reduces to the expressions shown in Equation set (3-61) describing the temperatures of particles indexed by the numbers 1 and 2 . Here, $\sigma$ is the Stefan-Boltzmann constant, and $m, C_{p p}, \epsilon, A_{s}$, and $T_{p}$ are the mass, specific heat, emissivity, surface area, and temperatures of the indexed particles, respectively.

$$
\begin{aligned}
& \frac{d T_{p}^{(1)}}{d t}=\sigma_{S-B} \frac{A_{s}^{(1)} \epsilon_{p}^{(1)}}{m^{(1)} C_{p p}^{(1)}}\left(\left(T_{p}^{(2)}\right)^{4}-\left(T_{p}^{(1)}\right)^{4}\right)=f_{1}\left(t, T_{p}^{(1)}, T_{p}^{(2)}\right) \\
& \frac{d T_{p}^{(2)}}{d t}=\sigma_{S-B} \frac{A_{s}^{(2)} \epsilon_{p}^{(2)}}{m^{(2)} C_{p p}^{(2)}}\left(\left(T_{p}^{(1)}\right)^{4}-\left(T_{p}^{(2)}\right)^{4}\right)=f_{2}\left(t, T_{p}^{(1)}, T_{p}^{(2)}\right)
\end{aligned}
$$

Due to the nonlinear dependence on particle temperature, obtaining analytic solutions for the temperature of the particles is nontrivial. Therefore, a standard fourthorder Runge-Kutta (RK4) method, shown as Equation set (3-62) with time step $h=10^{-3}$ and constant thermal and physical properties $\left(m, C_{p p}, \kappa_{p}\right.$, and $\left.R_{p}\right)$, is used for comparison. 


$$
\left\{\begin{array}{l}
T_{p}^{(1)}\left(t_{n+1}\right)=T_{p}^{(1)}\left(t_{n}\right)+\frac{1}{6}\left(k_{1}^{(1)}+2 k_{2}^{(1)}+2 k_{3}^{(1)}+k_{4}^{(1)}\right) \text { where } T_{p}^{(1)}\left(t_{0}\right)=T_{p 0}^{(1)} \\
T_{p}^{(2)}\left(t_{n+1}\right)=T_{p}^{(2)}\left(t_{n}\right)+\frac{1}{6}\left(k_{1}^{(2)}+2 k_{2}^{(2)}+2 k_{3}^{(2)}+k_{4}^{(2)}\right) \text { where } T_{p}^{(2)}\left(t_{0}\right)=T_{p 0}^{(2)}
\end{array}\right.
$$

where

$$
\left\{\begin{array}{l}
k_{1}^{(i)}=h f_{i}\left(t_{n}, T_{p}^{(1)}\left(t_{n}\right), T_{p}^{(2)}\left(t_{n}\right)\right) \\
k_{2}^{(i)}=h f_{i}\left(t_{n}+\frac{h}{2}, T_{p}^{(1)}\left(t_{n}\right)+\frac{k_{1}^{(1)}}{2}, T_{p}^{(2)}\left(t_{n}\right)+\frac{k_{1}^{(2)}}{2}\right) \\
k_{3}^{(i)}=h f_{i}\left(t_{n}+\frac{h}{2}, T_{p}^{(1)}\left(t_{n}\right)+\frac{k_{2}^{(1)}}{2}, T_{p}^{(2)}\left(t_{n}\right)+\frac{k_{2}^{(2)}}{2}\right) \\
k_{4}^{(i)}=h f_{i}\left(t_{n}+h, T_{p}^{(1)}\left(t_{n}\right)+k_{3}^{(1)}, T_{p}^{(2)}\left(t_{n}\right)+k_{3}^{(2)}\right)
\end{array} \text { for } i \in\{1,2\}\right.
$$

A simulation employing the same assumptions defined above was conducted with MFIX-DEM where only particle-particle radiation was solved. The physical and thermodynamic properties of the particle and gas are outlined in Table 3-6, and illustrated in Figure 3-34. As in the previous test cases, all values follow from reference [19]. The simulation was run for 145 seconds of physical time to allow the particles to achieve an equilibrium temperature $\left(\Delta T_{p} \leq 10^{-4}\right)$. The solids time step was kept constant $\Delta t_{s}=$ $0.856357 \times 10^{-6}$. The simulation was performed on a single AMD Opteron 265,

\section{$1.8 \mathrm{GHz}$ processor.}

Table 3-6: Physical and thermodynamic properties of the particles used to verify the radiative heat transfer model implemented into MFIX-DEM. The geometric configuration is illustrated in Figure 3-34.

\begin{tabular}{|c|c|c|}
\hline properties & particle 1 & particle 2 \\
\hline diameter $(\mathrm{cm})$ & 0.30 & 0.36 \\
\hline density $\left(\mathrm{g} / \mathrm{cm}^{3}\right.$ ) & 0.42 & 8.85 \\
\hline $\begin{array}{c}\text { thermal conductivity } \\
(\mathrm{cal} / \mathrm{sec} \cdot \mathrm{cm} \cdot \mathrm{K})\end{array}$ & 0.131453 & 0.002008 \\
\hline specific heat $(\mathrm{cal} / \mathrm{g} \cdot \mathrm{K})$ & 0.191204 & 0.013145 \\
\hline emissivity & 0.8 & 0.8 \\
\hline initial temperature $(\mathrm{K})$ & 298.15 & 453.15 \\
\hline x-position $(\mathrm{cm})$ & 2.5 & 2.5 \\
\hline
\end{tabular}




\begin{tabular}{|c|c|c|}
\hline y-position $(\mathrm{cm})$ & 3.0 & 3.43 \\
\hline z-position $(\mathrm{cm})$ & 0.18 & 0.18 \\
\hline
\end{tabular}

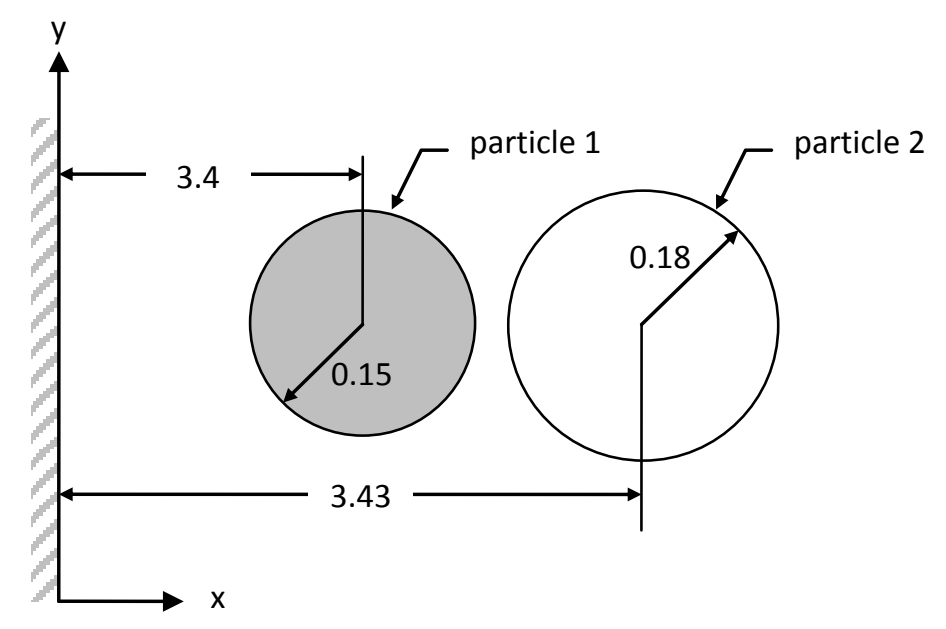

Figure 3-34: The geometric configuration of the particles used in the first simulation to verify the implementation of the particle-environment radiation model implemented into MFIX-DEM. All measurements are in centimeters.

The numerical and RK4 solutions for the particles' temperatures are shown in Figure 3-35.. Tp1 and Tp2 indicate the temperatures of particle 1 and particle 2, respectively. Solid symbols denote the particle temperatures calculated by MFIX-DEM, continuous lines represent the RK4 solutions, and open symbols indicate the percent relative error (see Equation (3-56)). Note the strong agreement between the RK4 and numerical solutions. The percent relative error is bounded above by $10^{-6}$, indicating the numerical solutions deviate less than $\pm 10^{-5}$ percent from the RK4 solution over the entire simulation. Therefore, the implementation of the particle-particle radiation model into the MFIX-DEM code is considered successful. 


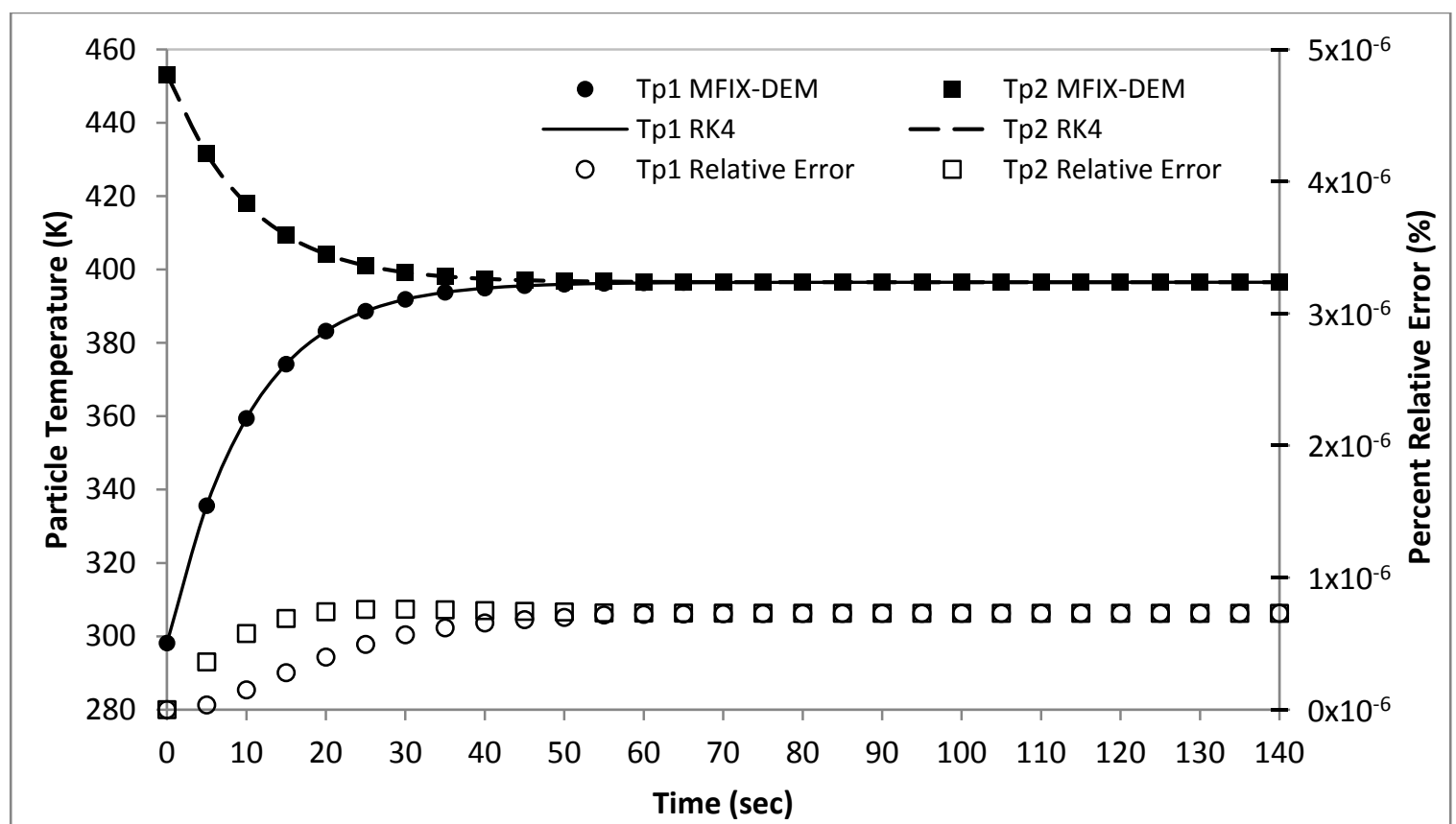

Figure 3-35: MFIX-DEM and fourth order Runge-Kutta (RK4) solutions to the internal energy equation for two particles not touching engaged in radiative heat exchange. Relative error between the two solutions is indicated by open symbols and is associated with the right vertical axis.

\subsection{Closing Remarks}

In this chapter, the various mechanisms of heat transfer present in gas-solids systems and the internal energy equation for a single, isothermal particle are defined. Subsequent sections review mathematical models for contact conduction, particle-fluidparticle conduction, particle-gas convection, and particle-particle radiation. For each of these mechanisms a specific model was chosen for implementation into an existing CFDDEM code based on model simplicity, wide acceptance in existing CFD-DEM heat transfer models, extendibility to particles of different sizes, and computational expense.

Necessary modifications were made to selected models before implementing them into MFIX-DEM. Batchelor and O'Brien's [52] contact conduction model was generalized to compute the contact radius from the geometric configuration of the particles, and an effective thermal conductivity for the particles was defined (see Section 3.2.2). The particle-fluid-particle conduction model of Rong and Horio [16] was extended 
to account for particles of different sizes (see Section 3.3.2). For particle-particle radiation, a new environment temperature was established (see Section 3.5.2).

Finally, the implementation of each model was verified for simple two particle test cases, or in the case of gas-particle convection, a single fixed particle in a flowing gas. Strong agreement was observed between the simulation data and the analytic or numerical solution. 


\section{Chapter 4: Particle-gas reactive chemistry interface}

Chemical reactions in gas-solids flows may occur within a single phase (homogeneous) or they may require the presence of at least two phases (heterogeneous) [90]. Presently, MFIX, the CFD code portion of the CFD-DEM model employed in this work (MFIX-DEM), is capable of modeling user-defined homogeneous and heterogeneous chemical reactions among continuum phases (i.e., Eulerian-Eulerian models only) [91,92]. The goal of this effort is to develop and implement a mathematical interface for managing user-defined heterogeneous chemical reactions among the gas and discrete solids phases in MFIX-DEM.

This chapter begins by briefly defining the (1) reaction rate, (2) specific heat, (3) heat of reaction, and (4) individual particle mass and species equations in Section 4.1. Three particle reaction models and their employment in existing CFD-DEM reactive chemistry models are presented in Section 4.2. Finally, the algorithmic implementation and verification of the mathematical interface for managing user-defined particle-gas chemical reactions developed in this effort are given in Sections 4.3 and 4.4.

\subsection{Foundations for particle-gas reactive chemistry}

In this section, specific concepts of reactive chemistry referenced in this work are introduced. This is done to provide a foundation for the topics managed by the mathematical interface for user-defined chemical reactions. Additional details concerning the fundamentals of reactive chemistry are found in references $[28,90,93,94]$. 


\subsubsection{Reaction rate}

Various interrelated definitions for the rate at which a chemical reaction progresses, termed the rate of reaction, are available [90]. Therefore, care is needed to avoid ambiguity. This work defines the rate of reaction with respect to species $\mathrm{A}$ as the number of moles of species A formed (or consumed) per unit time, per unit surface area of a particle (e.g., mol $\left.\cdot \mathrm{cm}^{-2} \cdot \mathrm{s}^{-1}\right)$. Specifically, if species $A$ is a reactant, then the rate of reaction is termed the rate of disappearance of species $\mathrm{A}$ and is notated as $-r_{\mathrm{A}}$. Likewise, if species $\mathrm{A}$ is a product of the reaction, then the reaction rate is termed the rate of formation of species $\mathrm{A}$ and notated as $r_{\mathrm{A}}$. The rate of disappearance is assigned a negative sign because the reaction rate is always specified as a non-negative number [93].

Defining the rate of reaction with respect to particle surface area is natural in the context of CFD-DEM as it allows for a 'per particle' treatment of particle-gas reactions. Other definitions (e.g., per volume of fluid or per reactor volume) may require averaging rates of formation and rates of disappearance of the solids phase species from a collection of particles contained within some control volume. This may, in effect, remove the distinct advantage of CFD-DEM modeling (i.e., the ability to track the conversion of individual particles during a chemical reaction).

The rate equation for a reaction (also referred to as a rate law) is an algebraic function which gives the reaction rate in terms of material properties [95]. An example rate law for the rate of formation of species A is shown in Equation (4-1). Here, $k$ is the rate coefficient (also called the rate constant), $\mathrm{C}_{\mathrm{A}}$ and $\mathrm{C}_{\mathrm{B}}$ are the concentration of reactants $\mathrm{A}$ and $\mathrm{B}$, and $\alpha$ and $\beta$ are empirically fitted constants. 


$$
-r_{\mathrm{A}}=k C_{\mathrm{A}}^{\alpha} C_{\mathrm{B}}^{\beta}
$$

Once the rate of reaction with respect to a particular species is specified, the rates of formation and disappearance of the other reactants and products are obtained from the reaction stoichiometry. For example, consider the generic reaction shown in Equation (4-2). Here, $a$ molecules of species A and $b$ molecules of species B are destroyed to produce $c$ molecules of species $\mathrm{C}$ and $d$ molecules of species $\mathrm{D}$. The terms in parentheses indicate whether the species belongs to the gas $(g)$ or solids $(s)$ phase. The proportional relationship linking the rates of disappearance of species $\mathrm{A}$ and $\mathrm{B}$ and the rates of formation of species C and D are given in Equation (4-3).

$$
\begin{gathered}
a \mathrm{~A}(\mathrm{~g})+b \mathrm{~B}(\mathrm{~s}) \rightarrow c \mathrm{C}(\mathrm{g})+d \mathrm{D}(\mathrm{s}) \\
\frac{-r_{\mathrm{A}}}{a}=\frac{-r_{\mathrm{B}}}{b}=\frac{r_{\mathrm{C}}}{c}=\frac{r_{\mathrm{D}}}{d}
\end{gathered}
$$

Reactions where the reactants are consumed to form products, but the products do not react to form the reactants are termed irreversible and require a single reaction rate. The example reaction shown in Equation (4-2) is irreversible as indicated by the symbol $(\rightarrow)$. However, rate equations are needed for both the forward and backward directions in reversible reactions, commonly indicated by the symbol $(\rightleftharpoons)$. If the example reaction in Equation (4-2) were reversible, a reaction rate for the backward direction, potentially a function of the concentrations of species C and D, is needed.

\subsubsection{Specific Heat}

The amount of heat required to increase a material's temperature one degree, while holding the pressure or volume constant is termed the heat capacity [96]. The heat capacity at constant pressure is notated as $C_{p}$, and the heat capacity at constant volume is 
notated $C_{v}$. The term specific heat is used to denote a heat capacity defined on a per unit mass basis. The specific heat of a material is a function of its temperature and commonly given as a polynomial [97]. Details concerning the calculation of specific heats in this effort are reserved for Section 4.3.3.

\subsubsection{Heat of reaction}

The amount of heat liberated or absorbed during a reaction, termed the heat of reaction $(\Delta H)$, is given by the difference in enthalpies between the products and reactants [90]. The change in enthalpy for endothermic reactions is positive, indicating that energy from the system is needed to facilitate the reaction. Conversely, the change in enthalpy for exothermic reactions is negative, signifying that energy is released to the system during the reaction. The heat of reaction for the generic reaction given by Equation (4-2) is illustrated by Equation (4-4). Here, $H_{\mathrm{C}}$ and $H_{\mathrm{D}}$ are the enthalpies of the products, and $H_{\mathrm{A}}$ and $H_{\mathrm{B}}$ are the enthalpies of the reactants.

$$
\Delta H=\left(H_{\mathrm{C}}+H_{\mathrm{D}}\right)-\left(H_{\mathrm{A}}+H_{\mathrm{B}}\right)
$$

The enthalpy for species $n$ at temperature $T\left(H_{n}(T)\right)$ is given by Equation (4-5). Here, $H_{n}^{\circ}\left(T_{R E F}\right)$ is species $n$ 's enthalpy of formation at reference temperature $T_{R E F}$, and $\Delta H_{Q n}$ is the enthalpy change caused by a change in temperature (i.e., from $T_{R E F}$ to $T$ ) [93]. The species change in enthalpy $\left(\Delta H_{Q n}\right)$ is calculated by integrating the material's specific heat $\left(C_{p n}\right)$ with respect to temperature, as shown by Equation (4-6). Details concerning the calculation of enthalpies in this effort are reserved for Section 4.3.4.

$$
H_{n}(T)=H_{n}^{\circ}\left(T_{R E F}\right)+\Delta H_{Q n}
$$




$$
\Delta H_{Q n}=\int_{T_{R E F}}^{T} C_{p n} d T
$$

In reactions where mass is transferred from one phase to another (e.g., solids and gas phase species react to produce a different gas phase species), accounting for the transfer of enthalpy with the transferred mass is needed. Consider the example reaction shown in Equation (4-2). Suppose that there is a net production of gas phase species C and, in turn, a net consumption of solids phase species B (i.e., there is a net transfer of mass from the solids phase to the gas phase). The rate of enthalpy transfer as a result of mass transfer is then given by Equation (4-7). As before, $-r_{\mathrm{B}}$ and $r_{\mathrm{D}}$ are the rate of disappearance and formation of solids species $\mathrm{B}$ and $\mathrm{D}$, and $H_{\mathrm{C}}$ is the enthalpy of species $\mathrm{C}$ at the local gas phase conditions. If there are multiple destination phase products, care is needed to ensure that the rate of enthalpy transfer $\left(\dot{H}_{f r}\right)$ is computed from the correct proportions and enthalpies of the various species generated.

$$
\dot{H}_{f r}=H_{\mathrm{C}}\left(\left(-r_{\mathrm{B}}\right)-r_{\mathrm{D}}\right)
$$

The usage of the transferred species' enthalpy at the destination phase's conditions is attributed to the wet bulb effect [98]. For example, as air passes over a wet wick covering a thermometer's bulb, some of the water evaporates. This causes the water's temperature to drop (i.e., the temperature reading on the thermometer drops). Once the temperature of the water stabilizes (i.e., there is a constant temperature reading from the thermometer), the heat loss from the water due to evaporation equals the heat gained by the water from the air. Otherwise, if energy from air was not transferred to the water, the water's temperature would continue to drop [99]. 
The heat of reaction and enthalpy transfer due to mass transfer are combined into source terms for the gas and particle internal energy equations $\left(\Delta H_{r g}\right.$ in Equation (1-6) and $\Delta H_{s g}$ in Equation (3-6)). Since the heat of reaction is calculated for a reaction in general, a methodology for distributing the energy among the gas and solids phases is needed. The approach for assigning the heat of reaction employed in this effort is reserved for Section 4.3.5.

\subsubsection{Mass and species equations for a particle}

The time rate of change of a particle's mass resulting from a chemical reaction or phase change is given by Equation (4-8). Here, $m_{p}^{(i)}$ is the mass of the indexed particle, and $R_{s n}^{(i)}$ is the rate of production (or consumption) of species $n$ for the indexed particle. The superscript over the summation, $N_{s}^{(i)}$, is the total number of species that comprise particle $(i)$. The sign convention is such that $R_{s n}$ is positive if species $n$ is produced and negative when consumed. Furthermore, $R_{s n}$ is obtained from expressions like Equations (4-1) and (4-3) and specific to the reaction of interest.

$$
\frac{d m_{p}^{(i)}}{d t}=\sum_{n=1}^{N_{s}^{(i)}} R_{s n}^{(i)}
$$

The time rate of change of the $n^{\text {th }}$ species' mass in particle ( $i$ ) is given by Equation (4-9). Here, $X_{p n}^{(i)}$ is species $n$ 's mass fraction of the indexed particle. For a particle comprised by $N_{s}^{(i)}$ species, $N_{s}^{(i)}$ equations of this form are needed to account for the composition of the particle. Employing the product rule and combing the result with Equation (4-8) yields an equation for the species mass fraction, shown as (4-10). 


$$
\begin{gathered}
\frac{d}{d t}\left(X_{p n}^{(i)} m_{p}^{(i)}\right)=R_{s n}^{(i)} \\
\frac{d X_{p n}^{(i)}}{d t}=\frac{1}{m_{p}^{(i)}}\left(R_{s n}^{(i)}-X_{p n}^{(i)} \sum_{n=1}^{N_{s}^{(i)}} R_{s n}^{(i)}\right)
\end{gathered}
$$

\subsection{Particle reaction models}

Three basic approaches for modeling the effect of a gas-solids reaction on a particle are surveyed in this section $[\mathbf{9 0 , 1 0 0}]$. Various considerations needed to execute each model in MFIX-DEM are investigated. The result is that the unreacted-core model is best suited as the default particle reaction model for implementation into MFIX-DEM.

\subsubsection{Progressive-conversion model}

In highly porous particles, gaseous reactants may diffuse uniformly throughout a particle before reacting with the pores' surfaces [94]. This process, illustrated in Figure 4-1, is captured by the progressive-conversion model wherein the concentration of solids phase reactant diminishes evenly in the particle as a reaction proceeds.

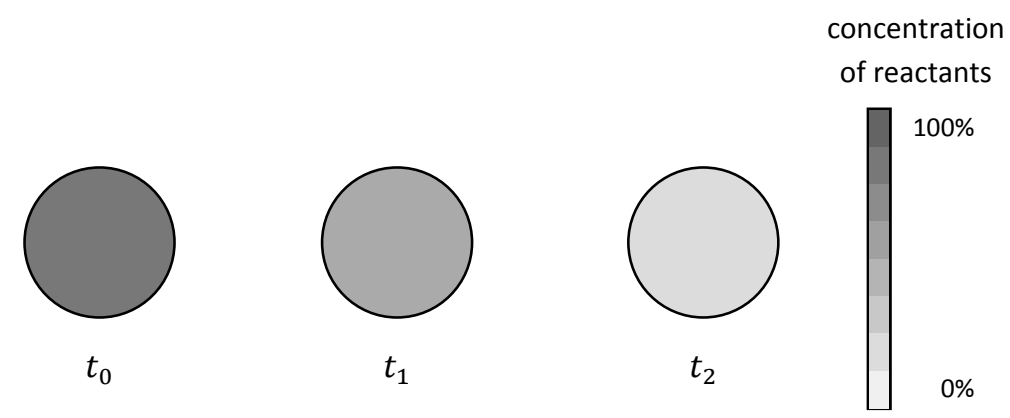

Figure 4-1: Illustration of the progressive-conversion model. The reaction occurs uniformly throughout a particle, leaving the particle size unchanged. In this example, the concentration of the reactant diminishes as the reaction proceeds.

The assumption of constant particle volume applied to the particle mass equation (Equation (4-8)) provides an expression for the time rate of change of the particle's density, shown as Equation (4-11). The sign convention for the rate of solids phase 
species production is such that a net production of solids mass causes the density of a particle to increase. Conversely, a net consumption of solids phase species results in a decrease of the particle's density.

$$
\frac{d \rho_{p}^{(i)}}{d t}=\frac{1}{\mathcal{V}_{p}^{(i)}} \sum_{n=1}^{N_{s}^{(i)}} R_{s n}^{(i)}
$$

The progressive-conversion model is limited to slow reactions between a gas and very porous solid and reactions where a solid is converted exclusively by heat $[\mathbf{9 0 , 9 4 ]}$. In the present effort, particles are assumed non-porous, and refining the particle model to include sub-particle pore structures is beyond the scope of this work. (More information on porous particle reaction models is found in reference [94].) Furthermore, physical experiments have shown that gas-solids reactions in non-porous solids typically progress from the particle's surface towards its center along a reaction front [90]. As a result, there is a separation of reacted and unreacted material within a particle, not a uniform conversion. Although the progressive-conversion model is implemented in the CFDDEM code of Simsek et al. [22], it was not selected for this work in favor of a more physical approach.

\subsubsection{Shrinking particle model}

The shrinking particle model assumes the size of a particle decreases as a reaction

proceeds. This is caused by an even gasification of the particle's components or a 'flaking-off' of solid products from the particle's surface. The shrinking particle model is illustrated in Figure 4-4 where the concentration of reactants in the particle remain constant as the particle's size decreases. 


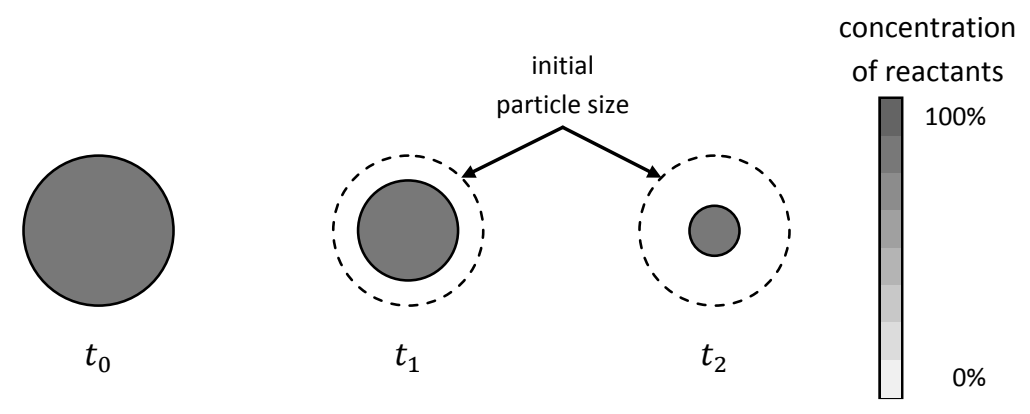

Figure 4-2: Illustration of the shrinking particle model. The reaction occurs at the particle's surface while the composition of the particle does not change. Any solid products of the reaction are assumed to 'flake off' of the particle's surface (i.e. no solid products collect on the surface of the particle).

By assuming the density of a particle is constant and that there are no solids phase products, the particle mass equation (Equation (4-8)) gives the time rate of change of the particle's radius, shown in Equation (4-12). The sign convention is such that the consumption of a particle's mass results in a decrease of the particle's radius. Since solids phase production is prohibited, $R_{s n}$ is always non-positive.

$$
\frac{d R_{p}^{(i)}}{d t}=\frac{1}{4 \pi \rho_{p}^{(i)} R_{p}^{(i)^{2}}} \sum_{n=1}^{N_{s}^{(i)}} R_{s n}^{(i)}
$$

The most notable limitation to the shrinking particle model is the opportunity for large disparities in particle sizes to develop (i.e., $R_{p}^{(i)} \ll R_{p}^{(j)}$ ). As the difference in particle sizes increase, the detection and storage of particle contact information may become computationally prohibitive. For example, in MFIX-DEM, each particle is the center of a neighborhood. Any particle whose center is contained within a particle's neighborhood is recorded as a potential contact partner. As the difference in particle sizes increase, the number of particles that are able to fit into a particle's neighborhood also increases. An exaggeration of how the computational expense could increase caused by an increase in size disparity among particles is illustrated Figure 4-3. A more robust 
approach to contact detection and information storage is needed before this limitation is overcome.

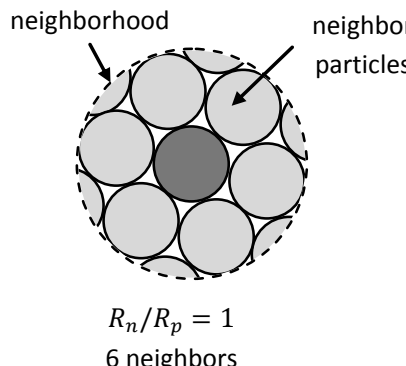

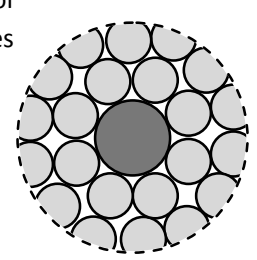

$R_{n} / R_{p}=0.6$

22 neighbors

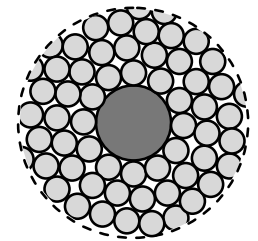

$R_{n} / R_{p}=0.33$

60 neighbors

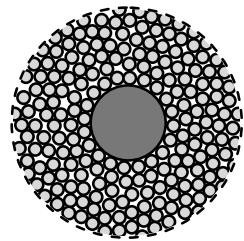

$R_{n} / R_{p}=0.167$ 202 neighbors

Figure 4-3: An example illustrating how the number of possible neighbor particles increases as the ratio of the neighbor's radius $\left(R_{n}\right)$ to the particle's radius $\left(R_{p}\right)$ decreases.

This model additionally assumes that any solids phase products or inert material either gasify or 'flake-off' of a particle's surface. This flaking-off of solids (ash) poses a difficult modeling task for CFD-DEM as discretizing and tracking any ash adds modeling complexity and computational expense. However, if the ash is not tracked (i.e., the ash is discarded or assumed negligible), then the conservation of solids mass is not maintained. Limiting simulations to those in which the initial particle contains no inert solids and all solids products are gasified is too restrictive for a general particle reaction model. Although the shrinking particle model is employed in several CFD-DEM reactive chemistry models $[\mathbf{1 8}, \mathbf{2 0}, \mathbf{2 2}, \mathbf{2 3}, \mathbf{1 0 1}]$, greater modeling flexibility is provided by a particle reaction model in which the production of solids phase species is permitted. As a result, the shrinking particle model is dismissed in favor of a more general approach.

\subsubsection{Shrinking, unreacted-core model}

In the shrinking, unreacted core model, a reaction starts on a particle's surface and proceeds toward the particle's center. As the reaction progresses, solids products from the reaction and any inert material initially in the particle form a continuous and even shell surrounding the unreacted core of the particle. Throughout the reaction, the unreacted 
core shrinks uniformly while the thickness of inert solids (ash layer) increases uniformly.

Illustrated in Figure 4-4, the outer radius of the particle and the concentration of reactants in the core remain constant as the core's radius decreases.

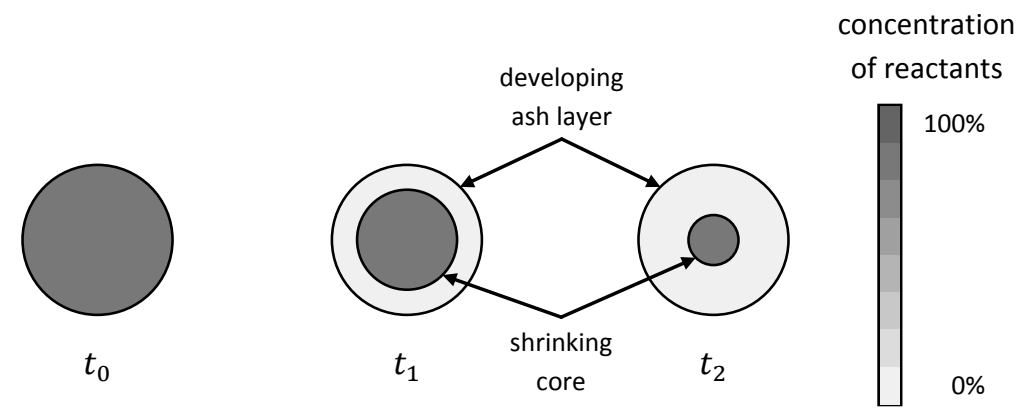

Figure 4-4: Illustration of the shrinking core model. The reaction occurs at the surface of the particle's unreacted core with an inert solid (ash) replacing the particle's solids. Although the unreacted core shrinks as the reaction proceeds, its composition remains constant.

Although not completely physical [90], the reaction front defines a sharp interface separating the ash layer from the unreacted core. An equation to specify the radial distance of the reaction front from the particle's center $\left(R_{c}\right)$ is shown as Equation (4-13) where $\rho_{c}$ is the density of the unreacted core, and $\hat{R}_{s n}$ is the rate of consumption of the solids phase reactants. This equation is obtained by considering only the reactants in the particle mass equation (i.e., Equation (4-8)).

$$
\frac{d R_{c}^{(i)}}{d t}=\frac{-1}{4 \pi \rho_{c}^{(i)} R_{c}^{(i)^{2}}} \sum_{n=1}^{N_{s}^{(i)}} \hat{R}_{s n}^{(i)}
$$

The definition of the unreacted core's density affects how its radius shrinks during the course of a reaction. For example, consider a particle-gas reaction where two solids phase species, B and C, are consumed. Furthermore, assume that there is an abundance of solid phase species $C$ within the particle such that the excess species $C$ remains in the particle after all the species $\mathrm{B}$ is consumed. The shrinking of the unreacted core employing three different definitions for the core's density is illustrated in Figure 4-5. In 
the figure, the mass of species $B$ and $C$ (i.e., $\mathrm{m}_{\mathrm{B}}$ and $\mathrm{m}_{\mathrm{C}}$ ) within the particle are indicated by the dashed and double lines. In this example, if the unreacted core's density $\left(\rho_{c}\right)$ is equated to the particle's initial density $\left(\rho_{p}\right)$, then the radius of the core shrinks to $R_{1}$. If the unreacted core's density is defined as the bulk density of solids reactants initially in the particle, shown as Equation (4-14) where $m_{s n}^{0}$ is the initial mass of the solids phase reactant $n$ and $\mathcal{V}_{p}$ is the particle's volume, then the radius of the core shrinks to $R_{2}$. Finally, if the unreacted core's density is defined as the bulk density of consumable solids phase reactants, given by Equation (4-15) where $m_{s n}^{c}$ is the total mass of solids phase reactant $n$ consumed during the reaction, then the radius of the unreacted core shrinks to zero.

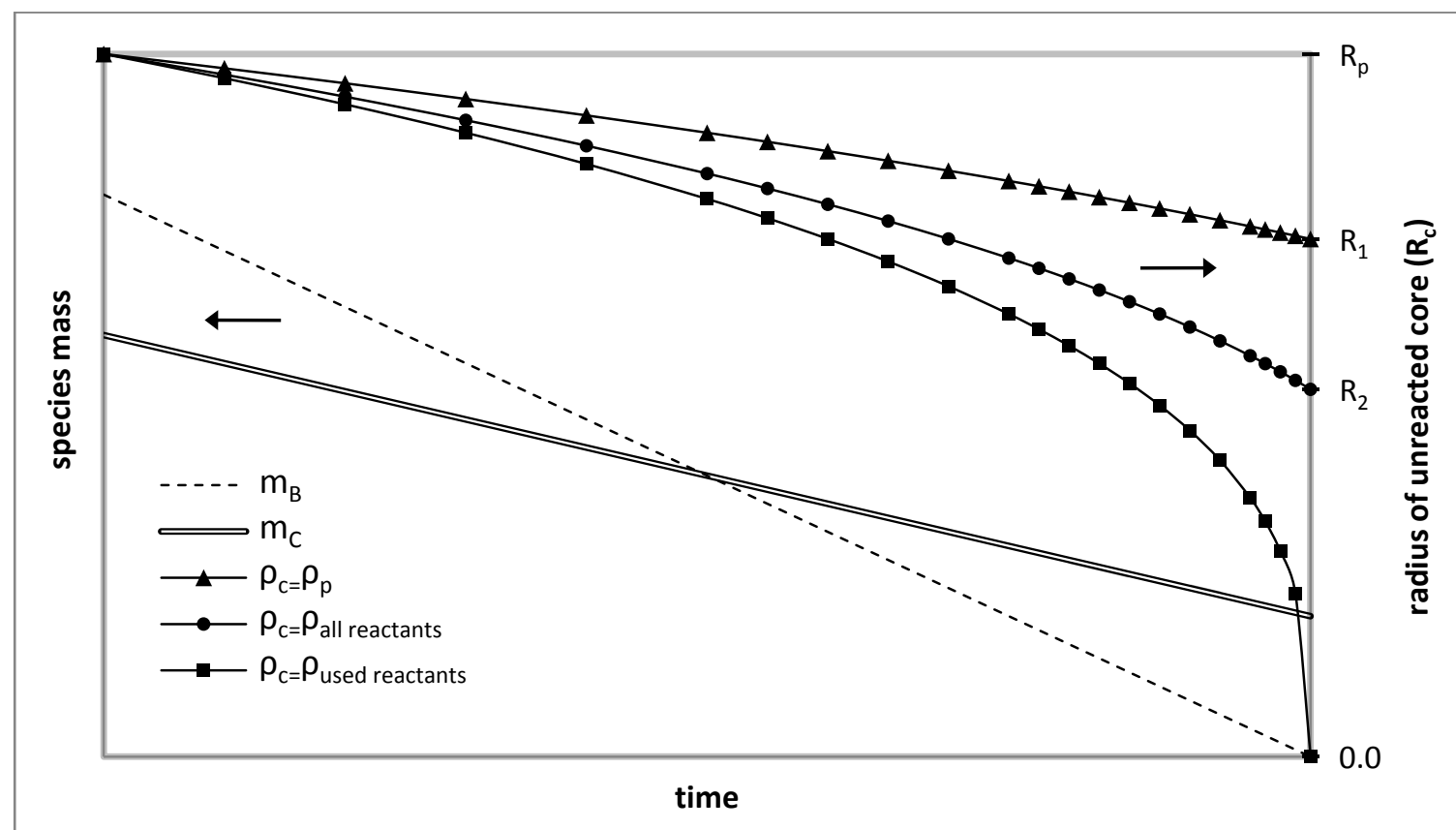

Figure 4-5: Example illustrating the connection between the radius of the unreacted core $\left(R_{c}\right)$ and the definition of the unreacted core's density $\left(R_{c}\right)$. If the unreacted core's density $\left(\rho_{c}=\rho_{p}\right)$ is equated to the particle's initial density $\left(\rho_{p}\right)$, then the core's radius shrinks to $R_{1}$. If the unreacted core's density ( $\rho_{c}=\rho_{\text {all reactants }}$ ) is defined as the initial mass of the solid phase reactants divided by the particle's volume, the core's radius shrinks to $\boldsymbol{R}_{2}$. Finally, if the unreacted core's density $\left(\rho_{c}=\rho_{\text {used reactants }}\right)$ is defined as the total mass of solid phase reactants consumed divided by particle's volume, then the core's radius shrinks to zero. 


$$
\begin{aligned}
& \rho_{c}=\frac{1}{\mathcal{V}_{p}} \sum_{n} m_{s n}^{0} \\
& \rho_{c}=\frac{1}{\mathcal{V}_{p}} \sum_{n} m_{s n}^{c}
\end{aligned}
$$

The above example leads to several observations. Note that if the initial composition of the particle is a single solids phase reactant, then all the definitions for the core density are the same. This is also true if there is no inert material initially present in the particle and the initial composition of the particle is balanced with the reaction stoichiometry (i.e., no solids phase reactants remain after the reaction is complete). However, if initially there is inert material in the particle and/or there is an abundance of one or more solids phase reactants, then the different solutions for the unreacted core's radius exist.

Additionally, the definitions for the unreacted core's density lead to several implicit assumptions. Consider the example illustrated in Figure 4-5. The unreacted core shrinks the least when the core's density is equated with the initial density of the particle. For this to occur, then the available solids reactants must exist in the outer region of the particle between $R_{1}$ and $R_{p}$. Similarly, the reactants must exist between $R_{2}$ and $R_{p}$ when the unreacted core's density is given by Equation (4-14). By using Equation (4-15) to define the unreacted core's density, reactants are assumed uniformly distributed throughout a particle. This last definition also implies that any surplus solids reactants within a particle is treated as inert material. 


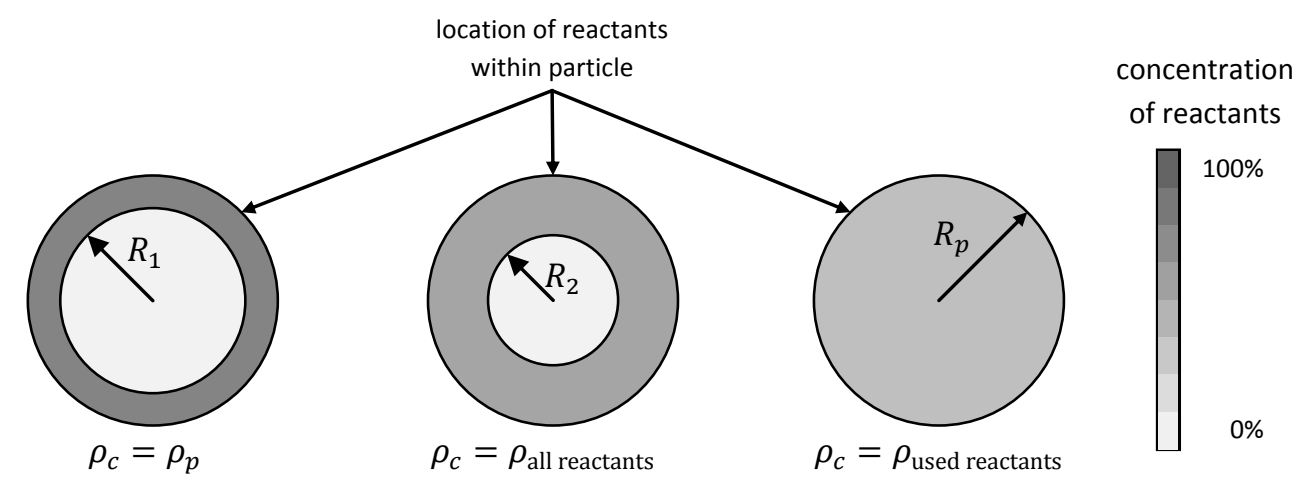

Figure 4-6: Example illustrating the implicit assumptions associated with the definition of the unreacted core's density $\left(R_{c}\right)$. If the unreacted core's density $\left(\rho_{c}=\rho_{p}\right)$ is equated to the particle's initial density $\left(\rho_{p}\right)$, then the consumable solids reactant exists between $R_{1}$ and $R_{p}$. If the unreacted core's density ( $\left.\rho_{c}=\rho_{\text {all reactants }}\right)$ is defined as the initial mass of the solid phase reactants divided by the particle's volume, the consumable solids exist between $R_{2}$ and $R_{p}$. Finally, if the unreacted core's density ( $\rho_{c}=\rho_{\text {used reactants }}$ ) is defined as the total mass of solid phase reactants consumed divided by particle's volume, then the consumable reactants are distributed uniformly through the particle.

The shrinking, unreacted core model was selected as the particle reaction model in this work. The selection was guided by (1) its near accurate physical account of particle-gas reactions [90], and (2) the ability to account for particles initially containing inert material. Although Liu, Chen, Zhou, and Zhao [21] claim to employ this model in their CFD-DEM, the authors cite a short simulation time (less than 15 seconds of physical time) to further assume that the diameter of the core is constant. This work makes no such assumption. The selection of the unreacted core's density is further addressed in Section 4.3.6. This concludes the survey of various particle reaction models. The following section outlines the implementation of the interface for managing particlegas reactions into MFIX-DEM.

\subsection{Implementation of interface}

The mathematical models managed in this effort by the reactive chemistry interface and discussed in the preceding sections, are summarized in Table 4-1. These models were implemented into MFIX-DEM, an existing CFD-DEM code. This required the modification and creation of several subroutines. Descriptions of new subroutines are 
outlined in Table 4-2, while the source code is reserved for Appendix E. Modifications to existing routines mainly consist of logical checks associated with call statements to the new routines. A call-tree showing the invocation of the reactive chemistry interface initialization routines is shown in Figure 4-7, and the invocation of the reactive chemistry interface model operational routines is shown by the call-tree in Figure 4-8.

Table 4-1: Summary of the models managed by the reactive chemistry interface considered in this work.

\begin{tabular}{|c|c|}
\hline $\begin{array}{c}\text { particle mass equation } \\
\text { (Section 4.1.4) }\end{array}$ & $\frac{d m_{p}^{(i)}}{d t}=\sum_{n=1}^{N_{s}^{(i)}} R_{s n}^{(i)}$ \\
\hline $\begin{array}{c}\text { particle species mass } \\
\text { fraction equation } \\
\text { (Section 4.1.4) }\end{array}$ & $\frac{d X_{p n}^{(i)}}{d t}=\frac{1}{m_{p}^{(i)}}\left(R_{s n}^{(i)}-X_{p n}^{(i)} \sum_{n=1}^{N_{s}^{(i)}} R_{s n}^{(i)}\right)$ \\
\hline $\begin{array}{c}\text { particle internal energy } \\
\text { equation (Section 3.1.3) }\end{array}$ & $m^{(i)} C_{p p}^{(i)} \frac{d T_{p}^{(i)}}{d t}=\dot{Q}_{p p}^{(i)}+\dot{Q}_{p f p}^{(i)}+\dot{Q}_{p g}^{(i)}+\dot{Q}_{r a d}^{(i)}-\Delta H_{r s}^{(i)}$ \\
\hline $\begin{array}{c}\text { shrinking, unreacted } \\
\text { core radius }\end{array}$ & $\frac{d R_{c}^{(i)}}{d t}=\frac{-1}{4 \pi \rho_{c}^{(i)} R_{c}^{(i)} \sum_{n=1}^{(i)}} \hat{R}_{s n}^{(i)}$ \\
\hline Section 4.2.3)
\end{tabular}

Table 4-2: A list of subroutines developed for the reactive chemistry interface implemented into MFIX.

\begin{tabular}{|c|c|}
\hline $\begin{array}{l}\text { Subroutine } \\
\text { file name }\end{array}$ & Description \\
\hline $\begin{array}{l}\text { DES_RXNS } \\
\text { des_rxns_mod.f }\end{array}$ & Global data storage for common reaction data. \\
\hline $\begin{array}{l}\text { CHECK_DES_RXNS } \\
\text { check_des_rxns.f }\end{array}$ & $\begin{array}{l}\text { Checks the data provided for the reactive chemistry } \\
\text { model, and sets various flags used to invoke the } \\
\text { necessary subroutines. }\end{array}$ \\
\hline $\begin{array}{l}\text { DES_CALC_H } \\
\text { calc_h.f }\end{array}$ & $\begin{array}{l}\text { Calculates the enthalpy of the species using the current } \\
\text { species temperature and the Burcat and Ruscic [102] } \\
\text { thermodynamic database. }\end{array}$ \\
\hline $\begin{array}{l}\text { DES_PHYSICAL_PROP } \\
\text { des_physical_prop.f }\end{array}$ & $\begin{array}{l}\text { Calculates the temperature dependent specific heat of } \\
\text { particles using the Burcat and Ruscic }[\mathbf{1 0 2}] \\
\text { thermodynamic database. }\end{array}$ \\
\hline $\begin{array}{l}\text { DES_RRATES } \\
\text { des_rrates.f }\end{array}$ & $\begin{array}{l}\text { Provides a set location within the code for a user to } \\
\text { implement their specific reaction kinetics. }\end{array}$ \\
\hline $\begin{array}{c}\text { DES_REACTION_MODEL } \\
\text { des_reaction_model.f }\end{array}$ & $\begin{array}{l}\text { Update the mass, density, and species composition of the } \\
\text { particles. }\end{array}$ \\
\hline
\end{tabular}




\begin{tabular}{|c|l|}
\hline $\begin{array}{c}\text { CALC_RRATES_DES } \\
\text { calc_rrates_des.f }\end{array}$ & $\begin{array}{l}\text { Invoke the DES_RRATES subroutine to calculate the } \\
\text { interphase mass transfer and heat of reaction for the gas } \\
\text { phase. }\end{array}$ \\
\hline
\end{tabular}

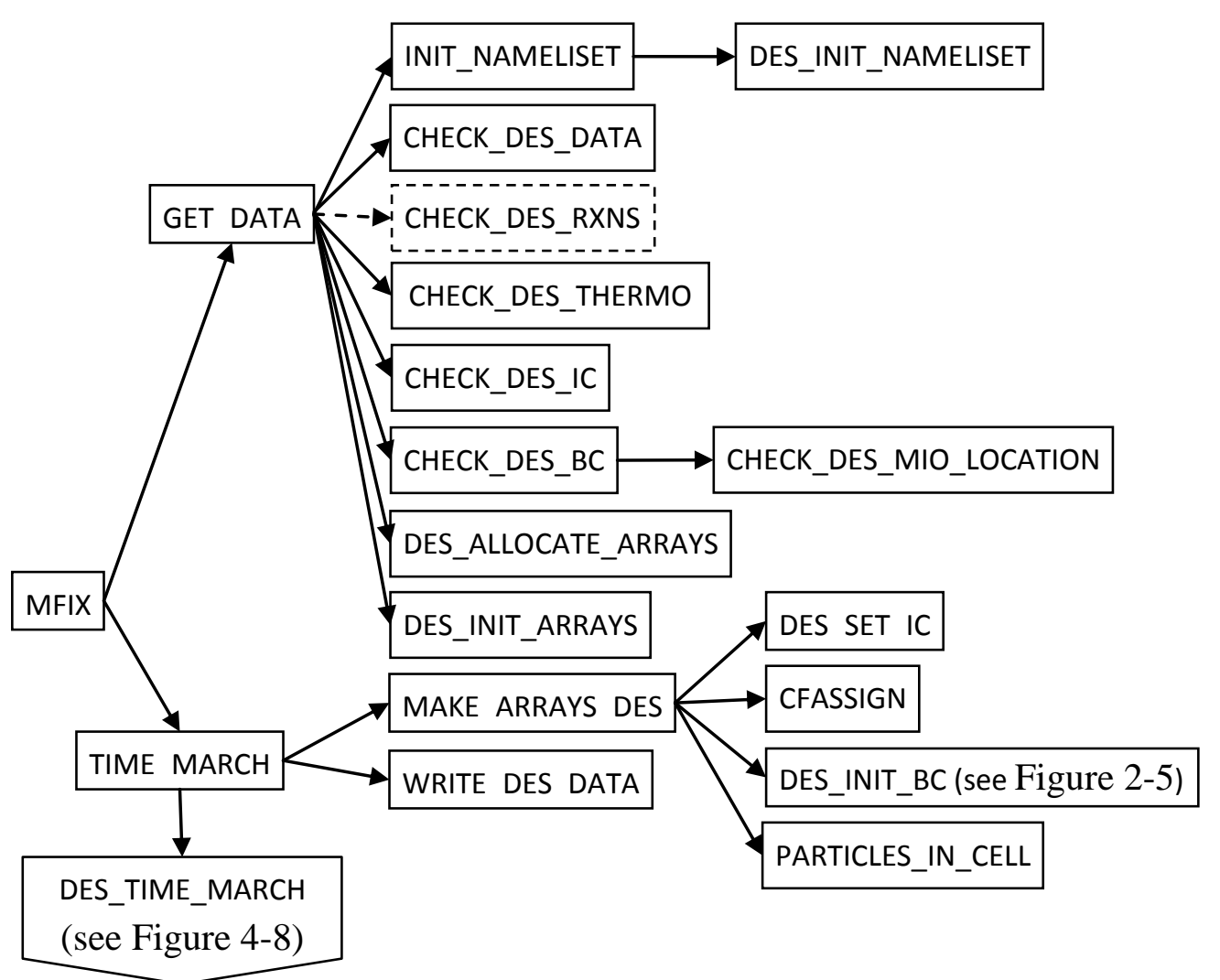

Figure 4-7: Call graph of main MFIX-DEM source code for initialization routines. Dashed lines indicate new calls and subroutines implemented for the reactive chemistry interface. 


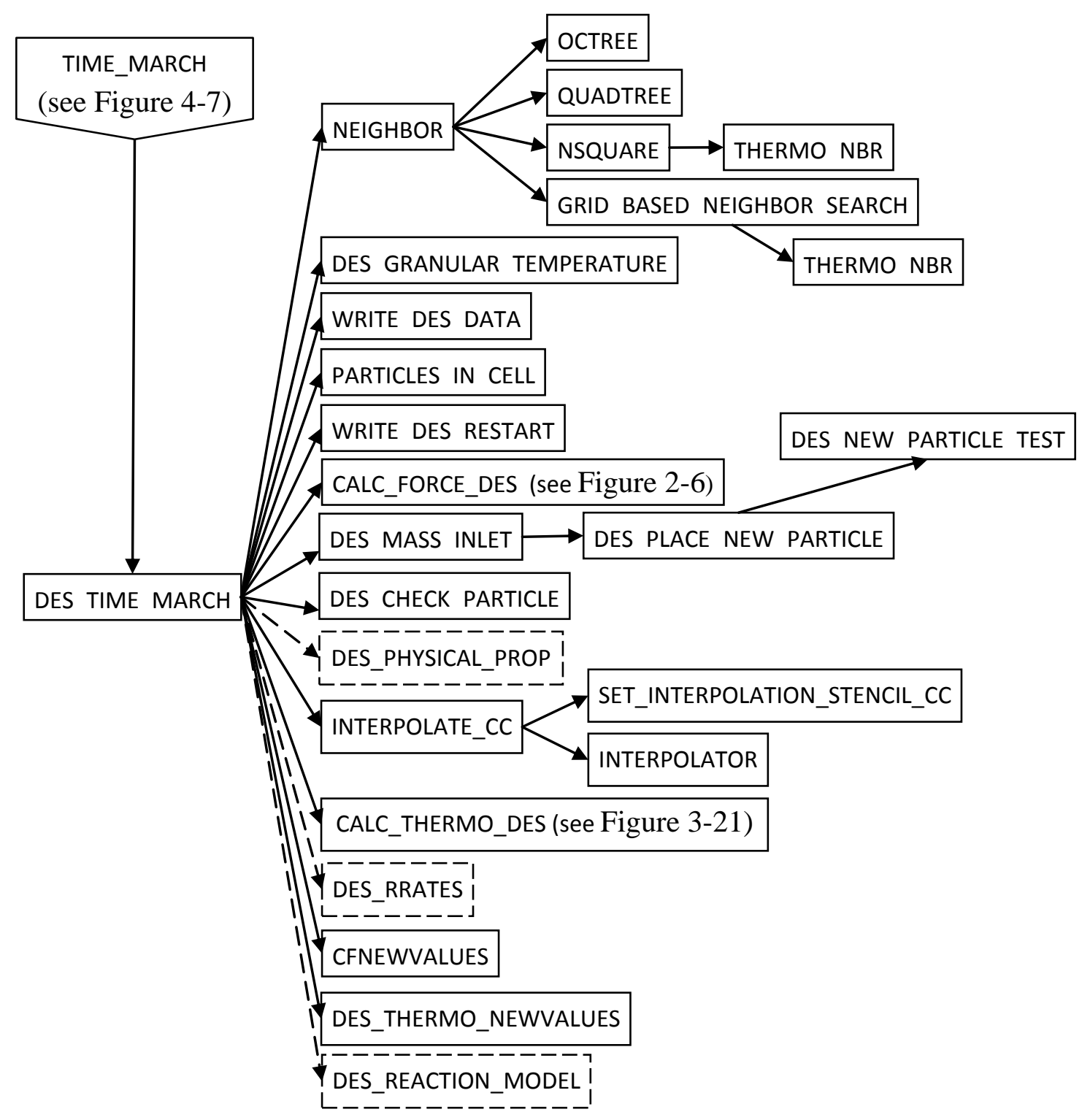

Figure 4-8: Call graph of MFIX discrete particle model routines. Dashed lines indicate new calls and subroutines implemented for the reactive chemistry interface.

A flow chart depicting the reactive chemistry interface implemented in MFIXDEM is presented in Figure 4-9. Processes added to the DEM algorithm for reactive chemistry are indicated by dashed lines. 


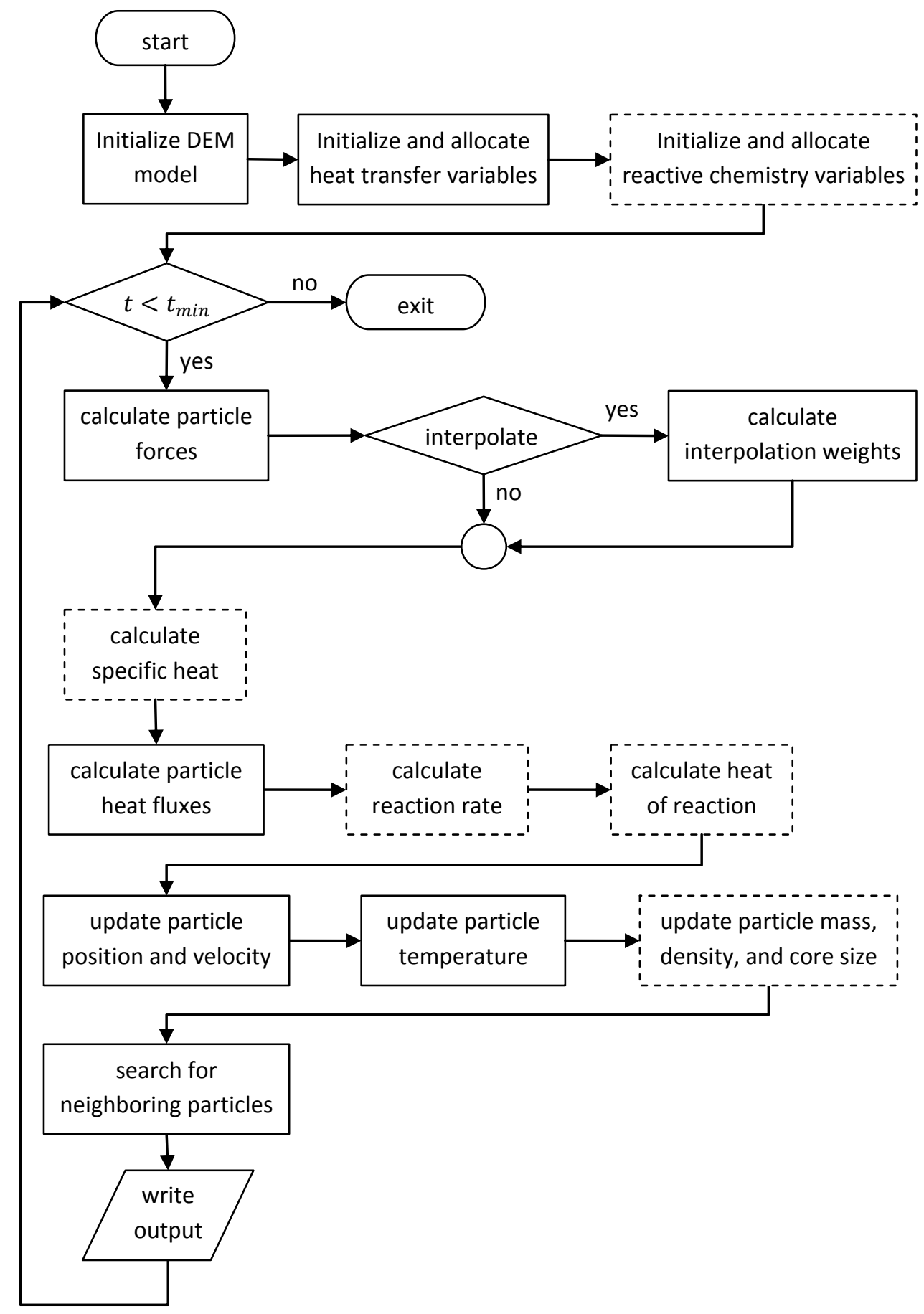

Figure 4-9: Flow chart illustrating the locations within MFIX-DEM where modifications have been made to include the reactive chemistry interface. 


\subsubsection{Gas-solids mass and species mass transfer}

The rates of disappearance and formation of gas and solids phases species, introduced in Section 4.1.1, result in an interphase coupling of the gas and particle mass balance equations as well as a coupling of the gas and particle mass balance species equations. Specifically, the rates of disappearance and formation of the gas and solids phase reactants and products result in source terms in the mass equations for the gas $\left(\sum_{n=1}^{N_{g}} R_{g n}\right.$ in Equation (1-1)) and particle $\left(\sum_{n=1}^{N_{s}} R_{s n}\right.$ in Equation (4-8)) as well as the species equations for the gas $\left(R_{g n}\right.$ in Equation (1-5)) and particle $\left(R_{s n}\right.$ in Equation (4-9)).

The approach used for interphase mass transfer follows directly from the method detailed in Section 3.6.1 for interphase energy transfer. Therefore, exchanging mass between the Eulerian grid and particles can occur through either a direct exchange between a particle and the Eulerian cell that contains its center, or through interpolating the information to the nearest Eulerian cells surrounding the particle (see Figure 3-24).

Equation (4-16) defines the species $n$ mass production (or consumption), $R_{g n}$. Here, $\mathcal{V}_{C V}$ is the volume of the control volume $(\mathrm{CV}), N_{C V}$ is the number of particles contained in the $\mathrm{CV}$, and $R_{g n}^{(k)}$ is the rate of gas phase species $n$ production (or consumption) due to a particle-gas reaction between particle $(k)$ and the gas phase. $K_{C V}^{(k)}$ is a function used to map the species mass to/from a particle's position from/to the computational nodes of the gas phase Eulerian grid.

$$
R_{g n}=-\frac{1}{v_{C V}} \sum_{k=1}^{N_{C V}} R_{g n}^{(k)} K_{C V}^{(k)}
$$

As in the coupling of the gas and solids phase energy equations, there are two

definitions for the mapping function, $K_{C V}^{(k)}$. By default, mass transfer between the gas and 
a particle is associated with the CV containing the particle's center (see Figure 3-25-(a)). A second option distributes mass among the control volumes surrounding the particle via linear interpolation (see Figure 3-25-(b)). If the interpolation method is employed, the gas phase species concentrations, if needed by the rate law (see Equation (4-1) in Section 4.1.1), is interpolated to a particle's position from the surrounding control volumes for consistency.

The information (mass and species mass) exchanged between the gas and particles follows the same approach used for energy exchange. The source terms for the mass and species mass equations for the particles and gas are managed differently to account for the disjoint updating procedure (see Section 3.6.1). When the gas phase equations are solved, all of the physical properties of the particles are constant. This is a direct result of their solutions lagging behind the solution of the gas phase. Similarly, when the particle phase equations are solved, all of the physical properties of the gas phase are constant.

\subsubsection{Mass, species mass, and core radius updating procedures}

As mentioned in the updating procedure for the particle internal energy equation in Section 3.6.3, there are various methodologies available for solving differential equations numerically $[\mathbf{8 7 , 8 8 , 8 9}]$. However, the time integration methods for the discrete solids phase momentum equation implemented in MFIX-DEM are limited to Euler's and Adams-Bashforth methods [35]. Therefore, the same methods are used to advance the solution for the particle's mass, species mass fraction, and core radius equations. Euler's method is the default approach selected by this work, and shown for the mass, species mass fraction, and unreacted core radius in Equations (4-17)-(4-19), respectively. Note 
that both the mass and species mass fraction equations have a dependence on the particle's mass. Therefore, the particle's mass is updated first so that the new value for particle mass is available for updating the species mass fraction equations. The AdamsBashforth method (not shown) is available for higher accuracy. Details concerning these methods are provided in references $[\mathbf{8 8 , 8 9}]$.

$$
\begin{gathered}
m_{p}^{(i)}\left(t+\Delta t_{s}\right)=m_{p}^{(i)}(t) \Delta t_{s}+\sum_{n=1}^{N_{s}^{(i)}} R_{s n}^{(i)} \\
X_{p n}^{(i)}\left(t+\Delta t_{s}\right)=X_{p n}^{(i)}(t)+\frac{\Delta t_{s}}{m_{p}^{(i)}\left(t+\Delta t_{s}\right)}\left(R_{s n}^{(i)}-X_{p n}^{(i)} \sum_{n=1}^{N_{s}^{(i)}} R_{s n}^{(i)}\right) \\
R_{c}^{(i)}\left(t+\Delta t_{s}\right)=R_{c}^{(i)}(t)+\frac{-\Delta t_{s}}{4 \pi \rho_{c}^{(i)}\left(R_{c}^{(i)}(t)\right)^{2}} \sum_{n=1}^{N_{s}^{(i)}} \hat{R}_{s n}^{(i)}
\end{gathered}
$$

\subsubsection{Specific heat calculations}

Specific heat with respect to pressure, introduced in Section 4.1.2, indicates the amount of heat required to increase a material's temperature one degree per unit mass. The present work uses a fourth order polynomial to represent specific heat, as shown in Equation (4-20). The polynomial coefficients are obtained from the Burcat and Ruscic [102] thermodynamic database. Here, $R$ is the gas constant, $T$ is the temperature, and $\alpha_{k}$ are the polynomial coefficients obtained from the Burcat and Ruscic [102] database. Using this database, the specific heat is obtained in units of cal $\cdot(\mathrm{mol} \cdot \mathrm{K})^{-1}$.

$$
C_{p n}=R \sum_{k=0}^{4} \alpha_{k} T^{k}
$$


The specific heat for a material composed of multiple species is approximated by combining the specific heats of the individual species weighted by their mass fraction. For example, the specific heat of a mixture composed of $n$ species is illustrated in Equation (4-21). Here, $T$ is the material temperature, and $X$ is the species mass fraction of the indexed species.

$$
C_{p}=\sum_{k=0}^{4} \sum_{i=1}^{n}\left(\alpha_{k i} X_{i}\right) T^{k}
$$

\subsubsection{Heat of reaction calculations}

The heat of reaction, $\Delta H$, introduced in Section 3.4, specifies the amount of heat liberated or absorbed during a reaction [90]. Recall that the heat of reaction is calculated from the difference in enthalpies between the products and reactants. The enthalpy for a material at temperature $T\left(H_{n}(T)\right)$ is obtained by combining the material's enthalpy of formation at reference temperature $T_{R E F}\left(H_{n}^{\circ}\left(T_{R E F}\right)\right)$ with the enthalpy change $\left(\Delta H_{Q n}\right)$ due to a change in temperature $\left(T_{R E F}\right.$ to $T$ ) [93] (see Equation (4-5)). The change in enthalpy is calculated by integrating the specific heat with respect to temperature, (see Equation (4-6)). This work uses forth order polynomials for specific heat with the polynomial coefficients obtained from the Burcat and Ruscic [102] thermodynamic database (see Section 4.3.1). Integrating the generic polynomial structure results in an explicit expression for change in enthalpy, shown as Equation (4-22). Here, $\alpha_{k}$ are the polynomial coefficients, $R$ is the gas constant, and $T$ and $T_{R E F}$ are material and reference temperatures, respectively. 


$$
\Delta H_{Q}=\int_{T_{R E F}}^{T} C_{p} d T=R \sum_{k=0}^{4} \frac{\alpha_{k}}{(k+1)}\left(T^{k+1}-T_{R E F}^{k+1}\right)
$$

Similar to specific heat, the enthalpy for a material composed of multiple species is approximated by combining the enthalpies of the individual species weighted by their mass fraction. For example, the enthalpy of a mixture composed of $n$ species is illustrated in Equation (4-23). Here, $X$ is the mass fraction of the indexed species. The enthalpy of formation for species $n$ at temperature $T_{R E F}$ divided by the gas constant $\left(H_{i}^{\circ}\left(T_{R E F}\right) / R\right)$ is obtained from the Burcat and Ruscic [102] thermodynamic database with units of temperature $(\mathrm{K})$.

$$
H=R\left(\sum_{i=1}^{n}\left(\frac{H_{i}^{\circ}\left(T_{R E F}\right)}{R}\right)+\sum_{k=0}^{4} \sum_{i=1}^{n}\left(\frac{\alpha_{k i}}{k+1} X_{i}\right)\left(T^{k+1}-T_{R E F}^{k+1}\right)\right)
$$

\subsubsection{Distribution of the heat of reaction}

The heat of reaction, $\Delta H$, introduced in Section 3.4, is calculated in general for each reaction. A methodology for distributing the energy among the gas and solids phases is needed. The approach for assigning the heat of reaction employed in this effort is taken from Syamlal, Rogers, and O’Brien [27]. In their work, the heat of reaction associated with the gas phase is computed as the difference in enthalpies of the gas phase products and reactants. Similarly, the heat of reaction associated with the solids phase is computed as the difference in enthalpies of the solids phase products and reactants. Considering the example reaction given in Equation (4-2), the gas and solids phase heats of reaction are calculated by Equations (4-24) and (4-25), respectively.

$$
\Delta H_{g}=H_{\mathrm{C}}-H_{\mathrm{A}}
$$




$$
\Delta H_{s}=H_{\mathrm{D}}-H_{\mathrm{B}}
$$

The rate of heat production from the reaction is calculated by multiplying the enthalpies in the heat of reaction equations by their respective rates of formation or disappearance. The rates of energy production (or consumption) for the example reaction (see Equation (4-2)) associated with the gas and solids phases are given by Equations (4-26) and (4-27), respectively. Again, $r_{C}$ and $r_{D}$ are the rates of formation of species $C$ and $\mathrm{D}$, and $r_{\mathrm{A}}$ and $r_{\mathrm{B}}$ are the rates of disappearance of species $\mathrm{A}$ and $\mathrm{B}$.

$$
\begin{aligned}
& \Delta \dot{H}_{g}=H_{\mathrm{C}} r_{\mathrm{C}}-H_{\mathrm{A}}\left(-r_{\mathrm{A}}\right) \\
& \Delta \dot{H}_{s}=H_{\mathrm{D}} r_{\mathrm{D}}-H_{\mathrm{B}}\left(-r_{\mathrm{B}}\right)
\end{aligned}
$$

The heat of reaction for each phase and enthalpy transfer due to mass transfer (see Section 4.1.3 and Equation (4-7)) are combined into source terms for the gas and particle internal energy equations ( $\Delta H_{r g}$ in Equation (1-6) and $\Delta H_{s g}$ in Equation (3-6)). Again, consider the example reaction shown in Equation (4-2). The net rate of heat generation for the gas phase is obtained by subtracting Equation (4-7) from Equation (4-26). The result, shown as Equation (4-28), is supplied to the internal energy equation for the gas phase (see Equation (1-6) in Section 1.1.1.4). Likewise, the net rate of heat generation for a particle is obtained by adding Equation (4-7) to Equation (4-27). The result, shown as Equation (4-29), goes into the particle internal energy equation. Note that the sign convention of the rate of enthalpy transfer $\left(\dot{H}_{f r}\right)$ in Equations (4-28) and (4-29) depends on the direction of mass transfer (i.e., solids to gas, or gas to solids).

$$
\begin{aligned}
& \Delta H_{r g}=\Delta \dot{H}_{g}-\dot{H}_{f r} \\
& \Delta H_{r s}=\Delta \dot{H}_{s}+\dot{H}_{f r}
\end{aligned}
$$




\subsubsection{Unreacted core density}

The radius in the shrinking, unreacted core model, introduced in Section 4.2.3, requires a closure model for the core's density. A general framework was created to provide easier customization of the reactive chemistry interface. This was achieved by providing a flag in the setup file (i.e., mfix.dat [84]) so that a user may define a specific core density. If the user does not specify the core density, it is equated to the particle's density by default. The generalization of the interface prevents Equations (4-14) and (4-15) from defining the default core density. Specifically, solids phase species are not explicitly identified as products or reactants within the code. Therefore, the amount of solids phase reactants (i.e., $m_{s n}^{0}$ in Equation (4-14) and $m_{s n}^{c}$ in Equation (4-15)) is not calculable within the confines of the reactive chemistry interface's implementation.

\subsection{Verification of implementation}

Implementation of the reactive chemistry interface was verified using the case of a single reacting particle in a uniform gas flow. The gas-solid reaction is shown in Equation (4-30), where $\mathrm{A}(g)$ and $\mathrm{B}(s)$ are gas and solids phase reactants and $\mathrm{C}(g)$ and $\mathrm{D}(s)$ are gas and solids phase products. The particle initially contains solids reactant $\mathrm{D}$, an inert solids phase material called I, and no solids product species D.

$$
\mathrm{A}(g)+2 \mathrm{~B}(s) \rightarrow \mathrm{C}(g)+\mathrm{D}(s)
$$

The reaction rate is given in terms of the rate of disappearance of species $\mathrm{B},\left(-r_{\mathrm{B}}\right)$ and is assumed constant. The proportional relationship linking the rates of disappearance of species $\mathrm{A}$ and $\mathrm{B}$ and the rates of formation of species $\mathrm{C}$ and $\mathrm{D}$ are given in Equation (4-39). In this case the mass and species equations for the particle (see Equations (4-8) 
and (4-9)) reduce to the expressions shown in Equations (4-32)-(4-35) describing the particle's mass and species mass fractions, respectively.

$$
\begin{gathered}
\frac{\left(-r_{\mathrm{A}}\right)}{\mathrm{M}_{s \mathrm{~A}}}=\frac{1}{2} \frac{\left(-r_{\mathrm{B}}\right)}{\mathrm{M}_{s \mathrm{~B}}}=\frac{r_{\mathrm{C}}}{\mathrm{M}_{s \mathrm{C}}}=\frac{r_{\mathrm{D}}}{\mathrm{M}_{s \mathrm{D}}} \\
\frac{d m_{p}}{d t}=\left(-r_{\mathrm{B}}\right)\left(\frac{1}{2} \frac{\mathrm{M}_{s \mathrm{D}}}{\mathrm{M}_{s \mathrm{~B}}}-1\right) \\
\frac{d}{d t}\left(X_{\mathrm{B}} m_{p}\right)=-\left(-r_{\mathrm{B}}\right) \\
\frac{d}{d t}\left(X_{\mathrm{D}} m_{p}\right)=\frac{1}{2} \frac{\mathrm{M}_{s \mathrm{D}}}{\mathrm{M}_{s \mathrm{~B}}}\left(-r_{\mathrm{B}}\right) \\
\frac{d}{d t}\left(X_{\mathrm{I}} m_{p}\right)=0
\end{gathered}
$$

Only heat transfer resulting from the reaction is considered (i.e., there is no convection, conduction, or radiation). In this case the internal energy equation describing the temperature of a particle (see Equation (3-6)) reduces to the expression shown in Equation (4-39). Here, $m$ is the mass of the particle. $C_{p p}, \Delta H_{s}$, and $H_{f r}$ are defined in Equations (4-37)-(4-39) and represent the specific heat, change in enthalpy caused by the reaction, and the transfer of enthalpy due to mass transfer, respectively. In these equations, $R$ is the gas constant, $\alpha_{i k}, H_{i}^{\circ}\left(T_{R E F}\right)$, and $\mathrm{M}_{s i}$ are the coefficients, reference enthalpies, and molecular weights of the indexed species obtained from the Burcat and Ruscic [102] thermodynamic database; $X_{i}$ are the species mass fractions of the indexed species, and $T_{g}$ and $T_{p}$ are the gas phase and particle temperatures, respectively.

$$
\frac{d T_{p}}{d t}=\frac{-1}{m_{p} C_{p p}}\left(\Delta \dot{H}_{s}+\dot{H}_{f r}\right)
$$




$$
\begin{aligned}
& \Delta \dot{H}_{s}=\left(-r_{\mathrm{B}}\right)(\left.\frac{1}{2} \frac{\mathrm{M}_{s \mathrm{D}}}{\mathrm{M}_{s \mathrm{~B}}} H_{\mathrm{D}}^{\circ}\left(T_{R E F}\right)-H_{\mathrm{B}}^{\circ}\left(T_{R E F}\right)\right) \\
&+R \sum_{k=0}^{4} \frac{\left(-r_{\mathrm{B}}\right)\left(\frac{1}{2} \frac{\mathrm{M}_{s \mathrm{D}}}{\mathrm{M}_{s \mathrm{~B}}} \alpha_{\mathrm{D} k}-\alpha_{\mathrm{B} k}\right)}{(k+1)}\left(T_{p}^{k+1}-T_{R E F}^{k+1}\right) \\
& \dot{H}_{f r}=\left(-r_{\mathrm{B}}\right)\left(1-\frac{1}{2} \frac{\mathrm{M}_{s \mathrm{D}}}{\mathrm{M}_{s \mathrm{~B}}}\right)\left(H_{\mathrm{C}}^{\circ}\left(T_{R E F}\right)+R \sum_{k=0}^{4} \frac{\alpha_{\mathrm{C} k}}{(k+1)}\left(T_{g}^{k+1}-T_{R E F}^{k+1}\right)\right) \\
& C_{p p}=R \sum_{k=0}^{4}\left(X_{p \mathrm{~B}} \frac{\alpha_{\mathrm{B} k}}{\mathrm{M}_{s \mathrm{~B}}}+X_{p \mathrm{D}} \frac{\alpha_{\mathrm{D} k}}{\mathrm{M}_{s \mathrm{D}}}+X_{p \mathrm{I}} \frac{\alpha_{\mathrm{I} k}}{\mathrm{M}_{s \mathrm{I}}}\right) T_{p}^{k}
\end{aligned}
$$

As before, analytic solutions for the particle's mass, species mass fractions, temperature, and unreacted core radius are obtained for a particle. All gas phase thermal and physical properties are assumed constant $\left(C_{p g}\right.$ and $\left.T_{g}\right)$. The particle's specific heat $\left(C_{p p}\right)$ is assumed constant with respect to temperature. The density of the unreacted core is calculated from the bulk density of consumable solids phase reactants, given by Equation (4-15). These assumptions result in five ordinary differential equations solved by separation of variables. The resulting solutions for the particle's mass, species mass fractions, temperature, and unreacted core's radius are shown in Equations (3-34)-(3-39), respectively. Here, $m_{p 0}, X_{p \mathrm{~B} 0}, X_{p \mathrm{D} 0}, X_{p \mathrm{I} 0}$, and $T_{p, 0}$ are the particle's initial mass, species mass fractions, and temperature, respectively.

$$
\begin{aligned}
& m_{p}(t)=m_{p 0}-t\left(-r_{\mathrm{B}}\right)\left(\frac{1}{2} \frac{\mathrm{M}_{s \mathrm{D}}}{\mathrm{M}_{s \mathrm{~B}}}-1\right) \\
& X_{p \mathrm{~B}}(t)=\frac{m_{p 0} X_{p \mathrm{~B} 0}-\left(-r_{\mathrm{B}}\right) t}{m_{p 0}-t\left(-r_{\mathrm{B}}\right)\left(\frac{1}{2} \frac{\mathrm{M}_{s \mathrm{D}}}{\mathrm{M}_{s \mathrm{~B}}}-1\right)}
\end{aligned}
$$




$$
\begin{gathered}
X_{p \mathrm{D}}(t)=\frac{m_{p 0} X_{p \mathrm{D} 0}+r_{\mathrm{D}} t}{m_{p 0}-t\left(-r_{\mathrm{B}}\right)\left(\frac{1}{2} \frac{\mathrm{M}_{s \mathrm{D}}}{\mathrm{M}_{s \mathrm{~B}}}-1\right)} \\
X_{p \mathrm{I}}(t)=\frac{m_{p 0} X_{p \mathrm{I} 0}}{m_{p 0}-t\left(-r_{\mathrm{B}}\right)\left(\frac{1}{2} \frac{\mathrm{M}_{s \mathrm{D}}}{\mathrm{M}_{s \mathrm{~B}}}-1\right)} \\
a_{1}=\left(-r_{B}\right) \frac{R}{\mathrm{M}_{s \mathrm{~B}}}\left(\frac{\alpha_{\mathrm{D} 0}}{2}-\alpha_{\mathrm{B} 0}\right), \quad \frac{1}{a_{1}}\left(\frac{b_{2}\left(a_{1} T_{p 0}+a_{2}\right)}{a_{1} t+b_{2}}-a_{2}\right) \text { where } \\
a_{2}=\left(-r_{\mathrm{B}}\right) R\left(\frac{1}{\mathrm{M}_{s \mathrm{~B}}}\left(\frac{1}{2} \frac{H_{\mathrm{D}}^{\circ}}{R}-\frac{H_{\mathrm{B}}^{\circ}}{R}\right)+\frac{T_{R E F}}{\mathrm{M}_{s \mathrm{~B}}}\left(\alpha_{\mathrm{B} 0}-\frac{1}{2} \alpha_{\mathrm{D} 0}\right)+\frac{1}{\mathrm{M}_{s \mathrm{C}}}\left(1-\frac{1}{2} \frac{M_{s \mathrm{D}}}{M_{s \mathrm{~B}}}\right)\left(\frac{H_{\mathrm{C}}^{\circ}}{R}\right)\right) \\
\text { and } b_{2}=\left(m_{p 0} R\right)\left(\frac{X_{\mathrm{B} 0} \alpha_{\mathrm{B} 0}}{\mathrm{M}_{s \mathrm{~B}}}+\frac{X_{\mathrm{D} 0} \alpha_{\mathrm{D} 0}}{\mathrm{M}_{s \mathrm{D}}}+\frac{X_{\mathrm{I} 0} \alpha_{\mathrm{I} 0}}{\mathrm{M}_{s \mathrm{I}}}\right) \\
R_{c}=\left(R_{p}^{3}-\frac{t\left(-r_{\mathrm{B}}\right)}{\frac{4}{3} \pi \rho_{c}}\right)^{1 / 3} \\
(4)
\end{gathered}
$$

A simulation employing the same assumptions as defined above was conducted with MFIX-DEM. The physical and thermodynamic properties of the particle, gas, and species components are outlined in Table 4-3 and Table 4-4. As the reaction is artificial, generic values were used for the thermodynamic properties and reaction rate. Realistic values are not necessary as this verification is designed to test the implementation of the various models, and not validate any specific chemical reaction. The simulation was run for 20 seconds of physical time to consume all of the available solids phase reactant (species B). The solids time step was kept constant at $\Delta t_{s}=0.856357 \times 10^{-6}$. The simulation was performed on a single AMD Opteron 265, 1.8GHz processor. 
Table 4-3: Physical and thermodynamic properties of the gas and particle used to verify the reactive chemistry interface implemented into MFIX-DEM.

\begin{tabular}{|c|c|}
\hline particle diameter $(\mathrm{cm})$ & 0.30 \\
\hline Initial core diameter $(\mathrm{cm})$ & 0.30 \\
\hline Initial particle density $\left(\mathrm{g} / \mathrm{cm}^{3}\right)$ & 2.5 \\
\hline unreacted core density $\left(\mathrm{g} / \mathrm{cm}^{3}\right)$ & 1.25 \\
\hline Initial particle temperature $(\mathrm{K})$ & 500 \\
\hline gas temperature $(\mathrm{K})$ & 298 \\
\hline reaction rate: $\left(-r_{\mathrm{B}}\right)$ (g of B consumed) & $1.1781 \times 10^{-3}$ \\
\hline gas constant: $R($ cal $/(\mathrm{mol} \cdot \mathrm{K}))$ & 1.98721 \\
\hline Reference Temperature: $T_{R E F}(\mathrm{~K})$ & 298 \\
\hline
\end{tabular}

Table 4-4: Physical and thermodynamic properties of the gas and particle species used to verify the reactive chemistry interface implemented into MFIX-DEM.

\begin{tabular}{|c|c|c|c|c|c|}
\hline Phase & Species & $\alpha_{0}$ & $\begin{array}{c}H^{\circ}\left(T_{R E F}\right) / R \\
(\mathrm{~K})\end{array}$ & $\begin{array}{c}\mathrm{M}_{s} \\
(\mathrm{~g} / \mathrm{mol})\end{array}$ & $\begin{array}{c}\text { Initial Mass } \\
\text { Fraction }\end{array}$ \\
\hline \multirow{3}{*}{ gas } & $\mathrm{A}(g)$ & 3.5 & 0 & 25 & 1.0 \\
\cline { 2 - 6 } & $\mathrm{C}(g)$ & 4.6 & -1666.67 & 30 & 0.0 \\
\hline \multirow{4}{*}{ particle } & $\mathrm{B}(s)$ & 1.4 & 0 & 15 & 0.5 \\
\cline { 2 - 6 } & $\mathrm{D}(s)$ & 1.5 & 0 & 25 & 0.0 \\
\cline { 2 - 6 } & $\mathrm{I}(s)$ & 3 & 0 & 20 & 0.5 \\
\hline
\end{tabular}

The numerical and analytical solutions for the particle's mass, species mass fraction, and temperature are shown in Figure 4-10, Figure 4-11, and Figure 4-12. Here, $\mathrm{mp}$ and $\mathrm{Tp}$ indicate the particle's mass and temperature; $\mathrm{XB}, \mathrm{XD}$, and $\mathrm{XI}$ represent the particle's species mass fractions, respectively. Solid symbols denote the values calculated by MFIX-DEM; continuous lines represent the analytic solution; and, open symbols indicate the error between the solutions. Percent relative error (see Equation (3-56)) is used in the analysis of the mass and energy equations. Absolute error, defined in Equation (4-46), was used in the analysis of the species mass fraction equations. Absolute error was needed because the analytic solution of species mass fraction for species B goes to zero, leading to a division by zero in Equation (3-56). 
Note the strong agreement between the analytic and numeric solutions. The percent relative error for the mass and species equations is bounded above by $10^{-5}$, indicating the numerical solution deviates less than $\pm 10^{-5}$ percent from the analytic solution over the entire simulation. The absolute error for the species mass fraction equations is bounded above by $10^{-6}$, indicating an agreement between the solutions up to six decimal places over the entire simulation.

$$
\text { Absolute Error }=\left|T_{p k}^{\text {analytic }}-T_{p k}^{\text {MFIX-DEM }}\right|
$$

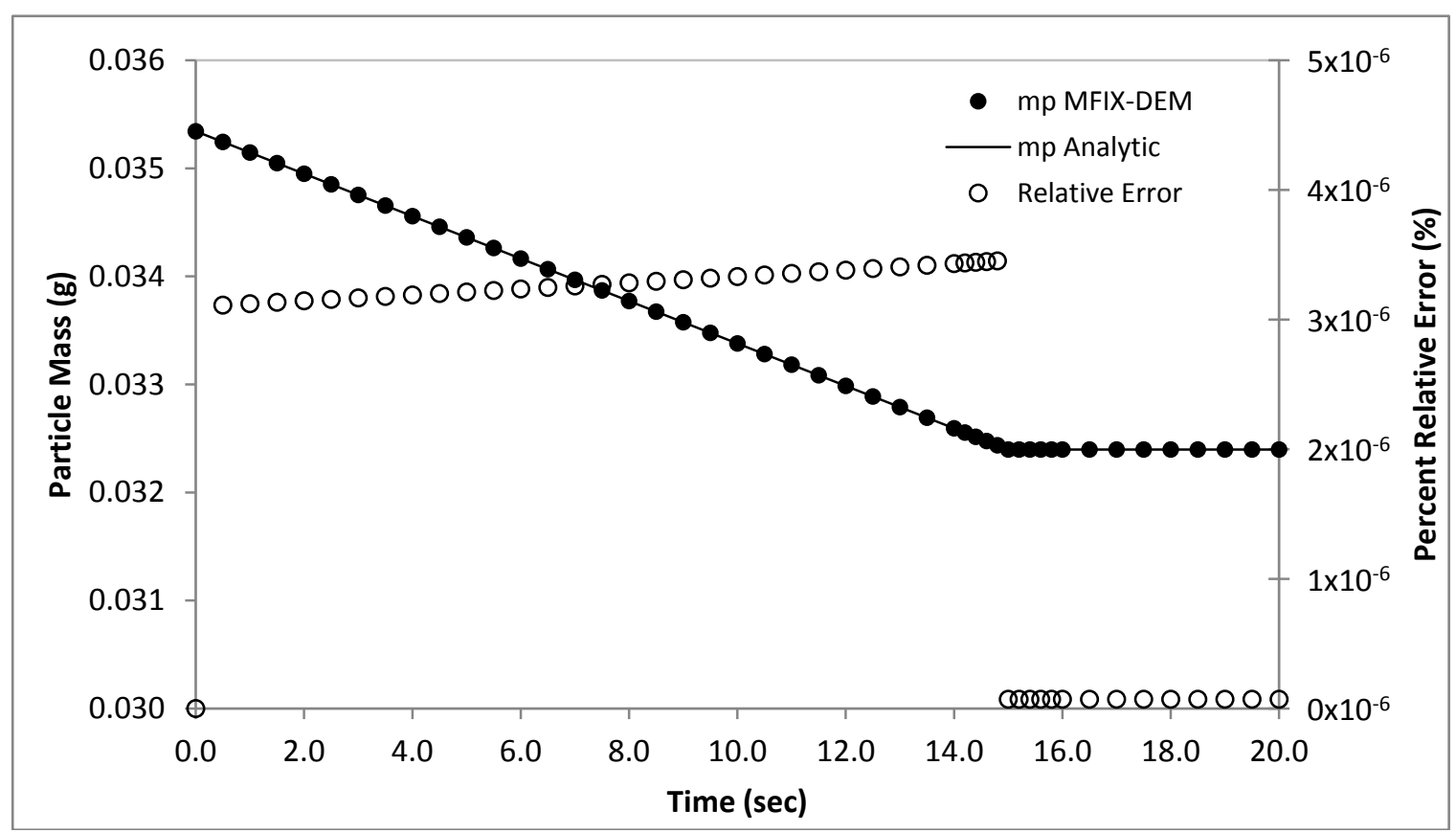

Figure 4-10: MFIX-DEM and analytic solutions to the particle mass equation engaged in reactive chemistry interface. $\mathrm{mp}$ is the mass of the particle. Relative error between the analytic and numeric (forward Euler method) solutions is indicated by open symbols and is associated with the right vertical axis. 


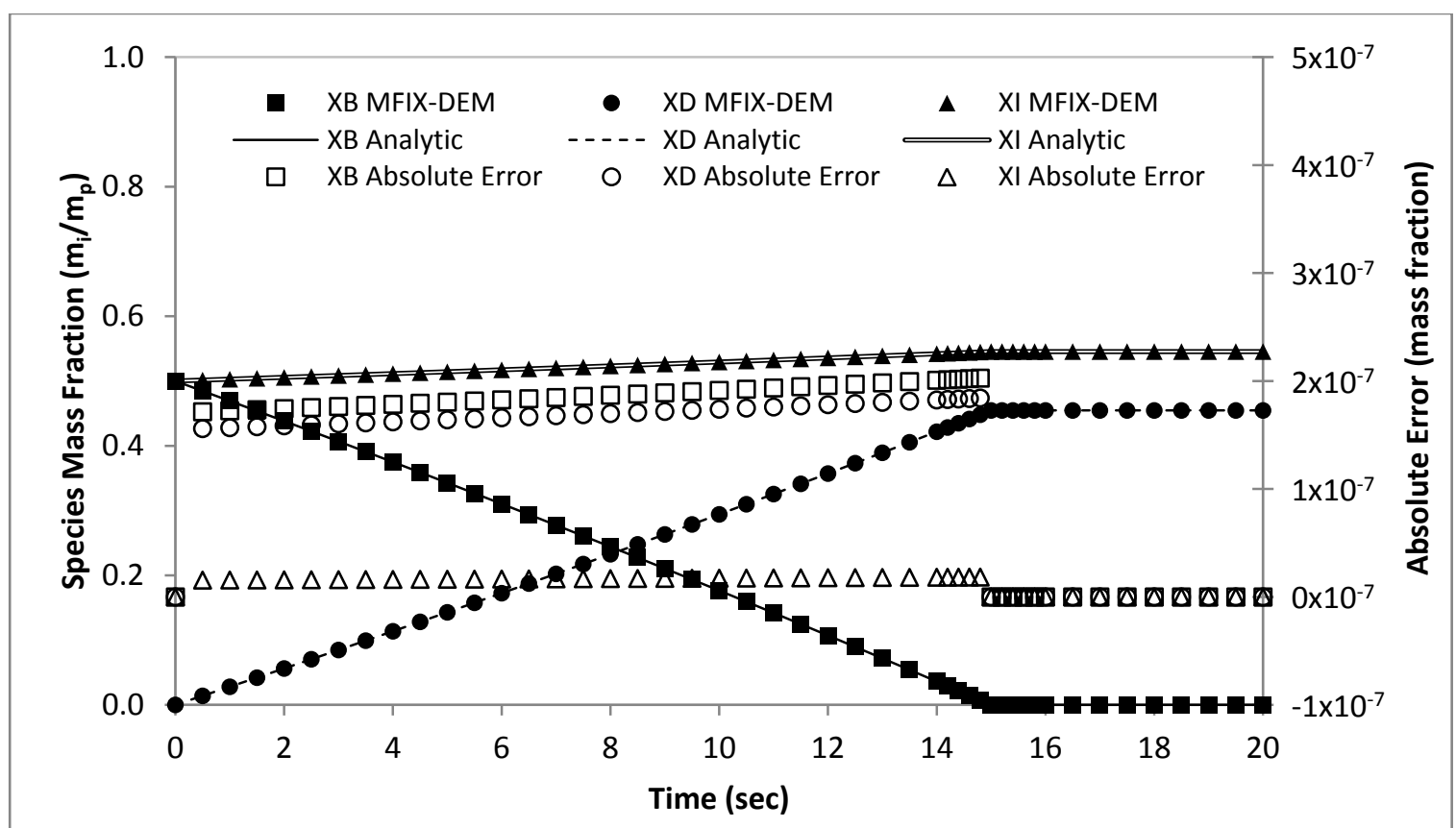

Figure 4-11: MFIX-DEM and analytic solutions to the particle species equations engaged in reactive chemistry interface. XB, XD, and XI are the species mass fractions for species $\mathrm{B}, \mathrm{D}$, and I, respectively. Absolute error between the analytic and numeric (forward Euler method) solutions is indicated by open symbols and is associated with the right vertical axis.

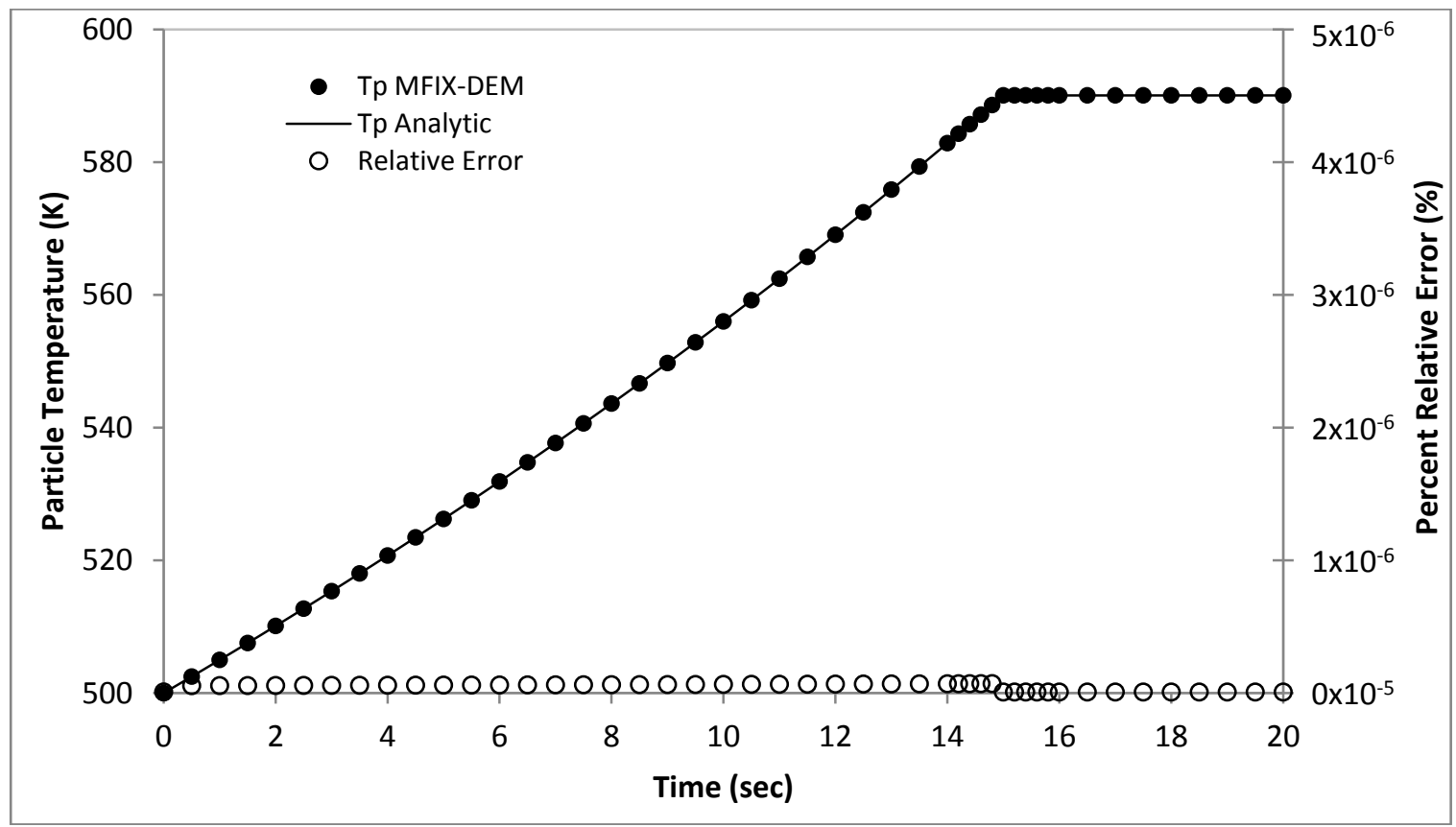

Figure 4-12: MFIX-DEM and analytic solutions to the particle energy equation engaged in reactive chemistry interface. $T p$ is the temperature of the particle. Relative error between the analytic and numeric (forward Euler method) solutions is indicated by open symbols and is associated with the right vertical axis. 
The numeric and analytic solutions for the radius of the unreacted core $(\mathrm{Rc})$ is shown in Figure 4-13. Solid symbols denote the values calculated by MFIX-DEM; continuous line represents the analytic solution; and, open symbols indicate the error between the solutions. Absolute error (see Equation (4-46)) was used in the analysis because the analytic solution of core radius goes to zero. Note the strong agreement between the analytic and numerical solutions up to approximately 15 seconds wherein the absolute error is bounded above by $10^{-6}$. However, the error is only bounded by $\pm 10^{-3}$ from this point forward. Neither forward Euler or Adams-Bashforth methods are able to capture the rapid decay of the unreacted core as the final amount of solids phase reactant (species B) is consumed. The reacting core's radius is not seen as a crucial modeling parameter. Therefore, a bounding of the absolute error from above by $\pm 10^{-3}$ is taken as acceptable for this effort.

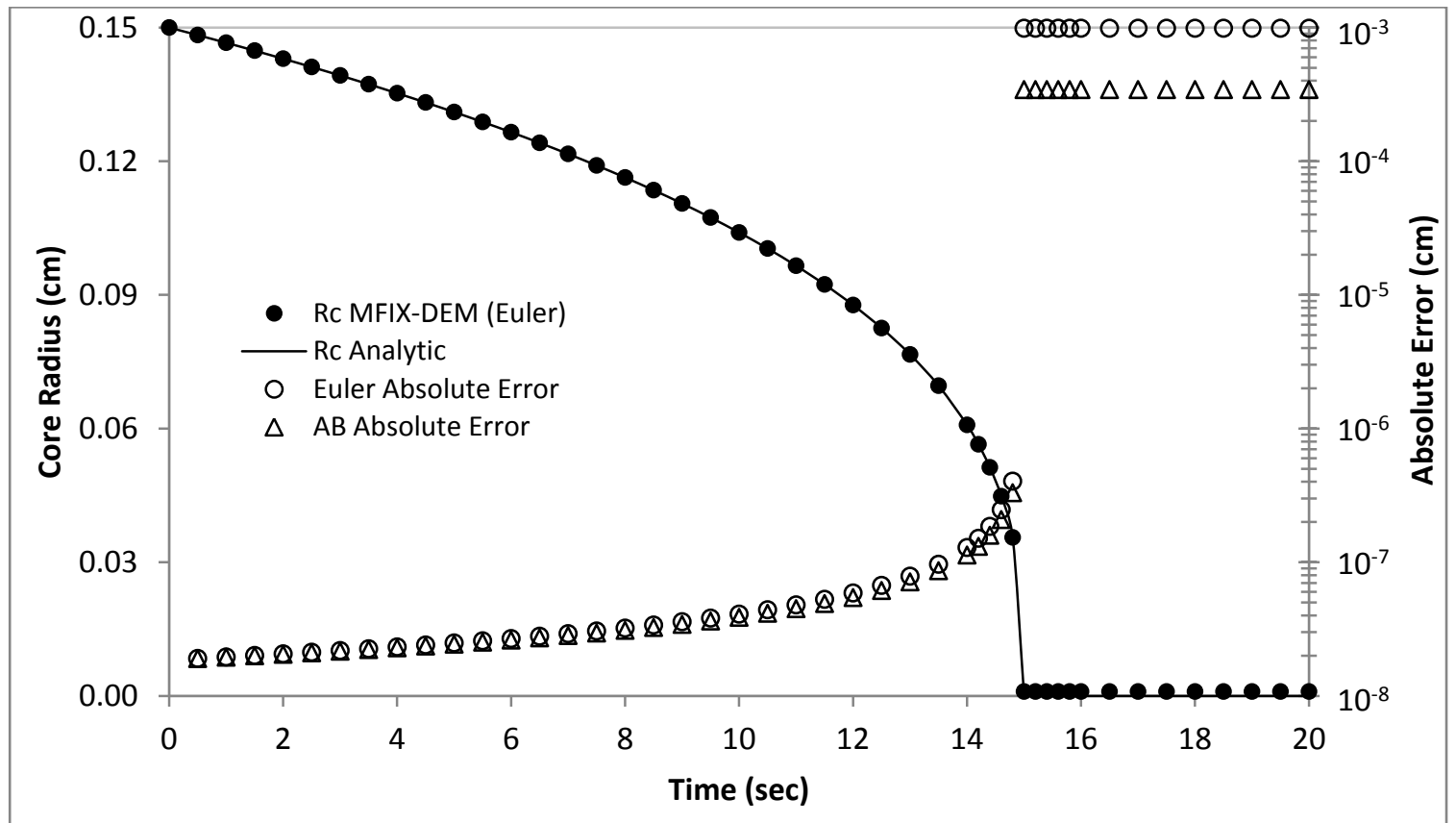

Figure 4-13: MFIX-DEM and analytic solutions to the radius of the shrinking, unreacted core engaged in reactive chemistry interface. $R c$ is the core radius. Absolute error between the analytic and numerical (forward Euler and Adams-Bashforth (AB) methods) solutions are indicated by open symbols and is associated with the right vertical axis. 
This verification shows a strong agreement between the analytic and numerical solutions for the mass, species mass fraction, and particle temperature equations. Similarly, agreement between analytic and numerical solutions for the shrinking, unreacted core is considered acceptable as it is a non-crucial modeling parameter. Therefore, the implementation of the reactive chemistry interface into the MFIX-DEM code is considered successful.

\subsection{Closing Remarks}

In this chapter, specific concepts of reactive chemistry referenced in this work was introduced. The subsequent section reviewed the mathematical models describing the effects of gas-solids reactions on a particle. The shrinking, unreacted core model (see Section 4.2.3) was selected for its relatively accurate physical account of particle-gas reactions and ability to account for particles initially containing inert material. Finally, the implementation of the reactive chemistry interface was verified for a single reacting particle. Strong agreement was observed between the simulation data and the analytic solution. 


\section{Chapter 5: Summary and recommendations}

In this effort, MFIX-DEM, an existing CFD-DEM, is enhanced to include discrete mass inflow-outflow boundary conditions (DMIBC or DMOBC), heat transfer, and an interface for managing user-defined chemical reactions. The following section provides a summary of these efforts. This chapter concludes by identifying several areas in which future work is offered to extend the functionality of the present effort.

\subsection{Summary of results}

\subsubsection{Discrete mass boundary conditions}

In Chapter 2, the algorithmic description of discrete mass inflow and outflow boundary conditions are presented. The inclusion of these functionalities allows models of more dynamic gas-solids systems by assigning user defined boundary conditions to the computational domain. An example wherein solids are fed into the bottom of a packed bed is given. This example illustrates the benefits of using a staging area outside of the computational domain. In doing so, new particles are fed into a system without disturbing the overall behavior of pre-existing particles. A second example is provided which overlaps a DMIBC and gas inflow boundary condition. This demonstrates how additional modeling flexibility is obtained through defining compound boundary conditions.

\subsubsection{Particle heat transfer}

Chapter 3 begins by defining the three modes of heat transfer and the internal energy equation for a single, isothermal particle. An examination of the assumption of isothermal particles is conducted which indicates that treating the particles as isothermal is appropriate. In the subsequent sections, an analysis of contact conduction, particle- 
fluid-particle conduction, particle-gas convection, and particle-environment radiation are presented.

Batchelor and O'Brien's [52] model is selected as the foundation for developing a contact conduction model in this work. The selection is guided by the algebraic simplicity of the model, and wide employment of this model for particle-particle conduction in CFD-DEM $[\mathbf{5 8 , 5 9 , 1 9 , 2 2 , 6 0 , 6 1 ]}$. Their model is extended in this work by defining an effective thermal conductivity between two particles. Additionally, Batchelor and O'Brien's [52] model is generalized by using the geometric configuration of touching particles to obtain the radius of their shared contact area. This results in a contact area representative of the specific collision model employed by the DEM (e.g., Cundall and Strack [44], Hertz [53], etc.), promoting greater modeling consistency throughout the CFD-DEM. The implementation of the particle-particle contact conduction model is verified for a simple two particle test case. Strong agreement is observed between the simulation data and the analytic solution.

Rong and Horio's [16] model is selected as the foundation for the particle-fluidparticle model developed in this work. This model is selected because of its relative simplicity, minimal computational expense, and extendibility to particles of different sizes. By establishing a standard point of reference, this model is additionally extended to particles of different size. The implementation of the particle-fluid-particle conduction model is verified by two simple test cases. In the first test case, two particles are close but not in contact, while in the second test, the particles are touching. Strong agreement is observed between the simulation data and the analytic solution for both test cases. 
Particle-gas convection is assumed to follow Newton's law of cooling. Ranz and Marshall's [69] Nusselt number correlation is chosen to model the convective heat transfer coefficient. Their correlation is selected because of its employment in numerous CFD-DEM models $[\mathbf{2}, \mathbf{1 6}, \mathbf{1 7}, \mathbf{2 1}, \mathbf{7 6}]$. Additionally, a general framework is created to facilitate implementation of additional correlations by users to provide greater customizability of the convective heat transfer model. This is achieved by providing a set location within the code for the user to implement their own correlation. The implementation of the particle-gas convection model is verified for a single fixed particle in a flowing gas. Strong agreement is observed between the simulation data and the analytic solution

A radiation model is developed based on an energy balance between two diffuse, gray surfaces [47]. The complexity of the model is reduced by assuming that neighboring particles form a spherical environment surrounding the particle of interest. An environmental temperature is established by averaging the temperature of the particles contained in a neighborhood surrounding the particle. Two limiting cases for the environment temperature produce definitions found in the literature $[\mathbf{1 6 , 1 8 , 1 9 , 2 0}]$. The implementations of the radiative heat transfer model is verified for a simple two particle test case. Strong agreement is observed between the simulation data and a fourth order Runge-Kutta solution.

\subsubsection{Particle-gas reactive chemistry interface}

In Chapter 4, specific concepts of reactive chemistry referenced in this work are introduced. The shrinking, unreacted core model is selected as the particle reaction model for its relatively accurate physical account of particle-gas reactions and ability to account 
for particles initially containing inert material. The implementation of the reactive chemistry interface is verified for a single reacting particle. Strong agreement is observed between simulation data and the analytic solutions for the particle's mass, species mass fraction, and internal energy equations. Agreement between the simulation data and analytic solution for the shrinking, unreacted core is considered acceptable.

\subsection{Recommendations for future work}

The discrete mass inflow and outflow boundaries provide significant flexibility to MFIX-DEM. Before this effort, a simulation started and ended with the same number of particles. With the inclusion of the discrete mass boundary conditions, the total amount of solids in a simulation is now permitted to fluctuate. However, there is a need for coupling the inflow/outflow boundaries so that as mass exits the system through the outlet, it reenters through the inlet. For example, the solids mass is constant in physical systems that employ a riser/standpipe combination. Solids exiting the riser are collected in the standpipe, then the solids in the standpipe are fed back into the riser. Therefore, the mass within the system is constant. In simulations of such physical systems, typically only the behavior within the riser is of interest. Computational expense is reduced by neglecting to model the standpipe. Constant solids mass within the simulation is enforced by directing out-flowing solids mass into a solids inlet (i.e., total solids flow out of the system equals the total solids flow into the system). Therefore, this author recommends developing an additional discrete mass boundary condition such that out-flowing solids are reintroduced as inflow solids.

System boundaries (walls) are assumed adiabatic (perfectly insulated) in this effort. As such, particle-wall heat transfer does not occur. This limitation is caused by the 
approach employed in MFIX-DEM to detect particle-wall interactions. Presently, in MFIX-DEM, particle-wall interactions are managed within the collision model. By separating the particle-wall contact detection algorithm from the collision model this information is then available to other models. Specifically, the conduction and radiation models developed in this effort could manage particle-wall heat transfer if the interaction information is made available. Therefore, this author suggests algorithmically restructuring where particle-wall detection is calculated in MFIX-DEM so that the information is available to all sub-models.

The computational complexity of CFD-DEM restricts its applicability to small scale systems wherein there are few particles. Larger scale models are achievable by parallelizing CFD-DEM codes so that the computational burden is distributed among multiple processors. Currently, such efforts are undertaken for the hydrodynamic models in MFIX-DEM [103]. To allow for large scale simulations with heat transfer and reactive chemistry, this author recommends including these sub-models in the current parallelization scheme.

Finally, verification tests are conducted in this effort to ensure proper implementation of the selected/modified models. However, verification does not indicate whether the selected models accurately predict the physical behavior they represent. Three-dimensional (3-D) simulations containing a large number of particles are needed to generate data sets comparable with physical experiments. Presently, such simulations are not computationally tractable. However, larger scale simulations may become feasible once the heat transfer models and mathematical interface for particle scale reactions are parallelized. Therefore, this author recommends conducting a model validation study 
once the models developed in this effort are incorporated into the current parallelization scheme. 


\section{Bibliography}

[1] A.H. Pelegrina and G.H. Crapiste, "Modelling the pneumatic drying of food particles," Journal of Food Engineering, vol. 48, no. 4, pp. 301-310, June 2001.

[2] Yasunobu Kaneko, Takeo Shiojima, and Masayuki Horio, "DEM simulation of fluidized beds for gas-phase olefin polymerization," Chemical Engineering Science, vol. 54, pp. 5809-5821, 1999.

[3] D. Geldart, "Types of gas fluidization," Powder Technology, vol. 7, no. 5, pp. 285292, May 1973.

[4] R. Krishna and J. M. van Baten, "Using CFD for scaling up gas-solid bubbling fluidised bed reactors with Geldart A powders," Chemical Engineering Journal, vol. 82, no. 1-3, pp. 247-257, March 2001.

[5] Sreekanth Pannala, Madhava Syamlal, and Thomas J. O'Brien, Computational GasSolid Flows and Reacting Systems: Theory, Methods, and Practice.: IGI Global, 2010.

[6] Madhava Syamlal, C. Guenther, A. Gel, and S. Pannala, "High performance computing: Clean coal gasifier designs using hybrid parallelization," in The 13th International Conference on Fluidization - New Paradigm in Fluidization Engineering, Gyeong-ju, Korea, May 16-21, 2010.

[7] V. Singh, S. Srivastava, R. Chaval, and V. Vitankar, "Simulation of Gas-Solid Flow and Design Modifications of Cement Plant Cyclones," in Fifth International Conference on CFD in the Process Industries CSIRO, Melbourne, Australia, 13-15 December 2006.

[8] B. G.M. van Wachem, J. C. Schouten, C. M. van den Blek, R. Krishna, and J. L. Sinclair, "Comparative analysis of CFD models of dense gas-solid systems," AIChE Journal, vol. 47, no. 5, pp. 1035-1051, May 2001.

[9] Y. Tsuji, T. Tanaka, and T. Ishida, "Lagrangian numerical simulation of plug flow of cohesionless particles in a horizontal pipe," Powder Technology, vol. 71, no. 3, pp. 239-250, September 1992.

[10] Y. Tsuji, T. Kawaguchi, and T. Tanaka, "Discrete particle simulation of twodimensional fluidized bed," Powder Technology, vol. 77, no. 1, pp. 79-87, October 
1993.

[11] B. H. Xu and A. B. Yu, "Numerical simulation of the gas-solid flow in a fluidized bed by combining discrete particle method with computational fluid dynamics," Chemical Engineering Science, vol. 52, no. 16, pp. 2785-2809, August 1997.

[12] R. Garg, C. Narayanan, D. Lakehal, and S. Subramaniam, "Accurate numerical estimation of interphase momentum transfer in Lagrangian-Eulerian simulations of dispersed two-phase flows," International Journal of Multiphase Flow, vol. 33, no. 12, pp. 1337-1364, December 2007.

[13] Takafumi Mikami, Hidehiro Kamiya, and Masayuki Horio, "Numerical simulation of cohesive power behavior in a fluidized bed," Chemical Engineering Science, vol. 53, no. 10, pp. 1927-1940, May 1998.

[14] Michael Webber, "Simulation of Cohesive Particle Flows in Granular and GasSolid Systems," University of Colorado, Boulder, PhD Thesis 2004.

[15] Wenqi Zhong, Yuanquan Xiong, Ahulin Yuan, and Mingyao Zang, "DEM simulation of gas-solid flow behaviors in spout-fluid bed," Chemical Engineering Science, vol. 61, no. 5, pp. 1571-1584, March 2006.

[16] Degang Rong and Masayuki Horio, "DEM simulation of char combustion in a fluidized bed," in Second International Conference on CFD in the Minerals and Process Industries, Melbourne, 1999, pp. 65-70.

[17] J. Li and D.J. Mason, "A computational investigation of transient heat transfer in pneumatic transport of granular particles," Powder Technology, vol. 112, no. 3, pp. 273-282, October 2000.

[18] Hoasheng Zhou, Gilles Flamant, and Daniel Gauthier, "DEM-LES simulation of coal combustion in a bubbling fluidized bed part 2: coal combustion at the particle level," Chemical Engineering Science, vol. 59, no. 20, pp. 4205-4215, October 2004.

[19] Z. Y. Zhou, A. B. Yu, and P. Zulli, "Particle scale study of heat transfer in packed and bubbling fluidized beds," AIChE Journal, vol. 55, no. 4, pp. 868-884, 2009.

[20] Yongming Geng and Defu Che, "An extended DEM-CFD model for char combustion in a bubbling fluidized bed combustor of inert sand," Chemical Engineering Science, vol. 66, no. 2, pp. 207-219, January 2011. 
[21] Daoyin Liu, Xiaoping Chen, Wu Zhou, and Changsui Zhao, "Simulation of char and propane combustion in a fluidized bed by extending DEM-CFD approach," Proceedings of the Combustion Institute, vol. 33, no. 2, pp. 2701-2708, 2011.

[22] E. Simsek, B. Brosch, S. Wirtz, V. Scherer, and F. Krull, "Numerical simulation of grate firing systems using a coupled CFD/discrete element method," Powder Technology, vol. 193, no. 3, pp. 266-273, August 2009.

[23] Michael Oevermann, Stephan Gerber, and Frank Behrendt, "Euler-Lagrange/DEM simulation of wood gasification in a bubbling fluidized bed reactor," Particuology, vol. 7, no. 4, pp. 307-316, August 2009.

[24] T. B. Anderson and Roy Jackson, "Fluid mechanical description of fluidized beds. Equations of motion," Industrial \& Engineering Chemistry Fundamentals, vol. 6, no. 4, pp. 527-539, 1967.

[25] R. Jackson, "Locally averaged equations of motion for a mixture of identical spherical particles and a Newtonian fluid," Chemical Engineering Science, vol. 52, no. 15, pp. 2457-2469, August 1997.

[26] Janine Galvin, "On the Hydrodynamic Description of Binary Mixtures of Rapid Granular Flows and Gas-Fluidized Beds," Department of Chemical and Biological Engineering, University of Colorado, Boulder, PhD Dissertation 2007.

[27] Madhava Syamlal, William Rogers, and Thomas J. O'Brien, "MFIX Documentation Theory Guide," U.S. Department of Energy, Morgantown, Software Documentation December 1993. [Online]. https://www.mfix.org/documentation/Theory.pdf

[28] P. W. Atkins, Physical Chemistry, 2nd ed. Oxford, Great Britain: Oxford University Press, 1982.

[29] Liang-Shih Fan and Chao Zhu, Principles of Gas-Solid Flows. Cambridge, United Kingdom: Cambridge University Press, 1998.

[30] Clayton Crowe, Martin Sommerfeld, and Yutaka Tsuji, Multiphase Flows with Droplets and Particles. Boca Raton, USA: CRC Press, 1998.

[31] G. K. Batchelor, An Introduction to Fluid Dynamics, Reprint edition, Ed. Cambridge, Great Britain: Cambridge Univ Pr, 1973.

[32] H. K. Versteeg and W. Malalasekera, An introduction to computational fluid 
dynamics: The finite volume method. Harlow, England: Pearson Education Limited, 1996.

[33] Dimitri Gidaspow, Multiphase Flow and Fluidization: Continuum and Kinetic Theory Descriptions, 1st ed. Boston, USA: Academic Press, 1994.

[34] M. P. Allen and D. J. Tildesley, Computer Simulation of Liquids. Oxford, United Kingdom: Oxford University Press, 1989.

[35] R. Garg, J. Galvin, T. Li, and S. Pannala. (2010, February) Documentation of opensource MFIX-DEM software for gas-solid flows. From URL https://mfix.netl.doe.gov/documentation/dem_doc_2010.pdf. [Online]. https://mfix.netl.doe.gov/documentation/dem_doc_2010.pdf

[36] Stefan Luding, "Molecular dynamics simulations of granular materials," in The Physics of Granular Media. Weinheim, FRG: Wiley-VCH Verlag GmbH \& Co. KGaA, 2005, ch. 13, pp. 299-322.

[37] N. G. Deen, M. Van Sint Annaland, M. A. Van der Hoef, and J. A. M. Kuipers, "Review of discrete particle modeling of fluidized beds," Chemical Engineering Science, vol. 62, no. 1-2, pp. 28-44, January 2007.

[38] Pawel Kosinski and Alex C. Hoffmann, "An extension of the hard-sphere particleparticle collision model to study agglomeration," Chemical Engineering Science, vol. 65, no. 10, pp. 3231-3239, May 2010.

[39] B. P. B. Hommans, J. A. M. Kuipers, W. J. Briels, and W. P. M. van Swaaij, "Discrete particle simulation of bubble and slug formation in a two-dimensional gas-fluidised bed: A hard-sphere approach," Chemical Engineering Science, vol. 51, no. 1, pp. 99-118, January 1996.

[40] Jie Li and J. A. M. Kuipers, "Effect of pressure on gas-solid flow behavior in dense gas-fluidized beds: a discrete particle simulation study," Powder Technology, vol. 127, no. 2, pp. 173-184, October 2002.

[41] Dhanujay Boyalakuntla, "Simulation of Granular and Gas-Solid Flows using Discrete Element Method," Carnegie Mellon University, Pittsburgh, PhD Thesis 2003.

[42] H.P. Zhu, Z.Y. Zhou, R.Y. Yang, and A.B. Yu, "Discrete particle simulation of particulate systems: A review of major applications and findings," Chemical 
Engineering Science, vol. 63, no. 23, pp. 5728-5770, December 2008.

[43] Aytekin Gel, Madhava Syamlal, Sofiane Benyahia, Sreekanth Pannala, and Thomas J. O'Brien, "Experiences with the open source model for disseminating information in computational gas-solids flow1-22," in The Fifth World Congress on Particle Technology, Orlando, Flordia, 2006, pp. 1-22.

[44] P. A. Cundall and O. D. L. Strack, "A discrete numerical model for granular assemblies," Geotechnique, vol. 29, no. 1, pp. 47-65, March 1979.

[45] Fei Wang, Yu Zhao, Qussai Marashdeh, and Liang-Shih Fan, "Horizontal gas and gas/solid jet penetration in a gas-solid fluidized bed," Chemical Engineering Science, vol. 65, no. 11, pp. 3394-3408, June 2010.

[46] Kai Zhang, Jiyu Zhang, and Bijiang Zhang, "Experimental and numerical study of fluid dynamic parameters in a jetting fluidized bed of a binary mixture," Powder Technology, vol. 132, no. 1, pp. 30-38, May 2003.

[47] A.F. Mills, Basic Heat \& Mass Transfer, 2nd ed.: Prentice Hall, 1998.

[48] M. M. Yovanovich, "Simple explicit expressions for calculation of the HeislerGrober charts," in ASME National Heat Transfer Conference, Houston, 1996.

[49] J. Sun and M. M. Chen, "A theoretical analysis of heat transfer due to particle impact," International Journal of Heat and Mass Transfer, vol. 31, no. 5, pp. 969975, May 1988.

[50] Michel Louge, Jamaludin Mohd Yusof, and James T. Jenkins, "Heat transfer in the pneumatic transport of massive particles," International Journal of Heat and Mass Transfer, vol. 36, no. 2, pp. 265-275, 1993.

[51] Yoshiyuki Shimizu, "Three-dimensional simulation using fixed coarse-grid thermal-fluid scheme and conduction heat transfer scheme in distinct element method," Powder Technology, vol. 165, no. 3, pp. 140-152, July 2006.

[52] G. K. Batchelor and R. W. O'Brien, "Thermal or Electrical Conduction Through a Granular Material," Proceedings of the Royal Society of London. Series A, Mathematical and Physical Sciences, vol. 355, no. 1682, pp. 313-333, July 1977.

[53] K. L. Johnson, Contact Mechanics. New York: Cambridge University Press, 1985.

[54] J. H. Zhou, A. B. Yu, and M. Horio, "Finite element modeling of the transient heat 
conduction between colliding particles," Chemical Engineering Journal, vol. 139, pp. 510-516, 2008.

[55] H. Zhou, G. Flamant, D. Gauthier, and Y. Flitris, "Simulation of Coal Combustion in a Bubbling Fluidized Bed by Distinct Element Method," Chemical Engineering Research and Design, vol. 81, no. 9, pp. 1144-1149, 2003 October, 9th Congress of the French Society of Chemical Engineering, October 2003.

[56] Itasca Consulting Group, Inc., "PFC2D Version 3.1," Itasca, Minneapolis, Manual 2004.

[57] Itasca Consulting Group, Inc., "PFC3D Version 3.1," Itasca, Minneapolis, Manual 2004.

[58] Watson L. Vargas and J. J. McCarthy, "Heat conduction in granular materials," AIChE Journal, vol. 47, no. 5, pp. 1052-1059, May 2001.

[59] F. P. Di Maio, A. Di Renzo, and D. Trevisan, "Comparison of heat transfer models in DEM-CFD simulations of fluidized beds with an immersed probe," Powder Technology, vol. 193, no. 3, pp. 257-265, August 20092009.

[60] P. A. Moysey and M. R. Thompson, "Modelling the solids inflow and solids conveying of single-screw extruders using the discrete element method," Powder Technology, vol. 153, no. 2, pp. 95-107, May 2005.

[61] K. F. Malone and B. H. Xu, "Particle-scale simulation of heat transfer in liquidfluidised beds," Powder Technology, vol. 184, no. 2, pp. 189-204, May 2008.

[62] Watson L. Vargas-Escobar, "Discrete Modeling of Heat Conduction in Granular Media," University of Pittsburg, Pittsburg, PhD Thesis 2002.

[63] C. Y. Wen and T. M. Chang, "Particle-to-particle heat transfer in air-fluidized beds," in Proceedings of the International symposium on fluidization, Eindhoven, June 6-9, 1967, pp. 491-505.

[64] C. Delvosalle and J. Vanderschuren, "Gas-to-particle and particle-to-particle heat transfer in fluidized beds of large particles," Chemical Engineering Science, vol. 40, no. 5, pp. 769-779, 1985.

[65] G. J. Cheng, A. B. Yu, and P. Zulli, "Evaluation of effective thermal conductivity from the structures of a packed bed," Chemical Engineering Science, vol. 54, pp. 4199-4209, 1999. 
[66] Jia Wei Chew, Jeffrey R. Wolz, and Christine M. Hrenya, "Axial segregation in bubbling gas-fluidized beds with Gaussian and lognormal distributions of Geldart Group B particles," AIChE Journal, vol. 56, no. 12, pp. 3049-3061, December 2010 .

[67] A. Mansoori, M. Saffar-Avval, H. Basirat Tabrizi, B. Dabir, and G. Ahmadi, "Interparticle heat transfer in a riser of gas-solid turbulent flows," Powder Technology, vol. 159, no. 1, pp. 35-45, November 2005.

[68] A. M. Xavier and J. F. Davidson, "Heat transfer to surfaces immersed in fluidised beds, particularly tube arrays," in Fluidization: Proceedings of the Second Engineering Foundation Conference, Cambridge, England, 2-6 April, 1978, pp. 333-338.

[69] W. E. Ranz and W. R. Marshall, "Evaporation from drops, Part 1 \& 2," Chemical Engineering Progress, vol. 48, pp. 141-146; 173-180, 1952.

[70] P. N. Dwivedi and S. N. Upadhyay, "Particle-fluid mass transfer in fixed and fluidized beds," Industrial \& Engineering Chemistry Process Design and Development, vol. 16, no. 2, pp. 157-165, April 1977.

[71] D. J. Gunn, "Transfer of heat or mass to particles in fixed and fluidised beds," International Journal of Heat and Mass Transfer, vol. 21, no. 4, pp. 467-476, April 1978.

[72] N. Wakao, S. Kaguei, and T. Funazkri, "Effect of fluid dispersion coefficients on particle-to-fluid heat transfer coefficients in packed beds : Correlation of Nusselt numbers," Chemical Engineering Science, vol. 34, no. 3, pp. 325-336, 1979.

[73] P. K. Agarwal, "Transport phenomena in multi-particle systems--II. Particle-fluid heat and mass transfer," Chemical Engineering Science, vol. 43, no. 9, pp. 2501$2510,1988$.

[74] D. Kunii and O. Levenspiel, Fluidization engineering, 2nd ed. Boston: Butterworth-Heinemann, 1991.

[75] S. Mankad, K. M. Nixon, and P. J. Fryer, "Measurements of particle-liquid heat transfer in systems of varied solids fraction," Journal of Food Engineering, vol. 31, no. 1, pp. 9-33, January 1997.

[76] T. Swasdisevi et al., "Prediction of gas-particle dynamics and heat transfer in a two-dimensional spouted bed," Advanced Powder Technology, vol. 16, no. 3, pp. 
275-293, 2005.

[77] Sakae Yagi and Daizo Kunii, "Studies on effective thermal conductivities in packed beds," AIChE Journal, vol. 3, no. 3, pp. 373-385, September 1957.

[78] John C. Chen, "Heat Transfer," in Handbook of Fluidization and Fluid-Particle Systems, Wen-Ching Yang, Ed.: CRC Press, 2003.

[79] J. Szekely and Robert J. Fisher, "Bed to wall radiation heat transfer in a gas-solid fluidized bed," Chemical Engineering Science, vol. 24, no. 5, pp. 833-849, May 1969.

[80] Charles Nelson Zeeb, "Two-Dimensional Radiative Heat Transdfer in Combustion Gases Via Monte Carlo," Colorado State University, Fort Collins, Thesis 1996.

[81] Z. Mansoori, M. Saffar-Avval, H. Basirat Tabrizi, and G. Ahmad, "Modeling of heat transfer in turbulent gas-solid flow," International Journal of Heat and Mass Transfer, vol. 45, no. 6, pp. 1173-1184, March 2002.

[82] Greg F. Naterer, Heat Transfer in Single and Multiphase Systems. Boca Raton: CRC Press, 2002.

[83] John R. Howell. (2008) A catalog of radiation heat transfer configuration factors. Document.

[84] MFIX Development Team. (2010, February) Multiphase Flow with Interphase eXchanges Version MFIX-2010-1 Readme. PDF Document.

[85] Madhava Syamlal, "MFIX Documentation Numerical Technique," U.S. Department of Energy, Morgantown, Software Documentation 1998.

[86] Sukas V. Patankar, Numerical Heat Transfer and Fluid Flow. USA: McGraw-Hill Inc., 1980.

[87] Arieh Iserles, A First Course in the Numerical Analysis of Differential Equations. Cambridge: Cambridge University Press , 1996.

[88] W. H. Press, B. P. Flannery, S. A. Teukoloky, and W. T. Vetterling, Numerical Recipes in Fortran: The Art of Scientific Computing, 2nd ed.: Cambridge Univeristy Press, 1992.

[89] David R. Kincaid and E Ward Cheney, Numerical Analysis: Mathematics of 
Scientific Computing, 3rd ed.: Brooks Cole, 2001.

[90] Octave Levenspiel, Chemical Reaction Engineering, Second Edition ed. New York, USA: John Wiley \& Sons, 1972.

[91] Madhava Syamlal and Thomas J. O’Brien, "Fluid dynamic Simulation of $\mathrm{O}_{2}$ decomposition in a bubbling fluidized bed," AIChE Journal, vol. 49, no. 11, pp. 2793-2801, November 2003.

[92] Thomas J. B'Brien, Madhava Syamlal, and Chris Guenther, "Computational fluid dynamic simulations of chemically reactive fluidized bed processes," in Third International Conference on CFD in the Mineral and Process Industries, Melbourne, Australia, 2003, pp. 496-474.

[93] H. Scott Fogler, Elements of Chemical Reaction Engineering, 2nd ed. Upper Saddle River, New Jersey: PRENTICE hALL, 1992.

[94] Julian Szekely, James W. Evans, and Hong Young Sohn, Gas-Solid Reactions. New York: Academic Press, 1976.

[95] D. C. Dixon, "The definition of reaction rate," Chemical Engineering Science, vol. 25, no. 2, pp. 337-338, February 1970.

[96] Harold C. Weber and Herman P. Meissner, Thermodynamics for Chemical Engineers, 2nd ed. New York, USA: John Wiley and Sons, 1957.

[97] Richard M. Felder and Ronald W. Rousseau, Elementary Principles of Chemical Processes, 2nd Edition, Ed. New York: John Wiley and Sons, 1986.

[98] M. Syamlal, "Derivation of Energy Equations in MFIX," U.S. Department of Energy, Morantown, MFIX Documentation 2007.

[99] Yunus A. Çengel and Michael A. Boles, Thermodynamics: An Engineering Approach, 4th ed. New York: McGraw-Hill, 2002.

[100] Shunji Homma, Shinji Ogata, Jiro Koga, and Shiro Matsumoto, "Gas-solid reaction model for a shrinking spherical particle with unreacted shrinking core," Chemical Engineering Science, vol. 60, no. 18, pp. 4971-4980, September 2005.

[101] T. Bluhm-Drenhaus, E. Simsek, S. Wirtz, and V. Scherer, "A coupled fluid dynamic-discrete element simulation of heat and mass transfer in a lime shaft kiln," Chemical Engineering Science, vol. 9, no. 1, pp. 2821-2834, May 2010. 
[102] Alexander Burcat and Branko Ruscic. (2005, July) Information Bridge: DOE Scientific and Technical Information. [Online]. http://www.osti.gov/bridge

[103] Pradeep Gopalakrishnan, "MFIX-DEM Parallelization," in NETL 2011 Workshop on Multiphase Flow Science, August 16-18, 2011 , Pittsburgh, 2011.

[104] J. Vanderschuren and C. Delvosalle, "Particle-to-particle heat transfer in fluidized bed drying," Chemical Engineering Science, vol. 35, pp. 1741-1748, 1980.

[105] Kenya Kuwagi, Muhammad Arif Bin Mokhtar, Toshihiro Takami, and Masayuki Horio, "Analysis of heat transfer between two particles for DEM simulations," in 2007 ECI Conference on the 12th International Conference on Fluidization - New Horizons in Fluidization Engineering, vol. 4, Vancouver, pp. 241-248.

[106] Y. T. Feng, K. Han, C. F. Li, and D. R. J. Owen, "Discrete thermal element modelling of heat conduction in particle systems: Basic formulations," Journal of Computational Physics, vol. 227, pp. 5072-5089, 2008.

[107] J. S. M. Botterill, Fluid-Bed Heat Transfer. New York, New York, United States of America: Academic Press, 1975.

[108] J. Baeyens, D. van Gauwbergen, and I. Vinckier, "Pneumatic drying: the use of large-scale experimental data in a design process," Powder Technology, vol. 83, no. 3, pp. 139-148, 1995.

[109] Esmail R. Monazam, Lawrence J. Shadle, Joseph S. Mei, and James Spenik, "Identification and characteristics of different flow regimes in a circulating fluidized bed," Powder Technology, vol. 155, no. 1, pp. 17-25, July 2005.

[110] Haosheng Zhou, Gilles Flamant, and Damiel Gauthier, "DEM-LES of coal combustion in a bubbling fluidized bed. Part I: gas-particle turbulent flow structure," Chemical Engineering Science, vol. 59, no. 20, pp. 4196-4203, October 2004.

[111] S. Benyahia, M. Syamlal, and T. J. O'Brien. (2008, August) www.mfix.org. [Online]. https://mfix.netl.doe.gov/documentation/MFIXEquations2005-4-4.pdf

[112] H. Kruggel-Emden, E. Simsek, S. Rickelt, S. Wirtz, and V. Scherer, "Review and extension of normal force models for the Discrete Element Method," Powder Technology, vol. 171, no. 3, pp. 157-173, February 2007.

[113] O. Molerus, "Heat transfer in moving beds with a stagnant interstitial gas," 
International Journal of Heat and Mass Transfer, vol. 40, no. 17, pp. 4151-4159, October 1997.

[114] D. Lathouwers and J. Bellan, "Modeling of dense gas-solid reactive mixtures applied to biomass pyrolysis in a fluidized bed," International Journal of Multiphase Flow, vol. 27, no. 12, pp. 2155-2187, December 2001.

[115] A. Ingram et al., "Multiple particle tracking in a fluidized bed," in 2007 ECI Conference on the 12th International Conference on Fluidization - New Horizons in Fluidization Engineering, Vancouver, 2007, pp. 449-456.

[116] Y. T. Feng, K. Han, and D. R. J. Owen, "Discrete thermal element modelling of heat conduction in particle systems: Pipe-network model and transient analysis," Powder Technology, vol. 193, pp. 248-256, 2009.

[117] Gregory Nellis and Sanford Klein, Heat Transfer. New York, United States of America: Cambridge niversity Press, 2009.

[118] V. D. Nguyen, C. Cogne, M. Guessasma, E. Bellenger, and J. Fortin, "Discrete modeling of granular flow with thermal transfer: Application to the discharge of silos," Applied Thermal Engineering, vol. 29, no. 8-9, pp. 1846-1853, Jume 2009.

[119] M. M. Avedesian and J. F. Davidson, "Combustion of carbon particles in a fluidised bed," Chemical Engineering Research and Design, vol. 51, pp. 121 - 131, 1973.

[120] M. M. Baum and P. J. Street, "Predicting the combustion behaviour of coal particles," Combustion Science and Technology, vol. 3, pp. 231-243, 1971.

[121] I. B. Ross and J. F. Davidson, "The combustion of carbon particles in a fluidised bed," Chemical Engineering Research and Design, vol. 60, pp. 108-114, 1982.

[122] United States Department of Energy, Multiphase Flow with Interphase eXchange, (Version mfix_05172011_MPPIC_MPI_CutCell) [Open source computer program]. Available at http://www.mfix.org (Accessed July 11, 2011). 


\section{Appendix A: Geometric definitions for collision calculations}

This section provides the geometric definitions for various terms needed for computing a two particle collision. A schematic of a collision is given in Figure A-1to provide context for the various terms.

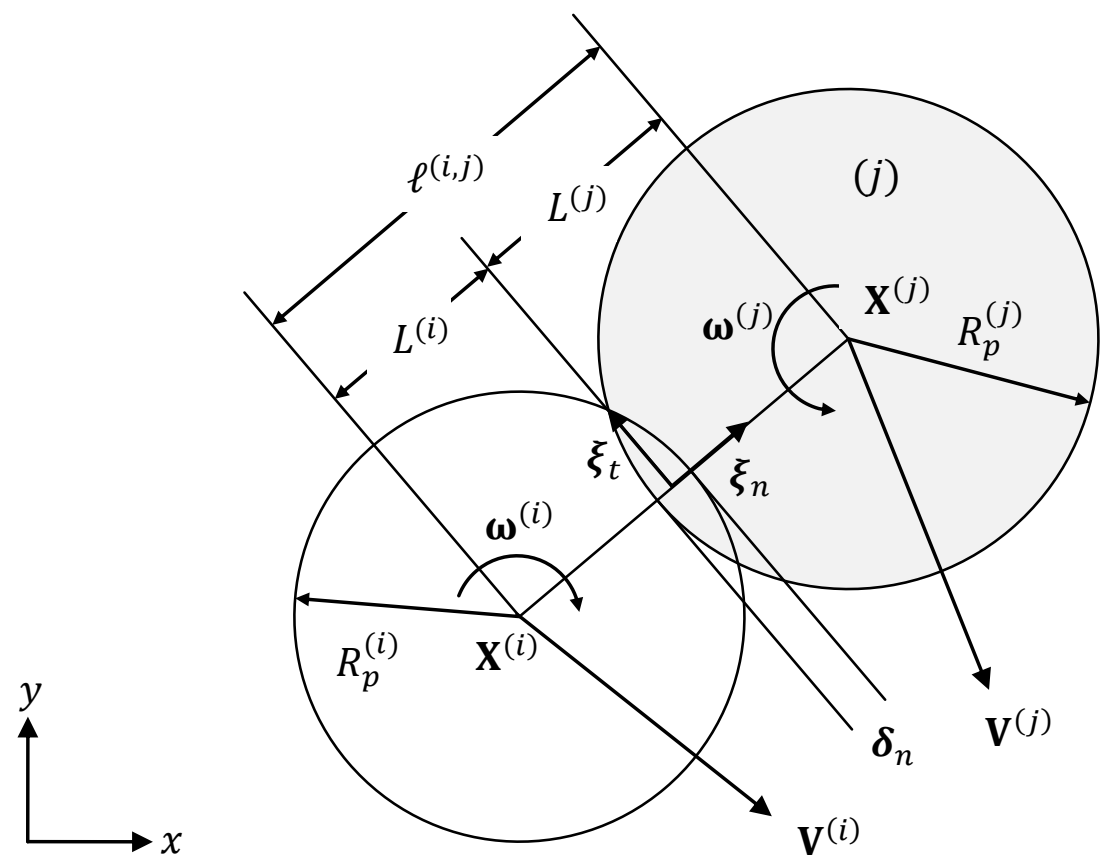

Figure A-1: Schematic demonstrating various terms needed in the calculation of the particle-particle collision force.

Consider the collision of two particles indexed by $(i)$ and $(j)$ were the particles' radii, positions, and linear and angular velocities are $R_{p}, \mathbf{X}, \mathbf{V}$, and $\boldsymbol{\omega}$, respectively. Then the follow defines:

the normal overlap

$$
\delta_{n}^{(i, j)}=R_{p}^{(i)}+R_{p}^{(j)}-\left\|\mathbf{X}^{(i)}-\mathbf{X}^{(j)}\right\|_{2}
$$


unit normal from $(i)$ to $(j)$

$$
\xi_{n}^{(i, j)}=\frac{\mathbf{X}^{(j)}-\mathbf{X}^{(i)}}{\left\|\mathbf{X}^{(j)}-\mathbf{X}^{(i)}\right\|_{2}}
$$

distance from center of particle ( $i$ ) to the contact point

$$
L^{(i)}=\frac{R_{p}^{(i)}-R_{p}^{(j)}+\left\|\mathbf{X}^{(j)}-\mathbf{X}^{(i)}\right\|_{2}}{2\left\|\mathbf{X}^{(j)}-\mathbf{X}^{(i)}\right\|_{2}}
$$

distance from center of particle $(j)$ to the contact point

$$
L^{(j)}=\left\|\mathbf{X}^{(j)}-\mathbf{X}^{(i)}\right\|_{2}-L^{(i)}
$$

relative velocity of the point of contact

$$
\mathbf{V}^{(i, j)}=\mathbf{V}^{(i)}-\mathbf{V}^{(j)}+\frac{1}{2}\left(L^{(i)} \boldsymbol{\omega}^{(i)}+L^{(j)} \boldsymbol{\omega}^{(j)}\right) \times \xi_{n}^{(i, j)}
$$

normal relative velocity of the point of contact

$$
\mathbf{V}_{n}^{(i, j)}=\left(\mathbf{V}^{(i, j)} \cdot \xi_{n}^{(i, j)}\right) \xi_{n}^{(i, j)}
$$

tangential relative velocity of the point of contact

$$
\mathbf{V}_{t}^{(i, j)}=\mathbf{V}^{(i, j)}-\mathbf{V}_{n}^{(i, j)}
$$

unit tangential direction

$$
\xi_{t}^{(i, j)}=\frac{\mathbf{v}_{t}^{(i, j)}}{\left\|\mathbf{v}_{t}^{(i, j)}\right\|_{2}}
$$


tangential displacement at initial contact, $t$

$$
\boldsymbol{\delta}_{t}^{(i, j)}=\mathbf{V}_{t}^{(i, j)} \min \left\{\frac{\left|\delta_{n}\right|}{\mathbf{V}^{(i, j)} \xi_{n}^{(i, j)}}, \Delta t_{s}\right\}
$$

tangential displacement at time $t+\Delta t$

$$
\boldsymbol{\delta}_{t}^{(i, j)}(t+\Delta t)=\boldsymbol{\delta}_{t}(t)+\mathbf{V}_{t}^{(i, j)} \Delta t
$$

corrected tangential displacement at time $t+\Delta t$

$$
\boldsymbol{\delta}_{t}^{(i, j)}(t+\Delta t)=\boldsymbol{\delta}_{t}^{(i, j)}(t+\Delta t)-\left(\boldsymbol{\delta}_{t}^{(i, j)}(t+\Delta t) \cdot \boldsymbol{\xi}_{n}^{(i, j)}\right) \boldsymbol{\xi}_{n}^{(i, j)}
$$

corrected unit vector in the tangential direction

$$
\xi_{t}^{\prime}=\left\{\begin{array}{cl}
\xi_{t} & \text { for } \xi_{t} \neq \mathbf{0} \\
\frac{\boldsymbol{\delta}_{t}}{\left\|\boldsymbol{\delta}_{t}\right\|_{2}} & \text { for } \quad \xi_{t}=\mathbf{0}, \quad \boldsymbol{\delta}_{t} \neq \mathbf{0}
\end{array}\right.
$$

Additional details concerning these definitions are found in references $[35,9]$. 


\section{Appendix B: Geometric definitions for conduction models}

This section provides the geometric definitions for the contact radius and bounds of integration required by contact conduction and particle-fluid-particle conduction models. A schematic of the particles' geometric configuration is given in Figure A-1 to provide context for various terms.

\section{B.1 Contact conduction definitions}

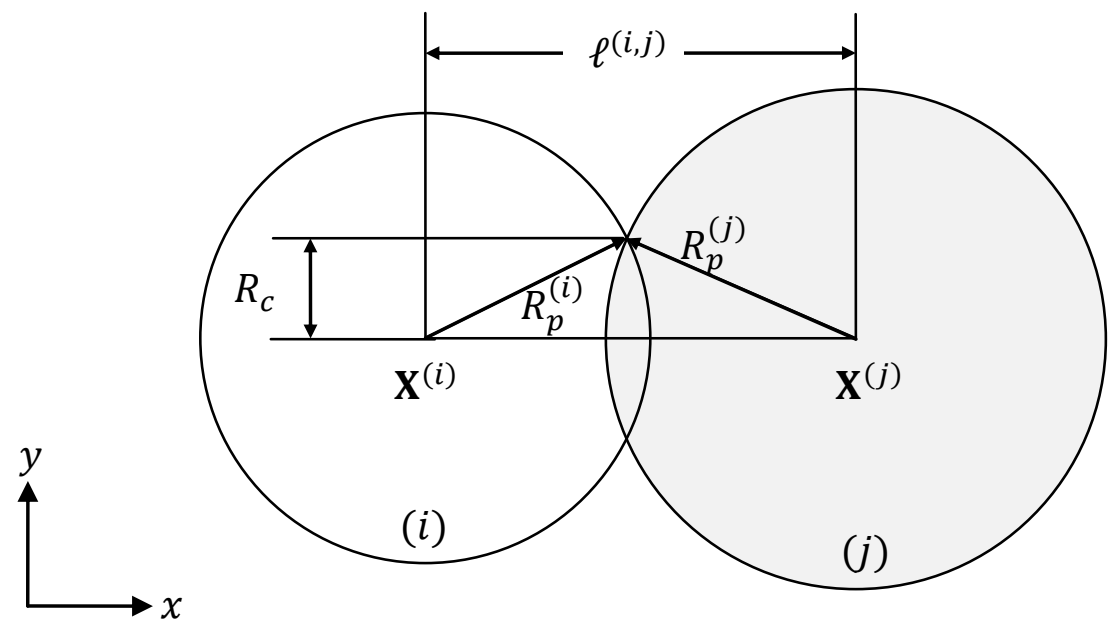

Figure B-1: Schematic demonstrating various terms needed in the calculation of the contact radius needed for computing contact conduction. In this illustration particle $i$ is smaller than particle $j$.

distance between particle centers

$$
\ell^{(i, j)}=\left\|\mathbf{X}^{(i)}-\mathbf{X}^{(j)}\right\|_{2}
$$

address the particle radii by the larger and smaller radii:

$$
\begin{aligned}
& R^{(l)}=\min \left\{R^{(i)}, R^{(j)}\right\} \\
& R^{(k)}=\max \left\{R^{(i)}, R^{(j)}\right\}
\end{aligned}
$$




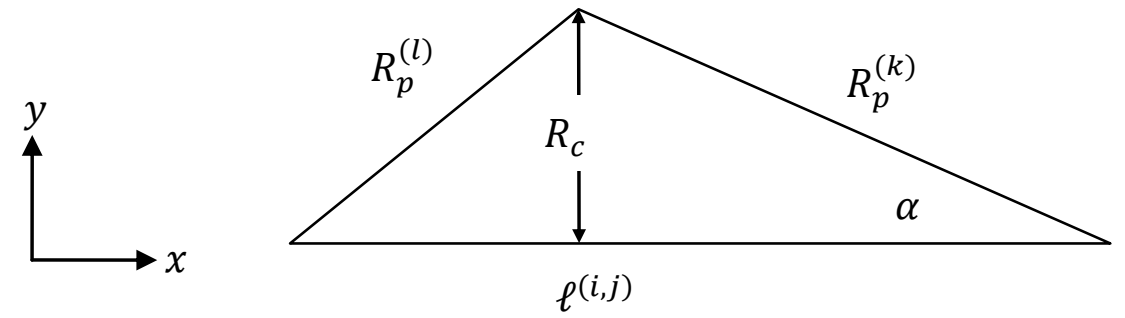

Figure B-2: Schematic demonstrating various terms needed in the calculation of the contact radius needed for computing contact conduction. In this illustration particle $i$ is smaller than particle $j$.

law of Cosines:

$$
\begin{gathered}
R^{(l)^{2}}=R^{(k)^{2}}+\ell^{(k, l)^{2}}-2 \ell^{(k, l)} R^{(k)} \cos \alpha \\
\cos \alpha=\frac{R^{(k)^{2}}-R^{(l)^{2}}+\ell^{(k, l)^{2}}}{2 \ell^{(k, l)} R^{(k)}}
\end{gathered}
$$

Pythagorean theorem:

$$
R_{c}^{2}+\left(R_{p}^{(k)} \cos \alpha\right)^{2}=R_{p}^{(k)^{2}}
$$

combining Equations (B-5) and (B-6) yields the contact radius:

$$
\begin{gathered}
R_{c}^{2}+\left(R_{p}^{(k)} \frac{R^{(k)^{2}}-R^{(l)^{2}}+\left(\ell^{(k, l)}\right)^{2}}{2 \ell^{(k, l)} R^{(k)}}\right)^{2}=\left(R_{p}^{(k)}\right)^{2} \\
R_{c}^{(k, l)}=\sqrt{R^{(k)^{2}}-\left(\frac{\left.R^{(k)^{2}}-R^{(l)^{2}}+\ell^{(k, l)}\right)^{2}}{2 \ell^{(k, l)}}\right)^{2}}
\end{gathered}
$$




\section{B.2 Particle-fluid-particle conduction definitions}

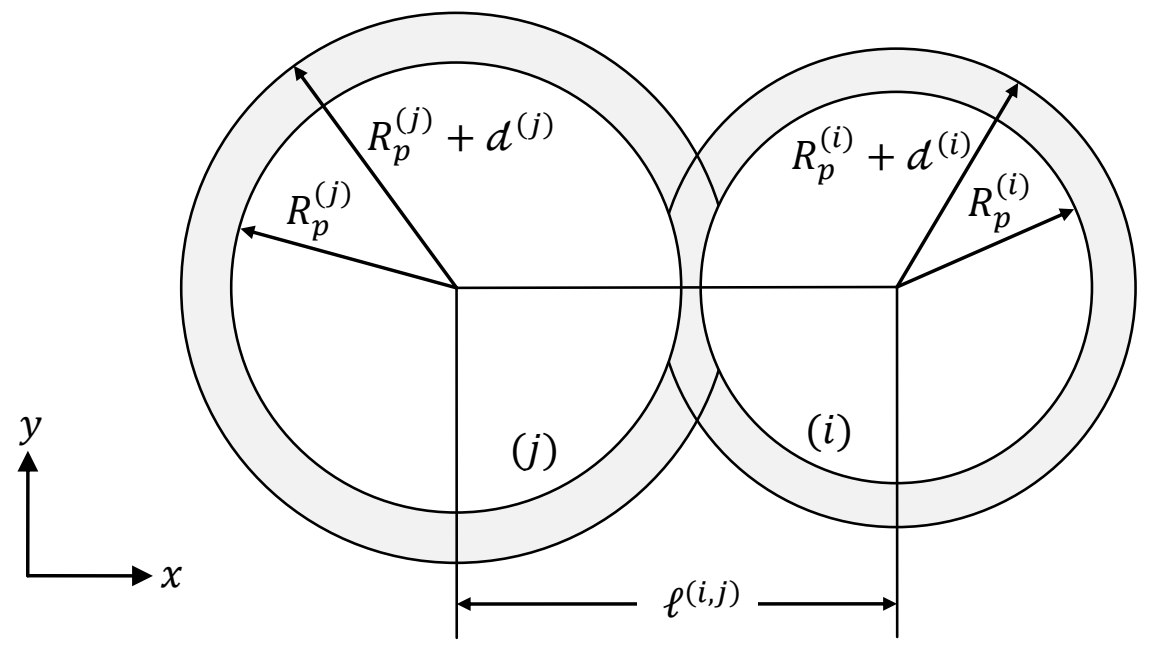

Figure B-3: Schematic demonstrating various terms needed in the calculation of the contact radius needed for computing contact conduction. In this illustration particle $i$ is smaller than particle $j$.

Abstracting to address the particle radii by the larger and smaller radii:

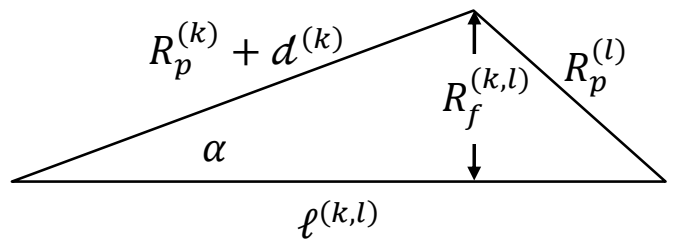

Figure B-4: Schematic demonstrating various terms needed in the calculation of the particle-fluidparticle conduction.

law of Cosines:

$$
\begin{gathered}
R^{(l)^{2}}=\left(R^{(k)}+d^{(k)}\right)^{2}+\ell^{(k, l)^{2}}-2 \ell^{(k, l)}\left(R^{(k)}+d^{(k)}\right) \cos \alpha \\
\cos \alpha=\frac{\left(R^{(k)}+d^{(k)}\right)^{2}-R^{(l)^{2}}+\ell^{(k, l)}}{2 \ell^{(k, l)}\left(R^{(k)}+d^{(k)}\right)}
\end{gathered}
$$

Pythagorean theorem:

$$
R_{f}^{2}+\left(\left(R^{(k)}+d^{(k)}\right) \cos \alpha\right)^{2}=\left(R^{(k)}+d^{(k)}\right)^{2}
$$


combining Equations (B-10) and (B-11) yields the outer region for particle-fluid-particle conduction:

$$
\begin{gathered}
R_{f}^{2}+\left(\left(R^{(k)}+d^{(k)}\right) \frac{\left(R^{(k)}+d^{(k)}\right)^{2}-R^{(l)^{2}}+\ell^{(k, l)}}{2 \ell^{(k, l)}\left(R^{(k)}+d^{(k)}\right)}\right)^{2}=\left(R^{(k)}+d^{(k)}\right)^{2} \\
R_{f}^{(k, l)}=\sqrt{\left(R_{p}^{(k)}+d^{(k)}\right)^{2}-\left(\frac{\left.\left.\left(R_{p}^{(k)}+d^{(k)}\right)^{2}-R_{p}^{(l)}\right)^{2}+\ell^{(k, l)}\right)^{2}}{2 \ell^{(k, l)}}\right)^{2}}
\end{gathered}
$$




\section{Appendix C: Discrete mass inflow boundary condition source code}

The section contains the source code for the discrete mass inflow and outflow boundary conditions. It has been written in the FORTRAN90/95 style.

\section{C.1 Module - DES_BC}

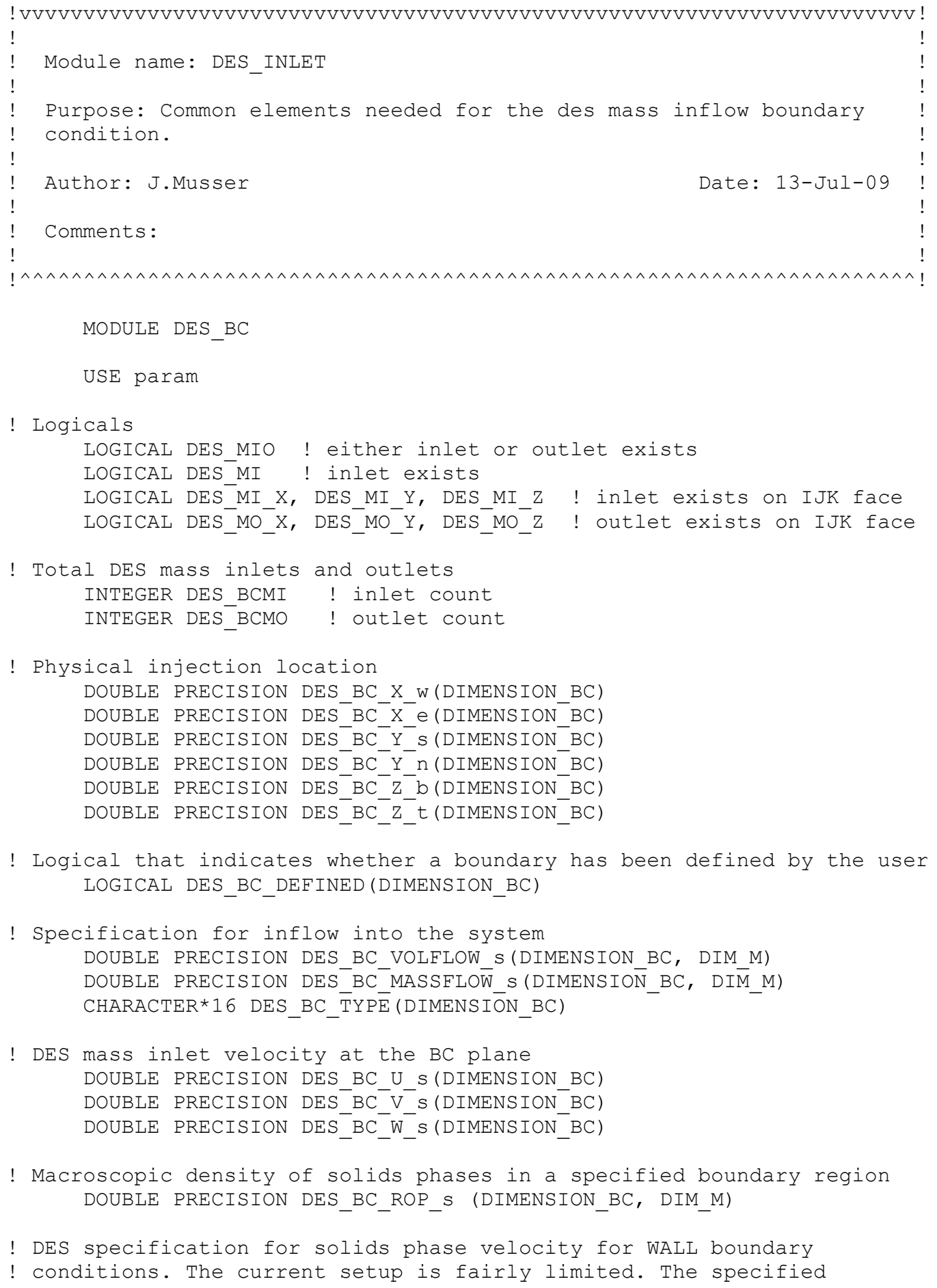


! boundary velocities are assigned to the indicated wall where a wall

! corresponds to one of the six planes in a cubic domain. Each wall

! corresponds to a number as follows west=1, east=2, bottom=3, top=4,

! south=5, north=6. See cfwallposvel for details. To specify a y or $z$

! velocity to the west wall set des_bc_vw_s $(1, M)$ or des_bc_ww_s $(1, M)$,

! respectively (note an $\mathrm{x}$ velocity is not valid for a west or east wall).

! Since these are user input, they are allocated here with a constant

! preset size, but their actual size is represented by (nwalls, mmax) DOUBLE PRECISION DES BC UW S (DIMENSION BC, DIM M) DOUBLE PRECISION DES_BC_VW_S (DIMENSION_BC, DIM_M) DOUBLE PRECISION DES_BC_WW_S (DIMENSION_BC, DIM_M)

! Limit on the total number of divisions (fineness) used to represent

! the particle number distribution at an inlet. INTEGER, PARAMETER : : NUMFRAC_LIMIT $=10000$

! Indicates if the boundary condition is mono or ploydisperse

!.F. = monodisperse and $. \mathrm{T} .=$ polydisperse LOGICAL, DIMENSION(:), ALLOCATABLE : : DES_BC_POLY ! (DES_BCMI)

! This array contains integers representing the mass/solid phase indices

! present at a specific boundary condtion in proportion to their

! respective number fraction at the inlet (i.e., it represents the

! particle number distribution of incoming solids at the inlet). The

! array is scaled in size according to the parameter NUMFRAC LIMIT. INTEGER, DIMENSION $(:,:)$, ALLOCATABLE : : DES_BC_POLY_LAȲOUT

! (DES_BCMI, NUMFRAC_LIMIT)

! DES boundary condition ID number (boundary number used in mfix.dat

! when the boundary is originally defined) INTEGER, DIMENSION(:), ALLOCATABLE : : DES_BC_MI_ID ! (DES_BCMI) INTEGER, DIMENSION (:), ALLOCATABLE : : DES_BC_MO_ID ! (DES_BCMO)

! Boundary classification

! Assigned a value of the face/edge associated with inlet in

! des_init_bc (see des_mi_classify)

! Possible values in $2 \mathrm{D}$ : 'YN', 'YS', 'XE', 'XW'

! Possible values in 3D: 'XZs', 'XZn', 'XYb', 'XYt', 'YZW', 'YZe' CHARACTER*3, DIMENSION (:), ALLOCATABLE : : DES MI CLASS ! (DES BCMI)

! Assigned a value of the face/edge associated with outlet in

! des init bc (see des mo classify)

! Possible values in $2 \mathrm{D}$ : 'XW', 'XE', 'YN', 'YS'

! Possible values in 3D: 'XW', 'XE', 'YN', 'YS', 'ZB', 'ZT' CHARACTER*2, DIMENSION(:), ALLOCATABLE : : DES'MO_CLASS ! (DES_BCMO)

! Assigned a value in des_init_bc (see des_mi_layout):

! When the inlet bc vêlocity is sufficienty $\quad$ high it is assigned

! 'RAND', otherwise it is assigned 'ORDR' CHARACTER*4, DIMENSION(:), ALLOCATABLE : : PARTICLE_PLCMNT

! Logical that can be flagged in the mfix.dat file to for $\overline{c e}$ the inlet

! to operate with an ordered boundary condition. This may be useful

! during long simulations or if the inlet appears to be taking a long

! time to randomly place particles. LOGICAL FORCE_ORD_BC

! Particle injection factor; how many solid time steps (dtsolid) pass ! before the next injection of a particle. if pi_count is greater than ! 1, then pi factor is set to 1 (i.e. multiple părticles enter every ! solids timè step). INTEGER, DIMENSION(:), ALLOCATABLE :: PI_FACTOR !(DES_BCMI)

! Particle injection count (injection number); how many particles are

! injected in one solids time step. pi_count is set to one if 


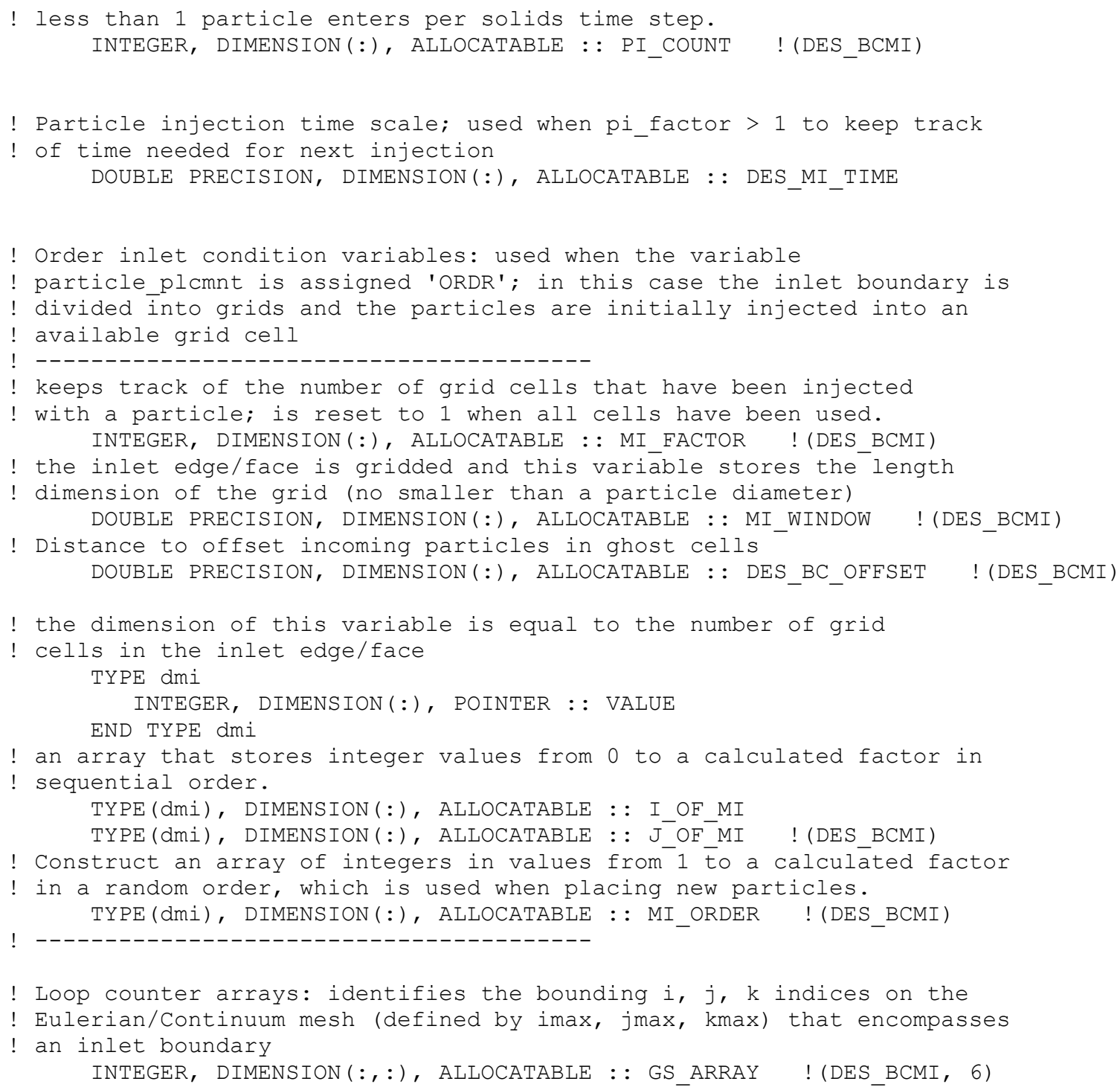




\section{C.2 : Routine - CHECK_DES_BC}

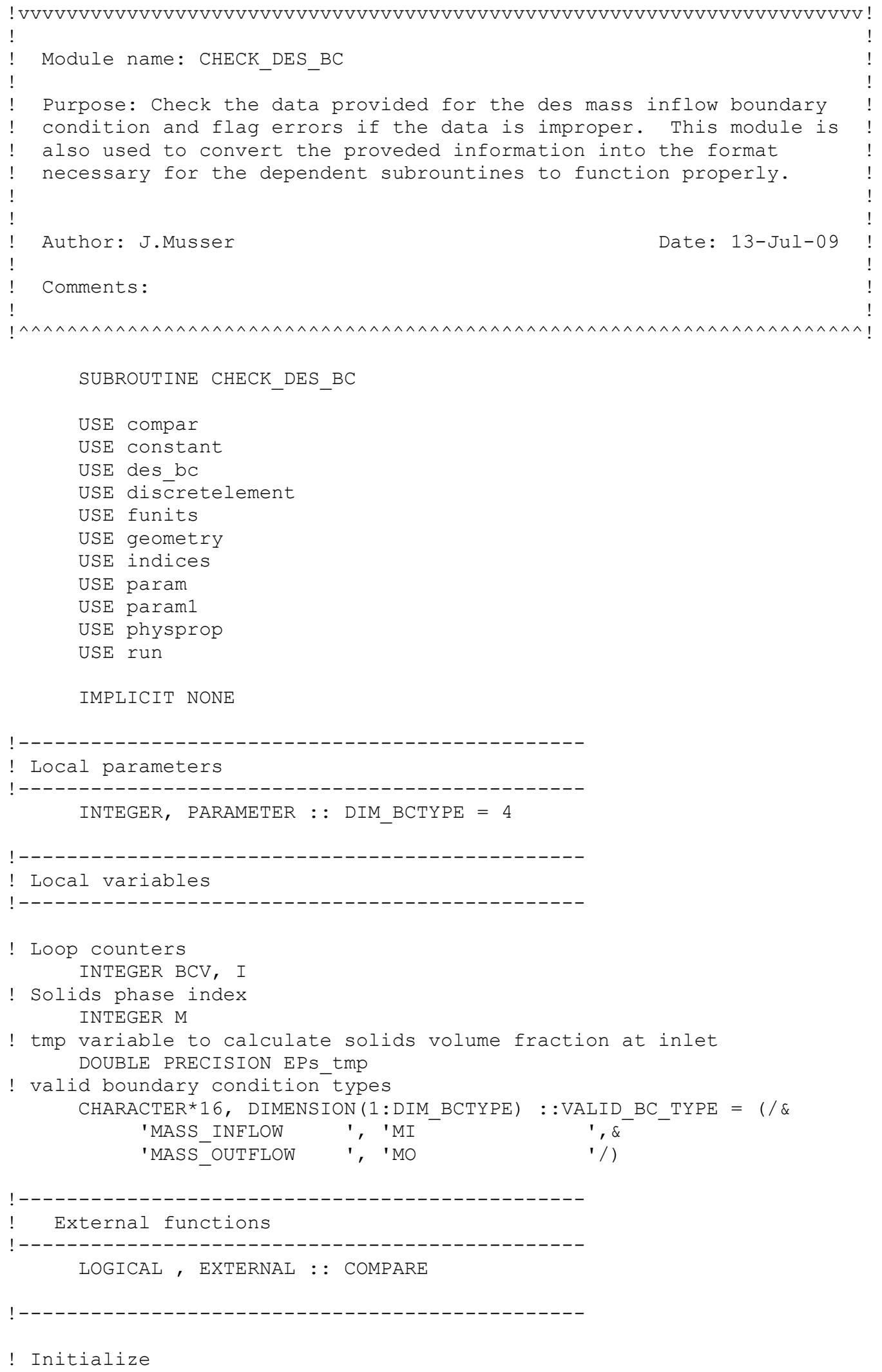




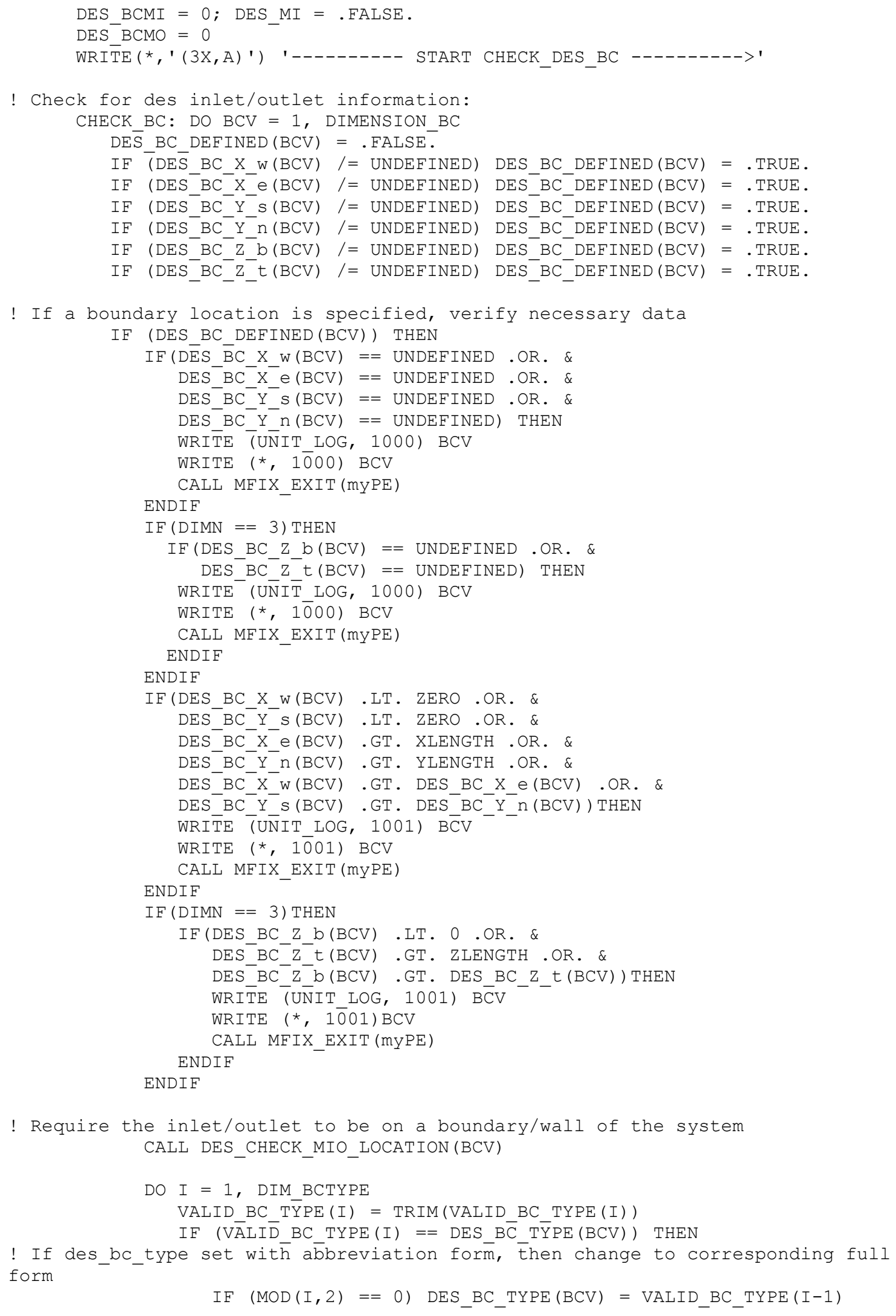


! Check if solids phase velocity at the BC plane is specified, if not,

! set solids velocity to zero and flag log file

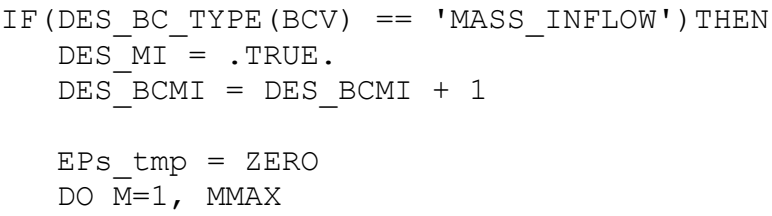

! Back check that if either DES_BC_MASSFLOW_s or DES_BC_VOLFLOW_s are

! well defined (defined and not zero), then ${ }^{-}$ROP s is also well defined. $\operatorname{IF}((\mathrm{DES} B C$ MASSFLOW_S $(\mathrm{BC} V, \mathrm{M}) /=$ UNDEFINED. AND. \& .NOT.COMPARE (DES_BC_MASSELOW_S (BCV,M), ZERO)) \& . OR. \&

(DES_BC_VOLFLOW_S (BCV,M) /= UNDEFINED.AND. \& .NOT. $\bar{T}$ OMPARE (DE $\bar{S}$ _BC_VOLFLOW_S (BCV, M), ZERO))) THEN

IF (DES BC ROP S (BCV, M) == UNDEFINED .OR. \& COMPARE (DE $\bar{S}$ BC ROP S (BCV, M), ZERO)) THEN

! A nonzero mass or volumetric flow rate is defined for BCV on mass

! phase M, and either ROP_s is zero or undefined. Flag error and exit. 


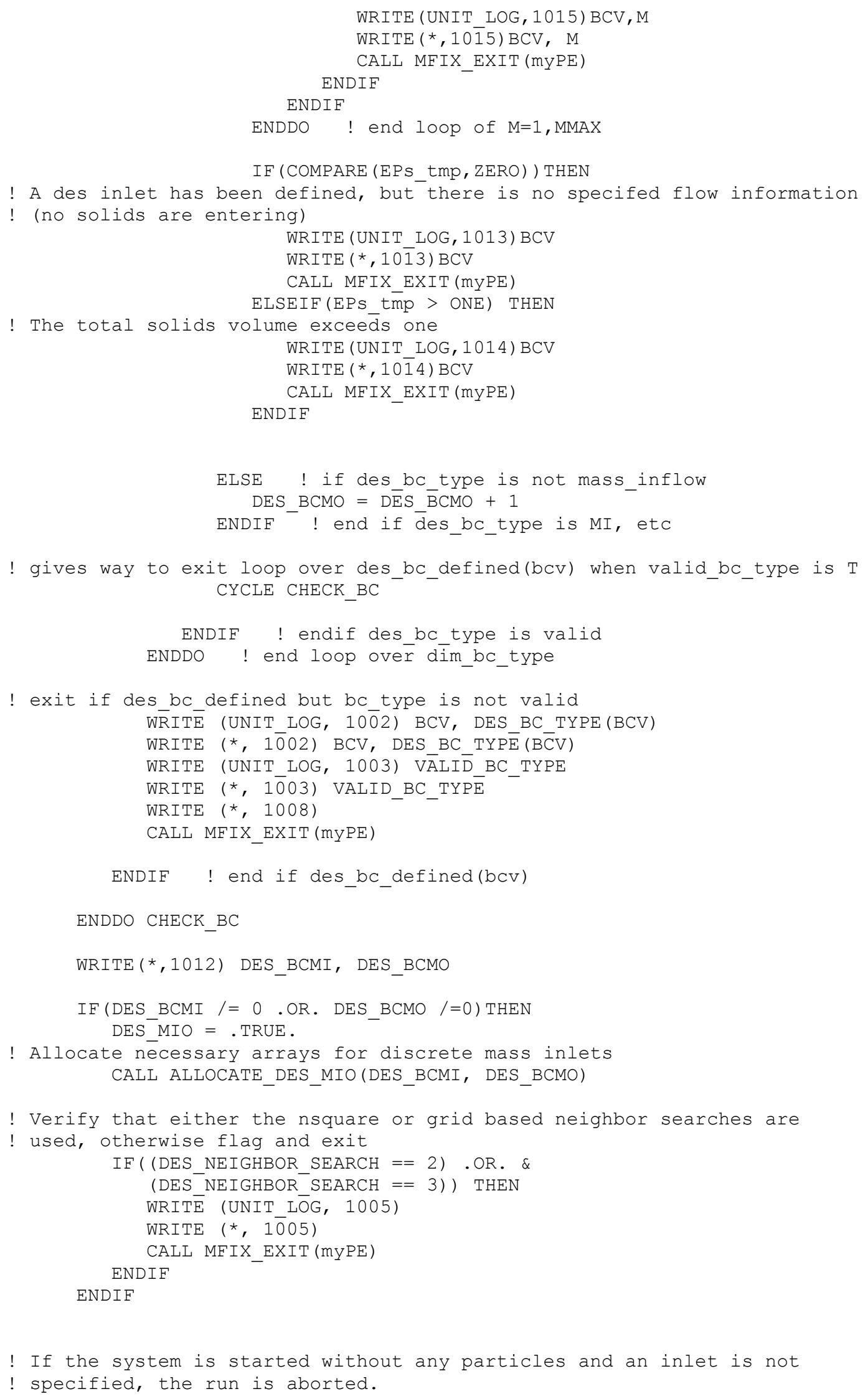




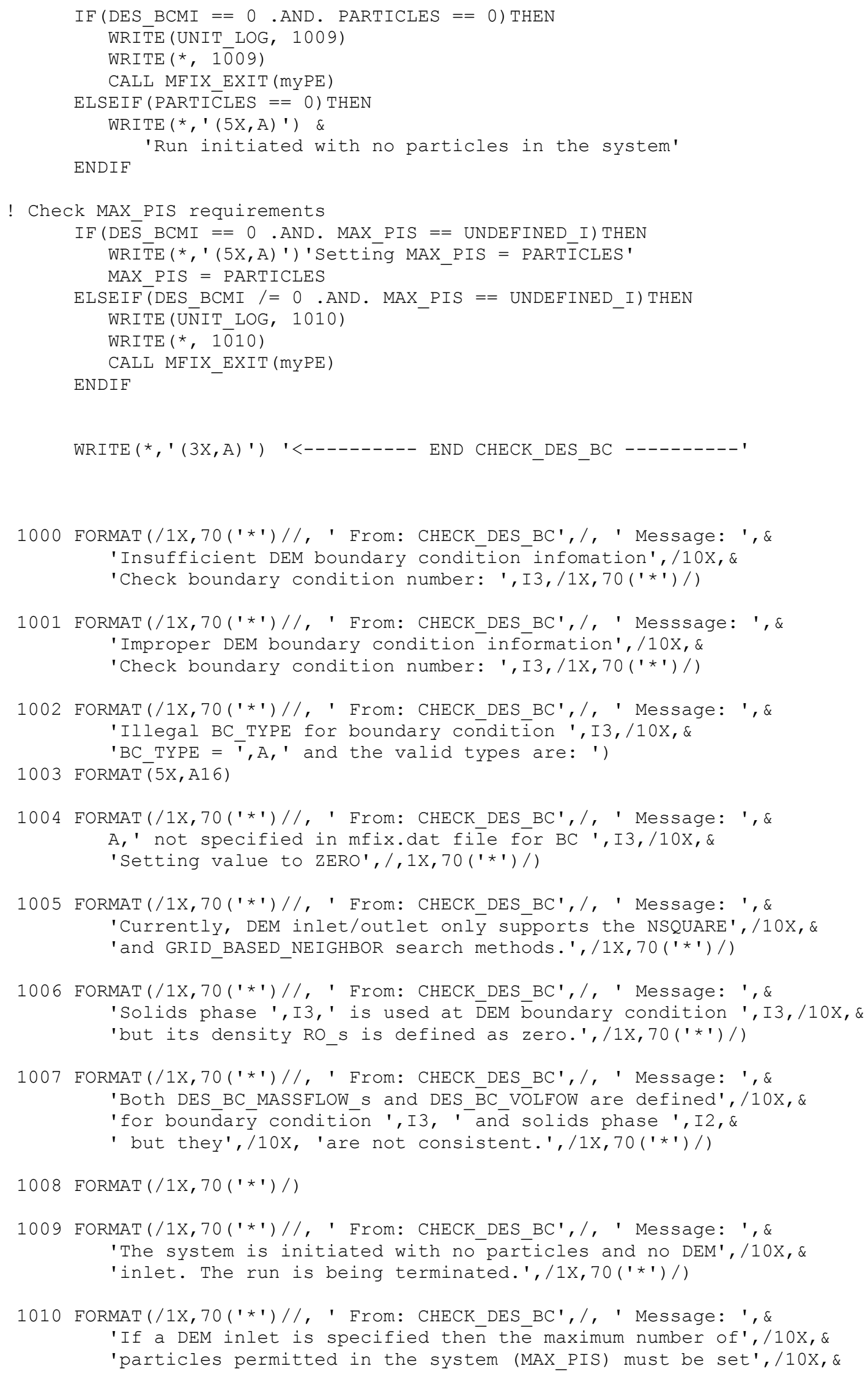


'in mfix.dat',/1x,70('*')/)

1011 FORMAT(/1X,70('*')//, ' From: CHECK DES BC',/,' Message: ', \& 'DES BC ROP s is defined (and not zero) for boundary ',/10X, \& 'condition ',I3,' ' and solids phase ',I2,' without defining',/10X, \& 'either DES_BC_MASSFLOW_S or DES_BC_VOLFOW.' /,1X,70('*')/)

1012 FORMAT (5X,'No. of mass inlet $\mathrm{BC}=1$, I4, /, \&

$5 \mathrm{X}$, 'No. of mass outlet $\mathrm{BC}=$ ', I4)

1013 FORMAT (/1X,70('*')//, ' From: CHECK DES BC',/,' Message: ', \& 'Boundary condition ', I3,' is identifed as an DEM inlet',/10X, \& 'but has no associated inflow information. Check mfix.dat', \& $\left./ 1 \mathrm{x}, 70\left({ }^{\prime} * '\right) /\right)$

1014 FORMAT (/1X,70('*')//, ' From: CHECK_DES_BC',/,' 'Message: ', \&

'The total solids volume fraction for boundary condition',/10X, \& I3,' exceeds one. Check the mfix.dat file.',/1X,70('*')/)

1015 FORMAT(/1X,70('*')//, ' From: CHECK DES BC',/,' 'Message: ', \& 'A nonzero mass or volumetric flow rāe has been specified', \& 'for',/10X, 'boundary condition ', I3, ' and solids phase ',I3,/10X, \& 'and the associated DES BC ROP $s$ is either undefined or zero',/10X, \& 'Check mfix.dat', /, 1X, $7 \overline{0}(' \bar{\star} ') / \overline{)}$

RETURN

END SUBROUTINE CHECK_DES_BC 


\section{C.3 : Routine - DES_CHECK_MIO_LOCATION}
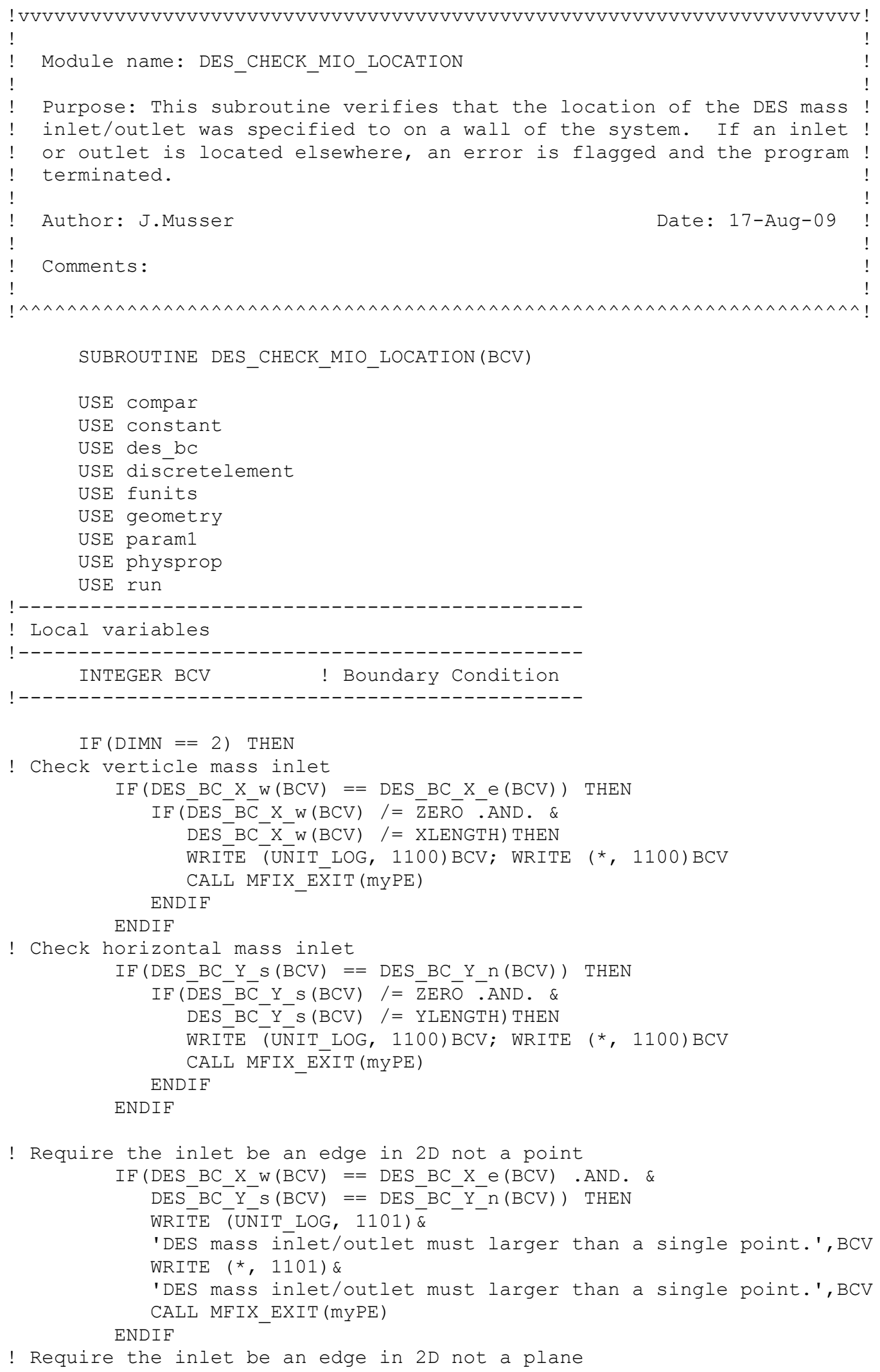


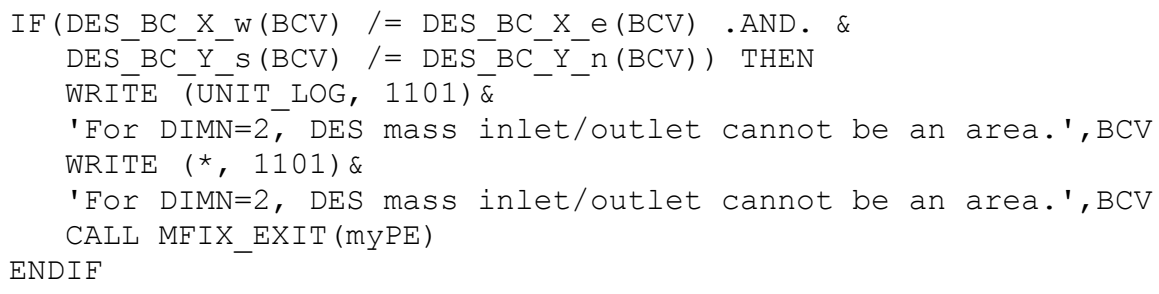




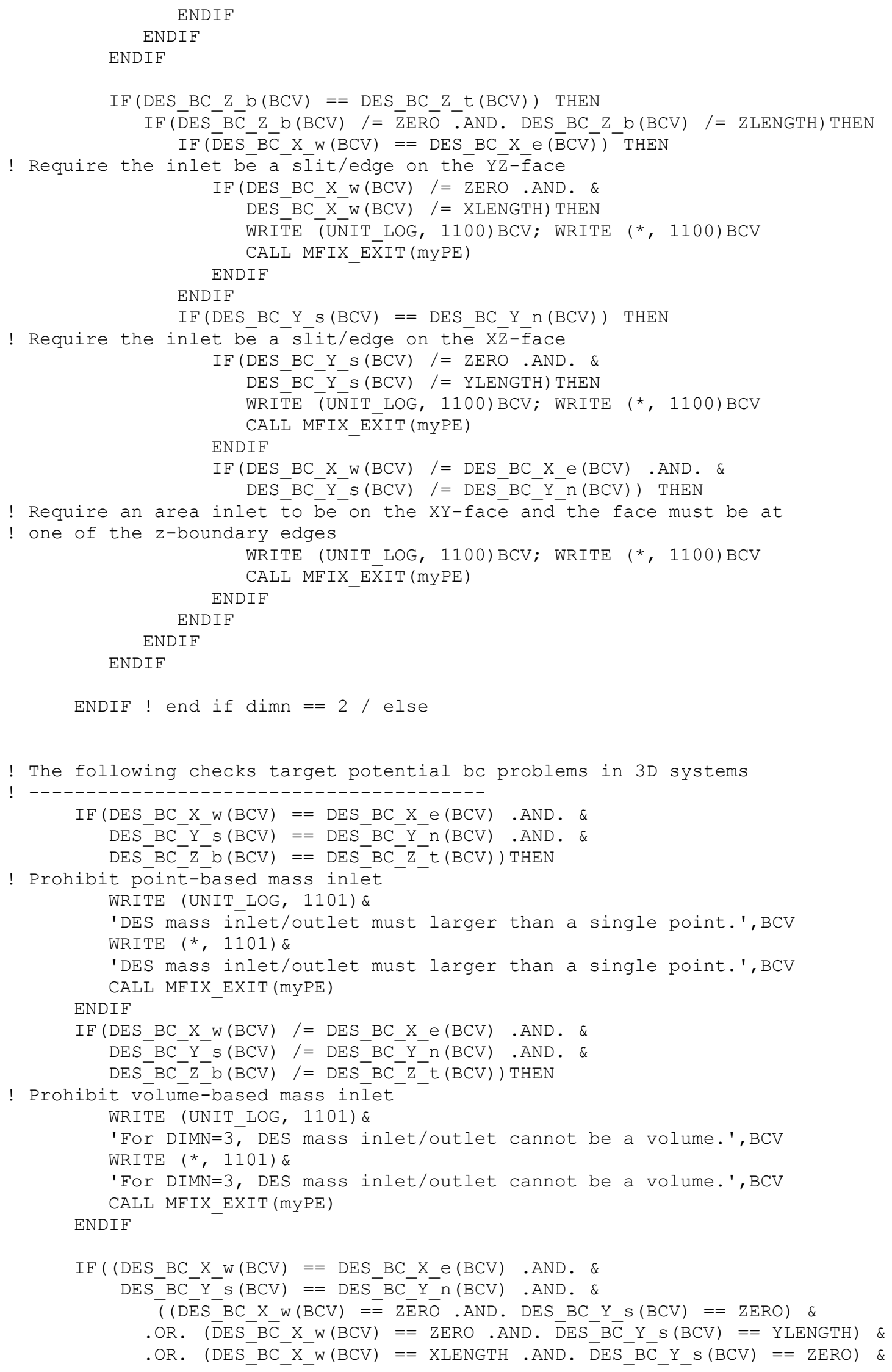




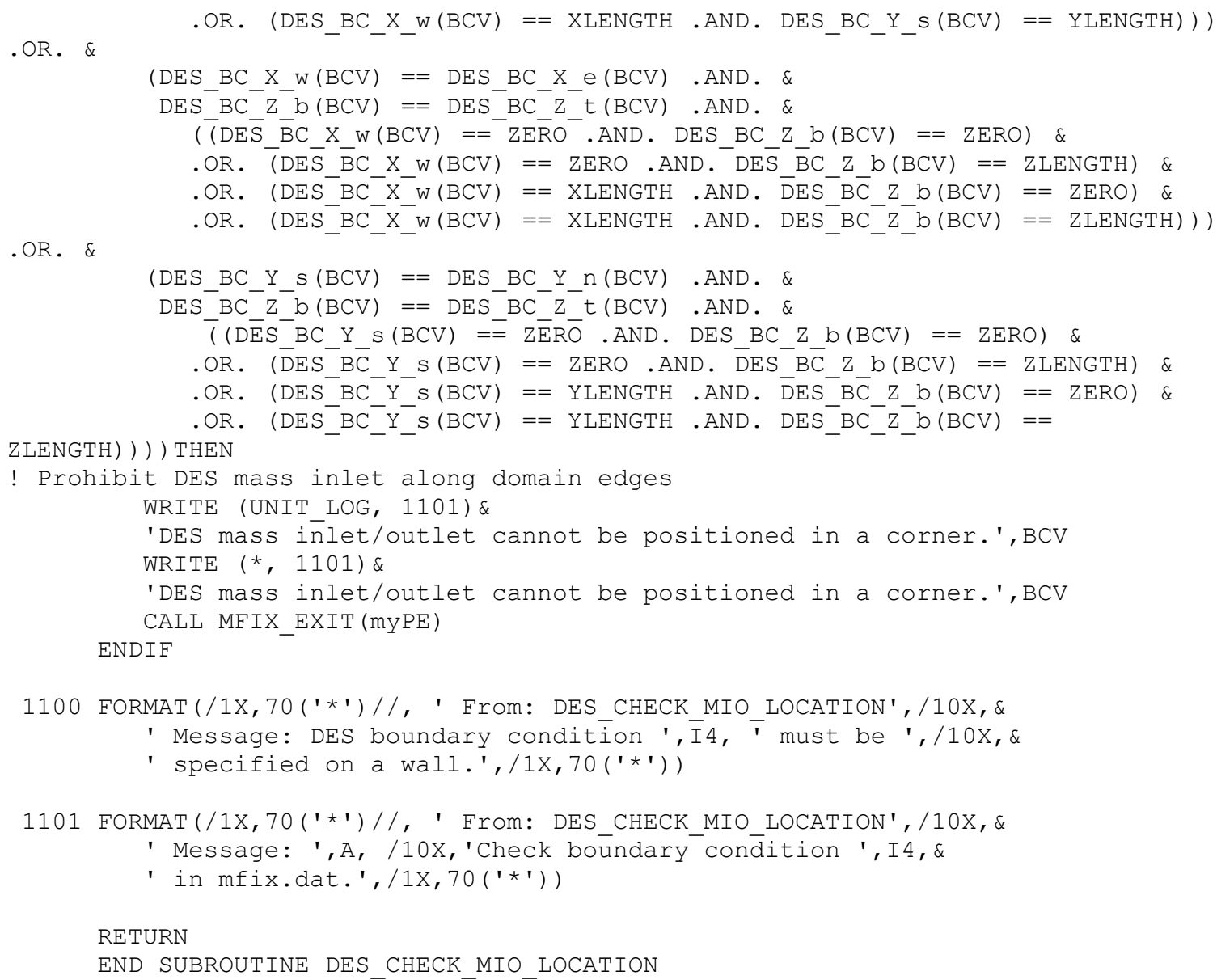




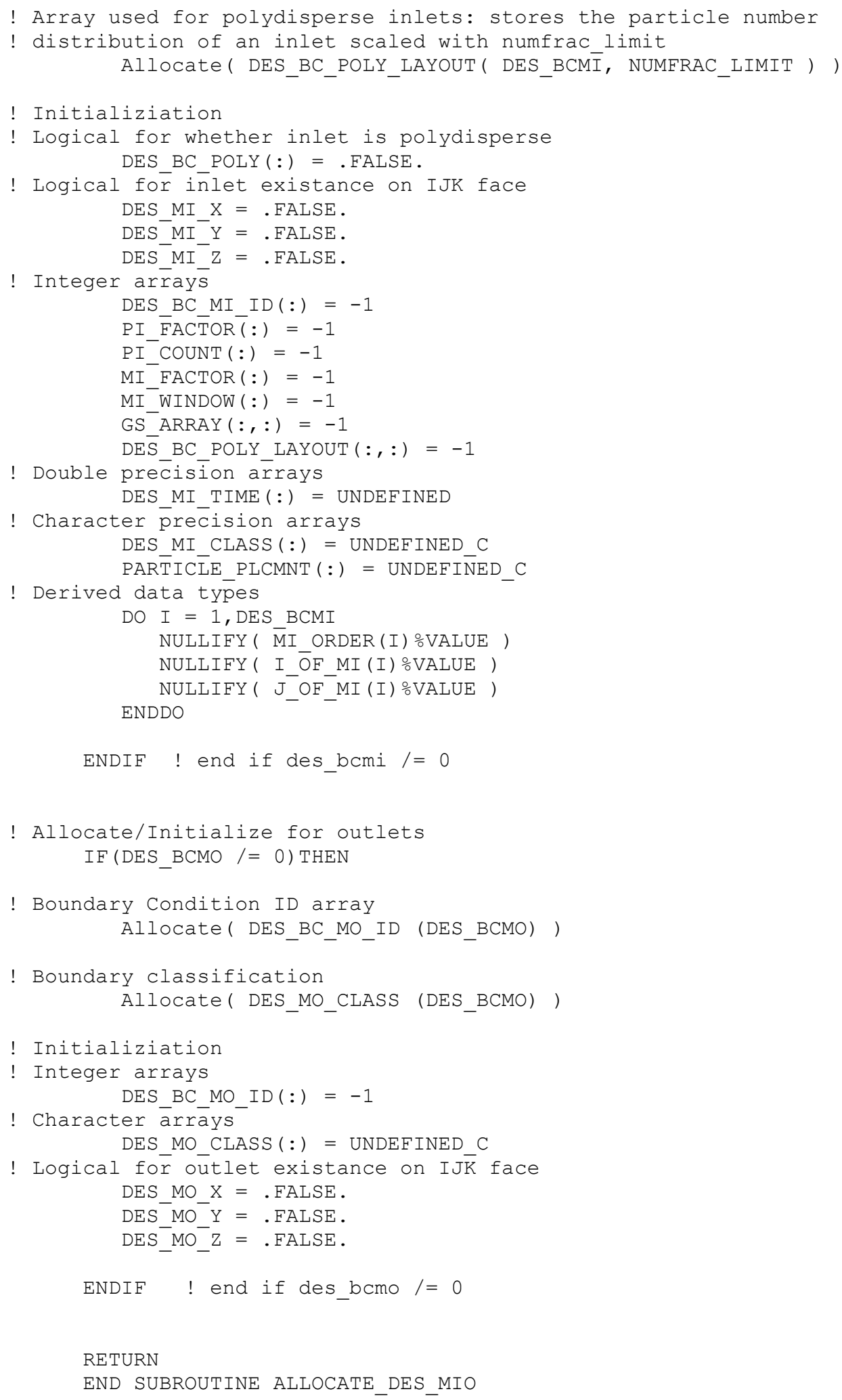




\section{C.5 : Routine - DES_INIT_BC}

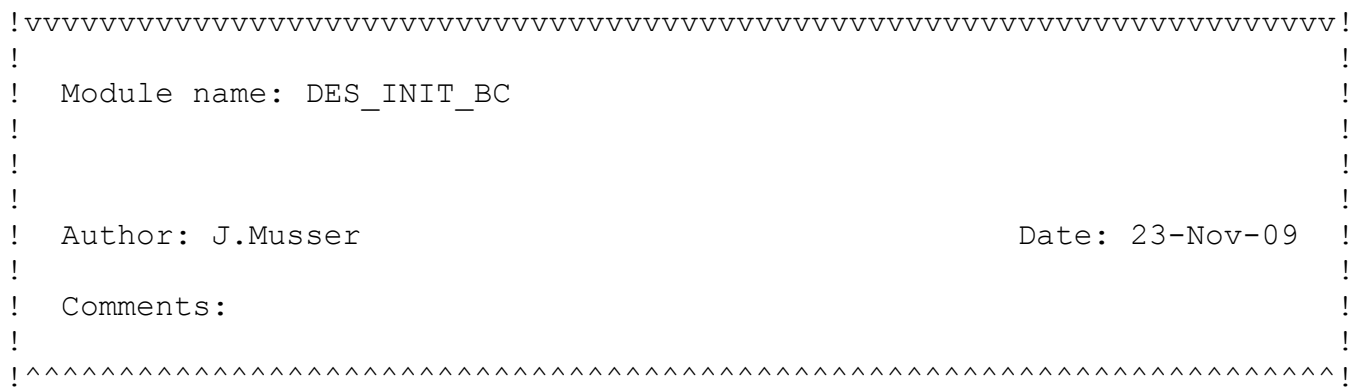

SUBROUTINE DES_INIT_BC

USE compar

USE constant

USE des_bc

USE discretelement

USE funits

USE geometry

USE indices

USE param

USE param1

USE physprop

USE run

IMPLICIT NONE

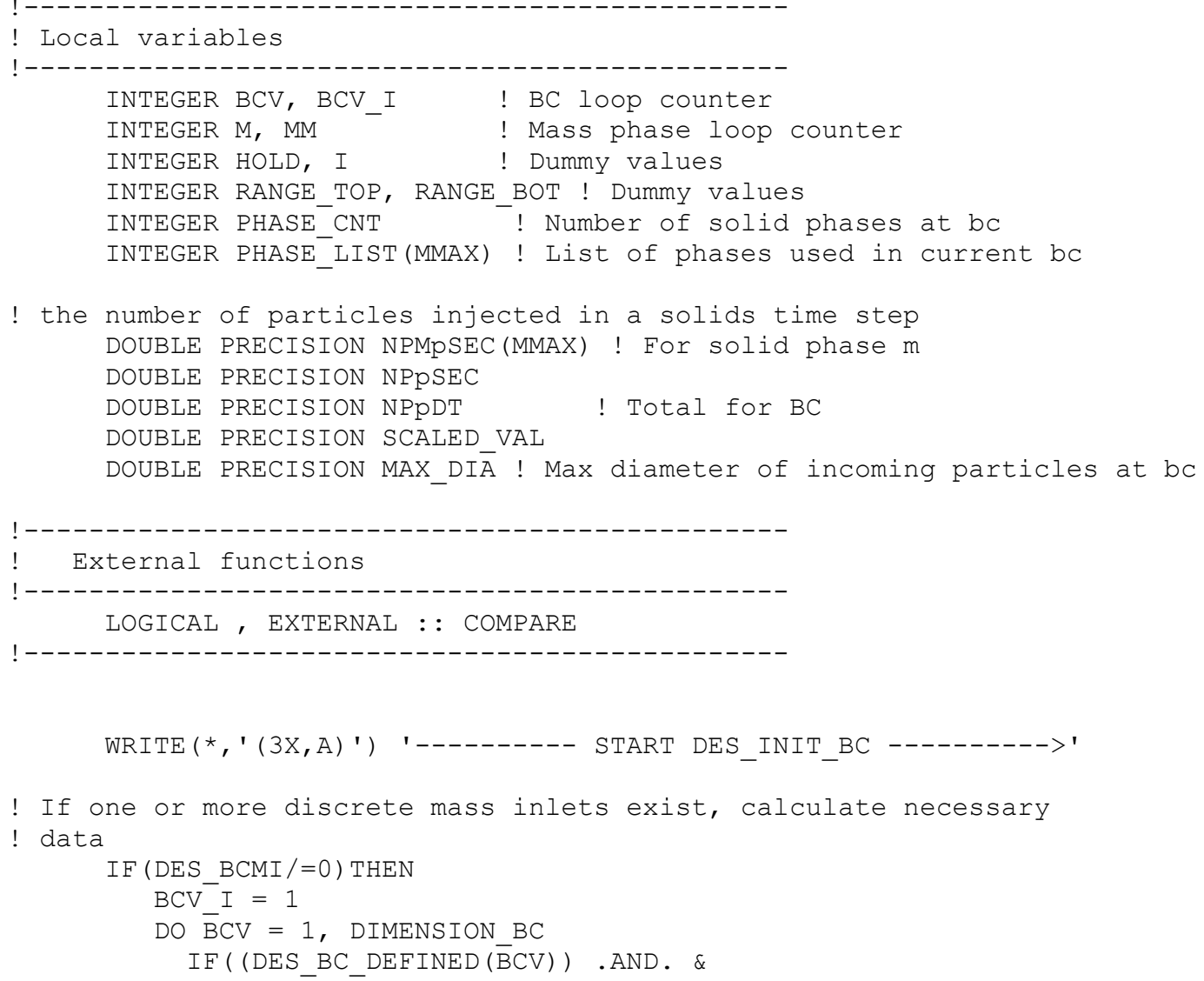




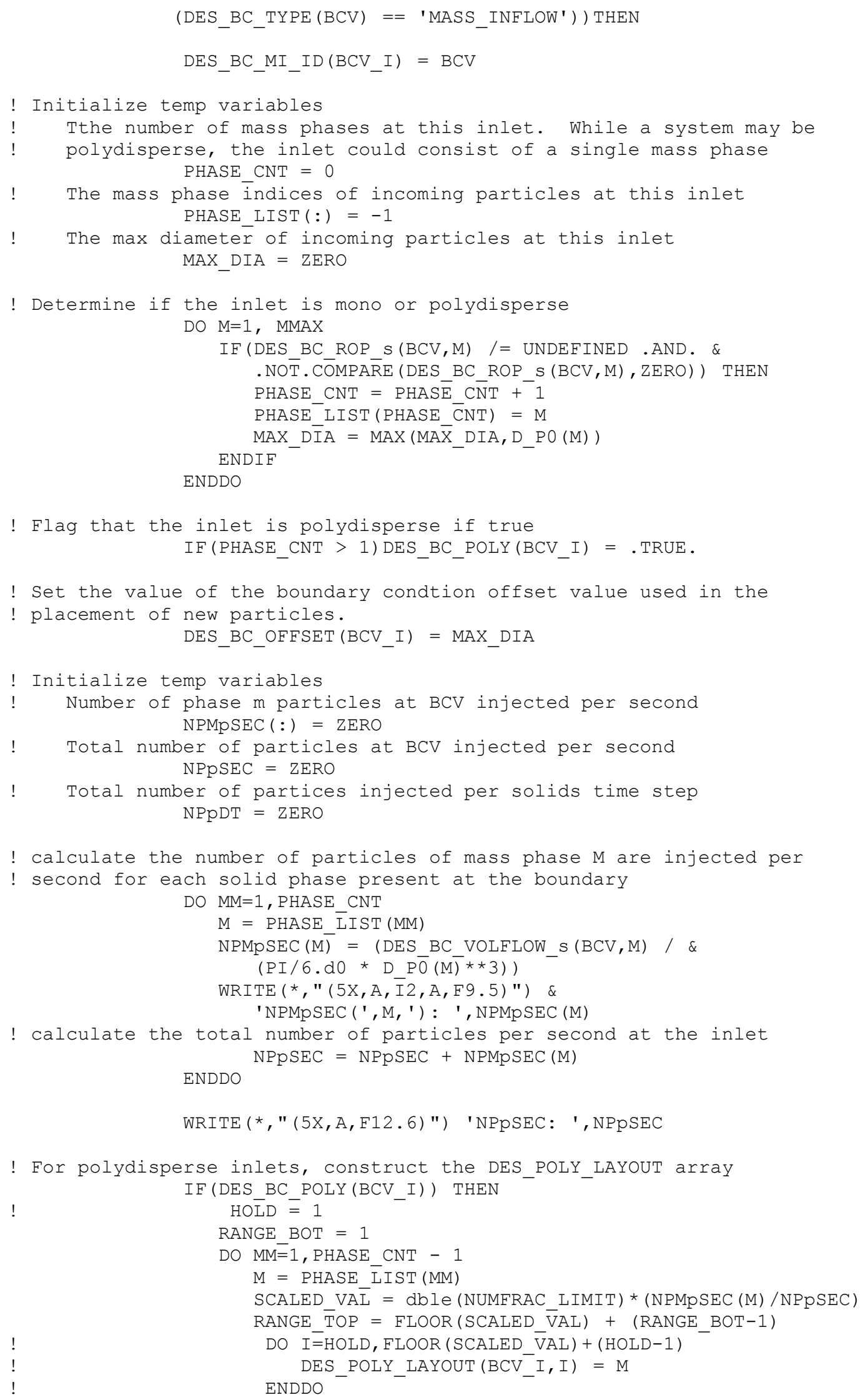




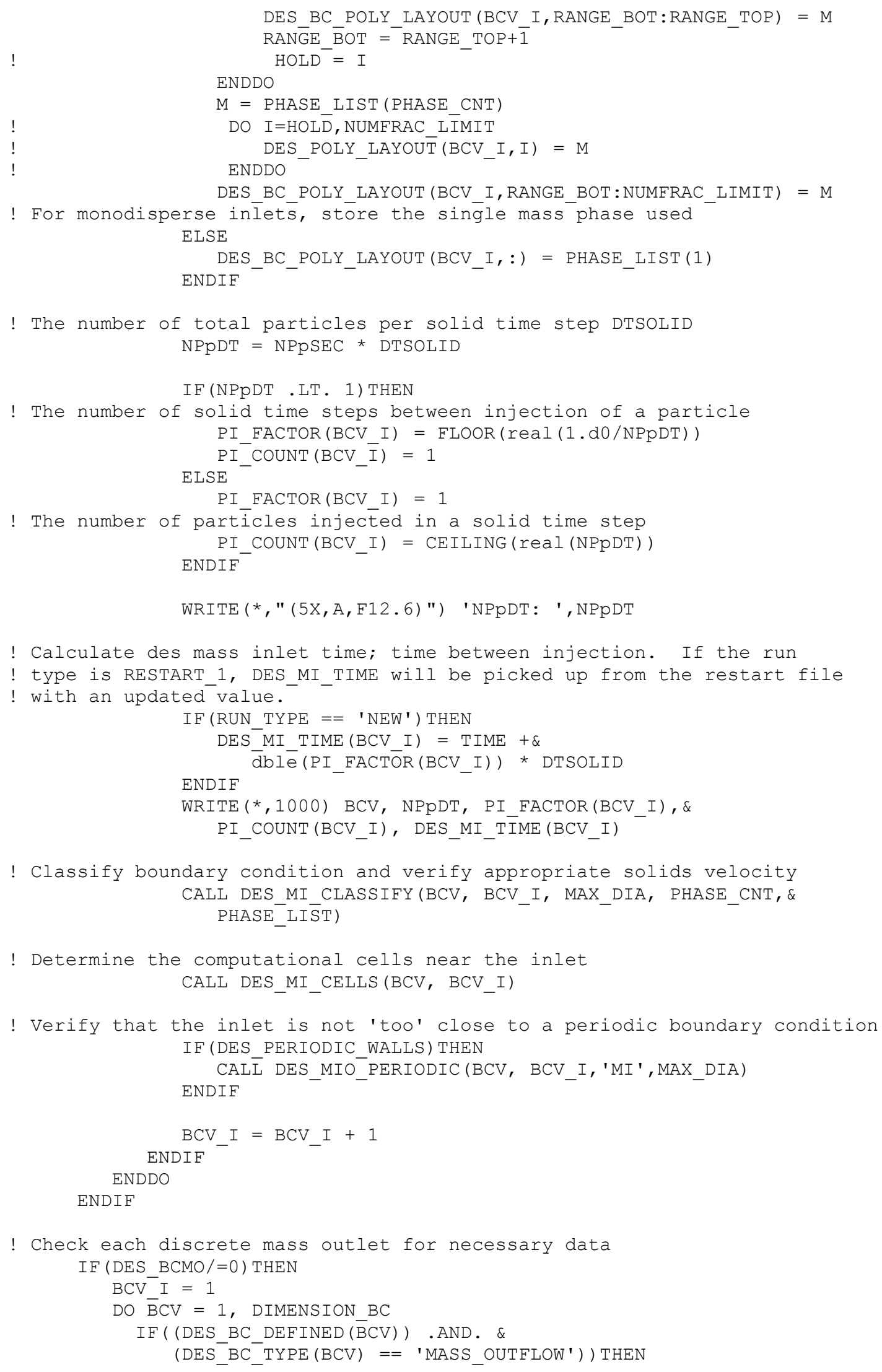




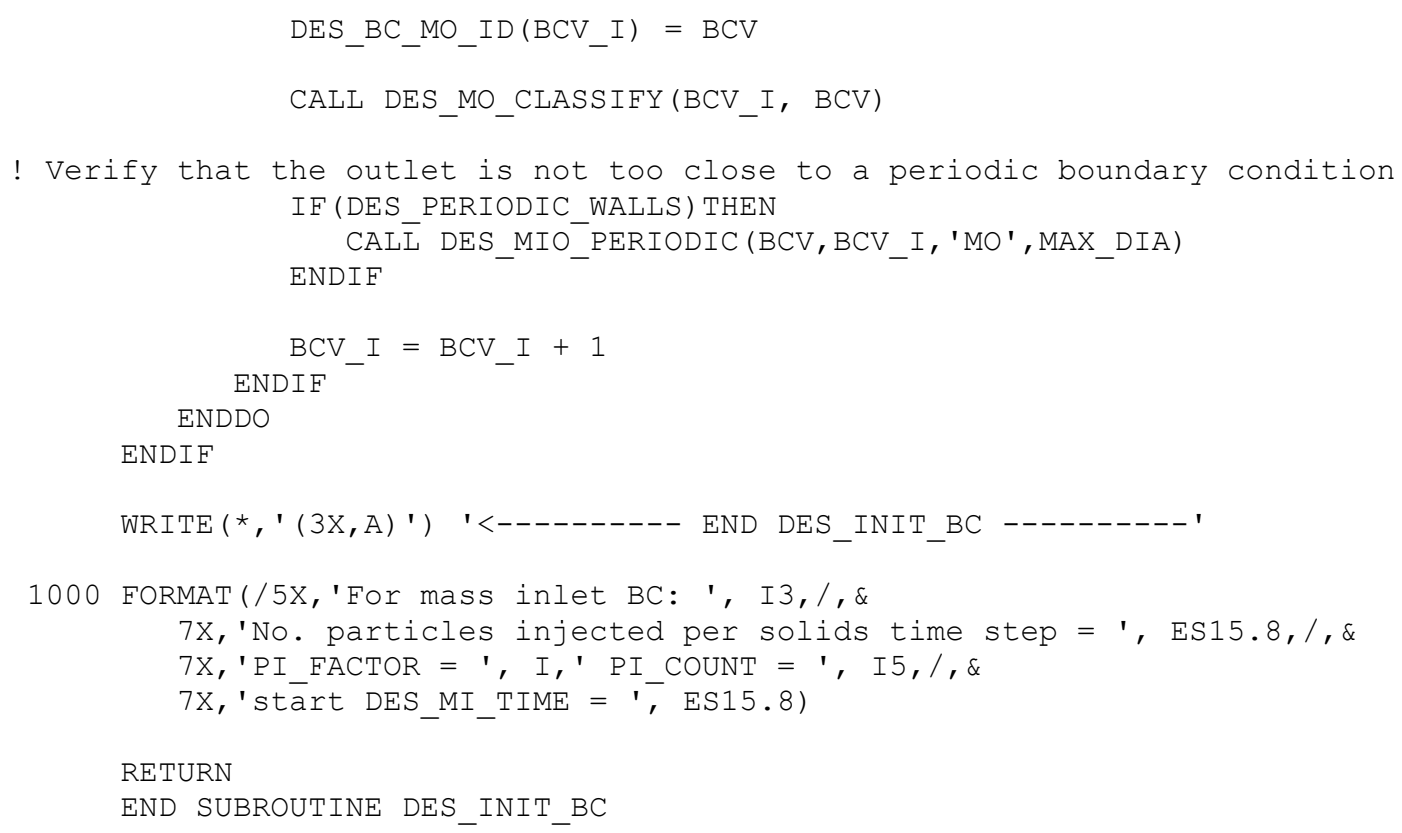




\section{C.6 : Routine - DES_MI_CLASSIFY}

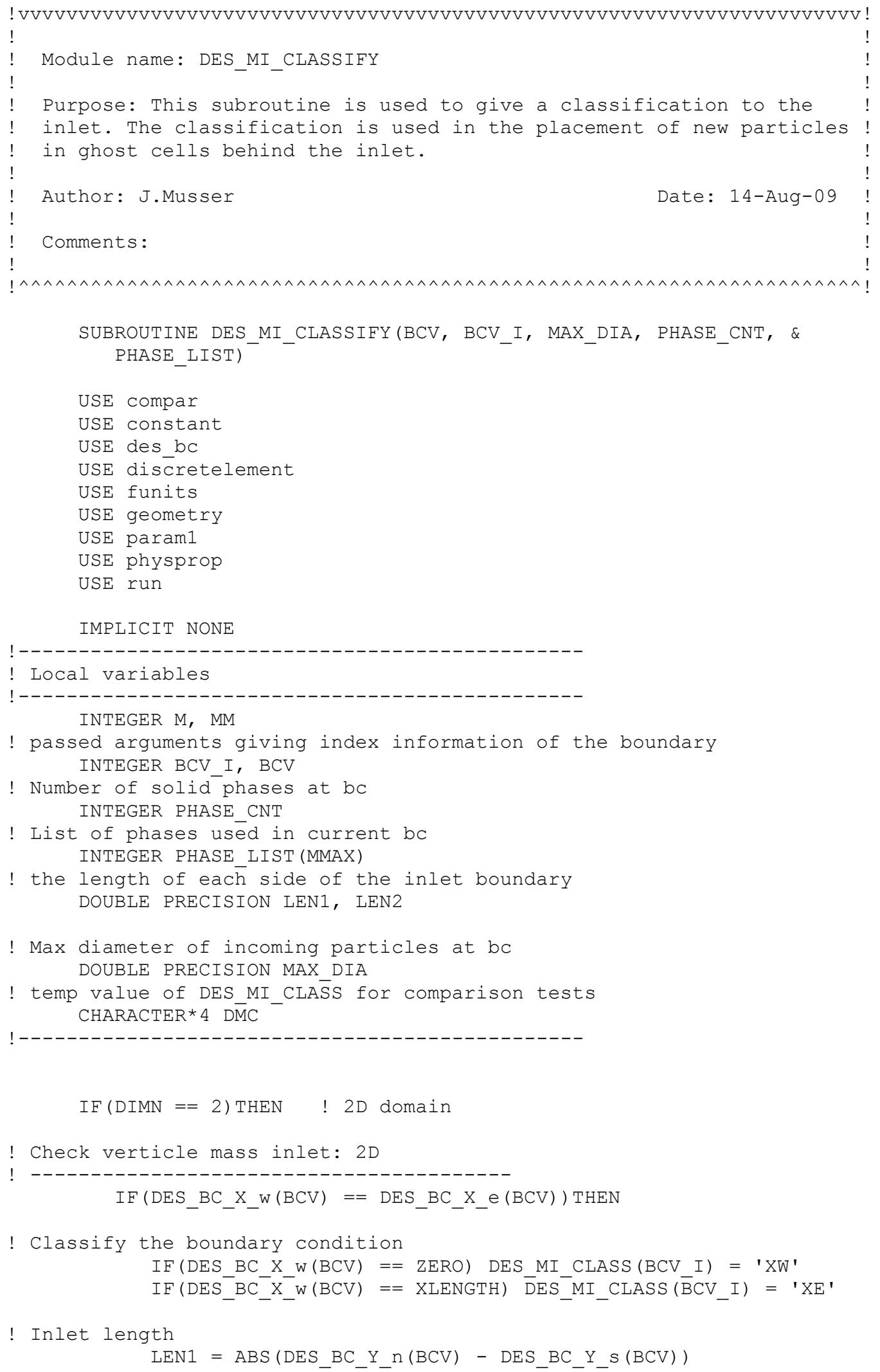


ENDIF

! Check horizontal mass inlet: 2D

$!-$

IF (DES_BC_Y_S $(B C V)==$ DES_BC_Y_n $(B C V))$ THEN

! Classify the boundary condition

$\operatorname{IF}(\mathrm{DES} B \mathrm{BC} Y \mathrm{Y}(\mathrm{BCV})==\mathrm{ZERO})$ DES MI_CLASS $(B C V I)=$ ' YS'

$\mathrm{IF}\left(\mathrm{DES}{ }_{-}^{-}{ }^{-}{ }_{-}^{-}{ }^{-}-\mathrm{S}(\mathrm{BCV})==\mathrm{YLENGTH}\right) \quad \overline{\mathrm{DES}}{ }_{-} \mathrm{MI}$ CLASS $(\overline{\mathrm{B} C V} \mathrm{I})={ }^{-} \mathrm{YN}$ '

! Inlet length ENDIF

LEN1 = DES_BC_X_e (BCV) - DES_BC_X_w $(B C V)$

! max dia is used for the 'depth/width' of the inlet

! (see des_bc_vel_assign for details)

CĀLL DES_BC_VEL_ASSIGN (BCV,BCV_I, LEN1, MAX_DIA, PHASE_CNT, PHASE_LIST)

! This subroutine determines the pattern that the particles will need to

! enter the system, if any. This routine only needs to be called if a

! run is new. If a run is a RESTART 1, all of the setup information

! provided by this subroutine is will be obtained from the * DES.RES file.

! This is done due to this routine's strong dependence on the

! RANDOM NUMBER() subroutine. IF (RUN TYPE $==$ 'NEW') THEN

CALI DES_MI_LAYOUT (LEN1, ZERO, BCV, BCV_I, MAX_DIA, \& ENDIF PHASE_CNT, PHASE_LIST)

ELSE ! 3D domain

! Check mass inlet on XZ face: 3D

! ----------------------------

! see comments following the yz face section for elucidation on this

! series of if statements $\mathrm{IF}(\mathrm{DES}$ BC_Y_S $(\mathrm{BCV})==\mathrm{DES} B \mathrm{BC} \mathrm{Y} n(\mathrm{BCV})$. AND. \&

DES BC_X_w (BCV) /= DES_BC_X_e(BCV) .AND. \&

$\mathrm{DES}{ }^{-} \mathrm{BC}^{-} \mathrm{Z}^{-} \mathrm{b}(\mathrm{BCV}) /=\mathrm{DES}^{-} \mathrm{BC}^{-} \mathrm{Z}^{-} \mathrm{t}(\mathrm{BCV}) \quad \&$

.$O R$. \&

DES_BC_Y_S $(B C V)==$ DES_BC_Y_n $(B C V)$. AND. \&

$\mathrm{DES}^{-} \mathrm{BC}^{-} \mathrm{X}^{-} \mathrm{W}(\mathrm{BCV})==\mathrm{DES}^{-} \mathrm{BC}^{-} \mathrm{X}^{-} \mathrm{e}(\mathrm{BCV})$. AND. \&

$\mathrm{DES}{ }^{-} \mathrm{BC}-\mathrm{X}-\mathrm{W}(\mathrm{BCV}) /=\mathrm{ZERO} \cdot \overline{\mathrm{A}} \mathrm{ND} . \&$

$\mathrm{DES} B C \mathrm{X}-\mathrm{W}(\mathrm{BCV}) /=\mathrm{XLENGTH} \&$

.$O R$ \& \&

DES_BC_Y_S $(B C V)==$ DES_BC_Y_n $(B C V)$. AND . \&

$\mathrm{DES}^{-} \mathrm{BC}^{-} \mathrm{Z}^{-} \mathrm{b}(\mathrm{BCV})==\mathrm{DES}^{-} \mathrm{BC}^{-} \mathrm{Z}^{-} \mathrm{t}(\mathrm{BCV})$. AND. \&

$\mathrm{DES}{ }_{\mathrm{BC}} \mathrm{Z}_{-} \mathrm{b}(\mathrm{BCV}) /=\mathrm{ZERO} \cdot \overline{\mathrm{A}} \mathrm{ND} . \&$

DES_BC_Z_b(BCV) /= ZLENGTH) THEN

! If a slit flow inlet is specified near an edge, expand the slit to

! the width of one particle diameter :

! see comments in the yz face section for further explanation IF (DES_BC_X_w $(B C V)==$ DES_BC_X_e (BCV)) THEN

$\operatorname{IF}\left(\overline{(}\left(\mathrm{DE} S \mathrm{~S}-\mathrm{X} W(\mathrm{BCV})-\mathrm{MAX} \overline{\mathrm{D} I A}{ }^{*} \mathrm{HALF}\right)\right.$. GE. ZERO) . AND. \& $\left(\left(D_{E S}^{-}{ }^{-} C^{-} X^{-} W(B C V)+\right.\right.$ MAX_DIA*HALF $)$. LE. XLENGTH) ) THEN $\mathrm{DES} B \overline{\mathrm{C}} \_\mathrm{X} \_\mathrm{W}(\mathrm{BCV})=\mathrm{DES} B \overline{\mathrm{BC}} \mathrm{X} \_\mathrm{w}(\mathrm{BCV})-\mathrm{MAX} \mathrm{DIA} * \mathrm{HALF}$ $\mathrm{DES} \mathrm{SBC}^{-} \mathrm{X}^{-} \mathrm{e}(\mathrm{BCV})=\mathrm{DES}^{-} \mathrm{BC}^{-} \mathrm{X}^{-} \mathrm{e}(\mathrm{BCV})+\mathrm{MAX}^{-} \mathrm{DIA} \mathrm{AHALF}^{*}$

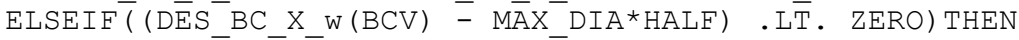
DES_BC_X_W $(\mathrm{BCV})=$ ZERO

$\mathrm{DES} \mathrm{BC}^{-} \mathrm{X}-\mathrm{e}(\mathrm{BCV})=\mathrm{MAX} \mathrm{DIA}$

$\operatorname{ELSEIF}((\mathrm{DE} S-B C \times$ e $(B C V) \overline{\text { MAX }}$ DIA*HALF $)$. GT. XLENGTH) THEN DES_BC_X_W $(\bar{B} C \bar{V})=X L E N G T H=$ MAX_DIA 


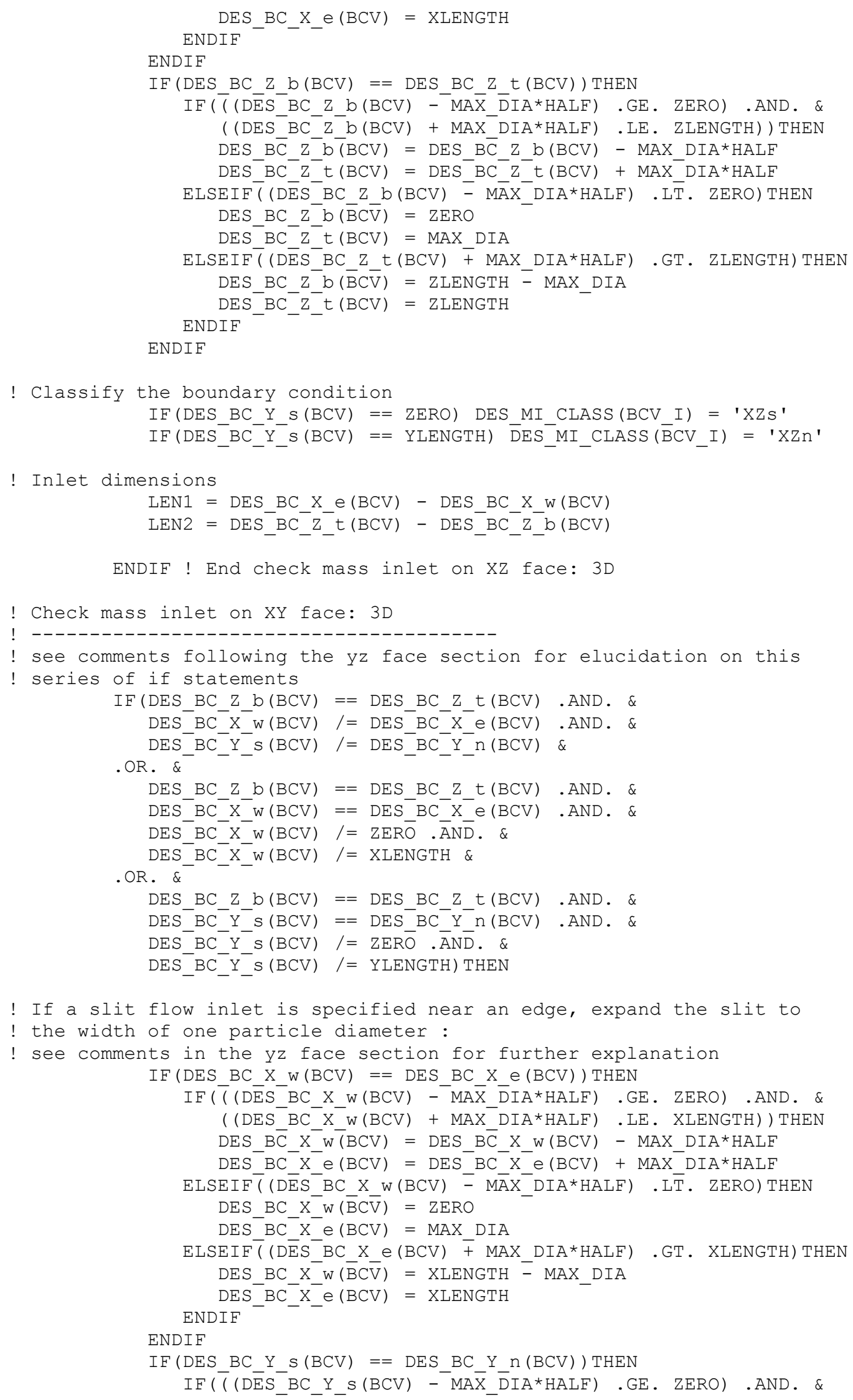




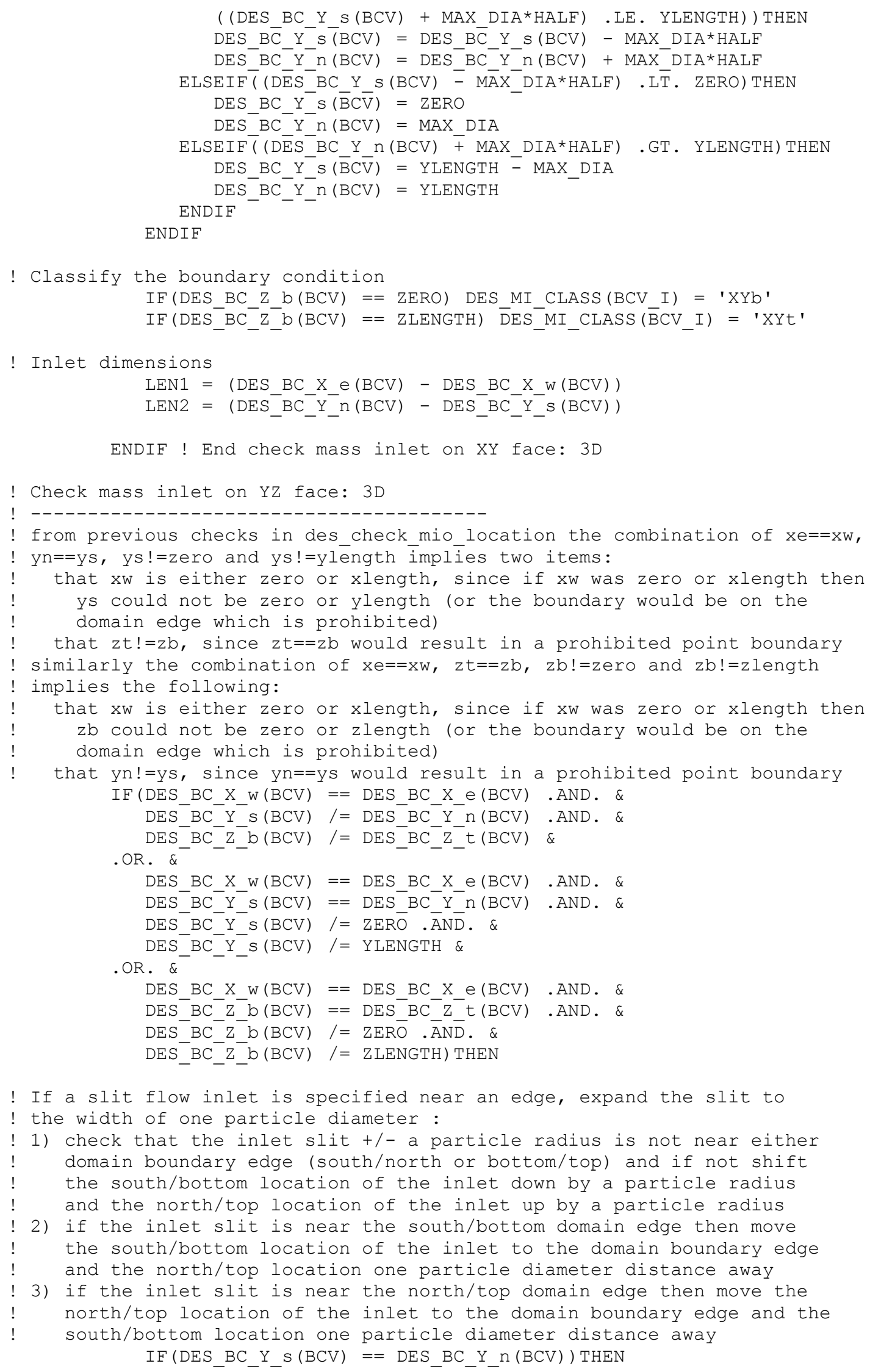

! If a slit flow inlet is specified near an edge, expand the slit to

! the width of one particle diameter :

! 1) check that the inlet slit +/- a particle radius is not near either domain boundary edge (south/north or bottom/top) and if not shift the south/bottom location of the inlet down by a particle radius and the north/top location of the inlet up by a particle radius

2) if the inlet slit is near the south/bottom domain edge then move the south/bottom location of the inlet to the domain boundary edge and the north/top location one particle diameter distance away

3) if the inlet slit is near the north/top domain edge then move the north/top location of the inlet to the domain boundary edge and the south/bottom location one particle diameter distance away IF (DES_BC_Y_S $(B C V)==$ DES_BC_Y_n $(B C V))$ THEN 


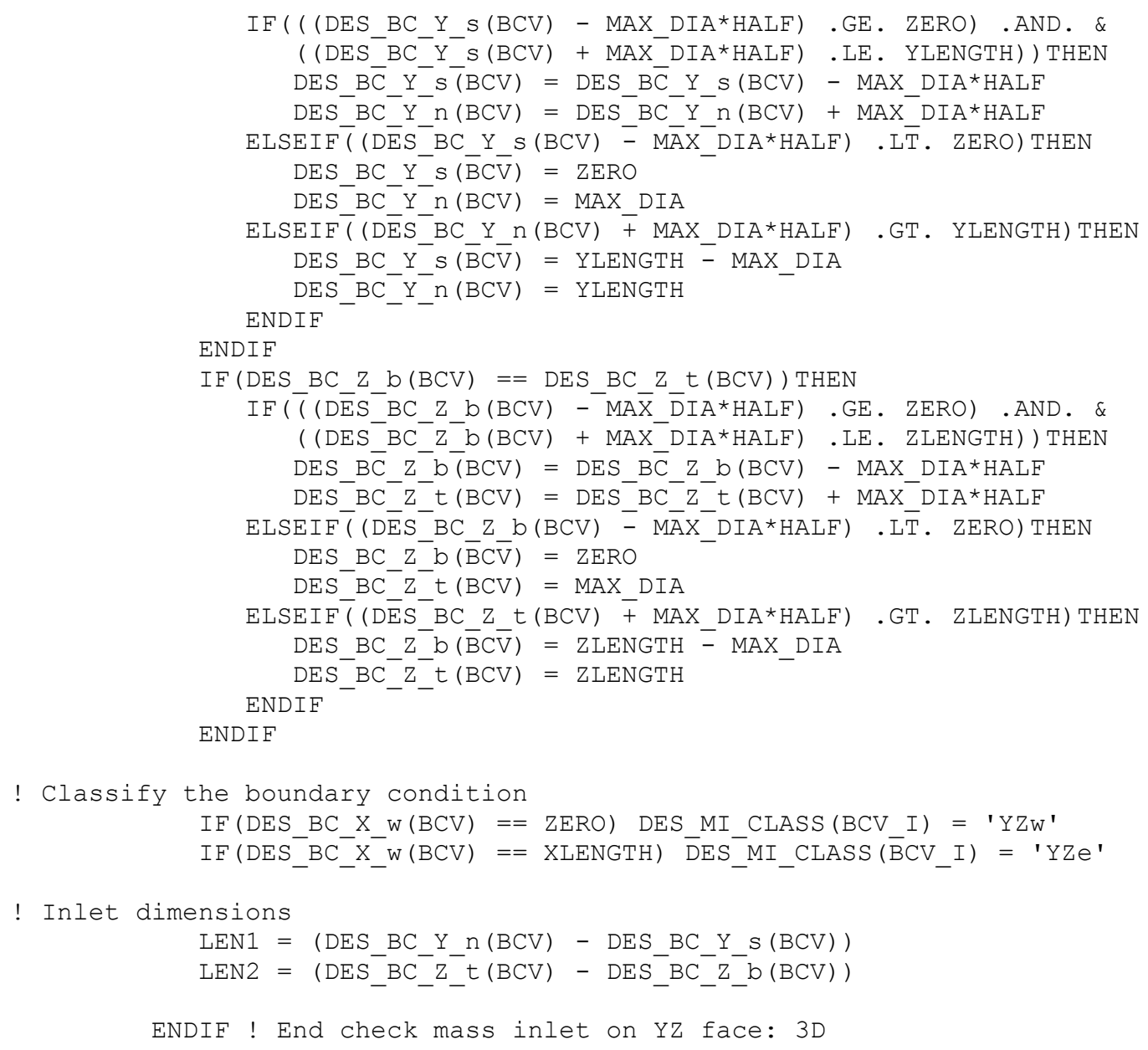

! No YS, YN, XZS or XZn inlet with Y direction periodic walls 


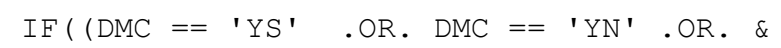

$\mathrm{DMC}==$ 'XZS' $^{\mathrm{O}} \mathrm{OR}$. DMC $==$ 'XZn'). AND. DES PERIODIC WALLS Y) THEN WRITE(UNIT_LOG, 1202 ) BCV, 'DES_PERIODIC_WĀLLS_Y'

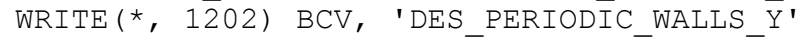
ENDIF

CALL MFIX_EXIT (mYPE)

! No XYb or XYt inlet with $Z$ direction periodic walls

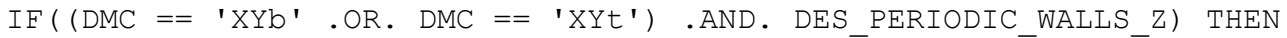
WRITE (UNIT LOG, 1202) BCV, 'DES PERIODIC W̄ WLLS Z'

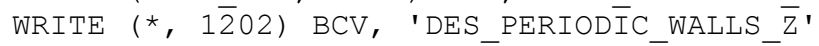

CALL MFIX_EXIT (mYPE)

ENDIF

! Set flags for individual mass inlets for used in the grid based search

! Flag that a X-face inlet exits

$\operatorname{IF}\left(\mathrm{DMC}==\mathrm{XWW}^{\prime} . \mathrm{OR} . \mathrm{DMC}==\mathrm{XE}^{\prime} . \mathrm{OR} . \&\right.$

$\mathrm{DMC}==$ ' $Y Z W '^{\prime} \mathrm{OR} . \mathrm{DMC}==$ 'YZe') THEN

DES_MI_X $=$.TRUE.

ENDIF

! Flag that a Y-face inlet exits

$I F(D M C==$ 'YS' . OR. DMC $==$ 'YN'.OR. \&

$\mathrm{DMC}==$ 'XZS' $^{\prime} \mathrm{OR} \cdot \mathrm{DMC}==$ 'XZn') THEN ENDIF

DES_MI_Y $=$.TRUE.

! Flag that a Z-face inlet exits

$\mathrm{IF}(\mathrm{DMC}==$ 'XYb' $\mathrm{OR}$. $\mathrm{DMC}==$ 'XYt') THEN

ENDIF

DES_MI_Z $=$.TRUE.

1202 FORMAT $(/ 1 \mathrm{X}, 70(' *$ ')//, ' From: DES_MI_CLASSIFY - ', /, \&

' Message : DEM inlet can not be $\bar{p}$ laced on a periodic', \&

' boundary.',/10X,' Check DEM boundary condtion ',I3, \&

' and ', A, '.', /1X,70('*')/)

RETURN

END SUBROUTINE DES_MI_CLASSIFY 
INTEGER LC EXIT

! Velocity minimizing the error for the PReVious and CuRrent iterations DOUBLE PRECISION CUR_VEL, PRV_VEL

! Incremental velocity stē size (dēlta velocity) DOUBLE PRECISION DELTA_VEL

! Incremental step velocity DOUBLE PRECISION STP VEL

! Total error for all solid phase generated by STP_VEL DOUBLE PRECISION ERR TOT

! Minimum error generated on current iteration DOUBLE PRECISION ERR_MIN

! Convergence tolerance DOUBLE PRECISION, PARAMETER : : TOL $=1.0 \mathrm{e}-4$

Initialize

MIN VEL = LARGE NUMBER

MAX_VEL $=$ ZERO

! BC inlet area

! To calculate velocity based on specified mass/volumetric inflow and

! bulk density the inlet needs to have dimension of length^2 (area).

! In the 2D case the depth of the inlet is taken to be the maximum

! particle diameter which is consistent with how the criteria for

! maximum and minimum velocity for the boundary are defined and how

! particles are seeded for a 2D inlet (particles are seeded along a

! line that has a depth equal to the maximum particle diameter).

! Alternatively, the depth of the inlet could be taken as zlength,

! but zlength is not guaranteed to be equal to the maximum particle

! diameter.

BCV_AREA $=$ LEN1 $*$ LEN2

! Calculate the individual velocities for each solid phase

$\mathrm{DO} \mathrm{MM}=1$, PHASE CNT

$\mathrm{M}=$ PHASE LIST $(\mathrm{MM})$

! Solids volume fraction for phase $M$

$\mathrm{EP} \_\mathrm{SM}=$ DES_BC_ROP_s $(\mathrm{BCV}, \mathrm{M}) / \mathrm{RO} \_\mathrm{s}(\mathrm{M})$

! Inlet velōity for solids phase M

TMP_VEL $(\mathrm{M})=(\mathrm{DES} B \mathrm{BC}$ VOLFLOW_S $(\mathrm{BCV}, \mathrm{M}) / \&$

$\bar{B} C V$ AREA ) / $\mathrm{EP}^{-} \mathrm{SM}^{-}$

! Check for min/max inlet vêlocity MIN_VEL $=$ MIN (ABS (TMP_VEL (M)), MIN_VEL) ENDDO

MAX_VEL $=\operatorname{MAX}(\mathrm{ABS}(\mathrm{TMP}-\mathrm{VEL}(\mathrm{M}))$, MAX_VEL)

! Determine necessary uniform inlet velocity

IF (MIN VEL $==$ MAX VEL) THEN

ELSE

$\mathrm{UI} \overline{\mathrm{V}} \mathrm{EL}=\mathrm{MIN} \_\mathrm{VEL}$

! Initialize Values

LC EXIT $=0$

PRV_VEL $=$ UNDEFINED

CUR_VEL $=$ ZERO

DO WHILE (ABS (CUR_VEL - PRV_VEL) >TOL)

! Initialize Values

! Store current velocity value in previous velocity PRV VEL = CUR VEL

! Set minimum error valūe to a large number ERR_MIN = LARGE_NUMBER

! Determine s̄ep size between tested velocity values DELTA_VEL $=($ MAX_VEL-MIN_VEL) /DBLE (NUM_VEL) 
! Check i=NUM VEL velocites between MAX VEL and MIN VEL for one that

! minimizes the total error between calculated bulk density values

! and the values provided in the mfix.dat file. The first loop (i=1)

! acts to initialize the values of cur vel and err min. DO $I=1, \quad($ NUM VEL +1$)$

! Initialize values STP VEL $=$ MIN VEL + DELTA VEL* $(I-1)$ ERR_TOT $=$ ZERO

! Calculate total êrror over all solid phases with respect to STP VEL $\mathrm{DO} \mathrm{MM}=1$, PHASE CNT $\mathrm{M}=$ PHASE LIST $(\mathrm{MM})$

! Calculate bulk density value based upon velocity CAL ROPSM $=($ RO $S(M) *$ DES BC VOLFLOW S $(B C V, M)) / \&$ (STP VEL * BCV AREA) ENDDO ERR TOT ${ }^{-}=$ERR_TOT+ABS (DES_BC_ROP_S (BCV,M) -CAL_ROPSM)

! Compare to determine if ERR_TOT is minimum error over all STP_VEL IF (ERR_TOT $==\bar{M} I N(E R R$ TOT, ERR MIN)) THEN ERR MIN $=$ ERR TOT ENDIF CUR VEL $=$ STP VEL

! Narrow search range for next iterative set. These values only change

! if cur_vel is updated which only occurs when the current stp_vel gives

! a smalier total error than previously MIN_VEL $=$ CUR_VEL - DELTA VEL ENDDO MAX_VEL $=$ CUR_VEL + DELTA_VEL

! Loop control to prevent hang up. * This should not be necessary * LC_EXIT $=$ LC_EXIT +1

IF $($ LC EXIT $>-100)$ THEN $\operatorname{WRITE}(*, 1256) \mathrm{BCV}$ EXIT ENDIF

ENDDO ENDIF

$\mathrm{UI}$ VEL $=$ CUR_VEL

! Assign the uniform inlet velocity to each solid phase DMCL $=$ DES_MI_CLASS $\left(\right.$ BCV_I $\left._{-}\right)$

$\mathrm{DO} \mathrm{MM}=1$, PHASE_CNT

$\mathrm{M}=$ PHASE_LIST $(\mathrm{MM})$

IF (DMCL $=\overline{=}$ ' $X W$ ' $. \mathrm{OR} . \mathrm{DMCL}==$ 'YZW') THEN DES BC U S (BCV) = UI VEL

$\operatorname{ELSEIF}\left(\mathrm{DMC} \bar{L}^{-}==\mathrm{XE}^{\prime}, \mathrm{OR} .{ }^{-} \mathrm{DMCL}==\right.$ 'YZe') THEN DES_BC_U_S $(B C V)=-U I \_V E L$

ELSEIF $\overline{(D M C L}-==$ 'YS' .OR. ${ }^{-}$DMCL $==$'XZS') THEN DES_BC_V_S $(B C V)=$ UI_VEL

ELSEIF $(D M C L==' Y N$ ' OR. DMCL $==$ 'XZn') THEN DES BC V S (BCV) = -UI VEL

ELSEIF $(\mathrm{DMC} \bar{C}-==$ 'XYb') THEN DES_BC_W_S $(B C V)=$ UI_VEL ELSEIF $\left(\mathrm{DMC} \overline{\mathrm{C}}{ }^{-}==\right.$'XYt') THEN ELSE DES_BC_W_S $(B C V)=-U I \_V E L$

WRITE (*, 1257) BCV, DMCL CALL MFIX_EXIT (mYPE) ENDIF ENDDO

! Output the new data values (screen/log file) $\operatorname{WRITE}(*, 1249)$

! Table Header 
WRITE $(*, 1250) \quad \mathrm{BCV}$

$\operatorname{WRITE}(*, 1251)$ UI VEL

$\operatorname{WRITE}(*, 1252)$

WRITE (UNIT LOG, 1250) BCV

WRITE (UNIT_LOG, 1251) UI_VEL

WRITE (UNIT_LOG, 1252)

! Column Labels

$\operatorname{WRITE}(*, 1253)$

$\operatorname{WRITE}(*, 1254)$

$\operatorname{WRITE}(*, 1253)$

WRITE (UNIT LOG, 1253)

WRITE (UNIT_LOG, 1254)

WRITE (UNIT_LOG, 1253)

! Fill Table Rows

$\mathrm{DO} \mathrm{MM}=1$, PHASE $\mathrm{CNT}$

$\mathrm{M}=$ PHASE LIST $(\mathrm{MM})$

! Calculate bulk density value based upon velocity

CAL ROPSM $=($ RO $S(M) *$ DES BC VOLFLOW $S(B C V, M)) / \&$

(UI VEL * BCV $\bar{V}$ AREA)

ERR ROP $\overline{S M}=\overline{A B S}(\mathrm{DES} B C$ ROP $S(B C V, M)-C A L$ ROPSM)

WRITE (*,1255) M, DES BC ROP ${ }^{-}{ }^{-}(B C V, M), C A L \overline{R O P S M}$, ERR ROPSM

$\operatorname{WRITE}(*, 1253)$

WRITE (UNIT_LOG, 1255) M, DES_BC_ROP_S(BCV,M), CAL_ROPSM, \& ERR ROP $\bar{S} M$

WRITE (UNNIT_LOG, 1253)

ENDDO

$\operatorname{WRITE}(*, "(/ /)))$

WRITE (UNIT_LOG," (//)")

1249 FORMAT (//,5X, 'FrOm: DES_BC_VEL_ASSIGN - ')

1250 FORMAT (5X, '|<--- Boundary Condition ', I2, 1X, 26 ('-' ), '>|' )

1251 FORMAT (5X,' ' Uniform Inlet Velocity: ',ES11.4,18(' ' ), '|')

1252 FORMAT (5X,' ' Adjusted DES_BC_ROP_s Values',25(' '), '।')

1253 FORMAT $(5 X, ' \mid ', 54('-'), '$ ' ' $)$

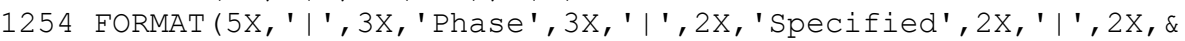

'Calculated', 2X, '|',2X, 'ABS Error', 2X, '।')

1255 FORMAT (5X, '।' , 4X, I2, 5X, '|' , 1X, ES11.4, 1X, '|' , 1X, ES11.4, 2X, '। ', \& $1 \mathrm{X}, \operatorname{ES} 11.4,1 \mathrm{X}, \mathbf{\prime}^{\prime}$ ')

1256 FORMAT $\left(/ 1 \mathrm{X}, 70\left({ }^{\prime} * \prime\right) / /, '\right.$ From: DES BC VEL ASSIGN -', /, \&

' Message : Tolerance not met on uniform inlet velocity for', \&

/10X, 'boundary ', I3, /1X,70('*')/)

1257 FORMAT (/1X,70('*' )//,' From: DES_BC_VEL_ASSIGN - ', /, \&

' Message : INVALID BOUNDARY CLASSIFICATION FOR BOUNDARY', I3, \&

/10X, 'with classification ',A,/1X,70('*')/)

RETURN

END SUBROUTINE DES_BC_VEL_ASSIGN 


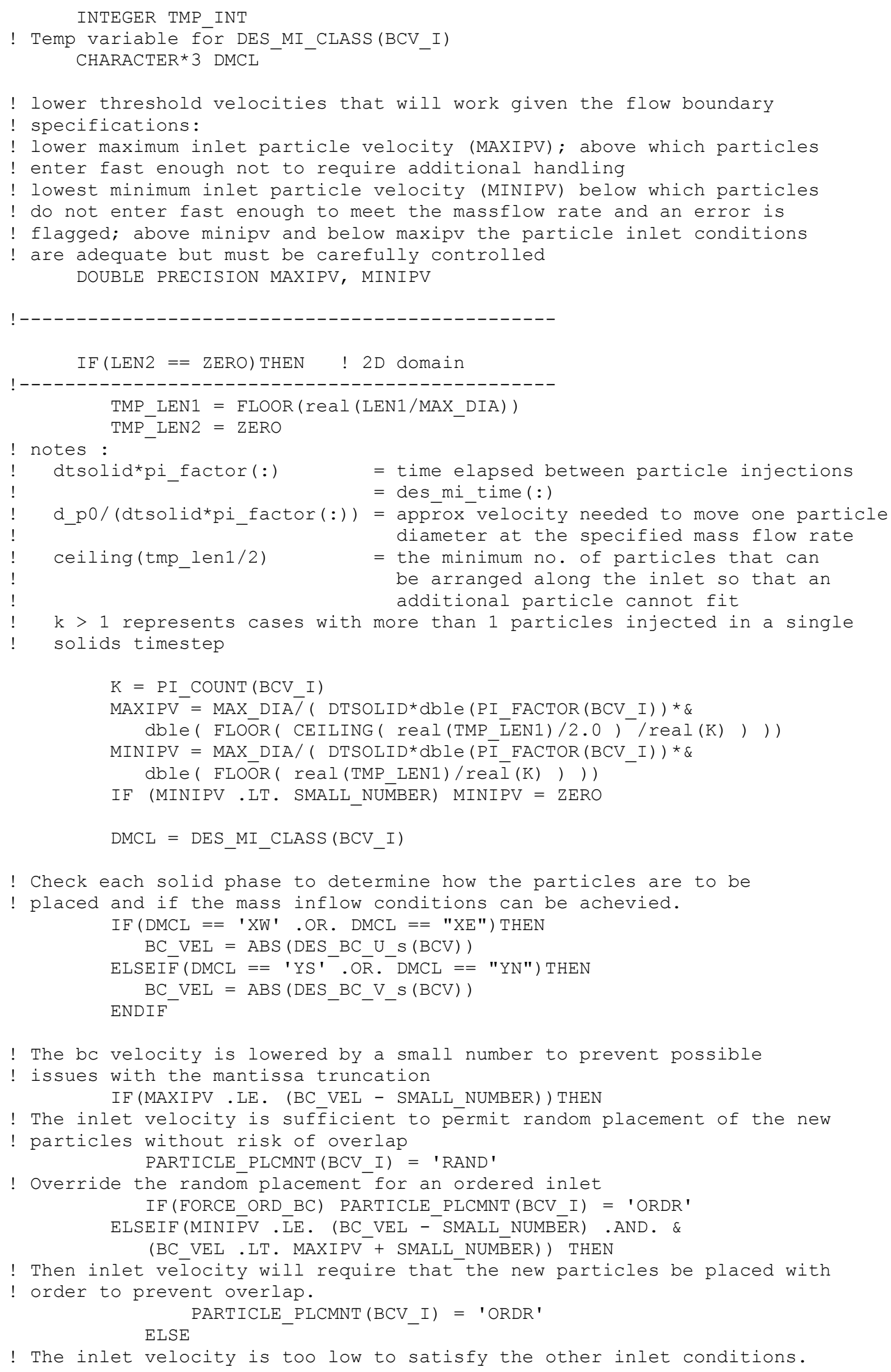


! Determine maximum possible ROP s values that will satisfy the inlet

! conditions. Flag error, prompt with new values, and exit. WRITE (UNIT LOG, 1300) BCV

WRITE $(*, 13 \overline{0} 0)$ BCV

$\mathrm{DO} \mathrm{MM}=1$, PHASE_CNT

$\mathrm{M}=$ PHASE_LIST $(\mathrm{MM})$

! Even though the system is $2 \mathrm{D}$, an area of the inlet is needed for

! the following calculation. This 'depth' is taken as max dia. MAX_ROPS $=\left(\right.$ DES_BC_VOLFLOW_S $\left.(B C V, M) * R O \_s(M)\right) / \&$

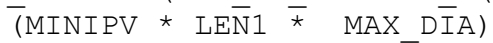

WRITE (UNIT_LOG, 1301) BCV, M, MAX_ROPS

ENDDO

WRITE (*,130 1 ) BCV, M, MAX ROPS

WRITE (UNIT_LOG, 1302)

$\operatorname{WRITE}(*, 13 \overline{0} 2)$

ENDIF

CALL MFIX_EXIT (mYPE)

IF (PARTICLE_PLCMNT (BCV_I) $==$ 'ORDR') THEN

! In 2D calculate the approx. pārticle line density (e.g., the no. of

! particles along the inlet) needed to achieve the specified particle

! mass flow rate and particle velocity;

TMP_FACTOR = CEILING $($ real $($ MAX DIA $/ \&$

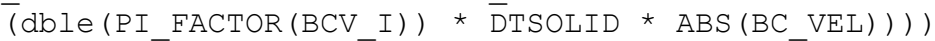

ALLOCATE ( MI ORDER (BCV I) OVALUE ( TMP FACTOR )')

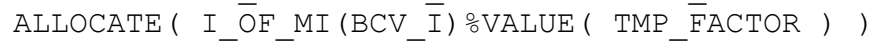

! Initialize

MI_ORDER (BCV_I) VALUE (:) $=-1$

MI $\operatorname{FACTOR}(\mathrm{BC} \overline{\mathrm{V}} \mathrm{I})=1$

! Dimension of grid cell; this may be larger than than the particle

! diameter but not smaller

MI_WINDOW (BCV_I) $=$ LEN1/dble (TMP_FACTOR)

$\mathrm{DO}^{-} \mathrm{I}=1, \mathrm{TMP}^{-} \mathrm{FACTOR}$ ENDD

I_OF_MI $\left(B C \bar{V} \_I\right) \circ \operatorname{VALUE}(I)=I-1$

! Construct an array of integers from 1 to TMP_FACTOR in a random

! order. This is used when placing new particles.

$\mathrm{LL}=1$

DO WHILE (MI_ORDER (BCV_I) \%VALUE (TMP_FACTOR) .EQ. -1)

CALL RANDOM NUMBER (TMP DP)

TMP INT $=$ CE ILING (real $($ TMP_DP*dble $($ TMP_FACTOR) ))

DO $\overline{\mathrm{L} C}=1, \mathrm{LL}$

IF (TMP INT .EQ. MI ORDER (BCV I) VALUE (LC) ) EXIT

IF ( LC .EQ. LL) THEN

MI_ORDER (BCV_I) VALUE $(\mathrm{LC})=$ TMP_INT

$\mathrm{LL}^{-}=\mathrm{LL}+1$

ENDIF

ENDDO

ENDDO

ENDIF ! endif particle_plcmnt(bcv_i) == 'ordr'

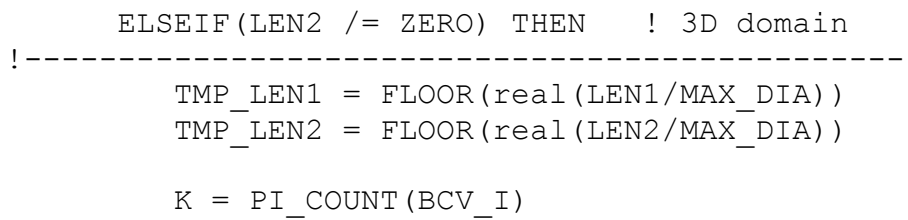

! In the 3D case the calculation for MAXIPV is conservative. That is, ! the actual bc velocity could be somewhat lower than the calculated

! value of MAXIPV and still allow for random particle placement 


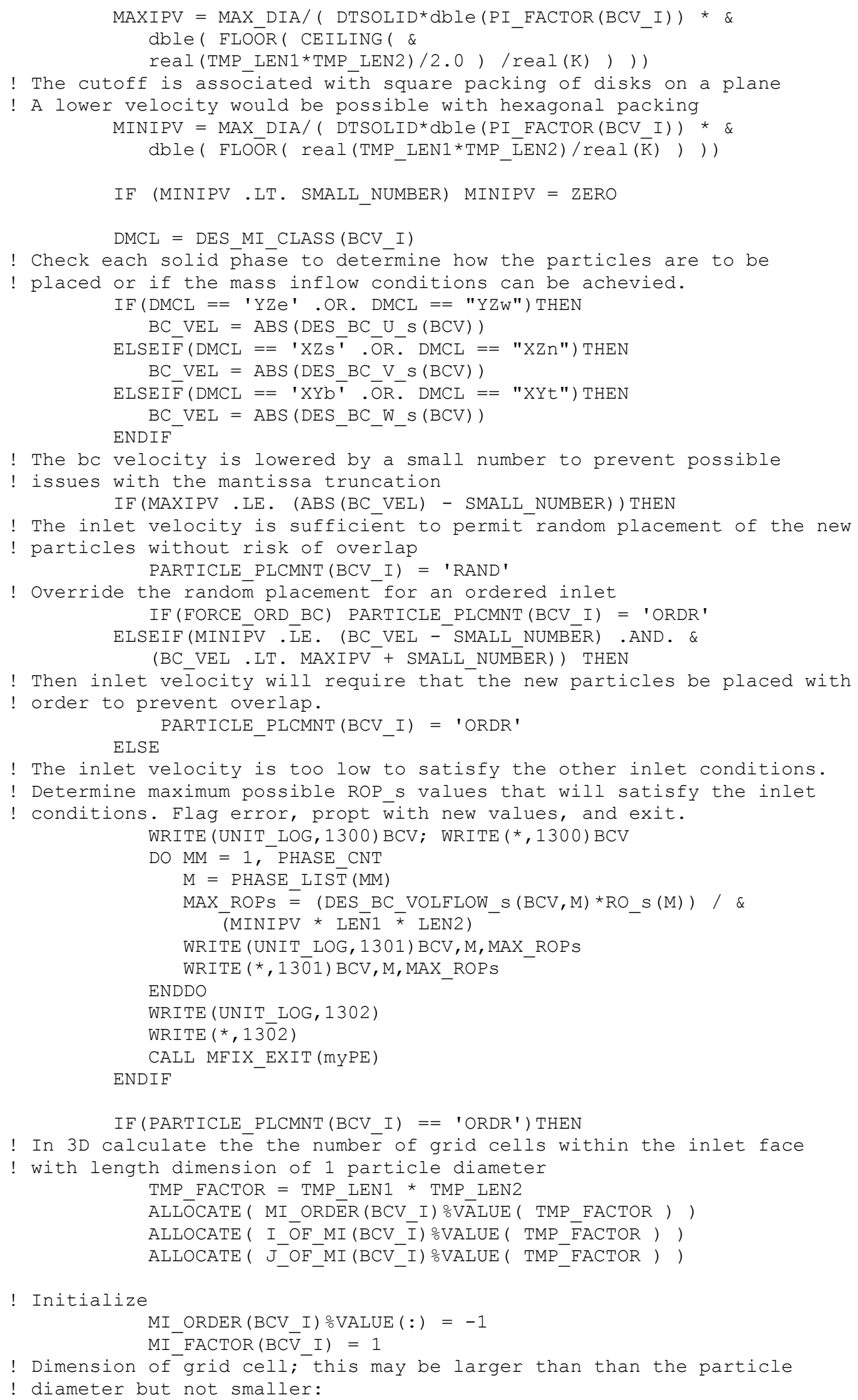




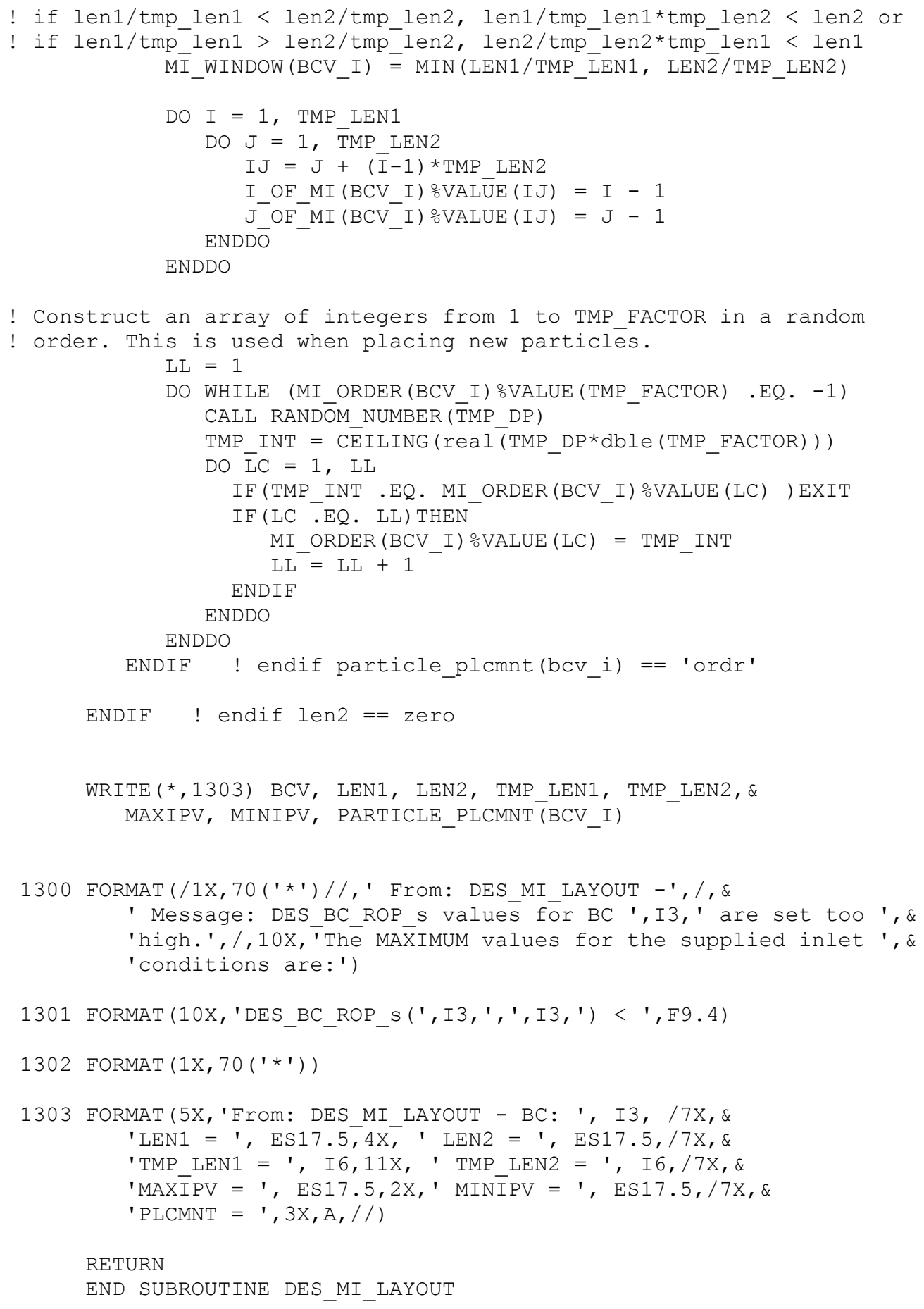




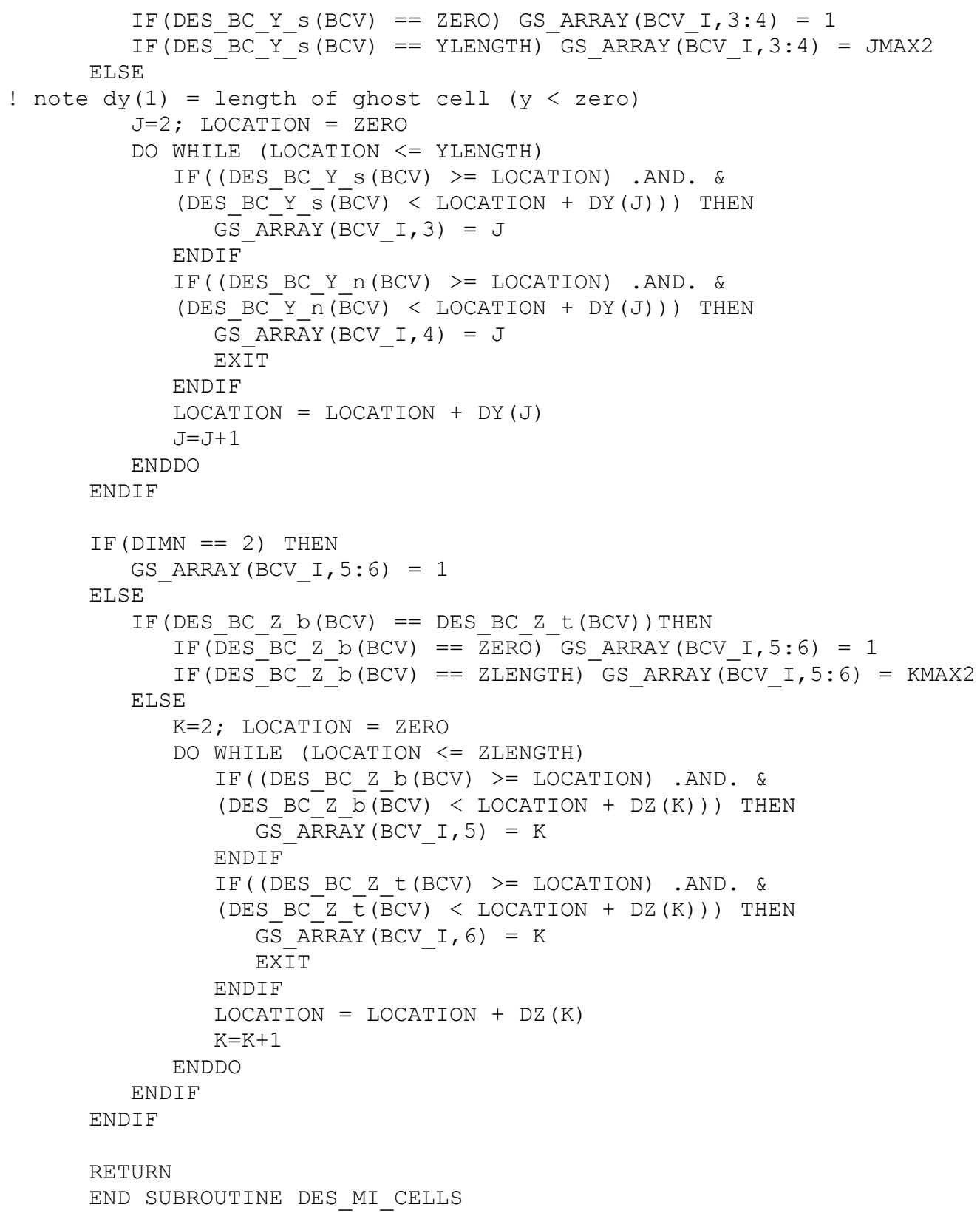




\section{C.10 : Routine - DES_MO_CLASSIFY}

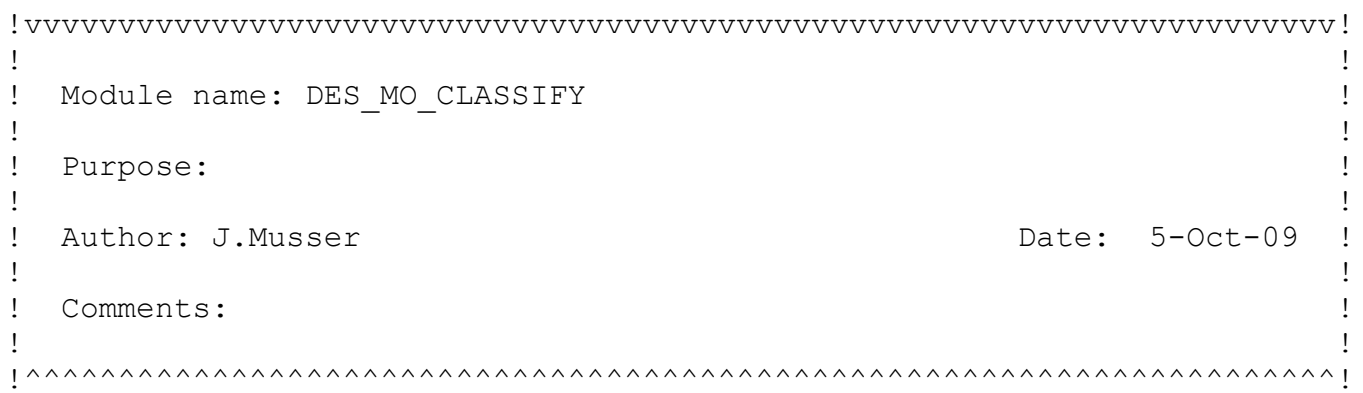

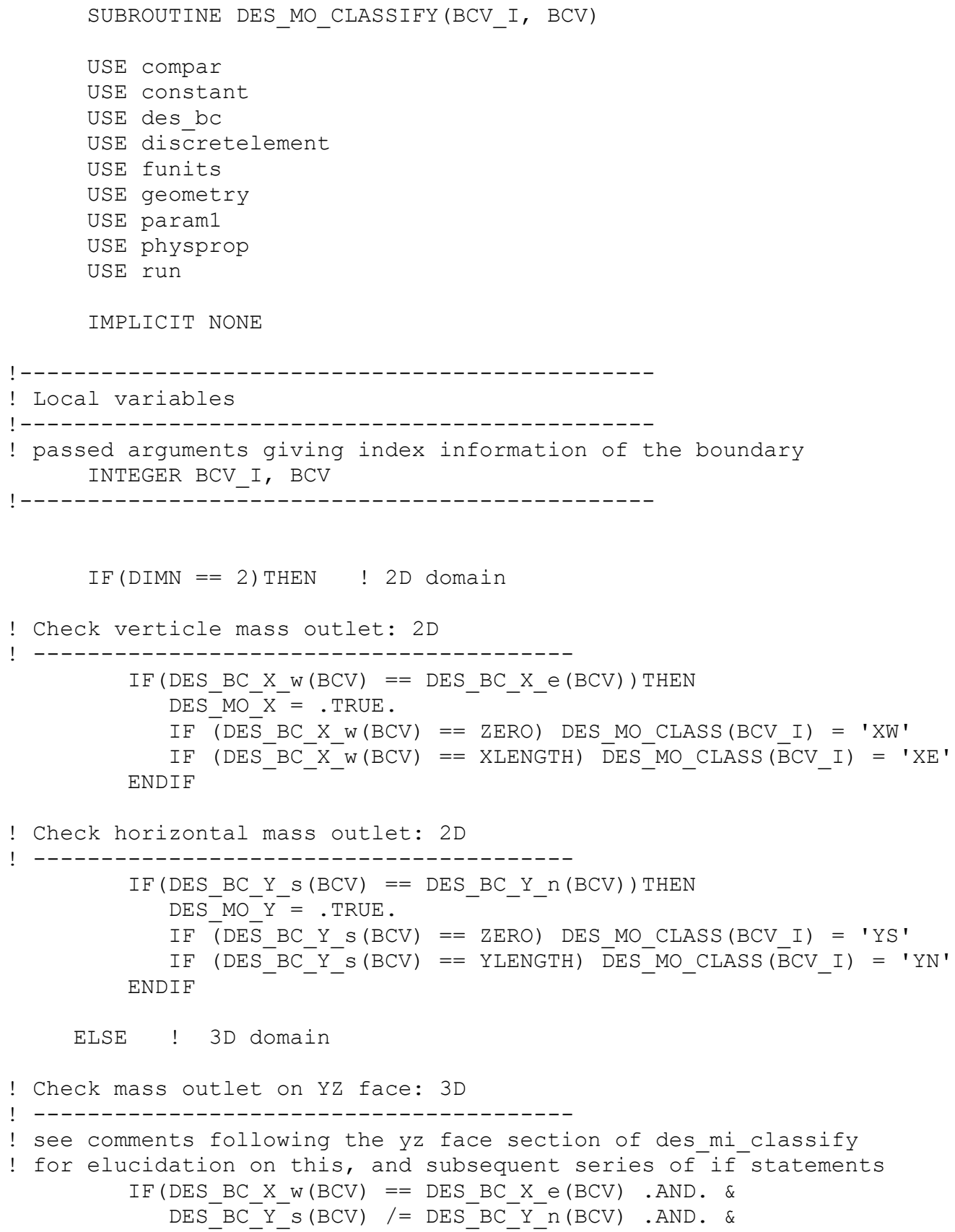




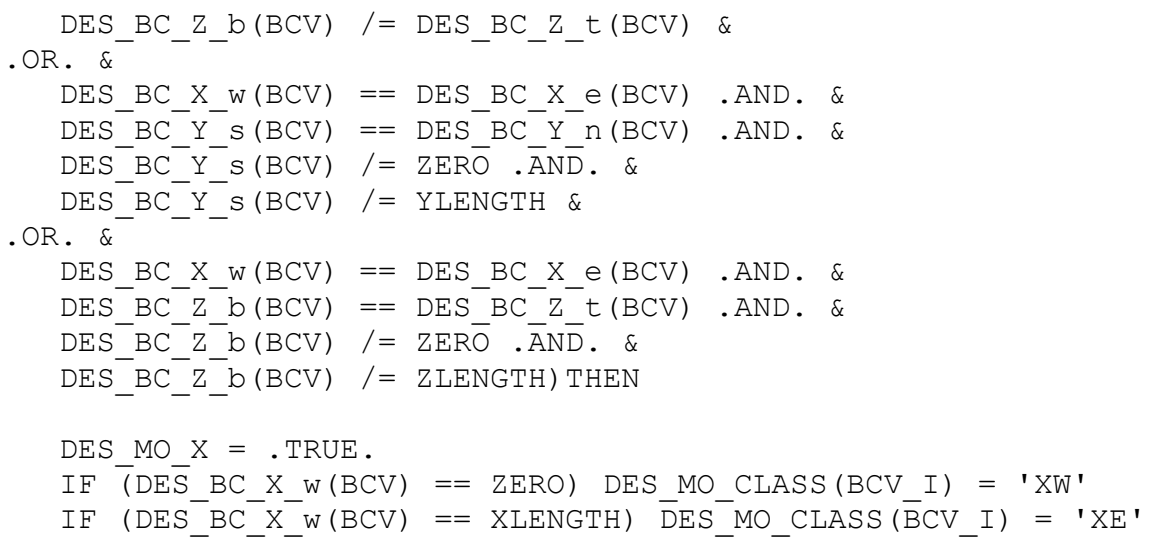

! End check mass outlet on YZ face: 3D

! Check mass inlet on XZ face: 3D

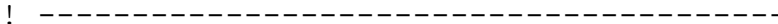

$\mathrm{IF}(\mathrm{DES} B \mathrm{BC} \quad \mathrm{Y} \mathrm{S}(\mathrm{BCV})==\mathrm{DES} \quad \mathrm{BC} \mathrm{Y} \cap(\mathrm{BCV})$. AND. \& 
ENDIF

! End check mass outlet on XY face: 3D

ENDIF ! endif dimn $==2$

! Verify that an inlet is not on a face that is connected to a periodic

! boundary condition. If so, write error message and exit.

! No Xew outlet with $\mathrm{X}$ direction periodic walls

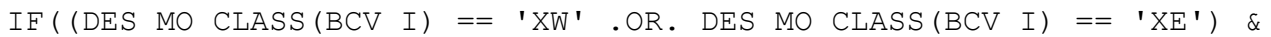
. AND. DES PERIODIC WALLS X) THEN

WRITE (UNIT LOG, $15 \overline{0} 0$ ) BCV , 'DES_PERIODIC_WALLS X'

WRITE (*, 1500) BCV, 'DES_PERIODIC_WALLS_ $\bar{X}$ ' ENDIF

CALL MFIX_EXIT (mYPE)

! No Ysn outlet with Y direction periodic walls

IF $\left(\left(D E S \_M O \_C L A S S\left(B C V \_I\right)==\right.\right.$ 'YS' .OR. DES_MO_CLASS $\left.\left(B C V \_I\right)==' Y N '\right)$ \& . AND. DES PERIODĪC WALLS Y) THEN

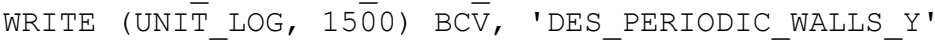

$\operatorname{WRITE}(*, 1 \overline{5} 00) \mathrm{BCV}$, 'DES_PERIODIC_WALLS_ $\bar{Y}$ '

ENDIF

CALL MFIX EXIT (myPE)

! No Zbt outlet with Z direction periodic walls

IF ( (DES MO CLASS (BCV I) == 'ZB'.OR. DES MO CLASS (BCVI) =='ZT') \& . AND. DES_PERIODIC_WALLS_Z) THEN

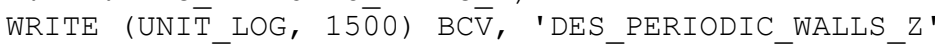

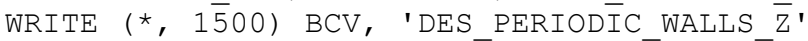

CALL MFIX_EXIT (mYPE)

ENDIF

1500 FORMAT (/1X,70('*')//,' From: DES MI CLASSIFY - ',/10X, \&

'DEM outlets can not be place $\bar{d}$ on a periodic boundary.', /10X, \&

'Check DEM boundary condtion ', I2,' and ',A,'.',/1X,70('*')/)

RETURN

END SUBROUTINE DES_MO_CLASSIFY 


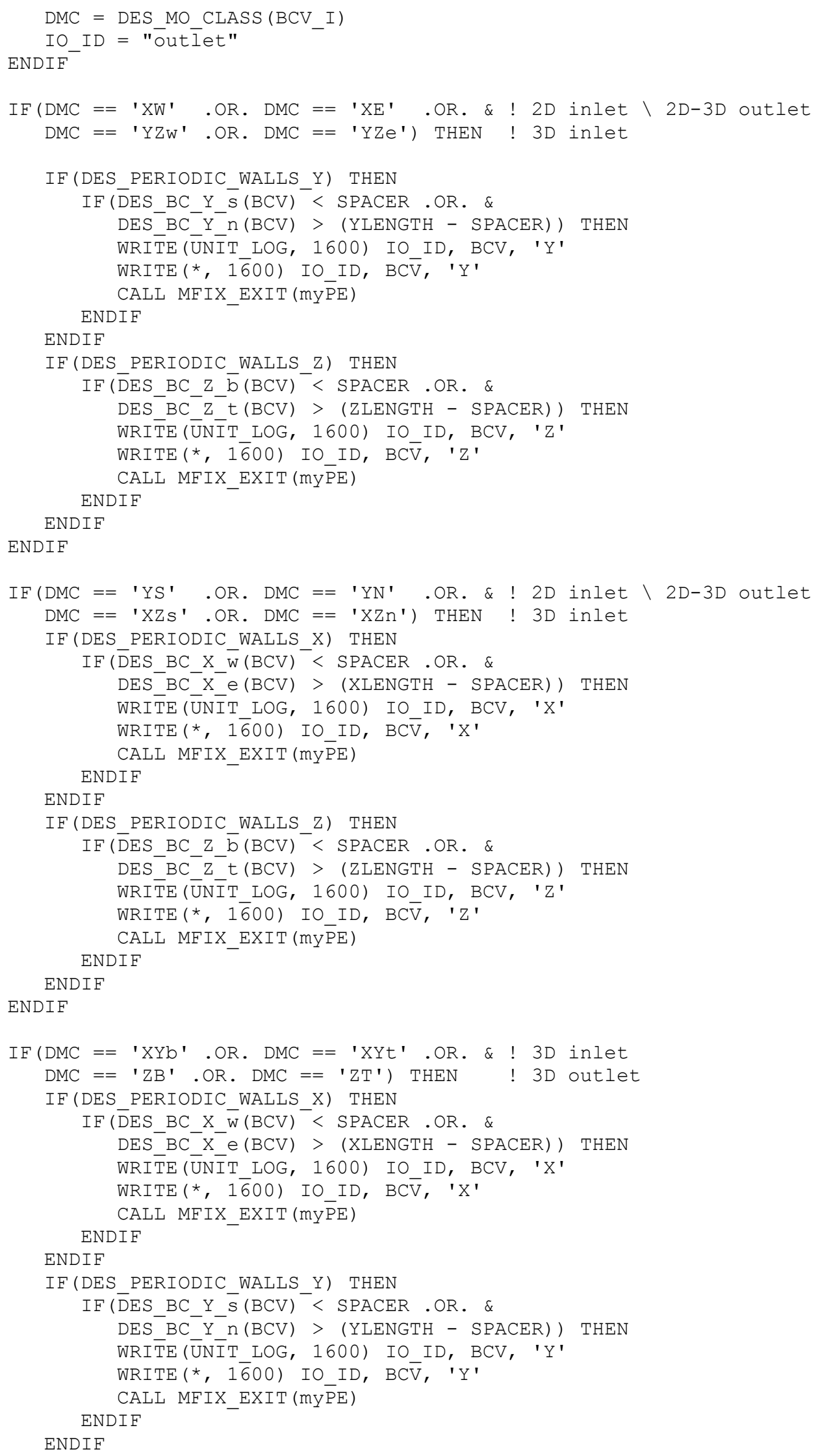


ENDIF

1600 FORMAT $(/ 1 X, 70(' * ') / /$, ' From: CHECK_DES_BC - ', /, \&

' Message: DES ',A, ' boundary ', $\overline{\mathrm{I}} 3,{ }^{\prime}$ ' is too close to', \&

' DES_PERIODIC_WALLS_', A, '.', /1X, $70(' * 1) /$ )

RETURN

END SUBROUTINE DES_MIO_PERIODIC 


\section{C.12 : Routine - DES_MASS_INLET}

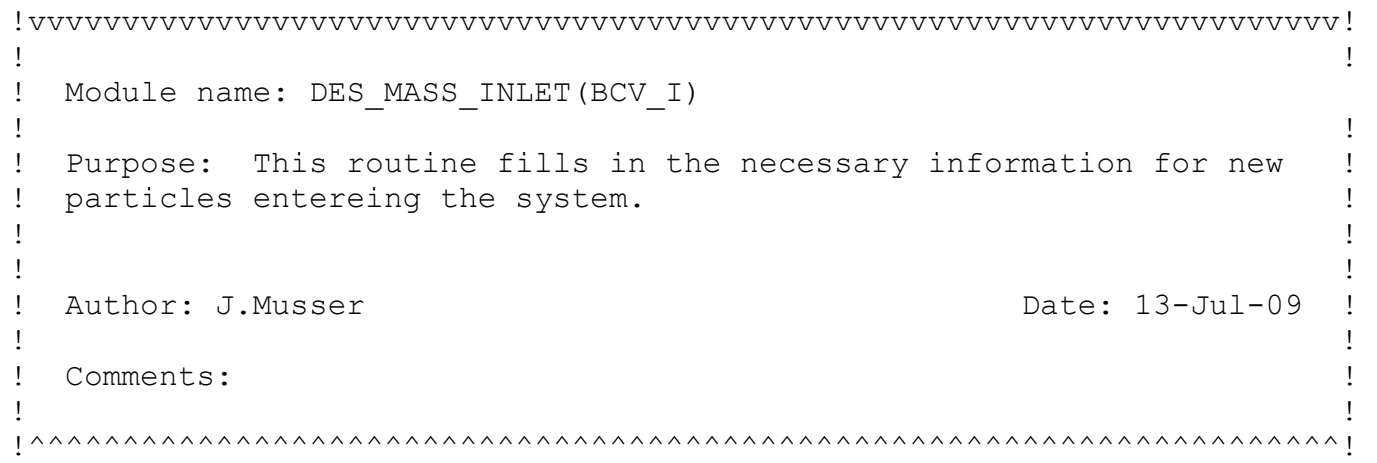

SUBROUTINE DES_MASS_INLET (BCV_I)

USE compar

USE constant

USE des bc

USE discretelement

USE funits

USE geometry

USE indices

USE param1

USE physprop

IMPLICIT NONE

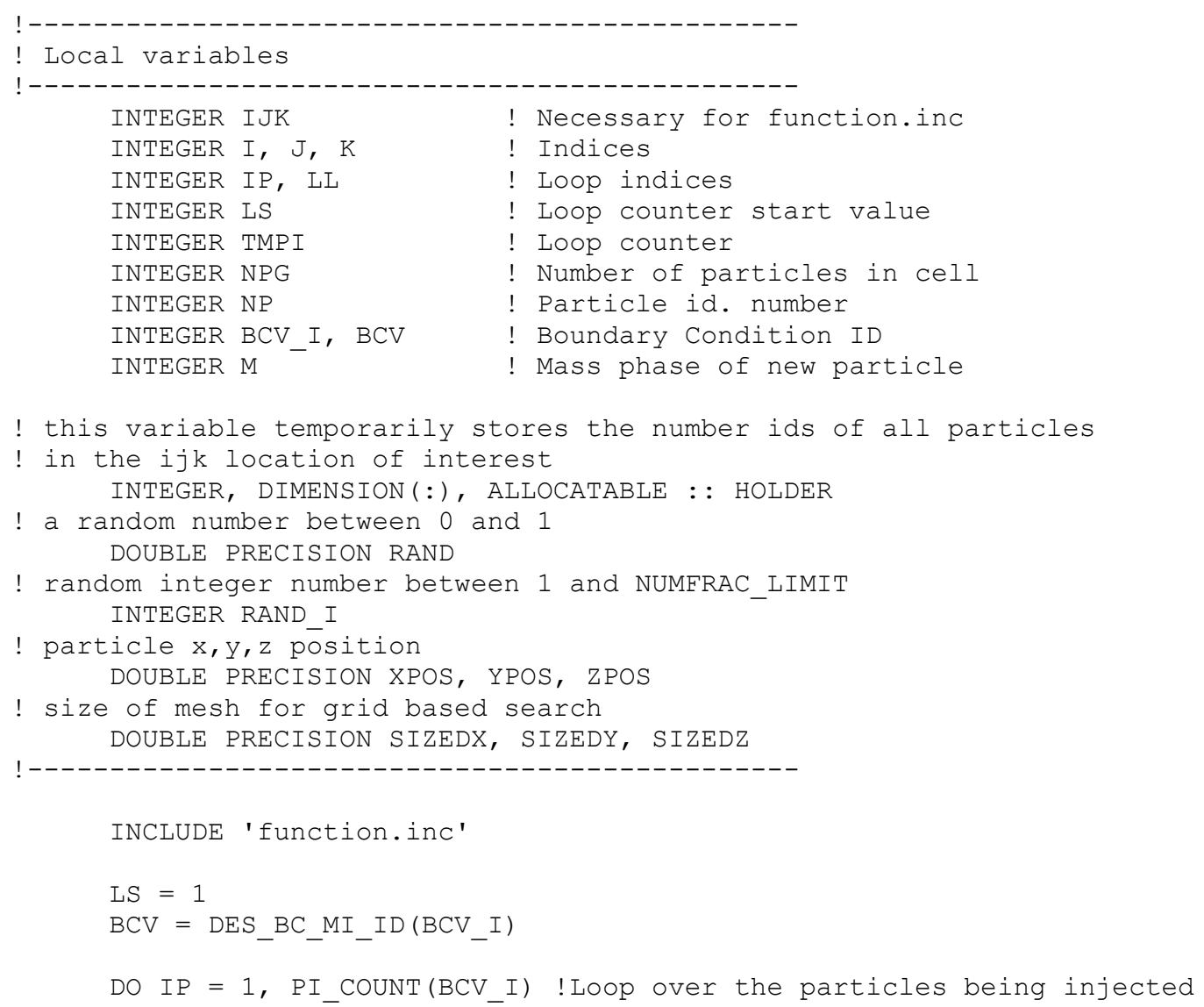


! Check to see if MAX PIS has been exceeded, if so, STOP

IF (PIS . GE. MAX PIS) THEN

WRITE (UNIT LOGG, 1000 )

$\operatorname{WRITE}(*, 1 \overline{0} 00)$

CALL MFIX EXIT (MYPE)

ENDIF

! Find the first free space in the particle existance array

DO NP = LS, MAX PIS

IF (.NOT.PEA $(\bar{N} P, 1))$ THEN

$\mathrm{LS}=\mathrm{NP}$

EXIT

ENDIF

ENDDO

! Set the flag in the particle existance array

$\operatorname{PEA}(\mathrm{NP}, 1)=$. TRUE.

! Set the flag that the particle is new. This allows it to be ignored

! by various subroutines $\mathrm{PEA}(\mathrm{NP}, 2)=$. TRUE.

! Increment the particle in system value by one $\mathrm{PIS}=\mathrm{PIS}+1$

! Obtain a mass phase to assign to the incoming particle IF (DES BC POLY (BCV I)) THEN

CAL $\bar{L}$ RĀNDOM NUMB ER (RAND)

! Scale and convert random number to an interger RAND I = CEILING (dble (NUMFRAC LIMIT)*RAND) ELSE $\mathrm{M}=\overline{\mathrm{DES}}$ BC POLY LAYOUT (BCV_I, RAND_I) ENDIF

$\mathrm{M}=$ DES_BC_POLY_LAYOUT $(\mathrm{BCV} I, 1)$

! Set the initial velocity values

$\operatorname{DES} \operatorname{VEL} \mathrm{OLD}(\mathrm{NP}, 1)=\mathrm{DES} B C \mathrm{U} S(\mathrm{BCV})$

$\mathrm{DES} \mathrm{V}^{-} \mathrm{VL}^{-} \mathrm{OLD}(\mathrm{NP}, 2)=\mathrm{DES}^{-} \mathrm{BC}^{-} \mathrm{V}^{-} \mathrm{S}(\mathrm{BCV})$

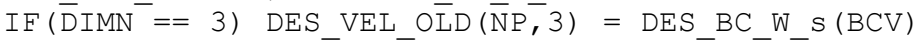

$\operatorname{DES} \operatorname{VEL} \_\mathrm{NEW}(\mathrm{NP}, \mathbf{:})=\mathrm{DES} \operatorname{VEL} \operatorname{OLD}(\mathrm{NP}, \mathbf{:})$

! Set the initial angular velocity values

$\mathrm{OMEGA} \operatorname{OLD}(\mathrm{NP},:)=0$

$\mathrm{OMEGA} \mathrm{NEW}(\mathrm{NP},:)=0$

! Set the particle radius value

DES_RADIUS $(\mathrm{NP})=\left(\mathrm{D}_{-} \mathrm{PO}(\mathrm{M}) * \mathrm{HALF}\right)$

! Set the particle density value RO_SOl (NP) $=$ RO_S $(\mathrm{M})$

! Set the particle mass phase $\operatorname{PIJK}(N P, 5)=M$

! Calculate the new particle's Volume, Mass, OMOI PVOL $(\mathrm{NP})=(4.0 \mathrm{~d} 0 / 3.0 \mathrm{~d} 0) * \mathrm{PI} * \mathrm{DES} \operatorname{RADIUS}(\mathrm{NP}) \star * 3$

$\operatorname{PMASS}(\mathrm{NP})=\operatorname{PVOL}(\mathrm{NP}) * \mathrm{RO} \operatorname{SOl}(\mathrm{NP})$

$\operatorname{OMOI}(\mathrm{NP})=5 . \mathrm{d} 0 /(2 . \mathrm{d} 0$ * PMASS $(\mathrm{NP}) \star$ DES_RADIUS $(\mathrm{NP}) \star * 2)$

! Set the initial position values based on mass inlet class CALL DES_PLACE_NEW_PARTICLE (NP, BCV_I) 


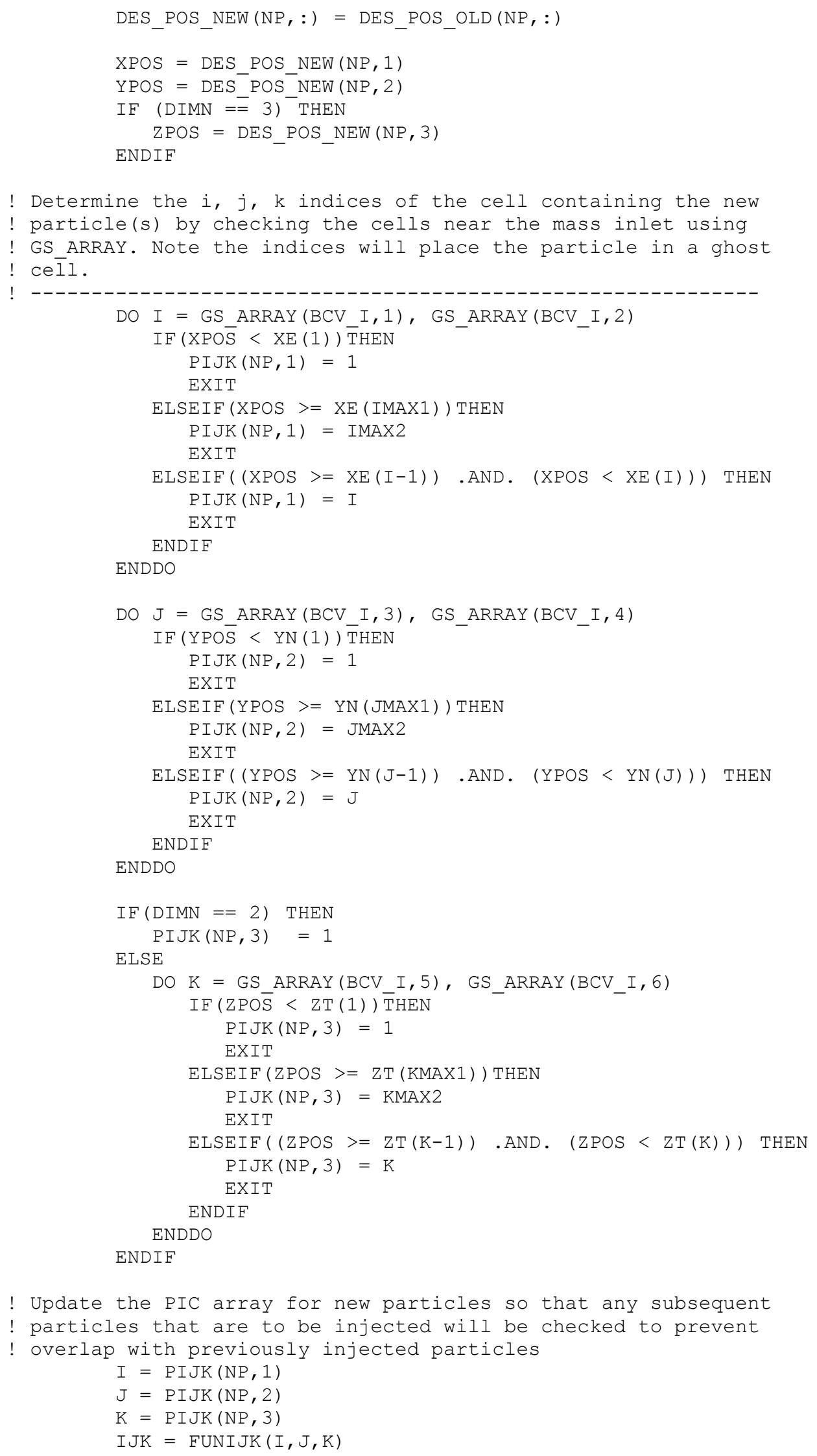




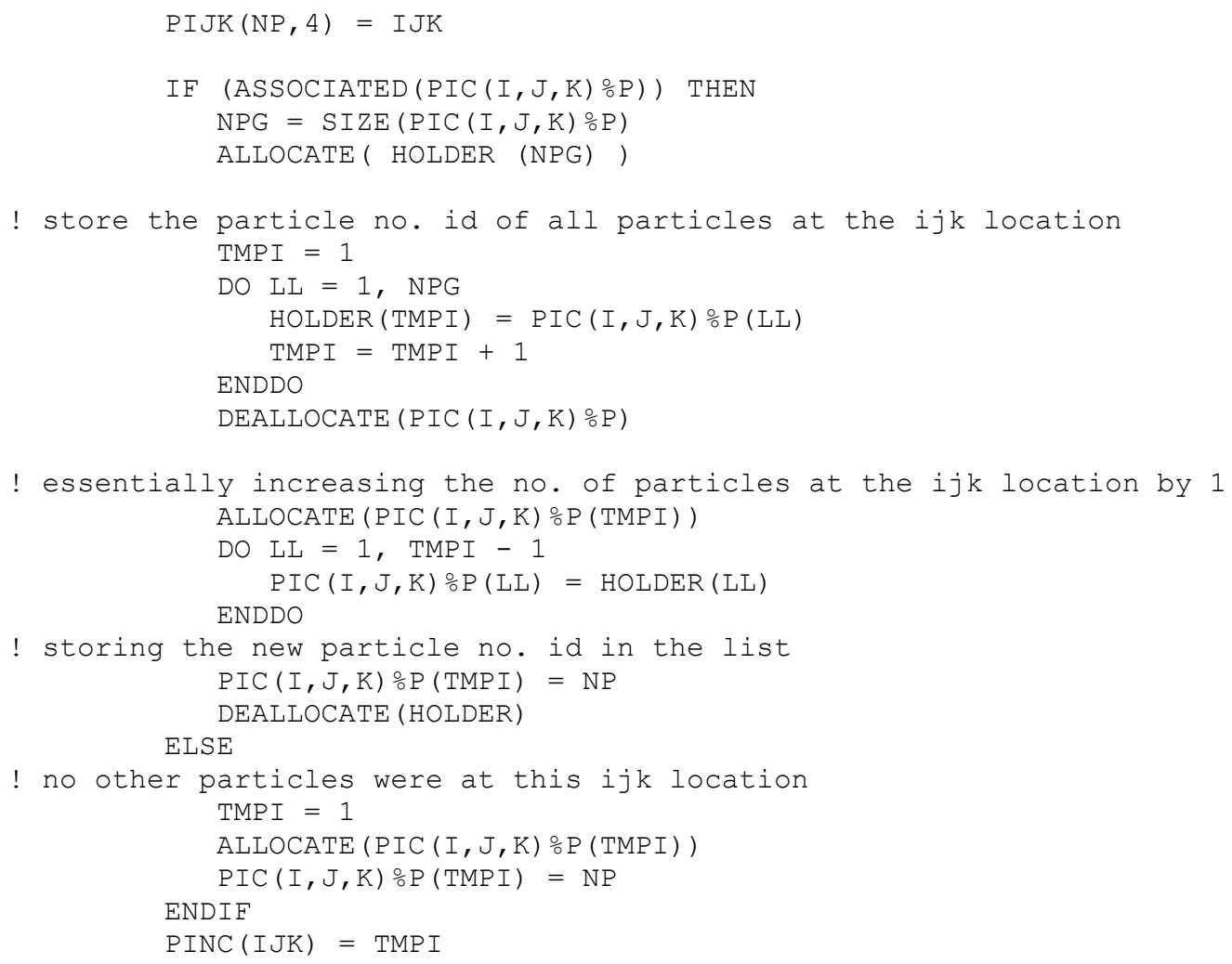

! If using des_neighbor_search option 4 (cell/grid based search) then

! determine the $i, j, k$ indices of the cell containing the new particle

! based on the mesh for the grid based search. If cell is outside the

! domain then either set the index to 1 or add 2 to the index to

! account for ghost cells.

! Note that this section is probably unnecessary since the routine

! particles_in_cell will pickup the same information before neighbor

! search is ever called and it is only in the neighbor search routine

! that this information should be needed

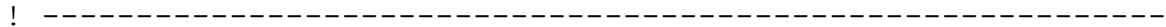

IF (DES NEIGHBOR SEARCH .EQ. 4) THEN

! determine size of the mésh

SIZEDX $=$ XLENGTH/DESGRIDSEARCH IMAX

SIZEDY $=$ YLENGTH/DESGRIDSEARCH_JMAX

IF (DIMN .EQ. 2) THEN

ELSE

SIZEDZ $=$ ONE

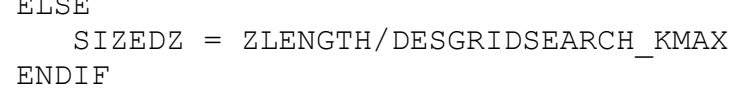




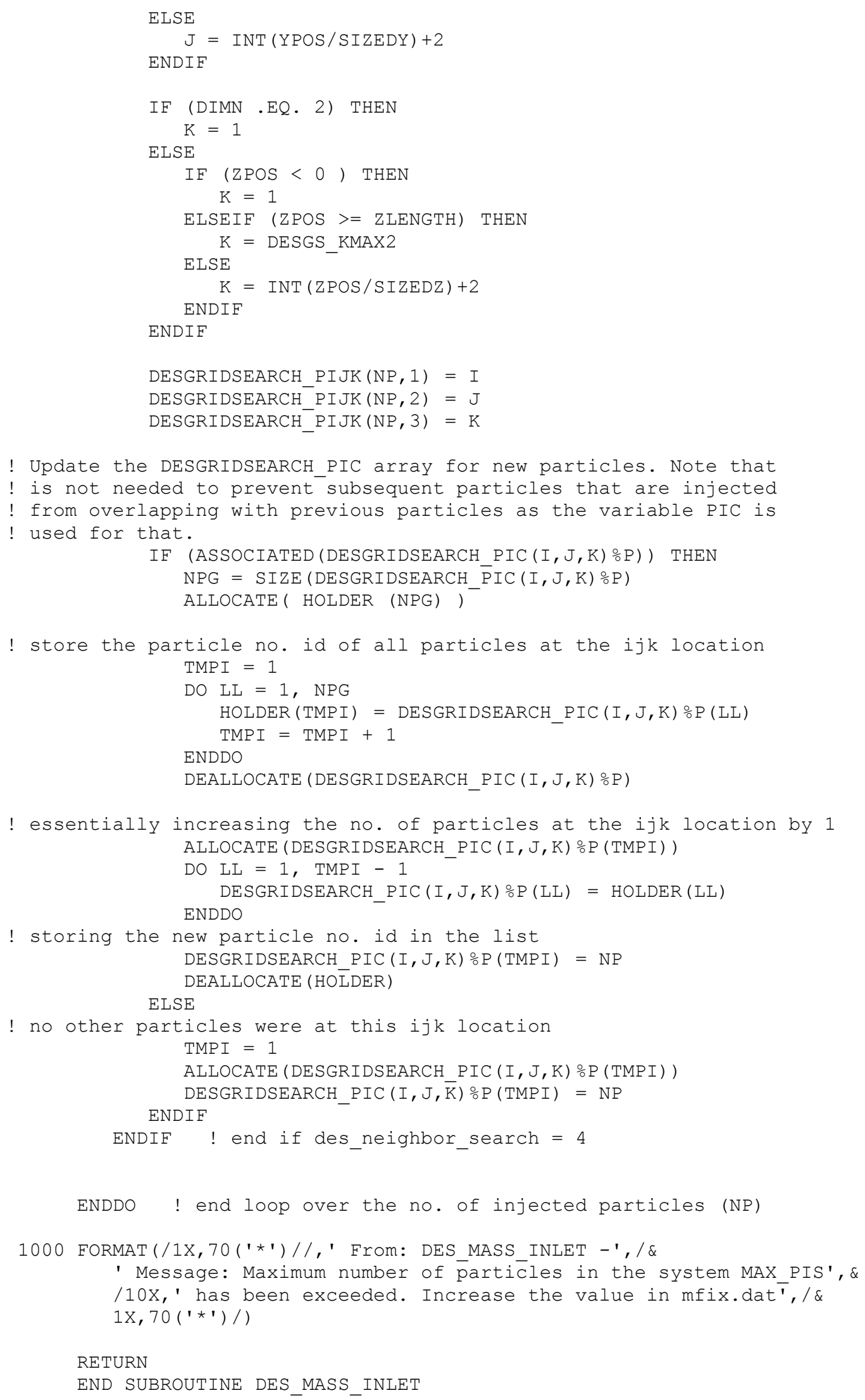




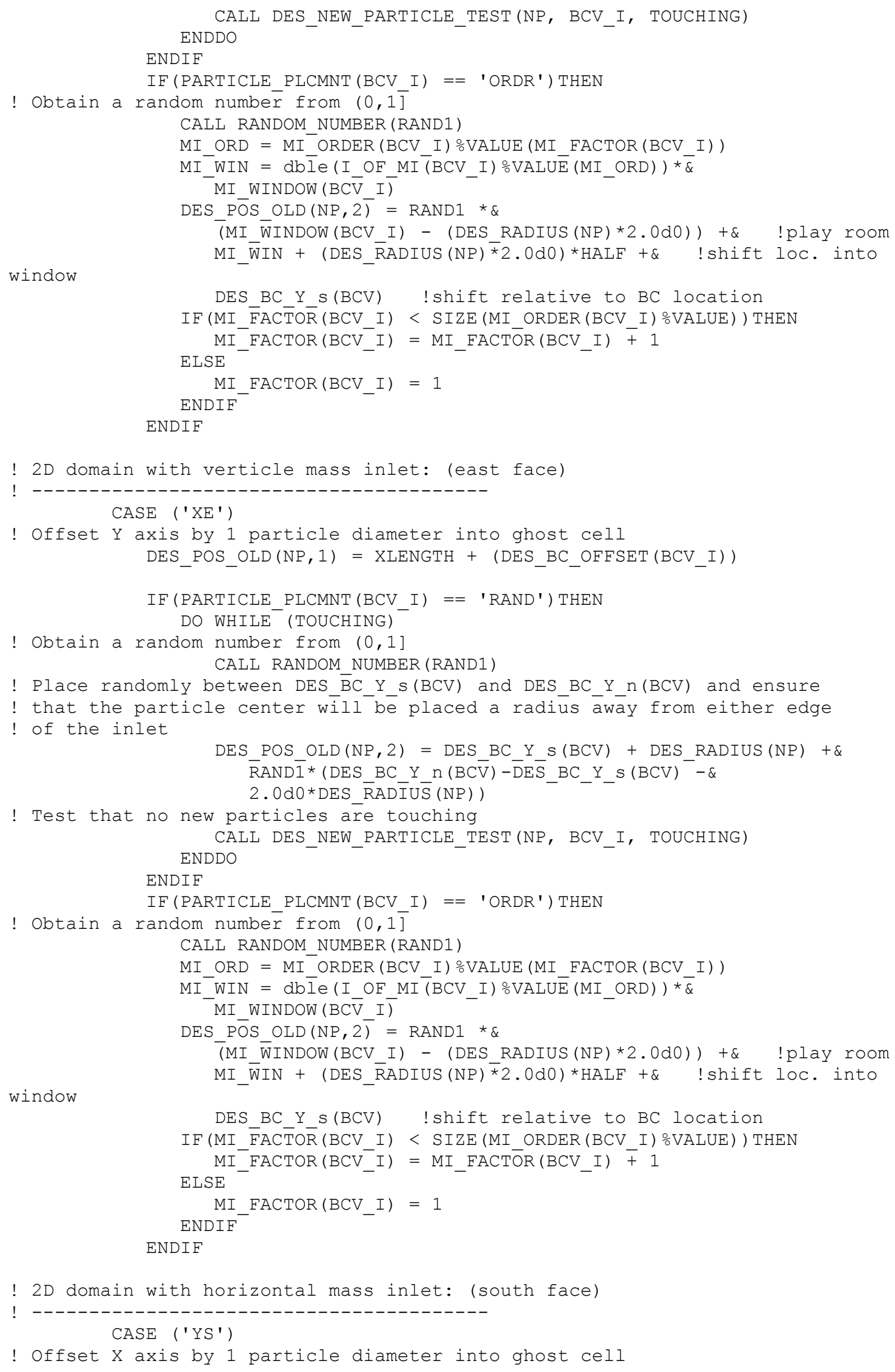




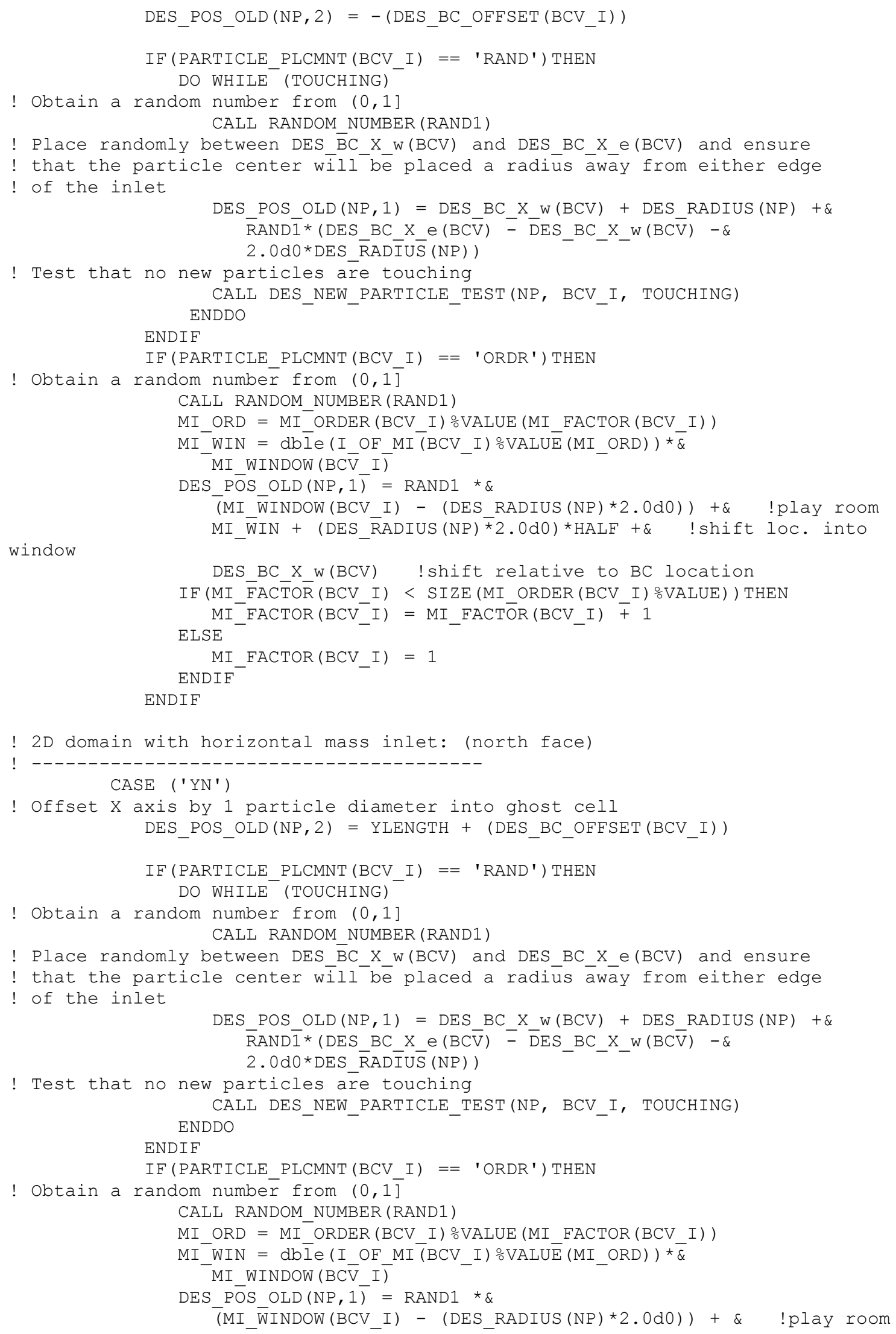


window

MI_WIN $+($ DES_RADIUS $(N P) * 2.0 d 0) * H A L F+\&$ !shift loc. into

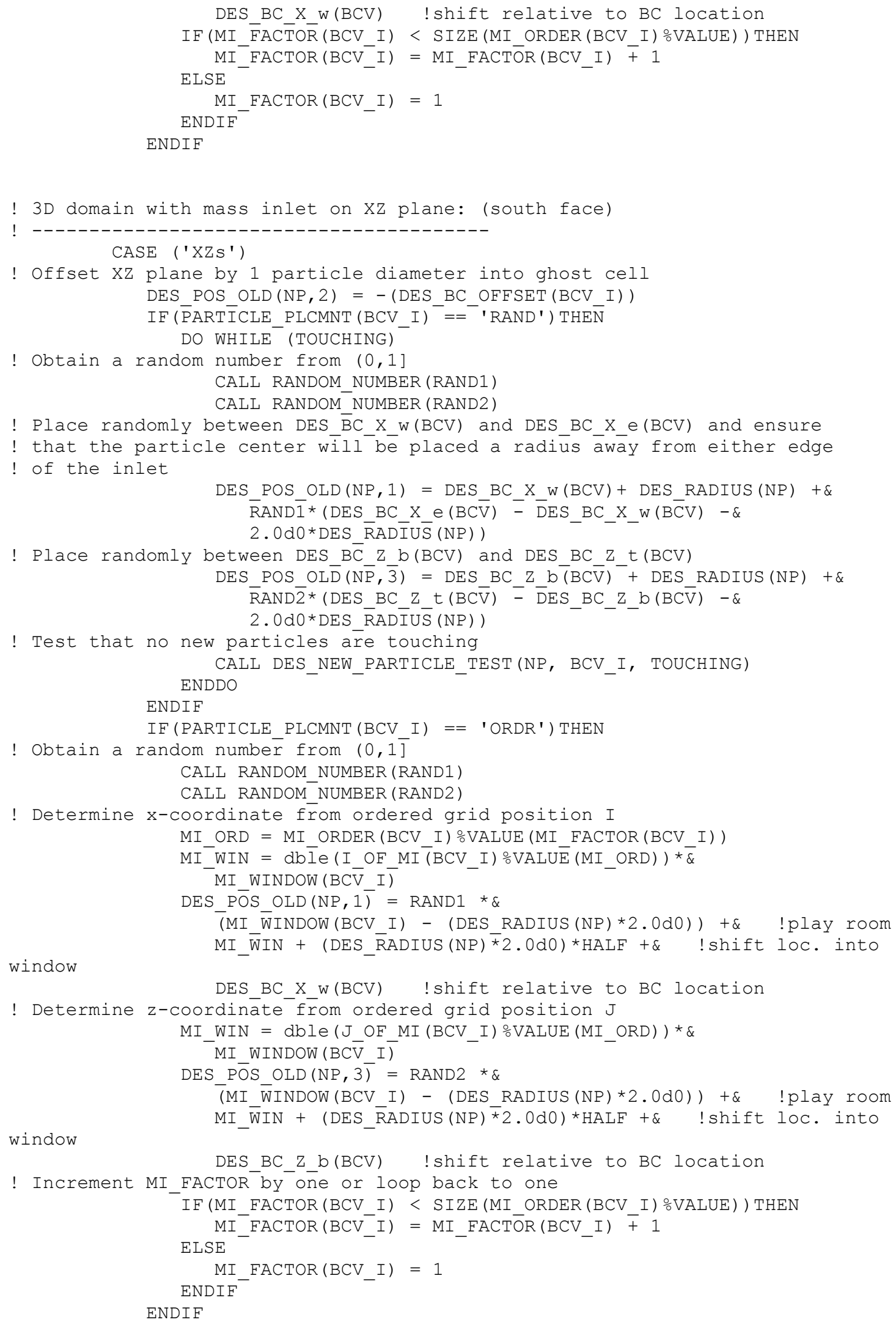


! 3D domain with mass inlet on $\mathrm{XZ}$ plane: (north face)

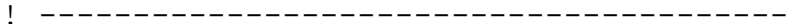

CASE ('XZn')

! Offset XZ plane by 1 particle diameter into ghost cell

DES POS OLD $(N P, 2)=$ YLENGTH + (DES BC OFFSET (BCV I ))

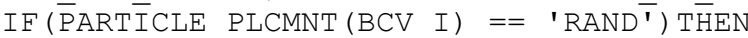

DO WHILE (TOUCHING)

! Obtain a random number from $(0,1]$

CALL RANDOM NUMBER (RAND1)

CALL RANDOM NUMBER (RAND2)

! Place randomly between DES $\bar{B} C \mathrm{X} W(\mathrm{BCV})$ and $\mathrm{DES} B \mathrm{BC}$ e (BCV) and ensure

! that the particle center will be placed a radius away from either edge

! of the inlet

DES POS OLD $(N P, 1)=D E S B C X w(B C V)+D E S$ RADIUS $(N P)+\&$

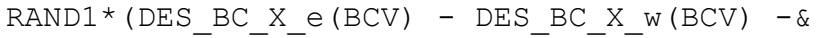

2.0d0*DES RADIUS (NP))

! Place randomly between DES BC $\bar{C}$ Z b (BCV) and DES BC_z_t (BCV)

DES POS OLD $(N \bar{N}, \overline{3})=$ DES_BC_z b (BCV) + DES RADIUS $(N P)+\varepsilon$

$\overline{\mathrm{R} A N D} \overline{2} *\left(\mathrm{DES} B \mathrm{BC} z \_t(B C \bar{V})=\overline{\mathrm{D}} \mathrm{ES} B \mathrm{BC} Z \mathrm{Z} \_\mathrm{b}(\mathrm{BC} \overline{\mathrm{V}})-\varepsilon\right.$

$2.0 \mathrm{~d} 0 * \mathrm{DES} \overline{\mathrm{R}} \mathrm{AD} \overline{\mathrm{I}} \mathrm{US}(\mathrm{NP}))$

! Test that no new particles are touching ENDDO

CALL DES NEW PARTICLE TEST (NP, BCV I, TOUCHING)

ENDIF

IF (PARTICLE PLCMNT (BCV I) $==$ 'ORDR') THEN

! Obtain a random number from $(0,1]$

CALL RANDOM NUMBER (RAND1)

CALL RANDOM NUMBER (RAND2)

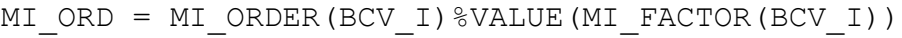

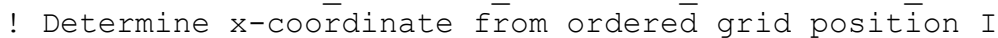

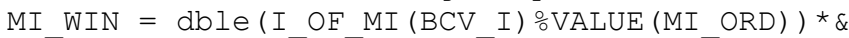
MI_WINDOW (BCV

DES POS OLD $(\mathrm{NP}, 1 \overline{)}=\operatorname{RAND} 1 * \&$

(MI_TIINDOW (BCV_I) - (DES_RADIUS $(\mathrm{NP}) * 2.0 \mathrm{dO}))+\& \quad$ !play room

window

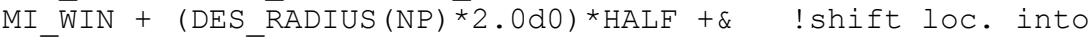

DES_BC_X_w(BCV) !shift relative to BC location

! Determine $z$-coordinate from ordered grid position J

MI WIN $=$ dble $(\mathrm{J}$ OF MI (BCV I) VALUE (MI ORD) $) * \&$

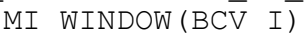

DES POS OLD $(\mathrm{NP}, 3 \overline{)}=\operatorname{RAND} 2 * \varepsilon$

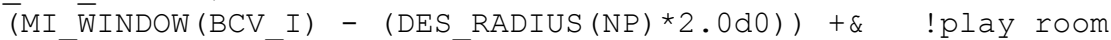

window

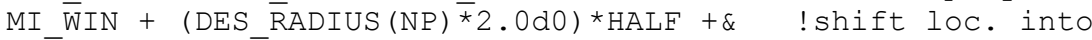

DES_BC_Z_b(BCV) ! shift relat
! Increment MI FACTOR by one or loop back to one

IF (MI FACTOR (BCV I) < SIZE (MI ORDER (BCV I) VALUE) ) THEN

ELSE

$\mathrm{MI}_{-}^{-} \mathrm{FACTOR}\left(\mathrm{BCV}_{-}^{-} \mathrm{I}\right)=\mathrm{MI}$ FACTOR $\left(\mathrm{BCV} \_\mathrm{I}\right) \overline{+} 1$

ENDIF

MI FACTOR (BCV I) $=1$

ENDIF

! 3D domain with mass inlet on XY plane: (bottom face)

! -------------------------------------

CASE ('XYb')

! Offset XY plane by 1 particle diameter into ghost cell

DES_POS_OLD $(N P, 3)=-\left(\right.$ DES_BC_OFFSET $\left.\left(B C V \_I\right)\right)$

IF (PARTICLE PLCMNT (BCV I) $==$ 'RAND') THEN

DO WHILE (TOUCHING)

! Obtain a random number from $(0,1]$ 


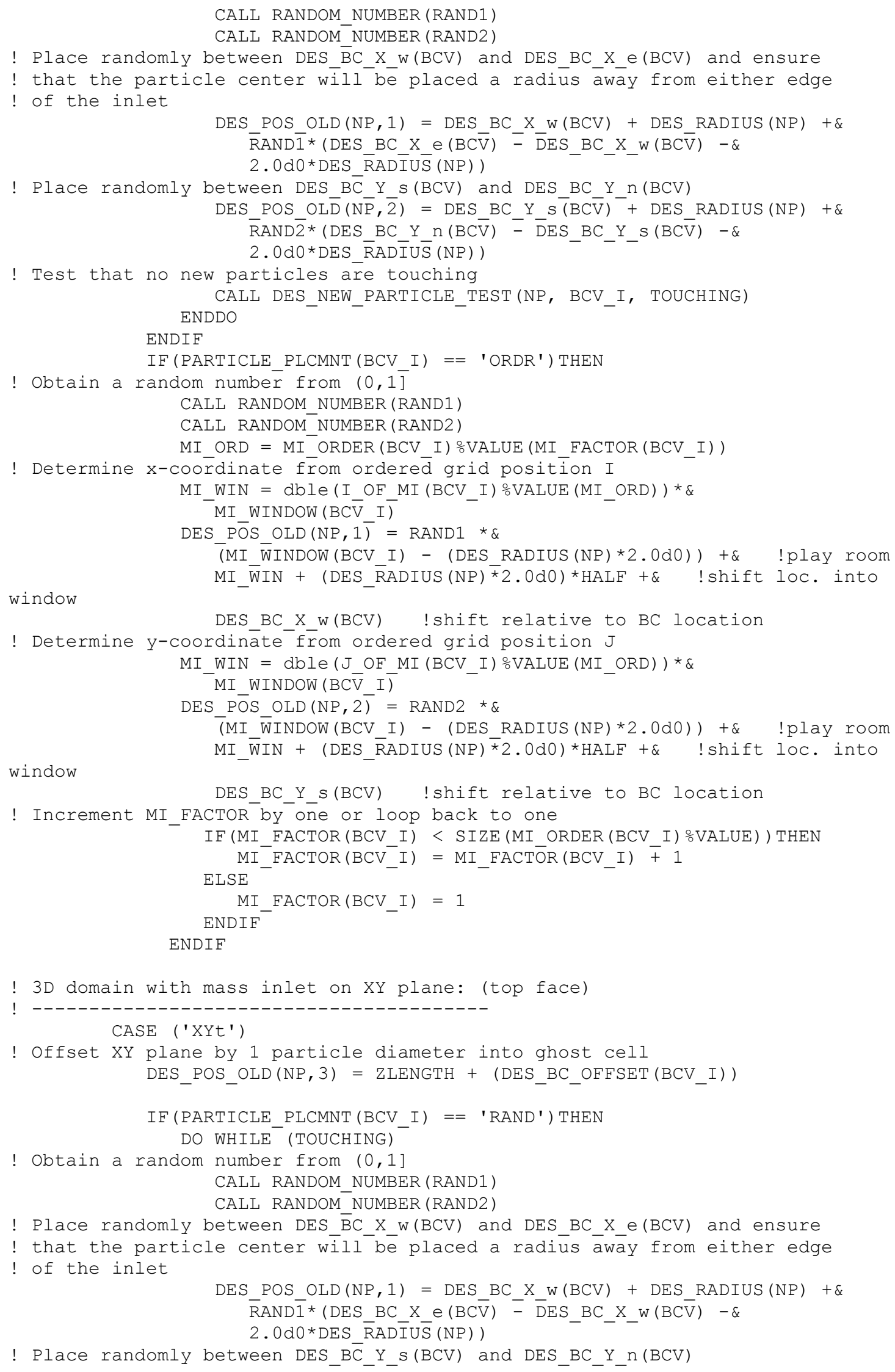




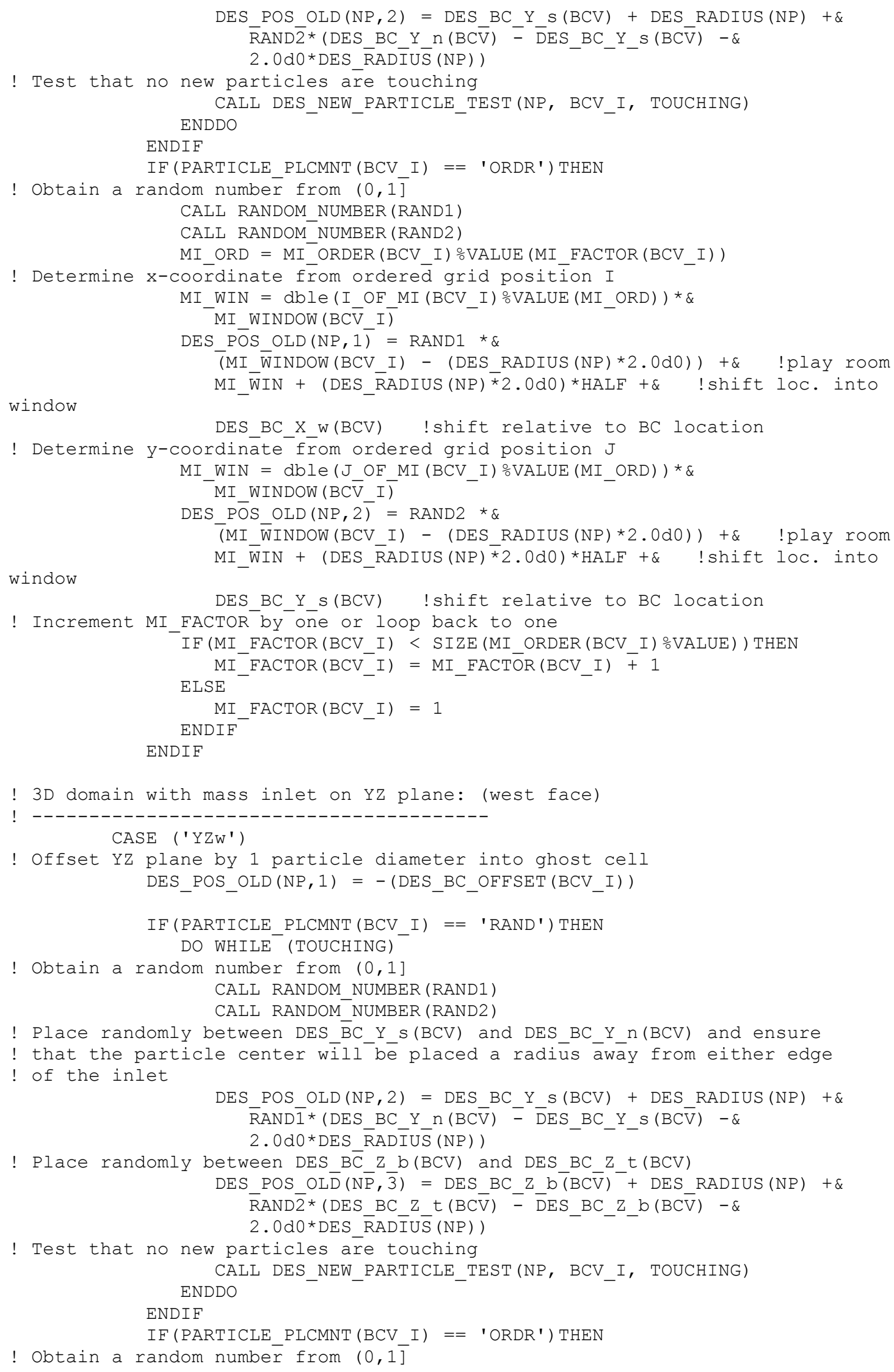




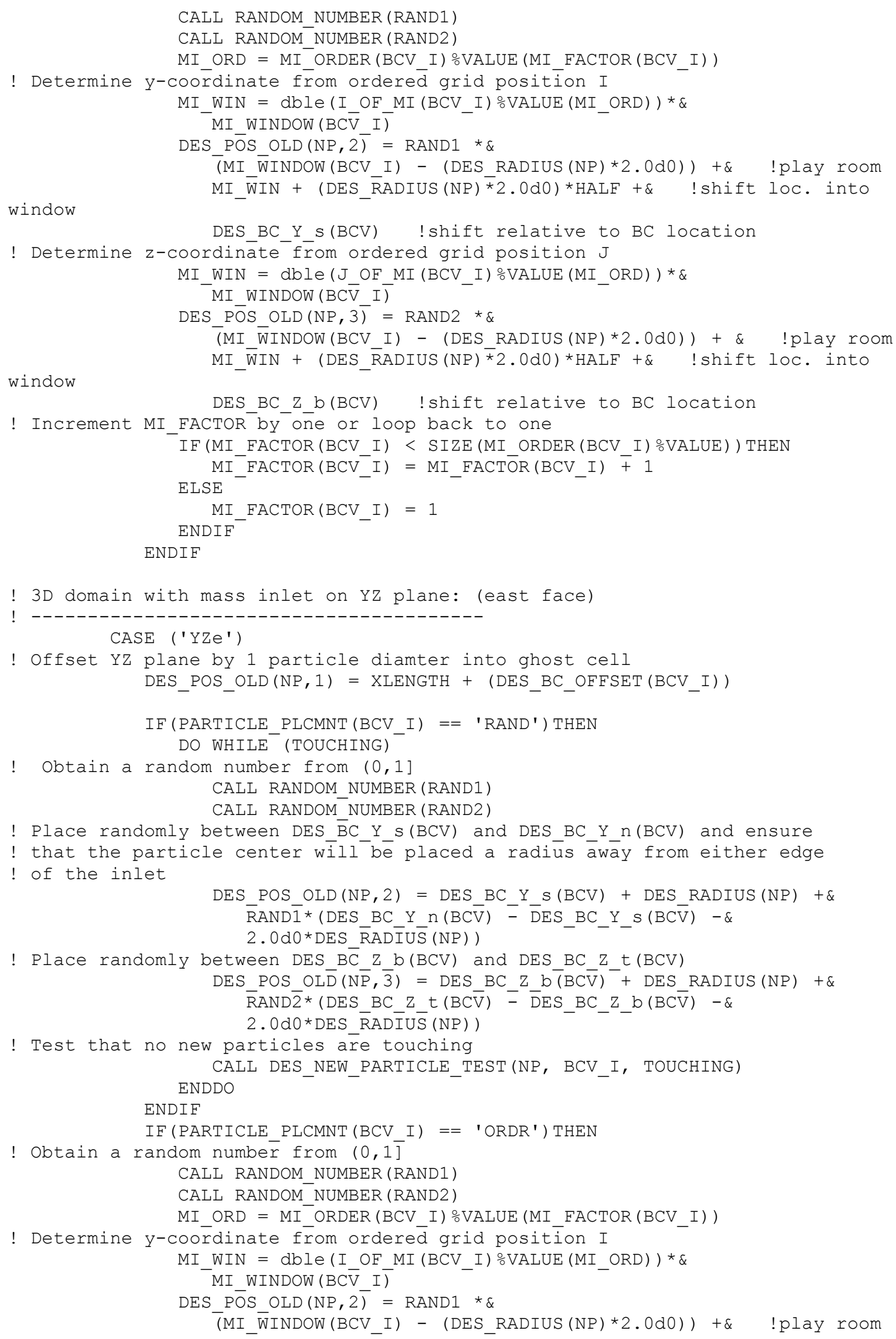




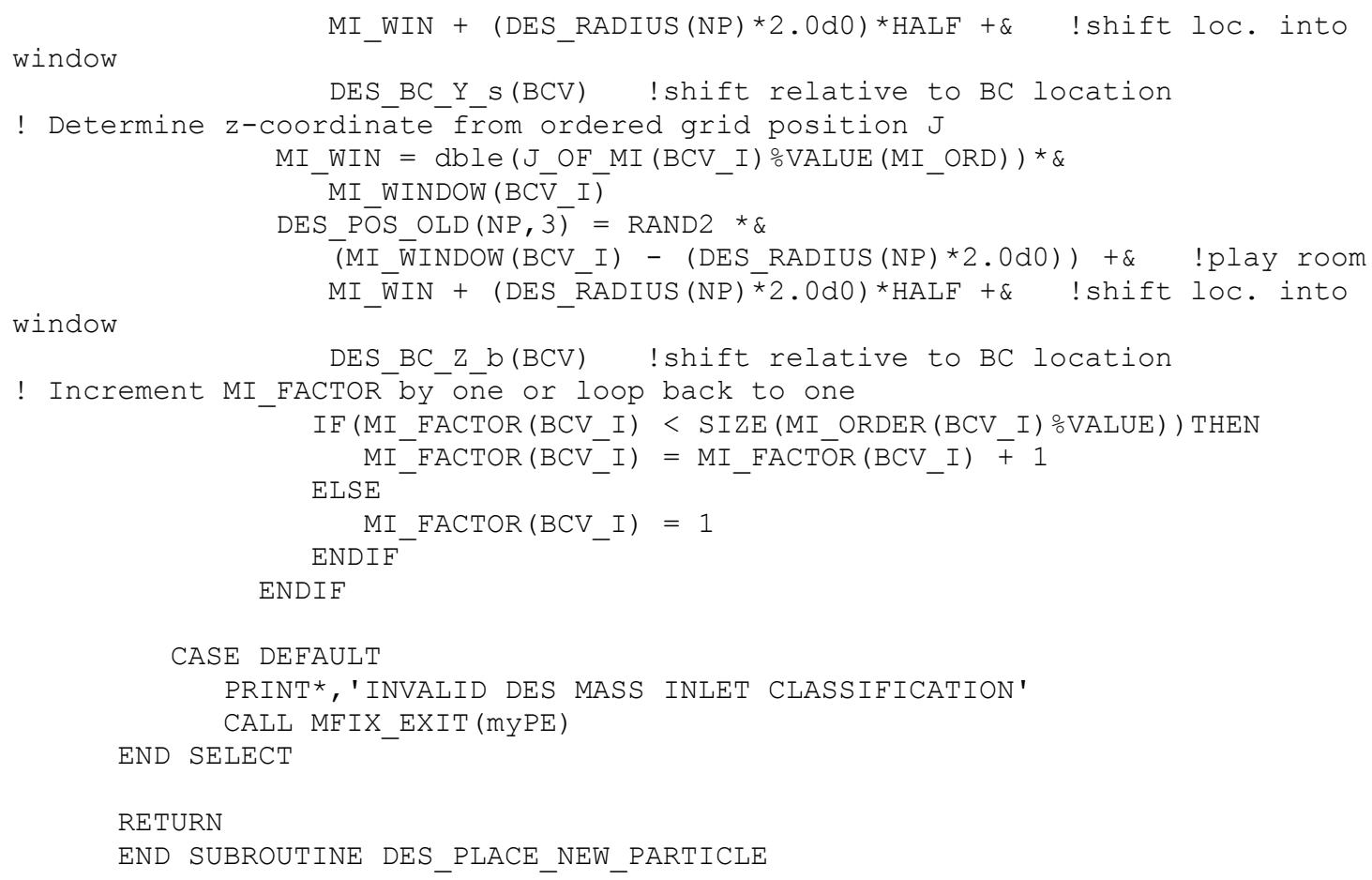




\section{C.14 : Routine - DES_NEW_PARTICLE_TEST}

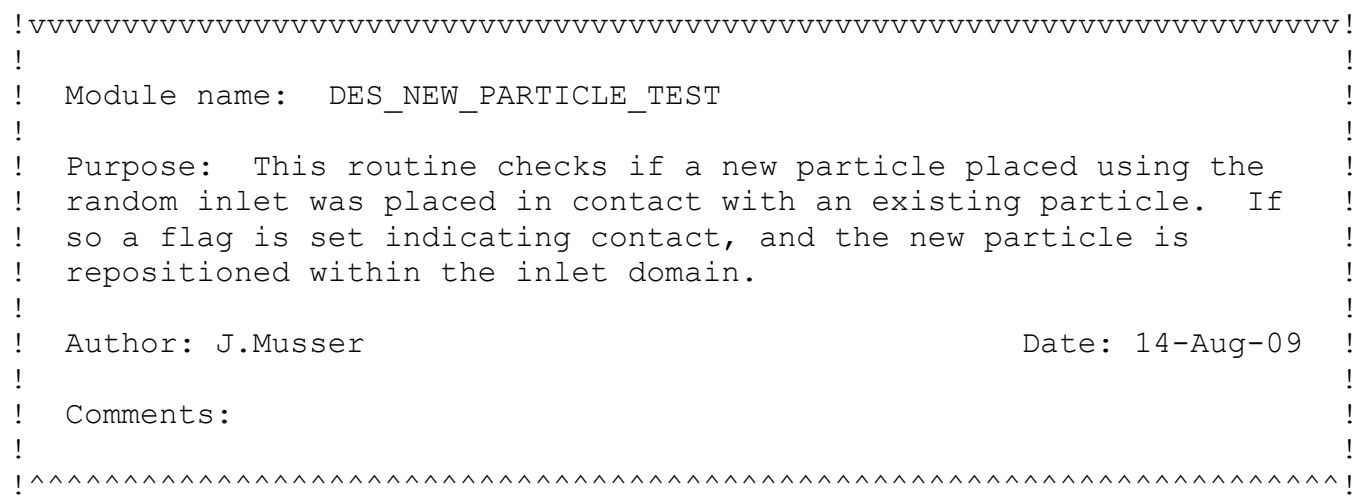

SUBROUTINE DES_NEW_PARTICLE_TEST (NP, BCV_I, TOUCHING)

USE compar

USE constant

USE des bc

USE discretelement

USE funits

USE geometry

USE indices

USE param1

USE physprop

IMPLICIT NONE

! Local variables

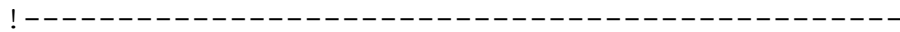

! particle number id of interest INTEGER NP

! particle number id of a potential overlapping/contacting particle INTEGER NP2

! index of boundary condition INTEGER BCV I

! total number of particles in current ijk cell and loop counter INTEGER NPG, LL

! i, j, $k$ indices along boundary used for loop counters INTEGER I, J, K, IJK

LOGICAL TOUCHING

DOUBLE PRECISION, EXTERNAL : : DES DOTPRDCT DOUBLE PRECISION DISTVEC(DIMN), DIST, R_LM

INCLUDE 'function.inc'

TOUCHING $=$.FALSE.

DO $\mathrm{K}=\mathrm{GS}$ _ARRAY $(\mathrm{BCV} I, 5), \mathrm{GS}$ ARRAY $(\mathrm{BCV} I, 6)$ DO $J=-G S$ ARRAY $(\bar{B} C V I, 3),-G S$ ARRAY $(\bar{B} C V I, 4)$ DO $I=-G S$ ARRAY $(\bar{B} C V I, 1),-G S \operatorname{ARRAY}(\bar{B} C V I I, 2)$

$I J K=F \bar{N} I J K(I, J, K)$ IF (ASSOCIATED (PIC ( I, J,K) P) ) THEN $N P G=\operatorname{SIZE}(P I C(I, J, K) \circ P)$

$\mathrm{DO} \mathrm{LL}=1, \mathrm{NPG}$ $N P 2=\operatorname{PIC}(I, J, K) \circ P(L L)$ 


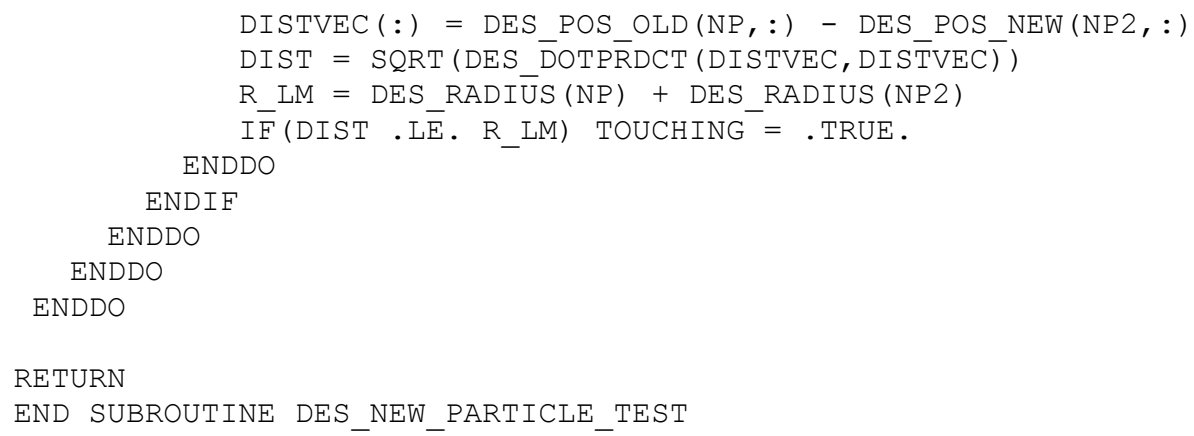




\section{C.15 : Routine - DES_CHECK_PARTICLE}

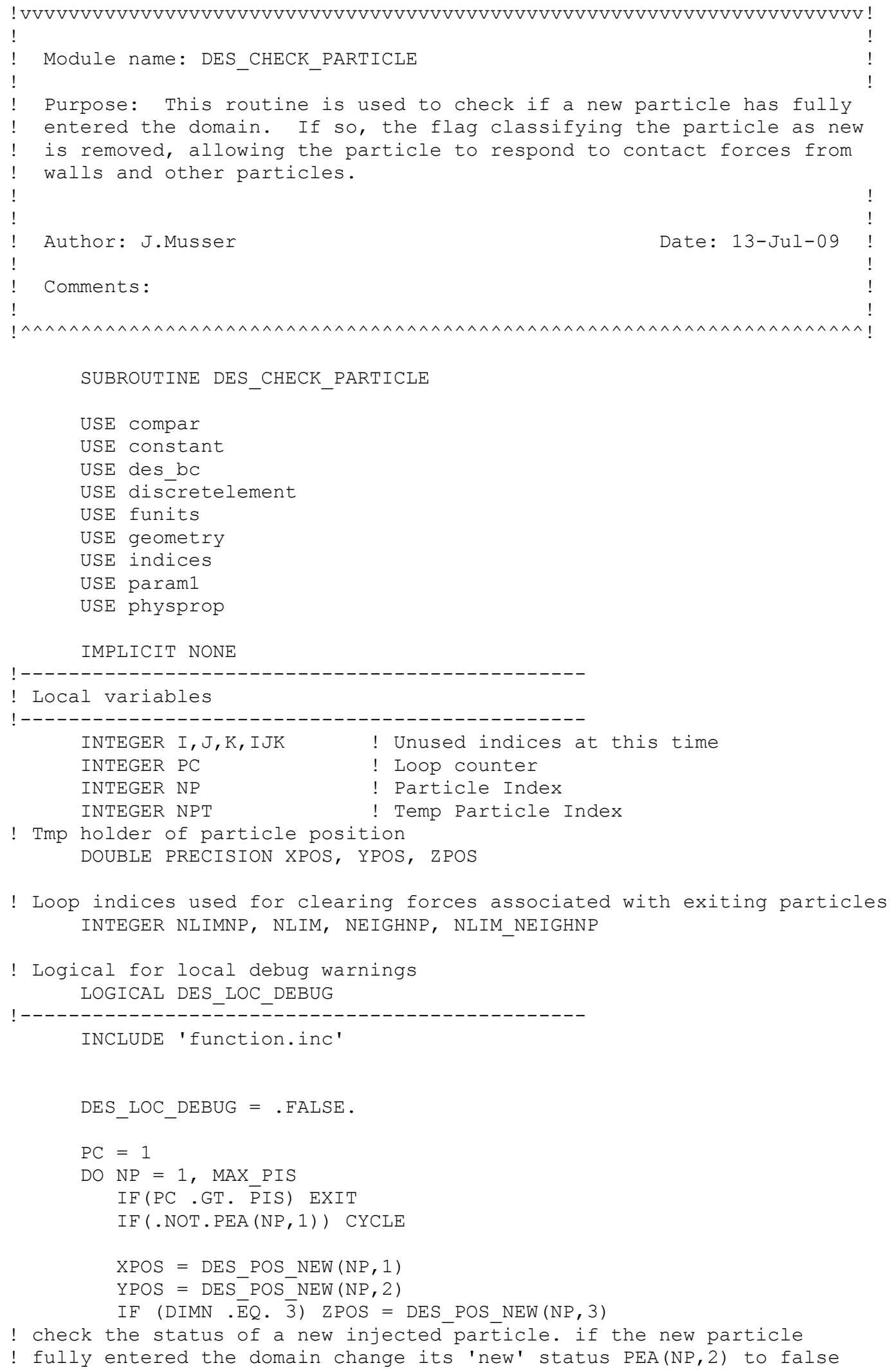




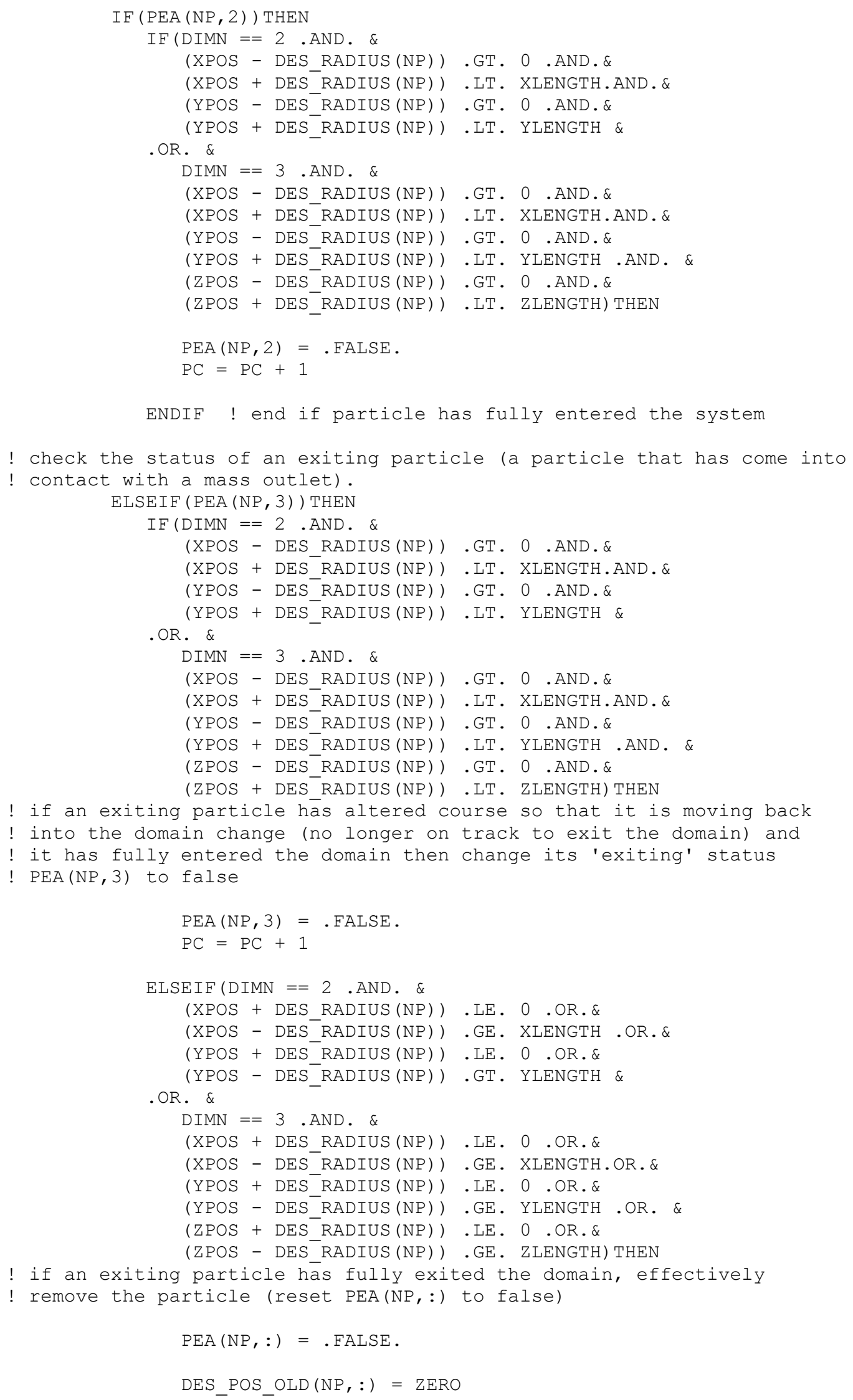




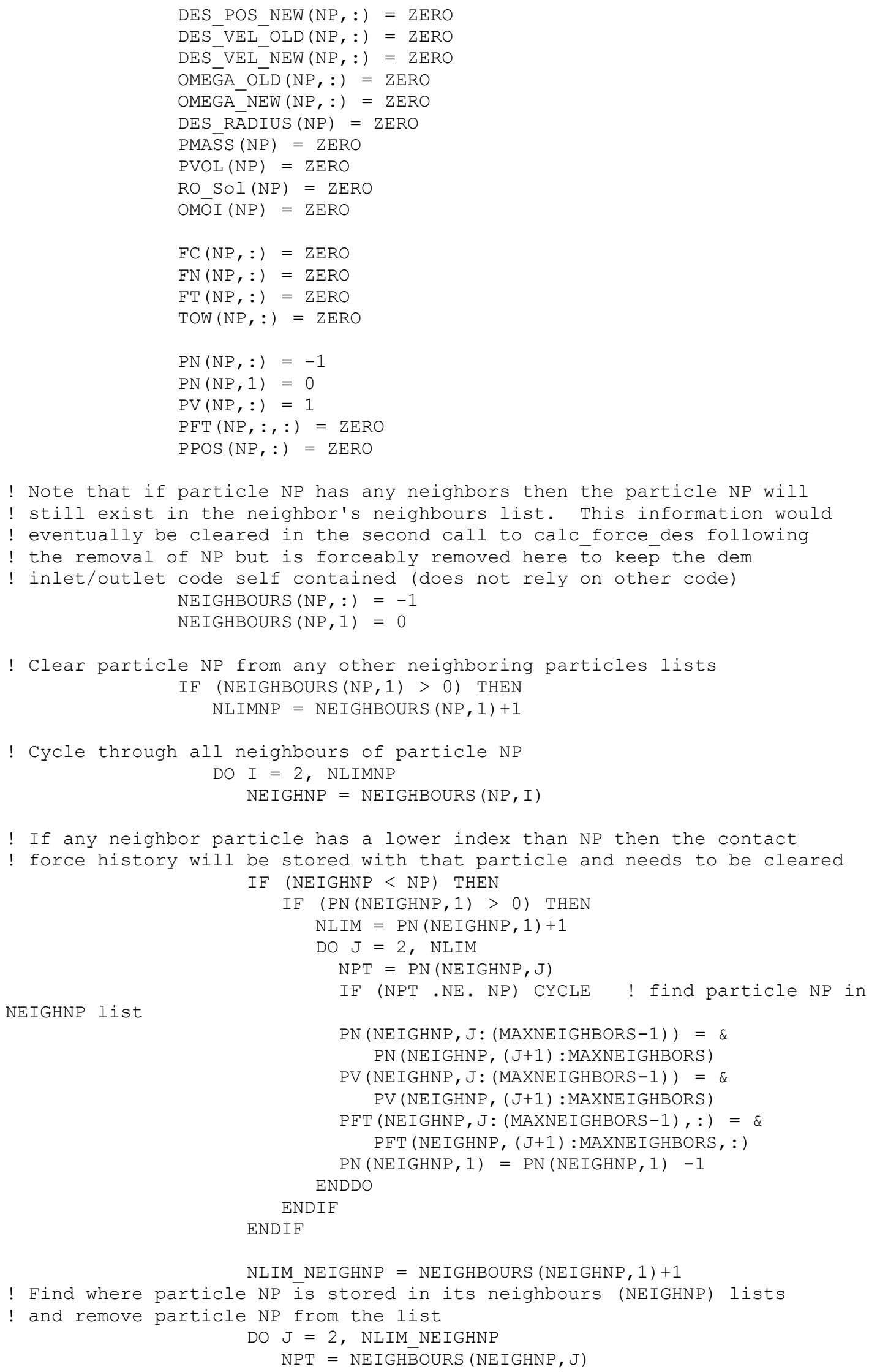




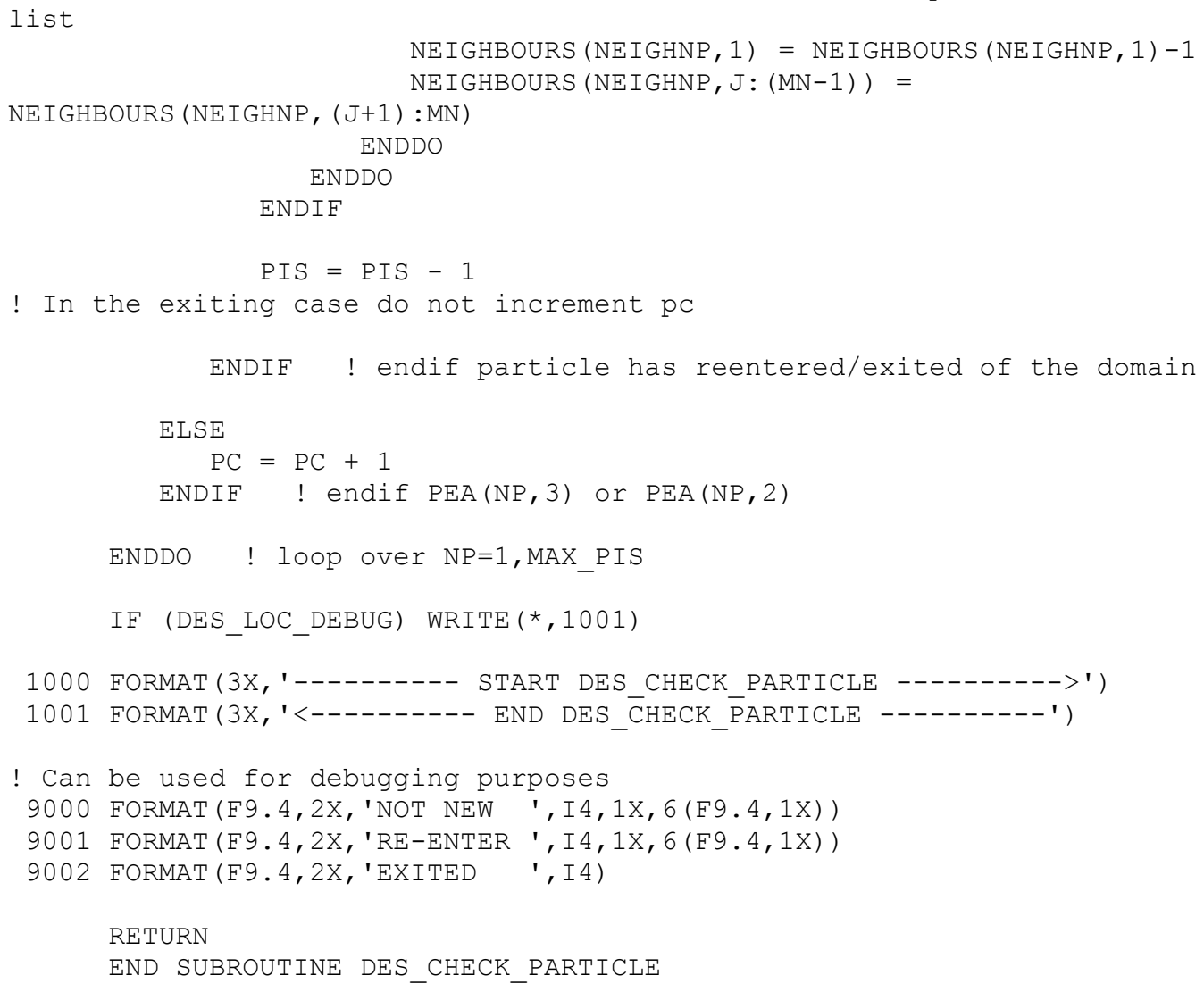




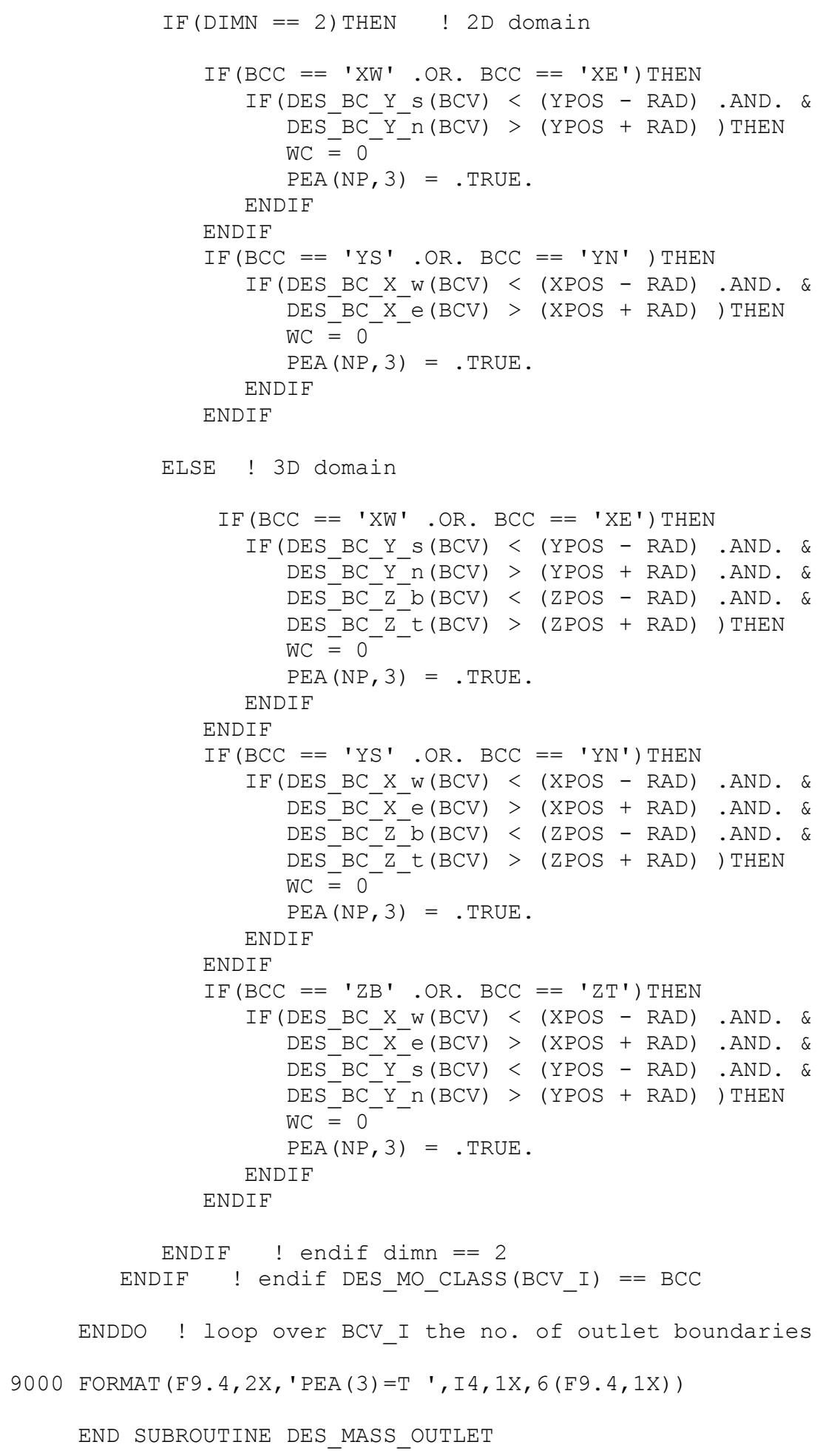




\section{Appendix D: Particle heat transfer model source code}

The section contains the source code for the particle heat transfer models. It has been written in the FORTRAN90/95 style.

\section{D.1 Module - DES_IC}

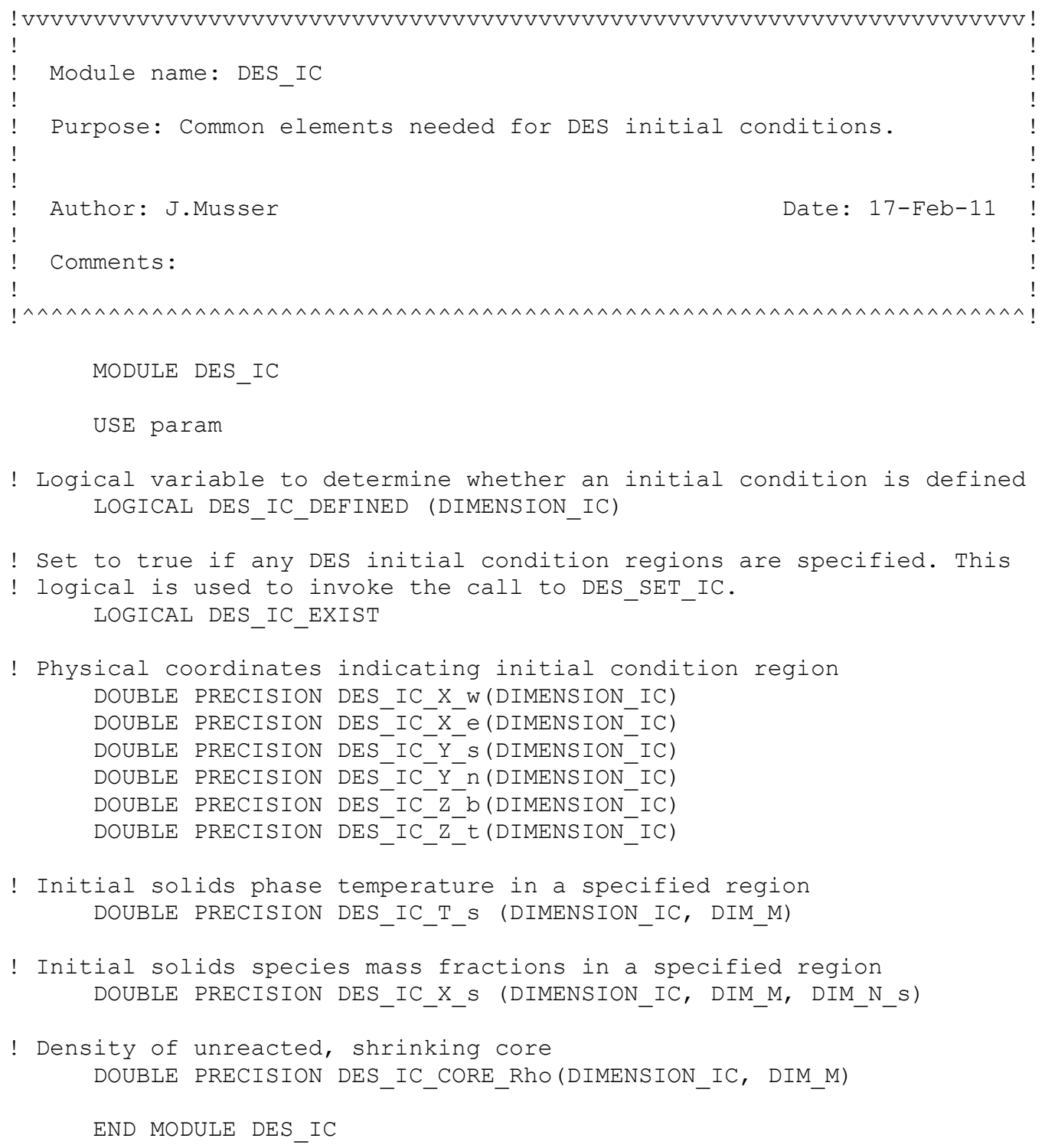




\section{D.2 Routine - CHECK_DES_IC}

! VVVVVVVVVVVVVVVVVVVVVVVVVVVVVVVVVVVVVVVVVVVVVVVVVVVVVVVVVVVVVVVVVVVVVV!

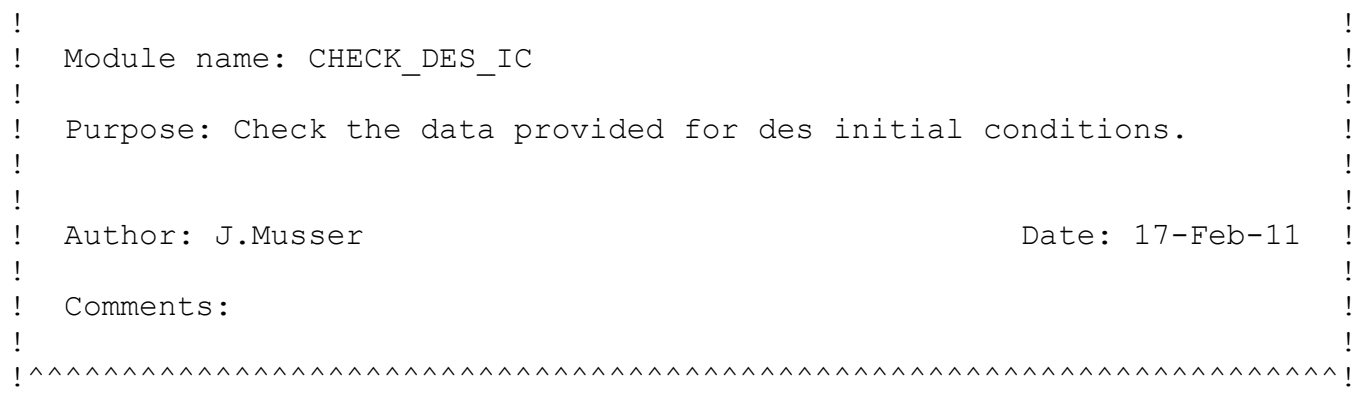

SUBROUTINE CHECK_DES_IC

USE des_ic

USE disčretelement

USE param

USE param1

USE des thermo

USE des_rxns

USE compar

USE constant

USE funits

USE geometry

USE indices

USE physprop

USE run

IMPLICIT NONE

INTEGER ICV

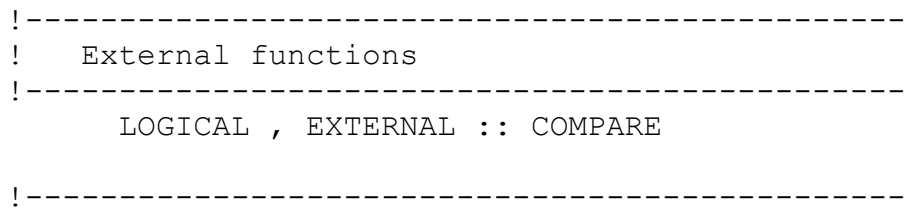

$\operatorname{WRITE}(*, '(1 \mathrm{X}, \mathrm{A})$ ') '-------- START CHECK_DES_IC $--------->$ '

! Initialize the initial condition logical.

DES IC EXIST $=$.FALSE.

! Check for des inlet/outlet information:

CHECK IC: $\mathrm{DO} I C V=1$, DIMENSION_IC

$\mathrm{DE} \overline{\mathrm{S}} \mathrm{IC}$ DEFINED $(\mathrm{ICV})=$. FALSE

IF (DES IC X w $(I C V) /=$ UNDEFINED) DES IC DEFINED (ICV) = . TRUE.

IF (DES IC X e (ICV) /= UNDEFINED) DES IC DEFINED (ICV) = . TRUE.

IF (DES $\left.{ }^{-} \mathrm{IC}^{-} \mathrm{Y}^{-} \mathrm{S}(\mathrm{ICV}) /=\mathrm{UNDEFINED}\right) \mathrm{DES}^{-} \mathrm{IC}^{-} \mathrm{DEFINED}(\mathrm{ICV})=. \mathrm{TRUE}$.

IF (DES_IC_Y ${ }^{-} \mathrm{n}(\mathrm{ICV}) /=$ UNDEFINED) DES_IC_DEFINED $(\mathrm{ICV})=. \mathrm{TRUE}$.

IF (DES_IC_Z_b(ICV) /= UNDEFINED) DES_IC_DEFINED(ICV) = . TRUE.

IF (DES IC_Z ${ }^{-}(I C V) /=$ UNDEFINED) DES IC DEFINED (ICV) = TRUE.

! If an initial condition is specified, verify necessary data

! Check that all dimensions have been defined

IF (DES_IC_DEFINED(ICV)) THEN

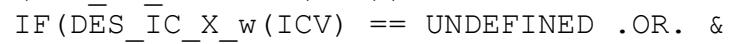

$\mathrm{DES}^{-} \mathrm{IC}^{-} \mathrm{X}_{-}^{-} \mathrm{e}(\mathrm{ICV})==\mathrm{UNDEFINED} . \mathrm{OR} \cdot \&$ 


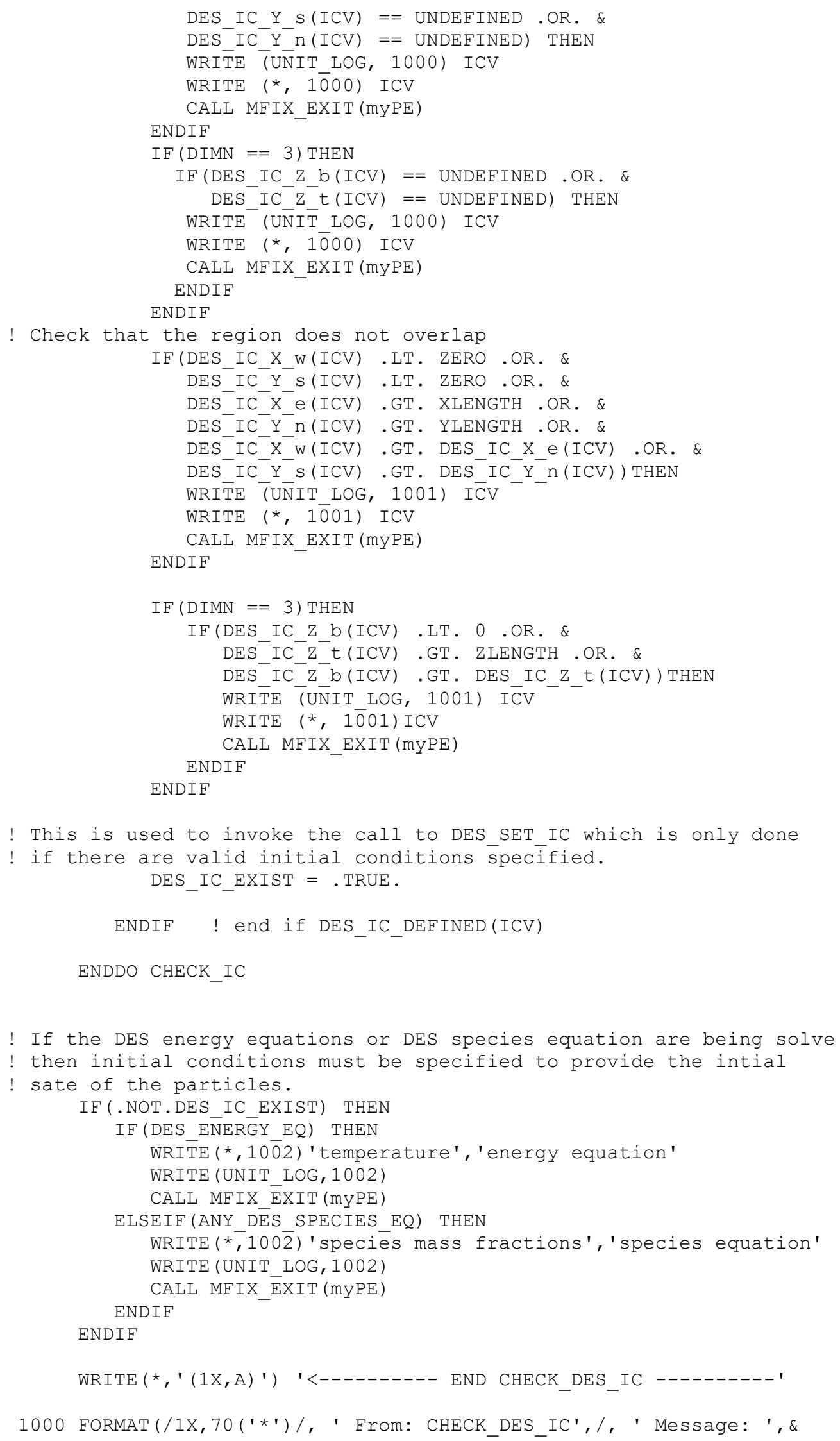




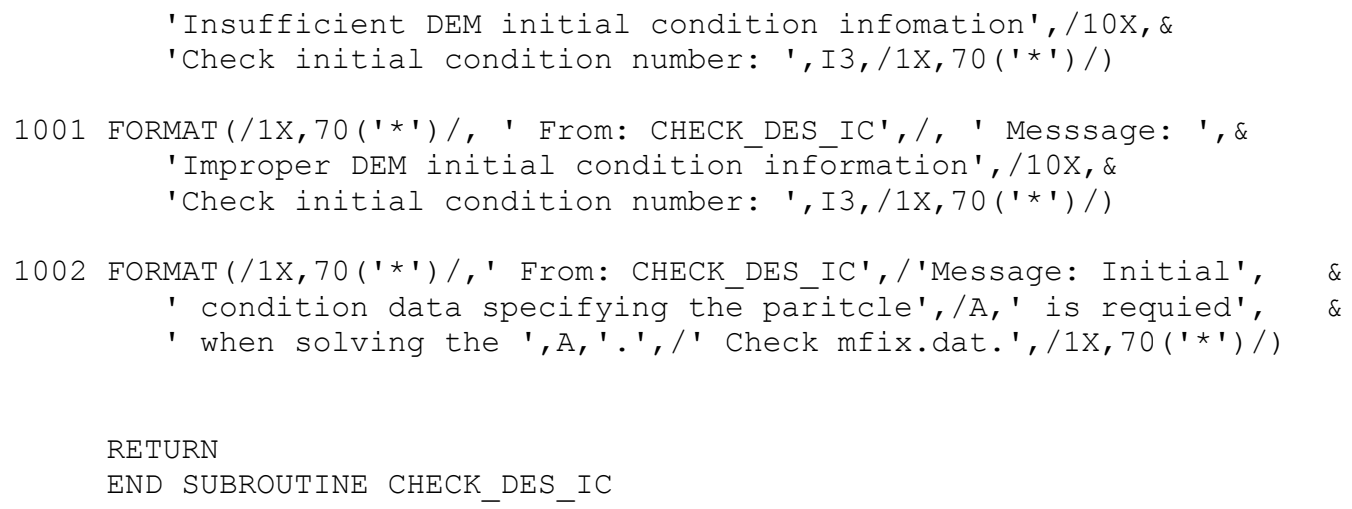




\section{D.3 Routine - DES_SET_IC}

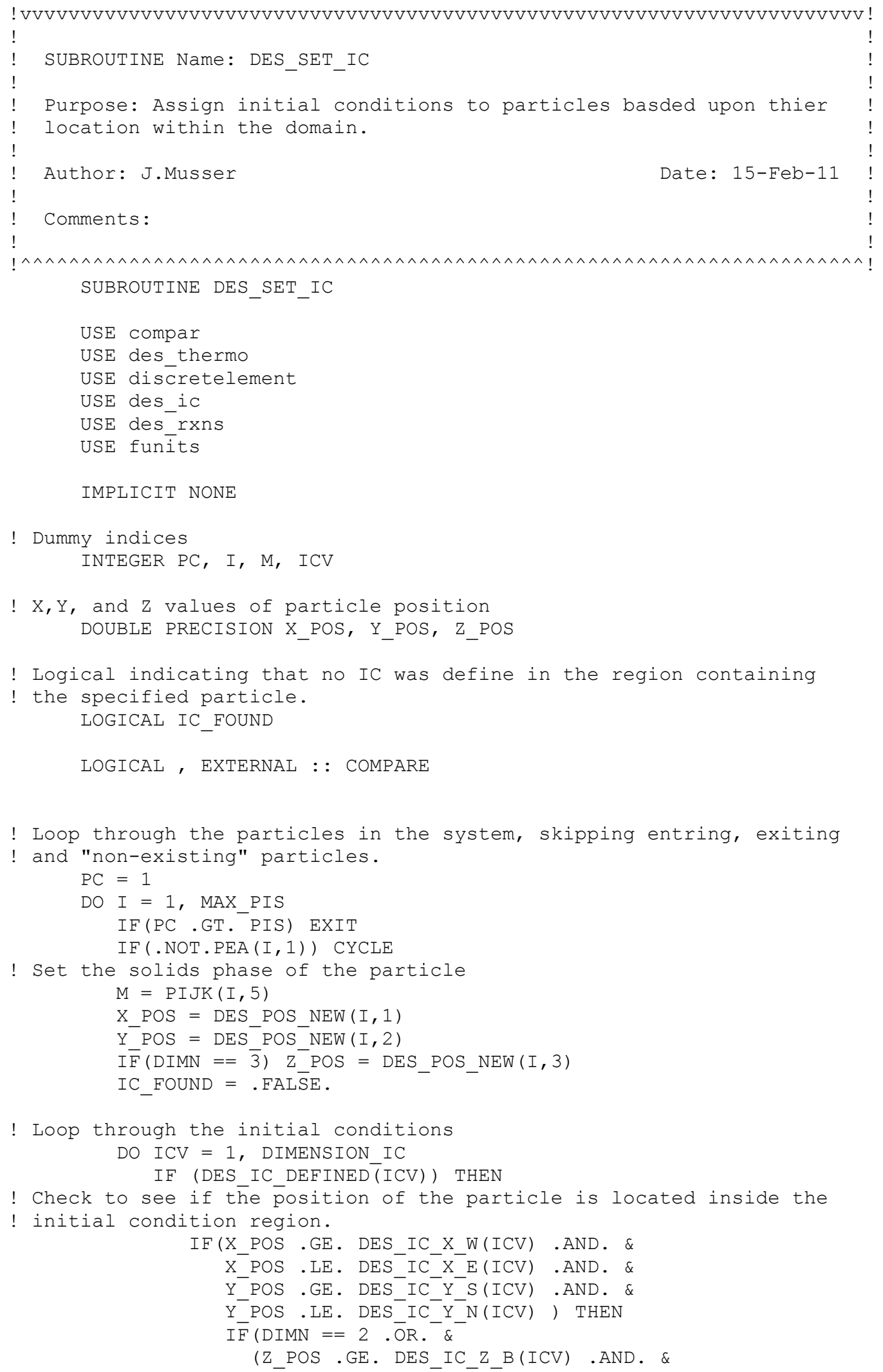




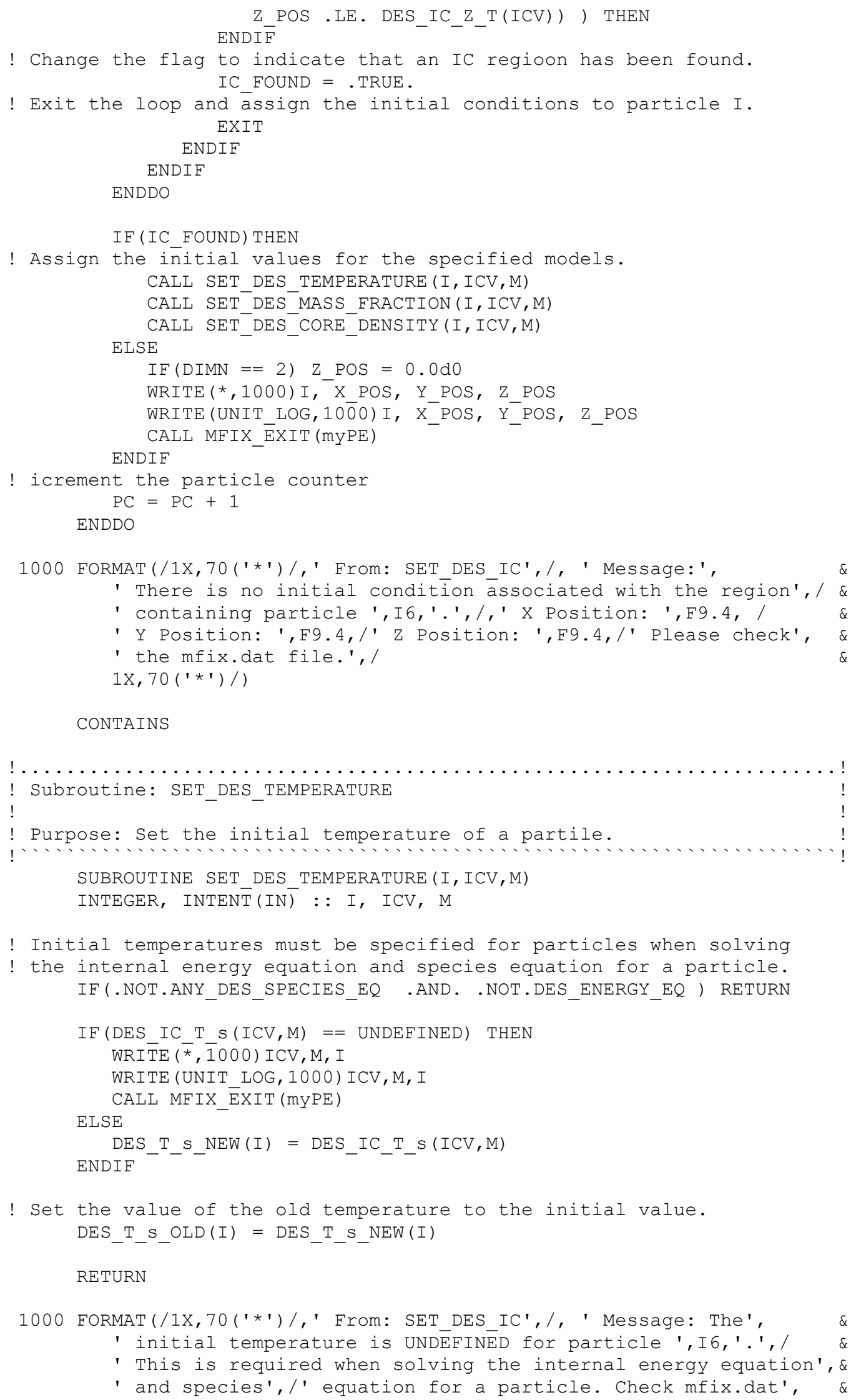


! file, the density of the particle is assigned to the core.

IF(.NOT.DES SPECIES EQ(M) .OR. TRIM(REACTION MODEL) $/=$ \&

'SHRINKIN̄G_CORE') RETURN

IF (DES IC CORE Rho (ICV,M) == UNDEFINED) THEN

IF (.NOT .WARN

$\operatorname{WRITE}(*, 1000)$

WRITE (UNIT LOG, 1000)

WARNED $=$. TRUE.

ENDIF

ELSE

CORE_RhO(I) = RO_SOI (I)

ENDIF

CORE_Rho $(I)=$ DES_IC_CORE_Rho $(I C V, M)$

RETURN

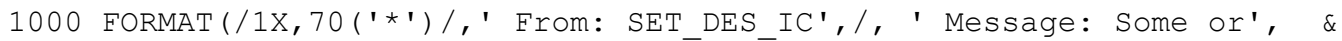

' all of the discrete solids phases do not have an',/ \&

' initial core density defined. This effects how the', \&

' shrinking,',/,' unreacted core model behaves.',/,' The', \&

' core density will be set to the particle density.',/, \& $\left.1 \mathrm{X}, 70\left({ }^{\prime} * 1\right) /\right)$

END SUBROUTINE SET_DES_CORE_DENSITY

END SUBROUTINE DES_SET_IC 


\section{D.4 Module - DES_THERMO}

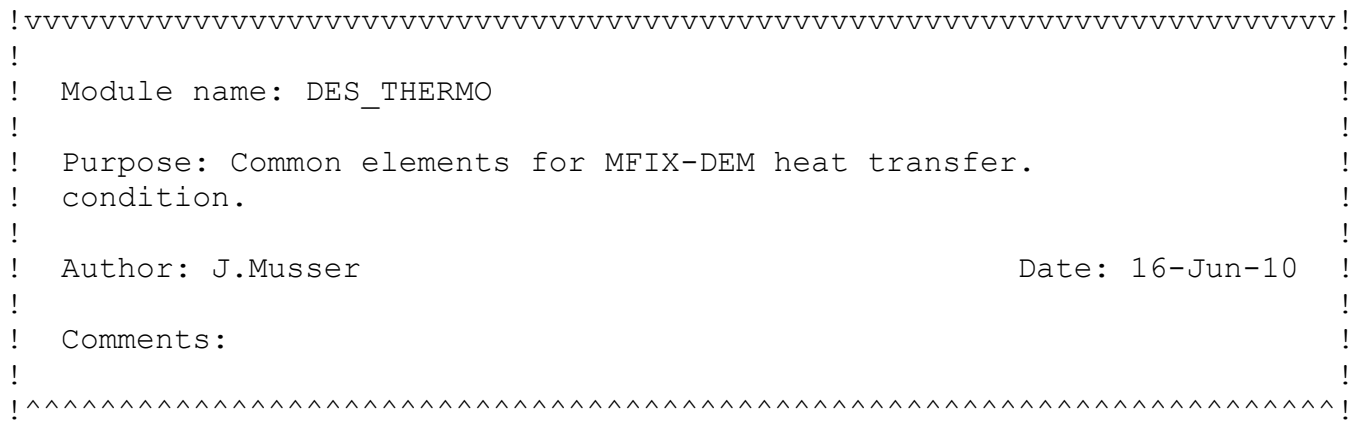

MODULE DES_THERMO

USE param

! Run time logicals

!--------------------------

! Slove DES Energy Equations

LOGICAL DES_ENERGY_EQ

! Specifies that the convection model is to be solved.

! Requires DES ENERGY EQ = . TRUE.

! Default [.TRŪE.] LOGICAL DES_CONV_EQ

! Specifies that the particle conduction models are to be solved.

! Requires DES_ENERGY_EQ = . TRUE.

! Default [.TRŪE.] LOGICAL DES_COND_EQ

! These logicals $\bar{c} a n$ be set in the mfix.dat file to omit specific

! particle-particle heat conduction models from being solved.

! If the DEM conduction model is not solved (DES_COND_EQ), the values

! are automatically set to .FALSE. in check_des_thermo.f.

! Default [.TRUE.] LOGICAL DES_COND_EQ_PFP ! particle-fluid-particle

! Default [.TRUE. $]$ LOGICAL DES_COND_EQ_PP ! particle-particle

! Specifies that the pärticle-environment radiation model is to be

! solved. Requires DES_ENERGY_EQ = . TRUE.

! Default [.TRUE.] LOGICAL DES_RADI_EQ

! Heat transfer correlation specified in mfix.dat

! Default [RANZ_1952] CHARACTER ${ }^{2} 4$ : : DES_CONV_CORR

! Specifies that a thermodynamic neighborhood for a particle be

! determined. This neighborhood can be much larger than the one

! established for determining collisions. It is used in conduction and

! radiation models. LOGICAL FIND_THERMO_NBRHD

! Particle properties

!--------------------------

! Particle temperature at current time step (S TIME) DOUBLE PRECISION, DIMENSION(:), ALLOCATAB̄EE : DES_T_S_OLD ! (PARTICLES)

! Particle temperature at previous time step (S_TIME - DT SOLID) DOUBLE PRECISION, DIMENSION (:), ALLOCATABLE : : DES_T_s_NEW ! (PARTICLES) 
! DES Specified constant solids thermal conductivity by solids phase DOUBLE PRECISION DES_K_SO (DIM_M)

! DES solids thermal conductivity by particle ! (not currently used) DOUBLE PRECISION, DIMENSION(:), ALLOCATABLE : : DES_K_S

! DES Specified constant solids specific heat by solids phase DOUBLE PRECISION DES_C_psO (DIM_M)

! DES specific heat of particles by particle DOUBLE PRECISION, DIMENSION(:), ALLOCATABLE : : DES_C_ps

! Emissivity of particles DOUBLE PRECISION DES_Em(DIM_M)

! Stefan-Boltzmann Constant DOUBLE PRECISION SB CONST

! Rates of heat transfer

!--------------------------

! Heat transfer TO the particle from the fluid. This value will be positive

! when the particle is heating and negative when the particle is cooling. DOUBLE PRECISION, DIMENSION(:), ALLOCATABLE : : QCV ! (PARTICLES)

! Heat transfer TO the particle from neighboring particles via the particle-

! particle conduction model. This value will be positive when the particle is

! heating and negative when the particle is cooling. DOUBLE PRECISION, DIMENSION(:), ALLOCATABLE : : epp ! (PARTICLES)

! Heat transfer TO the particle from neighboring particles via the particle-

! fluid-particle model. This value will be positive when the particle is

! heating and negative when the particle is cooling. DOUBLE PRECISION, DIMENSION(:), ALLOCATABLE : : epfp !(PARTICLES)

! Heat transfer $T O$ the particle from neighboring particles via radiation DOUBLE PRECISION, DIMENSION(:), ALLOCATABLE : : Qrd ! (PARTICLES)

! Internal heat generation resulting from a chemical reaction DOUBLE PRECISION, DIMENSION(:), ALLOCATABLE : : Qint !(PARTICLES)

! Previous time step's rate of heat transfer. Used for Adams-Bashforth

! time integration scheme DOUBLE PRECISION, DIMENSION(:), ALLOCATABLE : : Qtotal_OLD

! Thermodynamic Neighborhood

!-----------------------

! Thermodynamic neighborhood. This is the list of particles around a

! particle that may transfer energy with the given particle. INTEGER, DIMENSION $(:,:)$, ALLOCATABLE : : THERMO_NBRHD! (PARTICLES, MAXNEIGHBORS )

! This is the size of the thermodynamic neighborhood. Is is based on the

! maximum particle radius and the FLPC and RDPC DOUBLE PRECISION NBRHD_SZ

! Fluid Lens Proportion Constant used to calculate the radius of the

! fluid lens that surrounds the particle for particle-fluid-particle

! conduction. Default [ 0.2 ] DOUBLE PRECISION FLPC

! Radius of the radiation domain in relation of the particle diameter.

! Default [ 3.0 ] * DES RADIUS (-) DOUBLE PRECISION $\bar{R} D P C$

! Mininum separation distance between the surface of two contacting

! particles. This value is used to remove the singluarity that the

! particle-fluid-particle conduciton model develops at the contact

! interface. [4.0x10^(-10) meters] DOUBLE PRECISION DES_MIN_COND_DIST

END MODULE DES_THERMO 


\section{D.5 Routine - CHECK_DES_THERMO}

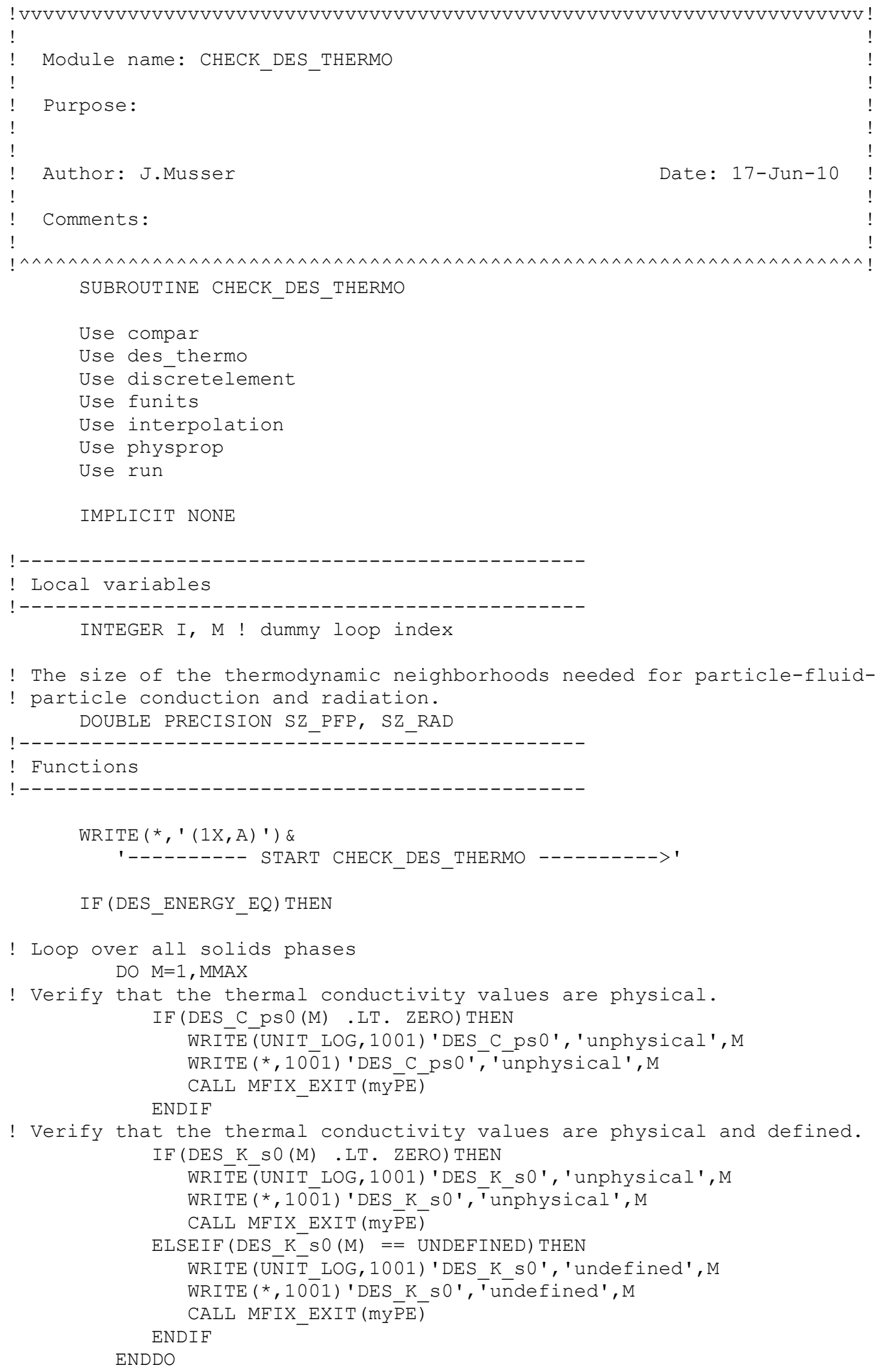


! Convection Equation:

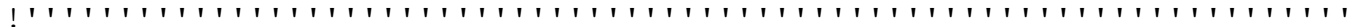

IF (DES CONV EQ) THEN

! Ensure that the continuum energy equations will be solved.

IF (.NOT.ENERGY EQ) THEN

$\operatorname{WRITE}(*, 100 \overline{0})$

ENDIF

CALL MFIX_EXIT (mYPE)

WRITE(*,' (6X,A)') 'DES convection model will be solved.'

SELECT CASE (TRIM (DES_CONV_CORR))

! Verify the selected convective heat transfer coefficient model

CASE ('RANZ 1952')

! Ranz, W.E. and Marshall, W.R., "Frication and transfer

! coefficients for single particles and packed beds,"

! Chemical Engineering Science, Vol. 48, No. 5, pp 247-253,

! 1925

! The Ranz and Marshall correlation needs no additional input from the

! user.

WRITE (*,' (9X, A, A) ') 'Heat transfer coefficient', \&

' model: Ranz and Marshall (1952)'

! If the heat transfer coefficient correlation provided by the user does

! not match one of the models outlined above, flag the error and exit.

CASE DEFAULT

WRITE $\left.(*, '(6 X, A))^{\prime}\right) \&$

' INVALID DES CONVECTION MODEL (DES CONV CORR) : '

WRITE (*,' (6X,A)') 'The available models include: '

WRITE (*,' (9X,A) ') 'RANZ 1952 - Ranz and Marshall (1952)'

END SELECT

CALL MFIX_EXIT

ELSE

WRITE(*,' (6X,A)') 'DES convection model will NOT be solved.' ENDIF ! END convection model checks

! Conduction Equations:

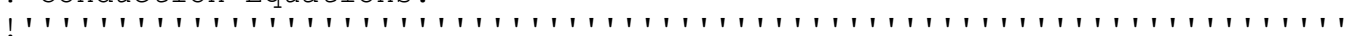

IF (DES COND_EQ) THEN

WRITE (*,' ' $6 \mathrm{X}, \mathrm{A})$ ') 'DES conduction model will be solved.'

! Particle-particle Conduction:

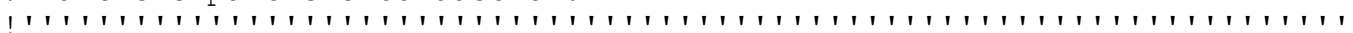

IF (DES COND EQ PP) THEN

DO $\overline{\mathrm{M}}=1, \mathrm{MM} A \mathrm{X}$

! Verify that a thermal conductivity value is specified for each solids

! phase and that the value is physical.

IF (DES_K_SO (M) = UNDEFINED) THEN

WRITE (UNIT_LOG, 1001 ) 'DES_K_sO', 'undefined', M

WRITE (*,1001) 'DES_K_s0',' 'undefined', M

CALL MFIX EXIT (my $\bar{P} E \overline{)}$

ELSEIF (DES K $\mathrm{K}^{-} \mathrm{SO}(\mathrm{M})$. LT. ZERO) THEN

WRITE (UNITT_LOG, 1001 ) 'DES_K_s0', 'unphysical', M

WRITE (*, 100 1 ) 'DES K s0',' 'unphysical', M

ENDIF

CALL MFIX EXIT (mY $\bar{P} E \overline{)}$

ENDDO

$\operatorname{WRITE}(*, '(6 \mathrm{X}, \mathrm{A})$ ') $\&$

'Solving the particle-particle conduction model.' 
! A secondary neighborhood is established surrounding a particle to

! determine the neighboring particles for possible particle-particle

! heat transfer. ELSE

FIND THERMO NBRHD $=$. TRUE.

WRITE (*, ' (5X,A) ') \& ENDIF

'NOT Solving the particle-particle conduction model.'

! Particle-fluid-particle Conduction:

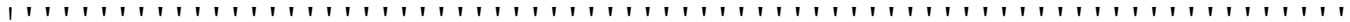

IF (DES COND EQ PFP) THEN

! Verify that either the simulation is coupled with a gas-phase

! simulation or that $K g 0$ has been provided in the dat file.

$I F\left(\mathrm{KGO}^{-}==\mathrm{UNDEFINED}\right.$. AND. \&

.NOT.DES_CONTINUUM_COUPLED) THEN

WRITE $(*, \overline{1} 002)$

WRITE (UNIT LOG, 1002)

ENDIF

CALL MFIX_EXIT (mYPE)

! Set the default value for the minimum distance separating particles'

! surfaces.

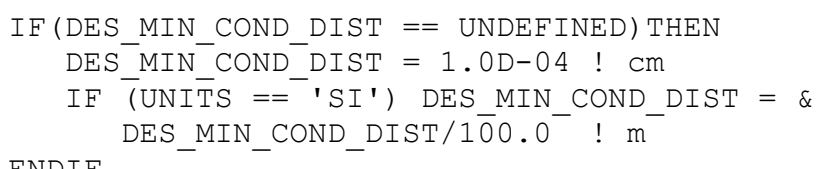
DES_MIN_COND_DIST/100.0 ! m

ENDIF

$\left.\operatorname{WRITE}\left(*,{ }^{\prime}(6 \mathrm{X}, \mathrm{A})\right)^{\prime}\right) \&$

'Solving the particle-fluid-particle conduction model.'

! A secondary neighborhood is established surrounding a particle to

! determine the neighboring particles for possible particle-fluid-

! particle heat transfer. ELSE

FIND_THERMO_NBRHD $=$.TRUE.

$\left.\operatorname{WRITE}(*, '(6 \mathrm{X}, \mathrm{A}))^{\prime}\right) \&$ ENDIF

'NOT Solving the particle-fluid-particle conduction model.'

ELSE

WRITE(*,' (6X,A)') 'DES conduction model will NOT be solved.'

! Set the logicals for the conduction sub-models to false.

! This should not be needed, but is done for precaution.

DES COND EQ PFP = . FALSE.

DES_CONDEE_PP $=$. FALSE.

ENDIF ! END conduction model checks

! Radiation Equation:

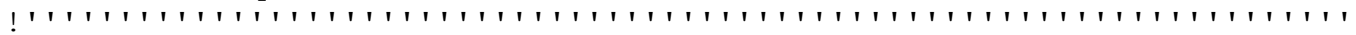

IF (DES_RADI_EQ) THEN

WRITE (*,' ' $6 \mathrm{X}, \mathrm{A})$ ') 'DES radiation model will be solved.'

! A secondary neighborhood is established surrounding a particle to

! determine the neighboring particles for possible radiative

! heat transfer.

FIND_THERMO_NBRHD $=$.TRUE.

! Set the value of the Stefan-Boltzman constant based on the untis

IF (UNITS $==$ 'SI') THEN

$\quad \quad W /\left(\left(m^{\wedge} 2\right) \cdot K^{\wedge} 4\right)$ ELSE

$\mathrm{SB}$ CONST $=5.6704 \mathrm{~d} 0 *(10.0 \mathrm{~d} 0 * *(-8))$

$! \quad \mathrm{cal} /\left(\left(\mathrm{cm}^{\wedge} 2\right) \cdot \sec \cdot \mathrm{K}^{\wedge} 4\right)$ 


\section{ENDIF}

$\mathrm{SB} \_$CONST $=1.355282 \mathrm{~d} 0 *(10.0 \mathrm{~d} 0 * *(-12))$

DO M=1, MMAX

! Verify that a emmisivity value is specified for each solids pase IF (DES_Em (M) $==$ UNDEFINED) THEN

WRITE (UNIT LOG, 1001) 'DES Em', 'undefined', M WRITE $(*, 10 \overline{0} 1)$ 'DES Em', 'undefined', M CALL MFIX_EXIT (my $\overline{\mathrm{P}} E)$

ELSEIF (DES Em $(M)$.LT. ZERO .OR. \& DES_Em $(\overline{\mathrm{M}})$.GT. ONE) THEN

WRITE (UNIT LOG, 1001) 'DES Em', 'unphysical', M WRITE (*,1001) 'DES_Em', 'unphysical', M CALL MFIX_EXIT (my $\bar{P} E)$ ENDIF ENDDO ELSE

WRITE(*,' (6X,A)') 'DES radiation model will NOT be solved.' ENDIF ! END radiation model checks

! Calculate the size of the thermodynamic neighbor (if needed) IF( FIND_THERMO_NBRHD) THEN SZ $P F \bar{P}=$ ZER $\bar{O}$

! If solving conductive heat transfer, the minimum neighborhood size

! is [1.3]*DES RADIUS by default. A buffer of 0.1 is added to ensure

! that the neighhor is seen as soon as possible. The size of the fluid

! lens for particle-fluid-particle heat transfer can be adjusted by

! specifying a different value in the mfix.dat file.

IF (DES_COND_EQ_PP .OR. DES_COND_EQ_PFP) \& $S Z$ PFP $=$ MAX_RADIUS * $(-2.05 \overline{\mathrm{d}} 0 \overline{\bar{X}}+\mathrm{FLPC})$ SZ RAD $=$ ZERO

! If radiation heat transfer is solved, the radius of the radiative ! heat transfer domain is [3.0]*DES RADIUS by default. The size of the

! radiative heat transfer domain on the particle may be adjusted by

! specifying a new value in the mfix.dat file. IF (DES RADI EQ) \& SZ $\overline{R A D}=$ MAX RADIUS * RDPC NBRHD_SZ $=$ MAX ( SZ_PFP, SZ_RAD ) $\operatorname{WRITE} \overline{(*}^{*},(6 \mathrm{X}, \mathrm{A}, \mathrm{F} 10.6)$ ') \& 'Radius of thermodynamic neighborhood is ', NBRHD SZ ENDIF ! FIND_THERMO_NBRHD

ELSE ! DES_ENERGY_EQ

WRITE (*,' $(6 \mathrm{X}, \mathrm{A})$ ') 'DES energy equations are not being solved.'

! Reinitialize the heat transfer logicals to false. DES_CONV_EQ = .FALSE. DES_COND_EQ $=$.FALSE. DES_RADI_EQ $=$. FALSE.

DES_COND_EQ_PFP $=$.FALSE. DES_COND_EQ_PP $=$.FALSE.

ENDIF ! DES_ENERGY_EQ

$\left.\operatorname{WRITE}(*, '(1 \mathrm{X}, \mathrm{A}))^{\prime}\right) \&$ '<-------- END CHECK_DES_THERMO ---------'

RETURN

1000 FORMAT $(/ 1 \mathrm{X}, 70(' \star$ ' $) /,$ ' From: CHECK_DES_THERMO',/, ' Message: ', \& 'The DES convection model requires that the energy ', \& 
'equations',/1X,' (ENERGY EQ) for the continuum phase must ', \&

'also be solved.', /1X, 70('*')/)

1001 FORMAT (/1X,70('*')/,' 'From: CHECK DES THERMO',/,' 'Message: ',\&

A,' is ',A,' for solids phase ',I2,''. Check mfix.dat.', \&

$\left./ 1 \mathrm{X}, 70\left({ }^{\prime} * '\right) /\right)$

1002 FORMAT (/1X,70('*')/,' From: CHECK_DES_THERMO',/,' Message:', \& ' The particle-fluid-particle model requires that either a'/, \&

' simulation be coupled with a gas-phase simultion or that', \&

' K_g0 be',/,' provided in the mfix.dat file. Check mfix.dat.', \& $\left./ 1 \mathrm{X}, 70\left({ }^{\prime} * \mathbf{\prime}^{\prime}\right) /\right)$

END SUBROUTINE CHECK_DES_THERMO 


\section{D.6 Routine - CALC_THERMO_DES}

! VVVVVVVVVVVVVVVVVVVVVVVVVVVVVVVVVVVVVVVVVVVVVVVVVVVVVVVVVVVVVVVVVVVVVV!

$!$
$! \quad$ Module name: CALC_THERMO_DES

SUBROUTINE CALC_THERMO_DES(I, INTERP_IJK, INTERP_WEIGHTS, FOCUS)

Use des_thermo

Use dis̄̄retelement

Use fldvar

Use interpolation

Use param1

IMPLICIT NONE

! Passed variables

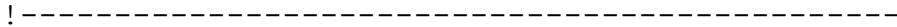

! Index of particle being looped over INTEGER, INTENT(IN) : : I

! IJK indicies of fluid cells involved in interpolation INTEGER, INTENT(IN) : : INTERP_IJK(INTERP_ORDER**DIMN)

! Weights associated with interpolation DOUBLE PRECISION, INTENT(IN) : : INTERP WEIGHTS (INTERP_ORDER**DIMN)

! Indicates that debugging information for the particle LOGICAL, INTENT(IN) : : FOCUS

! Local variables

!---------------------------------------------

! Index of neighbor particle of particle I such that I $<\mathrm{J}$ INTEGER J

! Generic index of neighbor particle of particle I INTEGER JJ

! index to track accounted for particles INTEGER PC

! Number of particles in the radiation domain INTEGER N RAD

! Distance between the centers of particle I and particle J (component) DOUBLE PRECISION DISTVEC (DIMN)

! Distance between the centers of particle I and particle J (magnitude) DOUBLE PRECISION CENTER DIST

! Distance between the surfacēs of particle I and particle J DOUBLE PRECISION SURFACE DIST

! Angle generated between the $\bar{l}$ ine connecting the particle centers of

! particle $I$ and $J$ and the line generated by the center of particle I

! and one of the premiter of the circle generated by intersection area

! of particle I and particle $\mathrm{J}$ DOUBLE PRECISION ANG IJ

! Radius of the circle created by the overlap of the spherical particles DOUBLE PRECISION R sij

! Environment temperature used in the radiative heat transfer model DOUBLE PRECISION TS_RAD 
! Functions

!---------

DOUBLE PRECISION, EXTERNAL : : DES_DOTPRDCT

! calculate heat transfer via convection IF(DES_CONV_EQ) CALL DES_CONVECTION(I, INTERP_IJK, \& INTERP WEIGHTS, FOCUS )

! Loop over thermodynamic neighbor for conduction and radiation IF (DES_COND_EQ.OR. DES_RADI_EQ) THEN

! Initialize variābles on entry TS_RAD $=$ ZERO

$\mathrm{N} \bar{R}_{-} A D=0$

! Distance between particle centers IF (THERMO_NBRHD $(I, 1)$. GT.0) THEN

DO JJ $=2, \operatorname{THERMO} \operatorname{NBRHD}(I, 1)+1$

! Set the index of the neighbor particle $\mathrm{J}=$ THERMO_NBRHD ( $\mathrm{I}, \mathrm{JJ})$

! Skip the neighbor if it does not exist (should not be a problem) IF (.NOT.PEA $(J, 1)$ ) CYCLE

! Only do conduction calculations for particles with an index value

! higher than the current particle. The opposite heat transfer will be

! applied to the neighbor. IF (J.GT.I) THEN

! Calculate the center distance between the two particles $\operatorname{DISTVEC}(:)=\operatorname{DES} \_P O S \_N E W(I,:)$ - DES_POS_NEW(J, : ) CENTER_DIST $=S Q \overline{R T}\left(D \bar{E} S \_D O T P R D C T(D I S T V E C, \bar{D}\right.$ DSTVEC) $)$

! If the radiation heat transfer model is being solved, check to see

! if the particle $J$ is within the domain of particle I. IF (DES_RADI_EQ) THEN

IF ( $\bar{C} E N T E \bar{R}$ DIST.LT.RDPC *DES_RADIUS ( I ) ) THEN

! Increment the number of neighbors in the radiation domain and sum

! the temperature into the environment temperature (TS_RAD) N_RAD $=\mathrm{N}_{-}$RAD +1
TS_RAD $=\overline{T S}$ _RAD + DES_T_S_NEW $(\mathrm{J})$
ENDIF ENDIF

! Calculate particle-particle conduction IF (DES_COND_EQ) THEN ENDIF

CALI DES_CONDUCTION ( I, J, CENTER_DIST, FOCUS)

ELSEIF (DES_RADI_EQ) THEN

! Calculate the center distance between the two particles $\operatorname{DISTVEC}(:)=\operatorname{DES} \operatorname{POS} \operatorname{NEW}(I,:)-\operatorname{DES} \operatorname{POS} \operatorname{NEW}(\mathrm{J},:)$ CENTER_DIST $=$ SQRT (DES_DOTPRDCT (DISTVEC,DISTVEC))

! If the radiation heat Eransfer model is being solved, check to see

! if the particle $J$ is within the domain of particle I. IF (RDPC*DES_RADIUS (I).GT.CENTER_DIST) THEN

! Increment the number of neighbors in the radiation domain and sum

! the temperature into the environment temperature (TS_RAD)

$$
\begin{aligned}
& \text { N_RAD }=\text { N_RAD }+1 \\
& \text { TS_RAD }=\bar{T} \text { S_RAD }+ \text { DES_T_S_NEW }(J)
\end{aligned}
$$
ENDIF

! calculate heat transfer via radiation IF (DES_RADI_EQ.AND. N_RAD.GT. O) THEN

! Average the temperature

TS_RAD = TS_RAD/N_RAD ENDIF

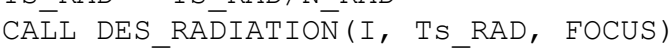

END SUBROUTINE CALC_THERMO_DES 


\section{D.7 Routine - DES_CONDUCTION}

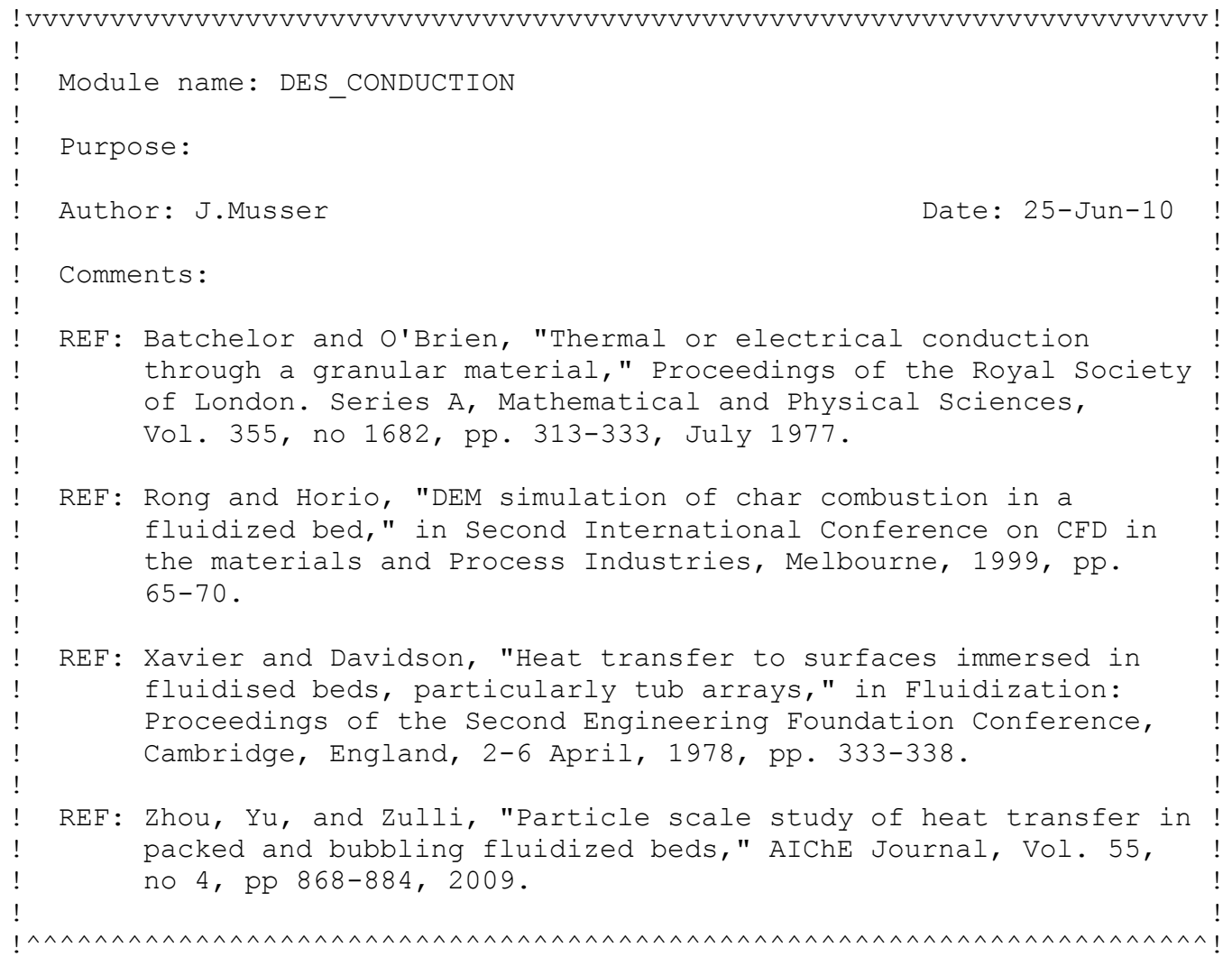

SUBROUTINE DES_CONDUCTION(I, J,C_DIST,FOCUS)

Use constant

Use des thermo

Use discretelement

Use fldvar

Use funits

Use param1

Use physprop

IMPLICIT NONE

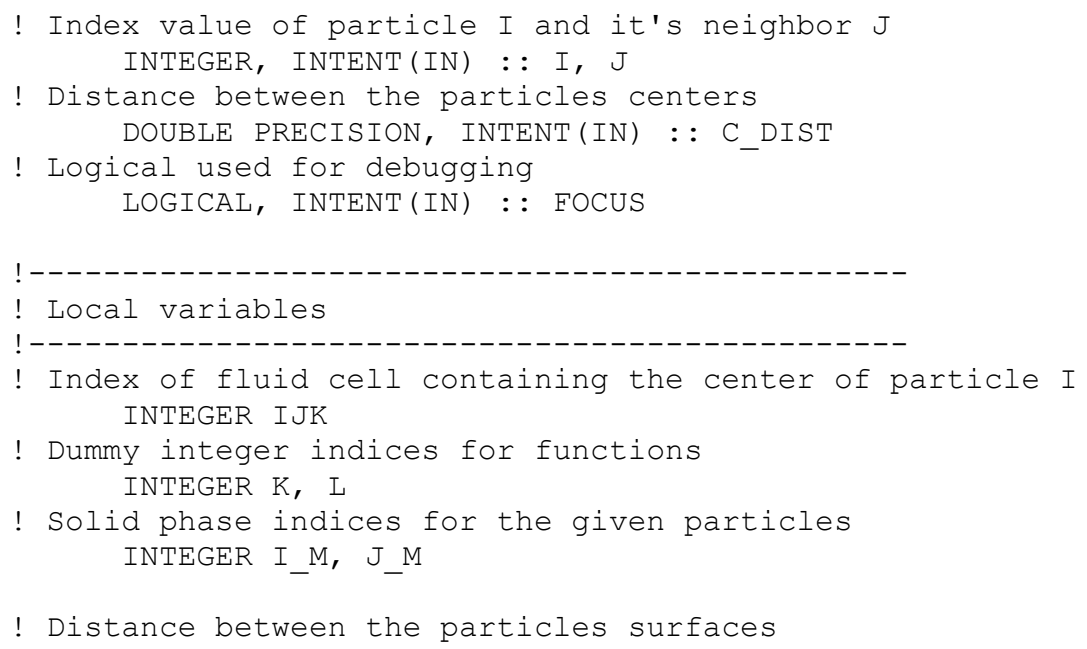


DOUBLE PRECISION S DIST

! Radius of smaller particle

DOUBLE PRECISION MIN_RAD

! Radius of larger particlēe

DOUBLE PRECISION MAX_RAD

! Rate of particle-particlēe conduction DOUBLE PRECISION Q pp

! Rate of particle-fluid-particle conduction DOUBLE PRECISION Q pfp

! Outer radius of region delineating particle-fluid-particle conduction DOUBLE PRECISION RD_OUT

! Inner radius of region delineating particle-fluid-particle conduction DOUBLE PRECISION RD_IN

! The radius of the fluid lens containing the larger particle DOUBLE PRECISION LENS RAD

! Surface area of particle DOUBLE PRECISION A_S

! Thermo DOUBLE PRECISION Kg

! Identify the fluid cell containing particle I $I J K=P I J K(I, 4)$

! Identify the solid phases of each particle $I M=\operatorname{PIJK}(I, 5)$

$\mathrm{J}^{-} \mathrm{M}=\operatorname{PIJK}(\mathrm{J}, 5)$

! Determine the radius of the larger and smaller particle MIN_RAD $=$ MIN (DES_RADIUS $(I), \operatorname{DES} \operatorname{RADIUS}(\mathrm{J}))$ MAX_RAD $=$ MAX $\left(D E S \_R A D I U S(I), D E S \_R A D I U S(J)\right)$

! Initialize the rates of conductive heat transfer $\mathrm{Q} \_\mathrm{pp}=\mathrm{ZERO}$

Q $\mathrm{pfp}=\mathrm{ZERO}$

! Calculate the particle-particle conduction

! REF: Batchelor and O'Brien, 1977 (MODIFIED) IF (DES_COND_EQ_PP .AND. C_DIST < (MAX_RAD + MIN_RAD) ) THEN

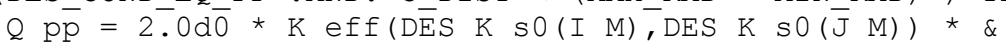

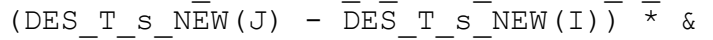
RADI $\bar{U} \bar{S}(\mathrm{MA} X$ RAD, MIN RĀD)

! Assign the heat flux to both particles. $\operatorname{epp}(I)=\operatorname{epp}(I)+Q \operatorname{pp}$ ENDIF $\operatorname{epp}(\mathrm{J}) \quad=\operatorname{epp}(\mathrm{J}) \quad-$ Q_pp

! Calculate the particle-fluid-particle conduction

! REF: Rong and Horio, 1999 (MODIFIED) IF (DES_COND_EQ_PFP) THEN

! Calculate the rädius of the fluid lens surrounding the larger particle LENS_RAD $=$ MAX_RAD * (1.0DO + FLPC $)$

! Calculate the outer radial distance (thickness) of the region for

! particle-fluid-particle heat conduction. RD OUT = RADIUS ( LENS RAD, MIN RAD)

! If the value returned is less̄ than zê̄o, then the fluid lens

! surrounding the larger particle does not intersect with the surface

! of the smaller particle. In this case, particle-fluild-particle

! conduction does not occur. IF ( RD_OUT .GT. ZERO) THEN

! Calculate the distance from the line connecting the particles' centers

! to the point of contact between the two particles. This value is

! zero if the particles are not touching and is the radius of the 


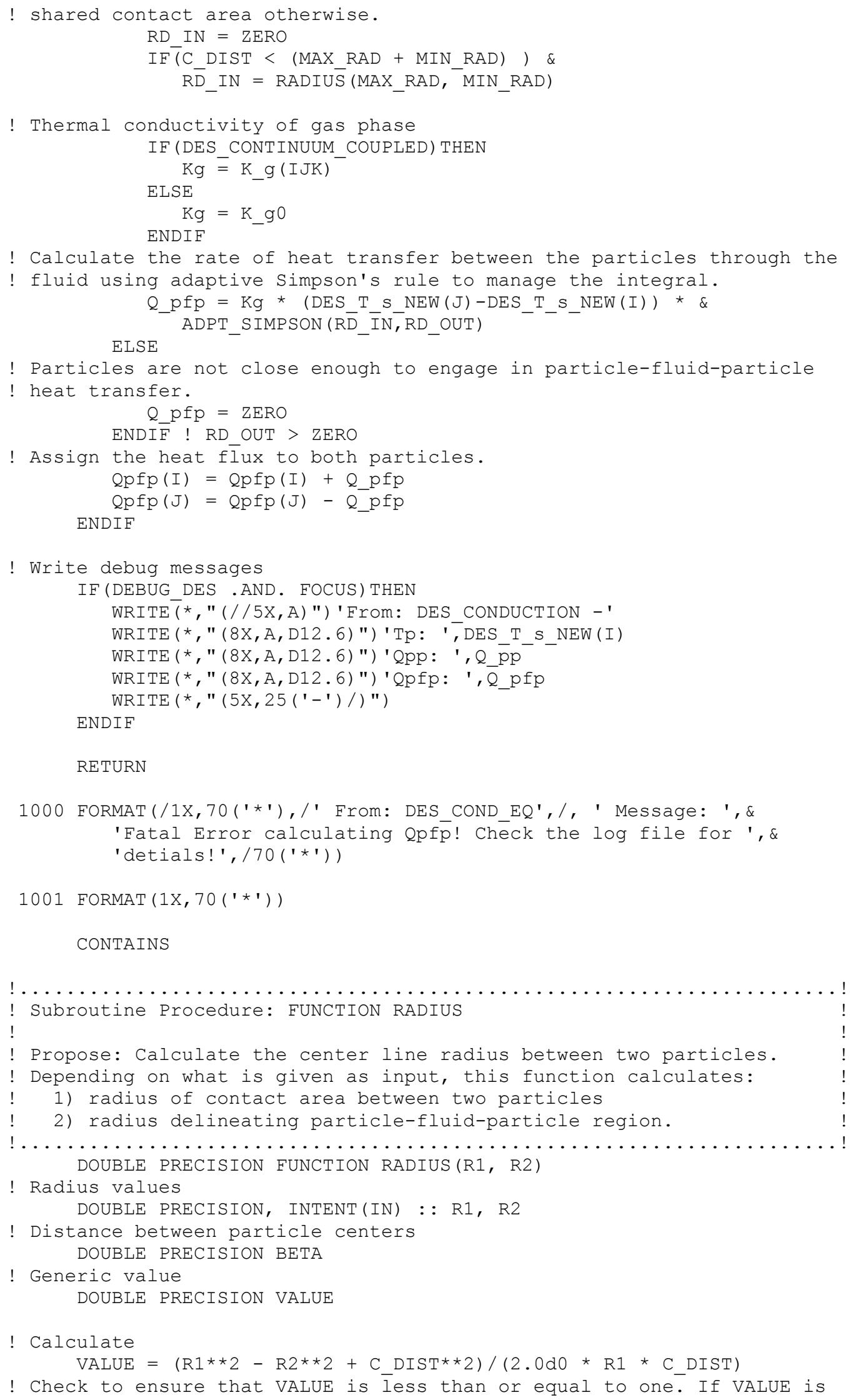


! greater than one, the triangle inequality has been violated. Therefore

! there is no intersection between the fluid lens surrounding the larger

! particle and the surface of the smaller particle.

! Thus, there is no particle-fluid-particle heat transfer.

IF ( VALUE .GT. 1.0d0) THEN

ELSE

RADIUS $=-1.0 \mathrm{~d} 0$

! Calculate beta (Law of cosines)

$\mathrm{BETA}=\operatorname{ACOS}(\mathrm{VALUE})$

! Calculate the radius

RADIUS $=$ RI * SIN (BETA)

ENDIF

RETURN

END FUNCTION RADIUS

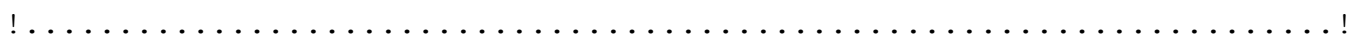

! Subroutine Procedure: FUNCTION K eff !

! Propose: Calculate the effective thermal conductivity of two

! particles with conductivities K1 and K2

DOUBLE PRECISION FUNCTION $K$ eff(K1, K2)

! Thermal conductivities

DOUBLE PRECISION, INTENT(IN) : : K1, K2

K_eff $=2.0 \mathrm{~d} 0 *(\mathrm{~K} 1 * \mathrm{~K} 2) /(\mathrm{K} 1+\mathrm{K} 2)$

RETURN

END FUNCTION K_eff

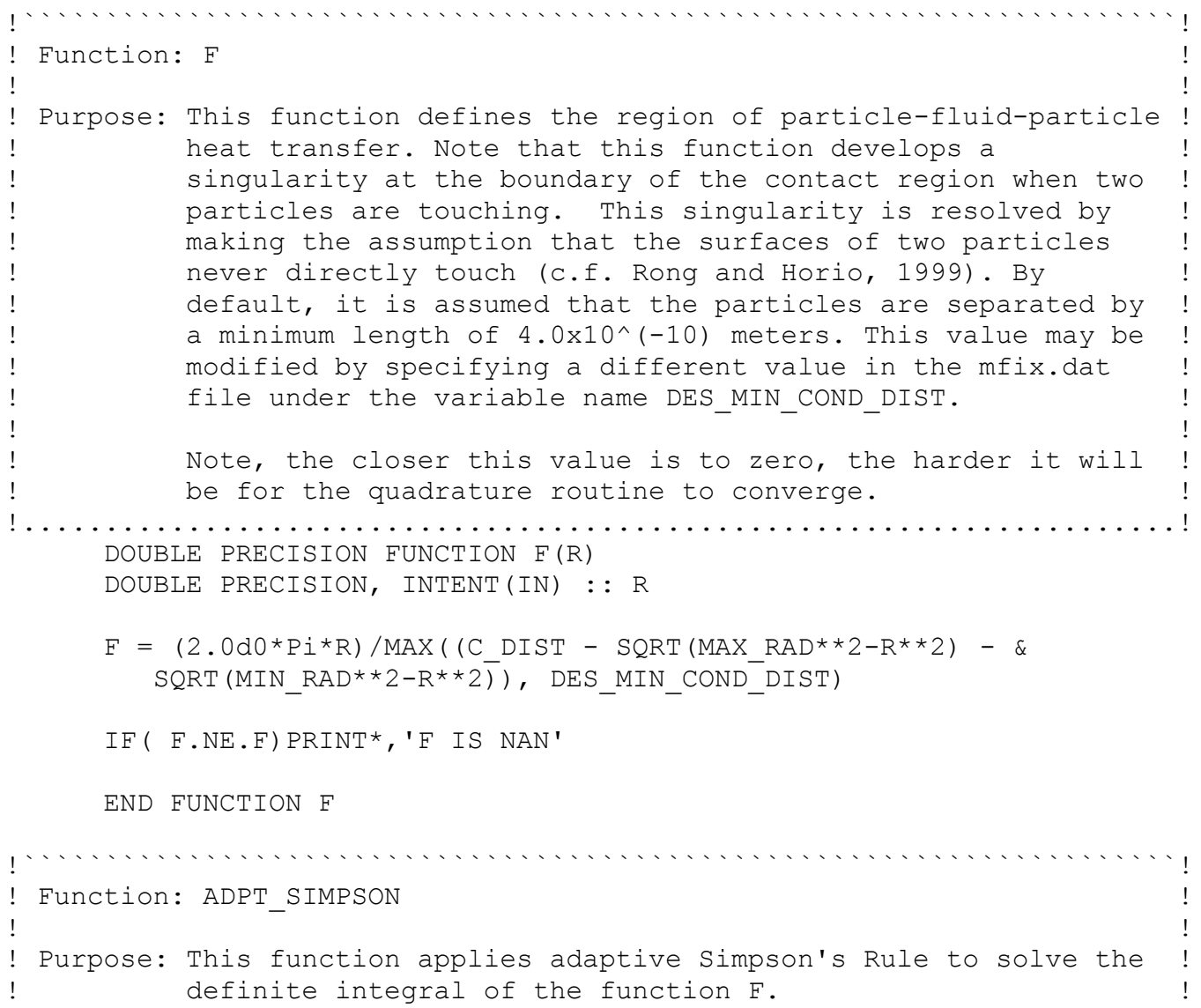




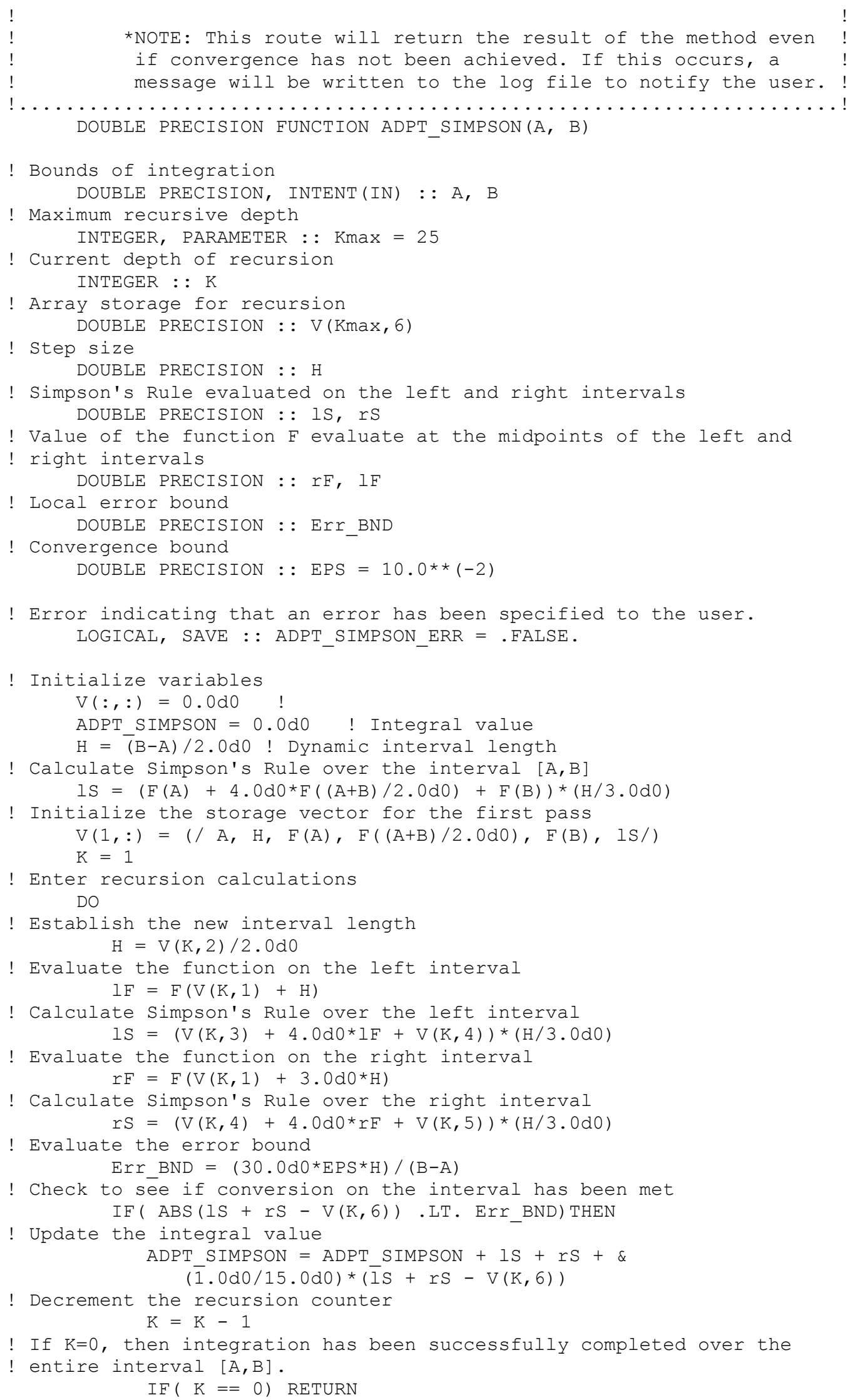

! Evaluate the error bound $\operatorname{Err} B N D=(30.0 \mathrm{~d} 0 * \mathrm{EPS} * \mathrm{H}) /(\mathrm{B}-\mathrm{A})$

! Check to see if conversion on the interval has been met IF ( ABS $(1 S+r S-V(K, 6))$. LT. Err BND) THEN

! Update the integral value 
$\operatorname{ELSEIF}((\mathrm{K}, \mathrm{GE} . \mathrm{Kmax}) . \mathrm{OR}$. \&

$(\mathrm{H}==(\mathrm{B}-\mathrm{A}) *(1.0 \mathrm{~d} 0 / 2.0 \mathrm{~d} 0) * *(\mathrm{Kmax}+3)))$ THEN

! Flag that the method did not converge.

IF (.NOT.ADPT SIMPSON_ERR) THEN

WRITE $(*, 1000)$

WRITE (UNIT LOG, 1000 )

ENDIF

ADPT_SIMPSON_ERR $=$. TRUE.

! Update the integral value

$\mathrm{ADPT}$ SIMPSON $=\mathrm{ADPT}$ SIMPSON $+1 \mathrm{~S}+\mathrm{rS}+\varepsilon$ $(\overline{1} .0 \mathrm{~d} 0 / 15.0 \mathrm{~d} 0) *(\overline{\mathrm{I}} \mathrm{S}+\mathrm{rS}-\mathrm{V}(\mathrm{K}, 6))$

! Decrement the recursion counter

$\mathrm{K}=\mathrm{K}-1$

ELSE

! Refine the subintervals through recursive splitting of the intervals

$\mathrm{V}(\mathrm{K}+1,:)=(/ \mathrm{V}(\mathrm{K}, 1)+2.0 \mathrm{~d} 0 * \mathrm{H}, \mathrm{H}, \mathrm{V}(\mathrm{K}, 4), \mathrm{rF}, \mathrm{V}(\mathrm{K}, 5), \mathrm{rS} /)$

$\mathrm{V}(\mathrm{K},:)=(/ \mathrm{V}(\mathrm{K}, 1), \mathrm{H}, \mathrm{V}(\mathrm{K}, 3), 1 \mathrm{~F}, \mathrm{~V}(\mathrm{~K}, 4), \mathrm{lS} /)$

! Increment level counter

$\mathrm{K}=\mathrm{K}+1$

ENDIF

ENDDO

1000 FORMAT (/1X,70('*'),/' From: DES COND EQ',/, ' Message: ',

'Integration of the particle-flui $\bar{d}$-particle equation did ', \&

'not',/' converge! No definite bound can be placed on the ', \&

'error.',/' Future convergence messages will be suppressed!', \& $\left./ 1 \mathrm{X}, 70\left({ }^{\prime}{ }^{\prime}\right)\right)$

END FUNCTION ADPT_SIMPSON

END SUBROUTINE DES_CONDUCTION 


\section{D.8 Routine - DES_CONVECTION}

! VVVVVVVVVVVVVVVVVVVVVVVVVVVVVVVVVVVVVVVVVVVVVVVVVVVVVVVVVVVVVVVVVVVVVV!

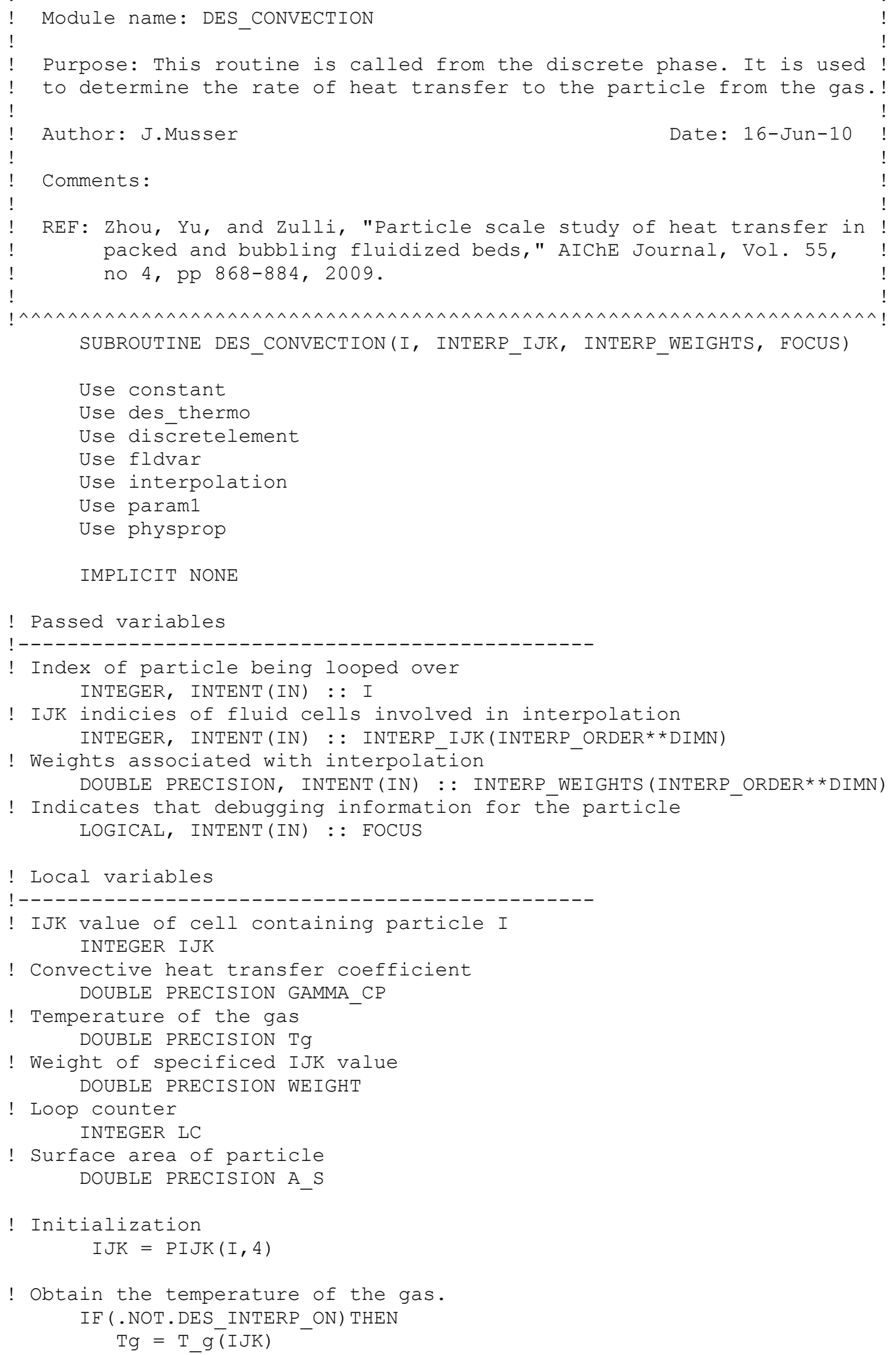




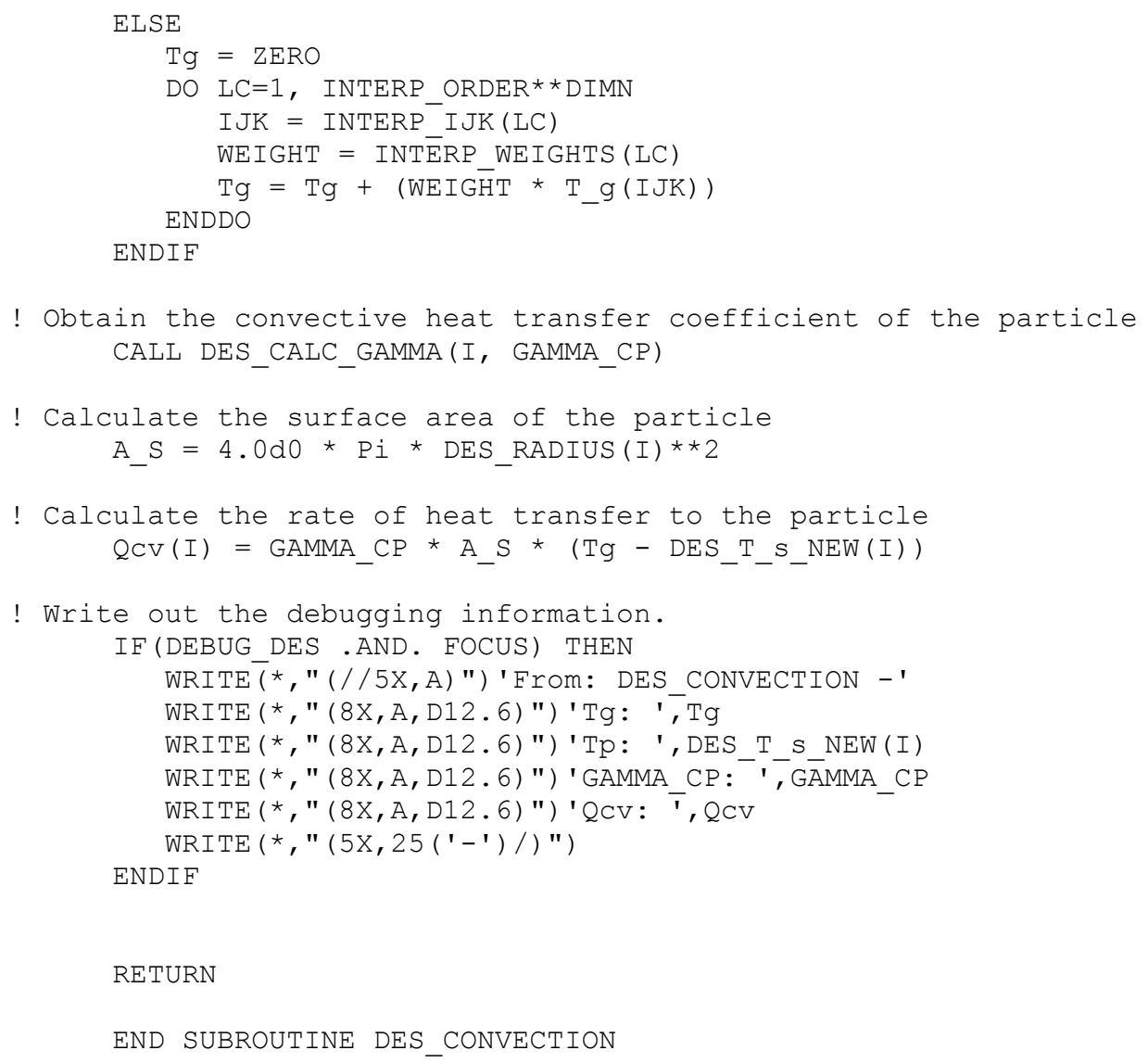




\section{D.9 Routine - DES_Hgm}

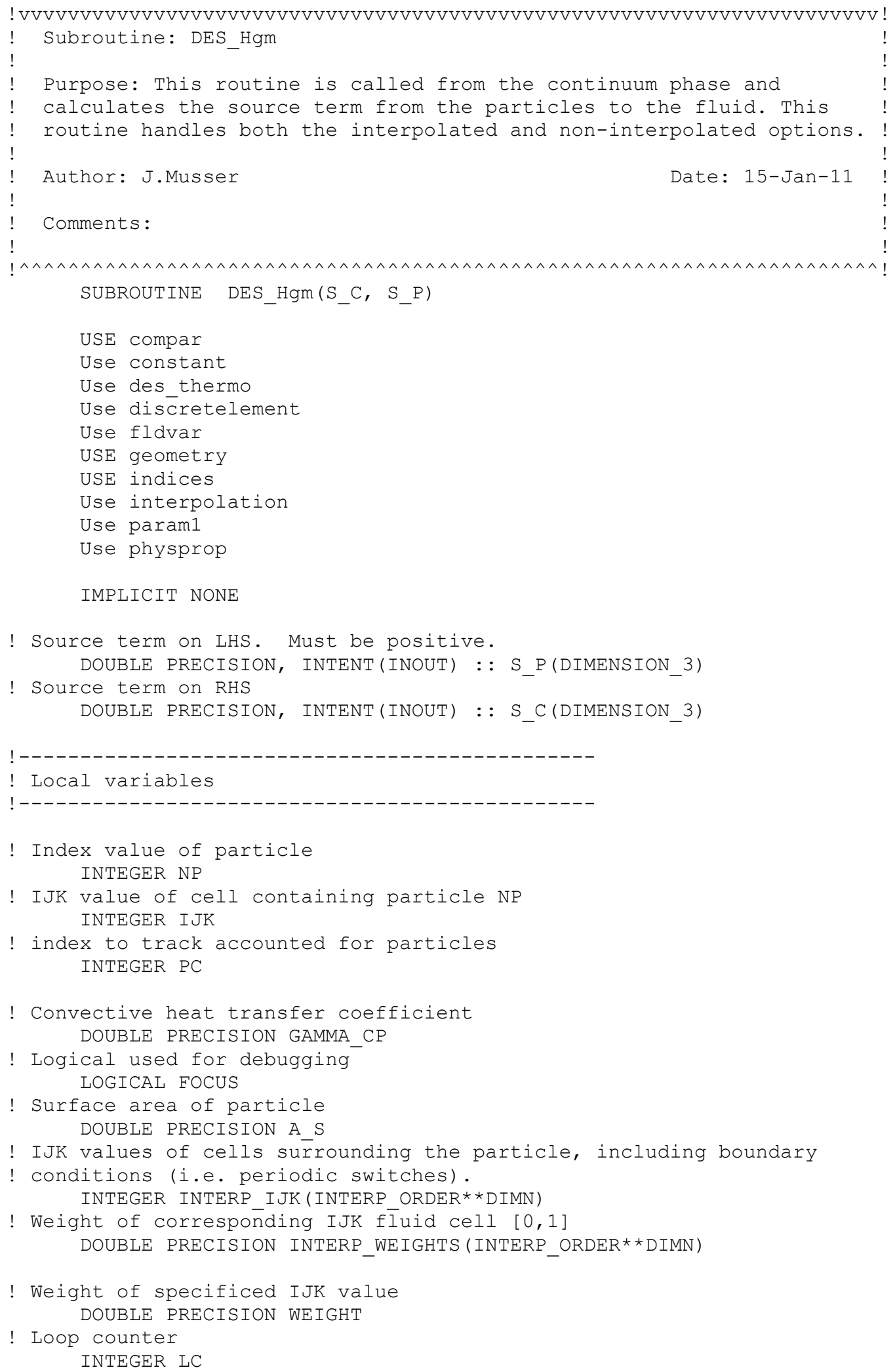


! Initialize the particle counter $\mathrm{PC}=1$

! Loop over the particles in the system

$\mathrm{DO} \mathrm{NP}=1$, MAX_PIS

! Exit the loop if all the particles in the system have been looped over IF (PC.GT. PIS) EXIT

! Cycle the loop if there is no particle associated with this index IF (.NOT.PEA $(N P, 1)$ ) CYCLE

! Set debug flag FOCUS $=$.FALSE.

IF (NP.EQ.FOCUS_PARTICLE) FOCUS $=$. TRUE.

! Obtain the convective heat transfer coefficient of the particle CALL DES_CALC_GAMMA (NP, GAMMA_CP)

! Calculate the surface area of the particle A_S $=4.0 \mathrm{~d} 0$ * Pi * DES_RADIUS $(\mathrm{NP}) * * 2$

! Obtain the temperature of the gas. IF (.NOT.DES_INTERP_ON) THEN

! Set the fluid cell index. $\mathrm{IJK}=\mathrm{PIJK}(\mathrm{NP}, 4)$

! Calculate the source term components $S \_P(I J K)=S \_P(I J K)+($ GAMMA_CP * A_S $)$ $S_{-}^{-} C(I J K)=S_{-}^{-} C(I J K)+($ GAMMA_CP $*$ A_S $*$ DES_T_S_NEW $(N P))$ ELSE

! Determine the IJK values for the surrounding fluid cells and calculate

! the cell-centered interpolation weights. CALL INTERPOLATE_CC (NP, INTERP ORDER, \& INTERP_SCHEME, INTERP_IJK, INTERP_WEIGHTS, FOCUS)

! Distribute the thermal energy to/f $\overline{r o m}$ the fluid cells DO LC $=1$, INTERP_ORDER $* *$ DIMN

! Set variable values IJK $=$ INTERP IJK (LC) WEIGHT = INTERP_WEIGHTS $(\mathrm{LC})$

! Portion the source term to céll IJK $S_{-P}(I J K)=S-P(I J K)+$ WEIGHT $*$ (GAMMA_CP $* A_{-} S$ )
$S^{-C}(I J K)=S C($ IJK) + WEIGHT $\star \varepsilon$ (GAMMA_CP ${ }^{-}$. A_S $*$ DES_T_S_NEW (NP)) ENDDO ! LC-lōop

ENDIF ! End interpolation routine

! Reset the focus particle logical FOCUS $=$. FALSE.

! Index the particle count by one $\mathrm{PC}=\mathrm{PC}+1$

ENDDO

END SUBROUTINE DES_Hgm 


\section{D.10 Routine -DES_CALC_GAMMA}

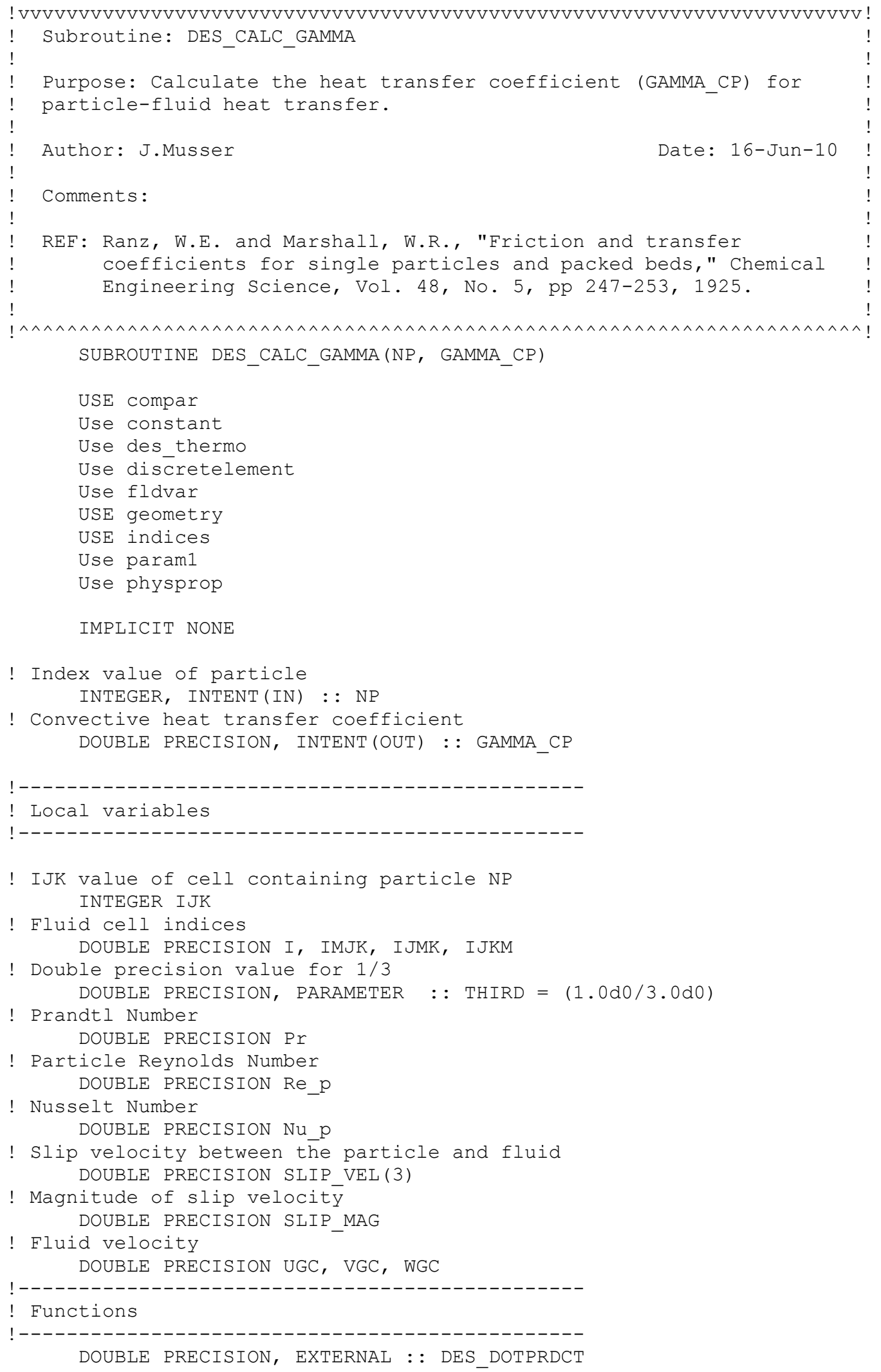




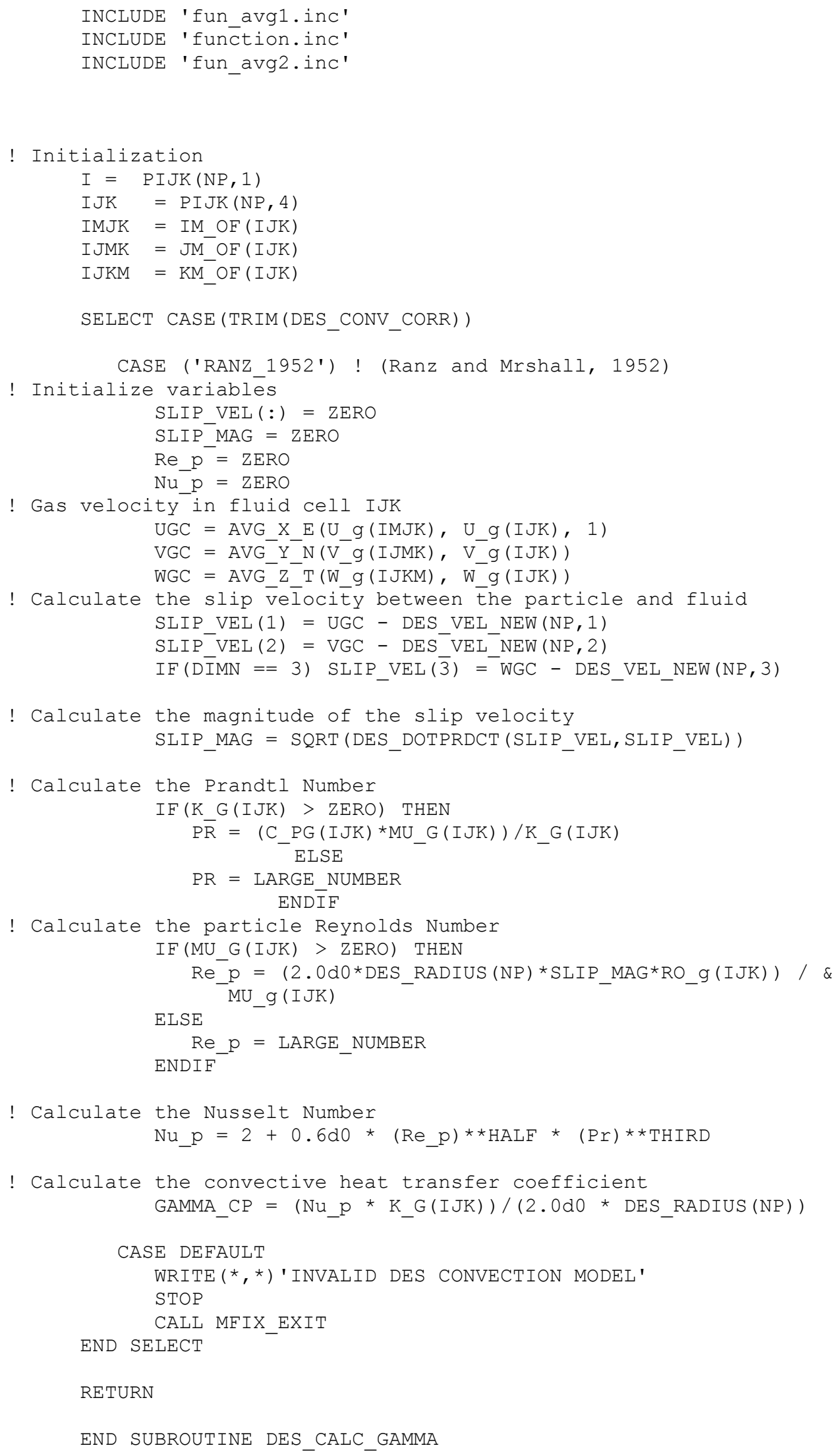




\section{D.11 Routine -DES_RADIATION}

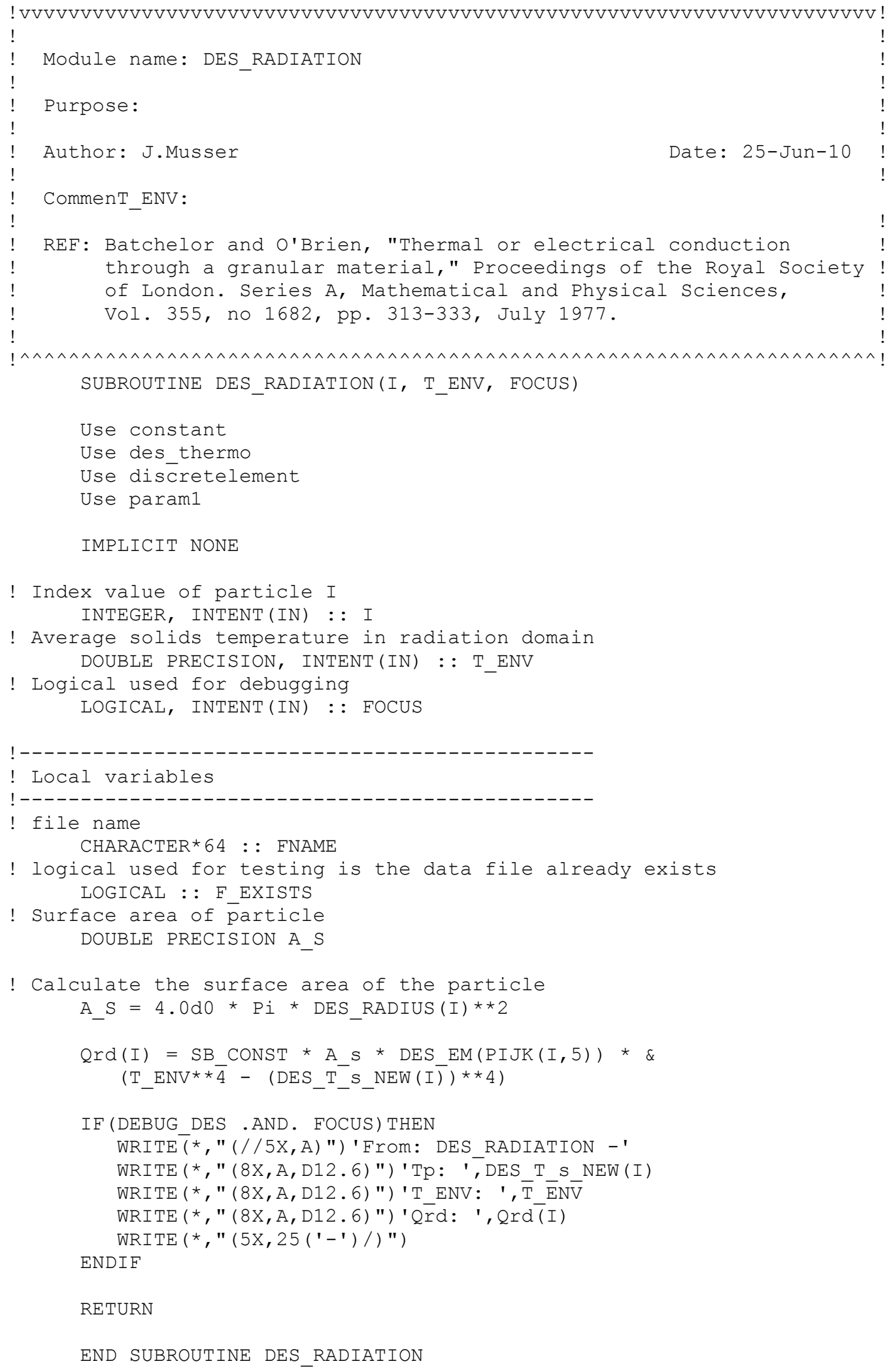




\section{D.12 Routine -DES_THERMO_NEWVALUES}

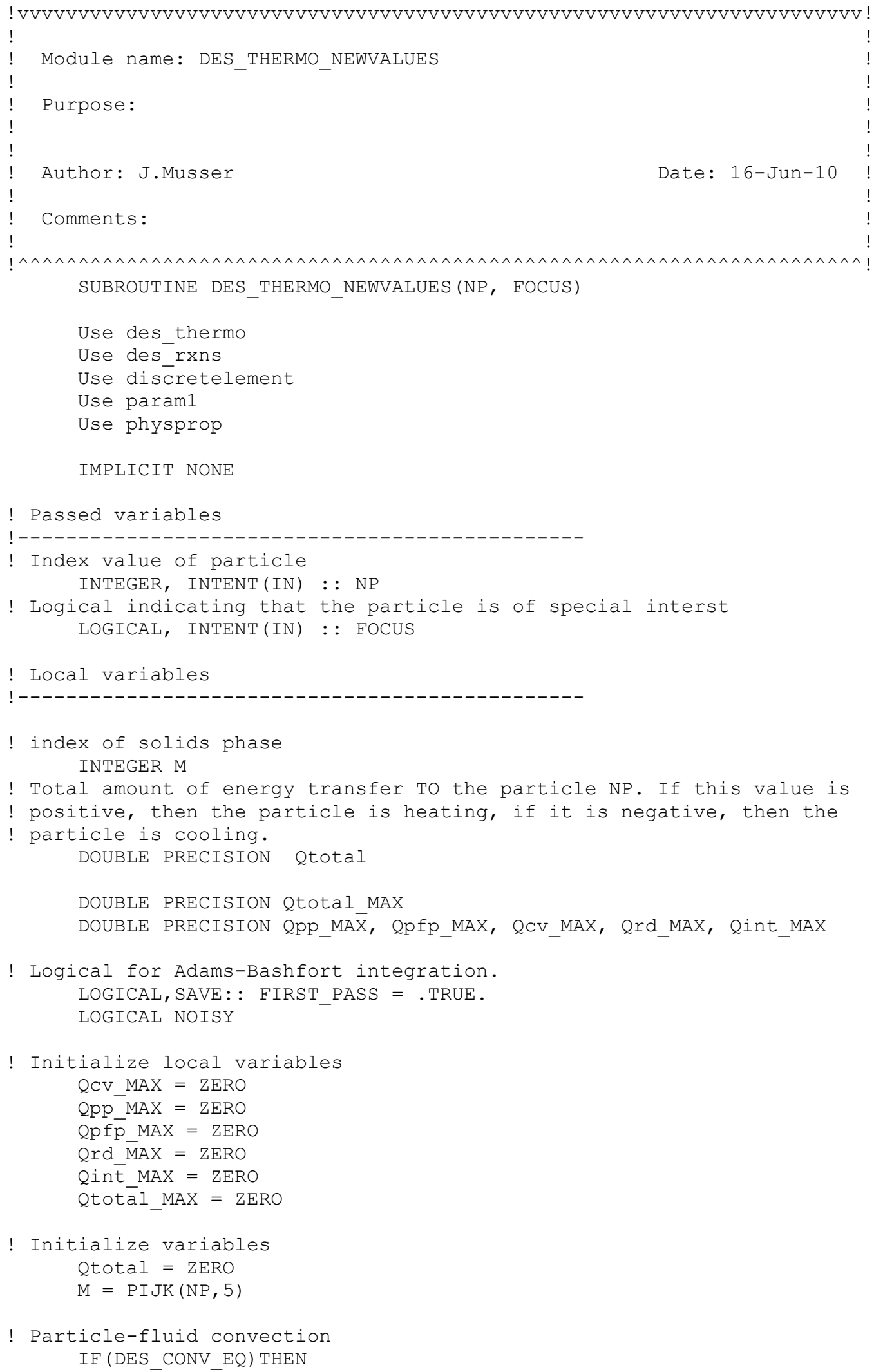




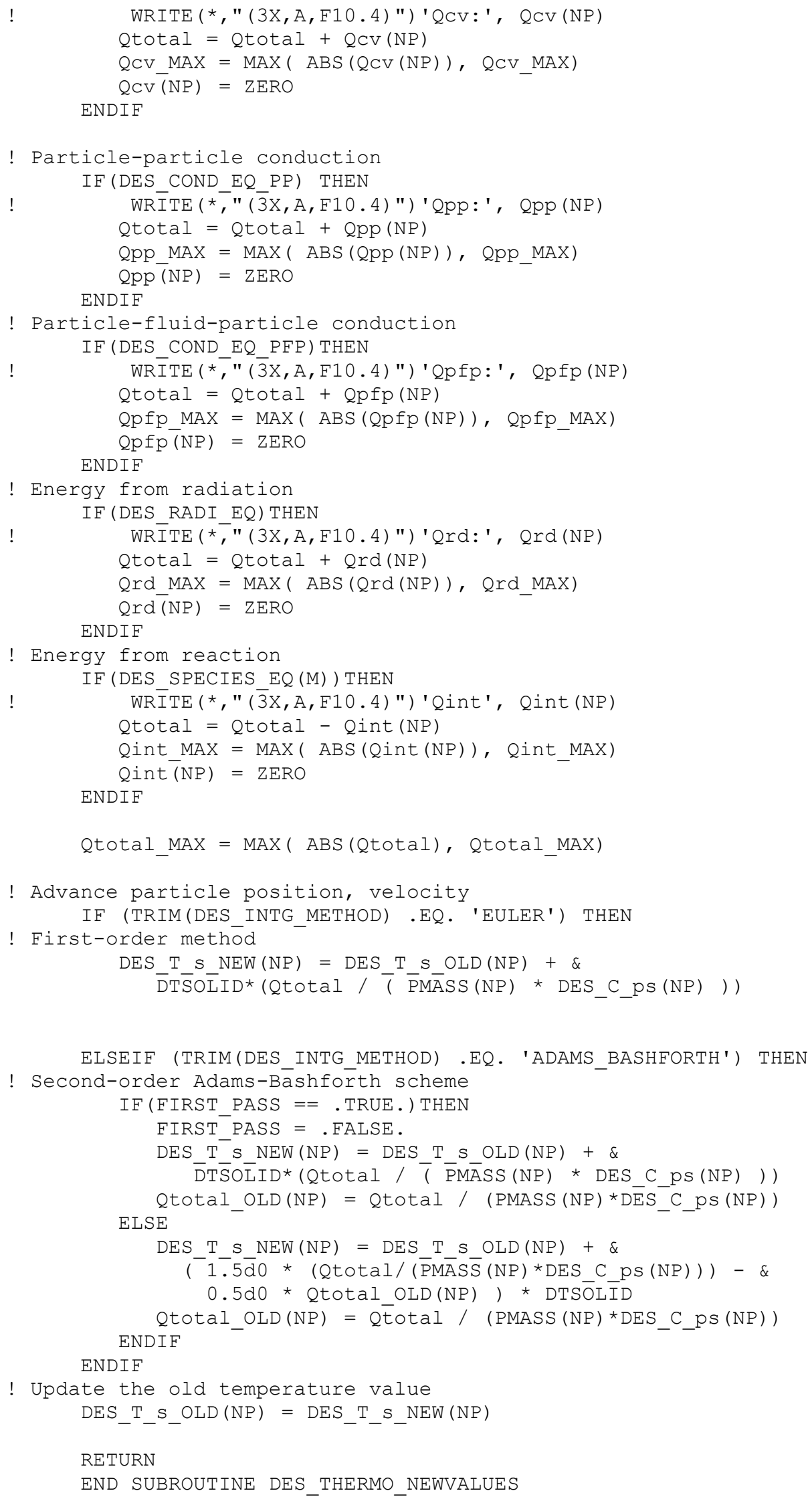




\section{D.13 Routine -THERMO_NBR}

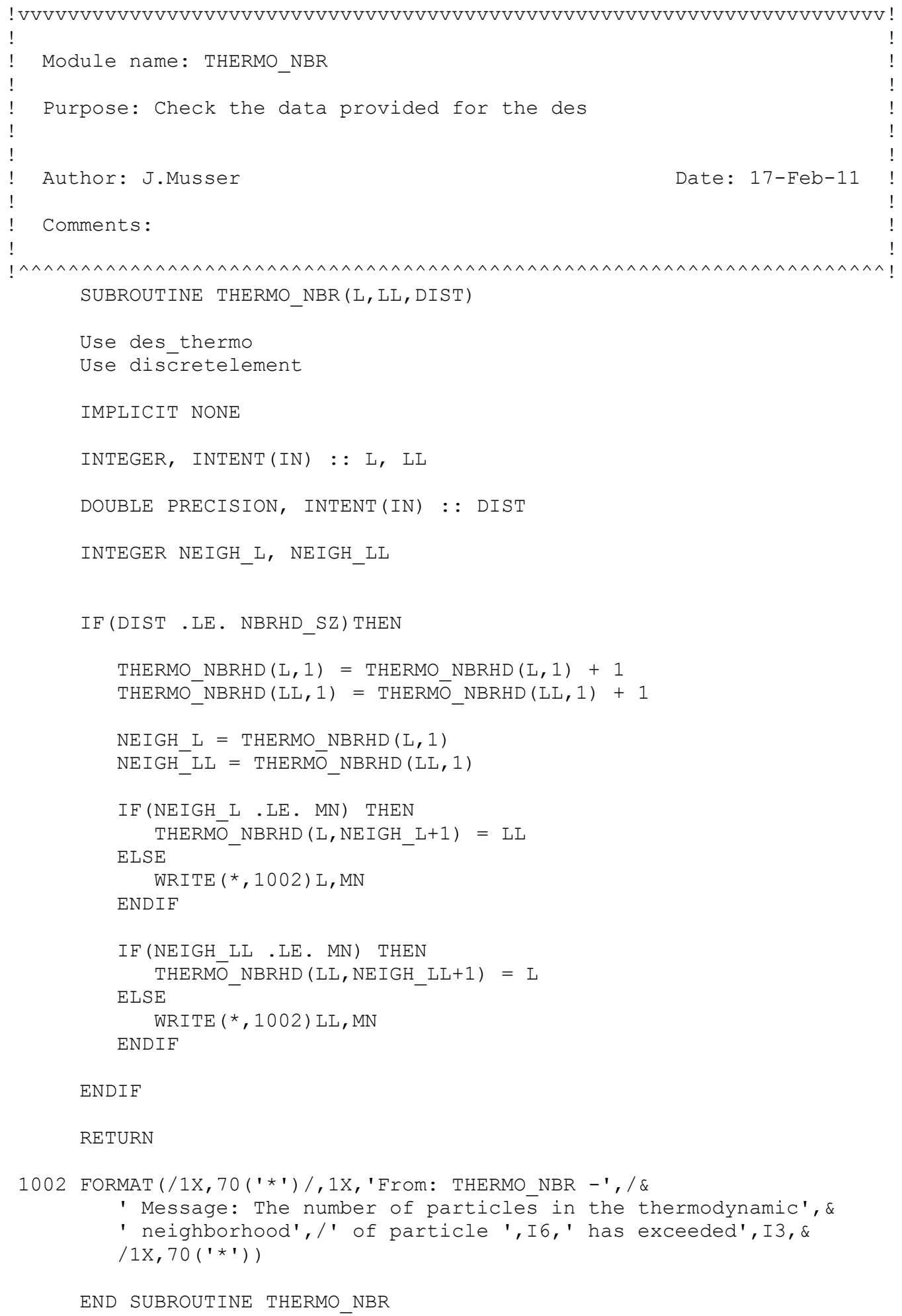




\section{D.14 Module -INTERPOLATE_CC}

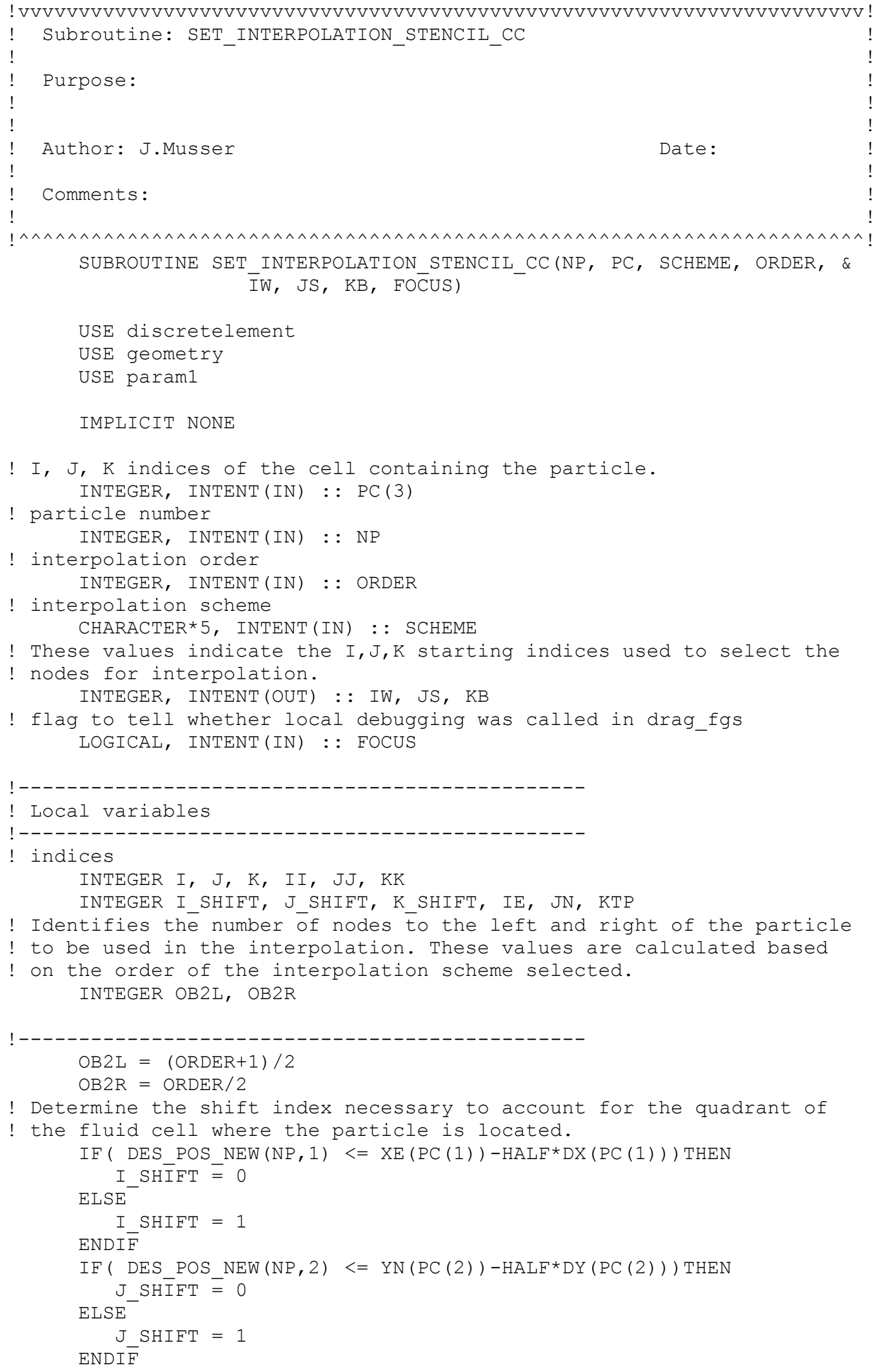




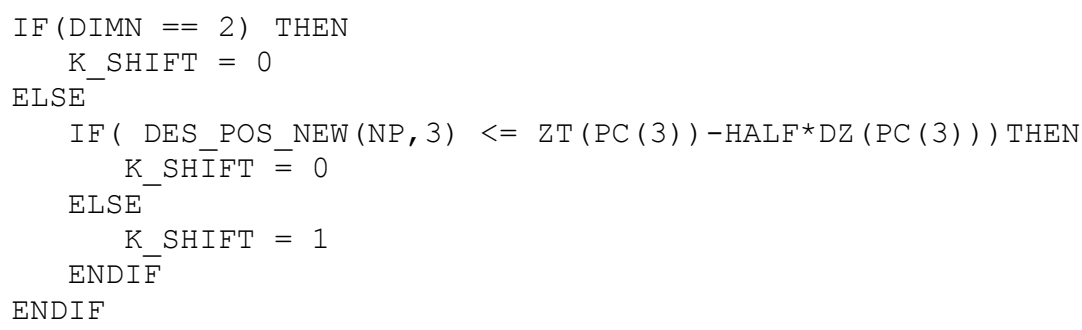

! If 2 dimension simulation, set the bottom index to 1 .

$$
\mathrm{KB}=1
$$

! Restric the bottom $\mathrm{K}$ index to a minimum of 1

$$
\mathrm{KB}=\operatorname{MAX}(1, \mathrm{PC}(3)-\mathrm{OB} 2 \mathrm{~L}+\mathrm{K} \text { SHIFT })
$$

! Restric the top $K$ index to a maximum of KMAX1 $\mathrm{KTP}=\operatorname{MIN}\left(\mathrm{KMAX} 2, \mathrm{PC}(3)+(\mathrm{OB} 2 \mathrm{R}-1)+\mathrm{K} \_\mathrm{SHIFT}\right)$ IF (.NOT.DES PERIODIC WALLS Z) THEN

! Shift indices for non periodic boundaries if needed. ELSE

! Shift indices for periodic boundaries if needed. ENDIF

IF $(\mathrm{KB} . \mathrm{EQ} \cdot 1) \mathrm{KB}=\mathrm{KTP}-$ ORDER + 1

\section{ENDIF}

RETURN

END SUBROUTINE SET INTERPOLATION_STENCIL_CC 


\section{D.15 Module -SET_INTERPOLATION_STENCIL_CC}

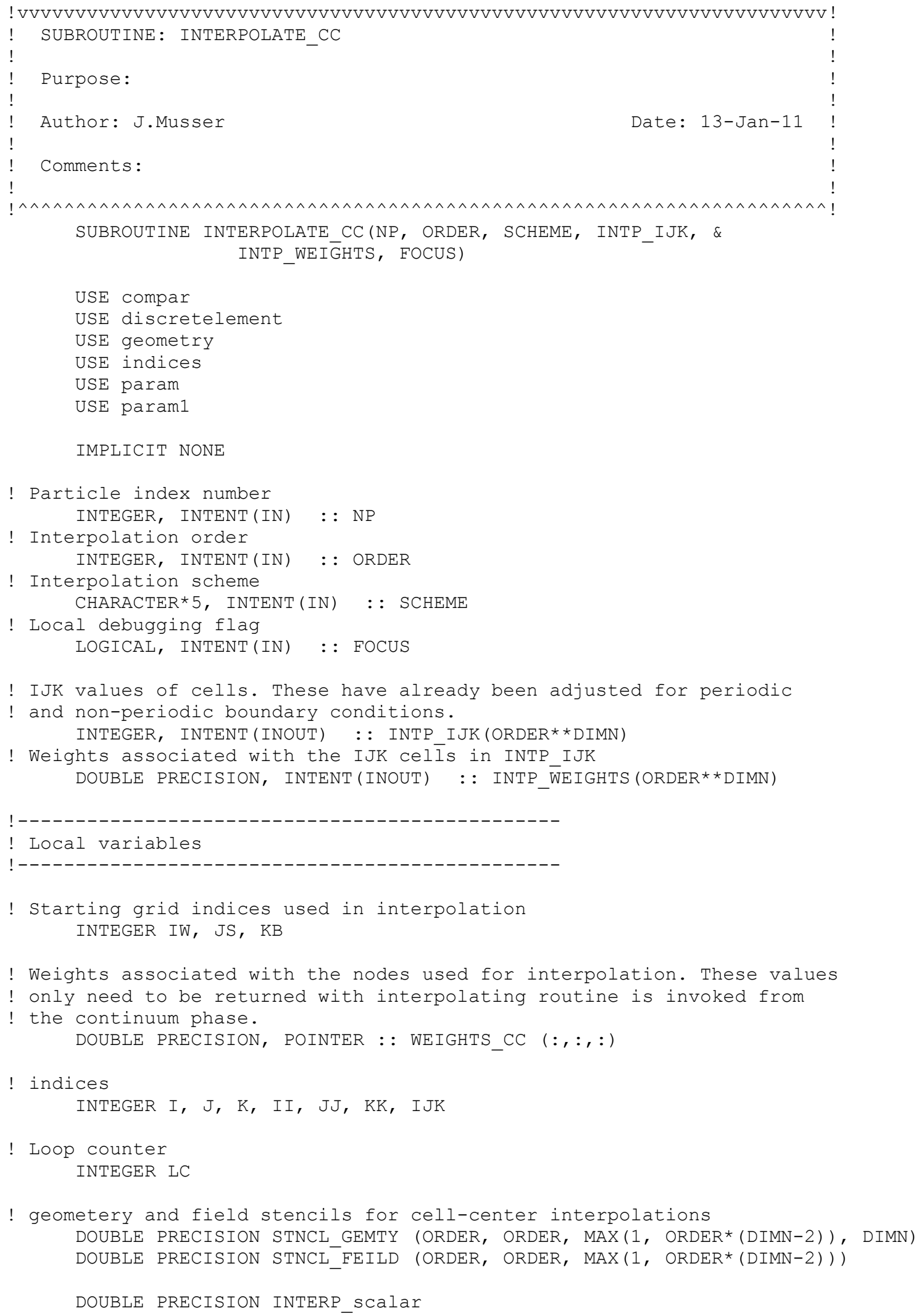




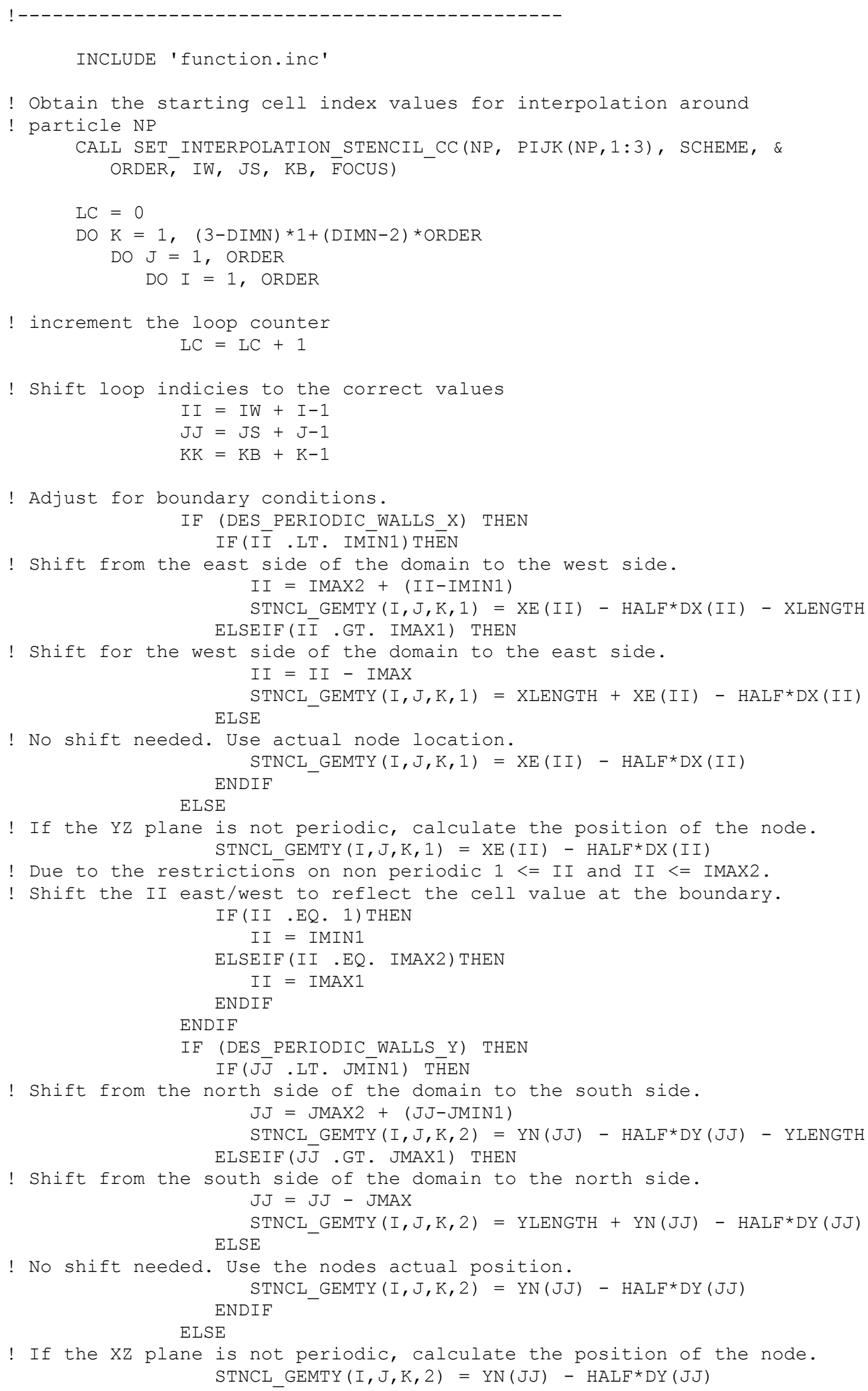

! If the Xz plane is not periodic, calculate the position of the node. STNCL_GEMTY $(I, J, K, 2)=$ YN (JJ) - HALF*DY (JJ) 
! Due to the restrictions on non periodic $1<=$ II and II <= IMAX2.

! Shift the II east/west to reflect the cell value at the boundary. IF (JJ .EQ. 1) THEN

$\mathrm{JJ}=\mathrm{JMIN} 1$

ELSEIF (JJ .EQ. JMAX2) THEN

ENDIF

$\mathrm{J} \mathrm{J}=\mathrm{JMAX} 1$

ENDIF

IF (DIMN .EQ. 3) THEN

IF (DES PERIODIC WALLS Z) THEN

IF $(\mathrm{K} \overline{\mathrm{K}} \cdot \mathrm{LT} \cdot \mathrm{KMIN} 1) \quad \mathrm{TH} E \mathrm{~N}$

! Shift from the top side of the domain to the bottom side.

$\mathrm{KK}=\mathrm{KMAX1}+(\mathrm{KK}-\mathrm{KMIN1})$

$\operatorname{STNCL} \operatorname{GEMTY}(I, J, K, 3)=\mathrm{ZT}(\mathrm{KK})-\operatorname{HALF} * \mathrm{DZ}(\mathrm{KK})-$ ZLENGTH

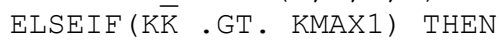

! Shift from the bottom side of the domain to the top side.

$\mathrm{KK}=\mathrm{KK}-\mathrm{KMAX}$

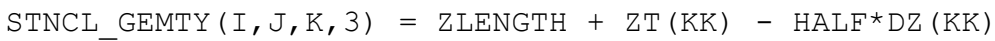

ELSE

! No shift needed. Use actual node location. ENDIF

$\operatorname{STNCL} \operatorname{GEMTY}(I, J, K, 3)=\mathrm{ZT}(\mathrm{KK})-\operatorname{HALF}{ }^{*} \mathrm{DZ}(\mathrm{KK})$

ELSE

! If the XY plane is not periodic, calculate the position of the node.

$\operatorname{STNCL} \operatorname{GEMTY}(I, J, K, 3)=\mathrm{ZT}(\mathrm{KK})-\mathrm{HALF}^{*} \mathrm{DZ}(\mathrm{KK})$

! Due to the restrictions on non periodic $1<=$ II and II <= IMAX2.

! Shift the II east/west to reflect the cell value at the boundary. IF (KK .EQ. 1) THEN

$\mathrm{KK}=\mathrm{KMIN} 1$

ELSEIF (KK .EQ. KMAX2) THEN

ENDIF

$\mathrm{KK}=\mathrm{KMAX1}$

ENDIF

ELSE

$\mathrm{KK}=1$

ENDIF

! Store the IJK in the interp array

INTP_IJK $(\mathrm{LC})=$ FUNIJK $(I I, J J, K K)$

! Assign a dummy value for the feild variable ENDDO

STNCL_FEILD $(I, J, K)=1.0 \mathrm{~d} 0$

ENDDO

! Call the interpolation routine. If the call to this routine originates

! from the continuum phase, the interpolation weights are of interest.

! Otherwise, the interpolated scalar value (INTERP_scalar) is desired. IF (DIMN.EQ.2) THEN

CALL interpolator(STNCL_GEMTY (1:ORDER, 1:ORDER, 1, 1:DIMN), \& STNCL_FEILD $(1:$ ORDER, $\overline{1}:$ ORDER, 1), \&

ELSE DES_POS_NEW (NP, 1:2), INTERP_scalar, ORDER, SCHEME, WEIGHTS_CC )

CALL interpolator( STNCL GEMTY (1:ORDER, 1:ORDER, 1:ORDER, 1:DIMN), \& STNCL_FEILD (1:ORDER, $\overline{1}$ :ORDER, 1:ORDER), \& ENDIF DES_POS_NEW(NP,:), INTERP_Scalar, ORDER, SCHEME, WEIGHTS_CC )

! Store interpolation weights in an array for calling routine $\mathrm{LC}=0$

$\mathrm{DO} K=1,(3-\mathrm{DIMN}) * 1+(\mathrm{DIMN}-2) * \mathrm{ORDER}$

DO $\mathrm{J}=1$, ORDER

DO $I=1$, ORDER

! increment the loop counter 


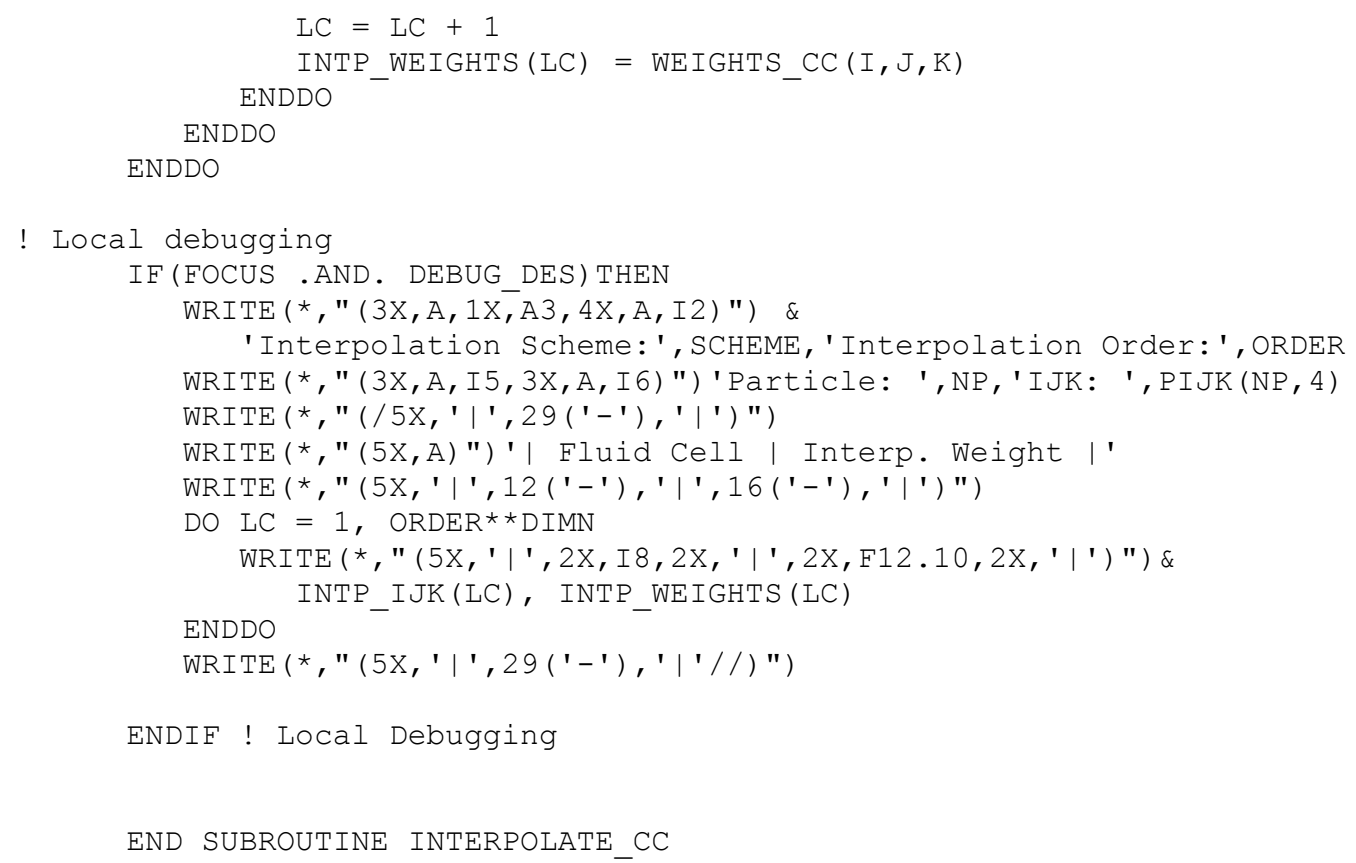




\section{Appendix E: Interface for managing reactive chemistry source code}

The section contains the source code for the interface for managing user-defined chemical reactions. It has been written in the FORTRAN90/95 style.

\section{E.1 Module - DES_RXNS}

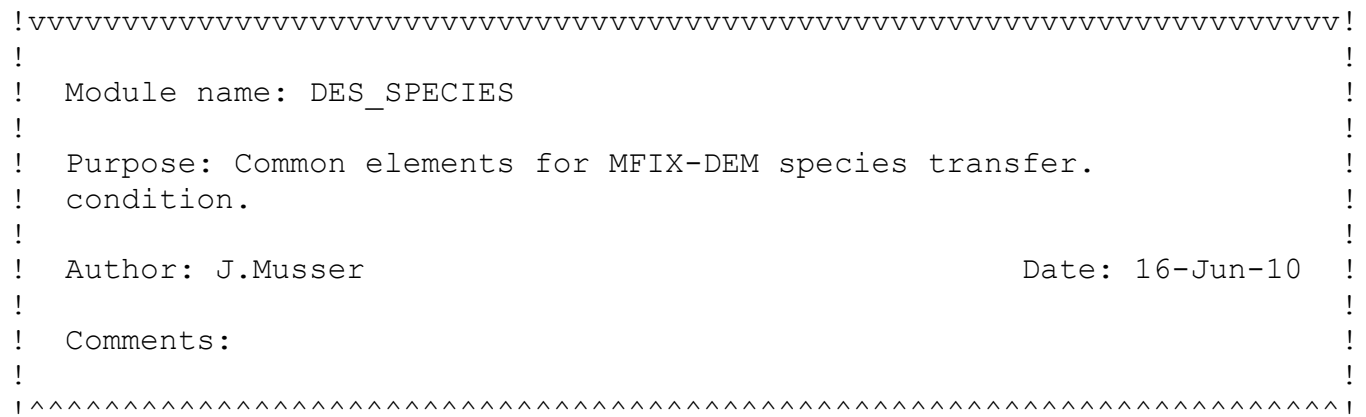

MODULE DES_RXNS

USE param

LOGICAL FIRST_CALL

! DES - Species Equation

LOGICAL DES_SPECIES_EQ(DIM_M)

LOGICAL ANY_DES_SPEC IES_EQ

! total number of discrete solids species for each phase INTEGER DES NMAX (DIM M)

! maximum number of species over all solids phases INTEGER MAX_DES_NMAX

! species names CHARACTER $(l e n=18)$ DES_SPECIES_NAME (DIM_N_ALL)

! molecular weight of discrete solids species DOUBLE PRECISION DES_MW_S (DIM_M, DIM_N_S)

! discrete solids species mass fractions (PARTICLES, 0:MAX DES NMAX)) DOUBLE PRECISION, DIMENSION(:, :), ALLOCATABLE : : DE $S$ X ${ }^{-} S$

! rate of production of solids species DOUBLE PRECISION, DIMENSION(:, :), ALLOCATABLE : : DES_R_sp

! rate of consumption of solids species DOUBLE PRECISION, DIMENSION(:, :), ALLOCATABLE : : DES_R_SC

! combined rate of production and consumption of solids species DOUBLE PRECISION, DIMENSION(:, :), ALLOCATABLE : : DES_SUM_R_S

! thermochemical data for discrese solids phases: DOUBLE PRECISION DES Thigh S(DIM M, DIM N S) DOUBLE PRECISION DES TIOW $\bar{S}($ DIM $\bar{M}$, DIM $\bar{N} \bar{S})$ DOUBLE PRECISION DES ${ }^{-} \mathrm{TCOM}^{-} \mathrm{S}$ (DIM M, DIM ${ }^{-} \mathrm{N}^{-} \mathrm{S}$ ) DOUBLE PRECISION DES Ahigh $\overline{-}$ (7, DIM_M, DIM_N_s) DOUBLE PRECISION DES_AlOW_S $(7$, DIM_M, DIM N $\bar{S}$ S) DOUBLE PRECISION DES HfrefoR S (DIM ${ }^{-} M$, DIM $^{-}{ }^{-}-\mathrm{S}$ ) DOUBLE PRECISION DES_IC_PSref $\bar{f} O R\left(D I \bar{M} \_M, D I \bar{M} \_\bar{N} \_S\right)$

! This indicates how a particle will respond to a chemical reaction. 


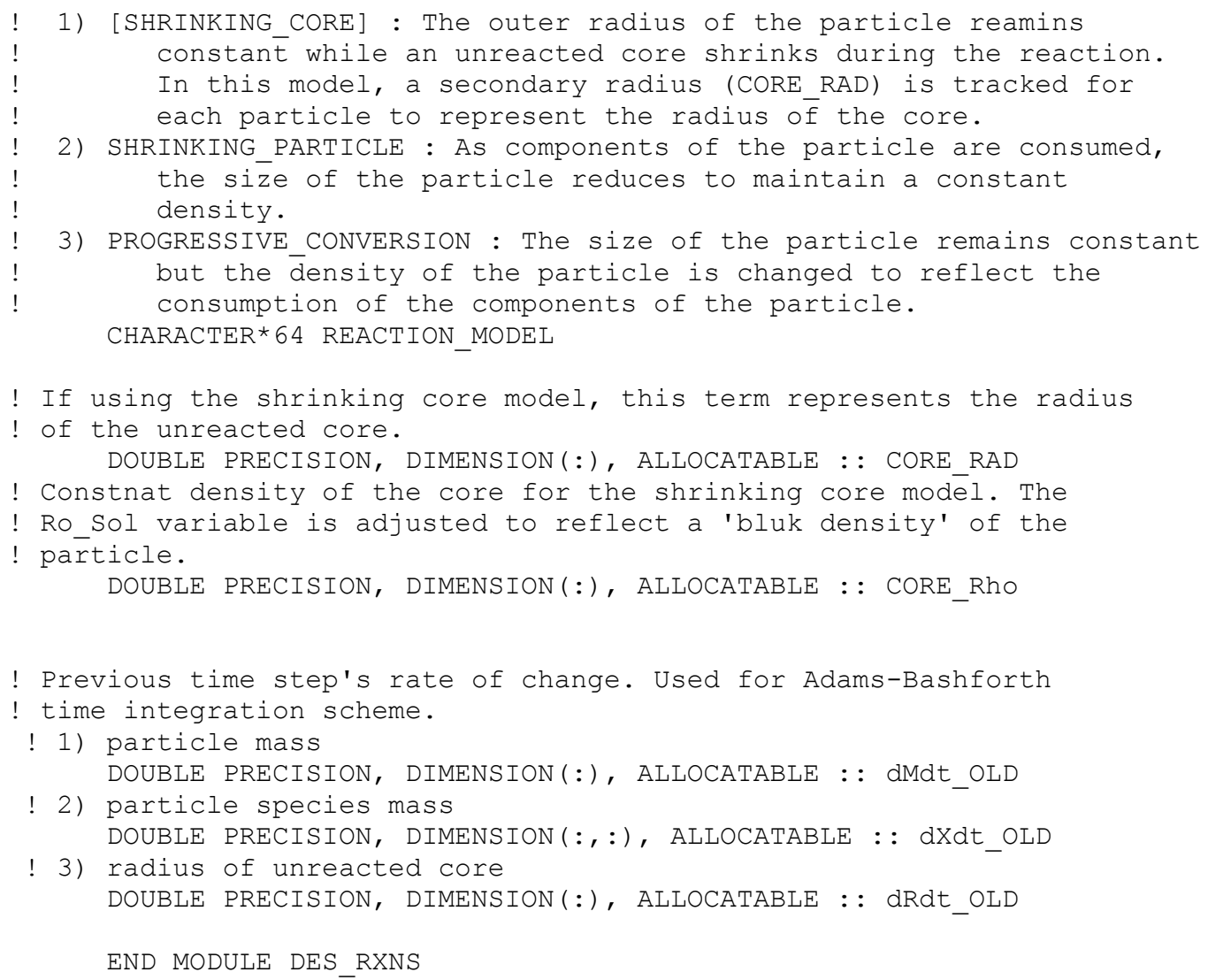




\section{E.2 Routine - CHECK_DES_RXNS}

! VVVVVVVVVVVVVVVVVVVVVVVVVVVVVVVVVVVVVVVVVVVVVVVVVVVVVVVVVVVVVVVVVVVVVV!

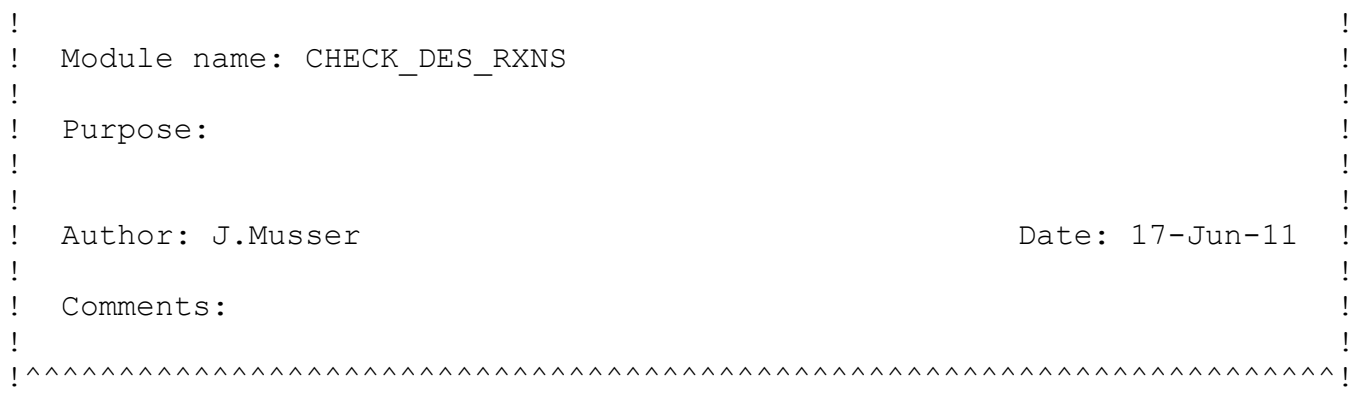

SUBROUTINE CHECK_DES_RXNS

Use compar

USE des rxns

Use discretelement

Use funits

Use run

IMPLICIT NONE

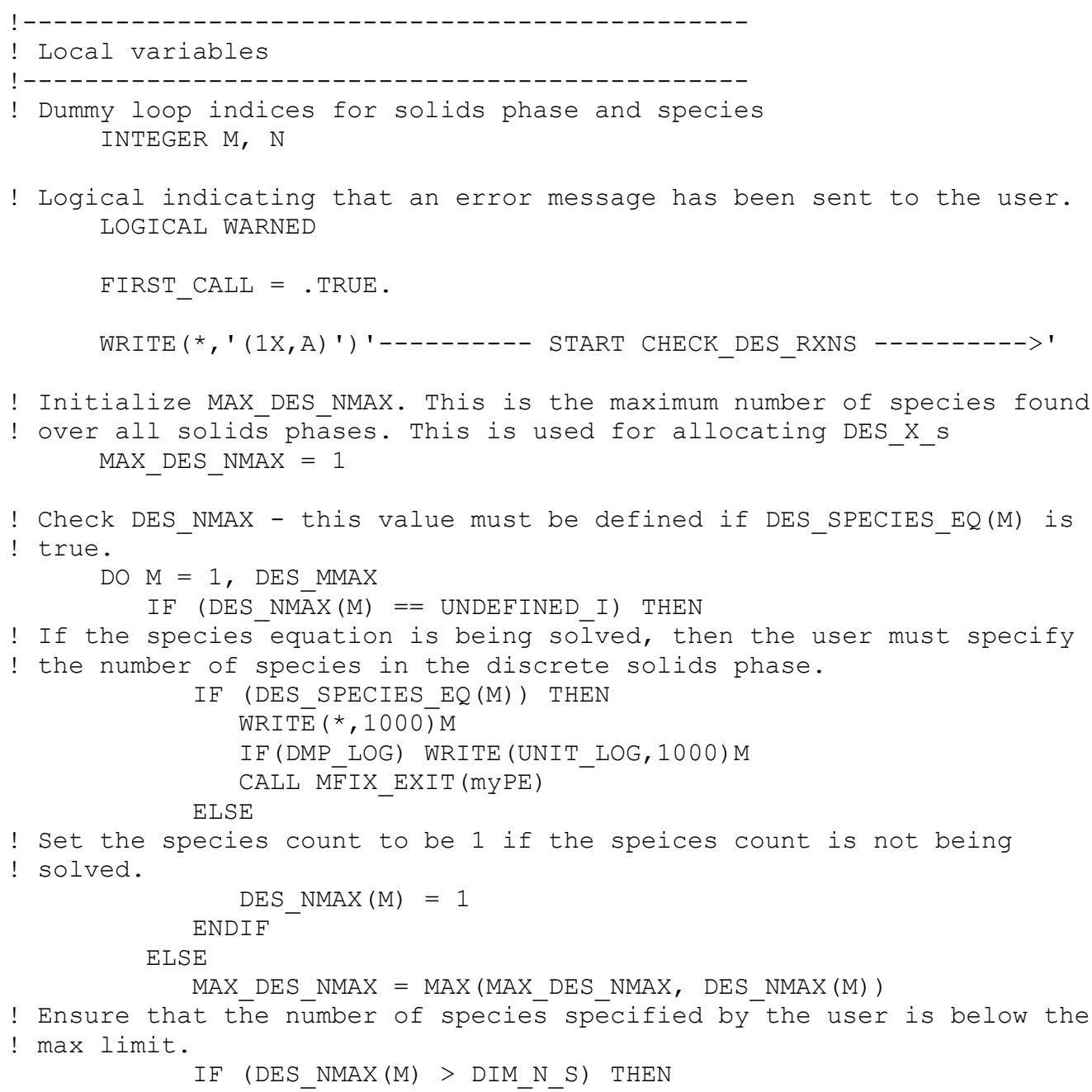




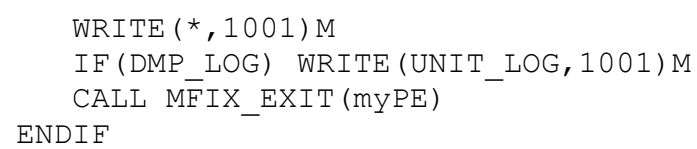




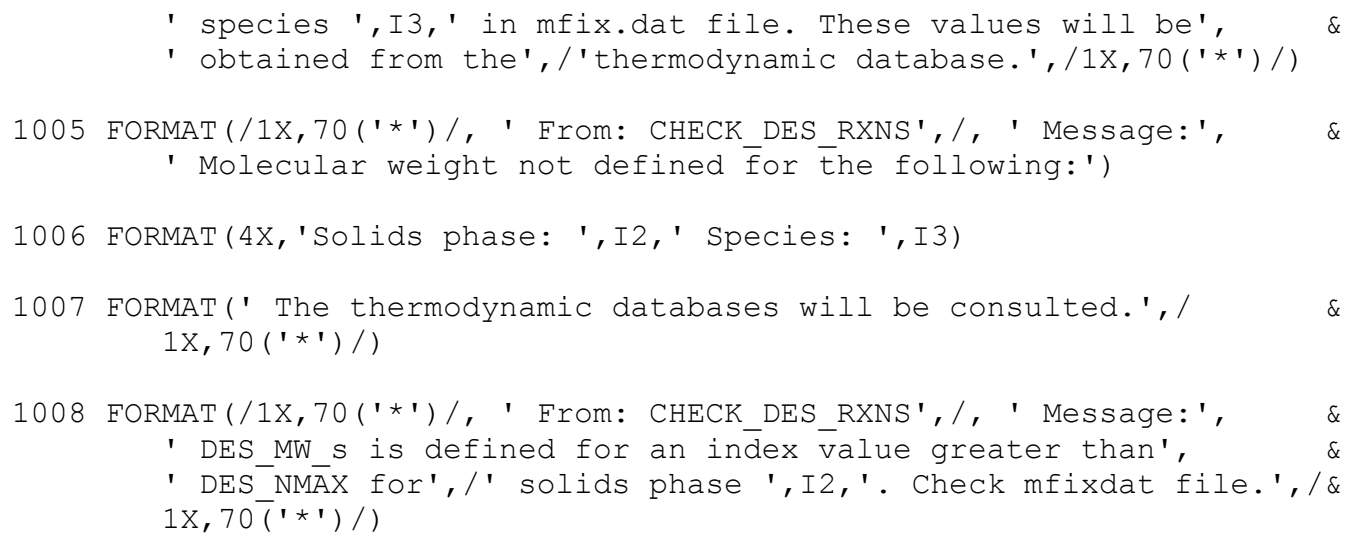




\section{E.3 Function - DES_CALC_H}

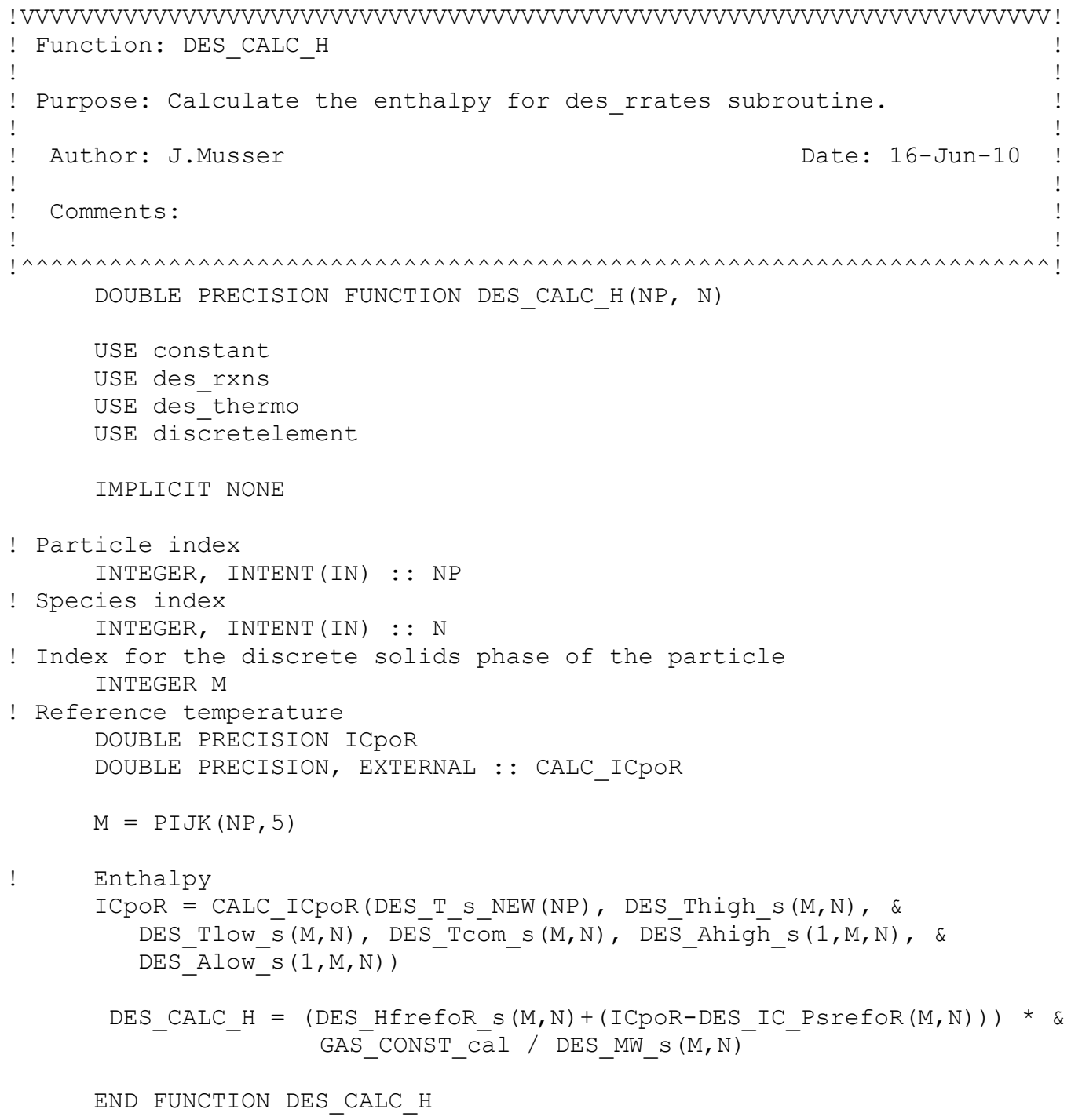




\section{E.4 Routine - DES_PHYSICAL_PROP}

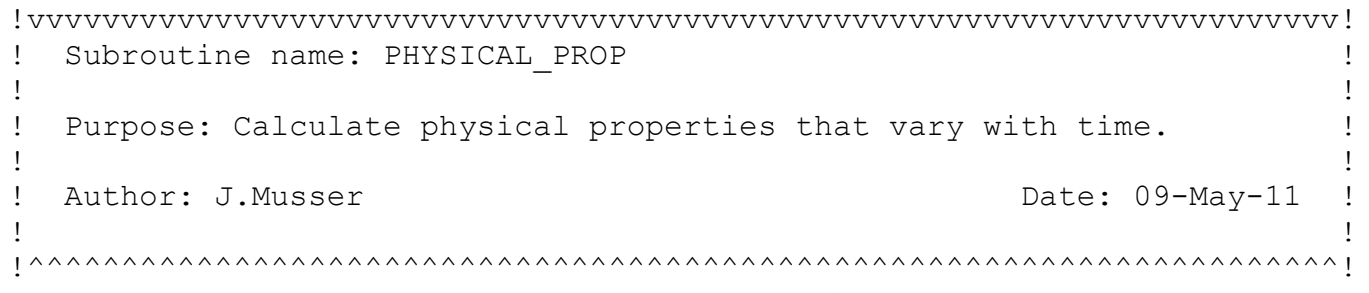

SUBROUTINE DES_PHYSICAL_PROP (NP, FOCUS)

Use des rxns

Use des_thermo

Use discretelement

Use funits

Use param

Use param1

Use physprop

Use run

IMPLICIT NONE

! Passed Variables

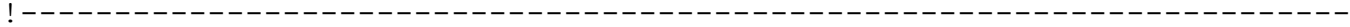

! Index of particle INTEGER, INTENT(IN) : : NP

! Logical indicating to write additional data about the particle for

! debugging purposes. LOGICAL, INTENT(IN) : : FOCUS

! Local Variables

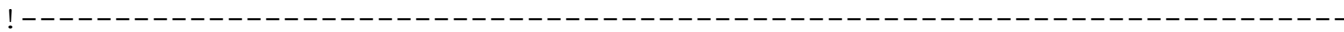

! Gas constant DOUBLE PRECISION, PARAMETER : : RGAS $=1.987207 \mathrm{DO}$ ! $\mathrm{cal} / \mathrm{mol} . \mathrm{K}$

! Dummy indices INTEGER M, N

! error indicator INTEGER IER

! External fuctions

! Calculate the specific heat from polynomical data obtained from the

! thermodynamic databases. DOUBLE PRECISION, EXTERNAL : : calc_CPOR

! Get the solids phase of the particle $\mathrm{M}=\mathrm{PIJK}(\mathrm{NP}, 5)$

! Specific heat

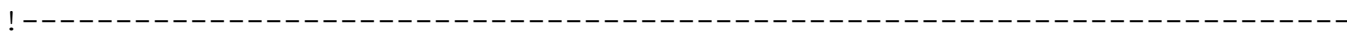

! This only needs calculated when solving the energy equations. IF (DES ENERGY EQ) THEN

! If a constānt valūe specific heat has not been defined in the mfix.dat

! file, calculate the temperature dependent value based on data from the

! thermodynamic databases. IF (DES_C PSO (M) == UNDEFINED) THEN

! Read the thermodynamic database if it has not already been done. IF (.NOT.DATABASE_READ) CALL READ_DATABASE(IER) DES_C_PS $(\mathrm{NP})=\mathrm{ZE} R \mathrm{E}$

! Calculate the specific heat based on the species composition of the

! particle and the data from the thermodynamic databases. 
$\&$

$\mathrm{DO} N=1, \operatorname{DES} \operatorname{NMAX}(\mathrm{M})$

$\mathrm{DES} C \mathrm{PS}(\mathrm{NP})=\mathrm{DES} \mathrm{C} P \mathrm{PS}(\mathrm{NP})+\mathrm{DES} \mathrm{XS}(\mathrm{NP}, \mathrm{N})$ * calc_CpoR(DES_T_S_NEW(NP), DES_Thigh_s $(M, N)$,

DES_TlOW_S $(M, N)$, DES_TCOM_S $(M, N)$,

DES_Ahigh_s $(1, M, N)$, DES_Alow_s $(1, M, N))$ *

ENDDO

$$
\text { RGA } \bar{S} / \mathrm{DES} \overline{\mathrm{M}} \mathrm{S} \mathrm{S}(\mathrm{M}, \mathrm{N})
$$

! Convert to SI units if needed. ELSE

IF (UNITS $==$ 'SI') DES_C_PS (NP) $=4183.925 \mathrm{D} 0 *$ DES_C_PS (NP)

! If a constant value specific heat has been assigned to the particle

! in the mfix.dat file, use this value.

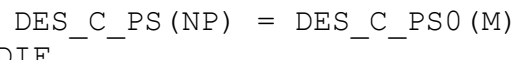

! $\quad \operatorname{WRITE}(*, "(3 \mathrm{X}, \mathrm{A}, \mathrm{I} 2, \mathrm{~A}, \mathrm{~F} 10.6) ")$ 'DES_C_PS ( ', NP, ' ) : ' , DES_C_PS (NP)

RETURN

END SUBROUTINE DES_PHYSICAL_PROP 


\section{E.5 Routine - DES_RRATES}

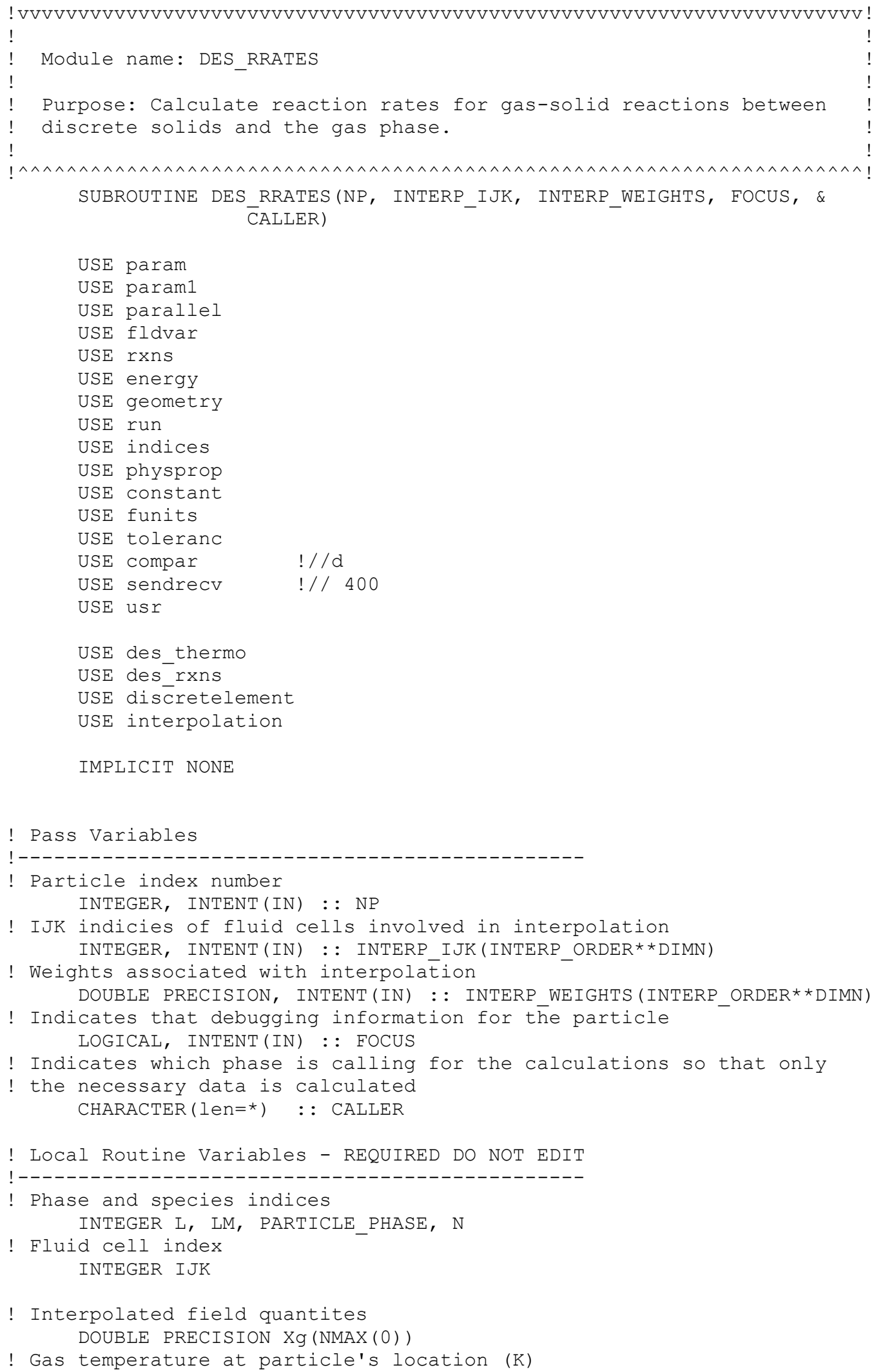




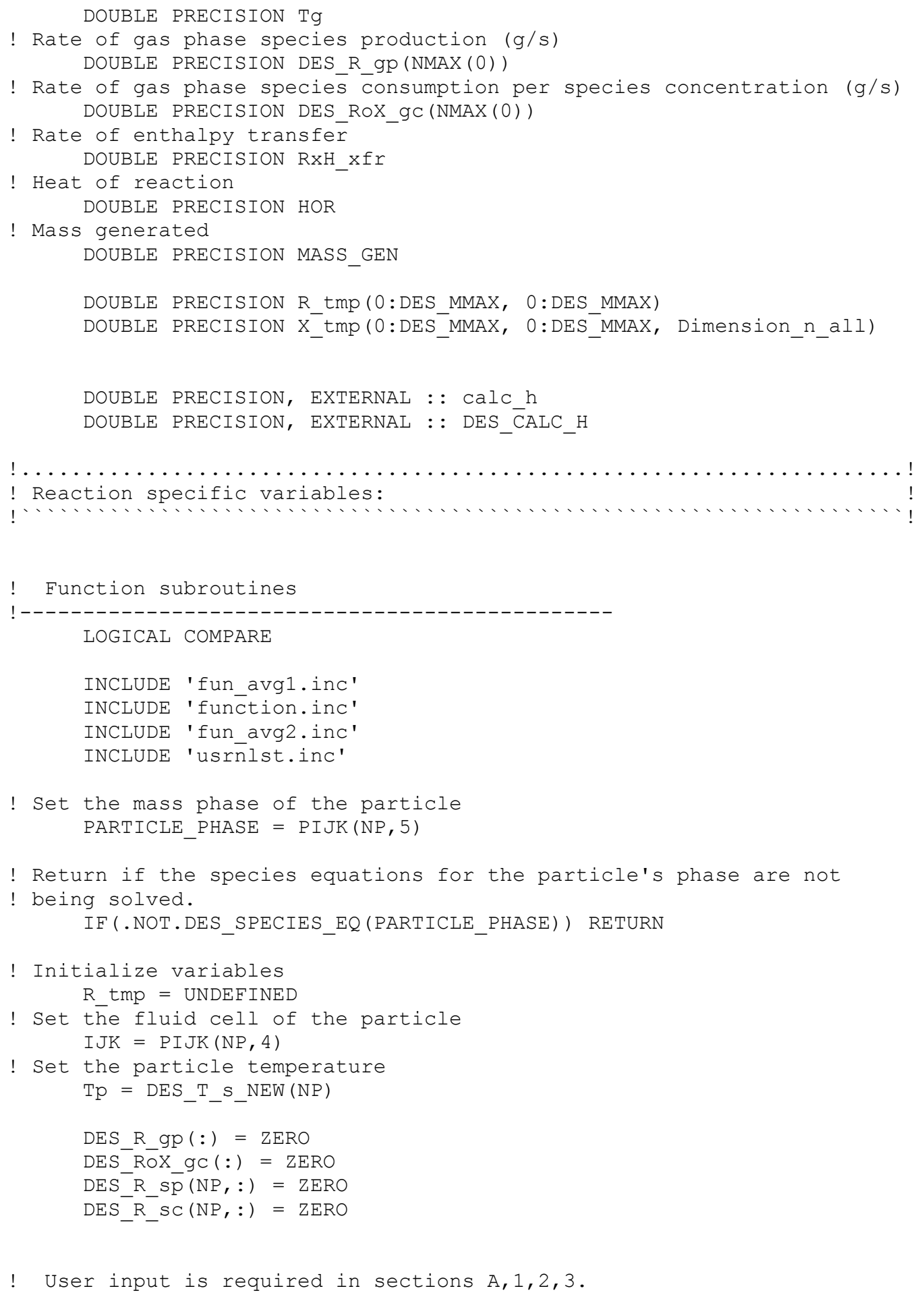

! User input is required in sections $A, 1,2,3$.

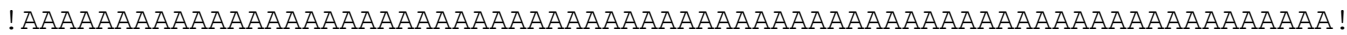

! A. Set gas phase quantities.

The gas phase species information is set to the array Xg(:) and the gas phase temperature is set to the variable Tg.

Additional quantites may also be set by using the function 


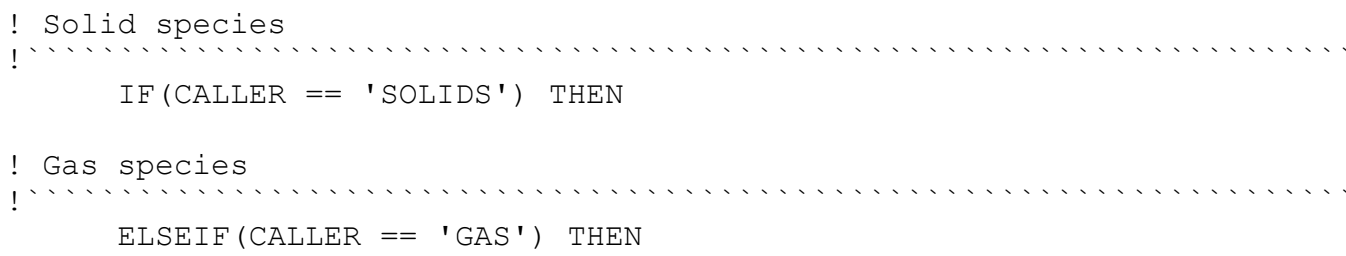




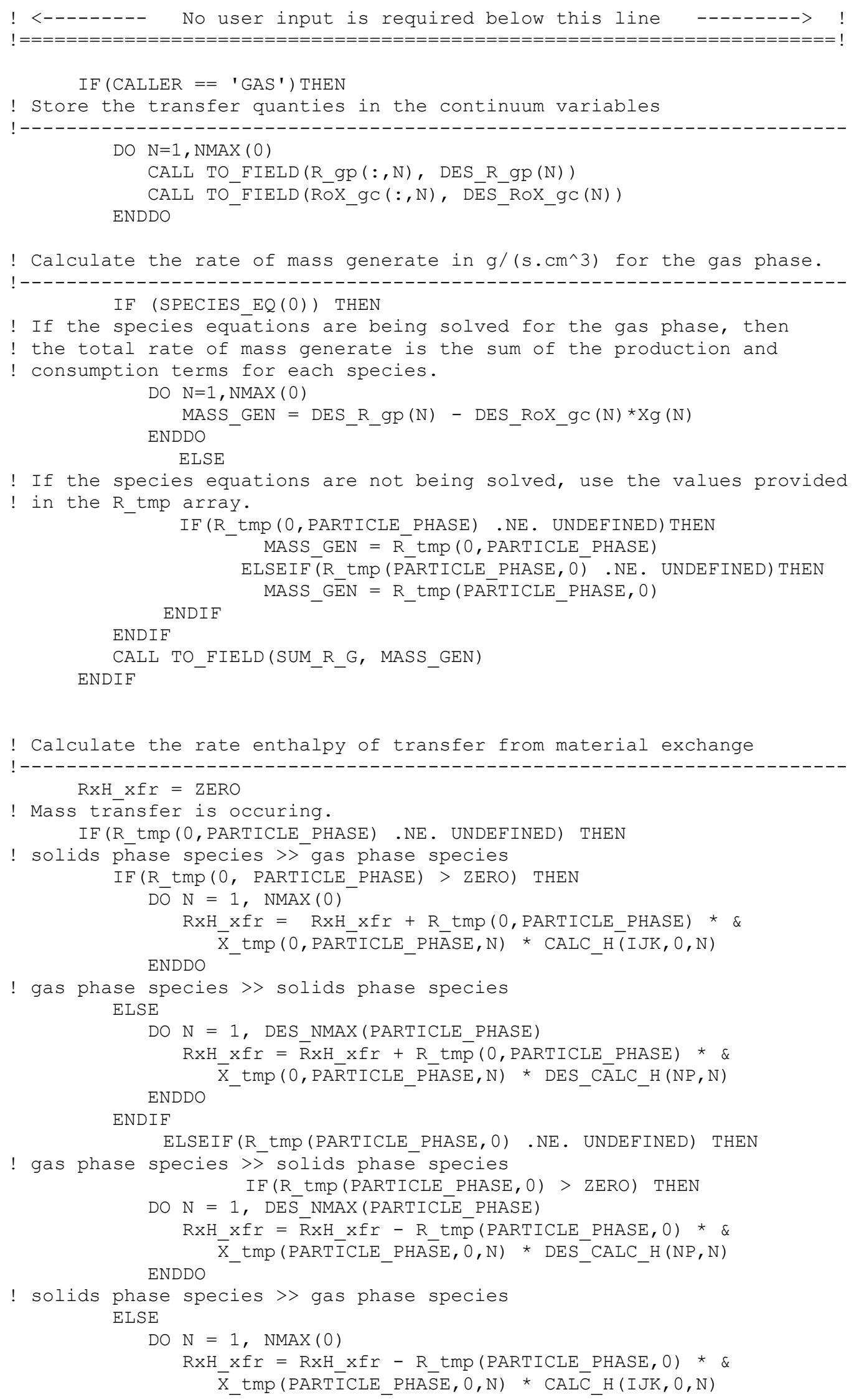




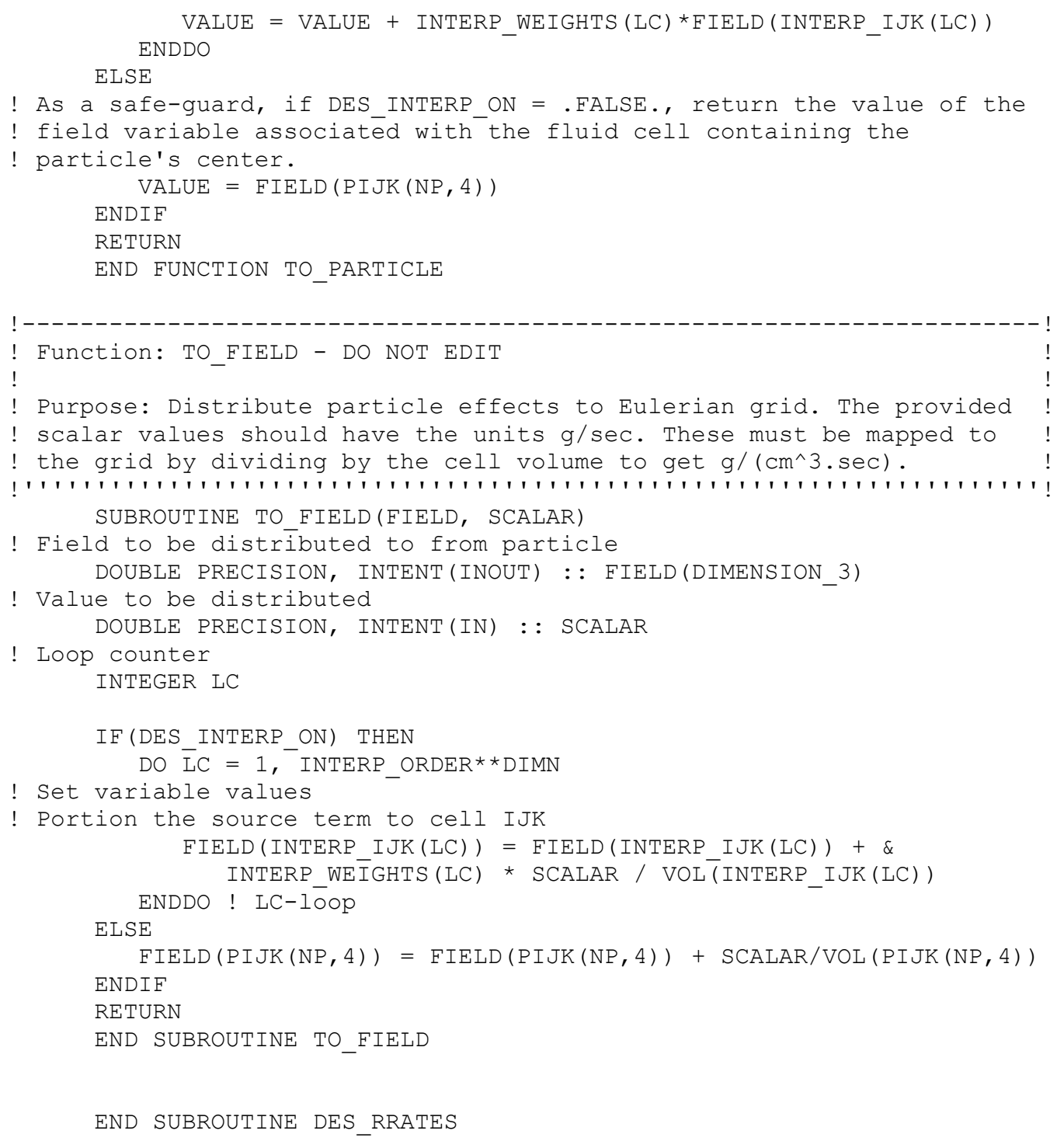




\section{E.6 Routine - DES_REACTION_MODEL}

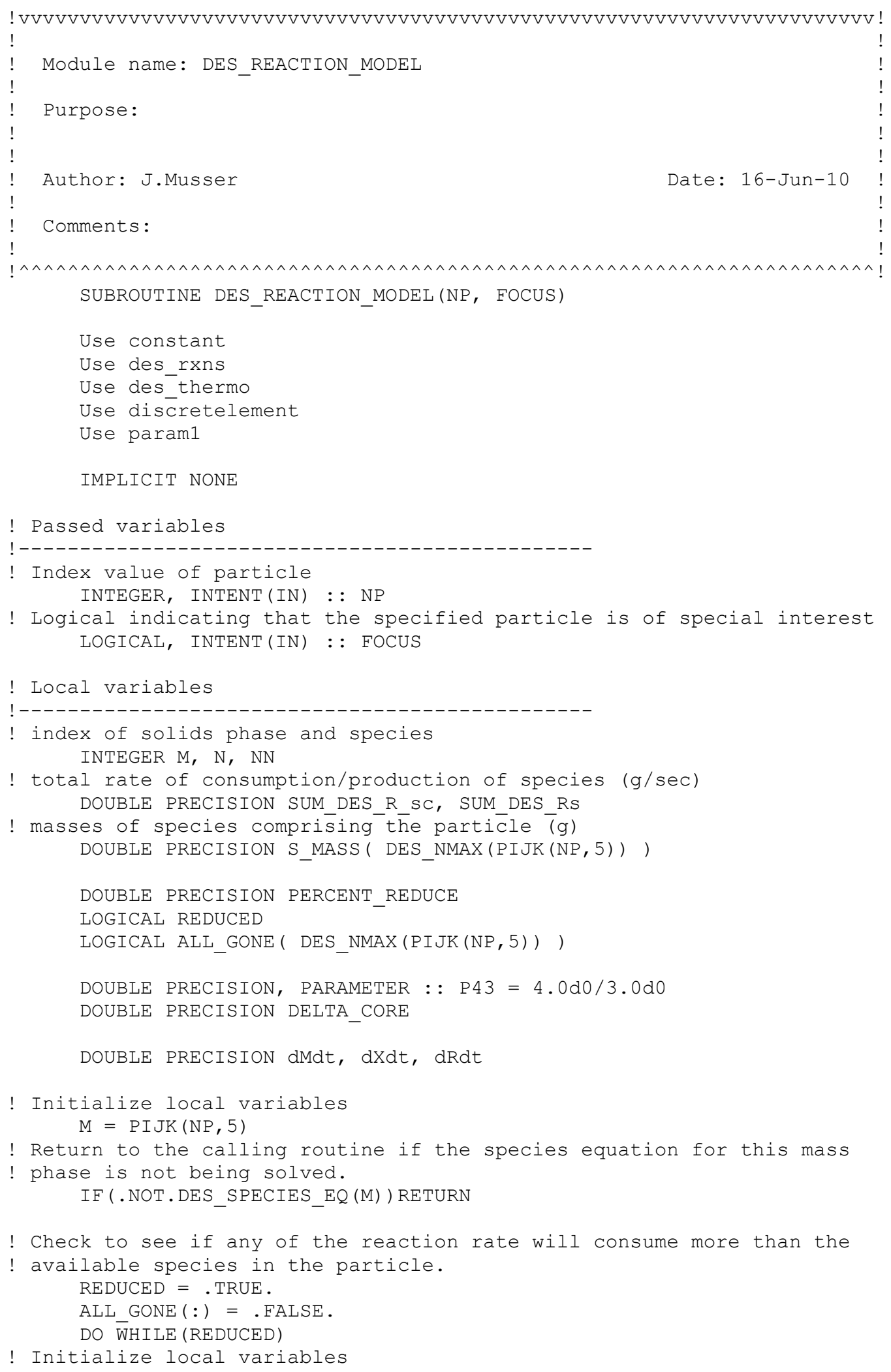




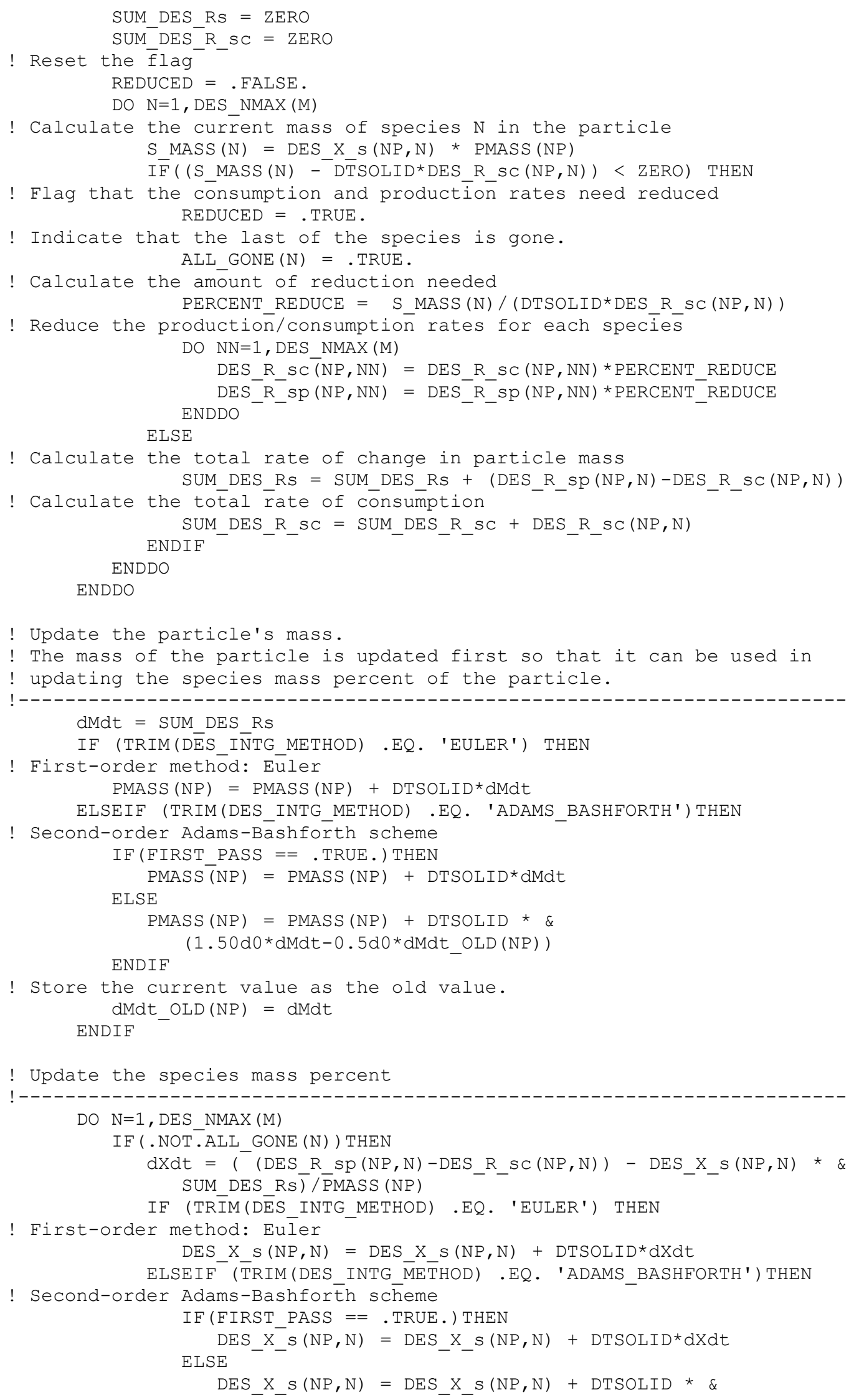




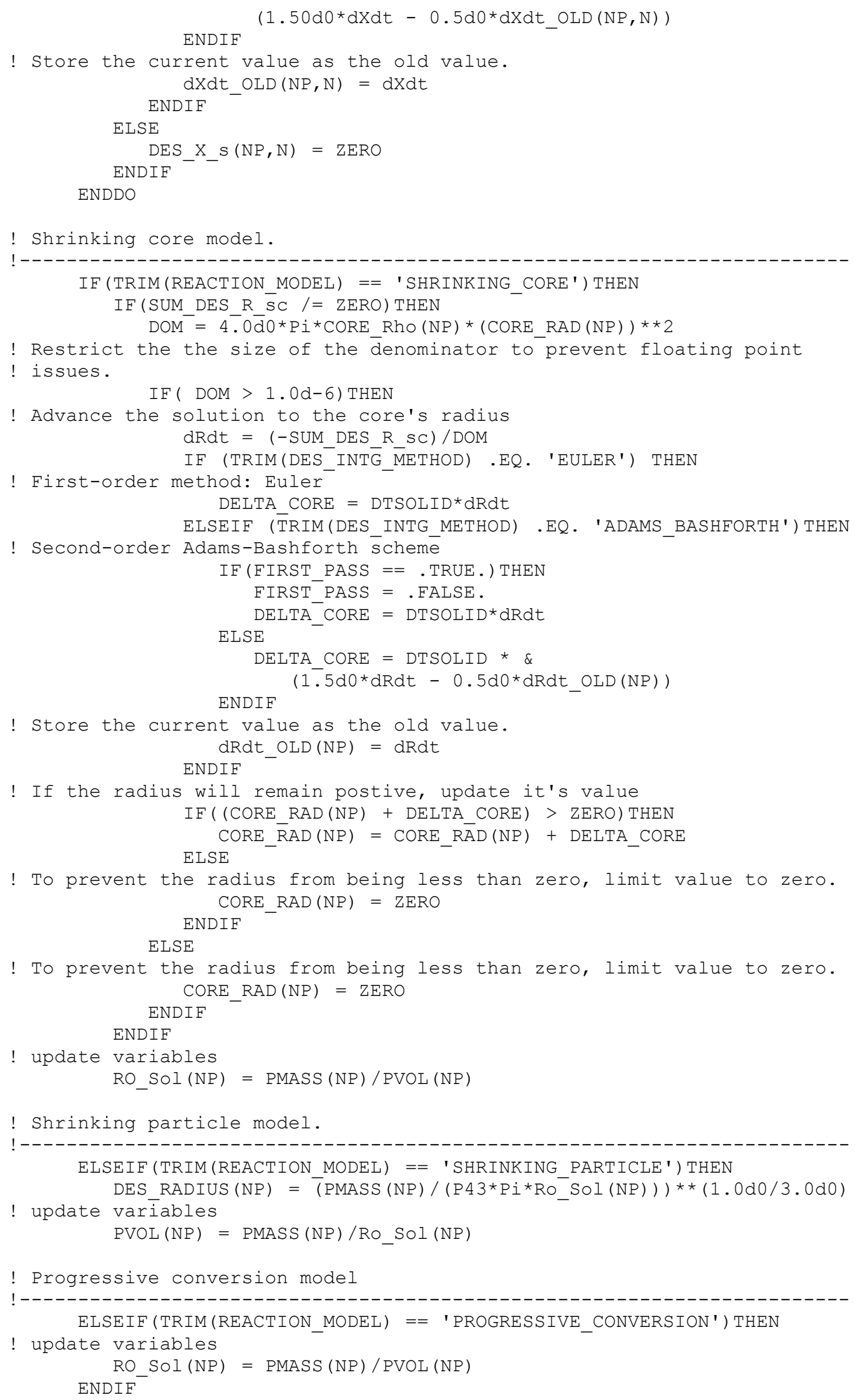




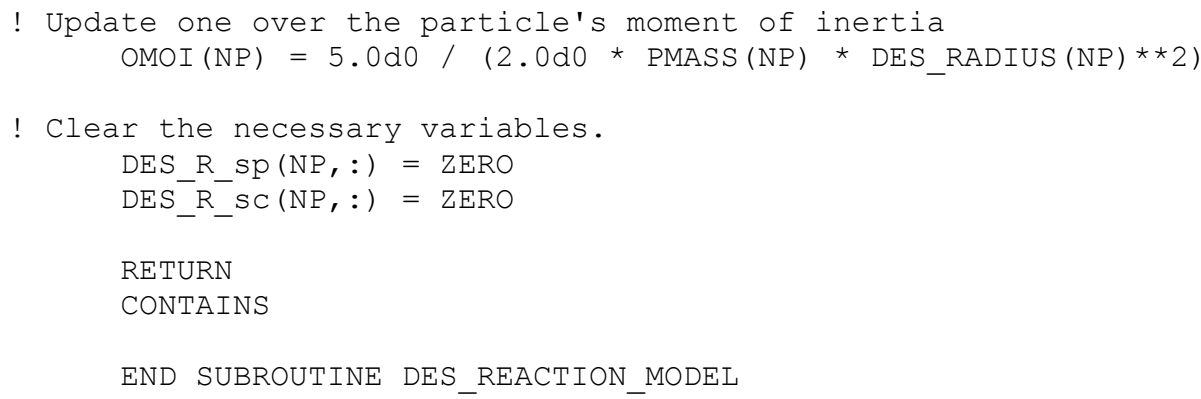

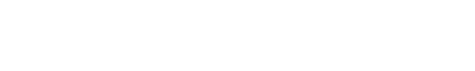

